\title{
Zirconium Oxidation on the Atomic Scale
}

\author{
Daniel Hudson \\ St Catherine's College
}

A thesis submitted for the degree of Doctor of Philosophy

Department of Materials, University of Oxford.

Michaelmas Term 2010

OxfordMaterials 


\section{Abstract}

This work was produced as part of a multidisciplinary study of the corrosion of zirconium alloys undertaken by a consortium of universities working in the MUZIC program; Oxford, Manchester and The Open University. The objective of the project as a whole was to further the understanding of the mechanisms of the breakaway oxidation process and to characterise these corrosion processes within a number of fuel rod cladding materials. This thesis describes laser 3D atom probe characterisation of the nano-scale chemical redistribution of oxygen and other solutes that occurs at the metal-oxide interface during corrosion, and a large body of technique development that was required to achieve this goal.

The development of the metal-oxide interface of ZIRLO, a Zr-Nb-Sn-Fe-O alloy, is followed by generating $3 \mathrm{D}$ atomic scale reconstructions at four different stages of corrosion. The formation of a sub-oxide $\mathrm{ZrO}$ layer is seen during pre-transition oxide development. The $\mathrm{ZrO}$ interfacial layer is consumed by the rapid formation of oxide after the breakaway transition. After transition the chemistry of the interface is similar to the early pre-transition case, although an oxygen-saturated layer of metal adjacent to the interface formed during corrosion remains. The $\mathrm{ZrO}$ interfacial layer $\left(\mathrm{Zr}-\mathrm{ZrO}-\mathrm{ZrO}_{2}\right)$ and the region of oxygen-saturated material ahead of the metal-oxide interface alter the distribution of minor alloying additions such as niobium and iron. The $\mathrm{ZrO}$ layer increases the acceptance of niobium into the oxide, which is otherwise seen to be rejected at the $\mathrm{Zr}-\mathrm{ZrO}_{2}$ interface along with iron. Niobium is seen to precipitate out of solution as nano-scale particles near the interface after around 100 days of corrosion. This is not seen in the bulk metal matrix of the corroded material due to the absence of other factors driving the process: the stress at the interface and a very high oxygen concentration in the metal ahead of the interface. The nano-scale niobium particles are found to be of a meta-stable composition. Iron is seen to redistribute in the corroded material and can be correlated with the local oxygen concentration. Similarities are seen in the behaviour of solutes within pre-transition ZIRLO and Zircaloy-4 (Zr-Sn-Fe-O). In both cases no redistribution of tin is seen at the metal-oxide interface. A $\mathrm{Zr}-\mathrm{Nb}-\mathrm{Ti}$ alloy with very poor corrosion resistance was also analysed in this way, and the similarities and differences with chemically-similar ZIRLO are discussed. The segregation of solutes to grain boundaries and solute clustering within the matrix are also examined before and after corrosion. 


\section{Acknowledgements}

I would like to express my gratitude to my supervisor, Prof. George D. W. Smith, for the continual guidance, support and the insatiable enthusiasm he provided throughout this project. I would also like to express my thanks to Dr. David Saxey, Prof. Alfred Cerezo, Dr. Baptiste Gault, and Mr. Terry Godfrey for their patient training and help developing the skills required to operate the atom probe, and interpret results. I would also like to thank my colleagues from the MUZIC consortium for their help and assistance, especially Prof. Chris Grovenor, Dr. Michael Preuss, Dr. Robert Comstock, Na Ni and Jianfei Wei. My time at Oxford was much enriched by the support of the FIM group as a whole, especially my office-mates, Ceri Williams and Tong Li, whose good humour helped keep the research going. I am also very grateful for the generous financial support provided by the EPSRC, UK MoD (DSTL) and St. Catherine's College, as well as the materials and facilities contributed to the project by EDF Energy, Westinghouse Nuclear Company, the NNL, Serco and Rolls Royce. Finally, I thank my family and my fiancée Katie for their constant support and encouragement throughout my research and the sacrifices they have made to make this possible.

\section{Other authors' contributions to this thesis}

Jianfei Wei, University of Manchester. Optical microscopy in Figure 24 (3.4.1.2), Fig. 25 (3.4.1.3). Corrosion testing in Fig. 27, Table 9 (3.4.2.2) and Table 10 (3.4.2.3)

$\mathrm{Na} \mathrm{Ni}$, University of Oxford. Transmission electron microscopy in Figure 26 (3.4.1.4), Table 40 and Fig. 78 (5.2.1.2), Fig. 146 (a) (6.4.1.1), Table 58 and Fig. 148 (6.4.1.3). EDX in Fig. 147.

David Saxey, formerly of the University of Oxford (now University of Western Australia). Scanning electron microscopy in Fig. 32 (3.5.3)

Baptiste Gault, formerly of the University of Oxford (now University of Sydney, Australia). Generation of simulated mass spectra in Appendix A.

The work of these authors is also acknowledged alongside these figures and in the text. 


\section{Preface}

The research described in this thesis was carried out by the author in the Department of Materials at the University of Oxford between October 2007 and October 2010 under the supervision of Prof G. D. W. Smith. All of the work is original, and where the work of others is included it has been referenced and acknowledged. No part of this thesis has been submitted for any other degree at this university or elsewhere.

Parts of this work have been published in peer-reviewed papers and/or presented at international conferences. Permission to reproduce this material, where necessary, has been granted. These papers are:

\section{0}

M. Preuss, P. Frankel, S. Lozano-Perez, D. Hudson, E. Polatidis, N. Ni, J. Wei, C. English, S. Storer, K.B. Chong, M. Fitzpatrick, F. Wang, J. Smith, C. Grosvenor, G.D.W. Smith, J. Sykes, A. Cerezo, B. Cottis, S. Lyon, L. Hallstadius, B. Comstock, A. Ambard and M. BlatYrieix, Towards a mechanistic understanding of corrosion mechanisms in zirconium alloys 16th International Symposium on Zirconium in the Nuclear Industry, ASTM, Chengdu, China, 2010. Journal of ASTM International: In Press.

Daniel Hudson, George D. W. Smith and Baptiste Gault, Optimisation of mass ranging for atom probe microanalysis and application to the corrosion processes in $\mathrm{Zr}$-alloys, IFES 2010, Proceedings of the 52th International Field Emission Symposium. Ultramicrscopy: In Press http://dx.doi.org/10.1016/j.ultramic.2010.11.007

Emmanuelle A. Marquis, Jonathan M. Hyde, David W. Saxey, Sergio Lozano-Perez, Vanessa de Castro, Daniel Hudson, Ceri A. Williams, Samuel Humphry-Baker and George D.W. Smith, Nuclear reactor materials at the atomic scale, Materials Today 1211 (2009) 30-37. 
Daniel Hudson, George D. W. Smith. Initial observation of grain boundary solute segregation in a zirconium alloy (ZIRLO) by three-dimensional atom probe. Scripta Materialia $\mathbf{6 1} 4$ (2009) 411-414.

Daniel Hudson, Na Ni, Sergio Lozano-Perez, David Saxey, Colin English, George D. W. Smith, John Sykes, Chris Grovenor. The Atomic Scale Structure and Chemistry of the Zircaloy-4 Metal-Oxide Interface. 14th International Conference on Environmental Degradation of Materials in Nuclear Power Systems, Virginia Beach, USA.

Daniel Hudson, Alfred Cerezo, George D. W. Smith. Zirconium oxidation on the atomic scale, IFES 2008, Proceedings of the 51th International Field Emission Symposium. Ultramicroscopy 1095 (2009) 667-671.

Oral and/or poster presentations have also been given at the following meetings:

$$
2010
$$

"Optimisation of mass ranging for atom probe compositional determination: Zr-alloy case study" 2010 International Field Emission Symposium, University of Sydney, Australia, $5^{\text {th }}$ $8^{\text {th }}$ July 2010 .

"Characterising the corrosion of $\mathrm{Zr}$ fuel rod cladding via pulsed-laser atom probe tomography" 2010 International Field Emission Symposium, University of Sydney, Australia, $5^{\text {th }}-8^{\text {th }}$ July 2010 .

"Nano-Scale Observations of Zirconium Alloy Corrosion using 3D Atom Probe" 16th International Symposium on Zirconium in the Nuclear Industry, Chengdu, China, 9-13 May 2010. 
"Materials science and the 3D atom probe" Winner of the poster for the furthering of public understanding of science, $3^{\text {rd }}$ year DPhil competition at the Department of Materials, University of Oxford, UK, $23^{\text {rd }}$ February 2010.

"The Corrosion and Microstructural Chemistry of Zirconium Alloys" Presentation for final round masterclass given by the Institute of Materials, Minerals and Mining 2010 Literature Review Competition prize, London, $22^{\text {nd }}$ February 2010.

"The surface migration of tin during laser atom probe analysis of zirconium-based alloys" New Frontiers of Atom Probe Applications - International Atom Probe Workshop 2009, University of Oxford, UK, $13^{\text {th }}-16^{\text {th }}$ September 2009.

"Zirconium oxidation on the atomic scale" 2008 International Field Emission Sympossium, University of Rouen, France, $29^{\text {th }}$ June $-5^{\text {th }}$ July 2009.

"Zirconium oxidation on the atomic scale" Universities Nuclear Technology Forum, University of Manchester, UK, $26^{\text {th }}$ March 2008. 


\section{Contents}

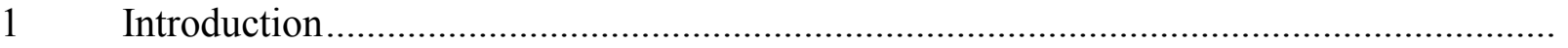

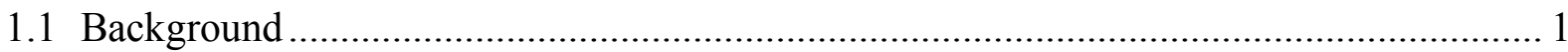

1.2 The current work

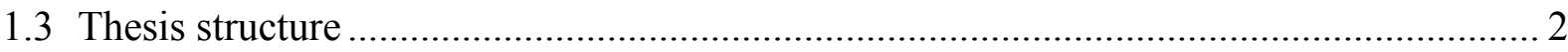

2 Literature review: oxidation of zirconium and its alloys ................................................. 4

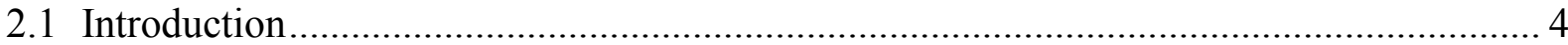

2.1.1 Structure of the literature review.............................................................. 4

2.1.2 Zirconium in the nuclear industry ............................................................... 5

2.1.3 Passivation and breakaway oxidation ............................................................ 7

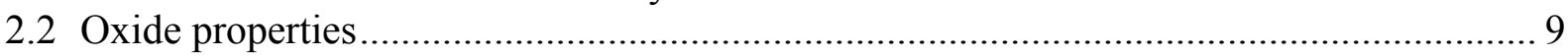

2.2.1 Monoclinic and tetragonal......................................................................... 10

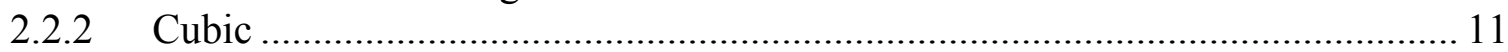

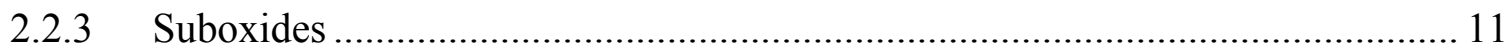

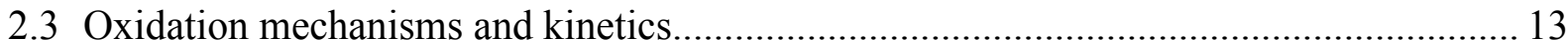

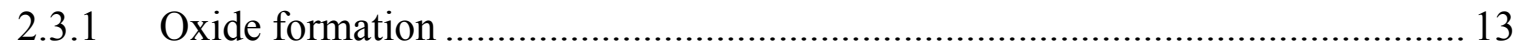

2.3.2 Nodular corrosion................................................................................ 15

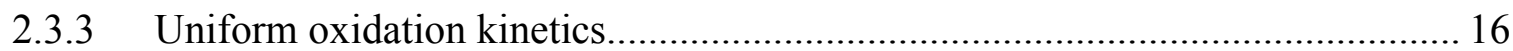

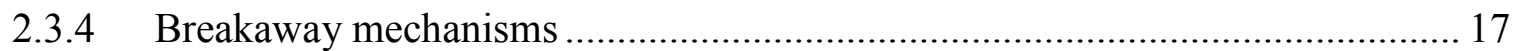

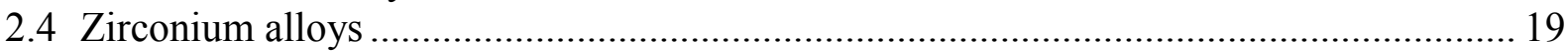

2.4.1 Summary of alloy development and usage ………………………………..... 19

2.4.2 Significant intermetallic phases ……………………………………….... 20

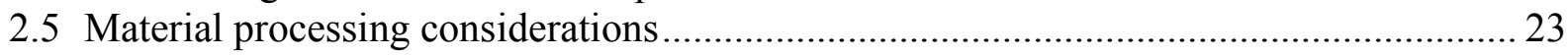

2.5.1 Annealing temperature, time and cold working ……………………………. 23

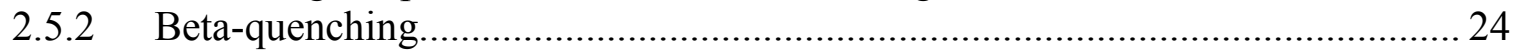

2.6 Effect of alloying elements in zirconium alloys ......................................................... 26

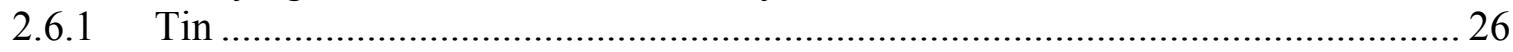

2.6.2 Niobium, vanadium and molybdenum ..................................................... 28

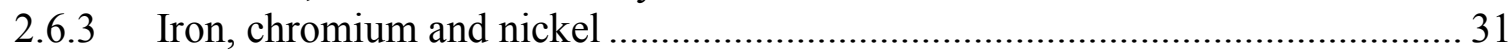

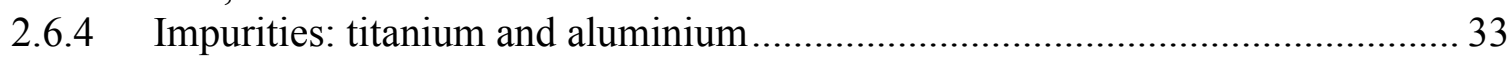

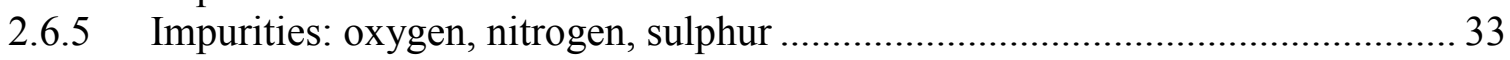

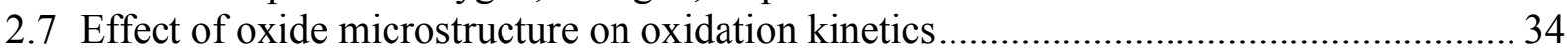

2.7.1 Correlation of structural changes with breakaway oxidation................................. 34

2.7.2 Second phase particles in the oxide.................................................................... 36

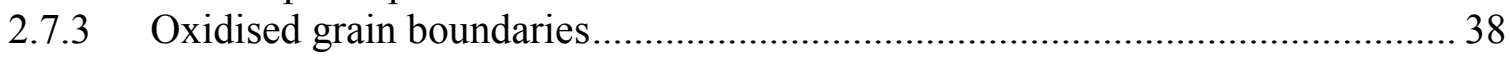

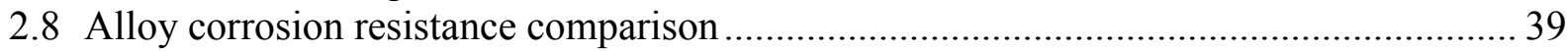

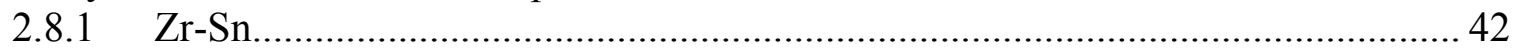

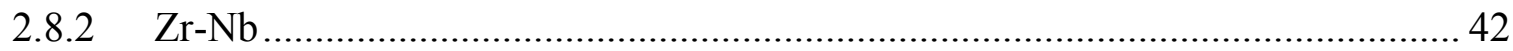

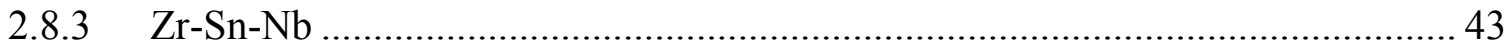

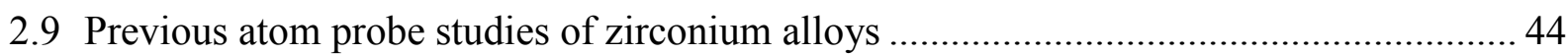

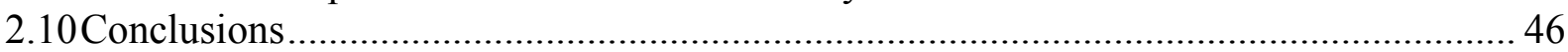

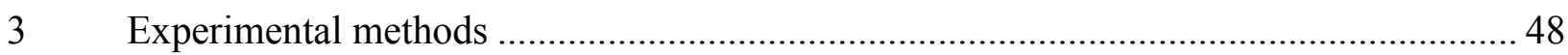

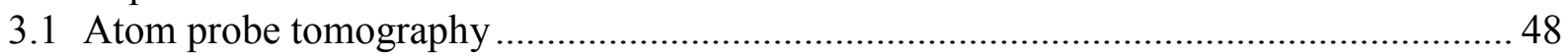

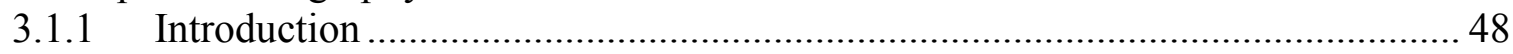

3.1.2 History and development of the instrument .................................................... 48

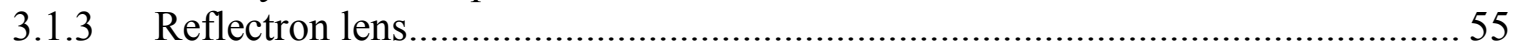

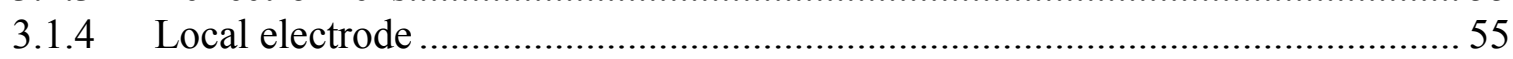

3.1.5 Micro-channel plate..................................................................................... 56

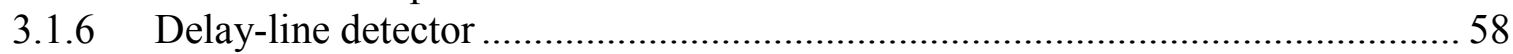

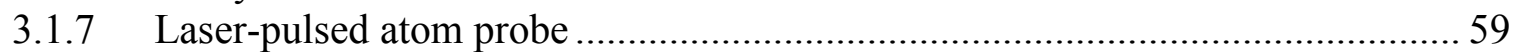




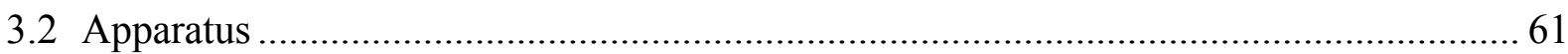

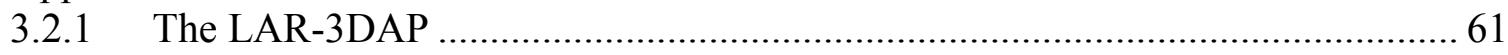

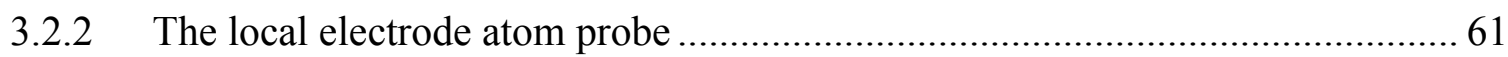

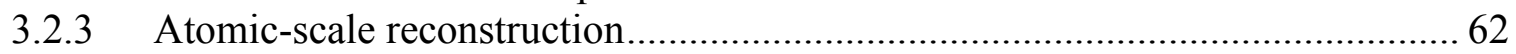

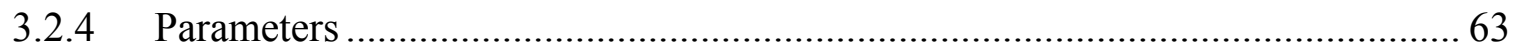

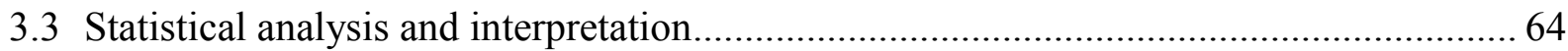

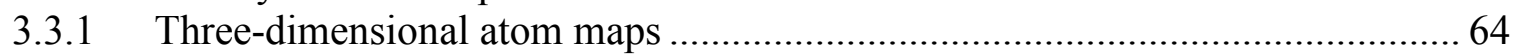

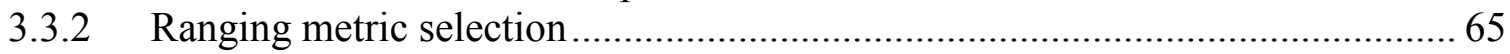

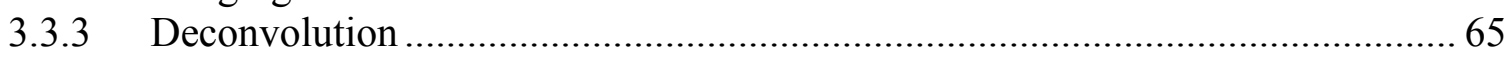

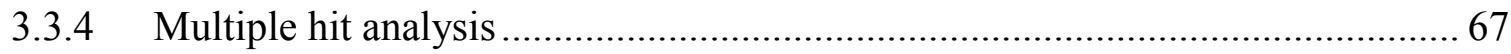

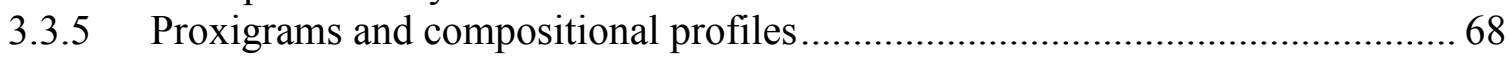

3.3.6 Important artefacts and aberrations ................................................................ 69

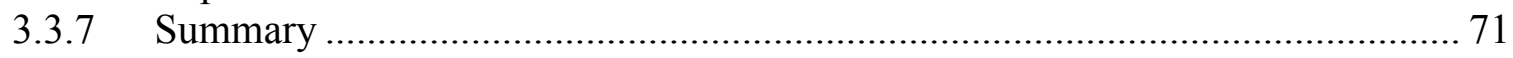

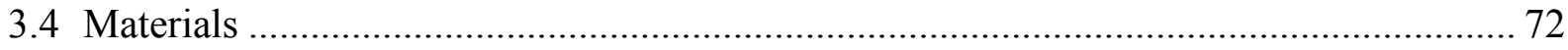

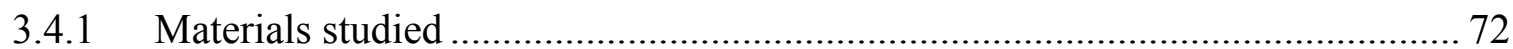

3.4.1.1 Commercially pure zirconium ................................................................... 73

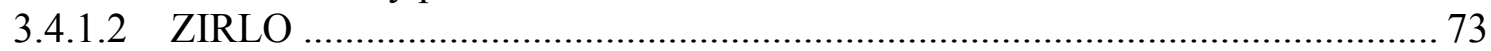

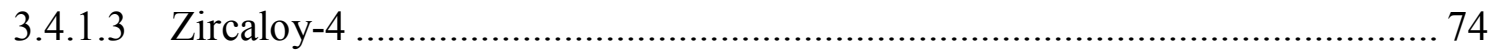

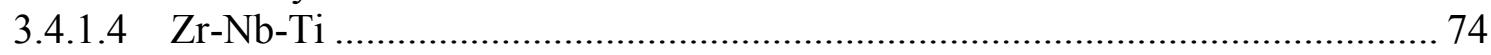

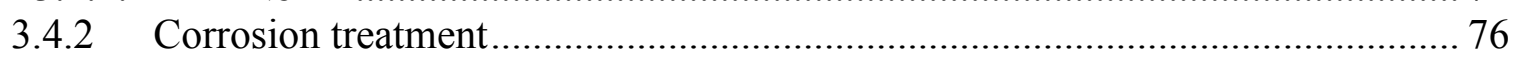

3.4.2.1 Commercially pure zirconium .................................................................. 76

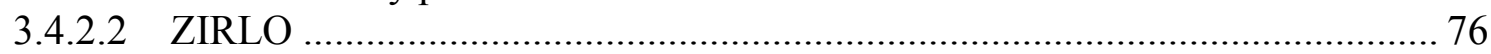

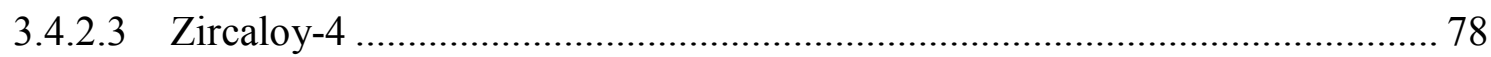

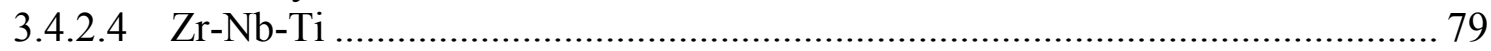

3.5 Atom probe specimen preparation ………………………………………………. 79

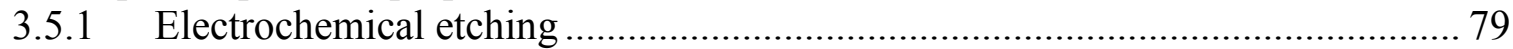

3.5.2 Focussed ion beam (FIB) milling ........................................................... 82

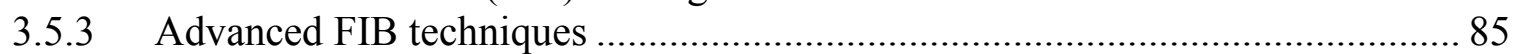

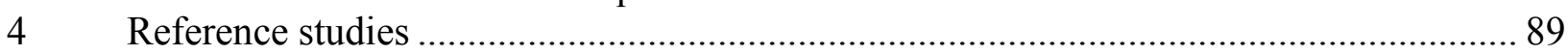

4.1 Determining suitable analysis conditions for voltage-pulsed LAR-3DAP ....................... 89

4.2 Voltage-pulsed mass spectra ..................................................................................... 90

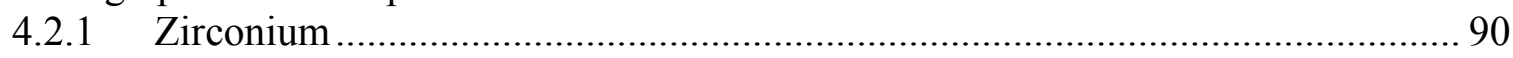

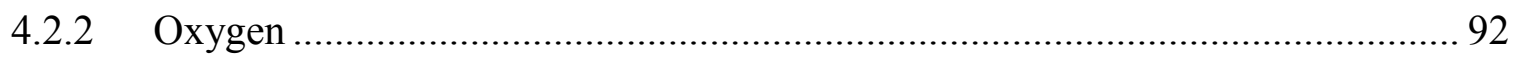

4.2.3 Hydrogen ................................................................................................... 92

4.3 Native oxides formed on commercially pure zirconium atom probe specimens .............. 95

4.4 Bulk hydrides formed on zirconium atom probe specimens .......................................... 97

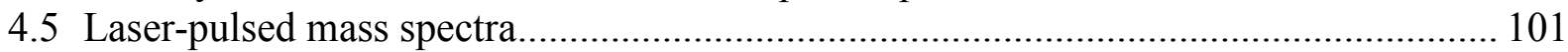

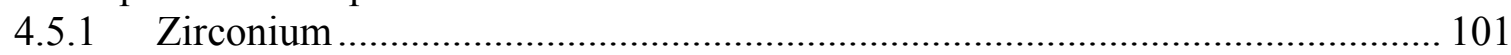

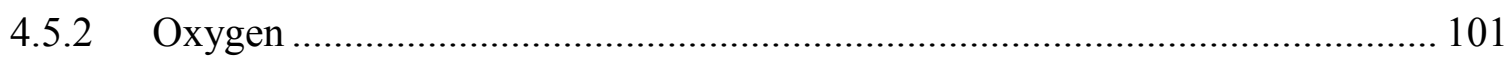

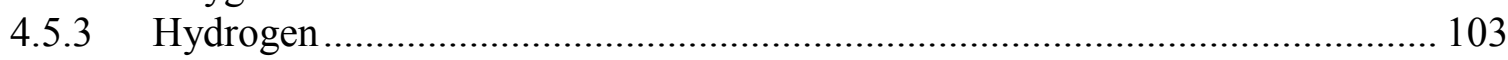

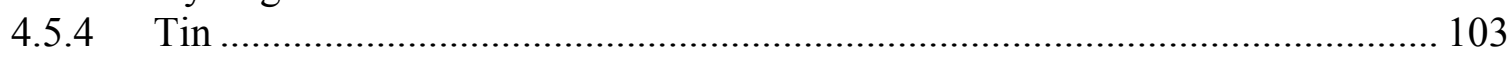

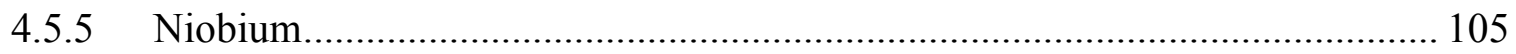

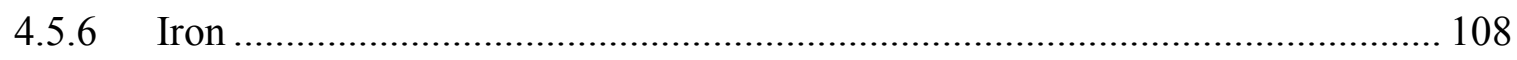

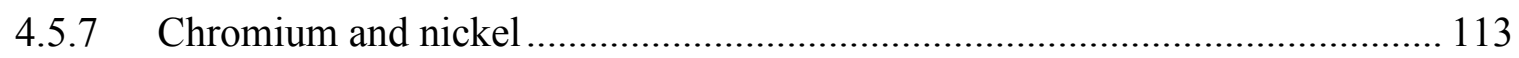

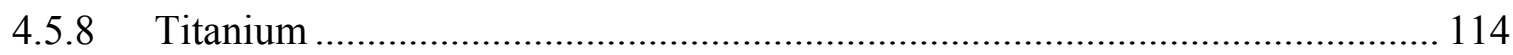

4.5.9 Ion complexes formed by laser pulsing........................................................ 116

4.6 Determining suitable analysis conditions for laser-pulsed LEAP ................................. 117

4.6.1 Evaporation field variation........................................................................... 119

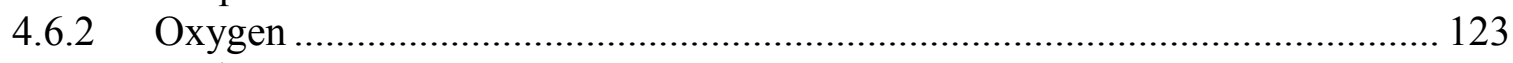

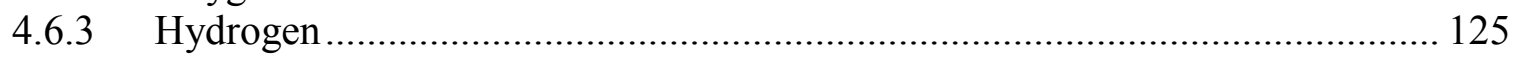

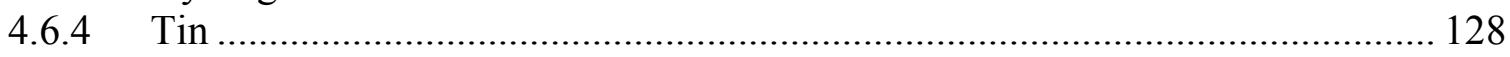




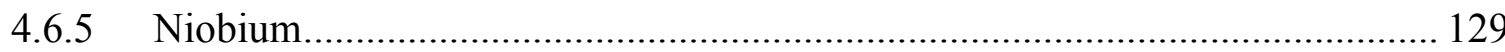

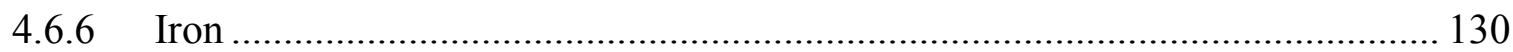

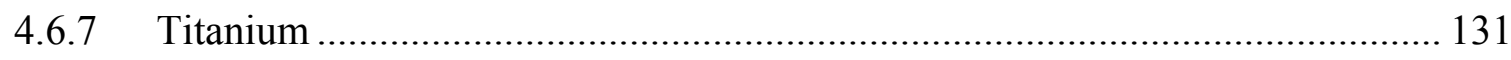

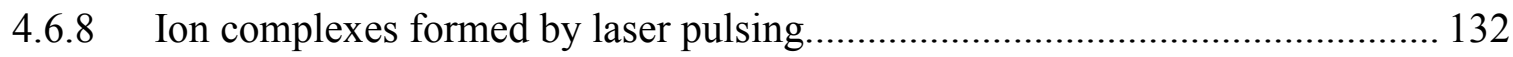

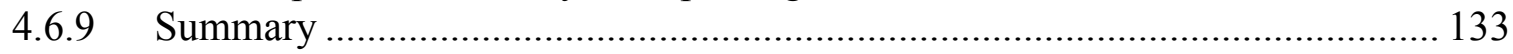

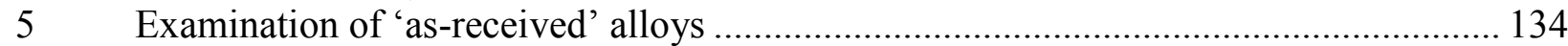

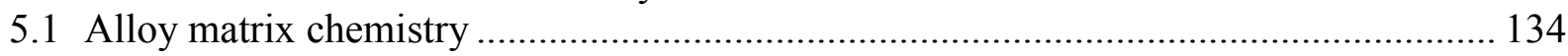

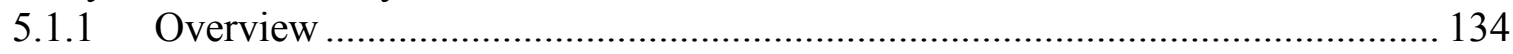

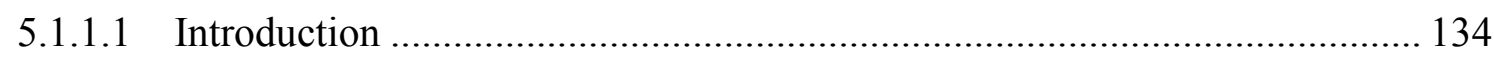

5.1.1.2 Matrix solute concentration .................................................................... 135

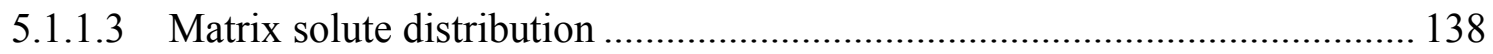

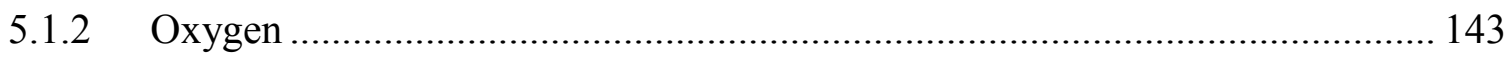

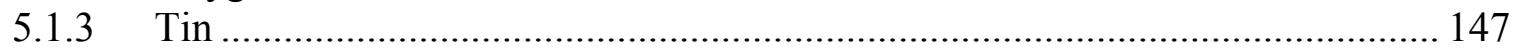

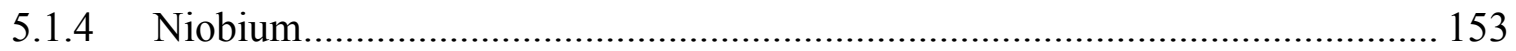

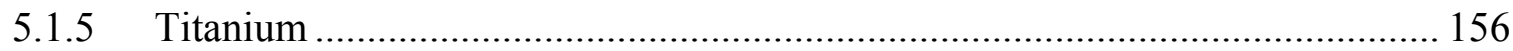

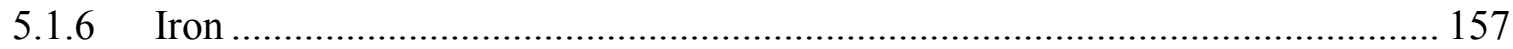

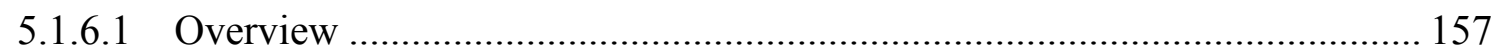

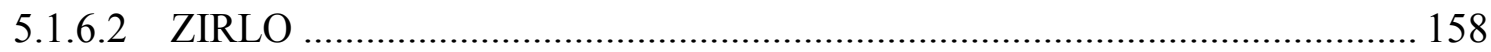

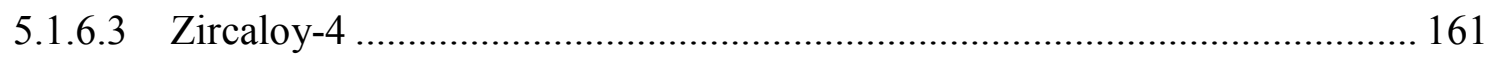

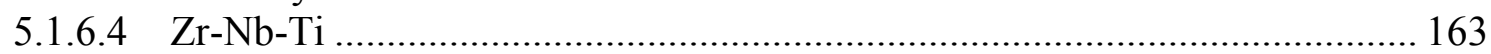

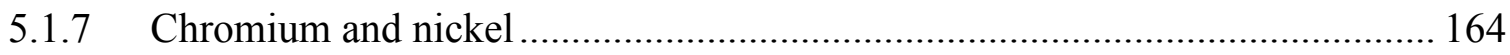

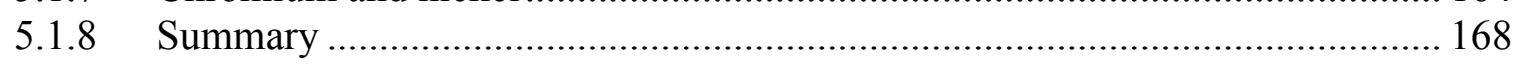

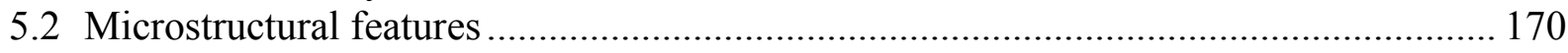

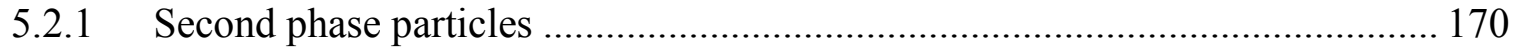

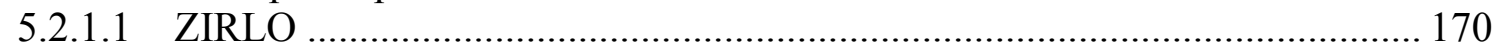

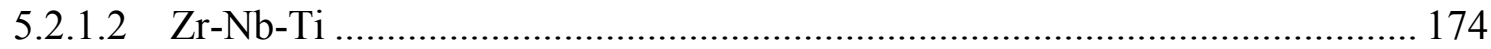

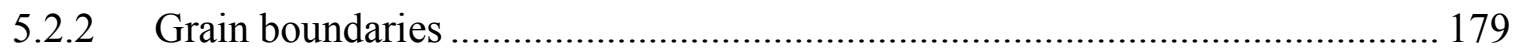

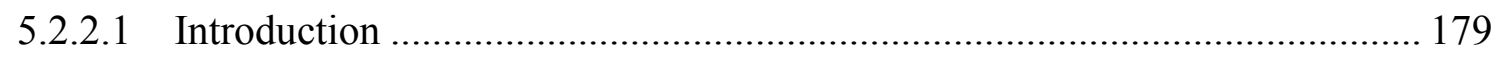

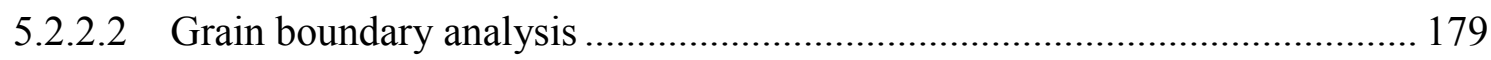

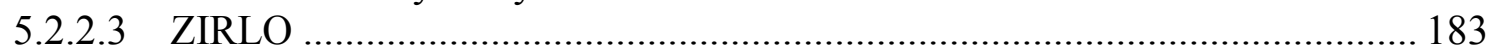

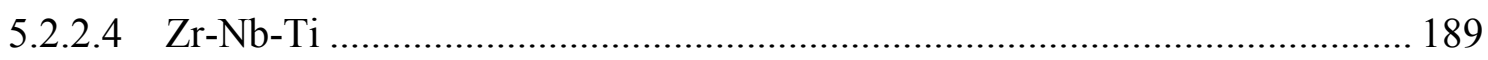

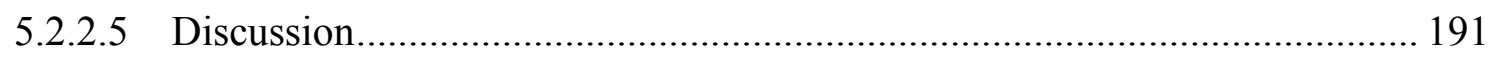

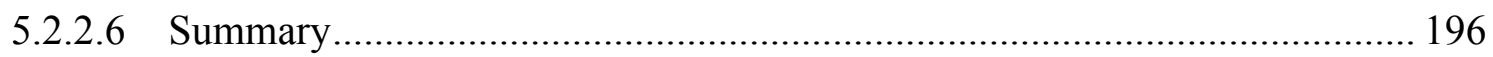

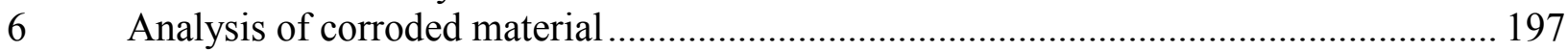

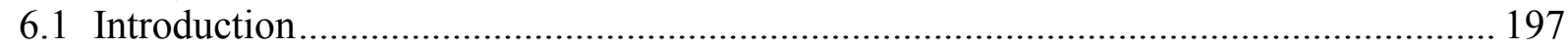

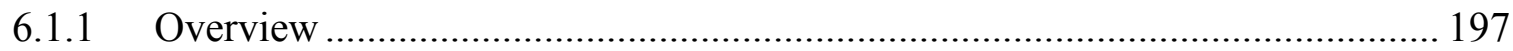

6.1.2 Notes on reproducibility of results ............................................................... 198

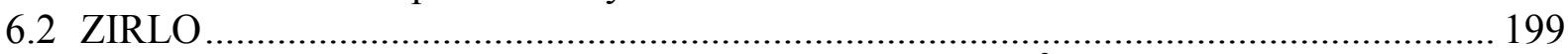

6.2.1 Pre-transition metal-oxide interface: $18.3 \mathrm{mg} \cdot \mathrm{dm}^{-2}$...................................... 199

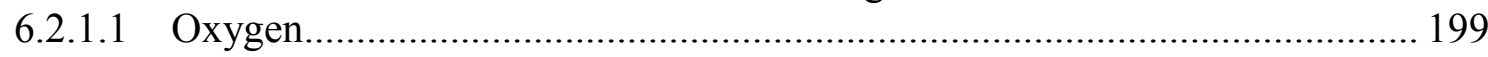

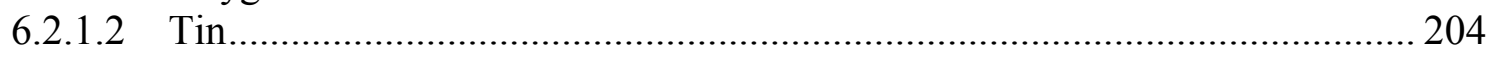

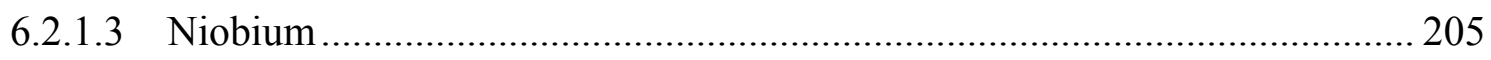

6.2.1.4 Iron and Chromium .......................................................................... 207

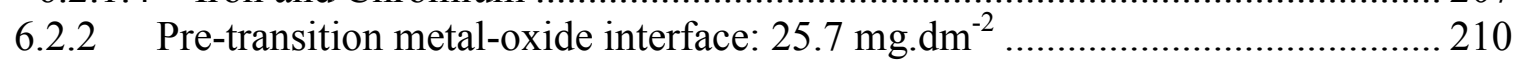

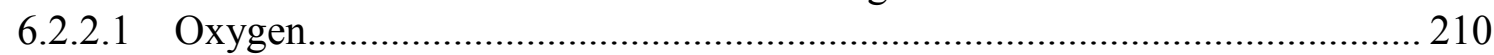

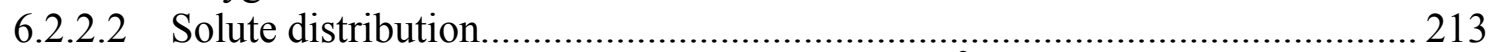

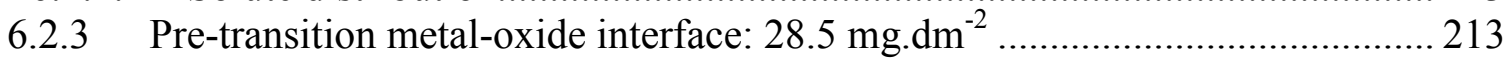

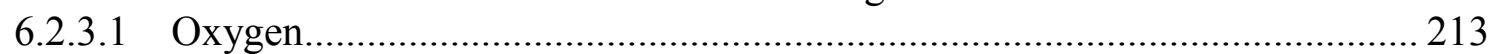

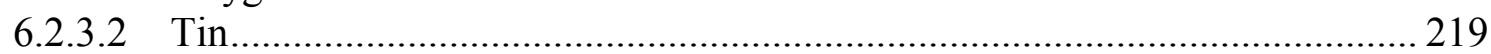

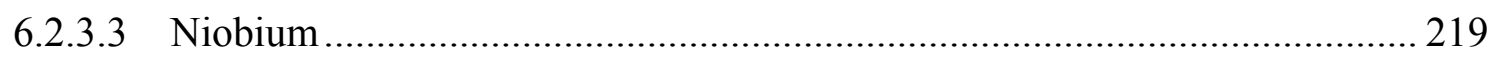

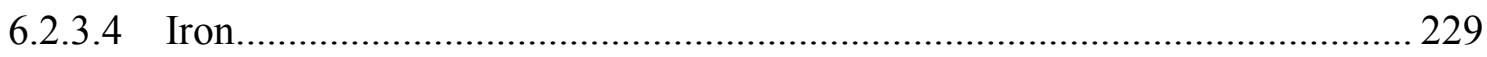




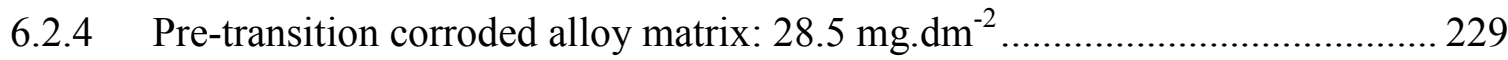

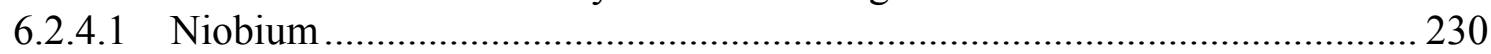

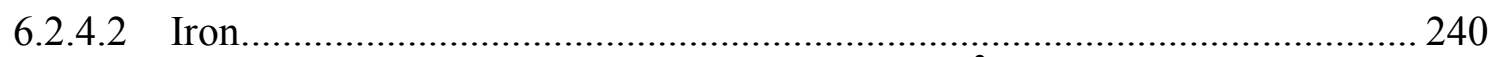

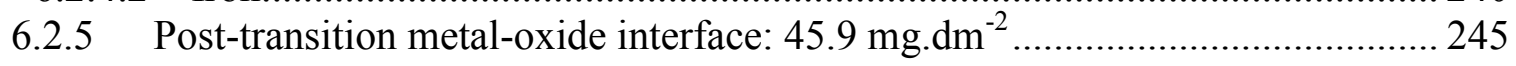

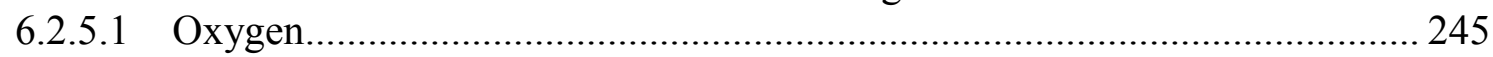

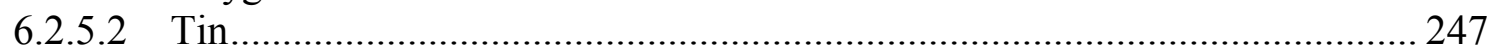

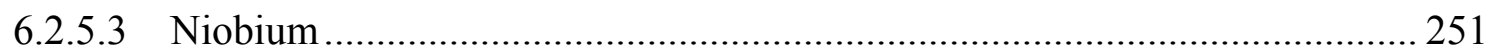

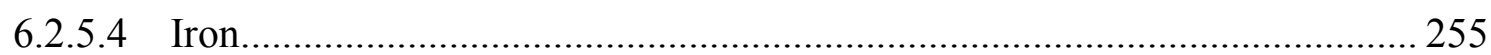

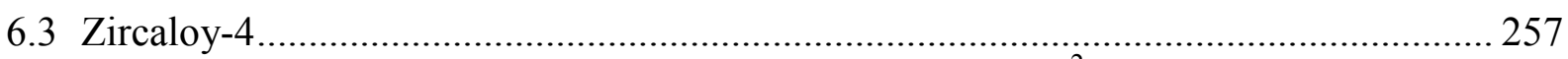

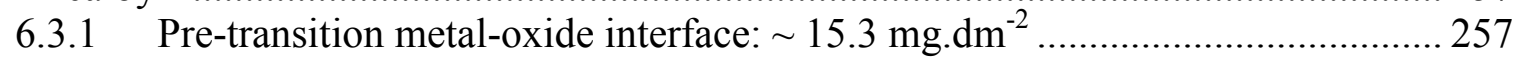

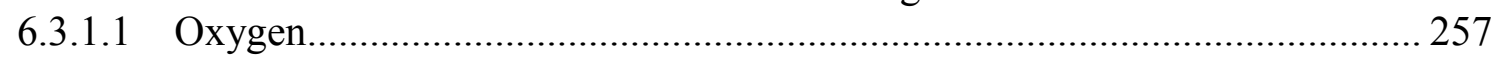

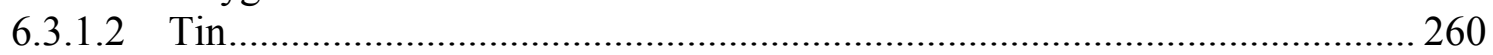

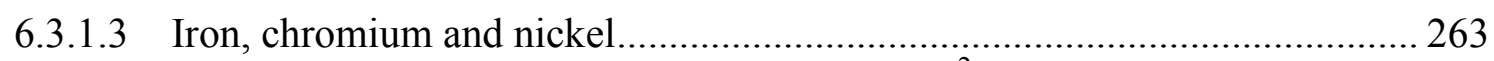

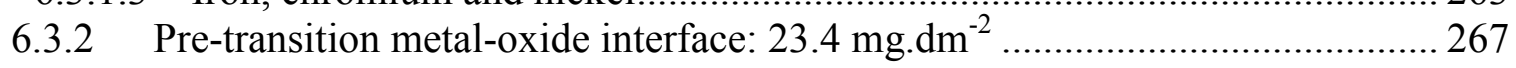

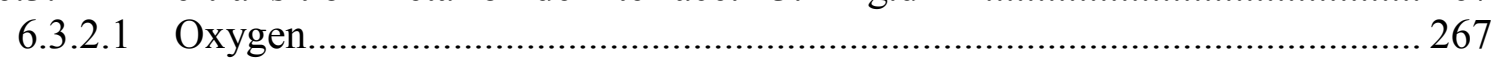

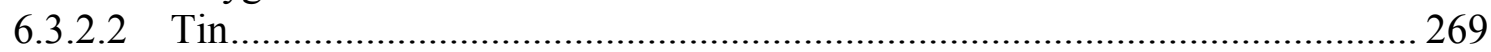

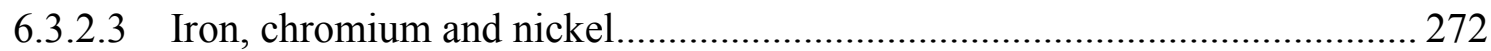

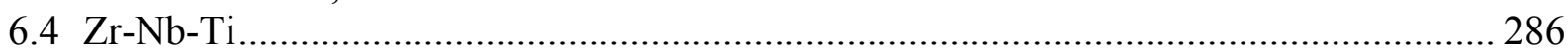

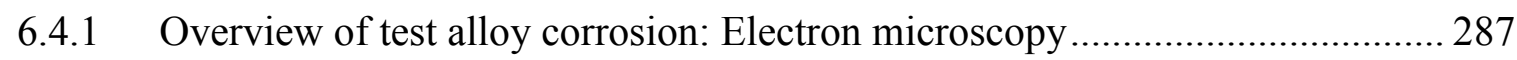

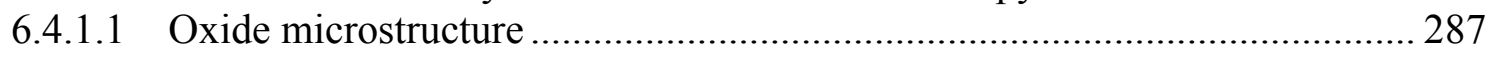

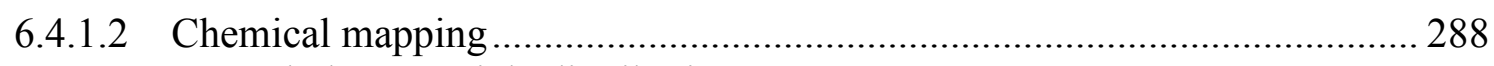

6.4.1.3 Second phase particle distribution ..................................................... 290

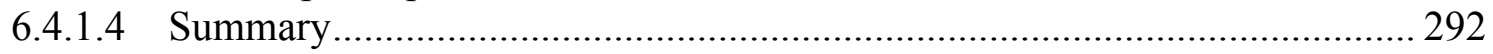

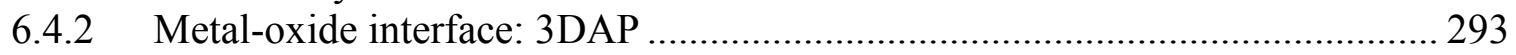

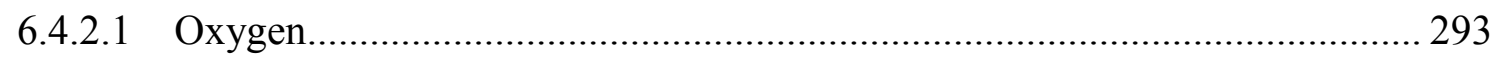

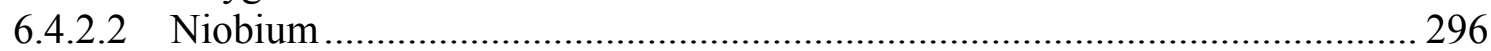

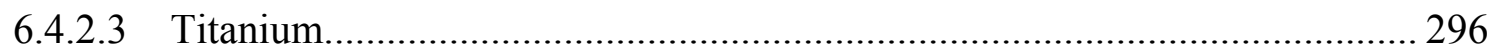

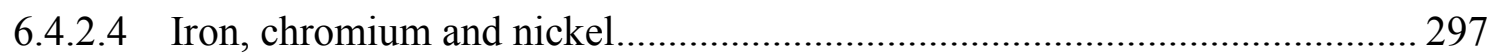

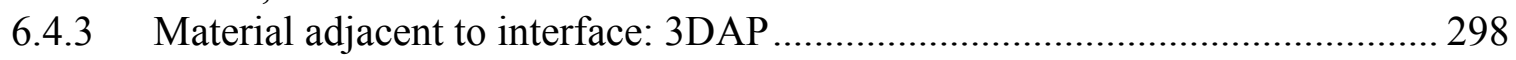

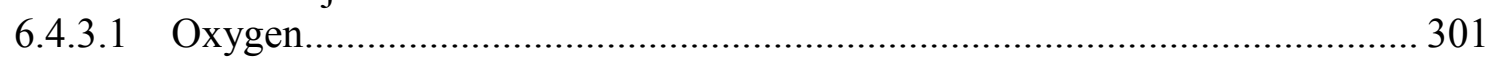

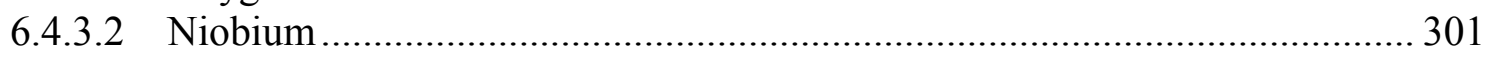

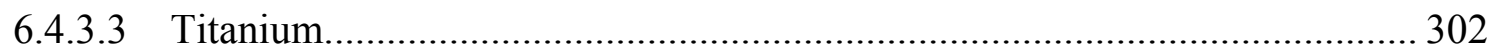

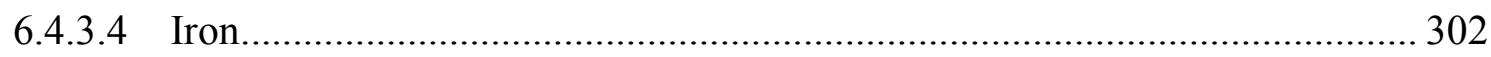

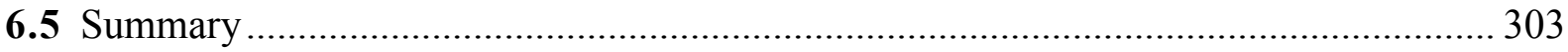

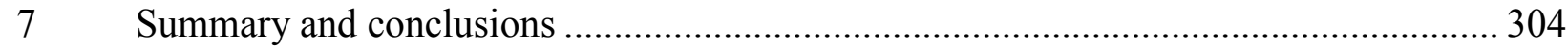

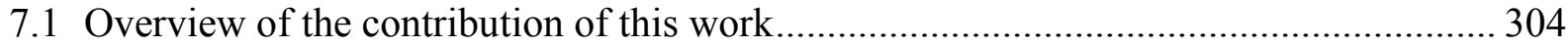

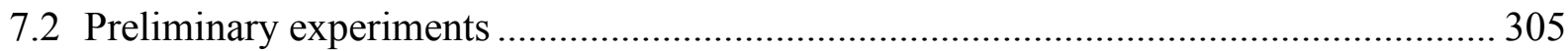

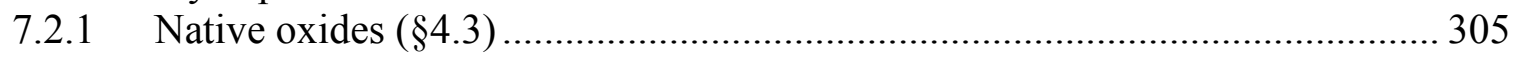

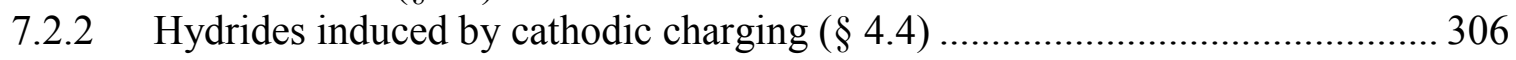

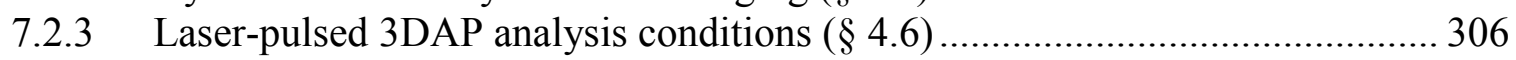

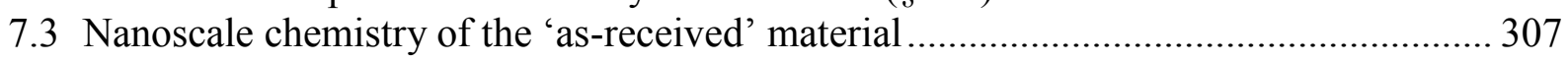

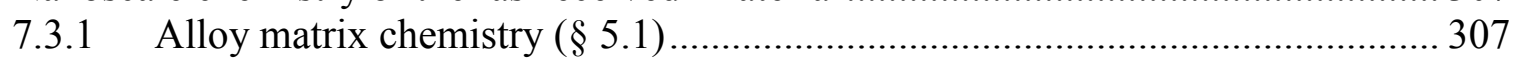

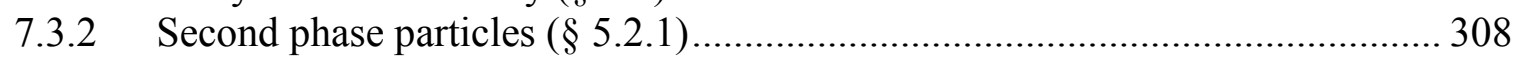

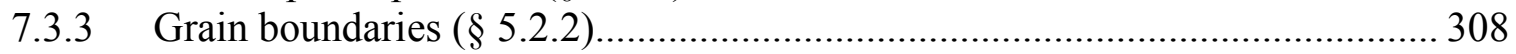

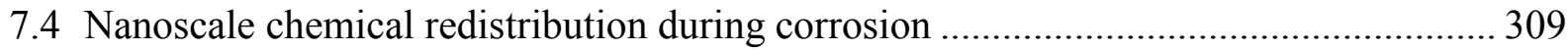

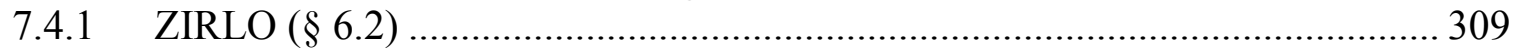

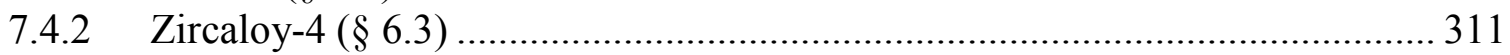

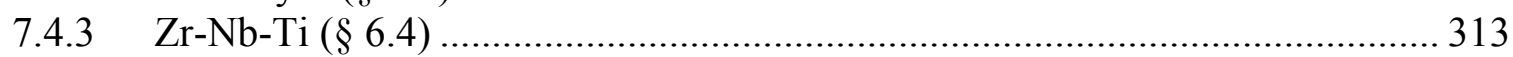

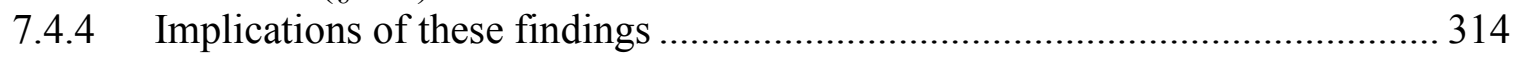

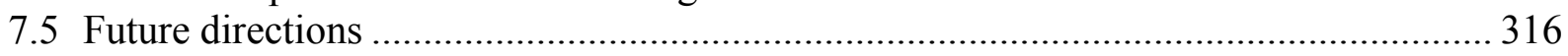

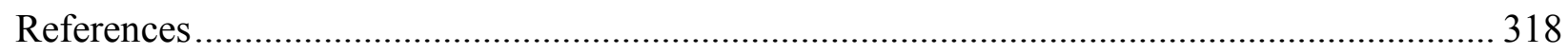




\section{$1 \quad$ Introduction}

\subsection{Background}

Zirconium-based alloys are widely used in nuclear reactor fuel rod cladding and structural applications. These alloys are chosen for this role due to their low thermal neutron cross-section, high corrosion resistance and good mechanical properties. The corrosion resistance of the material is due to the formation of a protective oxide film on the surface of the metal. Unfortunately, the protective nature of this film is seen to fail after the oxide reaches a critical thickness. This is known as 'breakaway', after which the oxidation proceeds at a faster, linear, rate. This increase in corrosion kinetics places a limitation on the lifespan of the fuel rod, and the fuel efficiency that can be achieved by the reactor.

\subsection{The current work}

The work described in this thesis formed part of a multidisciplinary project undertaken by a consortium of universities working in the MUZIC program; Oxford, Manchester and The Open University. The objective of the project as a whole was to further the understanding of the mechanisms of breakaway oxidation process and to characterise the corrosion processes within a number of fuel rod cladding materials in PWR conditions. This has involved several researchers separately concentrating on the analysis of the same corroded materials in order to produce complementary data. The characterisation performed using synchrotron-XRD, TEM, HR-TEM, nano-indentation, SEM, 3D-FIB cross-sectioning and 3D atom probe has allowed many different aspects of the corrosion process to be studied on a number of length scales. This thesis describes 
$3 \mathrm{D}$ atom probe characterisation of the nanoscale chemical redistribution of solutes that occurs during the corrosion process, focussing in particular on ZIRLO, a commercial $\mathrm{Zr}-\mathrm{Nb}-\mathrm{Sn}-\mathrm{O}-\mathrm{Fe}$ alloy. These results are compared with two other materials, Zircaloy-4, another commercial alloy and a test $\mathrm{Zr}-\mathrm{Nb}$-Ti alloy that demonstrates remarkably poor corrosion resistance, seemingly due to the addition of $0.2 \mathrm{wt} . \%$ titanium.

In order to conduct this study, a large amount of atom probe background experimental work was required to ascertain suitable 3DAP analysis conditions, develop specimen fabrication methods, identify the large number of peaks within mass-to-charge spectra, and implement data processing methods that allow the precise quantification of very low solute concentrations.

\subsection{Thesis structure}

Chapter 2 introduces the uses of zirconium in the nuclear industry $(\S 2.1)$ and the problems associated with its deployment in the application of nuclear fuel rod cladding in PWR conditions. This chapter goes on to describes the existing literature regarding the effect of zirconium alloy chemistry on corrosion resistance $(\S 2.2-2.8)$, as well as the contributions of previous atom probe studies $(\S 2.9)$. An overview of atom probe tomography is described in chapter 3, with further sections in this chapter devoted to the specification of the atom probes used in the present study (§ 3.2), methods of analysis and interpretation of results $(\S 3.3)$. The materials that were analysed in this study and the corrosion treatment they underwent $(\S 3.4)$ as well as the processes involved with the fabrication of specimens for the $3 \mathrm{D}$ atom probe $(\S 3.5)$ are also described in chapter 3 .

Chapter 4 details references studies that analysed native oxides and hydrides formed on atom probe specimens of commercial purity zirconium $(\S 4.3-4.4)$. The remainder of this chapter is devoted to finding suitable analysis conditions with which to perform laser-pulsed atom probe 
analysis of metallic zirconium specimens $(\S 4.5-4.6)$. This knowledge is put to use in chapter 5, where the metal matrix, second phase particle and grain boundary chemistry of three zirconium alloys are characterised. This information provides a benchmark for comparison of these materials after they have been subjected to various levels of corrosion. The analysis of corroded material is presented in chapter 6 . The main focus of this investigation is the nanoscale chemical redistribution that occurs within ZIRLO (§ 6.2) during corrosion, focussing on a $100 \mathrm{~nm}$ wide region centred on the metal-oxide interface. The changes in chemistry of the material remote from the interface are also presented to provide explanation of some of the behaviour observed. These changes in chemistry during corrosion are compared to those seen in two other alloys, Zircaloy-4 (§ 6.3), which like ZIRLO is a commercial fuel rod cladding material, and a test alloy containing titanium which suffers vastly inferior corrosion resistance (§ 6.4). A discussion of the results, together with a summary of the findings and conclusions are given in chapter 7 .

Appendices A and B describe work that was performed in the course of this project in order to standardise the mass ranging techniques used in atom probe tomography. Currently, this process is carried out manually, and there is no recognised convention on the definition of mass ranges. This variation can create large discrepancies in the detected solute concentration, especially those of low concentration solutes.

Appendix $\mathrm{C}$ is under an embargo. 


\section{Literature review: oxidation of zirconium and its}

\section{alloys}

\subsection{Introduction}

\subsubsection{Structure of the literature review}

The focus of this review is restricted to studies of the effects of chemical variations on oxidation resistance and the 'breakaway' process. The effect of irradiation is not discussed here, as the problem of breakaway oxidation is not dependent upon a radiation field. This later area was recently covered by another review [1]. Readers interested in this areas may also find a much earlier review by the same author of interest [2], demonstrating the development of ideas in this field between 1968 and 2005, as well as more recent articles relating to the effect of irradiation on these materials [3-5].

There is a large body of literature related to understanding the development of crystallographic texture and its effect on the corrosion properties of these alloys. This area is better understood than the effects relating to microscopic chemistry and interested readers may consult recent reviews [6-7] for more information on these topics.

The evolution and adsorption of hydrogen during the aqueous corrosion of zirconium alloys is also seen to be life limiting for high burn-up scenarios. It is instructive to consider the problem of hydrogen uptake and hydride precipitation separately from the central issue of this review, as the relationship between these processes and zirconium oxidation is a mutually dependent and complex one. Readers interested in issues regarding hydrogen-uptake are commended to a recent [8] and an historic [9] review on the subject. 
This review will introduce the role of zirconium in the nuclear industry $(\S 2.1 .2)$ and the efficiency limiting problem of break-away oxidation (\$2.1.3). The oxides formed on zirconium and its alloys are described (\$2.2) followed by details of the mechanics of oxidation and the effect of different reactor environments (\$2.3). The families of alloys that have developed to cater for the various reactor environments and the most significant intermetallic compounds these alloys contain are described in section 2.4. A brief summary of the effect of processing variables on the microstructure of these materials is presented in $\S 2.5$ as well as more detailed information on the role of individual solute additions within these materials (§2.6). After the problem and materials have been fully described, the focus of the review turns to the structure of the oxide that develops during the oxidation process and the correlation between the oxide microstructure and the oxidation rate, as well as the redistribution of solute during corrosion (§2.7). The corrosion resistance of various alloys is then compared with the observed oxide microstructure and their original precipitate morphology $(\S 2.8)$. The review concludes with a summary of previous atom probe studies on zirconium alloys $(\S 2.9)$, the state of knowledge of the oxidation mechanisms of these systems and how additional atom probe microscopy experiments with the new generation of instruments can assist in furthering the understanding of these processes $(\$ 2.10)$.

\subsubsection{Zirconium in the nuclear industry}

Zirconium alloys are widely deployed in nuclear reactor fuel rod cladding and structural applications. In water moderated reactors arrays of fuel rods, shown in Figure 1, containing uranium dioxide fuel are immersed in primary water. The primary water acts both as the moderator for the nuclear reaction, slowing down fast neutrons and so increasing their probability of producing further fission events, and conducting heat away to a secondary coolant system remote from the core. This heat transferred to the secondary coolant may be used to 
perform useful work. It is the role of the cladding to contain the fuel and keep it separated from the coolant.

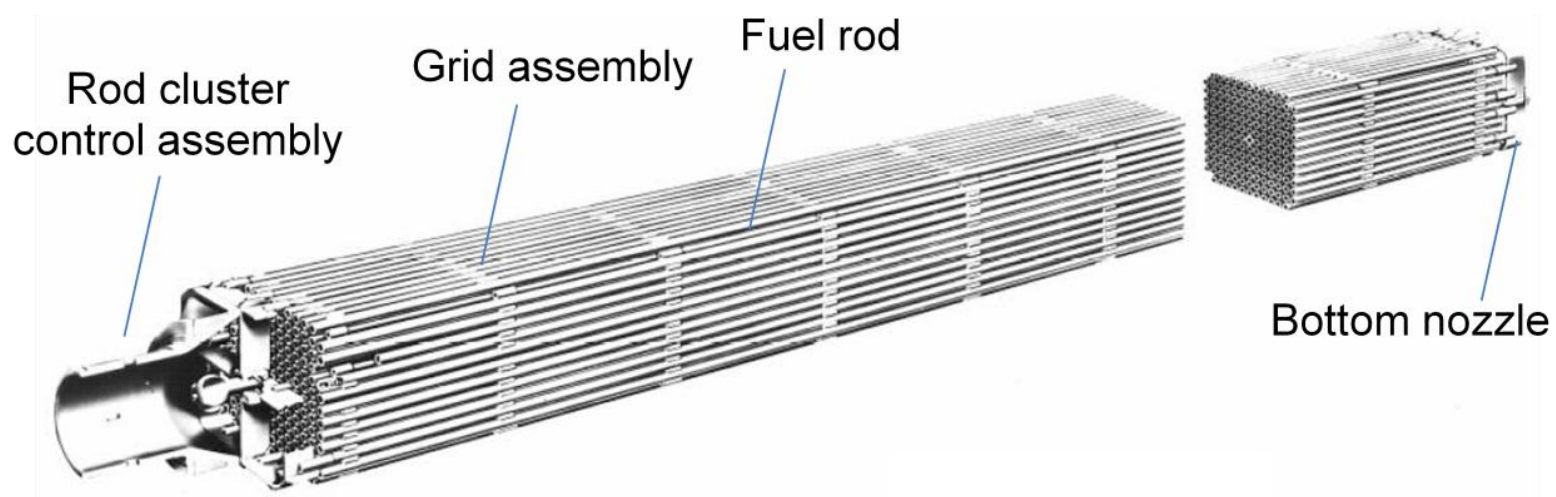

Figure 1: A cut-away perspective illustration of the arrangement of rods that make up a fuel assembly. Adapted from [10]. The fuel rod assembly is of the order of $4 \mathrm{~m}$ long.

Zirconium-based alloys are chosen for this role due to their relatively high transparency to the neutron flux generated by the nuclear reaction occurring within the fuel rod; the average thermal neutron cross section of zirconium is 0.18 barn [11]. Zirconium also possesses the desirable properties of high corrosion resistance in corrosive environments and good mechanical properties. Figure 2 shows a representation of the thermal neutron cross sections of several possible cladding materials and hafnium. The main difficulty in the preparation of pure zirconium is the separation of its fellow group IV element hafnium. Unfortunately, this extraction is essential due to the poisoning effect of hafnium on the neutron cross-section.

An extensive variety of alloys and alloy families has developed to cater for the various reactor environments. In general the corrosion resistance of these alloys is adequate for use in commercial nuclear reactors. However, as fuel burn-up requirements have grown tighter, in order to achieve higher reactor efficiency, the life-span of the cladding has become a limiting factor. This places more stringent specifications on the corrosion performance of these materials. 
Currently, only one third of the fuel encased in each rod can be used in a fuel cycle before the rod must be removed because of degradation of cladding. Although the reactor core is designed to allow periodic inspection and removal of these rods, the necessity to remove two thirds of the fuel unused in each cycle is both expensive and wasteful. Also, in applications such as in nuclear submarine reactors, periodic replacement of core components is less convenient than in civil power plants.

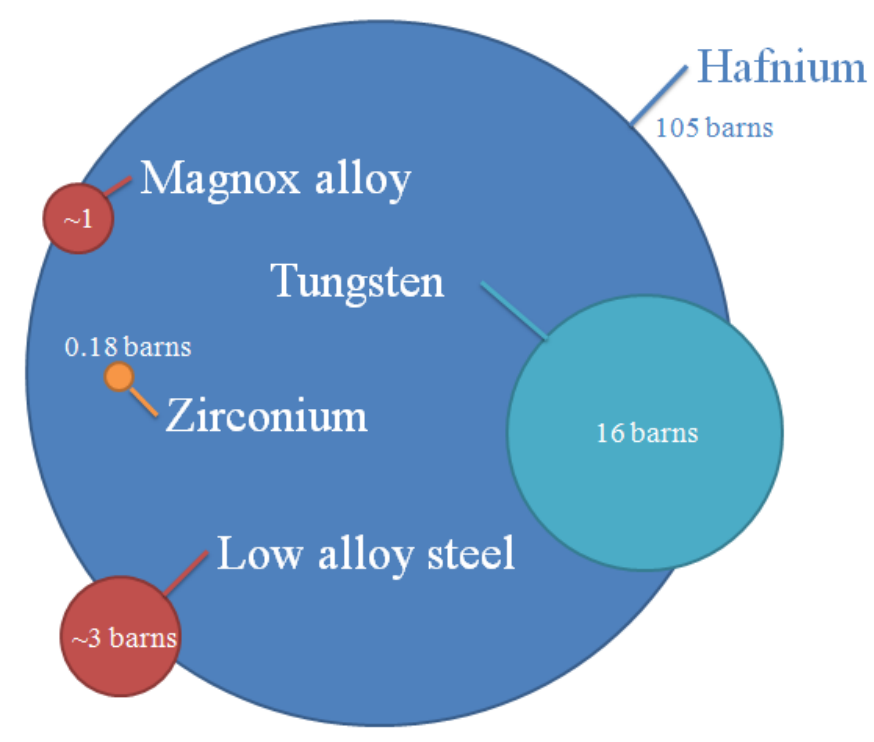

Figure 2: Representation of thermal neutron cross sections of various cladding materials in comparison with tungsten and hafnium, which is chemically similar to, and very difficult to separate from zirconium. Plot created from data within [11-13]

\subsubsection{Passivation and breakaway oxidation}

Like many reactive metals, a clean zirconium surface grows a passivating oxide film that has the effect of retarding further corrosion. It is such naturally occurring layers that allow highly reactive aluminium to be considered to be unreactive in the many engineering environments to 
which it is applied. Unfortunately, unlike aluminium, the passivating layer that grows on the surface of zirconium reaches a critical thickness and then loses its corrosion-protective properties [14]. This is known as breakaway oxidation, not because the oxide mechanically fails to such an extent that it breaks off from the surface, but because the corrosion rate increases dramatically after this transition. Figure 3 shows the exemplar oxidation kinetics for a generic zirconium alloy. The mechanisms by which this breakaway occurs are under much debate in the literature and the effect of alloying additions on this transition, as well as the overall corrosion kinetics, is poorly understood.

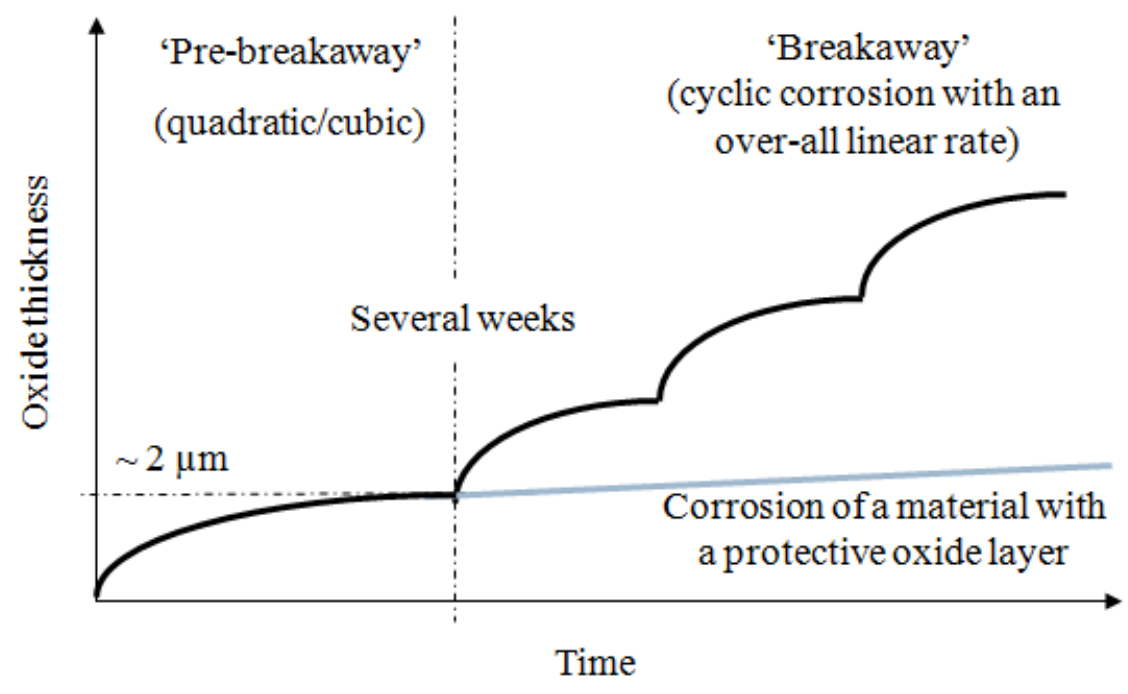

Figure 3: Typical oxidation kinetics of a generic zirconium-based alloy. The blue line shows the weight gain that would be expected for a material with a protective barrier layer. After the pretransition region where the oxidation kinetics follow a quadratic/cubic relationship, depending upon the oxidising environment, the protective layer breaks down and cyclic oxidation proceeds with an overall linear rate. The critical thickness before transition occurs (usually of the order of $2 \mu \mathrm{m})$ and the oxidation rates pre- and post-transition are dependent upon the alloy composition and processing, as well as the oxidising environment. 
The chemical distributions of solute within the alloy and oxide, and the microstructures of each of the phases, are of fundamental importance in understanding the differing corrosion rates and onset of breakaway corrosion seen in different alloy classes. The processing route and thermomechanical treatment applied to the alloys greatly affects the distribution and role of solute within the corrosion process. The difference in microstructure also affects the build-up and maintenance of stress at the metal-oxide interface, where oxide growth occurs. The microstructure of the resultant oxide is also strongly correlated to the further corrosion resistance of the material. The periodic oxide growth structure has been observed by several researchers [15], and the relative thickness of each layer can be correlated to the overall oxide growth rate.

\subsection{Oxide properties}

Zirconium dioxide is reportedly the only thermodynamically stable oxide of zirconium. Bonding in the oxide is almost completely ionic in character, implying that there can be few free electrons [1]. The band gap of $\mathrm{ZrO}_{2}$ is approximately 5-6 eV. [16], work function $4.0 \mathrm{eV}$ and relative permittivity 22 [17]. The oxide exists in three different solid phases, depending upon its environment. These are listed in Table 1. The corresponding phase diagram for these oxides is displayed in Figure 4.

\begin{tabular}{|c|c|c|c|}
\hline Phase & Formula & Symmetry & $\begin{array}{c}\text { Space } \\
\text { group }\end{array}$ \\
\hline$\alpha-\mathrm{ZrO}_{2} / \mathrm{m}-\mathrm{ZrO}_{2}$ & $\mathrm{ZrO}_{2}$ & Monoclinic & $\mathrm{P}_{1} / \mathrm{c}$ \\
$\beta-\mathrm{ZrO}_{2} / \mathrm{t}-\mathrm{ZrO}_{2}$ & $\mathrm{ZrO}_{2}$ & Tetragonal & $\mathrm{P} 4_{2} / \mathrm{nmc}$ \\
$\gamma-\mathrm{ZrO}_{2} / \mathrm{c}-\mathrm{ZrO}_{2}$ & $\mathrm{ZrO}_{2-\mathrm{x}}$ & Cubic & $\mathrm{Fm} 3 \mathrm{~m}$ \\
\hline
\end{tabular}

Table 1: The three phases of zirconium dioxide [18] 


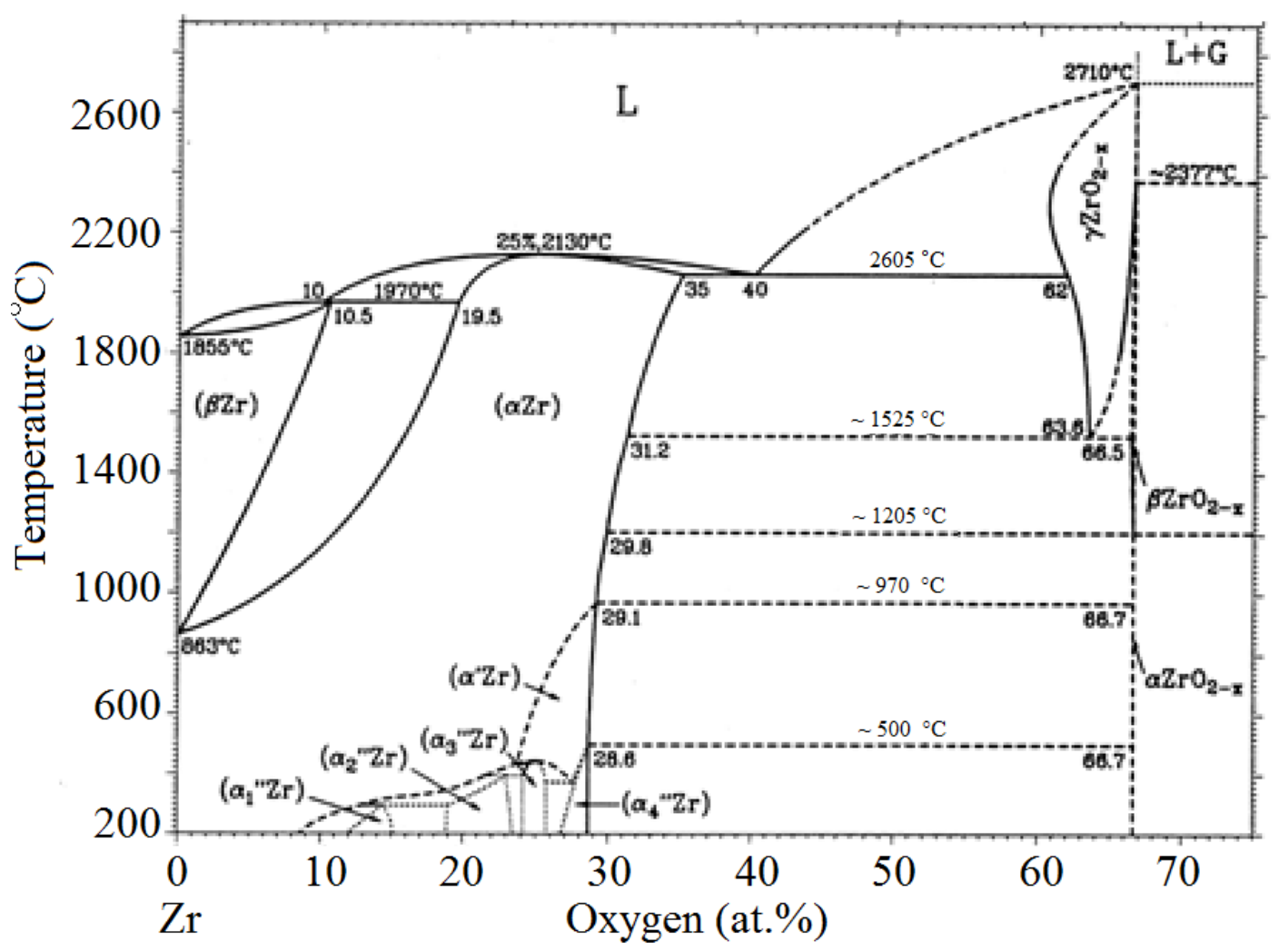

Figure 4: The Zr-O system phase diagram [19].

\subsubsection{Monoclinic and tetragonal}

Monoclinic $\mathrm{ZrO}_{2}$ is the most frequently observed oxide phase after oxidation in PWR conditions and occupies the greatest volume fraction. The tetragonal phase, which occupies a smaller volume fraction, and also a lower specific volume, is distributed inhomogeneously within the oxide film. The tetragonal phase is less stable at standard PWR reactor conditions than the monoclinic. Chemically stabilised zirconia ceramics undergo degradation from t- $\mathrm{ZrO} 2$ to $\mathrm{m}-\mathrm{ZrO}_{2}$ when exposed to moisture, even at low temperatures $100{ }^{\circ} \mathrm{C}$ [20]. However, $\mathrm{t}-\mathrm{ZrO}_{2}$ can be stabilised over $\mathrm{m}-\mathrm{ZrO}_{2}$ by the effects of hydrostatic pressure or compressive stress [21] as well as possible unknown factors [22]. Stresses in the oxide due to the Pilling-Bedworth ${ }^{1}$ ratio are

\footnotetext{
${ }^{1}$ The ratio of the oxide volume compared to the volume occupied by the metal from which it was produced.
} 
compressive [23]. The metallic phase at the interface is reported to be under tensile stress [24] and it is possibly these stresses that stabilise the tetragonal oxide at the interface.

\subsubsection{Cubic}

The hypo-stoichiometric cubic phase is not typically observed in the temperature and pressure regimes of PWR as the necessary stabilising factors are not present. Small volume fractions of this phase are observed in high temperature corrosion experiments [25].

\subsubsection{Suboxides}

Many sub-oxide phases have been reported in zirconium-related literature, using a variety of techniques and considering various sections of the oxide. It is useful to draw a distinction between the literature describing phases arising from partially, or highly ordered interstitial oxygen in alpha-zirconium and oxide phases that are not isomorphous with the HCP $\alpha$-Zr phase. We may then reserve the term 'sub-oxide' to describe oxide phases dissimilar from $\alpha-Z r$ or any form of $\mathrm{ZrO}_{2}$. These observations usually relate to regions adjacent to the metal-oxide interface of corroded material.

Moseley and Hudson [26] observe structures that they characterise as $\mathrm{ZrO}$ and $\mathrm{Zr}_{3} \mathrm{O}$ using electron diffraction and develop as cogent argument for the need of these phases as an explanation for efficient oxygen packing over the composition gradient of the oxide-metal interface. Iltis and Michel [27] also present [in French] electron diffraction that demonstrates the existence of an ordered phase which the authors purport to be $\mathrm{Zr}_{3} \mathrm{O}$. Iltis, along with other authors [28], later conducted a multi-scale characterisation of the metal-oxide interface of Zircalloy-4. In this study $\omega-\mathrm{Zr}$, not $\mathrm{Zr}_{3} \mathrm{O}$, was identified at the oxide-metal interface. $\omega-\mathrm{Zr}$ is a 
meta-stable hexagonal phase. The authors identified a compositional plateaux by taking an EDX line scan across the interface and suggest that the omega phase has the composition of approximately 60 at.\% Zr, 40 at.\% $\mathrm{O}$ and 0.5 at.\% Sn. The size of the grain was taken to be about $200 \mathrm{~nm}$. Motta et al. [15] also observe a phase described as $\mathrm{Zr}_{3} \mathrm{O}$ at the metal-oxide interface region using micro-beam synchrotron radiation diffraction. The authors identified the $\mathrm{Zr}_{3} \mathrm{O}$ as having a hexagonal (rhombic) structure with lattice parameters $\mathrm{a}=0.556 \mathrm{~nm}$ and $\mathrm{c}=$ $3.119 \mathrm{~nm}$.

There is some confusion in the literature regarding a consistent description of sub-oxide phases of zirconium. At this stage, several distinctions should be elucidated. The high solid solution saturation of oxygen in $\alpha-Z r$ described by Figure 4 suggests that at higher levels of uptake some interstitial ordering may be expected. The result of which would be the creation of an order or semi-ordered $\alpha-\operatorname{Zr}(\mathrm{O})$, which could sensibly be denoted as $\alpha^{\prime} \mathrm{Zr}$. Oxygen occupies the octahedral interstitial sites in $\alpha$-Zr. An HCP unit cell contains $6 \mathrm{Zr}$ atoms and 6 octahedral sites [29]. Two of these sites must be filled order to achieve solid solution saturation, a condition that might be expected near the oxide-metal interface in $\alpha$-Zr. In such circumstances oxygen atoms are preferentially sited in order to minimise their higher order bond energies. This ordering is often referred to in the literature as $\mathrm{Zr}_{3} \mathrm{O}$ [15]; although it seems likely that such an arrangement would possess a large degree of variation in stoichiometry. A more suitable description would be as a specific ordered phase, such as $\alpha$ ' Zr in which the process of ordering perturbs the lattice parameters from those of $\alpha-\mathrm{Zr}$ This labelling notation is used by Abriata in Figure 4. In contrast, reported sub-oxide phases such as $\mathrm{ZrO}$ cannot be based on the $\mathrm{HCP} \alpha-\mathrm{Zr}(\mathrm{O})$ as they are well above the solubility limit of oxygen in alpha-zirconium. If such phases exist, they must possess a different crystal structure, although it is likely that it is closely related to the HCP structure and remains equiaxed to it. Tetragonal $\mathrm{ZrO}_{2-\mathrm{x}}$ is also often referred to as a sub-oxide as it is sub- 
stoichiometric. This is misleading; $\mathrm{t}-\mathrm{ZrO}_{2-\mathrm{x}}$ is a well defined form of zirconium dioxide that is stable at high temperature and pressure.

\subsection{Oxidation mechanisms and kinetics}

\subsubsection{Oxide formation}

Oxidation of zirconium proceeds by chemical diffusion of component oxygen from the free surface to the metal-oxide interface [30-32]. Metallographic investigation have shown that a post-breakaway oxide film consists of a black [33], hypo-stoichiometric, highly conductive layer next to the oxide-metal interface, and a white oxide layer with high resistivity near the outer surface [17]. The concept of a barrier layer that consists of a $30 \mathrm{~nm}$ thick dense layer at the metal-oxide interface has been suggested [34]. These authors suggest that a uniform oxide formed at the metal surface is initially a quasi-amorphous sub-stoichiometric oxide, and this oxide crystallises to fine equiaxed tetragonal grains. The mode of growth changes from recrystalisation to preferential crystal growth. This leads to a columnar monoclinic grain structure [34]. Small oxide grains are due to a high nucleation rate [22]. Well developed columnar grains are seen to be related to high corrosion resistance [34].

Mott-Schottky ${ }^{2}$ analysis shows that a passive film formed anodically on zirconium in PWR-like environments is n-type in electronic character, corresponding to a preponderance of oxygen/hydrogen vacancies and/or zirconium interstitials, with the former being likely, in the barrier layer region [35]. The n-type electronic character of the film is consistent with the diagnostic criteria offered by a point defect model [35]. There is a relatively high electron

\footnotetext{
${ }^{2}$ The Mott-Schottky relationship results from an absence of charge rearrangement or interaction at a metal oxide interface. Mott-Schottky analysis can be developed from the comparison of capacitance ${ }^{-2}$ vs. potential curves.
} 
concentration in the barrier layer, which is independent of temperature and originates from oxygen vacancies [17].

The conductivity mechanism of oxide films on tubes of various zirconium alloys grown in water and steam was investigated by Frank [17] using I-V measurements. He asserted that neither the Schottky emission $^{3}$ mechanism nor the Poole-Frenkel ${ }^{4}$ effect could be proved. A high constant carrier concentration with extremely low, but temperature dependent mobility could be proved using the Mott-Gurney ${ }^{5}$ relation. Cox found a complex behaviour of current in Zircaloy-2 oxides when measuring I-V curves, showing that the electronic current fits a Schottky emission process whereas the ionic current follows the $\operatorname{Tafel}^{6}$ relationship [36]. The potential measured on the metal is always negative indicating that electron transport is usually the more difficult, hence rate determining, process [1, 37]. Howlander et al. [37] also measured the electrical conductivity of Zircaloy-2 and 4 oxide films. The current-voltage characteristics of all oxide films showed nonOhmic behaviour where the bulk current (electronic in nature) was associated with the Schottky and/or the Poole-Frenkel processes. Cox argued that the electronic conductivity was controlled by minor alloying elements by comparing the characteristics of unalloyed and alloyed oxides. He concluded that both electrons and ions were equally important in the rate-controlling process. Howlader et al. [37] and Frank [17] concluded that electron conduction dominates the electrical conductivity of zirconium alloy oxide films and so slow-diffusing negative oxygen ions control

\footnotetext{
${ }^{3}$ In Schottky emission electrons are emitted from a metal surface over a potential barrier reduced by the presence of an externally applied electric field.

${ }^{4}$ The Poole-Frenkel effect is an internal Schottky effect where the electrical conductivity of insulators and semiconductors increase in strong electric fields.

${ }^{5}$ The Mott-Gurney relationship relates the theory of space-charge-limited current between plane parallel electrodes. Current density is proportional to free carrier mobility multiplied by the dielectric constant of the oxide, and the applied potential squared, divided by the oxide thickness cubed.

${ }^{6}$ Tafel relates the rate of an electrochemical reaction to its overpotential.
} 
the oxidation process. Tupin et al. [38] describes the process of oxide growth by a mechanism involving oxygen vacancy diffusion, this has been shown by Tupin to provide a good interpretation of the oxidation of Zircaloy-4 [39].

\subsubsection{Nodular corrosion}

As well as the undesirable change in oxidation rate during uniform corrosion, under certain circumstances zirconium alloys also suffers from a localised rapid corrosion, known as nodular corrosion. This has been observed in BWR reactor conditions, but not in PWR. A comparison of PWR and BWR conditions is given in Table 2. The conditions in BWR allow bulk boiling, whereas only localised boiling occurs in PWR. In recent years the corrosion potential in BWR has been reduced to similar levels found in PWR by the addition of hydrogen. Previously, products of radiolysis had increased the potential. Two approaches to the problem of nodular corrosion in alloys have been suggested; one suggestion is that nodular corrosion occurs at SPPs, the other is that corrosion nucleates in solute depleted regions of the matrix [40]. It has been noted that nodules nucleate and grow, but do not tend to increase in number [41], suggesting their occurrence may be correlated with specific nucleation sites.

\begin{tabular}{|c|c|c|c|}
\hline PWR & $\begin{array}{c}\text { Temperature } \\
/{ }^{\circ} \mathrm{C}\end{array}$ & $\begin{array}{c}\text { Pressure } \\
/ \mathrm{MPa}\end{array}$ & Water chemistry (deliberate additions) \\
\hline BWR & $290-360$ & $15.5-17$ & $\mathrm{LiOH}(2.2-0.7 \mathrm{ppm}), \mathrm{H}_{3} \mathrm{BO}_{3}(1600-500 \mathrm{ppm})$, \\
$\mathrm{H}_{2}(3.5 \mathrm{ppm})$
\end{tabular}

Table 2: Summary of conditions in PWR and BWR reactors, adapted from [41]. 


\subsubsection{Uniform oxidation kinetics}

The uniform oxidation behaviour is often described by Equation 1. Where the increase in mass, $\Delta m$, is proportional to the increase in cladding thickness, $\xi, k$ is a rate constant, the exponent, $n$, is determined experimentally and $t$ is time [42].

$$
(\Delta m)^{n}=k t
$$

The rate of oxidation is a function of composition, thermal history and temperature of oxidation. In commercially pure zirconium and in many dilute zirconium alloy systems the oxidation kinetics are characterised by a two-stage process. The first regime is most clearly seen in Figure 5 curve (b), and the first half of Figure 5 curve (a). The rate follows a kinetics curve that has been fitted between a cubic and parabolic rate. It is suggested that during pre-transition oxide formation the diffusion of oxygen through the existing oxide layer is the rate limiting factor [22]. A parabolic rate is expected for oxidation due to the diffusion of oxygen through the bulk material [43], a thermally activated process. However, the pre-transition kinetics at reactor temperature $\left(300-360^{\circ} \mathrm{C}\right)$ have repeatedly shown an approximately cubic rate law [44], $\mathrm{n} \sim 3$, rather than the parabolic rate, $\mathrm{n} \sim 2$, predicted for a process controlled by bulk diffusion of oxygen through the oxide [1].

Cox [45] postulated that stress has an effect on corrosion behaviour. This was later explained by Rudling [46] and Yoo et al. [30], who suggest that a better mechanism for the understanding of zirconium oxidation can be attained by considering the stress gradient at the metal-oxide interface as well as the chemical potential, where the mechanically driven diffusion opposes the chemically driven diffusion. This causes the overall kinetics to deviate from the parabolic rate law, as observed experimentally. 


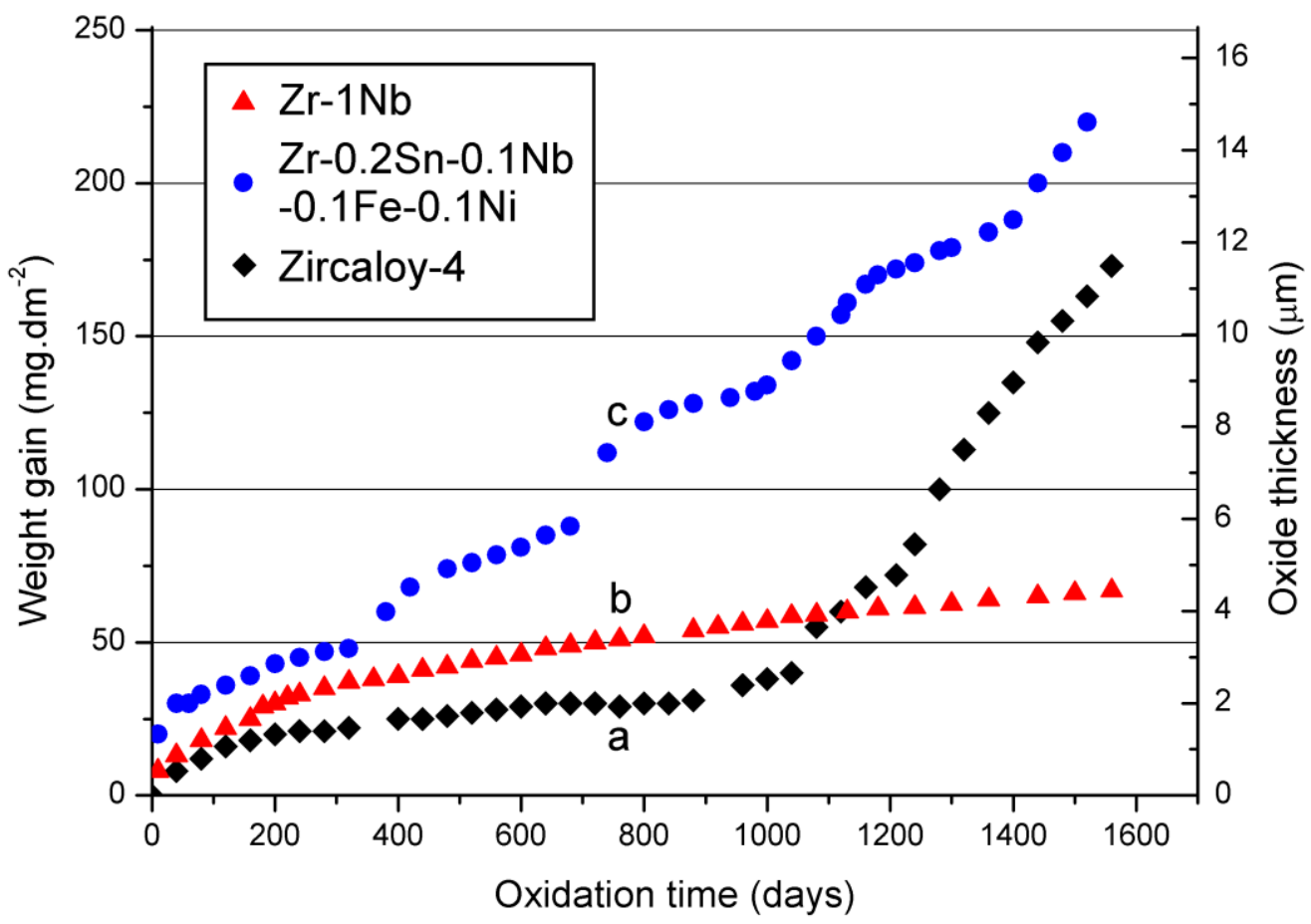

Figure 5: The oxidation kinetics of three zirconium-based alloys in a PWR-type environment (350 ${ }^{\circ} \mathrm{C}, 17 \mathrm{MPa}$ water with PWR-like additions). a) Zircaloy-4 (black squares); b) Zr - 1 wt.\% $\mathrm{Nb}$ (red circles); c) Ozhenite, a $\mathrm{Zr}-0.2 \mathrm{Sn}-0.1 \mathrm{Nb}-0.1 \mathrm{Fe}-0.1 \mathrm{Ni}$ (all wt. \%) alloy (blue triangles). Re-plotted from experimental data [47].

After the pseudo-parabolic pre-transition period [48], a kinetic transition occurs when the thickness of the oxide layer exceeds a critical, material specific, value. Figure 5 curve (a) demonstrates this transition in oxidation kinetics after $\sim 1000$ days. After this transition the scale takes on a periodic structure with a linear overall rate, similar to that most clearly seen in Figure 5 curve (c). This regime is known as 'breakaway oxidation' due to the increase in oxidation rate.

\subsubsection{Breakaway mechanisms}

In general, it is not possible to grow oxide films thicker than two micrometres without a change in the oxidation kinetics to either an approximately linear or a cyclic kinetic stage (post- 
transition). The mechanism for the breakaway transition is not entirely understood, with arguments within the literature about the relative importance of different observed effects that have been correlated with transition. These effects are summarised in Table 3 .

\begin{tabular}{|c|c|}
\hline Observation & Explanation \\
\hline $\begin{array}{l}\text { Tetragonal to } \\
\text { monoclinic } \\
\text { oxide } \\
\text { transformation }\end{array}$ & $\begin{array}{l}\text { There is much interest in the effect of the oxide structure on its } \\
\text { morphology [20, 49-53]. The t/m phase ratio is observed to } \\
\text { reduce as oxidation proceeds in PWR conditions [52], indicating } \\
\text { either a change in growth mechanism or a degradation of t- } \\
\text { phase to m-phase oxide. The monoclinic to tetragonal phase } \\
\text { transition results in a volume contraction of between } 3-5 \% \text { at } 1 \\
\text { bar [54] due to the higher density of the tetragonal phase [55]. } \\
\text { This transformation allows for the accommodation of some of } \\
\text { the stresses built up at the metal-oxide interface by oxide } \\
\text { growth. Studies of } t \rightarrow \text { m degradation have reported that the } \\
\text { transition is governed by a martensitic transformation, resulting } \\
\text { in micro-cracks around twin boundaries [45]. There is evidence } \\
\text { from XRD of cyclic variations in the levels of tetragonal and } \\
\text { monoclinic oxide across the oxide thickness with a definite fixed } \\
\text { period [56]. }\end{array}$ \\
\hline $\begin{array}{l}\text { Formation of } \\
\text { cracks by purely } \\
\text { mechanical } \\
\text { breakdown }\end{array}$ & $\begin{array}{l}\text { The transition between kinetic regimes is associated with the } \\
\text { appearance of cracks and pores in the oxide layer }[39,57-58] \text {. } \\
\text { The arguments are whether the cyclic behaviour is caused by } \\
\text { cracks normal to the oxide surface that can pass most of the } \\
\text { way through the oxide [59], or by fine pores that form a network } \\
\text { within the oxide [60]. A purely mechanical breakdown of the } \\
\text { material by stresses built up by oxide development would likely } \\
\text { cause mechanical failure by crack development perpendicular to } \\
\text { the metal-oxide interface. Such cracking is not typically seen, } \\
\text { and when it is, is usually attributed to poor specimen preparation } \\
\text { techniques. Cracks are frequently seen parallel with the metal } \\
\text { oxide interface, but have not be convincingly shown to form a } \\
\text { network in three dimensions }\end{array}$ \\
\hline $\begin{array}{c}\text { Coalescence of } \\
\text { pores within the } \\
\text { oxide }\end{array}$ & $\begin{array}{c}\text { Recent TEM work has shown that nanometre-scale pore } \\
\text { coalescence can be correlated with the different stages in the } \\
\text { corrosion process [14], and that these are sites of high local } \\
\text { stress may give rise to crack initiation [61]. It is suggested that } \\
\text { pores nucleate at the metal oxide interface and develop to form } \\
\text { paths for enhanced oxidising species diffusion as oxidation } \\
\text { progresses [14]. }\end{array}$ \\
\hline
\end{tabular}

Table 3: Proposed mechanisms by which breakaway occurs. 


\subsection{Zirconium alloys}

\subsubsection{Summary of alloy development and usage}

A great variety of zirconium-based alloys have been developed since the first zirconium clad fuel rods. The two primary alloy systems are $\mathrm{Zr}-\mathrm{Sn}$ and $\mathrm{Zr}-\mathrm{Nb}$ with the tin based alloy being favoured initially America and Western Europe and the niobium-based alloy principally developed in the former USSR and later in the Canadian CANDU pressurised heavy water reactors [62]. In both of these alloy groups, zirconium comprises a minimum of $97.5 \mathrm{wt} . \%$ of the bulk material. Minor elements used as alloying additions in these two families include $\mathrm{Fe}, \mathrm{Cr}, \mathrm{Ni}, \mathrm{V}, \mathrm{Mo}$, and $\mathrm{Cu}$. Some values for the alloying additions used in specific instances of the major industrial zirconium alloys are given in Table 4.

In western literature, the Zircaloy family developed from tin-based alloys with a variety of carefully controlled and restricted alloying additions. All but Zircaloy-2 (Zry-2) and Zircaloy-4 (Zry-4) are now redundant. Zry-2 is mainly used in fuel cladding in boiling water reactors (BWR). Zry-4 is used in fuel cladding and for fuel components in pressurised water reactors (PWR), BWR and cladding in heavy water reactor (HWR) applications [40]. Table 4 shows that the primary differences between Zry-4 and Zry-2 are that Zry-4 contains slightly higher levels of iron and no nickel.

Niobium containing alloys used for cladding materials in PWR usually contain around $1 \mathrm{wt} . \%$ niobium with fewer additions, such as E110 and M5. Zr-2.5Nb is mainly used as pressure tubing in heavy water reactors (HWR) [40]. 


\begin{tabular}{|c|c|c|c|c|c|c|c|c|}
\hline Material & $\mathrm{Sn}$ & $\mathrm{Nb}$ & $\mathrm{Fe}$ & $\mathrm{Cr}$ & $\mathrm{Ni}$ & $\mathrm{O}$ & $\mathrm{Zr}$ & Ref \\
\hline Zircaloy-2 & 1.32 & - & 0.17 & 0.10 & 0.07 & $\mathrm{NR}$ & balance & {$[37]$} \\
& 1.5 & - & 0.12 & 0.1 & 0.05 & $\mathrm{NR}$ & balance & {$[63]$} \\
& $1.3-$ & - & $0.12-$ & $0.09-$ & $0.04-$ & $\mathrm{NR}$ & Balance & {$[64]$} \\
& 1.6 & & 0.16 & 0.12 & 0.06 & & & \\
\hline 'Improved' & 1.48 & - & 0.23 & 0.10 & 0.09 & $\mathrm{NR}$ & balance & {$[37]$} \\
\hline Zircaloy-2 & & & & & & & & \\
\hline Zircaloy-4 & 1.29 & - & 0.21 & 0.11 & - & $\mathrm{NR}$ & balance & {$[37]$} \\
& 1.52 & - & 0.22 & 0.11 & - & 0.13 & balance & {$[65]$} \\
& $1.20-$ & - & $0.12-$ & $0.05-$ & - & $\mathrm{NR}$ & balance & {$[63]$} \\
& 1.74 & & 0.18 & 0.15 & & & & \\
& $1.3-$ & - & $0.19-$ & $0.09-$ & - & $\mathrm{NR}$ & balance & {$[64]$} \\
& 1.6 & & 0.23 & 0.11 & & & & \\
\hline M5 & - & 1.0 & - & - & - & 0.1 & balance & {$[66]$} \\
\hline E110 & - & 0.97 & 0.01 & - & - & 0.05 & balance & {$[65]$} \\
\hline ZIRLO® & 0.99 & 0.98 & 0.11 & 0.03 & 0.01 & NR & balance & {$[40]$} \\
& 1.0 & 1.0 & 0.1 & $\mathrm{NR}$ & - & 0 & balance & {$[67]$} \\
\hline E635 & $1.0-$ & 1 & $0.35-$ & - & - & NR & balance & {$[68]$} \\
& 1.3 & & 0.4 & & & & & \\
\hline
\end{tabular}

Table 4: Typical compositions of common zirconium alloys. All in wt.\%. NR: Not recorded.

In the 1990s a new alloy group was developed that combined both tin and niobium as major alloying additions. ZIRLO ${ }^{\circledR}$ is the most widely used $\mathrm{Zr}-\mathrm{Sn}-\mathrm{Nb}$ alloy [62]. ZIRLO is used in structural and cladding applications in PWRs [40] and has generally supplanted Zircaloy-4; under standard PWR conditions ZIRLO is seen to have improved corrosion resistance over Zry-4 $[15,56,69]$.

\subsubsection{Significant intermetallic phases}

Pure zirconium undergoes a phase transformation at $863{ }^{\circ} \mathrm{C}$ [19]. The $\alpha$-phase, a hexagonal close-packed structure with lattice parameters of $\mathrm{a}=0.32312 \mathrm{~nm}$ and $\mathrm{c}=0.51477 \mathrm{~nm}$ [63], exists below this temperature. Above the transition point the metal adopts a body-centred cubic structure $(\beta$-phase) with lattice parameter $\mathrm{a}=0.36090 \mathrm{~nm}[63]$. The $\beta$-phase of the pure metal melts at $1855^{\circ} \mathrm{C}$ [19]. The majority of alloying additions tend to be more soluble in this $\beta$-phase, and this is discussed further in section 2.6. 
$\alpha$-zirconium has a very low solubility for most transition metals [70], but it also has a high affinity for these metals, and so the formation of second phase particles (SPPs) in favoured. The common phases found in the major alloys are listed in Table 5. The 2009 review by Tedenac and Perrot [71] describes the Zr-Nb-Fe precipitate literature in further detail. Table 6 details the observation of all these, and other minor phases, in various common alloys. The SPPs containing $\mathrm{Fe}, \mathrm{Cr}$ and Ni can only occupy a volume fraction of less than $0.3 \%$ in most alloys, assuming that almost all the transition metals are precipitated out of the matrix. $\beta-\mathrm{Nb}$ can be expected to have a higher volume fraction of up to $0.4 \%$ based on the maximum solid solution level of niobium remaining in solution.

The composition, size and distribution of intermetallics are functions of the bulk chemical composition of the material and thermal history. The differences in the character of the intermetallics are a major factor in the quality of the material in terms of its corrosion resistance and performance. In particular, it has been reported that a fine distribution of precipitates is the main reason for the good corrosion resistance of ZIRLO $[67,72]$ in PWR conditions.

\begin{tabular}{|c|c|c|c|c|c|c|c|}
\hline Phase & Symmetry & $\begin{array}{l}\text { Space } \\
\text { group }\end{array}$ & $\begin{array}{l}\text { Atoms } \\
\text { per unit } \\
\text { cell }\end{array}$ & $\begin{array}{c}\mathrm{a} \\
(\mathrm{nm})\end{array}$ & $\begin{array}{c}\mathrm{C} \\
(\mathrm{nm})\end{array}$ & $\begin{array}{l}\text { Unit cell } \\
\text { volume } \\
\left(\mathrm{nm}^{3}\right)\end{array}$ & $\begin{array}{l}\text { Density } \\
\left(\mathrm{g} \cdot \mathrm{cm}^{-3}\right)\end{array}$ \\
\hline$\alpha-Z r$ & Hexagonal & - & $1 \cdot 6$ & 0.3231 & 0.5148 & 0.14 & 6.51 \\
\hline$\beta-\mathrm{Nb}$ & Cubic & - & $1 \cdot 2$ & 0.33 & - & 0.036 & 8.59 \\
\hline $\mathrm{Zr}(\mathrm{Nb}, \mathrm{Fe})_{2}$ & Hexagonal & $\mathrm{P} 63 / \mathrm{mmc}$ & $3 \cdot 12$ & 0.5366 & 0.8792 & 0.658 & 7.5 \\
\hline$(\mathrm{Zr}, \mathrm{Nb})_{3} \mathrm{Fe}$ & Orthorhombic & - & - & 0.88 & 0.33 & 1.10 & - \\
\hline$(\mathrm{Zr}, \mathrm{Nb})_{4} \mathrm{Fe}_{2}$ & Cubic & $\begin{array}{l}\text { Fm-3m } \\
\text { or F-43m }\end{array}$ & $6 \cdot 16$ & 1.215 & 1.794 & - & 7.1 \\
\hline \multirow[t]{2}{*}{$\mathrm{Zr}(\mathrm{Fe}, \mathrm{Cr})_{2}$} & $\begin{array}{l}\text { Hexagonal } \\
\left(\mathrm{T}<994^{\circ} \mathrm{C}\right)\end{array}$ & $\mathrm{P} 63 / \mathrm{mmc}$ & $3 \cdot 12$ & 0.505 & 0.825 & 0.558 & 7.12 \\
\hline & $\begin{array}{c}\text { Cubic } \\
\left(T>994^{\circ} \mathrm{C}\right)\end{array}$ & $\mathrm{Fd}-3 \mathrm{~m}$ & $4 \cdot 3$ & 0.72 & 0.373 & - & 6.98 \\
\hline $\mathrm{Zr}_{2}(\mathrm{Fe}, \mathrm{Ni})$ & Tetragonal & $14 / \mathrm{mcm}$ & $4 \cdot 3$ & 0.6385 & 0.5596 & 0.228 & 6.94 \\
\hline
\end{tabular}

Table 5: Common phases in zirconium alloys. Amended from [73] using data from [74] and data regarding the orthorhombic phase from [75]. 
Most precipitates in the $\mathrm{Zr}-\mathrm{Sn}-\mathrm{Nb}-\mathrm{Fe}-\mathrm{Cr}$ alloy used by Liu et al. [75] are $\mathrm{HCP} \mathrm{Zr}(\mathrm{Nb}, \mathrm{Fe})_{2}$, $\mathrm{Zr}(\mathrm{Nb}, \mathrm{Fe})_{2}$ precipitates were found in material containing $0.8 \mathrm{wt} . \% \mathrm{Nb}$, whereas $\beta$ - $\mathrm{Nb}$ particles were observed in materials containing higher ratios of niobium [76]. The distribution of elements for each iron-containing $\beta$-Nb particle varied [77]. The majority of $\beta-\mathrm{Nb}$ particles consist of $\mathrm{Zr}$, $\mathrm{Nb}$ and $\mathrm{Fe}$ or $\mathrm{Cr}$, although some contain only $\mathrm{Zr}$ and $\mathrm{Nb}$ in detectable quantities.

\begin{tabular}{|c|c|c|}
\hline Material & $\begin{array}{c}\text { Intermetallic phases } \\
\text { observed }\end{array}$ & Ref. \\
\hline Zircaloy-4 & $\begin{array}{c}\mathrm{Zr}(\mathrm{Fe}, \mathrm{Cr})_{2} \\
\mathrm{ZrCr}_{2}\end{array}$ & {$[75]$} \\
\hline Zircaloy-2 & $\begin{array}{c}\mathrm{Zr}(\mathrm{Fe}, \mathrm{Cr})_{2} \\
\mathrm{Zr}_{2}(\mathrm{Fe}, \mathrm{Ni})\end{array}$ & {$[75,78]$} \\
\hline Zr-Sn-Nb-Fe-Cr & $\begin{array}{c}\mathrm{Zr}(\mathrm{Fe}, \mathrm{Cr})_{2} \\
\mathrm{Zr}(\mathrm{Nb}, \mathrm{Fe}, \mathrm{Cr})_{2}\end{array}$ & {$[79]$} \\
\hline ZIRLO® & $\begin{array}{c}\beta-\mathrm{Nb} \\
\mathrm{Zr}(\mathrm{Nb}, \mathrm{Fe})_{2}\end{array}$ & {$[75]$} \\
\hline $\mathrm{Zr}-1 \mathrm{Nb}$ & $\begin{array}{c}\beta-\mathrm{Nb} \\
\mathrm{Zr}(\mathrm{Nb}, \mathrm{Fe})_{2}\end{array}$ & \\
\hline $\mathrm{Zr}(\mathrm{Nb}, \mathrm{Fe})_{2}$ & {$[75]$} \\
& $\begin{array}{c}\mathrm{Zr}, \mathrm{Nb})_{2} \mathrm{Fe} \\
(\mathrm{Zr}, \mathrm{Nb})_{3} \mathrm{Fe}{ }^{*}\end{array}$ & \\
\hline
\end{tabular}

Table 6: The phases present in various zirconium based alloys, listed in order of abundance.

Zircaloy-2 may respond differently than Zircaloy-4 to reduced matrix solute composition because $\mathrm{Zr}_{2}(\mathrm{Fe}, \mathrm{Ni})$ has a different oxidation rate to $\mathrm{Zr}(\mathrm{Fe}, \mathrm{Cr})_{2}[40]$. $\mathrm{Zr}(\mathrm{Fe}, \mathrm{Cr})_{2}$ oxidises more slowly than $\alpha-\mathrm{Zr}$ in Zircaloy systems. This is expected as $\mathrm{Fe}$ and $\mathrm{Cr}$ are more noble than $\mathrm{Zr}$ and are reduced by un-oxidised $\mathrm{Zr}$ in their vicinity [40]. When oxidised this phase was reported to transform to an orthorhombic structure from hexagonal close packed with little effect on the surrounding oxide morphology [50]. In instances of the material not containing chromium, $\mathrm{Zr}_{2} \mathrm{Fe}$ is formed by slow quenching but is metastable at low temperatures. $\mathrm{Zr}_{3} \mathrm{Fe}$ is formed by more rapid quenching [80]. At equilibrium $\mathrm{Zr}_{3} \mathrm{Fe}$ can dissolve up to $12 \%$ nickel [80], presumably as $\mathrm{Zr}_{3}(\mathrm{Fe}, \mathrm{Ni})$. 


\subsection{Material processing considerations}

\subsubsection{Annealing temperature, time and cold working}

Measuring the size distribution of intermetallics is not a convenient parameter for controlling manufacture. This has prompted the development of a normalised annealing parameter, $\sum A_{i}$, which is generally considered to be a reflection of second phase particle distribution [40]. This allows the comparison of materials treated at different temperatures and time periods [51]. Thorvaldsson et al. [81] proposed that the corrosion resistance of Zircaloy-4 can be related to this accumulated annealing parameter, which is an index of the total amount of heat treatment received in the $\alpha$-region after the last $\beta$-quench treatment [79]. The formulation of the parameter is given in Equation 2, where $T$ is temperature and $t$ time of anneal $i, Q$ is the activation energy and $R$ is the ideal gas constant. A typical value for $\mathrm{Q} / \mathrm{R} \sim 40,000 \mathrm{~K}$ [41].

$$
\sum_{i} A_{i}=\sum_{i} t_{i} e^{\left(\frac{-Q}{R T_{i}}\right)}
$$

Equation 2 has a number of drawbacks, the first being that it is only valid after the last $\beta$ treatment, a fact that has caused confusion in some literature. The parameter is also not independent of the $\beta$-quench that preceded the alpha phase annealing, especially the cooling rate [40]. The assumption that a high temperature, short time anneal is equivalent to a low temperature, long time anneal is not always valid, especially in extremes [82]. Most importantly, the annealing parameter, $\sum A_{i}$, is not affected by cold working. However, corrosion resistance increases after cold working. This effect is probably due to work hardening increasing the yield strength of the substrate [83]. It must also be considered that cold working increases the rate of approach towards phase equilibrium [84]. So, use of the normalised annealing parameter is 
limited in certain circumstances. More complex heating programmes with multiple $\beta$-quenches or significant cold working are not suitably described by the parameter [79].

The final treatment stage in the fabrication process is usually performed within two temperature ranges. A stress relieved state (SR) is mostly used for Zry-4 in PWR. It consists of a final treatment of $475{ }^{\circ} \mathrm{C}$ for 2 hours under a protective atmosphere after which the grain shape remains elongated and highly deformed from previous cold rolling cycles [41]. The recrystallised state (RX) uses a higher heat treatment of $550-600{ }^{\circ} \mathrm{C}$ with the effect of reducing dislocation density and producing equiaxed grains [41]. It has been found that crystal texture strongly affects the corrosion resistance of cladding. A high proportion of basal poles orientated in the direction of the sheet, perpendicular to the rolling direction, provides the lowest corrosion weight gain [83, 85]. Charquet et al [86] noted also that corrosion resistance is considerably improved when the (0002) poles are close to the sheet normal. However, the textures of cladding tubes are relatively independent of fabrication method and basal poles do not change orientation significantly with annealing [87].

\subsubsection{Beta-quenching}

Whereas $\alpha$-annealing predominantly affects SPP size and distribution in the matrix, beta quenching tends to have a far more severe implication for the materials microstructure, as well as affecting the SPP properties. An obvious expectation for this process is that quenching might leave $\mathrm{Fe}, \mathrm{Cr}$ and $\mathrm{Ni}$ in solid solution of alloys containing these constituents, and this has been found to be the case [40].

A comparison of the microstructure of oxide films of sub-micron thickness after $\beta$-quench and $\alpha$-anneal heat treatments is given in Figure 6. The schematic suggests possible changes in the electronic properties of the oxide that may affect its further growth. Intermetallics in the oxide 
retain their metallic nature, before themselves becoming oxidised, as previously discussed. The alloying element distributions in the oxide have important effects on the oxidation rate of the alloy [88]. The electrical resistivity of the oxide film on Zry-2 tends to increase with an increase in nodular corrosion resistance [89], and $\beta$-quench samples have the highest resistivity. Figure 6 offers a qualitative explanation for this, where the smaller SPP distribution presents a series of barriers to electron conduction through the oxide.

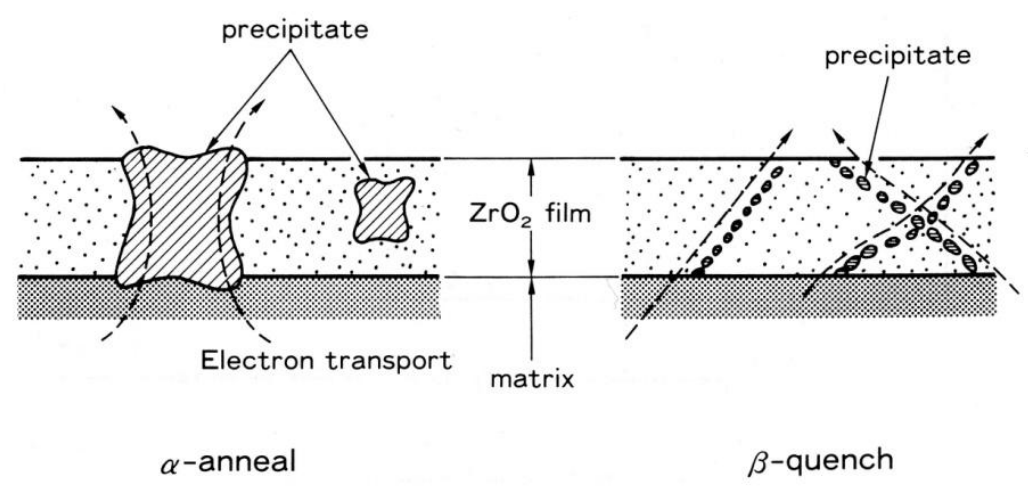

Figure 6: A schematic of microstructures in oxide films [89] . Larger precipitates in $\alpha$-annealed material act as continuous condition paths for electrons through the oxide layer. The fine precipitate distribution in $\beta$-quenched material leads to regions of high resistivity between the embedded metallic particles in the oxide layer.

The $\beta$ to $\alpha$ phase transformation in Zircaloy commonly yields a Widmanstätten microstructure [90]. This microstructure can manifest itself in two types of morphology, referred to as the basket-weave [90] and parallel-plate structures, as noted by Ökvist and Källström in Zry-2 [91]. The type of structure produced appears to be dependent on the rate of cooling. Holt [92-93] found that by increasing the cooling rate of Zry-4 from 2 to $200 \mathrm{Ks}^{-1}$ finer Widmanstätten plates are produced, while cooling at $2000 \mathrm{Ks}^{-1}$ produced quenched martensitic structure. Wadekar [94] 
used water quenching with an estimated cooling rate of $300 \mathrm{Ks}^{-1}$ to achieve a martensitic structure in a binary Zr-Sn alloy.

It was found that $\beta$-quenched Zircaloy cladding had more tetragonal oxide close to the metaloxide interface than material with increased annealing parameter [40]. The authors suggest that this is likely to be due to the decomposition of the oxide to a more stable form after annealing. $\beta$ phase grain size in the metal is correlated to the holding time in $\beta$-phase prior to quenching, and $\alpha$-phase plate width is correlated to the cooling rate of quenching from the beta phase [90].

\subsection{Effect of alloying elements in zirconium alloys}

\subsubsection{Tin}

Figure 7 demonstrates the moderate solubility of tin in $\alpha-\mathrm{Zr}$; it is unsurprising that there are no common tin-containing SPPs in any dilute $\mathrm{Zr}$-Sn systems. Up to $1 \% \mathrm{Sn}$ there is no influence of tin on the SPP nature and composition $[50,73,84]$, and up to $1.3 \mathrm{wt} . \%$ tin does not influence the ternary phase diagram $\mathrm{Zr}-\mathrm{Nb}-\mathrm{Fe}$ in a significant way [95].

Tin is a solid solution strengthener and $\alpha-\operatorname{Zr}$ stabiliser [90, 94]. Tin may also stabilise monoclinic zirconium dioxide [50]. There is disagreement in the literature as to the ideal level of tin for optimised corrosion resistance. Sell et al. [64] found their ideal value to be 1.1-1.7 wt.\% tin, whereas a lower level of tin of about $0.5 \mathrm{wt} . \%$ was found to be preferable for corrosion resistance in a system with $0.2 \mathrm{wt} \%$ added niobium, although mechanical properties were degraded [96]. Most authors state that using lower concentrations of tin in the Zircaloy family has the effect of improving corrosion resistance, but that this is detrimental to mechanical properties such as the yield stress and creep resistance [62, 97]. A similar effect has been 
observed in ZIRLO, although a minimum level of tin was required [98]. Qualitatively, the work hardening behaviour and tensile test curve of $\mathrm{Zr}$-Sn alloys was similar for alloys with tin concentration in the range $0.5-1.5$ wt. $\%$ [94] and a linear relationship between $0.2 \%$ yield stress and tin content to the power of $2 / 3$ has been observed [94]. This relationship suggests the operation of the Mott-Nabarro model of solid solution strengthening in which clustering of solute atoms is envisaged. These clusters are considered to be an effective obstacle against dislocation motion. Sn clusters are observed in TEM images of $\beta$-quenched alloys [94].

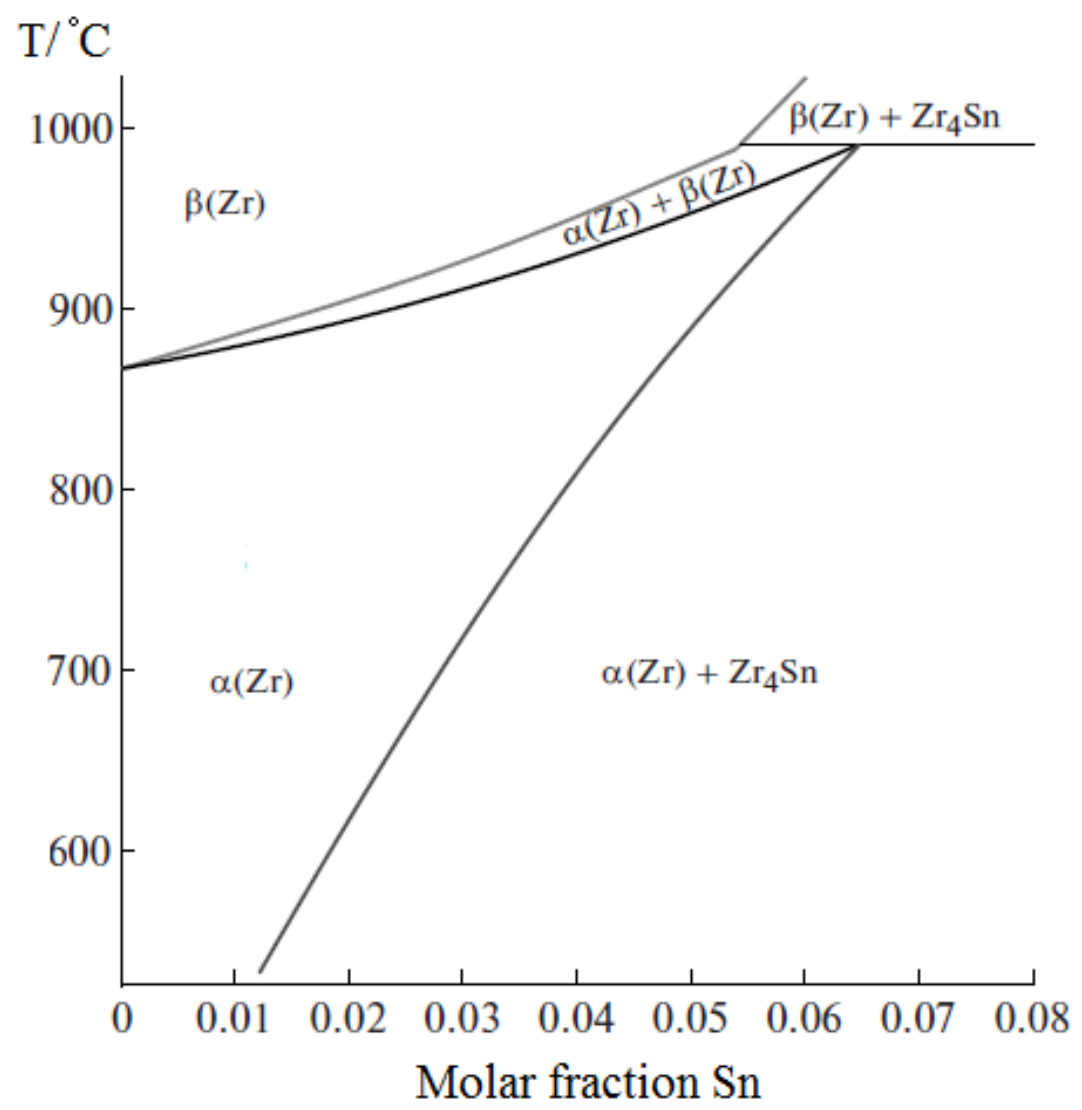

Figure 7: Partial phase diagram of the zirconium-rich region of the binary Zr-Sn system [99]. 


\subsubsection{Niobium, vanadium and molybdenum}

A calculated phase diagram for the $\mathrm{Zr}-\mathrm{Nb}$ and $\mathrm{Zr}-\mathrm{Nb}-\mathrm{O}$ systems is given in Figure 8 [95]. The maximum solubility limit of niobium in the matrix of $\mathrm{Zr}-\mathrm{Nb}$ alloy is $0.6 \%$ [79], this can be seen at around $900 \mathrm{~K}$ in Figure 8 . Other authors' TEM investigation of a ternary Zr-Nb-Fe(-O) system have separately shown the solubility of niobium in alpha zirconium to be $0.37 \pm 0.05 \mathrm{wt} . \%$ [73], much lower than the usual value for Zr-Nb. Niobium acts as a $\beta$-stabiliser [100], an effect that can be counteracted by the addition of the $\alpha$-stabiliser. The addition of the $\alpha$-stabiliser oxygen in Figure 8 reduces the solubility of niobium in the $\alpha$-phase and increasing the $\alpha / \beta$ transition temperature.

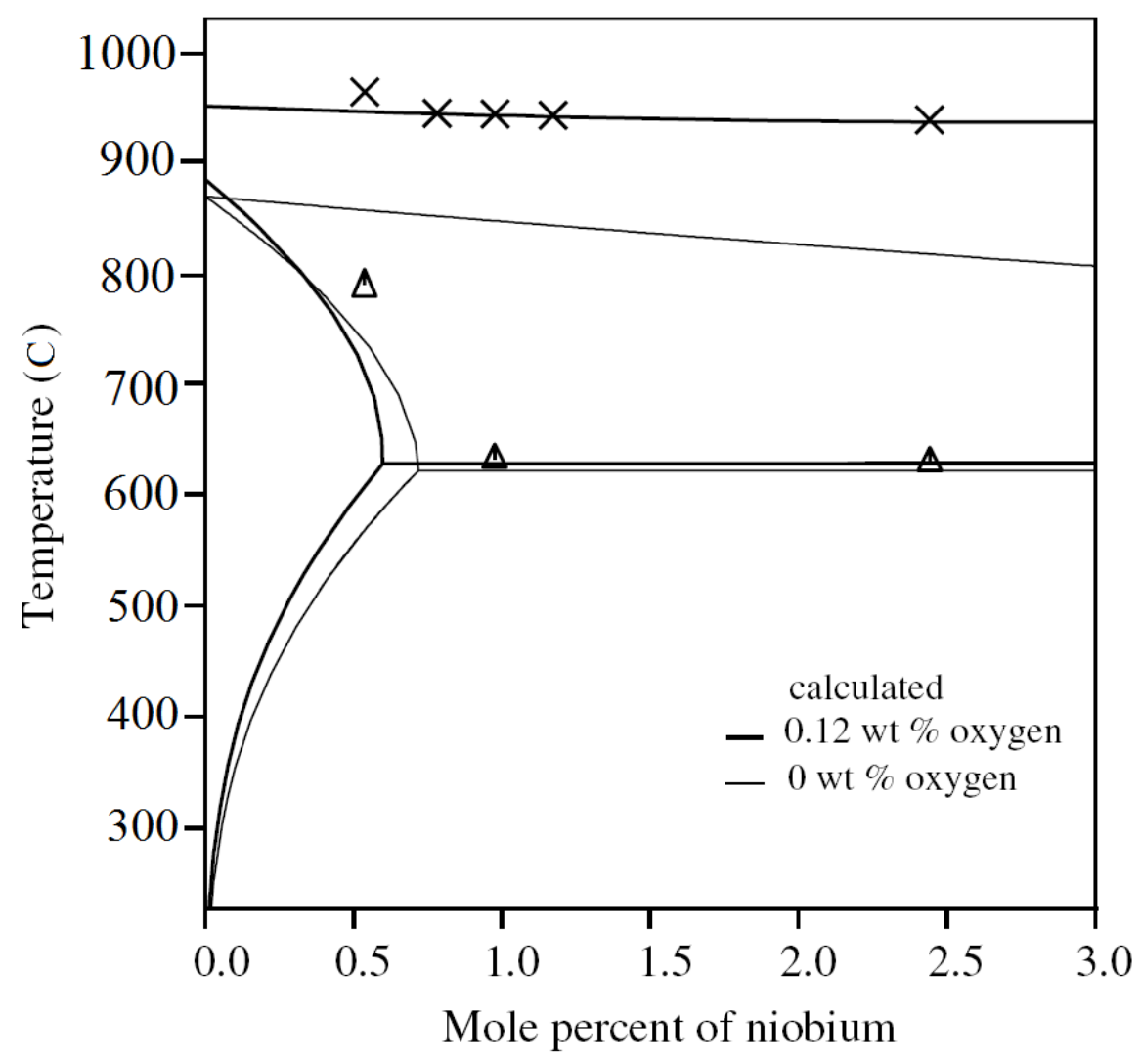

Figure 8: Calculated isoplethal section of $\mathrm{Zr}-\mathrm{Nb}-0.12 w t . \% \mathrm{O}$ (black line) and $\mathrm{Zr}-\mathrm{Nb}$ (grey line) phase diagrams as calculated by Thermo-Calc [101]. Experimental data: Triangle: $a \rightarrow(a+b)$ and cross: $(a+b) \rightarrow$ b. Adapted from [95]. 
The corrosion resistance of niobium-containing alloys has been seen to be controlled by the $\mathrm{Nb}$ solubility in $\alpha-Z r$ and the type of $\beta$-phases, which are determined by the annealing temperature [76]. Zr-Nb alloys containing niobium only in solid solution showed a good corrosion resistance and their corrosion resistance was not affected by the annealing temperature. In the middle range of niobium content, $0.5-0.8 \mathrm{wt} . \% \mathrm{Nb}$, where niobium-containing precipitate were formed in the matrix, the corrosion resistance was increased by increasing the niobium content. In the high $\mathrm{Nb}$ content of 1.0-2.0 wt.\%, the corrosion rate was sensitive to the type of the second phase formed which was determined by the annealing temperature [76]. A comparison of the microstructure of various binary $\mathrm{Zr}-\mathrm{Nb}$ alloys is given in Figure 9. The niobium content can be visually correlated to number density and size of SPPs. Sabol et al. [62] compared the corrosion resistance of 0.5-1 wt.\% $\mathrm{Nb}$ alloys and concluded that their results strongly supported the significance of matrix composition in controlling alloy corrosion. After aging was completed about $0.5 \%$ niobium was retained in the matrix [62], this is comparable to the maximum solubility of $\mathrm{Nb}$ in $\alpha-\mathrm{Zr}$ from the phase diagram in Figure 8. Deformation of $\alpha-\mathrm{Zr}$ at high temperature produces dislocation networks. This increases the diffusivity of niobium within the matrix, which is normally very sluggish. Above $573 \mathrm{~K}$ strain has been seen to induce $\beta-\mathrm{Nb}$ precipitation [102].

Another general trend is that increasing the $\mathrm{Nb}$ and Fe content decreases the grain size [73]. As we know from the Hall-Petch relationship, this has beneficial effects on the macroscopic mechanical properties of a material. A qualitative demonstration of the decrease in grain size with increased niobium content can be seen in Figure 10.

The addition of molybdenum and vanadium to zirconium-based alloys to Zry-4 improves the mechanical [96] and corrosion [62] properties, as long as thermal processing allows the additions to be precipitated out of the matrix. Despite these advantages it was found that the same results could be obtained using niobium, but with a less sensitivity to thermal history [62], which is advantageous in terms of allowing greater flexibility in production techniques. 


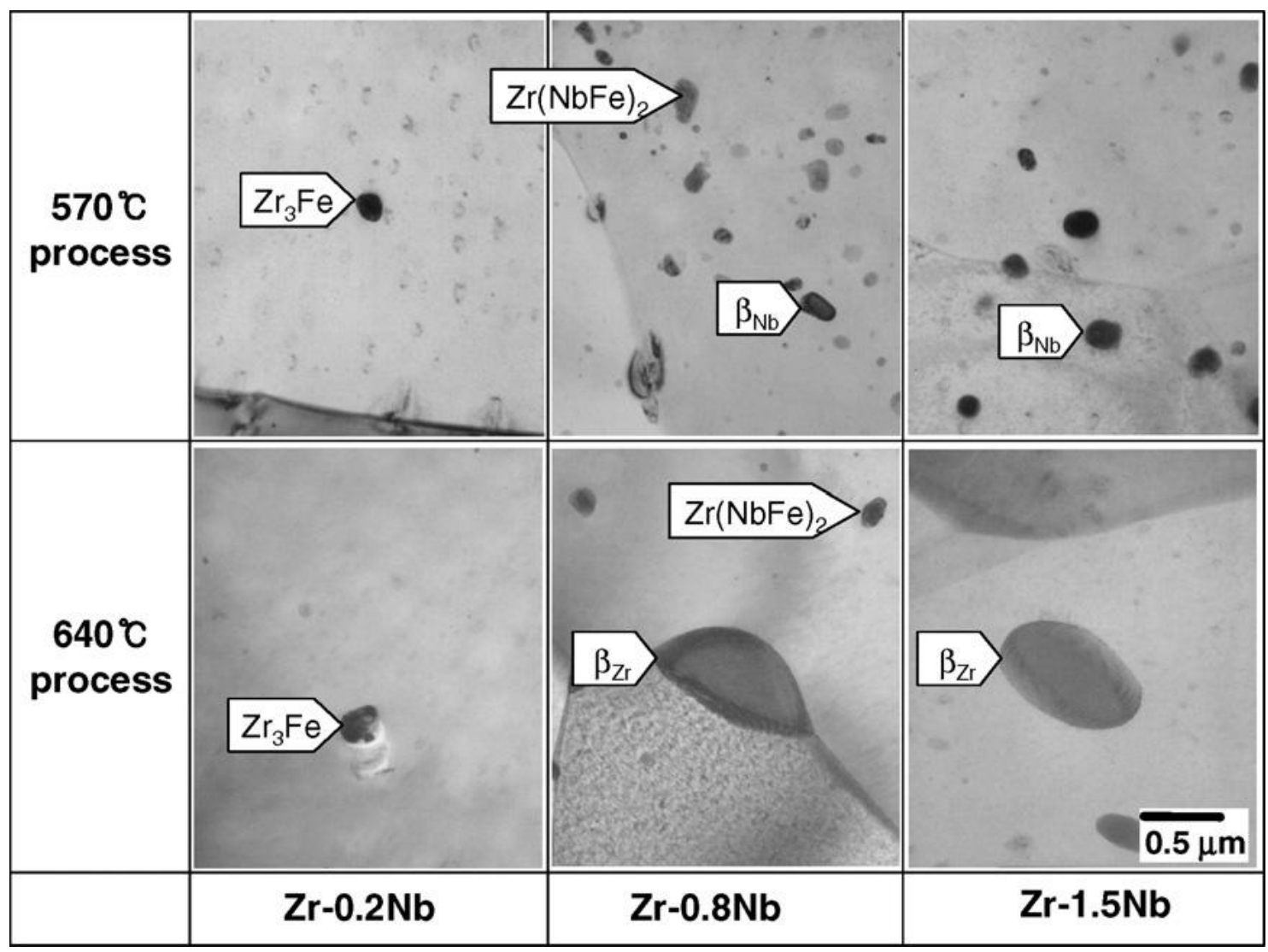

Figure 9: TEM micrographs of $\mathrm{Zr}-\mathrm{Nb}$ annealed at (570 / 640) ${ }^{\circ} \mathrm{C}$ with various $\mathrm{Nb}$ content [76].
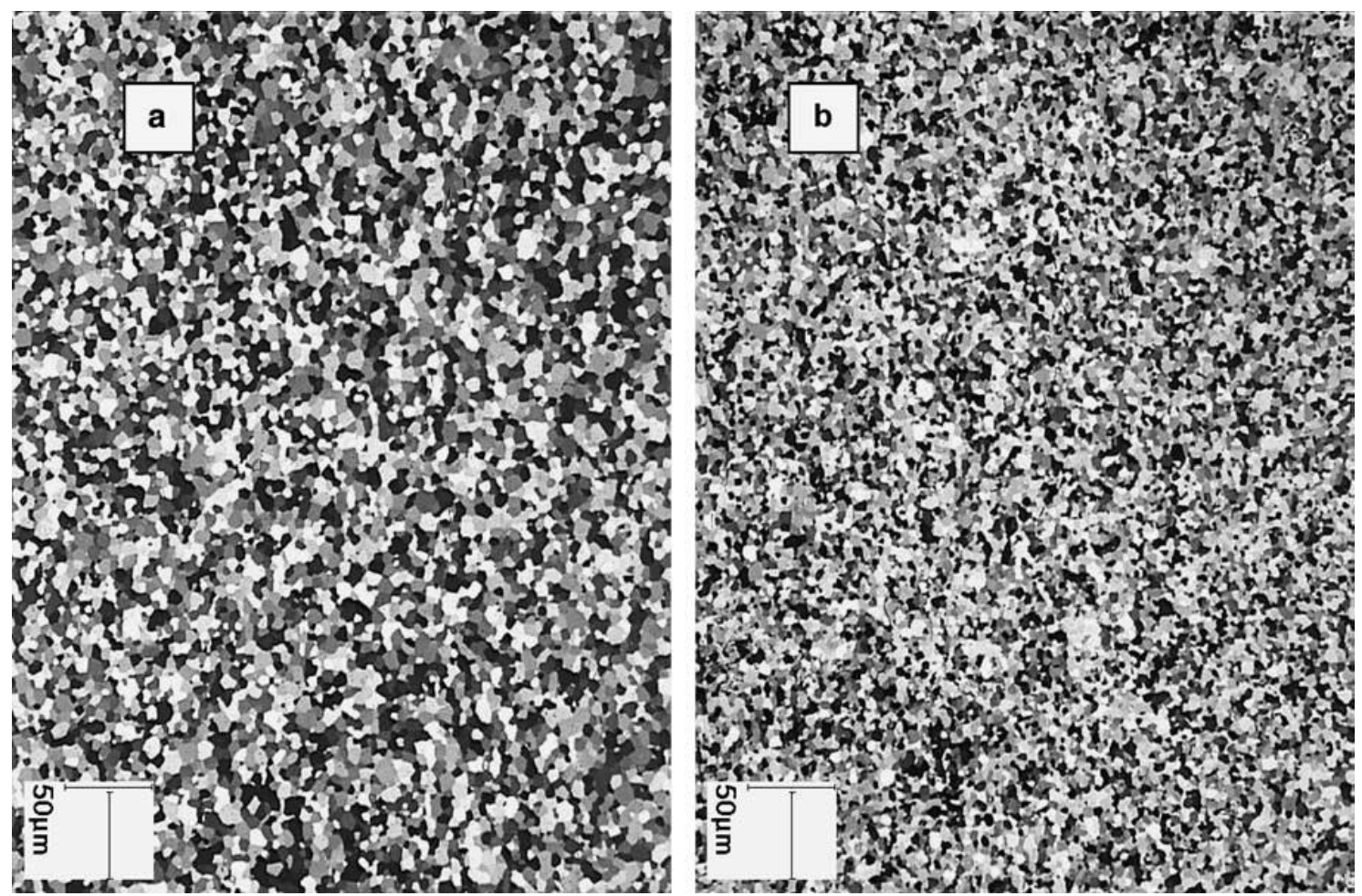

Figure 10: Polarised optical microscopy of claddings: (a) 0.07 wt.\% Nb, (b) $0.56 w t . \% \mathrm{Nb}$ [73]. 


\subsubsection{Iron, chromium and nickel}

Iron has vanishingly low solubility in $\alpha$-zirconium, and so forms intermetallics [84]. Iron is more soluble in $\beta-\mathrm{Zr}$ [77]. The main influence of iron is on phase boundaries [84]. Small increases in iron content reduces hydrogen uptake, whereas nickel increases it. Chromium and nickel also have low solubility in $\alpha$-Zr. The heavy segregation of $\mathrm{Fe}$ and $\mathrm{Cr}$ to intermetallics causes solute deficiency in the $\alpha$-matrix [77]. The phase diagram in Figure 11 demonstrates the low solubility of $\mathrm{Fe}$ in $\alpha-\mathrm{Zr}$; the miscibility and phase domain behaviour of chromium are qualitatively very similar to iron.

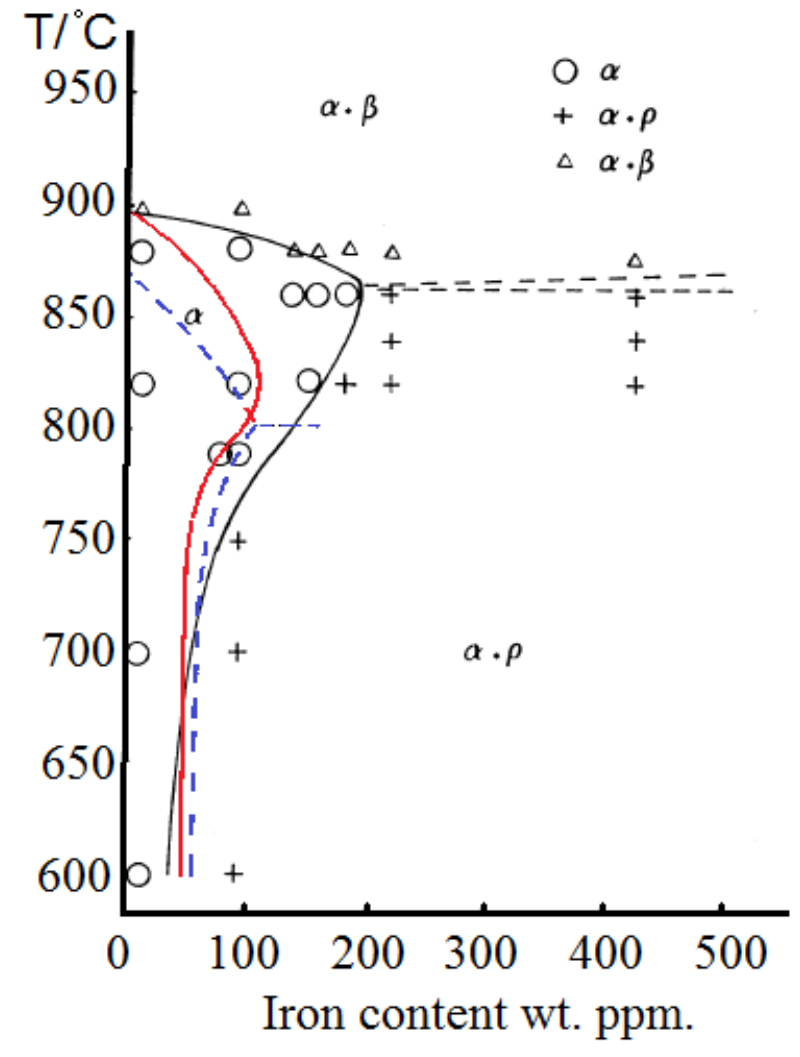

Figure 11: Partial phase diagram for the zirconium rich region of the binary Zr-Fe system (blue dashed line) and proposed $\mathrm{Zr}-1.4 \mathrm{wt} \% \mathrm{Sn}-\mathrm{Fe}$ system (red and black lines).The black line is based upon the experimental points; a-Zr with Fe in solution (circles), two phase region with a$\mathrm{Zr}$ with Fe in solution and iron containing intermetallics (crosses), and b-Zr with iron in solution (triangles). Adapted from [1]. 
Iron additions of $0.1 \mathrm{wt} . \%$ improve workability of ZIRLO [62]. Increasing Fe and $\mathrm{Cr}$ in Zry-4 improves mechanical properties without affecting corrosion resistance [96]. The improvement in strength is to be expected as, in general, increasing the iron content decreases the grain size [73]. New alloys with improved corrosion resistance in PWRs are generally low in Fe (and Sn), or have Fe in the form of more radiation resistant SPPs than those in the Zircaloys [1], that is, SPPs that tend not to become amorphous under irradiation. Iron chromium and nickel have been shown to increase the local oxygen vacancy level within the oxide $[16,103]$ and have also been shown to control its electronic conductivity [104].

Barberis et al. [80] suggest that grain boundaries may be pinned by SPPs because of the bent and wavy boundaries they observed in $\mathrm{Zr}-0.5 \mathrm{Fe}-0.5 \mathrm{Cr}$ and $\mathrm{Zr}-0.5 \mathrm{Cr}-0.5 \mathrm{Ni}$ systems. Intermetallics were found at the $\alpha-\mathrm{Zr}$ grain boundary along with $\beta-\mathrm{Nb}$ in all heat-treated materials examined by Northwood [77]. These small precipitates found had a definite but unknown orientation to the boundary [77]. These observations are unsurprising given that within most systems heterogeneous nucleation of SPPs is more likely than nucleation within the bulk matrix.

Wang et al [83] suggest that $\mathrm{Sn}$ solute distribution is uniform but $\mathrm{Ni}, \mathrm{Fe}$ and $\mathrm{Cr}$ vary in the Zircaloy matrix and that the fluctuation or depletion of solutes is likely to be responsible for nodular corrosion resistance. Similarly, Harada et al. [50] observed that uniform corrosion properties seem to be affected by solute elements but not by SPPs. During oxidation tests by Cox [105] in oxygen and low pressure steam, the insoluble transition metals did not decrease the diffusion controlled pre-transition oxidation rates, but did result in the generally earlier onset of the linear post-transition oxidation rates. 


\subsubsection{Impurities: titanium and aluminium}

Small additions of titanium and aluminium are seen to be severely detrimental to the corrosion performance of zirconium and its alloys [106]. The reason for this is not mechanistically understood and particularly surprising for titanium, which is chemically very similar to zirconium. The addition of a $0.2 \mathrm{wt} . \%$ titanium to an alloy with good corrosion resistance, $\mathrm{Zr}$ $1 \mathrm{wt} . \% \mathrm{Nb}$, resulted in an huge increase in weight gain during corrosion testing. After 1 day in water at $360{ }^{\circ} \mathrm{C}$ this material had undergone a transition in oxidation rate and developed an oxide with weight gain $1189 \mathrm{mg} . \mathrm{dm}^{-2}$ [107]. In comparison, a similarly treated $\mathrm{Zr}-1 \mathrm{wt} . \% \mathrm{Nb}$ alloy in the same conditions underwent a weight gain of $63.6 \mathrm{mg} \cdot \mathrm{dm}^{-2}$ in 189 days [108]. The oxidation rate increases by a factor of $\sim 3500$ becuase of this $0.2 \mathrm{wt} . \%$ addition of titanium.

\subsubsection{Impurities: oxygen, nitrogen, sulphur}

Some alloying additions are unintentional, and introduced either due to high affinity and availability from the environment or the manufacturing process. Nitrogen is commonly introduced by the Kroll process, which is not a common route for nuclear grade alloys. Nitrogen causes embrittlement of the alloy [109]. Oxygen is an alpha stabiliser at $1200 \mathrm{wt}$ ppm and is brought in to solid solution from the environment by sponge zirconium. Sulphur is also an impurity introduced during production of non-nuclear grade alloys. The solubility limit of sulphur in $\alpha-Z r$ is about 20 wt. ppm [110]. While sulphur in solid solution markedly enhances creep strength due to solid solution hardening, the presence of sulphide precipitates has been seen to have an additional beneficial influence on the corrosion behaviour [110] in high temperature steam. 


\subsection{Effect of oxide microstructure on oxidation kinetics}

\subsubsection{Correlation of structural changes with breakaway oxidation}

Oxides with a columnar structure composed of primarily monoclinic grains have been shown to offer better corrosion resistance than alternative configurations with less regular structure. Figure 12 shows a representation of the development of an oxide. Initial fast nucleation of grains occurs at the interface (a).The smaller grains in the vicinity of the metal-oxide interface are reported to be mainly tetragonal phase oxide [15]. The average grain size of the tetragonal oxides is smaller than that of the matrix by a factor of ten [52]. Grains with orientation such that growth can occur more rapidly normal to the plane of the interface grow preferentially (b-c). 


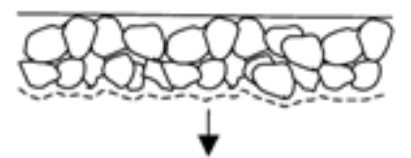

(b)

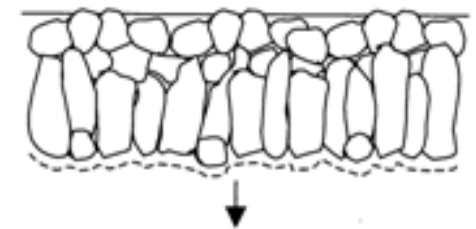

(c)

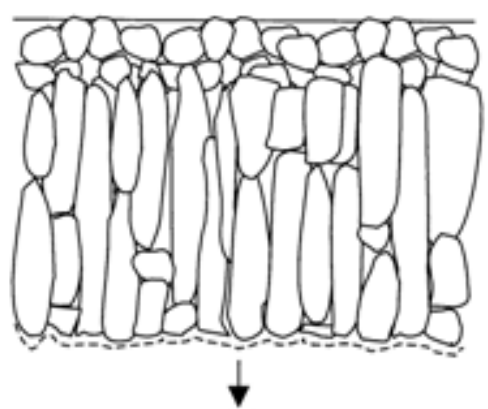

(d)

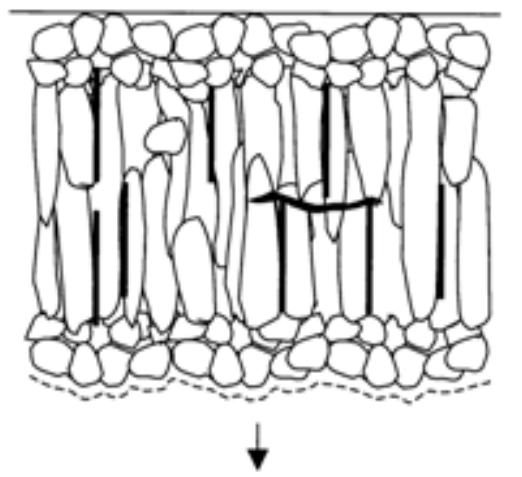

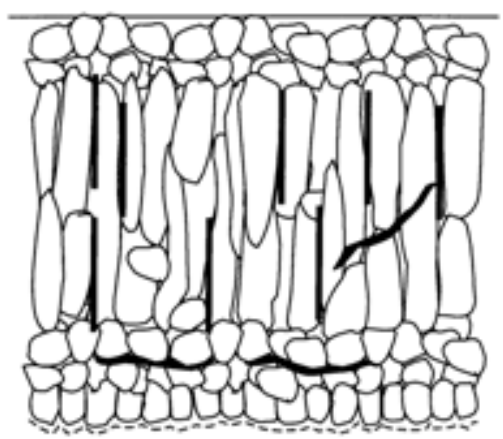

(f)

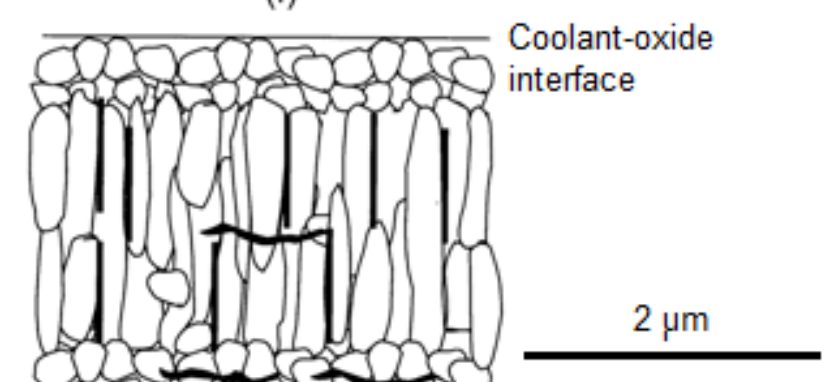

Eqiaxed grains (often described as having a high tetragonal phase fraction)

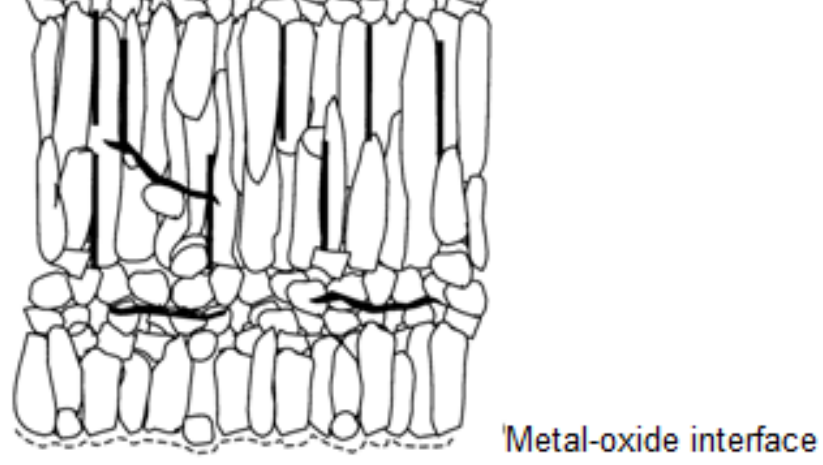

Figure 12: Schematic diagram of the observed oxide grain structure. Adapted from [15].

The black bold lines are intergranular cracks due to induced growth stresses, this author suggests that a network of cracks is created allowing a free path to close to the metal-oxide interface [15]. It should be noted that lateral cracking within the body of the oxide actually acts as a barrier to the oxidising ionic species diffusing. A network of cracks from the oxide/water interface into the body of the oxide is required to shorten the diffusion path.

TEM studies of real oxide microstructures show a more complicated picture than the idealised cyclic growth suggested by Figure 12, although it has many features that are seen in real 
materials. Three schemes by which rate transition occurs in real alloys have been proposed by Cox, and these are described in Table 7.

\begin{tabular}{|c|c|}
\hline Type of transition & Situation / conditions \\
\hline Sudden & A rapid transitions such as Zry-4 in PWR conditions (Figure 5a) \\
\hline Slow transition & $\begin{array}{c}\text { May be a fast process nucleating and spreading slowly over the } \\
\text { surface, or a genuinely slow but uniform process [2]. There is not } \\
\text { usually enough evidence to distinguish between them [1]. }\end{array}$ \\
\hline $\begin{array}{c}\text { Para-linear } \\
\text { breakdown }\end{array}$ & $\begin{array}{c}\text { A steady kinetic change from approximately cubic, through } \\
\text { parabolic, to linear without any actual oxidation rate increase } \\
\text { throughout the whole process [111]. This is sometimes, but not } \\
\text { always, seen during the oxidation of Zr-Nb binary alloys [1] (Figure } \\
5 \mathrm{~b}) .\end{array}$ \\
\hline
\end{tabular}

Table 7: Three schemes by which rate transition occurs in zirconium alloys, as noted by Cox [1].

\subsubsection{Second phase particles in the oxide}

Intermetallic precipitates have been observed to influence corrosion behaviour significantly [34]. The alloying element distributions in the final oxide have an important impact on the oxidation rate of the alloy [88]. SPP size and distribution in the oxide film affect stress gradient opposes the chemical potential gradient and decreases the rate of corrosion [70].

Several authors suggest SPPs provide short-circuit routes for oxidation [112-113] with ions migrating around intermetallics. Others dispute this and claim that matrix solute concentration is more important $[86,114]$.

Electrochemical investigations show that intermetallics are nobler than the surrounding zirconium. So SPPs act as a cathode relative to the $\mathrm{Zr}$ anode [70]. Kubo and Uno [89] used EDX to show that particles in the oxide dissolved during corrosion in high temperature steam. From their observations they concluded that corrosion resistance improvements are due not to shortcircuit oxidation effects, but to changed oxide properties. Oxidation in the region of a SPP begins with the oxidation of $\alpha$-zirconium and is later accompanied by iron diffusion in to the 
surrounding oxide. It is generally agreed that SPP in the oxide film readily oxidise [70]. This process is illustrated in Figure 13. Metallic precipitates do not oxidise until they are completely surrounded by the oxide [115], before this their metallic character is retained within the oxide [116]. EDX showed that $\mathrm{Ni}$ and Cr also diffused away from precipitates in the oxide, although at a lesser rate than $\mathrm{Fe}$ [89]. Anada et al. [117] reported that a transformation from columnar grains to equiaxed grains was observed predominantly around the oxidised precipitates and that oxidation of the precipitates was the cause of an accelerated corrosion.

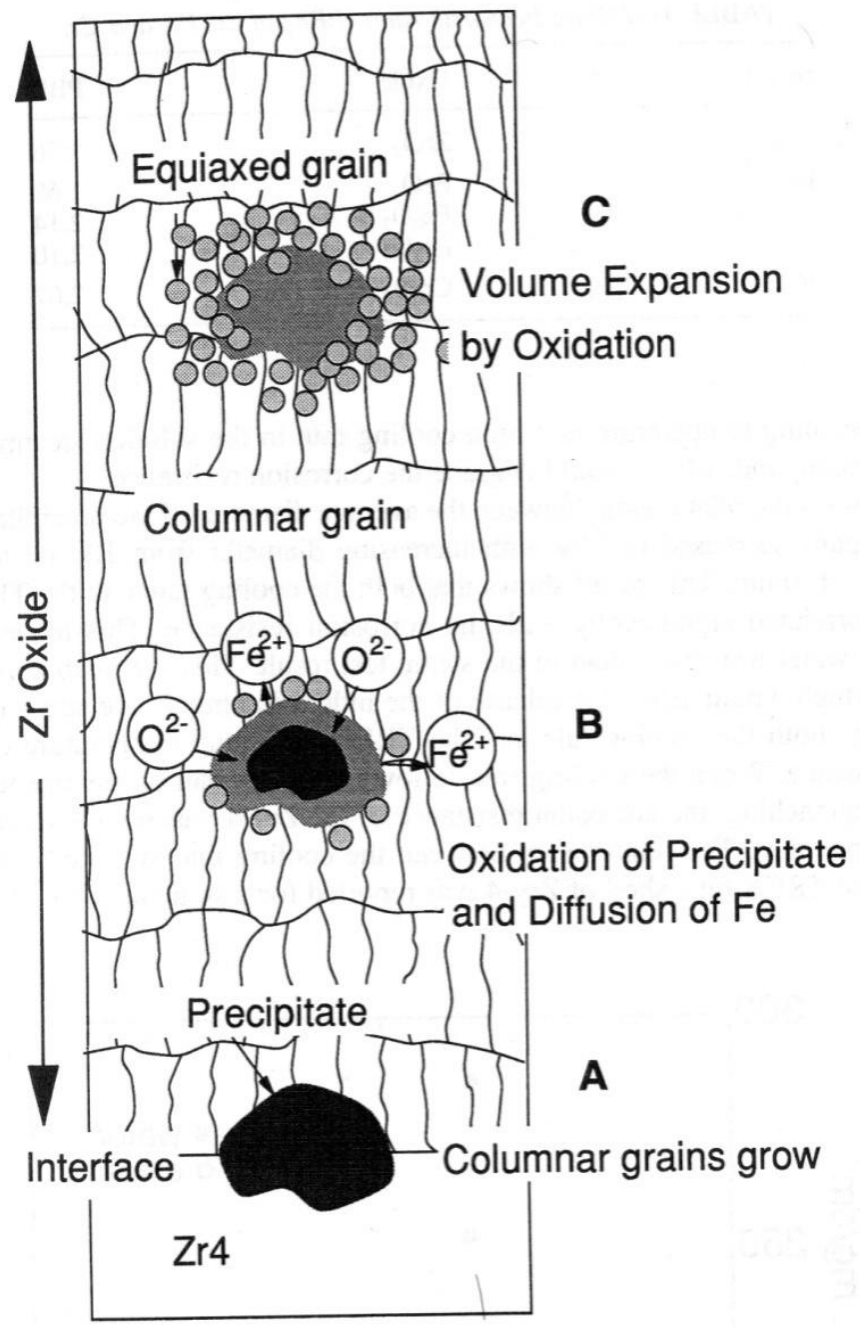

Figure 13: Schematic of the effect of iron diffusion from intermetallics precipitates in the oxide film on Zircaloy-4 [117]. 


\subsubsection{Oxidised grain boundaries}

The distribution of alloying additions within the grain boundaries of the alloy substrate allow for localised concentration of dopant that would be taken up by oxide grown on these regions. This localised doping would be expected to alter the vacancy and electronic conductivity of the oxide.

It has been suggested that oxygen transport in the oxide occurs principally by grain boundary diffusion [49], known as short circuit oxidation. Many species, including oxygen, show diffusion rates many orders of magnitude higher in grain boundaries than in the bulk. It has been calculated that for oxygen $\mathrm{D}_{\text {boundary }} \sim 10^{8} \mathrm{D}_{\text {bulk }}$ at reactor temperatures [49]. This is the case both before and after the rate transition.

Cathodoluminescence (CL) has been used to image the oxide films formed on binary zirconium based alloys [59]. Only the Zr-Fe alloy showed strong CL variations. The strong CL signals from the oxide originated from grain boundaries and were especially strong near $\mathrm{Zr}-\mathrm{Fe}$ intermetallic precipitates. No localised CL signals were observed at grain boundaries when examining asreceived zirconium, but were strong near cracks in the oxide. For the as-received, $\beta$-annealed sample oxidised in air at $600{ }^{\circ} \mathrm{C}$, very strong CL signals originated from the grain boundaries and cracks [59]. The segregation of iron to the grain boundaries in the Zr-Fe alloy seems to be a continuous process as the oxide grows in the $600{ }^{\circ} \mathrm{C}$ temperature range [59]. High intensity $\mathrm{CL}$ emissions at the cracks were not observed when using a $\mathrm{Zr}$-Sn-Fe alloy. The authors consider that this suggests either that tin may be slowing down the diffusion of other solute, such as iron, to the free surface or it is quenching the luminescence. The observation of iron at grain boundaries by this method is somewhat indirect, as it detects the elements grown as an oxide film on top of the substrate, and it is possible that further redistribution occurs during this process. 


\subsection{Alloy corrosion resistance comparison}

In a PWR-like environment, Zry-4 is seen to corrode at a greater rate to ZIRLO and Zr-2.5Nb, as shown in Figure 14. The sampling period of Figure 14 is too coarse to demonstrate the periodic features seen in a similar environment in Figure 5. A comparison of these figures also demonstrates the marked variation that can be seen in the corrosion performance of materials with the same nominal composition with very slight variations in experimental conditions.

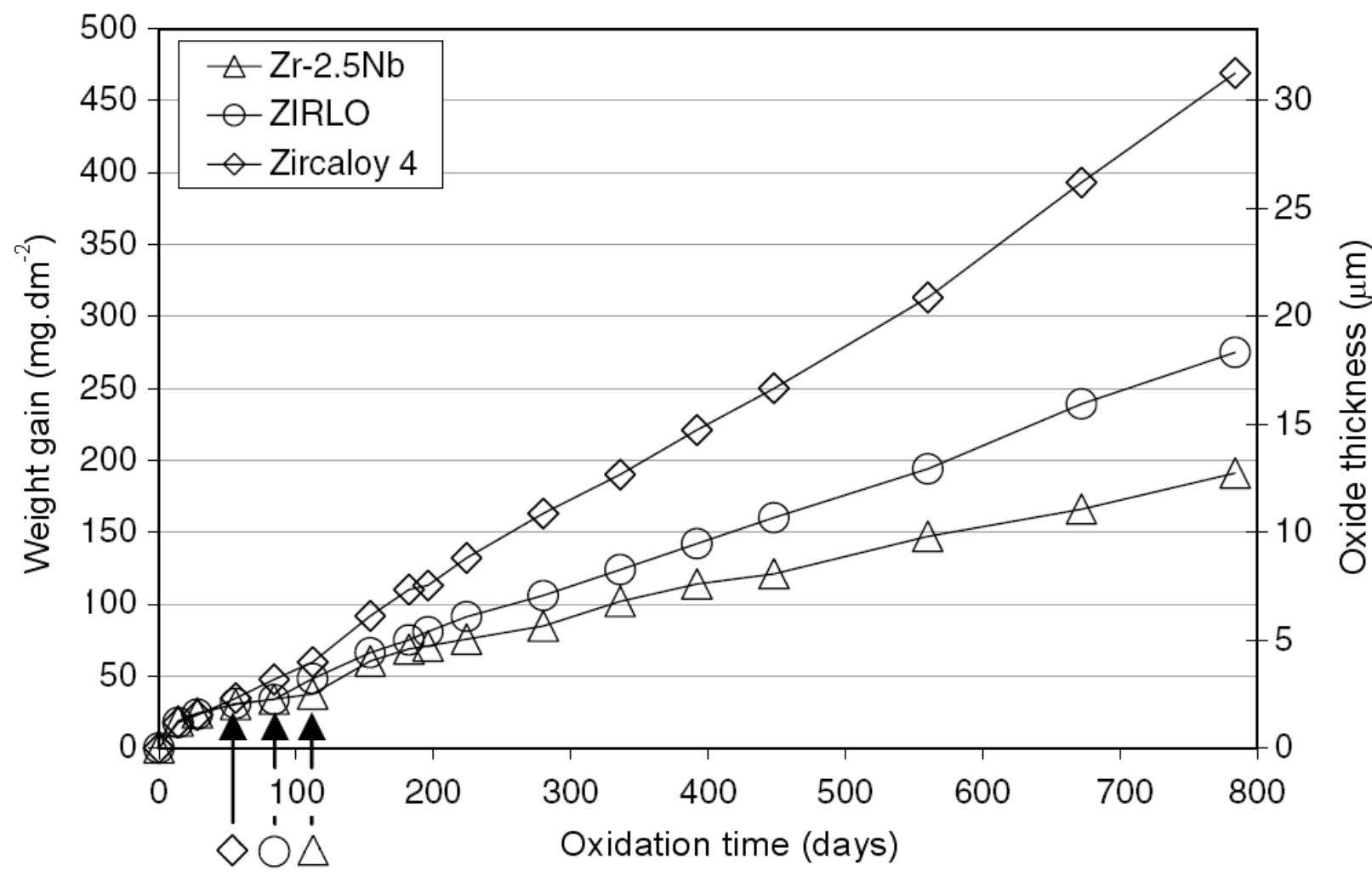

Figure 14: Corrosion weight gain vs. exposure time of Zircaloy-4, ZIRLO and Zr-2.5Nb in 360

${ }^{\circ}$ C pure water. Solid arrows show the transition for each material. Adapted from [15, 69]. 


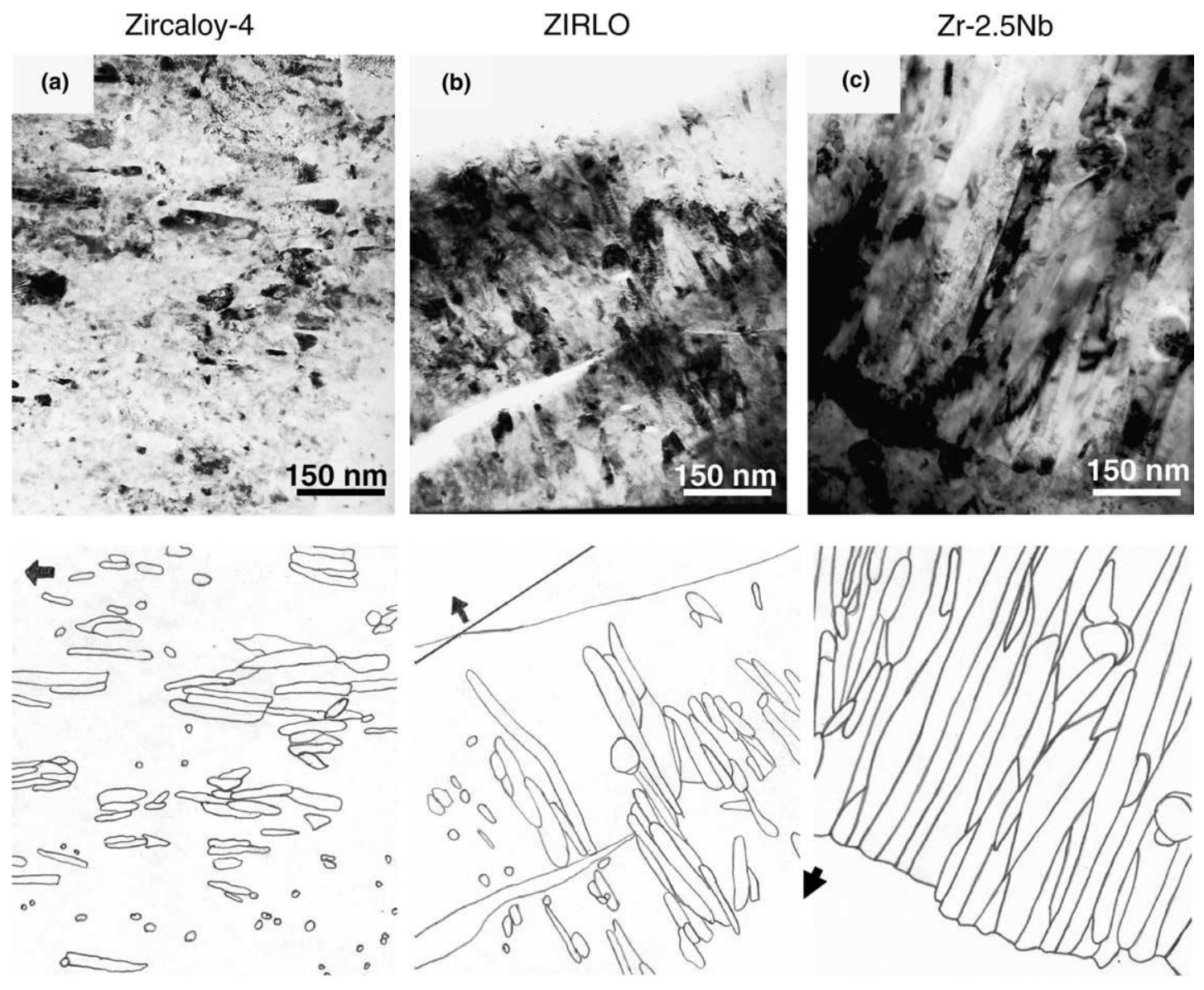

Figure 15: Grain size, shape and orientation comparison near the oxide/metal interface of (a) Zircaloy-4, (b) ZIRLO and (c) Zr-2.5Nb alloy oxides formed in $360^{\circ} \mathrm{C}$ pure water environments. The cartoons below each bright-field images show the hand-drawn sketch of the grain boundaries. Black arrows indicate oxide growth direction [69].

The microstructure of the oxides formed on these materials near the metal-oxide interface is shown in Figure 15. The percentage of tetragonal oxide was highest in the first two micrometres or so, near the interface, and was higher in Zircaloy-4 than in ZIRLO [69]. Comparing the layer periodicity from alloy to alloy, the average spacing between layers was inversely proportional to the post-transition corrosion rate, indicating a clear benefit to delaying the oxide transition [69]. Experimental results for this were produced in an earlier work by the same authors [56] using various zirconium alloys and are reproduced in Figure 16. The character of the metal-oxide 
interface of Zircaloy-4 has been noted to exhibiting an undulating morphology, whereas the Zr$2.5 \mathrm{Nb}$ demonstrates a more jagged "jigsaw" interface [23]. The most regular columnar structure was seen in $\mathrm{Zr}-2.5 \mathrm{Nb}$, the best performing alloy. The relation between differences in oxide morphology and alloy composition is not immediately apparent, and is masked by the interplay between numerous variables. The regularity of the oxide structure upon the corrosion resistance of the alloys is more marked.

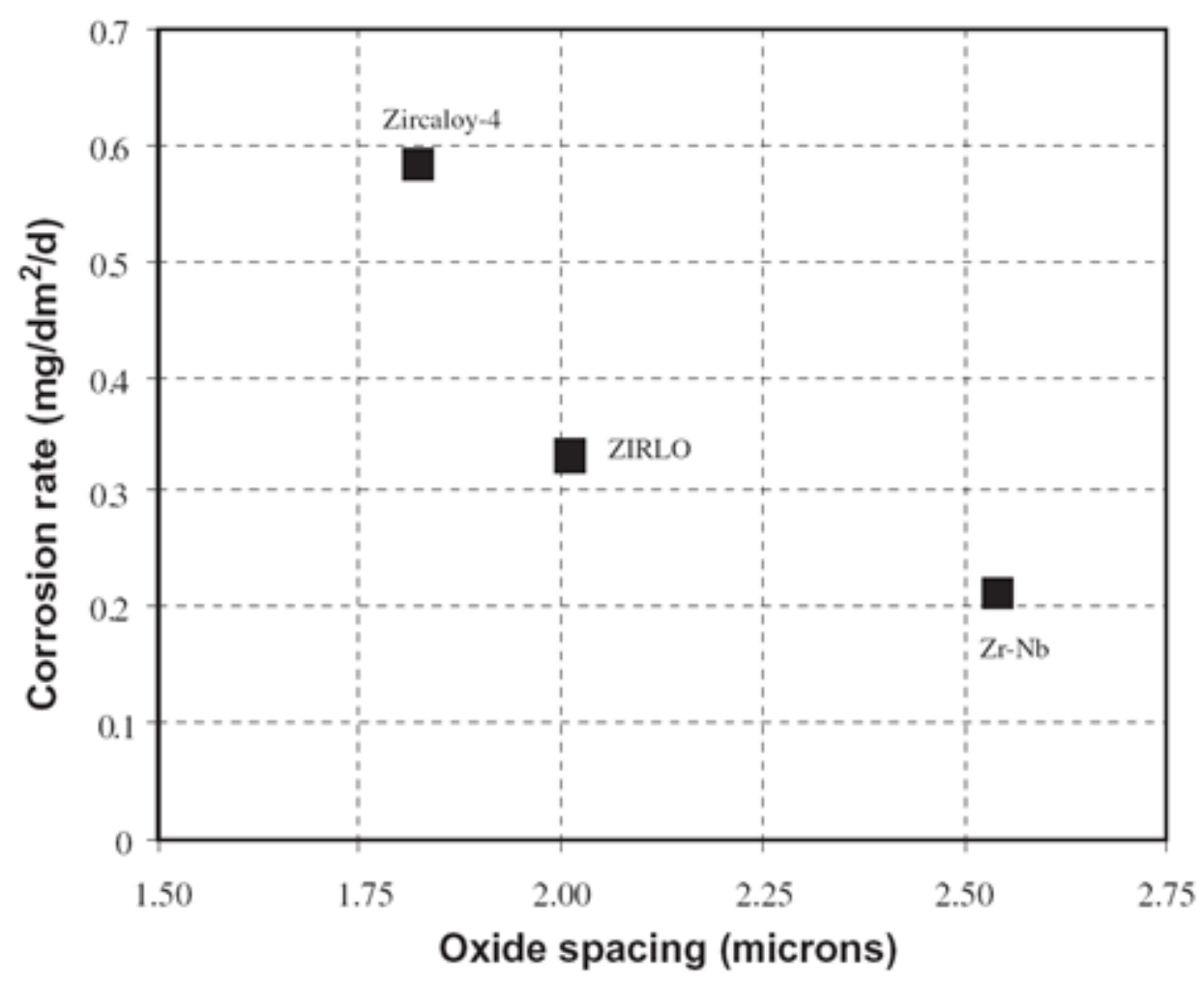

Figure 16: Post-transition corrosion rate of various zirconium alloys in $360{ }^{\circ} \mathrm{C}$ water versus average thickness of oxide layer. Oxide spacing refers to the distance between successive layers of equiaxed grains as seen in Figure 12. Adapted from [56]. 


\subsection{1 $\mathrm{Zr}-\mathrm{Sn}$}

Zr-Sn based systems, such as the Zircaloy family, are qualitatively the most similar to zirconium in their corrosion performance, the alloying additions imparting the benefit of a reduced corrosion rate. Zircaloy-4 shows a columnar oxide structure, with a fine tetragonal phase observed at the metal-oxide interface [1].

The oxides that develop on these alloys tend to be uniform films that are not susceptible to early spalling [118]. Improved uniform corrosion resistance of Zircaloys in PWR conditions requires complete precipitation of Fe and $\mathrm{Cr}$ [79, 119]; highly annealed structures with most alloying elements in large, widely spaced, intermetallics have optimal uniform corrosion resistance [40].

Like many factors we have encountered, there is a variation of behaviour between the Zr-Sn and Zr-Nb systems. Harada et al. [50] reported that the composition of SPPs varied with bulk content but had no correlation to uniform corrosion resistance in Zircaloys. However, many authors have observed a correlation between intermetallic distribution and macroscopic corrosion performance $[34,51,89,120-122]$. Small high density intermetallics have better corrosion resistance than larger, lower number density SPPs [51, 120-122]. A large number of particles, those with a diameter less than $300 \mathrm{~nm}$, were excluded from their analysis. The effect of SPPs on corrosion resistance may be due to their effect on electronic conductance [34]; resistivity of Zircaloy-2 increased with a decrease in the mean diameter of SPPs [89].

\subsection{2 $\mathrm{Zr}-\mathrm{Nb}$}

The main benefit of $\mathrm{Zr}-\mathrm{Nb}$ based systems over $\mathrm{Zr}-\mathrm{Sn}$ is that the kinetic transition is not so sharp in $\mathrm{Nb}$-containing alloys and it occurs after a longer period of time [39], as seen previously in 
Figure $5 \mathrm{~b}$. However the $\mathrm{Nb}$-containing alloys are oxidised faster than recrystallised Zircaloy in oxygen and in water vapour $[24,58,123]$.

$\mathrm{Zr}-\mathrm{Nb}$ alloys have very different corrosion properties to $\mathrm{Zr}$-Sn alloys. This is mainly due to the change of microstructure with heat treatment [40] as demonstrated earlier in Figure 9. Microstructural evolution in $\mathrm{Zr}-\mathrm{Nb}-(\mathrm{Fe}-\mathrm{Sn})$ alloys is governed by the most slowly diffusing elements in the system, niobium [84]. In the $\mathrm{Zr}-\mathrm{Nb}$ system the required level of annealing is strongly related to the amount of niobium addition [96]. Although it is necessary to perform long term annealing in order to reach thermodynamic equilibrium in the $\mathrm{Zr}-\mathrm{Nb}-(\mathrm{Fe}-\mathrm{Sn})$ system [84], generally $\mathrm{Nb}$-containing $\mathrm{Zr}$ based alloys demonstrated increasing weight gain with increased levels of annealing [96]. A non-equilibrium, uniform distribution of fine iron-containing $\beta-\mathrm{Nb}$ particles was found to be an important factors for improving corrosion resistance of these alloys in PWR environments [79].

\subsubsection{Zr-Sn-Nb}

Cold worked ZIRLO-type alloys are optimised after only one hour anneal at a temperature of $550{ }^{\circ} \mathrm{C}$ [98]. The Zr-Sn-Nb system shares similar corrosion properties to the $\mathrm{Zr}-\mathrm{Nb}$ system. It has been suggested that the diffusion of niobium governs the process, and that a fine distribution of $\beta-\mathrm{Nb}$ is also the main reason for the good corrosion resistance of ZIRLO [67, 124]. It should also be remembered that the ZIRLO system also contains Fe and Cr. After relatively short annealing periods these elements are precipitated out of solution along with the niobium [77]. 


\subsection{Previous atom probe studies of zirconium alloys}

One dimensional atom probe analysis (1DAP) has been used by Kruger et al. [125] and Wadman et al. [126-127] to directly measure solute matrix composition. 1DAP is a challenging technique, especially using zirconium; the tiny needles with end radii of the order of $50 \mathrm{~nm}$ analysed by this process are frequently subject to fracture. This means that the conclusions drawn by these authors are based upon small data sets. Kruger et al. observed alloying element depletion in the matrix of Zry-2 and Zry-4. Contrary to expectations, this technique measured lower values after $\beta$-quenching Zircaloy-2 than after high $\alpha$-annealing. Kruger et al. attributed the improved nodular-corrosion resistance from the former to direct oxidation of tiny precipitates. They found a 5-10 nm thick oxide film on the surface of their atom probe specimens. The detected levels of $\mathrm{Cr}, \mathrm{Fe}$ and Ni were reportedly higher in this film that the zirconium matrix. Wadman et al. [127] found the several nanometres of oxide grown on the tip of Zircaloy needles to have a composition of $\mathrm{ZrO}$. Using TEM they identified this layer as being amorphous but containing small crystallites. Wadman et al. did not report the same enrichment of alloying elements in the oxide as observed by Kruger and neither set of workers found segregation of transition elements to the metal-oxide interface. Wadman et al. [126] found the $\alpha$-Zr matrix to be depleted of Fe and $\mathrm{Cr}$ in Zry-4. The matrix composition measurements of Kruger [125] and Wadman [127] both agreed nominally with the solubility limits of Charquet et al. [128]. Wadman demonstrated the sensitivity of the technique to changes in analysis conditions such as temperature and pulse fraction $^{7}$ [129]. Comparison of values is only valid for specimens probed using the same analysis conditions. The precision of the measurements of small concentration elements is limited by the small quantities of data. For a particular specimen of Zr-4 exposed to air for $312 \mathrm{hrs,} \mathrm{only} 380$

\footnotetext{
${ }^{7}$ The relative magnitude of the electrical pulse used in 1DAP to liberate ions from the end of the needle compared to the standing potential on the tip.
} 
ions were detected before the specimen failed. Of these, 1 ion was ranged as an iron containing species, meaning the matrix concentration of $\mathrm{Fe}=0.26 \pm 0.26 \%{ }^{8}$. No chromium was detected in the matrix of this Zry-4 sample [127].

Sano and Takeda [130] used the 1D atom probe to analyse $\mathrm{Sn}$ profiles in a range of $\mathrm{Zr}-\mathrm{Sn}$ alloys. It was found that for Zircaloy-4 and Zr-0.6Sn binary alloy all of the tin was in random solid solution over the length scale measured. For a higher tin Zr-1.39Sn binary alloy small tin clusters composed of a few tens of atoms were found in the matrix. No tin-rich phases were observed. This could be due either to their non existence or to their low number density compared to the small region of analysis, typically a cylinder with radius of $1-2 \mathrm{~nm}$ and length of around $50 \mathrm{~nm}$. It is also possible that specimens containing intermetallics may be more prone to fracture during atom probe analysis than those without.

Zircaloy materials were found to be sensitive to surface oxidation during atom probe specimen preparation [131]. Specimens were prepared using electrochemical etching in all three cases, using a solution consisting of varying proportions of acetic acid and perchloric acid $[125,130]$ or perchloric acid, iso-butanol and methanol [132]. These specimens typically allowed 5,000 30,000 ions to be collected for analysis by combining the data from several specimens. The method successfully allows the analysis of matrix solute concentration but would benefit from a greater quantity of data to improve the precision of results. 1DAP techniques are not suitable for analysing SPPs in zirconium alloys as the number density of particles compared to the volume of analysis is highly unfavourable. Analysing conditions must be carefully controlled and great care taken when comparing results obtained on different instruments and using different conditions.

\footnotetext{
${ }^{8}$ In atom probe analysis the error in the detected quantity of atoms, $n$, is that dictated by Poisson statistics and is commonly quoted as 1 standard deviation.
} 


\subsection{Conclusions}

Breakaway corrosion occurs in all alloy classes to some extent. There are several morphological changes that have been observed in the oxides undergoing this transition, as discussed in Table 3, yet a precise mechanism that may be used to describe the breakaway transition is as yet unknown. Such a mechanism is likely to involve all of these phenomenological observations, to different degrees, depending upon the local conditions and alloy. Different modes of transition are seen between different alloys, as was described in Table 7. The different alloy classes have been shown to develop oxides with similar features, but varying degrees of regularity. Highly textured columnar structures in the oxide are seen to impart improved corrosion resistance.

A good empirical understanding has developed of the thermal/mechanical treatments required to produce alloys with desired substrate microstructures (section 2.2), and the alloying addition chemistry is also well understood empirically (section 2.6). This has not translated into a mechanistic understanding of how alloying additions effect the breakaway transition in oxidation kinetics, or how the microstructure of the substrate causes the change in morphology of the oxide. The preferred precipitate distribution in the substrate varies largely upon the environment to which the material is to be subjected, as well as the alloy bulk composition.

Many authors have suggested that another factor, such as the matrix composition may be important in explaining these discrepancies. However, the precise quantitative determination of the matrix solute concentration is experimentally challenging with few techniques giving the spatial resolution and chemical precision required and as yet the matrix alloy chemistry and its redistribution during corrosion are not understood.

The high spatial resolution, chemical sensitivity and superior data collection rate of the current generation of $3 \mathrm{D}$ atom probe instruments allows for a systematic study of the solute distribution 
in the 'as-received' substrate alloy matrix and subsequent redistributed during the corrosion process. This redistribution occurs where the new oxide forms: at the metal-oxide interface. The change in chemical properties at this interface will affect the on-going corrosion resistance of the alloy and so have a direct effect upon the oxidation process. Nano-scale analysis of different alloys at various stages in the corrosion process allows the key similarities and differences due to the addition of various solutes to be established. After a series of preliminary studies in chapter 4, the characterisation of the solute distributions within the metal matrix of uncorroded materials, and the redistribution of these solutes at the metal-oxide interface of three alloys are described in chapters 5 and 6 , respectively. 


\section{Experimental methods}

\subsection{Atom probe tomography}

\subsubsection{Introduction}

$3 \mathrm{D}$ atom probes (3DAP) represent a unique category of instrument that allow the construction of three dimensional maps displaying the chemical distribution of individual atoms within a small region of interest (ROI). The dimensions of this region are of the order of $100 \mathrm{~nm} \times 100 \mathrm{~nm} \times$ several hundred nanometres for the current generation of instruments. These instruments acquire data at a typical rate of 1,000 ions per second creating datasets of many tens of millions of ions during a matter of hours. Atomic spatial-resolution can be achieved in the specimen depth direction and near atomic resolution can be achieved laterally in homogeneous regions of metal matrix away from features such as boundaries and intermetallics, which create ion trajectory aberrations (discussed later in section 3.3.6) [133]. In favourable conditions atomic planes may be observed in ordered metallic structures [134]. The chemical sensitivity of these instruments is uniform for all atomic species and the efficiency of the detector is such that over a third of all of the atoms within the ROI are detected. As such, the 3D atom probe is a powerful tool for conducting experiments pertaining to nanoscale chemistry.

\subsubsection{History and development of the instrument}

The development of the atom probe has been a gradual affair, built on the dedication and skill of various groups of scientists from around the world. The first one-dimensional atom probe (1DAP) was built by Muller in 1967. The apparatus was a development on the concept of a field 
ion microscope (FIM). FIM consist of a needle-like conductive specimen held at a high electric potential in a vacuum chamber. The high potential results in an electric field that is strong enough to ionise atoms at the end of the tip. Equation 3 demonstrates that this field, $F$, is inversely proportional to the radius, $r$, of the tip.

$$
F=\frac{V}{k_{f} r}
$$

Where $V$ in Eq. 3 refers to the applied electric potential and $\mathrm{k}_{f}$ is a numerical constant. In a FIM the ions are projected radially from the tip and impact a phosphor screen, producing a magnified image of the specimen apex. It was found that superior image resolution could be obtained by cooling the emitter to cryogenic temperatures [131]. The 1DAP used a hole in the phosphor screen to allow atoms from a specific site to pass through into a time-of-flight mass spectrometer. This resulted in a 1D composition profile of the ions emitted from a selected area of the surface of the needle.

The removal of substrate atoms that are bound to the surface of the specimen is referred to as field evaporation. Two models have been developed to explain the process of field evaporation: the image force model and the charge exchange model. These are described in section 2.4 .2 of reference [131], some important points from which are briefly summarised here. In both models, an applied electric field promotes the stability of ionic species over neutral atoms with increasing distance from the surface of the metal specimen. However, the image force model provides a more accurate description of the behaviour of most transition metals, which are predicted to predominantly form doubly charged ionic species.

In both models an energy barrier must be overcome for a neutral atom to leave a metal surface. For the image force model this energy barrier equates to the magnitude of the Schottky hump, which is assumed to lie outside the neutral atomic curve. This activation energy for producing 
an $\mathrm{n}$-charged ion in zero field, $\mathrm{Q}_{\mathrm{n}(\mathrm{F}=0)}$, is given as being the sum of the heat of sublimation of the neutral atom, $\gamma$, and the sum of its ionisation energies, $I_{n}$, less the work function of the emitting surface, $\varphi_{n}$. In the presence of an applied field, F, this relationship also takes on a term which reduces the activation energy proportional to the square root of the applied field. The activation energy in the presence of field $\mathrm{F}$ is given in Eq. 4. The form of this function is shown in Figure 17.

$$
Q_{n}=\gamma+\sum_{n} I_{n}-n \varphi_{e}-\sqrt{\frac{n^{3} e^{3} F}{4 \pi \epsilon_{0}}}
$$

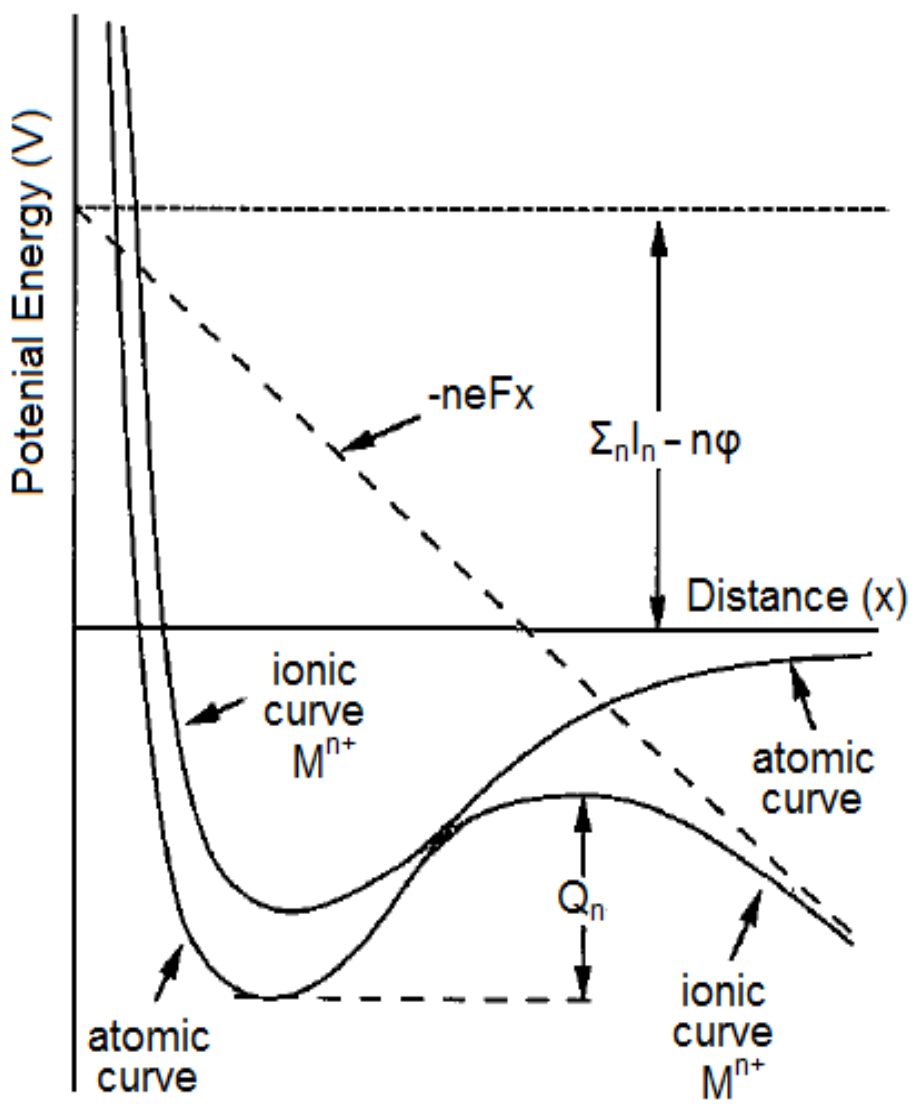

Figure 17:Potential energy diagram for the image force model of field evaporationin the presence of an applied electric field. Reformatted from [131]. 
A correction factor to take account of the change in surface atom binding energy with field should also be applied. The rate constant, K, for thermally activated field evaporation takes the form of an Arrhenius equation, shown in Eq. 5 [135] using the activation energy $Q_{n}$ described above.

$$
K=v \exp \left(\frac{-Q_{n}}{k_{B} T}\right)
$$

By contrast, another process that takes place in the atom probe, field ionisation, is a quantum mechanical process that proceeds independently of temperature. Field ionisation governs the ionisation, and subsequent radial projection of free atoms and free molecules in the vicinity of the emitter that are not bound to the specimen surface. Material that has been adsorbed onto the surface is field desorbed, a process similar to the field evaporation described above.

An overview of the processes involved in field ionisation is provided in section 2.3 .2 of reference [131], from which some important points are summarised here. The potential energy experienced by a tunnelling is given below in Eq. 6 . The form of this potential function is shown in Figure 18.

$$
V(x)=\frac{e^{2}}{4 \pi \epsilon_{0}\left|x_{i}-x\right|}+e F x-\frac{e^{2}}{16 \pi \epsilon_{0} x}+\frac{e^{2}}{4 \pi \epsilon_{0}\left(x_{i}+x\right)}
$$

where $\mathrm{F}$ is the electric field strength, $\mathrm{e}$ is the elemental charge, and $\mathrm{x}_{\mathrm{i}}$ is the distance from the centre of the positive ion to the plane of the surface. The successive terms in this equation represent the potential funnel of the ion plus external field energy minus electron image potential plus repulsion energy of the electron from the ion image. The form of this function is shown in Figure 18. The rate of tunnelling increases as the gas atom approaches the surface until a critical distance, $x_{c}$, is reached, at which point the potential energy of an outer shell electron of the gas 
atom coincides with the Fermi energy of the metal and tunnelling of an electron from the atom to the surface may occur. A forbidden zone exists in which ionisation cannot occur at distances closer to the metal than the critical distance.

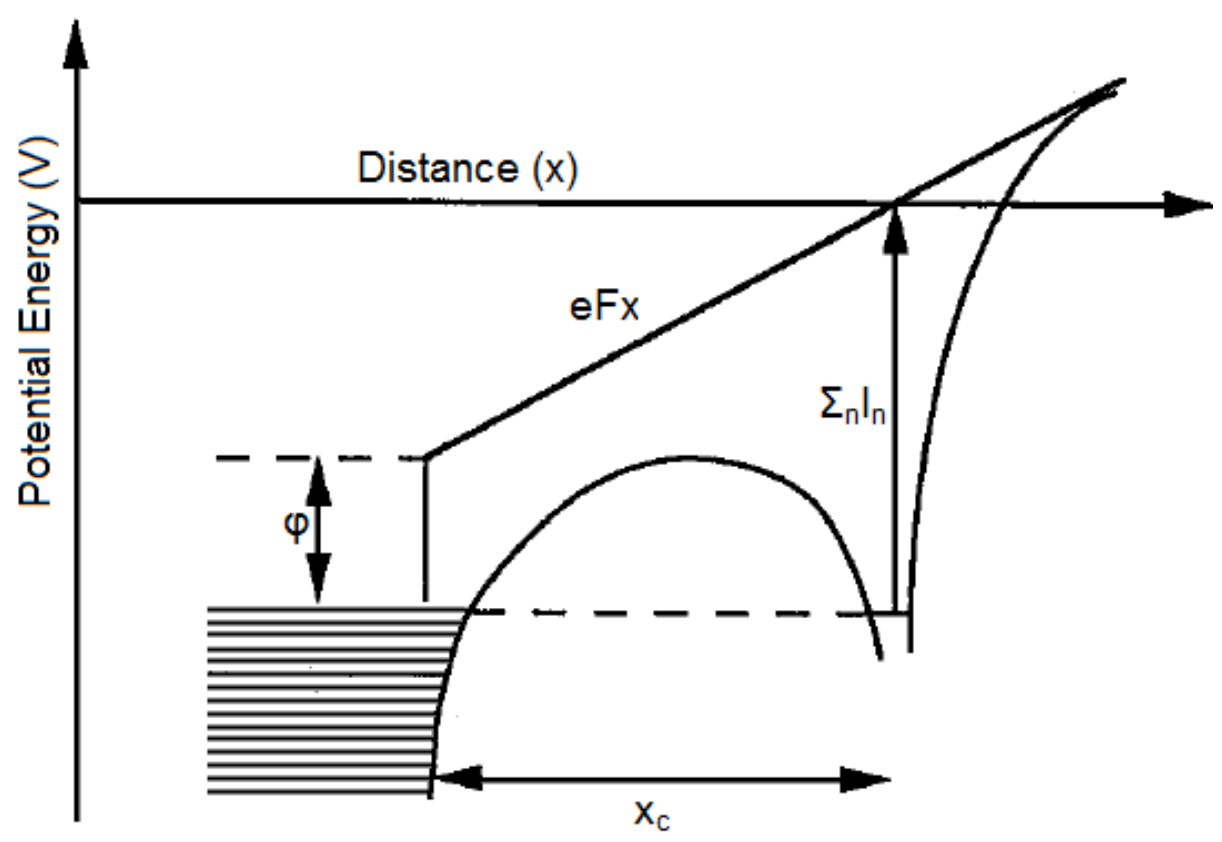

Figure 18: Potential energy diagram for field ionisation near a clean metal surface [131].

A narrow, $0.015-0.0025 \mathrm{~nm}$ thick, region of space exists at the critical distance in which there is a moderate probability of tunnelling occurring. The presence of field adsorbed layers on the surface of the metal and the atomic scale geometry of the sample influence the field ionisation process. The ionisation probability for free atoms in close proximity to the surface is modified because the presence of the adsorbed atom modifies the barrier through which an electron of the free atom has to tunnel. The modification is more intense because the barrier is relatively narrow, and so the adsorbed atom occupies a large part of it. At low fields, the ionisation probability at lower-field sites is so low as to be virtually negligible and so nearly all the observed ionisation takes place at localised higher-field sites, such as regions of high curvature. 
The first operational 3DAP was produced by Cerezo and colleagues in 1988 [136]. The position sensitive atom probe PoSAP replaced the phosphor screen with a position sensitive detector. Time-of-flight mass spectrometry is achieved by applying a positive standing voltage below that required to ionise atoms from the specimens together with a high frequency pulse set at a level such that a small fraction of the pulses will result in an ionisation event occurring. The mass-tocharge-state ratio $(\mathrm{m} / \mathrm{n})$ can be deduced as the time of the application of each pulse is known, as is the time of the atom impacting the detector. The mass-to-charge state ratio is given in Equation 7:

$$
\frac{m}{n}=2 e V \frac{t^{2}}{d^{2}}
$$

Here, $V$ is the combined amplitude from the standing voltage and the pulse voltage, $e$ is the charge of an electron, $t$ is the time of flight and $d$ is the flight length. Equation 7 assumes that the distance between the specimen and detector remains constant. As the run progresses and ionisation continues to occur this distance will increase marginally, but the variation in $d$ will only be $\sim 1 \mu \mathrm{m}$ over a flight path of several centimetres.

Eq. 7 also assumes that all ions are created using an instantaneous pulse, resulting in immediate acceleration, and constant velocity thereafter. Suitable design modifications to the pulsing system limits $\Delta t$ due to uneven square wave pulsing in which the leading edge of the pulse is at a higher amplitude than the remainder of the pulse and so reduces the evaporation period. Other energy compensation techniques have been developed utilising lenses to limit unwanted spread in $m / n$ resulting from the tails of pulses. The overall time of flight of the ions is of the order of microseconds, so the duration of each pulse must be restricted to the nanosecond range in order to minimise the spread in flight time caused by the pulse duration. 
Attempts to produce an atom probe that applied negative standing and pulse voltages to the specimen resulted in field emission of electrons from the specimen at lower applied fields than that required to evaporate ions. The effect of this was that specimens were destroyed by resistive heating caused by the electron flux before field evaporation of the specimen material was achieved. This process can, however, be used to field desorb weakly bonded surface adsorbates [131].

The high electron flux emitted by such specimens makes them useful for other areas of research, such as field emitters for electron [137] and surface scanning microscopy [138], but hampers the ability of a 3DAP detector to identify emitted ionic species because of the high electron flux [131]. Subsequent variants of the 3DAP have used the application of positive potential to the specimen, which avoids the possibility of field emission. 


\subsubsection{Reflectron lens}

Electrostatic lenses have been developed to achieve improved 3DAP mass resolution. The reflectron lens is an electrostatic mirror that compensates for the spread in ion energy caused by non-instantaneous evaporation time from the specimen. Such lenses typically have the effect of improving the mass resolution from $\Delta \mathrm{m} / \mathrm{m}=1 / 70 \mathrm{FWHM}$ to around 1/500 FWHM [139]. A schematic demonstrating the principle of the lens is given in Figure 19.

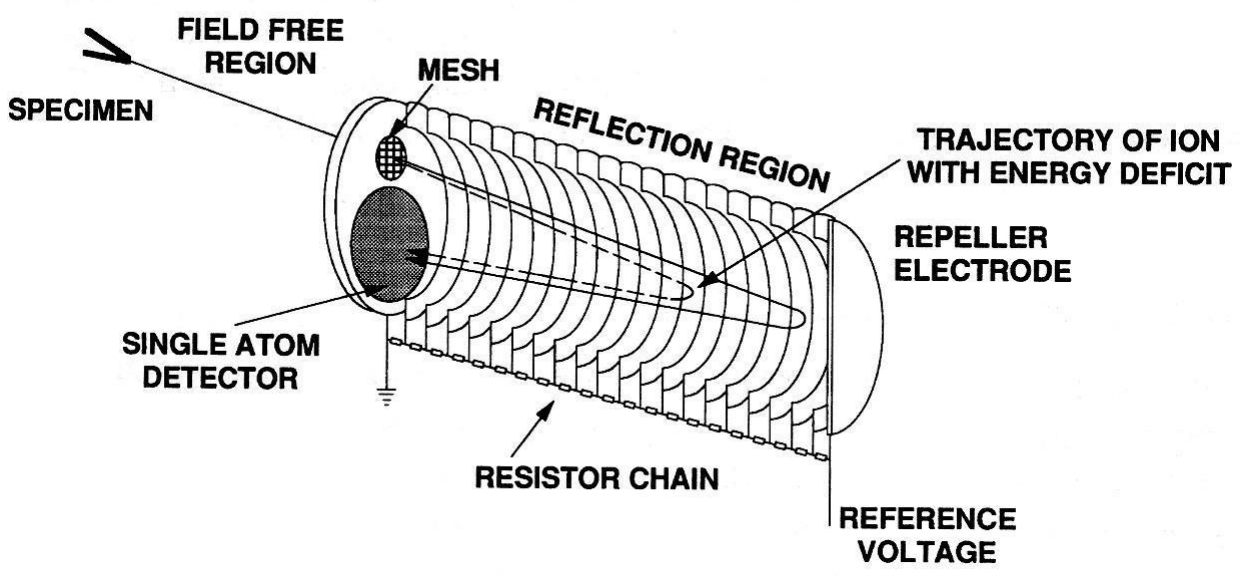

Figure 19: Reflectron lens schematic [131].

\subsubsection{Local electrode}

The local electrode is a conical electrode placed in close proximity to the atom probe specimen [140]. During analysis the electrode sits between the specimen and the detector, as shown in Figure 20. Ions travel through a 10-50 micron aperture in the electrode. The effect of this configuration is that the path over which ions are accelerated is reduced, improving the mass resolution. Other advantages from this configuration are that it affords a superior field of view and reduces the standing and pulse voltages required to produce an ionising field by around $50 \%$. 
Lower pulse voltages mean that higher pulse repetition rates can be achieved as the generation of a lower current is level is permitted. These currents become very high (hundreds of amps) for several kilovolt potentials applied and removed in nanoseconds. The high pulse repetition rate applied to the local-electrode $(200 \mathrm{kHz})$ allows a greater analysis data acquisition rate at a lower level of evaporation per pulse. This allows a slight reduction in the field at the apex of the specimen, reducing the stress placed upon the specimen.

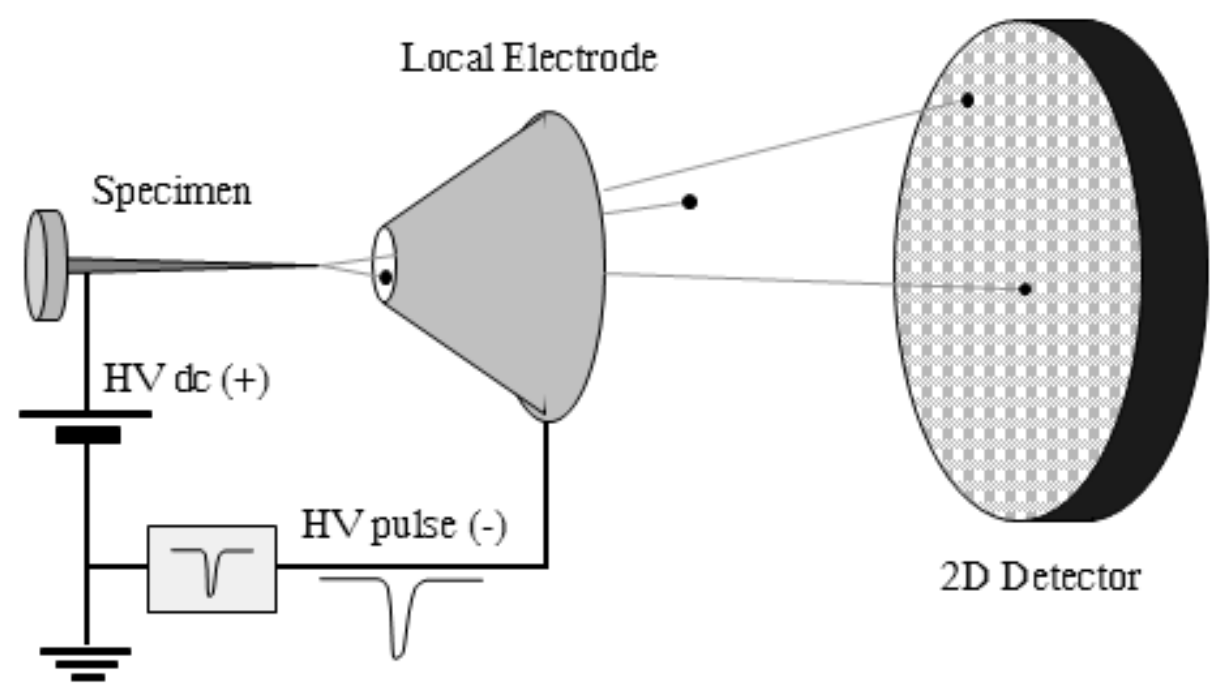

Figure 20: The configuration of a typical local electrode within the 3D atom probe. Schematic courtesy of Dr. D. Saxey, Oxford Materials.

\subsubsection{Micro-channel plate}

The atom probe detectors used in this study consist of a chevron micro-channel plate (MCP) arrangement in front of a delay-line detector (described below in section 3.1.6). The MCP performs the function of a signal amplifier, similar to a photomultiplier, magnifying the weak input signal from a single particle impact to creating a usable electrical signal, a cascade of secondary electrons. The MCP consists of an array of hollow channels, a few microns in 
diameter. Each of these acts as a miniature electron multiplier, generating a cascade of secondary electrons following a primary ion impact event. Each plate has many slots of a few microns in thickness parallel to each other. These act as channels for electron flow under a large imposed field. The gap between the two plates creates a spread in the electron cloud between the first and second plates. This ensures that several channels in the second plate multiply the signal. This double plate arrangement significantly increases the gain of the detector. A schematic of this arrangement is shown in Figure 21.

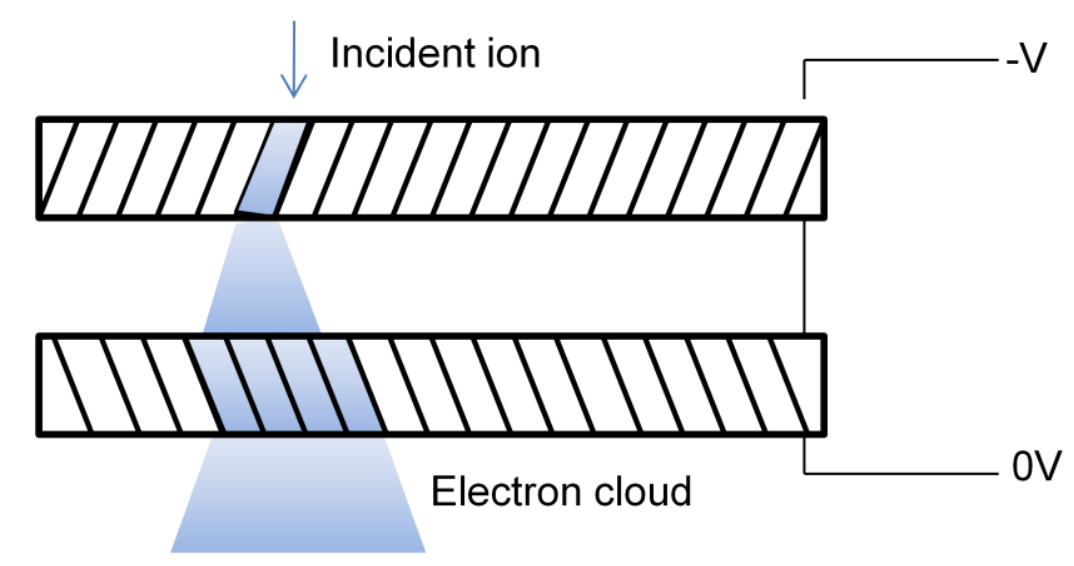

Figure 21: A chevron micro-channel plate arrangement.

The gain of the MCP has a broad spread of statistical amplitudes. The amplitude of the signal is not used in the calculation of the incident particle mass, which is achieved solely by time of flight mass spectrometry; or the incident particle $(x, y)$ position, which is calculated from the position of the charge cloud. The amplitude of the signal events is required to meet a threshold level. Low energy MCP noise that falls below this amplitude is ignored by the signal processing systems. 


\subsubsection{Delay-line detector}

Single particle positioning is achieved using a delay-line detector (DLD) arrangement. The sandwich arrangement of multiple DLDs allows for the accurate determination of flight time and impact position. A delay line is a single conductive channel in a zig-zag pattern. Figure 22 shows a two layer DLD used in combination with a circular MCP. Only the area behind the MCP is active. A particle incident at point $P$, with $2 \mathrm{D}$ coordinates $(x, y)$ produces four signals from the DLD $\left(t_{1}, t_{2}, t_{3}, t_{4}\right)$. These signals are the times at which the charge produced by the particle interaction with the MCP takes to reach the end of the DLD. The incident time of $P$ can be found by taking the arithmetic mean of the four output signals. The $x$ and $y$ coordinates are proportional to $t_{2}-t_{1}$ and $t_{4}-t_{1}$ respectively. A third delay line is used in modern 3DAP detectors. This delay line is positioned as a parallel third slice of the DLD sandwich, and its lines run at $45^{\circ}$ to the other two DL elements [141]. This yields a further two unique time coordinates for each hit event $\left(t_{5}, t_{6}\right)$. This additional information provides a further degree of freedom that can be used to identifying and disentangle the information acquired when a multiple hit event occurs [142]. 


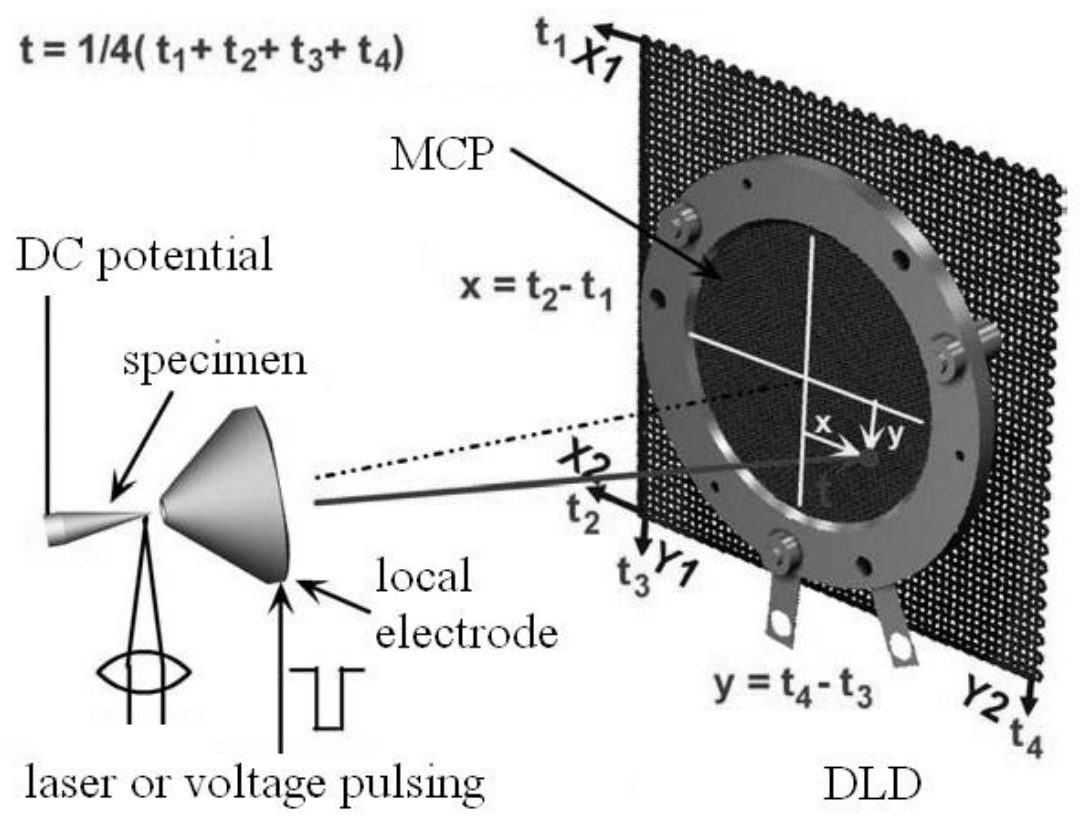

Figure 22: Complete detector configuration for a local electrode atom probe, including DLD. Adapted from [143].

\subsubsection{Laser-pulsed atom probe}

Pulsed laser atom probing (PLAP) was developed by Kellogg and Tsong in 1980 [144]. PLAP replaces the voltage pulses, which take the field at the specimen apex to above that required to cause ionisation, with thermal pulses, which increase the field evaporation process probability at the standing voltage potential [131]. A comparison of the modes by which laser and voltage pulsing promote field evaporation is shown in Figure 23. The excitation of free carriers within the skin depth of the material by incident radiation decays to heat on the picosecond timescale [145]. Thermal pulsing has the advantages that it allows the technique to be applicable to a wider range of materials, including semiconductors. The reasons for this are two-fold; the electronic conductivity requirements are reduced, high conductivity is required to allow the prompt propagation of nanosecond voltage pulses, and reduced mechanical strength requirements, as there is no need to withstand the elastic shockwave induced by voltage pulsing. 


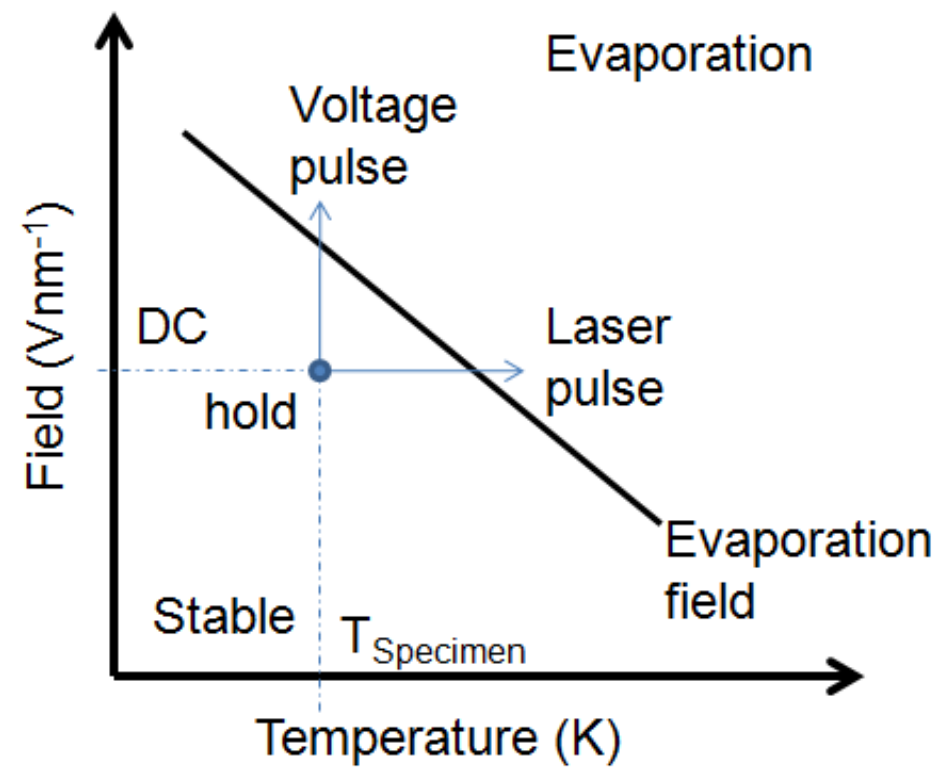

Figure 23: Schematic showing the operating mode of the laser and voltage pulsed atom probe techniques. The blue circle represents the position of the specimen on the evaporation curve during the holding part of the pulsing cycle. The Blue arrows mark the effect of voltage and thermal (laser) pulsing on the evaporation field experienced by the specimen: to take it above the required field and increase the probability of field evaporation from near nil to that which will induce the desired evaporation rate [131].

Laser pulsing removes the uncertainty in ion energy due to the voltage pulse shape, instead there is uncertainty due to the laser pulse width and the dissipation of energy within the specimen. This energy dissipation is a complex function of the specimen geometry and thermal conductivity. This has led to concerns that the comparison of results between different specimens analysed using PLAP should be treated cautiously. However, for the majority of specimens, those with a sharp apex and good thermal conductivity, it can be assumed that the tip is illuminated uniformly. In the $3000 \mathrm{HR}$ LEAP a high repetition rate $(200 \mathrm{kHz})$ femtosecond pulse laser is used. The femtosecond pulsing produces a suitably small fractional uncertainty in the spread of ion evaporation, given that the time-of-flight is of the order of a microsecond. 


\subsection{Apparatus}

\subsubsection{The LAR-3DAP}

Oxford has two 3D atom probes. One of these, the LAR-3DAP, is fitted with a large area reflectron. This instrument has three chambers. A load-lock, operating in the low to intermediate vacuum range, allows 3 specimens to be introduced into the chamber at a time. When the load lock has been pumped down to below $5 \times 10^{-6}$ mbar, the specimens can be transferred to a storage chamber. When the pressure in the storage chamber is below $5 \times 10^{-8}$ mbar a single specimen can be admitted to the analysis chamber. The analysis chamber contains two detectors; a phosphor screen and CCD camera arrangement for recording FIM images, and a delay-line detector for atom probe analysis. The two detectors are positioned perpendicular to each other. The needle-like specimen can be rotated so that it faces the relevant detector. Although the instrument is fitted with femtosecond laser pulsing capability, this is still the subject of development. In this study the LAR-3DAP was used only for some preliminary studies (described in sections 4.1, 4.3 and 4.4) using voltage pulsing. The pulse generator of the LAR3DAP operates at $20 \mathrm{kHz}$ with $\sim 8 \mathrm{~ns}$ pulse duration. The pulse-pair resolution of the instrument is $20 \mathrm{~ns}$. The field of view is typically $20 \mathrm{~nm}$ diameter.

\subsubsection{The local electrode atom probe}

The local electrode atom probe (LEAP) is a 3D atom probe with enhanced field of view of around $50 \mathrm{~nm}$. This is afforded by the implementation of a local electrode between the specimen and detector. The LEAP has a similar chamber configuration to the LAR-3DAP, but is capable of storing more specimens and transferring six of these into the load-lock in a single pumping 
cycle. The LEAP is a commercially developed instrument with both reliable voltage and femtosecond laser pulsing capability. The maximum repetition frequency of the pulses is 200 $\mathrm{kHz}$. The combination of the higher pulse rate and enhanced field of view allows the data acquisition rate of the LEAP to be many times greater than the LAR-3DAP. The 3 ns pulse-pair resolution of the LEAP is also superior to the LAR-3DAP.

\subsubsection{Atomic-scale reconstruction}

Unprocessed atom probe data consists of a sequence of records containing the times that a pulse was detected at the end of a series of delay lines along with the standing voltage and pulse amplitude when the ion was detected. The position the ion hits the detector corresponds to its original position on the hemispheric tip surface. This lateral resolution is limited by aberrations due to deviations in the tip geometry from the assumed hemispheric shape, and to surface migration. The lateral resolution is usually taken to be between $0.5-1 \mathrm{~nm}$. The position of the detected ion along the axis of the tip ( $z$ direction) is calculated from the order in which it was detected. This makes the reasonable assumption that atoms at the surface are ionised before those underneath them. The lateral resolution in the $z$ direction is taken to be that of a single atomic layer. The mass-to-charge-state ratio can be calculated from the voltage and time-of-flight data, as discussed previously in section 3.1.2. Specialist software is commercially available to reconstruct $3 \mathrm{D}$ datasets from raw atom probe data. The Imago IVAS package was used in this project as a front end for a version of CERN's ROOT software. 


\subsubsection{Parameters}

The reconstruction of atom probe data is based on five physical parameters. These are the image compression factor, the density of the material being analysed, the atom probe detector efficiency, and the initial tip radius and tip shank angle. The image compression factor (ICF) is due to the non-radial nature of projection from the tip; it is assumed that the surface is hemispherical, when in fact it is a needle with a finite shank angle. To account for this, the magnification of the image must be reduced by a constant factor. The ICF and detector efficiency are both considered to be constant for a specific atom probe instrument [146]. The LAR-3DAP has a ICF of 1.7 and the LEAP has a factor of 1.4. The detector efficiencies of these instruments are $35 \%$ and $38 \%$ respectively. The detection efficiency is lower than previous generations of instrument because a substantial fraction of the ions become incident upon grids within the reflectron.

For all alloys discussed in this work, the atomic density is approximated to that of pure alpha phase zirconium. This assumption is necessary due to a limitation of the reconstruction software. It is expected that this assumption affects the reconstruction of metallic specimens little, as the alloys are all at least 98 wt.\% zirconium. However, the assumption of constant density of the material under analysis poses a problem for the analysis of metal-oxide interfaces or intermetallic phases, where the densities of the two phases are distinct. The Pilling-Bedworth ratio of $\mathrm{ZrO}_{2}$ is 1.56 . In order to correctly reconstruct such datasets an estimate of the initial radius from TEM would be useful. This is not possible for micro-tip coupon specimens where the physical construction of the specimen prevent TEM investigation. The initial tip radius can be estimated from atom probe analysis by rearrangement of Eq. 3 into Eq. 8 .

$$
r=\frac{V}{k_{f} F_{e x}}
$$


$k_{f}$ is a constant $\sim 5$. $V$ is the applied voltage when evaporation 'turns on.' $F_{e x}$ is the expected electric field (textbook value for a given material) required to cause field evaporation. The measurement of the radius by this method often compares poorly with examination by TEM [147], a difference of up to $50 \%$ is not unusual. The radius also evolves as the run progresses; the rate of increase is dependent upon the shank angle.

\subsection{Statistical analysis and interpretation}

\subsubsection{Three-dimensional atom maps}

After successful reconstruction, the resulting dataset comprises a correctly scaled, threedimensional collection of points, each with a fourth value representing the ion mass-to-chargestate ratio. The term 'atom map' is somewhat of a misnomer as the map actually offers a useful way of displaying the position of the ionic species collected during the analysis, which may consist of either monatomic or molecular species, or both. Appropriate mass-charge ranging of ionic species is necessary to assign identities to the ions detected. This is achieved by inspection of the mass-to-charge spectrum of the dataset, or a particular sub-volume of ions. For dilute zirconium alloy systems this identification process is non-trivial and will be discussed further in section 3.3.2, below.

The reconstructed atom map data are highly flexible, allowing numerous ways of interrogating and displaying the collected data. There are a great variety of useful data analysis techniques that can be implemented to extract information about the physical chemistry of the specimen material. The data interpretation techniques that were used in this project are described below in sections 3.3.2 to 3.3.5. Some of the more important artefacts that affect the type of atom probe data examined in this study are discussed in section 3.3.6. 


\subsubsection{Ranging metric selection}

Mass ranges are the definitions of where windowed peaks begin and end on the mass spectrum. The definition of mass ranges is a key issue in the identification of ions in order to perform accurate and precise compositional analysis. These atomic identities are also used in the reconstruction protocol to assign an adequate in-depth increment to each detected ion [148]. Despite the importance of range selection, and the impact that the choice and application of ranging criteria has on both the accuracy of the reconstructed volume and its apparent composition, no standardised protocols exist with regard to the ranging metrics used by atom probe microscopists. The process is also currently conducted manually and with little consistency of methodology, even within a single research group.

A variety of automated ranging metrics are described and characterised in Appendix A. This is achieved by using the various metrics to calculate the composition of a series of simulated datasets. The composition of these datasets can be known with absolute certainty and compared with the measured composition given by the different ranging metrics. The results of this analysis inform the choice of ranging metric applied to real atom probe data in order to find the concentration of low level solute. These automated methods are used to analyse the iron concentration in sections 5 and 6.

\subsubsection{Deconvolution}

Deconvolution is the name given to the method of distinguishing between different ionic species that occupy the same position in the mass-charge spectrum. For an illustrative example, the mass-to-charge spectrum in Figure 24 contains 3 peaks, a, b and c. The spectrum is that of a binary alloy containing $A$ and $B$ atoms. The $A^{+}$ions have three isotopes with mass-to-charge 
ratios of 2,4 and $5(a, b, c)$. The relative isotopic abundance of isotopes is 60 at. $\%, 20$ at.\% and 20 at.\% respectively. $B$ is a mono-isotopic species with a mass-to-charge ratio of 5 .

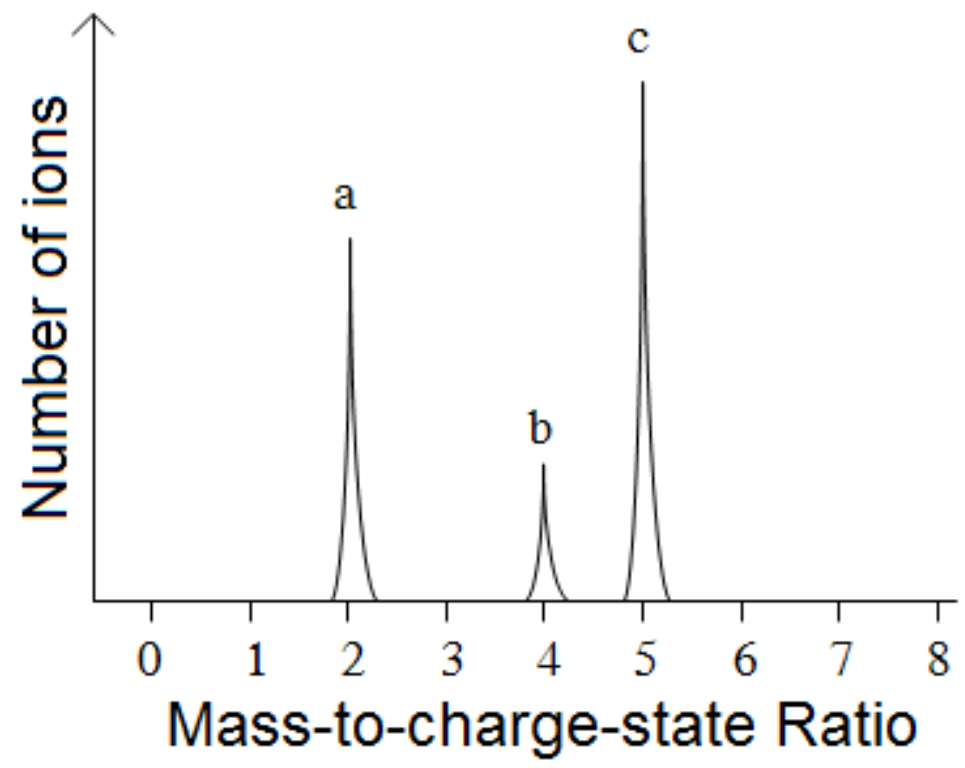

Figure 24: Example of the use of deconvolution in determining the amount of $A$ and $B$ atomic species in peaks $a, b$ and c. Similar to that in [131].

The amount of solute A and B is easily determined in this example,

$$
\begin{aligned}
& A=\frac{(a+b)}{(0.6+0.2)} \\
& B=c-0.2 A .
\end{aligned}
$$

Unfortunately there are several more complicated series of peak overlaps in the zirconium alloy systems that we shall encounter later. For our example above it should be noted that although the concentration of $A$ and $B$ could be determined trivially there was a loss of information compared to the case where the overlap had not occurred. This manifests itself in two ways:

- It is not possible to label individual atoms within peak $c$ as $A$ or $B$ atoms, only the proportion of each. This means that any segregation of $A$ and $B$ will be harder to 
distinguish from interrogation of the $3 \mathrm{D}$ atom map as the map can only display the atoms ranged within peak $c$, not $\mathrm{A}$ or $\mathrm{B}$ atoms within $c$.

- Not all the information from the spectrum was used in the calculation of the number of atoms. This increases the uncertainty in the measured composition of $\mathrm{A}$ and $\mathrm{B}$. For some situations this could be a significant disadvantage. These cases include where a minor solute overlaps with a major component of a system.

IVAS Version 3.4.1 contains automated deconvolution tools. These were not used for the majority of the work detailed in this project as this software takes away some choices from the user which can limit its flexibility. These choices include the ability to select the isotopes of a species that are used to determine its concentration. Such functionality is useful when considering areas of the spectrum where multiple species overlap, or where it is suspected peaks contain a contribution from an unknown species. For the most part, deconvolution was carried out numerically, away from the IVAS environment.

\subsubsection{Multiple hit analysis}

Ions detected during atom probe tomography are liberated by discrete pulses of energy, rather than a continuous stream of ions emitted during DC FIM. This allows time of flight mass spectrometry to be conducted.

Ions can be liberated singularly, or sometimes more than one ion is field evaporated from the specimen during the same pulse. Of these multiple hit events, a fraction will not be fully resolvable by the detector and a proportion of the signal lost. A potential problem arises for quantitative compositional analysis as the proportion of ions evaporated as multiple hits is different for different species. This in turn means that a different proportion of hit information is 
lost regarding different species - altering the observed composition. In order to minimise the number of multiple hit ion events the evaporation rate is controlled so that the average rate of ionisation is in the range of 1 ion per $200-500$ pulses.

Multiple hit events can provide useful information about the identity of overlapping species. An example of this is at $28 \mathrm{Da}$, where $\mathrm{Fe}^{2+}$ and $\mathrm{CO}^{+}$overlap. In some circumstances, the distribution of iron can be better observed by plotting only the multiple hit events, as a greater proportion of the multiple hits at this mass position are iron than $\mathrm{CO}$, as can be determined from the isotopic ratio between $27,28,28.5$ in the multi-hit spectra.

\subsubsection{Proxigrams and compositional profiles}

Sub-volumes, or 3D regions of interest, can be defined within reconstructed atom probe datasets. The bounds and orientation of these regions can be varied so that they either contain only one region, or are across an interface between regions of different composition. In the first case, the atoms within selected volumes can be displayed as separate mass-to-charge spectra. This is particularly useful if the sub-volume selected is chemically homogeneous. However, where there is variation of composition within a defined region it can be useful to represent these data as a one-dimensional compositional profile. This can be generated by splitting the region into slices separated by a small increment along a selected axis of the volume. The composition of each individual section is calculated and saved as a single data point. For such data representations, the uncertainty in the individual slice composition is dependent upon the number of atoms within the slice. A compromise must be found between thick slices, where the uncertainty in the composition is lower but the measurement of variations is coarser, and thinner slices. Such profiles are convenient for measuring the composition across planar features, such as sections of 
grain boundaries, and when examining 1D concentration gradients in volumes of matrix material.

When the concentration profile of a feature with a more complex geometry is of interest a proxigram may be used. 'Proxigram' is a combination of 'proximity' and 'histogram.' Proxigrams are a method of displaying local concentration of species against proximity to a defined surface [149]. The profile produced is normal to the surface at all points. The surface is known as an 'isosurface', usually a surface of uniform composition. The proxigram is a useful tool when examining precipitates above a certain size of a few nanometres - this threshold is well below the size of the precipitates found in commercial zirconium allows. An isosurface can easily be defined around the boundary of such features.

\subsubsection{Important artefacts and aberrations}

Reconstruction algorithms normally assume a homogeneous magnification of the tip surface structure. This is not the case where there is a variation in the geometry of the tip from that of a hemisphere. This occurs when precipitates are analysed as the evaporation field of the precipitate tends to be different from that of the matrix. Because of this there is preferential evaporation of the material with the lower evaporation field. This process leads to changes in curvature around the precipitate. This will lead either to localised convergence or divergence of ion trajectories from the precipitate region [150]. This has the effect of altering the observed size of the particle on the detector and also its density. Another case where the geometry of the tip is altered by a microstructural feature is in the case of high angle grain boundaries. The grain misorientation at the interface creates additional curvature and generates an apparent increase in density at the grain boundary on the detector. 

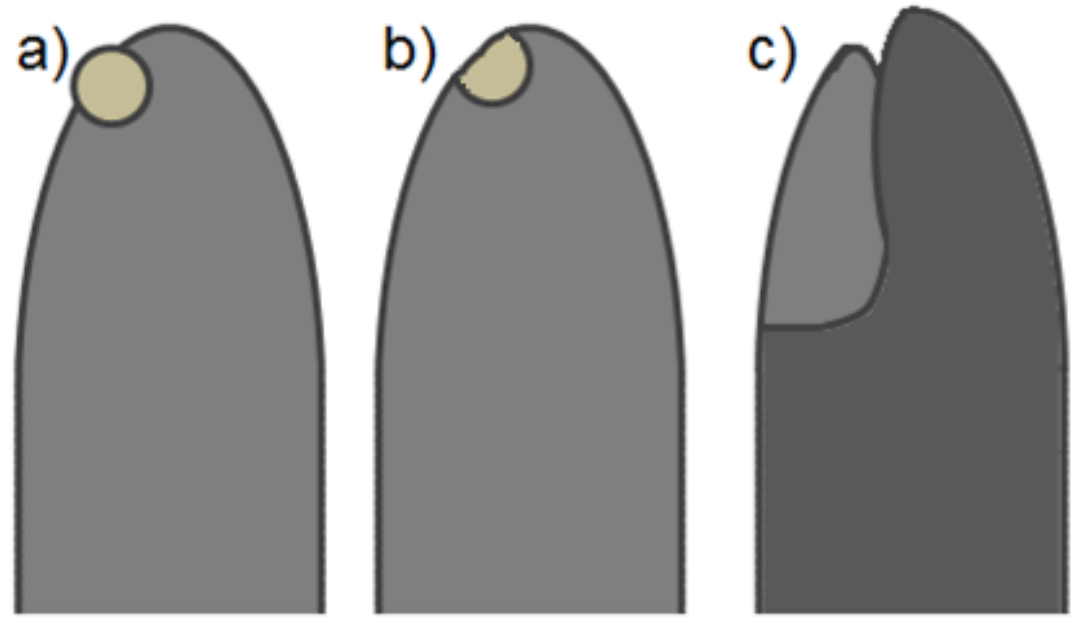

\section{Intermetallic particle}

$\square=$

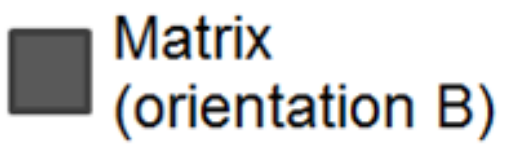

Figure 25: Atom probe specimen containing various microstructural features: An intermetallic with a) higher and b) lower evaporation field than the matrix, the outer surface of the precipitate has a larger radius of curvature that the specimen matrix. c) Shows a specimen containing a grain boundaries. There is a high angle mismatch between orientation $A$ and $B$. The extent of the difference in curvature is exaggerated for this example.

There are several other common problems with the reconstruction or interpretation of atom probe results, however, these can be mitigated by careful consideration or additional data manipulation. These are summarised as follows:

- Improper selection of analysis conditions leading to retention of atomic species. This causes a perturbation in the apparent concentration of species. Calibration experiments must be performed to ensure that species are not subject to preferential evaporation.

- Poor thermal conductivity leading to the delay of ions liberated during laser pulsing creating 'thermal tails' in mass spectra. If two peaks have a similar mass-to-charge value this can lead to interference between the two and if a basic ranging method is used then one species may be incorrectly defined within the window of the other. This is a special case of incorrect ranging, or the requirement to deconvolute overlapping peaks. 
- Incorrect choice of reconstruction parameters, leading to dimensionally inaccurate reconstructions. This causes the incorrect scaling of micro-structural features within reconstructions. It can be corrected by ensuring atomic planes in reconstructions are not curved and that micro-structural features are the correct shape; spherical features are not oval in appearance, linear features are not curved. Geometric information about the tip end form can be acquired using TEM before analysis; this information can then be fed into the reconstruction.

\subsubsection{Summary}

Atom probe data can be reconstructed to give 3-dimensional datasets that aim to represent faithfully the distribution of individual atoms over a volume of up to $100 \mathrm{~nm} \times 100 \mathrm{~nm} \times 1000$ nm. Modern computational techniques allow the manipulation of these datasets to acquire precise details of microstructural features at almost an atomic scale. A variety of such methods have been developed for the inspection of different regions and interrogating them in order to obtain different types of information. In the course of this project, original work has been performed that adds to, and complements, existing data analysis techniques regarding background correction and ranging metric application. The design and testing of these methods and metrics is described in Appendix A.

Artefacts introduced by atom probe tomography are less amenable to quantitative theoretical analysis than those encountered in more common electron microscopy techniques. The results chapters (sections 4,5 and 6) describe in detail the steps that have been taken to identify and account for artefacts and inaccuracies within reconstructed datasets. 


\subsection{Materials}

\subsubsection{Materials studied}

Four zirconium-based alloys were investigated during the course of this study: commercially pure zirconium, ZIRLO, Zircaloy-4 and a Zr-Nb-Ti test alloy. Compositional information for the batches of material used can be found in Table 8 .

\begin{tabular}{|c|c|c|c|c|c|c|c|c|}
\hline \multicolumn{9}{|c|}{ Bulk composition } \\
\hline & \multicolumn{2}{|c|}{$\begin{array}{l}\text { Commercially 'pure' } \\
\text { zirconium }\end{array}$} & \multicolumn{2}{|c|}{ ZIRLO } & \multicolumn{2}{|c|}{ Zircaloy-4 } & \multicolumn{2}{|c|}{$\mathrm{Zr}-\mathrm{Nb}-\mathrm{Ti}$} \\
\hline & (wt.\%) & (at.\%) & (wt.\%) & (at.\%) & (wt.\%) & (at.\%) & (wt.\%) & (at.\%) \\
\hline $\mathrm{Nb}$ & 0 & 0 & 0.98 & 0.96 & 0 & 0 & 0.98 & 0.96 \\
\hline Sn & 0 & 0 & 0.99 & 0.76 & 1.28 & 0.98 & 0 & 0 \\
\hline $\mathrm{Fe}$ & 0.02 & 0.03 & 0.11 & 0.18 & 0.22 & 0.36 & 0.05 & 0.08 \\
\hline $\mathrm{Cr}$ & 0.02 & 0.03 & $<0.01$ & $<0.02$ & 0.11 & 0.19 & - & - \\
\hline $\mathrm{Ni}$ & - & - & $<0.01$ & $<0.02$ & - & - & - & - \\
\hline $\mathrm{N}$ & 0.01 & 0.06 & 0.04 & 0.25 & - & - & - & - \\
\hline $\mathrm{C}$ & 0.025 & 0.19 & 0.014 & 0.11 & 0.016 & 0.12 & - & - \\
\hline $\mathrm{Hf}$ & 0.25 & 0.13 & - & - & - & - & - & - \\
\hline $\mathrm{Ti}$ & 0 & 0 & 0 & 0 & 0 & 0 & 0.21 & 0.4 \\
\hline $\mathrm{O}$ & 0.1 & 0.57 & 0.19 & 1.1 & 0.13 & 0.74 & - & - \\
\hline $\mathrm{H}$ & 0.001 & 0.09 & - & - & - & - & - & - \\
\hline Si & - & - & - & - & 0.01 & 0.03 & & \\
\hline $\mathrm{Zr}$ & balance & balance & balance & balance & balance & balance & balance & balance \\
\hline
\end{tabular}

Table 8: Bulk compositional information regarding the four alloys studied. '- 'is inserted in elements where data has not been provided. Data courtesy of Goodfellow Cambridge Ltd, EDF Energy and Westinghouse Electric Company Ltd. 


\subsubsection{Commercially pure zirconium}

Commercially pure (>99.2 wt.\%) zirconium was used as a reference material so that the added complexities of alloying additions could be introduced later, after a better understanding of specimen fabrication and analysis of the pure zirconium mass spectra, had been obtained. The material was supplied by Goodfellow Cambridge in the form of a $0.125 \mathrm{~mm}$ drawn wire. This wire was further stress relieved at $500{ }^{\circ} \mathrm{C}$ for 30 minutes in an inert atmosphere.

\subsubsection{ZIRLO}

Standard ZIRLO sheet was supplied by the Westinghouse Electric Company. The sheet had undergone numerous hot- and cold-rolling stages. Between each rolling stage the material was annealed at around $595{ }^{\circ} \mathrm{C}$ with the intention of fully recrystallising the material. Suitable methods for performing this process are described in detail by [151]. The final anneal created a fine, equiaxed microstructure. An optical micrograph of this material is shown in Figure 26.

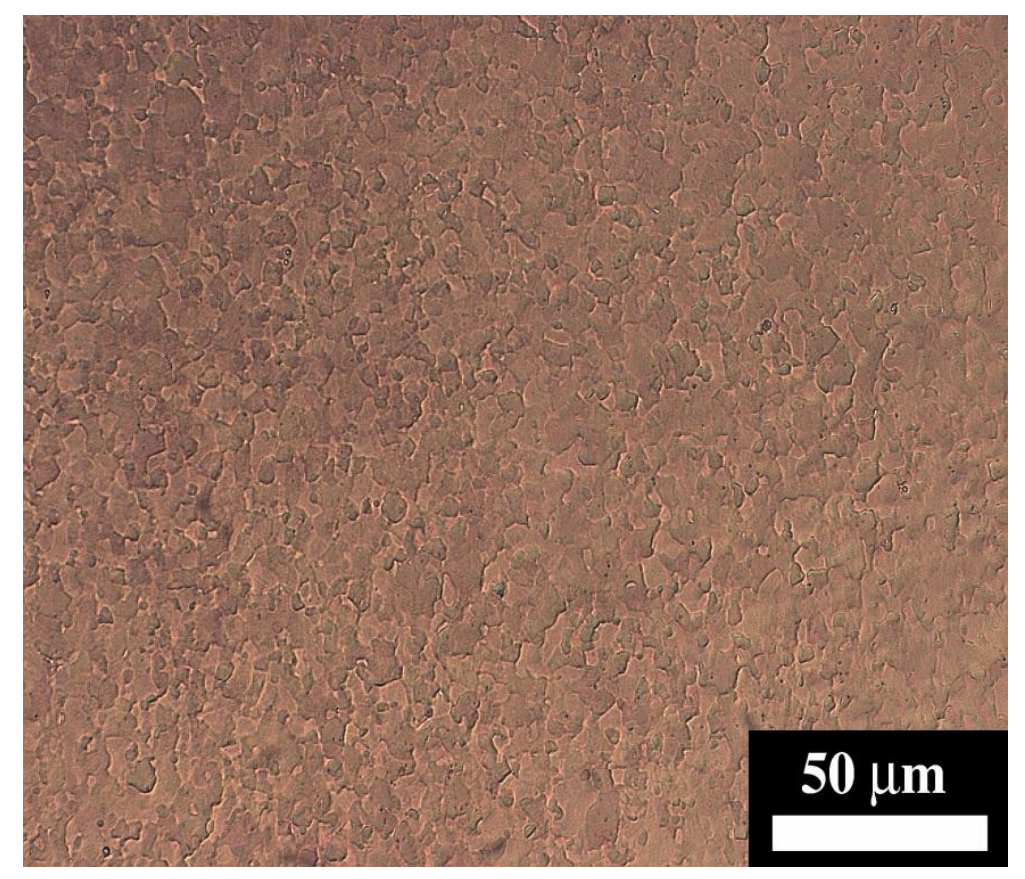

Figure 26: Electrochemically etched ZIRLO RXA sheet material. Courtesy of Jianfei Wei, UoM. 


\subsubsection{Zircaloy-4}

Zircaloy-4 specimens from two different autoclave studies were analysed. Tubular material was prepared and subjected to a final stage recrystallising heat treatment in argon at $620{ }^{\circ} \mathrm{C}$ for 2 hours before being subjected to autoclave exposure. This material was used in an initial study of the metal-oxide interface of autoclave corroded material. Further work was conducted on recrystallised sheet material that underwent a similar thermal processing route. The microstructure of this material is shown in Figure 27. Both materials were provided by Westinghouse Electric Company.

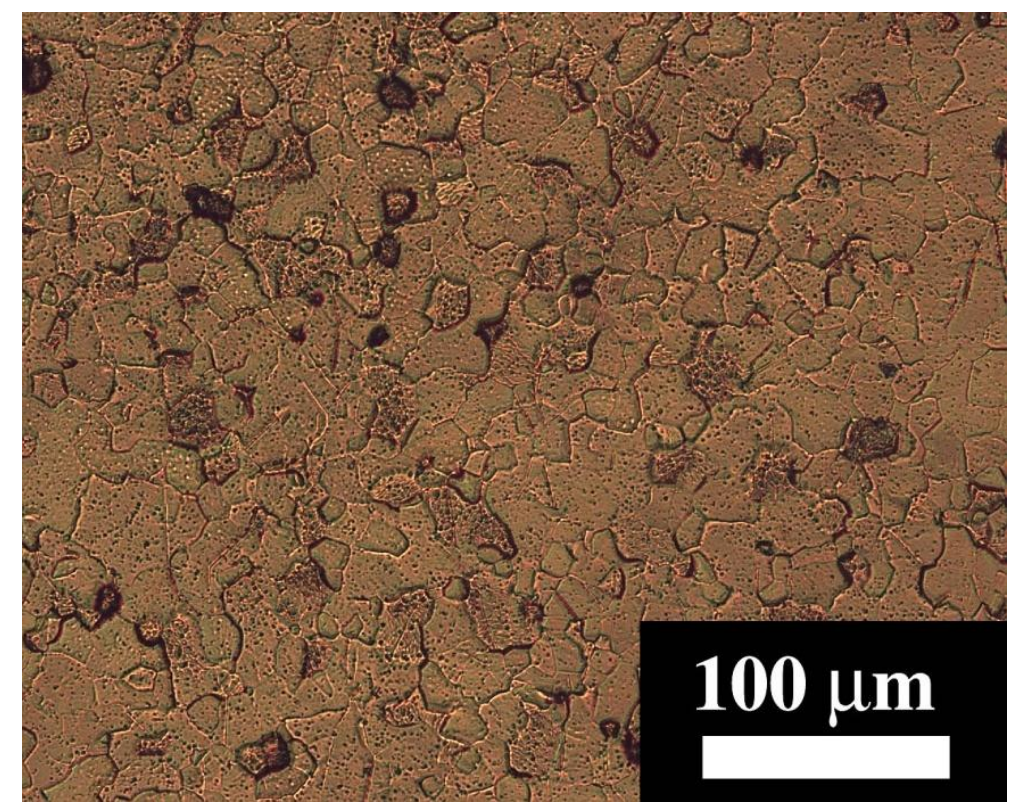

Figure 27: Optical micrograph of electrochemically etched Westinghouse Zircaloy-4 RXA sheet material. Courtesy of Jianfei Wei, University of Manchester.

\subsubsection{Zr-Nb-Ti}

The $\mathrm{Zr}-\mathrm{Nb}$-Ti alloy was processed as a $350 \mathrm{~g}$ test ingot and deformed to a strip. Processing of the resulting button included beta-quenching followed by hot rolling to an intermediate size. The 
sample was reduced to a final size by two iterations of cold rolling and annealing. The intent of the final anneal was to fully recrystallise the strip. The recrystallising conditions used were similar to those used to process ZIRLO. However, in this case the resulting material microstructure has grains elongated by a factor of 5 that are finer than the grains in the ZIRLO material. A TEM micrograph showing the grain structure of this material is shown in Figure 28.

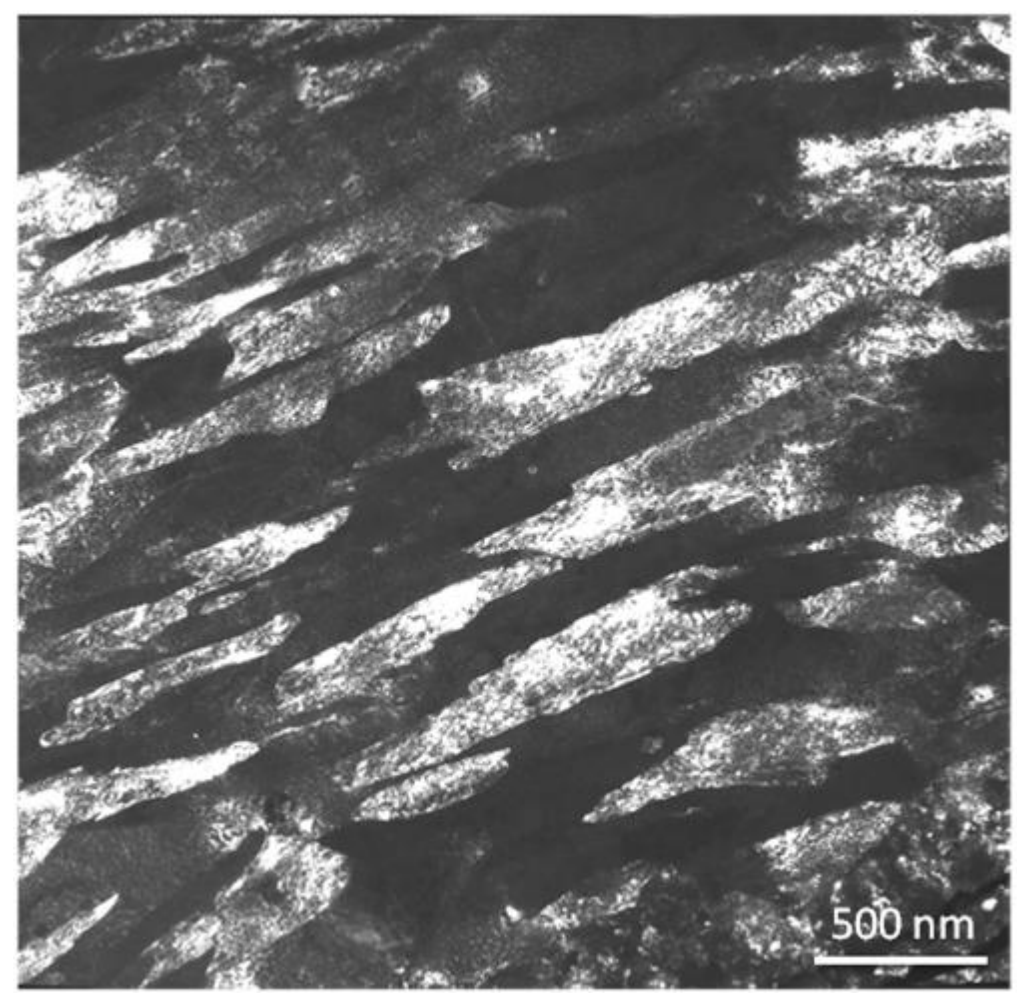

Figure 28: Bright field TEM micrograph of the $\mathrm{Zr}-\mathrm{Nb}-\mathrm{Ti}$ test alloy metal grain structure. Courtesy of Na Ni, Oxford Materials. 


\subsubsection{Corrosion treatment}

\subsubsection{Commercially pure zirconium}

Laboratory studies on the commercially pure drawn wire were conducted by subjecting atomically clean atom probe needle specimens to corrosion by air at room temperature and by boiling water. The atomically clean needle surface was prepared by field ion microscopy or atom probe analysis of the atom probe specimen, removing the original surface layer. Room temperature air experiments allow the description of very early stage oxidation that cannot practically be attained in real life corrosion environments or during autoclave experiments. Boiling water treatments were applied in order to replicate more severe corrosion, and to develop methods of analysing such specimens before progressing to full-scale autoclave experiments. The results of these laboratory experiments are described in section 0 .

\subsubsection{ZIRLO}

Recrystallised annealed sheet ZIRLO was subjected to aqueous corrosion in a static autoclave. This corrosion testing was conducted by Jianfei Wei of Manchester University at an EDF Energy facility near Paris. The static autoclave was operated at $360{ }^{\circ} \mathrm{C}, 18 \mathrm{MPa}$ using primary water chemistry (pure $\mathrm{H}_{2} \mathrm{O}$ with additions of $2 \mathrm{ppm} \mathrm{LiOH}$ and 1000 wt.ppm boric acid) as a corrosive medium. Many identical specimens were placed in the autoclave and subsequently removed after incremental periods of time. Figure 29 demonstrates the first transition in the corrosion kinetics of the ZIRLO specimens. Specimens were chosen from this large matrix so that a wide range of corrosion conditions would be encountered whilst limiting the number of atom probe specimens to be examined. A description of the condition of each sample is given in Table 9. Several specimens were examined for each data point, with the objective of looking at the metal-oxide 
interface and the regions of metal and oxide closely adjacent to the interface (within a few hundred nanometres). The specimen preparation of metal-oxide interface specimens is more time consuming and skilled than the specimen preparation methods required for bulk metal specimens. These processes are described in $\S 3.5 .3$.

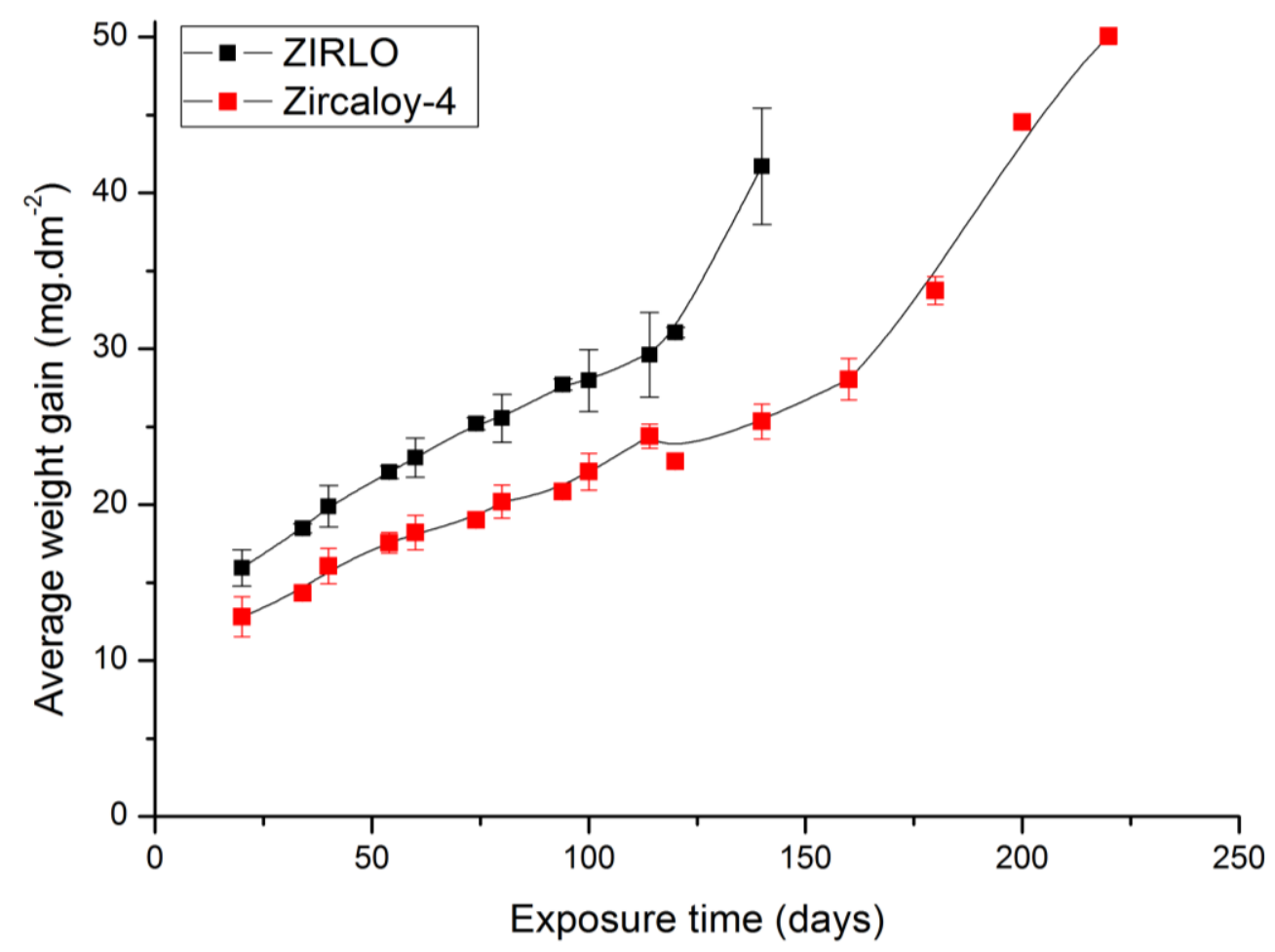

Figure 29: The corrosion of ZIRLO and Zircaloy-4 in a $360{ }^{\circ} \mathrm{C}$ water static autoclave. Data supplied by Jianfei Wei, Manchester University.

\begin{tabular}{|c|c|cc|c|}
\hline Sample ID & Weight gain & \multicolumn{2}{|c|}{ Duration } & Description of oxidation progress \\
$\#$ & (Mg.dm ${ }^{-2}$ ) & (days) & (hours) & \\
\hline SZLRX 17 & 18.25 & 34 & 816 & Pre-transition oxide \\
SZLRX 1 & 25.66 & 114 & 2736 & Further corroded pre-transition oxide \\
SZLRX 12 & 28.52 & 100 & 2400 & $\begin{array}{c}\text { Pre-transition oxide nearest break- } \\
\text { away }\end{array}$ \\
SZLRX 4 & 45.91 & 140 & 3360 & Post-transition oxide \\
\hline
\end{tabular}

Table 9: Description of the conditions of the corroded ZIRLO specimens. 
Specimen SZLRX1 presented lower-than-trend weight gain. The weight gain of this sample after 114 days was more typical of a specimen having undergone 75 - 80 days exposure.

\subsubsection{Zircaloy-4}

Initial investigations relating to the metal-oxide interface of Zircaloy- 4 were conducted using Westinghouse Zry-4 supplied by EDF energy. The recrystallised tube specimens were subjected to corrosion testing for 90 days $(2160 \mathrm{hrs})$ in $360{ }^{\circ} \mathrm{C}$ primary water containing water 2 wt.ppm Li and 1000 wt.ppm boric acid.

Recrystallised Zircaloy-4 sheet specimens were also examined after 7 and 114 days of autoclave exposure treatment administered by Jianfei Wei and Peng Wang of Manchester University. These specimens are also listed in Table 10. The weight gain curve for the Westinghouse sheet Zircaloy-4 used in this study was shown in comparison to ZIRLO in Figure 29. It is seen that the Zircaloy-4 material performed better than ZIRLO in the autoclave environment tested in this study, a result that would not be expected from the literature. The water chemistry of this autoclave is similar to the conditions experienced by the tube material $-360{ }^{\circ} \mathrm{C}$ at $18 \mathrm{MPa}$ in a static autoclave using primary water chemistry (pure $\mathrm{H}_{2} \mathrm{O}$ with additions of 2 ppm $\mathrm{LiOH}$ and 1000 wt.ppm boric acid). The tube specimen corroded for 90 days have a higher average weight gain than the sheet material exposed to 114 days exposure.

\begin{tabular}{|c|c|cc|c|}
\hline Sample ID & Weight gain & \multicolumn{2}{|c|}{ Duration } & Description of oxidation progress \\
$\#$ & (Mg.dm $^{-2}$ ) & (days) & (hours) & \\
\hline 7day Zry-4 & 15.3 & 7 & 168 & Early-oxidation pre-transition oxide \\
Z4RX1 & 23.43 & 114 & 2736 & Pre-transition oxide \\
\hline
\end{tabular}

Table 10: Description of the conditions of the corroded ZIRLO specimens 


\subsubsection{4 $\mathrm{Zr}-\mathrm{Nb}-\mathrm{Ti}$}

Atom probe specimens were prepared from a sample that had increased in weight by 1189 mg.dm ${ }^{-2}$, equivalent to an average oxide thickness of $\sim 80$ microns [14]. This corrosion occurred after only 1 day ( $24 \mathrm{hrs})$ in primary water at $360{ }^{\circ} \mathrm{C}$. The corrosion rate experienced by this material was $\sim 400$ times greater than that seen in similar alloys such as ZIRLO, M5 or Zr-1Nb. The processing route to which the material was subjected was similar to that of the ZIRLO used in this study. The large difference in corrosion performance is attributed to the presence of 0.21 wt.\% titanium added to this material.

\subsection{Atom probe specimen preparation}

Three methods of atom probe sample preparation were used during the course of this project. These are described are described in sections 3.5.1 Electrochemical etching, 3.5.2 Focussed ion beam (FIB) milling and 3.5.3 Advanced FIB techniques. The three methods were used to create specimens from different types of material and balanced the ease of preparation with the requirements related to analysing localised sites within materials.

\subsubsection{Electrochemical etching}

The preparation of field ion microscope needles for the 3-D atom probe is a critical step in the analysis process. The acquisition of useful quantities of high quality data is dependent upon the creation of needles with good structural integrity, the absence of points of mechanical weakness, and an even, hemispherical end form to allow correct reconstruction. As mentioned previously the tip apex must be less than $\sim 100 \mathrm{~nm}$ in radius in order to allow the creation of the high electric fields necessary for field evaporation. 
Electrochemical etching can be used to create needle specimens from conductive material. The etching is usually a two stage process. Initially a rod or wire is placed as the anode in an electrochemical cell. A typical arrangement of such an apparatus is shown in Figure 30. The electrolyte in the cell is supported by a dense fluid that does not take part in the etching process. The cathode is formed by a gold wire. The specimen can be inserted and retracted from the etchant in order to avoid the formation of a passive layer on the area under electrochemical attack. The rate of removal of material from the specimen can be controlled by the choice of electrolyte and the potential difference applied across the cell. The temperature of the solution also has an effect; lower temperatures tend to produce a smoother finish due to the development of a polishing layer near the interface. The conditions used for the electrochemical etching are given in Table 11.

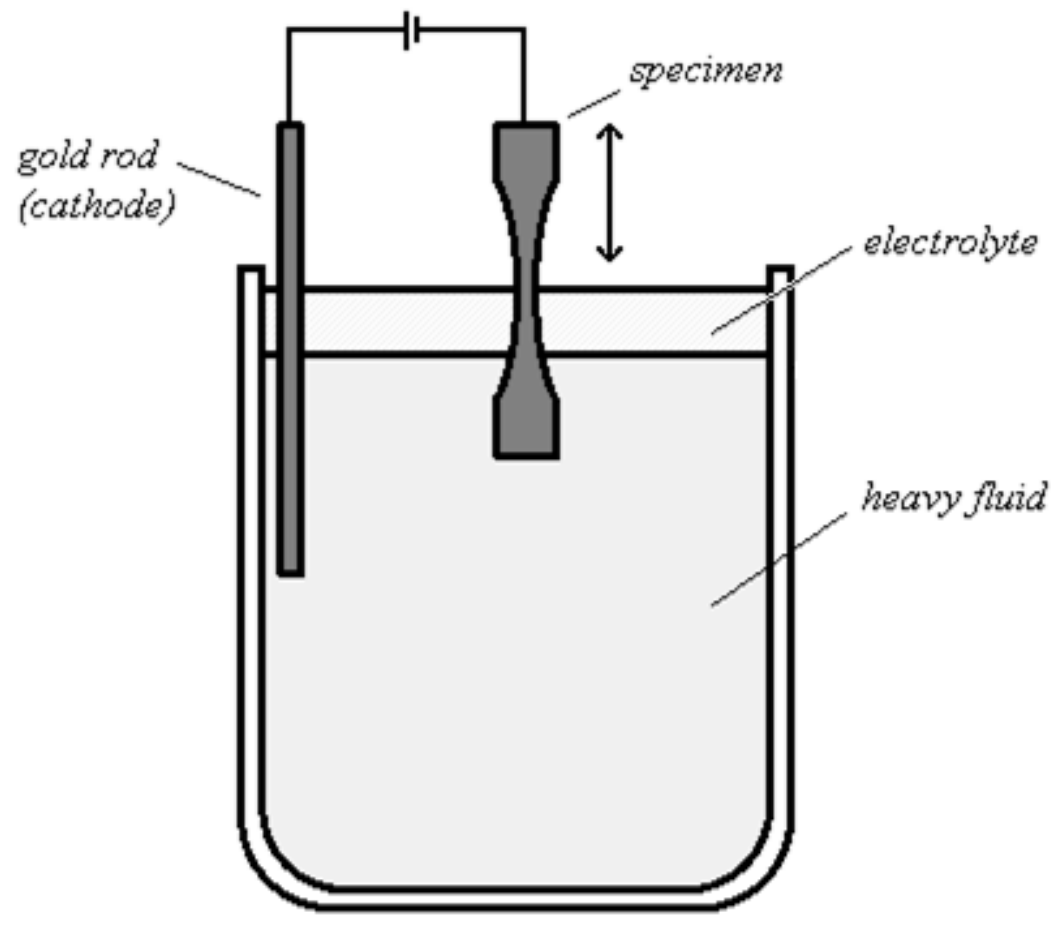

Figure 30: An electrochemical cell (stage 1) arrangement used to etch away the mid-section of a conductive electrode. 


\begin{tabular}{|c|c|c|c|}
\hline Stage & Cell chemistry & Applied p.d. & Time \\
\hline 1 Start & $\begin{array}{c}25 \% \text { perchloric } \\
\text { acid (60\%), 75\% } \\
\text { acetic acid (100\%) }\end{array}$ & $20 \mathrm{~V}$ & $\sim 3 \mathrm{~min}$ \\
\hline 1 Middle & " & $10 \mathrm{~V}$ & $\sim 3 \mathrm{~min}$ \\
\hline 1 End & $\begin{array}{c}\text { "\% perchloric acid } \\
(60 \%), 98 \% 2- \\
\text { butoxyethanol }\end{array}$ & $10 \mathrm{~V}$ & $\sim 3 \mathrm{~min}$ \\
\hline 2 Start & " & $4 \mathrm{~V}$ & $\sim 3 \mathrm{~min}$ \\
\hline 2 End & \multicolumn{2}{|c|}{} \\
\hline
\end{tabular}

Table 11: The experimental conditions used during electrochemical etching.

The result of this stage in the process is the gradual removal of material from the mid-section of the sample material, eventually causing it to etch through the mid-point. This produces two needle-like specimens. At this stage, the specimen end radii are of the order of a micron. Further thinning is required, and this can be most easily achieved by drop-polishing. The specimen is held in a small vice that may be moved towards or away from a drop of fluid using a fine control. The specimen may pierce the drop and may also be withdrawn from the drop. The process may be monitored using an optical stereomicroscope. Figure 31 shows a schematic of a specimen during drop polishing. Table 11 also details the conditions used in this arrangement, which are designed to strip material from the specimen at a much reduced rate compared to the previous stage.

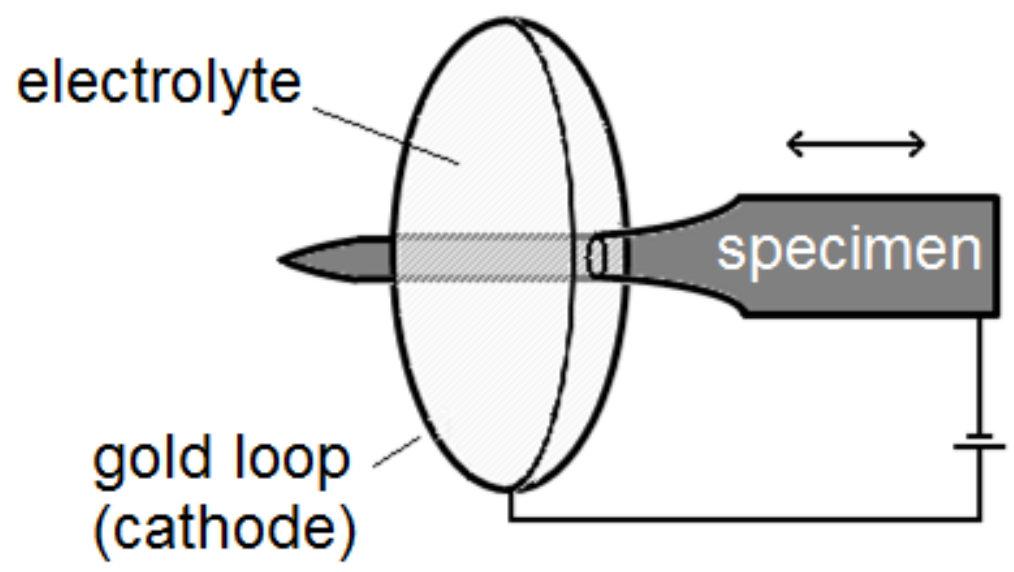

Figure 31: A schematic of a specimen during drop-polishing (stage 2). 
The careful removal of additional material from near the tip creates a second region of thinning, similar to the necking that resulted from the first stage. This area is further thinned until a sharp point is formed.

The main disadvantages of this electrochemical polishing are the need for good specimen conductivity, making it unsuitable for non-metallic specimens, and the anisotropic polishing of materials that can be achieved when applied to hexagonal crystal structure metals. The process is also a minor source of heat and allows a possible route for hydrogen and oxygen uptake. Hydrogen, being a more mobile species, is able to diffuse further into the bulk specimen in the same amount of time.

\subsubsection{Focussed ion beam (FIB) milling}

Focused ion-beam milling can be used to further sharpen samples after preparation by electropolishing. Although electropolishing alone is capable of producing tips of the required geometry for analysis using 3DAP, sharpening tips using FIB has some additional benefits. FIB allows precise control of tip geometry, such as the end radius and shank angle. Hexagonal $\alpha-\mathrm{Zr}$ has a tendency to electropolish anisotropically, forming specimens with an elongated axialdimension. It can also remove any secondary sharp points that have formed near the tip apex, which might interfere with the laser targeting system.

The FEI 200 FIB is a focussed ion beam milling instrument with a single liquid gallium source. It also allows the deposition of platinum to enable the construction of thermally and electrically conductive islands, as well as the deposition of a protective layer against ion implantation damage. Imaging can be performed using secondary ion and secondary electron detectors. The ion current is varied to control the rate of milling. This instrument can deliver beam currents between $10 \mathrm{pA}$ and $20 \mathrm{nA}$ and can achieve a spatial resolution of $\sim 20 \mathrm{~nm}$. Characterisation of 
the finished tips using the FIB as an ion-microscope is undesirable, as the act of characterisation mills away material from the surface under examination. The role of the single beam FIB must therefore be restricted to use as a milling tool.

Tip sharpening is conducted by the application of a beam using an annular milling pattern down the shank of a tip. The inner and outer radii of the pattern can be progressively altered to cause the reduction in inner radius and shank angle. If a single pattern was used, with a large outer radius and a small inner radius, then the tip would have a larger shank angle. There are situations where this might be desirable, such as the creation of tips from poor thermally conducting material. In this case, the additional shank provides a greater area for the diffusion of heat from the apex and the atom probe laser beam would still fully illuminate the tip during analysis. Despite this advantage this is not a good approach as this would lead to a large increase in the specimen radius during analysis.

Although the imaging resolution of the instrument is very good, there is some beam spread. The effect of this is that the tips created are sharper than the nominal inner radius of the beam scan. The final desired inner radius and the actual diameter of the tip were compared using bright field TEM, these data are given in Table 12 (over leaf). Table 12 also indicates the variation in geometry of the tips produced using this method and instrument. The variation in shank angle for these tips was less apparent. All of these specimens had a half-shank angle of $(4 \pm 2)^{\circ}$.

After the final stage of polishing in the FIB the tips were removed and transported in air to either the atom probe, where they would be stored at $\sim 1 \times 10^{-8}$ mbar, or a storage desiccator, where they would be stored at around 5.3 mbar. The sputtering of environmental oxygen onto the tip of a charged field emitter has been informally conjectured to explain the unexpected deterioration of some atom probe specimens in transit between FIB and atom probe. Precautions were taken to 
prevent the build up of static charge around the specimens. These include using earthed tweezers and lining specimen cases with metal foil to act as a Faraday cage.

\begin{tabular}{|ccc|}
\hline $\begin{array}{c}\text { Nominal } \\
\text { radius }(\mathrm{nm})\end{array}$ & $\begin{array}{c}\text { Nominal } \\
\text { diameter }(\mathrm{nm})\end{array}$ & $\begin{array}{c}\text { Measured } \\
\text { diameter }(\mathrm{nm})\end{array}$ \\
\hline 80 & 160 & $130 \pm 20$ \\
80 & 160 & $140 \pm 20$ \\
80 & 160 & $160 \pm 30$ \\
140 & 280 & $180 \pm 40$ \\
140 & 280 & $250 \pm 30$ \\
140 & 280 & $290 \pm 30$ \\
\hline
\end{tabular}

Table 12: Comparison between nominal and actual tip geometry prepared using a FEI FIB 200.

Focussed ion beam milling allows the fine control of tip end geometry. It also removes any surface impurities that may be left after electrochemical etching. However, the FIB methods discussed thus far are only suitable for the creation of atom probe specimen from conductive materials, and often involve cases where the selection of the region of interest is not critical. These methods are not suitable for the investigation of the metal-oxide interface of zirconium, or any other specific pre-identified region of interest. It was necessary to develop a separate method for such cases, as the immediate locality of the metal-oxide interface is important in furthering our understanding of the oxidation mechanisms of zirconium. 


\subsubsection{Advanced FIB techniques}

Specimens can be prepared from the metal-oxide interface by cutting out a wedge along the interface, similar to a TEM lift-out technique [152-153]. This requires the preparation of a crosssection by cutting, mounting and polishing. The wedge-shaped beams are equilateral triangular prisms with lengths between $20-30 \mu \mathrm{m}$ and widths of $2-3 \mu \mathrm{m}$ [154]. A prism is created from the bulk by applying two long cuts parallel to the interface and at $30^{\circ}$ to the surface normal, along with a cut at one end to create a prism attached to the bulk by a single face. Such a structure is shown in Figure 32, along with a smaller part-finished prism where only one long cut has been created.
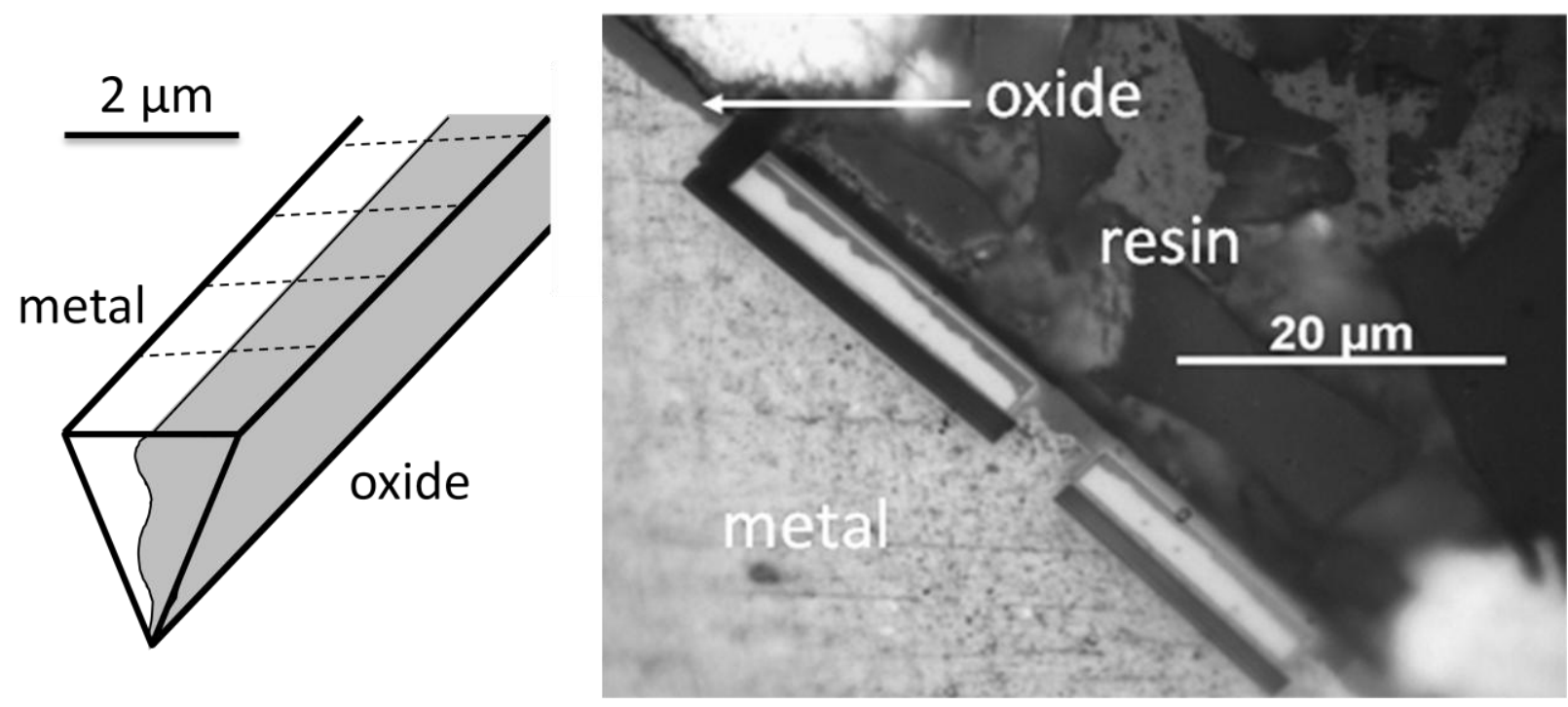

Figure 32: Left) Schematic of a lifted-out prism showing the geometry of the specimen and metal-oxide interface. Right) Optical micrograph showing a prism containing a metal-oxide interface, and a second, incomplete prism (right). 
A number of possible options exist for the geometry for metal-oxide interface lift-out specimens. Specimens have been created where with interfaces parallel and perpendicular to the axis of the tip. The stages above describe the steps in the production of specimens with the interface parallel to the axis of the tip. Specimens can be created from the prismatic lift-outs prepared by fibbing directly into a flat oxidised surface, rather than a cross-sectioned metal-oxide interface. This specimen production technique has the advantage that it requires less mechanical preparation of the specimen, and the final formation of the end-apex region containing the interface is less challenging. Figure 33 shows the geometry of lift-out sections prepared using this method.

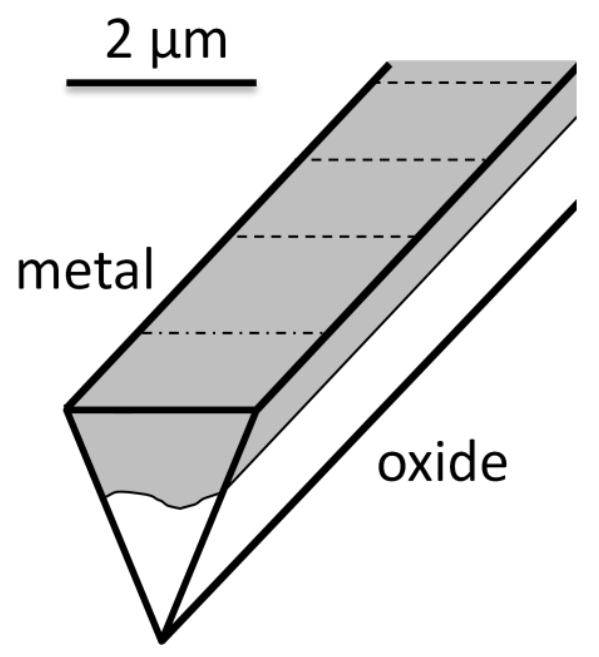

Figure 33: An alternative prism geometry to Figure 32, where the wedge has been dug into the plane of the oxide.

These prisms can be manufactured using the FEI FIB 200 instrument. However, the work necessary to create atom probe specimens from these prisms is greatly aided by use of a more advanced instrument. The Carl Zeiss NVision 40 microscope is a dual beam FIB; a combined FIB and high resolution SEM. It is also fitted with a micromanipulator, a small probe that can be finely controlled and brought into contact with the specimen and welded in place using tungsten or amorphous carbon deposition. Using the NVision FIB, the prism may be welded to the 
manipulator and cut free from the bulk of the material (Figure 34a). A microtip coupon is then introduced into the FIB chamber; this is a silicon wafer with numerous posts attached to it. Each post can be transformed into an atom probe needle and analysed separately in the LEAP. The prism is brought into contact with the top of a post and welded on at one side. The remainder of the prism is then cut free, creating a wedge welded to a post (Figure 34b). This step can be repeated to create more specimens from the prism. The wedge is further secured by deposition of tungsten on to the opposite side (previously welded). Annular milling, similar to that performed in conventional FIB preparation, is used to create a tip from the block. The high resolution SEM, which can achieve a pixel size below $10 \mathrm{~nm}$, assists greatly when used to monitor the progression of the milling during the milling process. The electron column can be used during milling to verify progress made and any further action required in real time. 


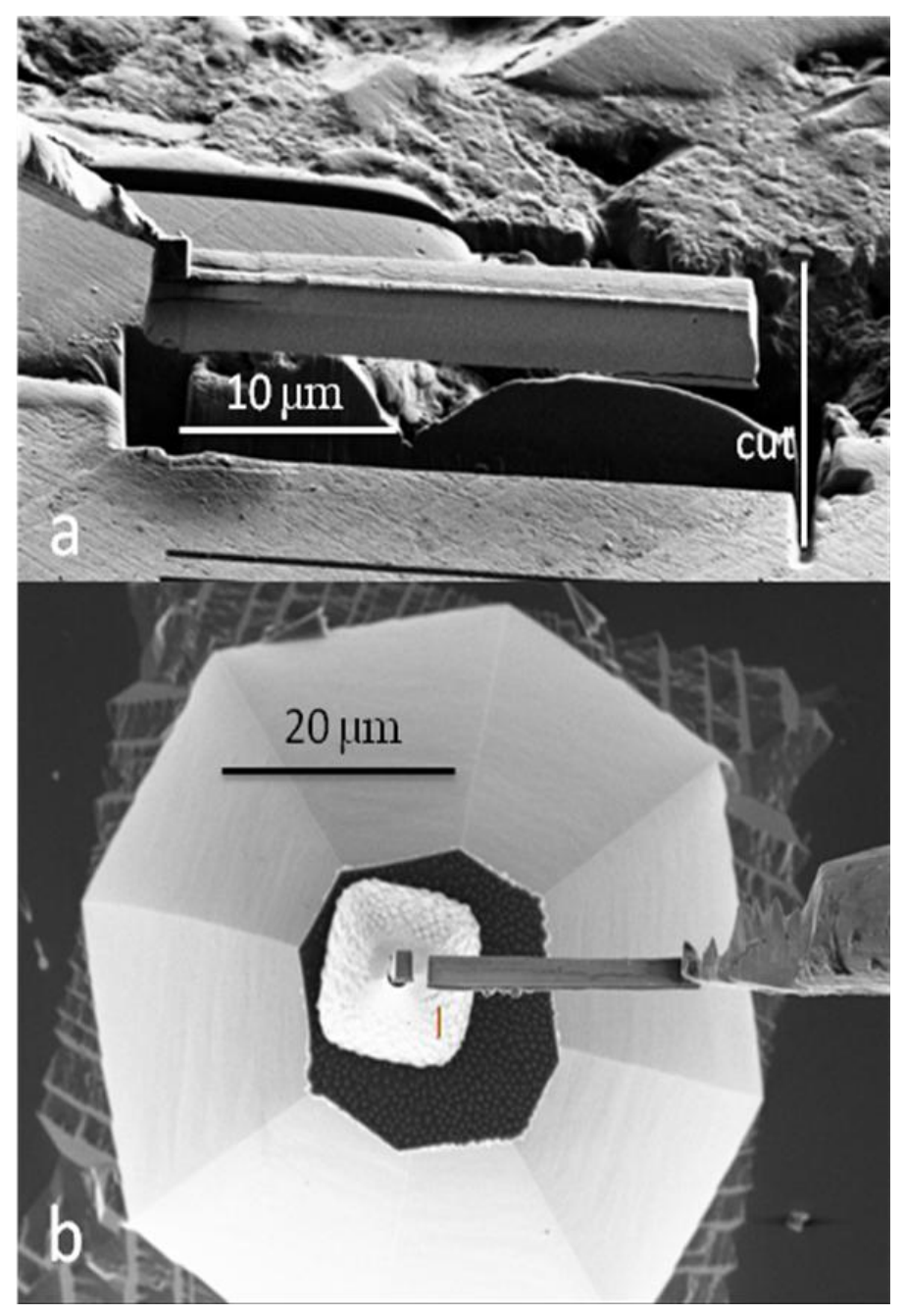

Figure 34: Stages of dual beam FIB preparation. a) A manipulator is welded to the prism and cut away from the bulk. The manipulator is visible, welded to the left hand side of the beam. b) The prism welded to and cut away from a mirco-tip post. Courtesy of Dr. David Saxey. 


\section{$4 \quad$ Reference studies}

This chapter describes the selection of analysis conditions for the LAR-3DAP (§ 4.1) and the identification of mass spectra of commercially pure zirconium analysed using the LAR-3DAP with voltage-pulsed atom probe tomography $(\S 4.2)$. This analysis platform was used to characterise the very early stage formation of native oxides $(\$ 4.3)$ and the analysis of laboratory induced hydrides $(\S 4.4)$. Chapter 4 also describes work performed to identify the mass spectra of zirconium alloys analysed by laser-pulsed 3DAP $(\S 4.5)$ and the variation of the measured solute concentration with analysis conditions ( $\$ 4.6)$. This acts as a foundation for the analysis of materials described in chapters 5 and 6.

\subsection{Determining suitable analysis conditions for voltage-pulsed LAR- $3 D A P$}

As discussed previously, cooling the specimens improves the lateral resolution of the instrument as it reduces surface migration and also reduces the lateral kinetic energy of the field evaporated ions. Unfortunately, cooling also increases the incidence of fracture. Specimens were analysed between $\mathrm{T}=40 \mathrm{~K}$ and $120 \mathrm{~K}$ in the LAR-3DAP. It was found that no useful quantity of data could be obtained using this instrument below $50 \mathrm{~K}$. At temperatures above $100 \mathrm{~K}$ there was no notable increase is specimen longevity. $90 \mathrm{~K}$ was found to be a suitable balance between these two requirements. A lower temperature would need to be used for tin-containing alloys as it has been shown by previous workers that there is a potential problem with solute retention at higher temperatures [155].

The target evaporation rate from the LAR-3DAP was set between the range $0.2 \%$ and $1 \%$ at the beginning of data acquisition. This means that the control software aimed to keep the standing 
potential such that between 0.2 and $1 \%$ of the pulses applied to the specimen caused an ion to be detected. During acquisition this rate was gradually increased until the median value was up to $1 \%$. For the LAR-3DAP this means 200 ions per second were detected. A higher data rate is preferred as it reduces the proportion of detector hits due to dark current and so increases the signal to noise ratio. However, this also increases the proportion of ions detected as multiple hit species, which can lead to a loss of information that varies for different atomic species. This evaporation rate is still relatively low and yields only a few percent $(3-4 \%)$ of ions as multiple hit events.

The pulse fraction, the amplitude of the voltage pulse over the standing voltage applied to the tip, was typically between $15 \%$ and $20 \%$. A lower pulse fraction causes the tip to undergo less stress, and consequently last longer. However, if the pulse fraction is insufficient then this will lead to the preferential evaporation of species with lower evaporation fields. The commercially pure material examined using voltage pulsing in the LAR-3DAP contained only $\mathrm{Zr}, \mathrm{Fe}, \mathrm{O}$ and $\mathrm{H}$.

\subsection{Voltage-pulsed mass spectra}

\subsubsection{Zirconium}

Zirconium has an average relative atomic mass of 91.22 derived from five naturally occurring isotopes, listed in Table 13. This 5 isotope fingerprint of zirconium aids the identification of zirconium-containing species. Figure 35 demonstrates the correspondence between expected and observed zirconium spectra. This is the same pattern seen in all $\mathrm{ZrX}_{\mathrm{i}}$ ions, where $\mathrm{X}_{\mathrm{i}}$ is a monoisotopic species or one where a single isotope is dominant (e.g. $\left.\mathrm{X}_{\mathrm{i}}=\mathrm{H}, \mathrm{C}, \mathrm{N}, \mathrm{O}\right)$. Metal ions were observed in the $3+, 2+$ and $1+$ charge states. In most analysis conditions triply-charged zirconium ions are the dominant species detected. The effect of analysis conditions on 
evaporation field is described in section 4.6.1. Zirconium- and oxygen-containing species are tabulated in Table 14 (below, in section 4.2.2).

\begin{tabular}{|c|c|c|}
\hline Isotope & $\begin{array}{c}\text { Atomic } \\
\text { mass } \\
(\mathrm{Da})\end{array}$ & $\begin{array}{c}\text { Relative } \\
\text { abundance } \\
(\%)\end{array}$ \\
\hline${ }^{90} \mathrm{Zr}$ & 89.9043 & 51.46 \\
${ }^{91} \mathrm{Zr}$ & 90.9053 & 11.23 \\
${ }^{92} \mathrm{Zr}$ & 91.9046 & 17.11 \\
${ }^{94} \mathrm{Zr}$ & 93.9061 & 17.40 \\
${ }^{96} \mathrm{Zr}$ & 95.9082 & 2.80 \\
\hline
\end{tabular}

Table 13: The naturally occurring isotopes of zirconium [156].
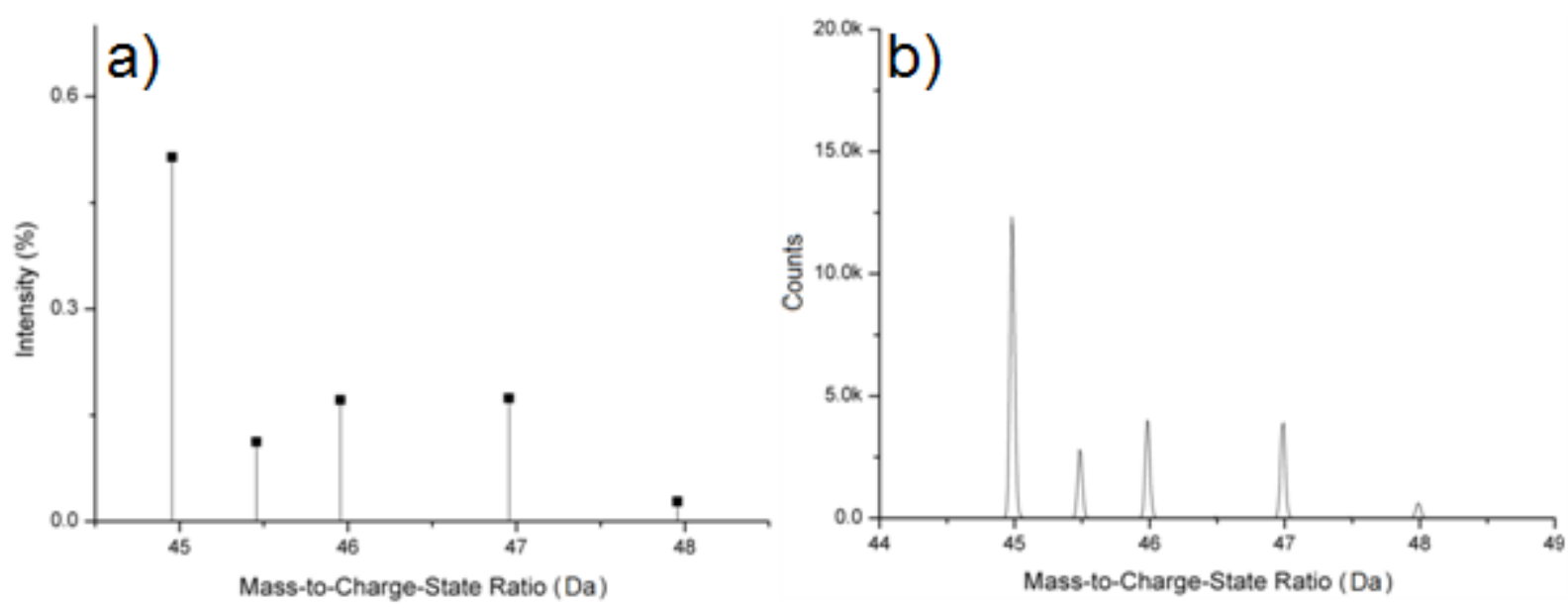

Figure 35. A comparison of a) the isotopic distribution of $\mathrm{Zr}^{2+}$; and b) the corresponding spectra obtained by $3 D A P($ right). 


\subsubsection{Oxygen}

The majority of oxygen in commercially pure zirconium metal matrix is detected in the form of a $\mathrm{ZrO}^{+}$species. All of the oxygen containing species and the charge-states at which they are observed are given by Table 14 .

\begin{tabular}{|c|c|c|c|}
\hline Species & Zr & ZrO & O \\
\hline Charge state & $1,2,3$ & 2,3 & 1 \\
\hline $\begin{array}{c}\text { Relative } \\
\text { abundance (\%) }\end{array}$ & $\sim 99$ & $\sim 1$ & $<1$ \\
\hline
\end{tabular}

Table 14. Zirconium- and oxygen-containing ionic species detected by voltage-pulsed APT.

\subsubsection{Hydrogen}

Hydrogen was detected as $\mathrm{ZrH}$, as well as $\mathrm{H}, \mathrm{H}_{2}$ and $\mathrm{H}_{3}$. The source of this $\mathrm{ZrH}$ species is difficult to identify unequivocally; it is either from the vacuum system of the atom probe, or is a representative of the level of hydrogen in the sample. For specimens that have been prepared using chemical etching, another possible source of hydrogen is introduced. However, $\mathrm{ZrH}$ was not detected in the majority of specimens, and appeared to be concentrated in localised regions of datasets when it was observed. A number of such samples, containing a high proportion of $\mathrm{ZrH}$ ions, were detected. The analysis of these regions is discussed in more detail in section 4.4. In these datasets, the doubly-charged $\mathrm{ZrH}^{2+}$ and $\mathrm{Zr}^{2+}$ spectra overlap, and so each $\mathrm{ZrH}$ peak is shifted half a mass-to-charge unit higher than its corresponding zirconium peak. The two sets of peaks, an example of which is shown in Figure 36, can be deconvoluted to find the total amount of hydrogen. 


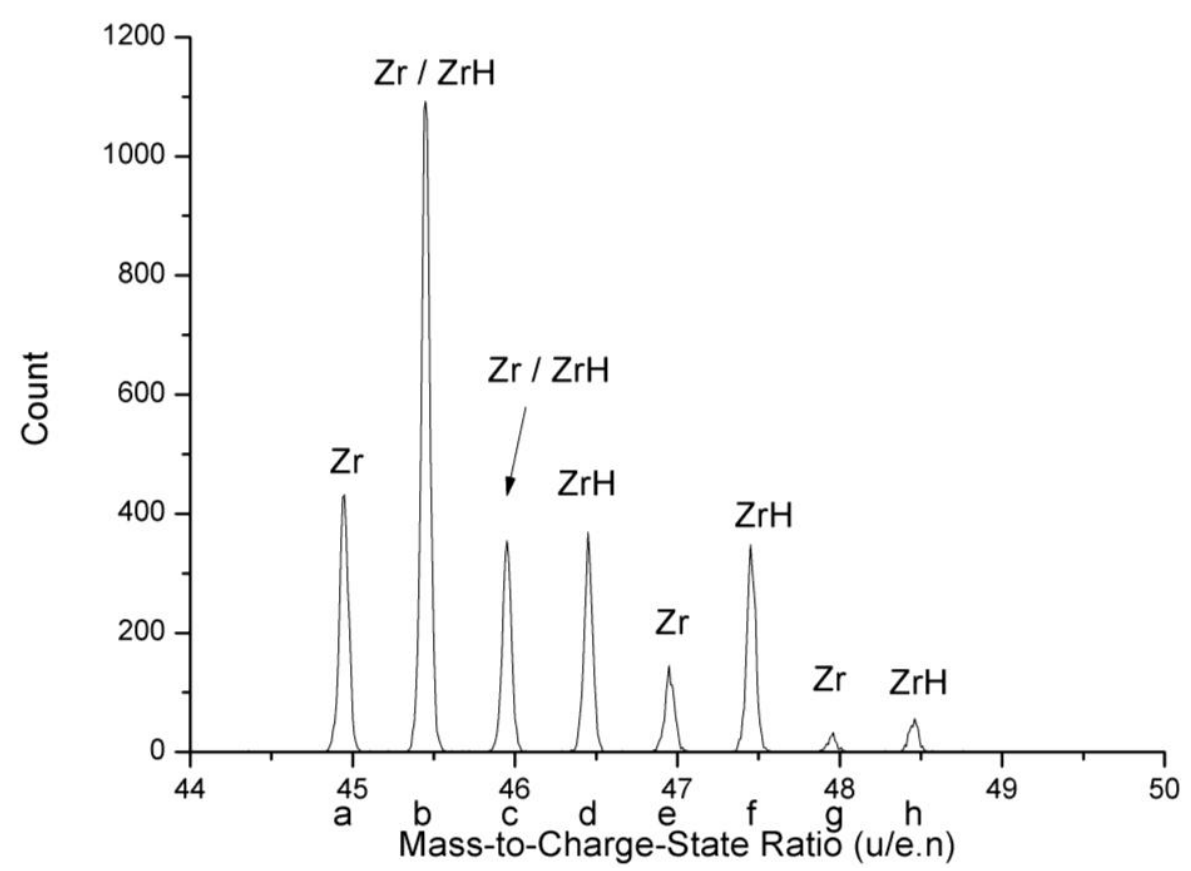

Figure 36: A spectrum taken from a region with overall composition ZrH showing the overlapping of $\mathrm{Zr}^{2+}$ and $\mathrm{ZrH}^{2+}$ spectra.

Figure 36 shows a region of the mass-to-charge spectrum of a zirconium specimen where a large proportion of the $\mathrm{Zr}^{2+}$ ions are evaporated together with a hydrogen ion. The individual isotopes are labelled, $\mathrm{a}-\mathrm{h}$ (see horizontal axis). The contribution of the number of atoms of each species from these two overlapping ion-types is given by (with reference to the peak labelling in Figure 36):

$$
N_{Z r}=\sum_{i=a}^{h} i
$$

$$
N_{H} \approx N_{Z r}-\frac{a+e+g}{x_{90}+x_{94}+x_{96}}
$$


where $i$ is a dummy variable, $a$ to $h$ are the sum of counts from the peaks as labelled in Figure 36 , and $x_{j}$ is the isotopic abundance of zirconium with mass $j . N_{Z r}$ and $N_{H}$ are the total number of atoms of zirconium and hydrogen. In all cases $\mathrm{x}_{90}+\mathrm{x}_{94}+\mathrm{x}_{96}=0.7167$.

The summation in eq. 11 is obvious by inspection, as all the ions contain exactly one zirconium atom. Eq. 12 loses possible information by subtracting a calculated value for the ions that contain only zirconium, and no hydrogen, from the total number of ions. The uncertainly in these measurements (one standard deviation) are given by:

$$
\begin{gathered}
\sigma_{\mathrm{Zr}}=\sqrt{\mathrm{N}_{\mathrm{Zr}}\left(1-\frac{\mathrm{N}_{\mathrm{Zr}}}{\mathrm{N}}\right)} \\
\sigma_{H}=\sqrt{\sigma_{Z r}^{2}+\sigma_{X}^{2}} \\
\text { where } \sigma_{X}=\sqrt{N_{x}\left(1-\frac{N_{x}}{N}\right)}
\end{gathered}
$$

where $\mathrm{N}=\mathrm{N}_{\mathrm{Zr}}+\mathrm{N}_{\mathrm{H}}$ and $\sigma_{\mathrm{x}}$ is the uncertainty associated with $\mathrm{N}_{\mathrm{X}}=(\mathrm{a}+\mathrm{e}+\mathrm{g}) /\left(\mathrm{x}_{90}+\mathrm{x}_{94}+\mathrm{x}_{96}\right)$. For dilute solutions of hydrogen this can be approximated to:

$$
\sigma_{\mathrm{H}} \approx \sqrt{\mathrm{N}_{\mathrm{X}}}
$$

as the uncertainty in the measurement of $\mathrm{N}_{\mathrm{Zr}}$ is negligible compared to the uncertainty in $\mathrm{N}_{\mathrm{H}}$. 


\subsection{Native oxides formed on commercially pure zirconium atom probe specimens}

Atom probe specimens made from commercially pure zirconium wire were subjected to corrosion in a variety of media. The cumulative concentration profiles in Figure 37 show the oxygen against zirconium atom count taken along the specimen axis for a variety of oxidation treatments: a) boiling water for $3 \mathrm{~s}, \mathrm{~b}$ ) air for $10 \mathrm{~min}$. and c) the native oxide created by the electro-polishing process. The gradient of the curve yields the instantaneous $\mathrm{O} / \mathrm{Zr}$ ratio. This ratio in the native oxide and the metal matrix is listed in Table 15 for treatments a-c. The oxygen content of the matrix is due to the processing of the wire, and is very similar for all of the treatments, $\sim 0.5$ at. $\%$. The native oxide formed by these three very different processes is also very similar, as can be seen from the close agreement of the curves a-c towards zero zirconium atoms (the specimen surface) in Figure 37.

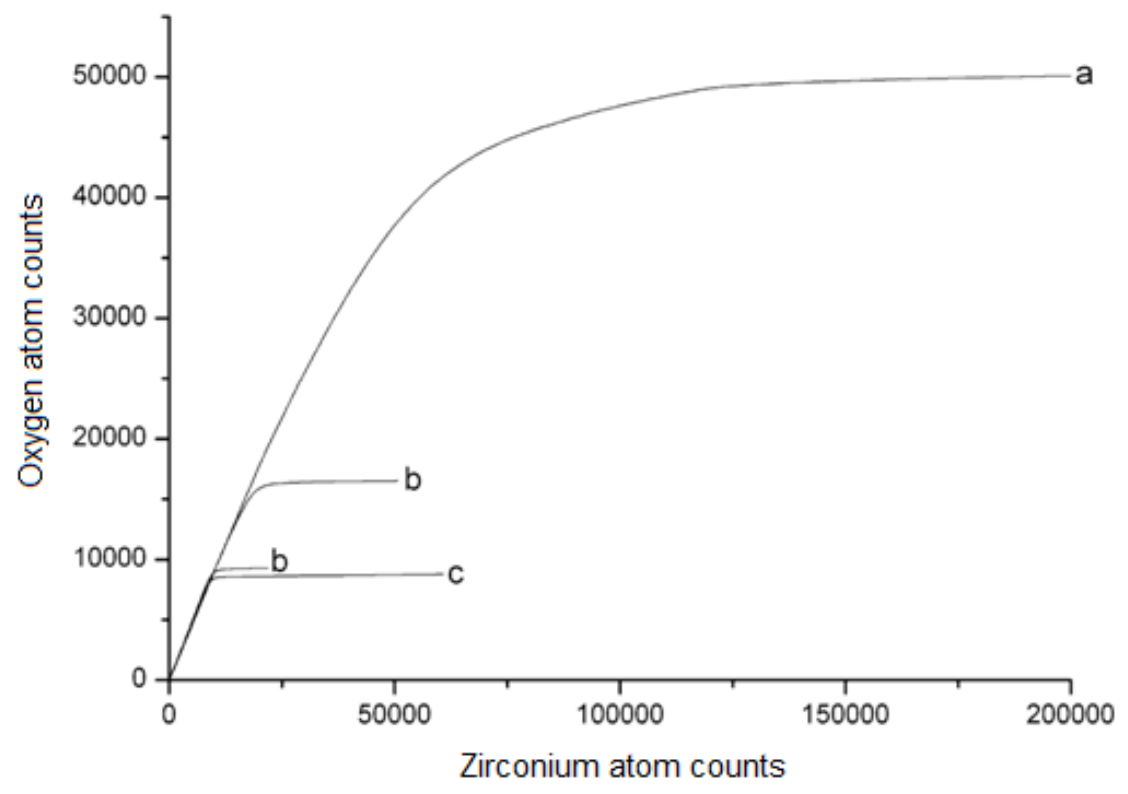

Figure 37: O/Zr ladder diagrams for specimens oxidised (a) in boiling water for 3 , (b) by the sample preparation process and (c) in $150^{\circ} \mathrm{C}$ air for $10 \mathrm{~min}$. 


\begin{tabular}{|cccc|}
\hline $\begin{array}{c}\text { Oxidation } \\
\text { method }\end{array}$ & $\begin{array}{c}\text { Tempera } \\
\text { ture }\end{array}$ & $\begin{array}{c}\mathrm{O} / \mathrm{Zr} \text { ratio } \\
\text { in oxide }\end{array}$ & $\begin{array}{c}\mathrm{O} / \mathrm{Zr} \text { ratio in } \\
\text { matrix }\end{array}$ \\
\hline (a) & $100{ }^{\circ} \mathrm{C}$ & 0.902 & 0.0054 \\
Water & & \pm 0.001 & \pm 0.0002 \\
(b) & Ambient & 0.907 & 0.0047 \\
$\begin{array}{c}\text { Polishing } \\
\text { (c) }\end{array}$ & & \pm 0.001 & \pm 0.0003 \\
Air & & 0.925 & 0.0046 \\
& & \pm 0.001 & \pm 0.0003 \\
\hline
\end{tabular}

Table 15: Summary of data from Figure 37.

The $\mathrm{O} / \mathrm{Zr}$ ratio of all of the native oxides formed on these commercially pure zirconium samples is $\sim 0.9$, which agrees well with the compositional measurements performed by Wadman et al. [157] on Zircaloy-4 needles oxidised in air and water ( 0.91 and 0.89 , respectively). This oxide layer is better described as oxygen deficient $\mathrm{ZrO}$ than the expected stable oxide of $\mathrm{ZrO}_{2}$.

Figure 37 shows a sharp transition in oxygen concentration between the metal and oxide regions of samples $b$ and $c$, and the thickness of the scale on these samples is very similar. The transition in the oxygen concentration of the water corroded sample is more gradual, suggesting that a small amount of oxygen has diffused into the metal ahead of the oxide, even during this very rapid oxidation experiment.

The kinetics of the very early stages of the oxidation process were analysed by atomically cleaning a specimen by $3 \mathrm{DAP}$ and exposing it to air at room temperature and pressure for a variety of time periods. The same specimen was repeatedly oxidised and analysed to ensure that the crystallographic structure of the specimen was similar in all cases. The oxidation kinetics of many metals show an initially high rate of growth which for zirconium alloys continues as a parabolic/cubic oxidation regime [109]. Figure 38 shows the initial kinetics for zirconium as analysed by 3DAP. A discontinuity is seen between $0-5$ minutes, suggesting that there is almost immediate formation of a native oxide layer when atomically clean zirconium is exposed to air at room temperature. Over the course of an hour this film increases in thickness. The rate 
of growth is linear within experimental limits, suggesting that the oxide layer that initially forms is non-protective against further growth since the oxide growth rate is not dependent upon film thickness. At some later point this initial mechanism is superseded by the parabolic growth of zirconium dioxide.

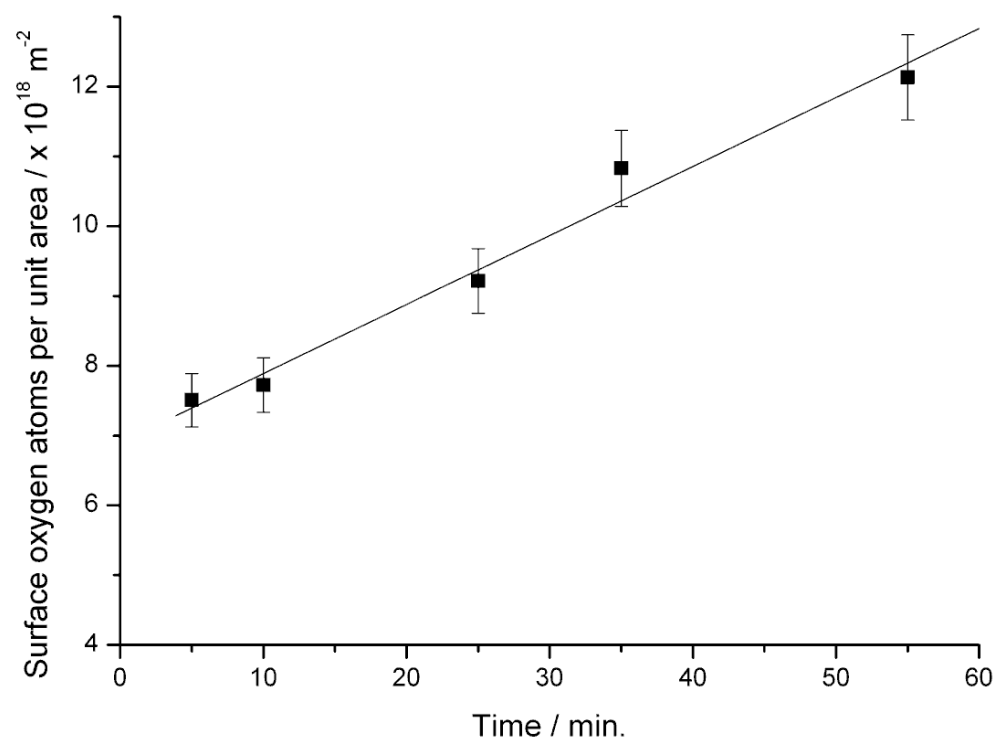

Figure 38: The initial oxidation kinetics of an atomically clean zirconium surface in room temperature air. Adjusted correlation coefficient $R^{2}=0.972$ for linear fit shown. Adjusted $R^{2}=$ 0.965 for parabolic trend.

\subsection{Bulk hydrides formed on zirconium atom probe specimens}

Hydrides were induced in atom probe needle specimens by cathodic charging using the electrochemical solutions described in section 3.5. A potential of $-10 \mathrm{~V}$ DC was applied to the specimen to perform the charging in this case, although this is likely to be much higher than the minimum potential required, which is likely to be of the order of -1 V [158]. The hydride regions analysed here correspond well with the stoichiometry expected of meta-stable $\mathrm{ZrH}$ hydride, as demonstrated by Table 16. Other workers looked at a similar system, TiH, but used FIB 
preparation of hydrided samples. They found that the FIB method caused partial hydride dissolution [159].

\begin{tabular}{|ccccc|}
\hline Species & $\begin{array}{c}\text { Total ions } \\
(\#)\end{array}$ & $\begin{array}{c}\text { 1 s.d. } \\
(\#)\end{array}$ & $\begin{array}{c}\text { Concentration } \\
\text { (at.\%) }\end{array}$ & $\begin{array}{c}\text { 1 s.d. } \\
\text { (at.\%) }\end{array}$ \\
\hline $\mathrm{Zr}$ & 1014996 & 711 & 50.265 & 0.035 \\
$\mathrm{O}$ & 6883 & 83 & 0.341 & 0.004 \\
$\mathrm{H}$ & 997421 & 710 & 49.394 & 0.035 \\
\cline { 1 - 3 } $\mathrm{H} / \mathrm{Zr}$ ratio & 0.983 & 0.001 & & \\
\cline { 1 - 2 } & & & & \\
\hline
\end{tabular}

Table 16: The composition of the hydride region shown in Figure 39.
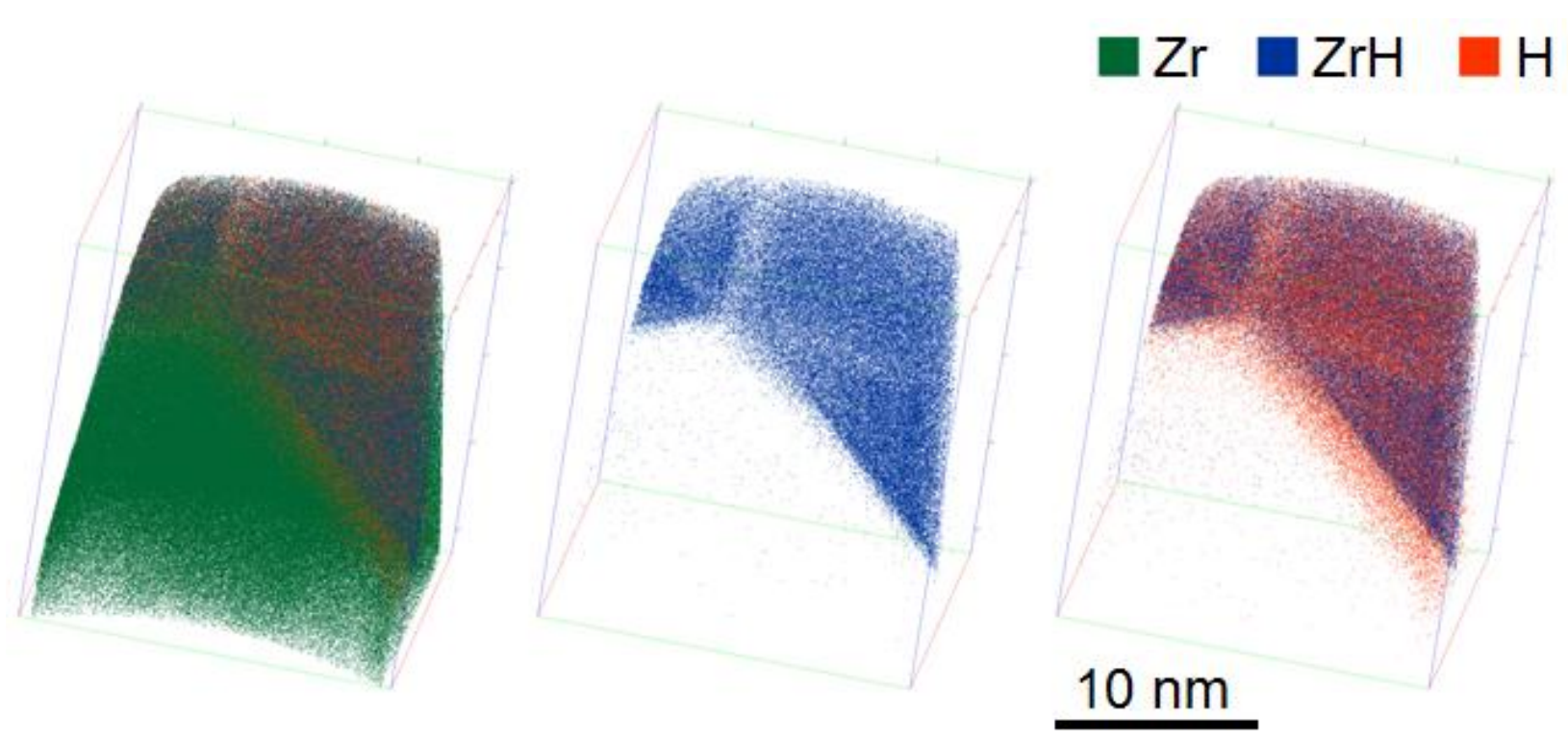

Figure 39: 3D atom maps showing a bulk hydride region (blue) with composition $\mathrm{ZrH}$ in a commercially pure zirconium specimen.

The 3D atom maps in Figure 39 display the $\mathrm{ZrH}^{2+}$ ionic species at $46.5 \mathrm{Da}$ and $47.5 \mathrm{Da}$ as blue dots. The $\mathrm{Zr}$ ionic species are represented as green dots and the $\mathrm{H}$ species as red dots. There is a sharp interface of the $\mathrm{ZrH}$ species. The atom probe detection efficiency is less around a major pole, which is visible in the blue and red atom maps as the light region running almost vertically on the page (the specimen axis). The profile in Figure 40 is produced using a proxigram around a 
volume contained within a region bounded by 45 at.\% hydrogen. As was seen in Figure 39, the light $\mathrm{H}^{+}$ions are observed around the periphery of the hydride. The $\mathrm{H}$ ions undergo a more gradual decay over ' $12-16 \mathrm{~nm}$ '. This is likely due to trajectory aberrations around the hydride causing the very light $\mathrm{H}^{+}$species to undergo a much greater spreading in their flight path at the interphase boundary, which causes a magnification effect. This effect is not so pronounced for the $\mathrm{ZrH}$ species, which are a similar weight to $\mathrm{Zr}$ ions.

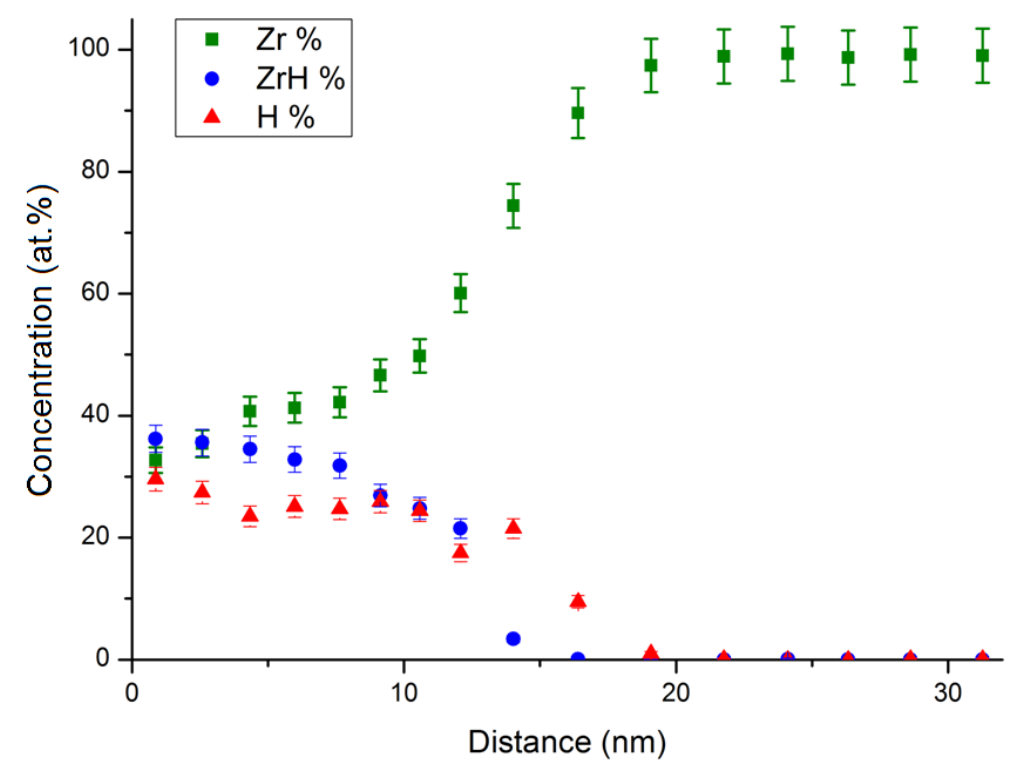

Figure 40: Concentration profile across the Zr-ZrH interface for the commercially pure $\mathrm{Zr}$ sample shown in Figure 39.

Figure 41 and Figure 42 compare the mass spectra around $\mathrm{Zr}^{2+}$ in hydrided pure zirconium and ZIRLO specimens. The isotopic ratios of the spectra match very closely, suggesting that there is a fixed relationship for $\mathrm{H}^{+} / \mathrm{ZrH}^{2+}$ ionic species evaporation in this type of hydride in zirconium alloy systems. This $\mathrm{Zr}^{+} / \mathrm{ZrH}^{2+}$ 'finger print' can therefore act as a useful identifier when looking for $\mathrm{Zr}$ hydrides in future studies. 

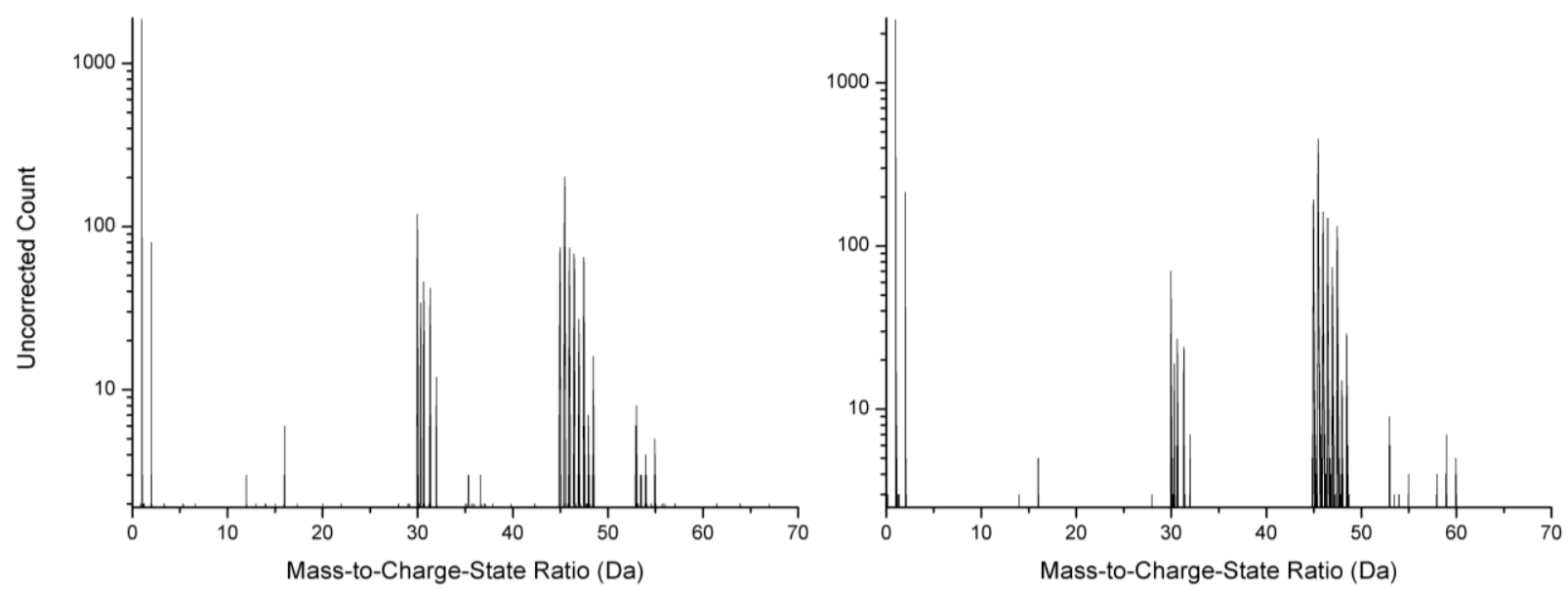

Figure 41: Mass spectra for ZrH hydride regions within; left) commercially pure zirconium, and left) ZIRLO.

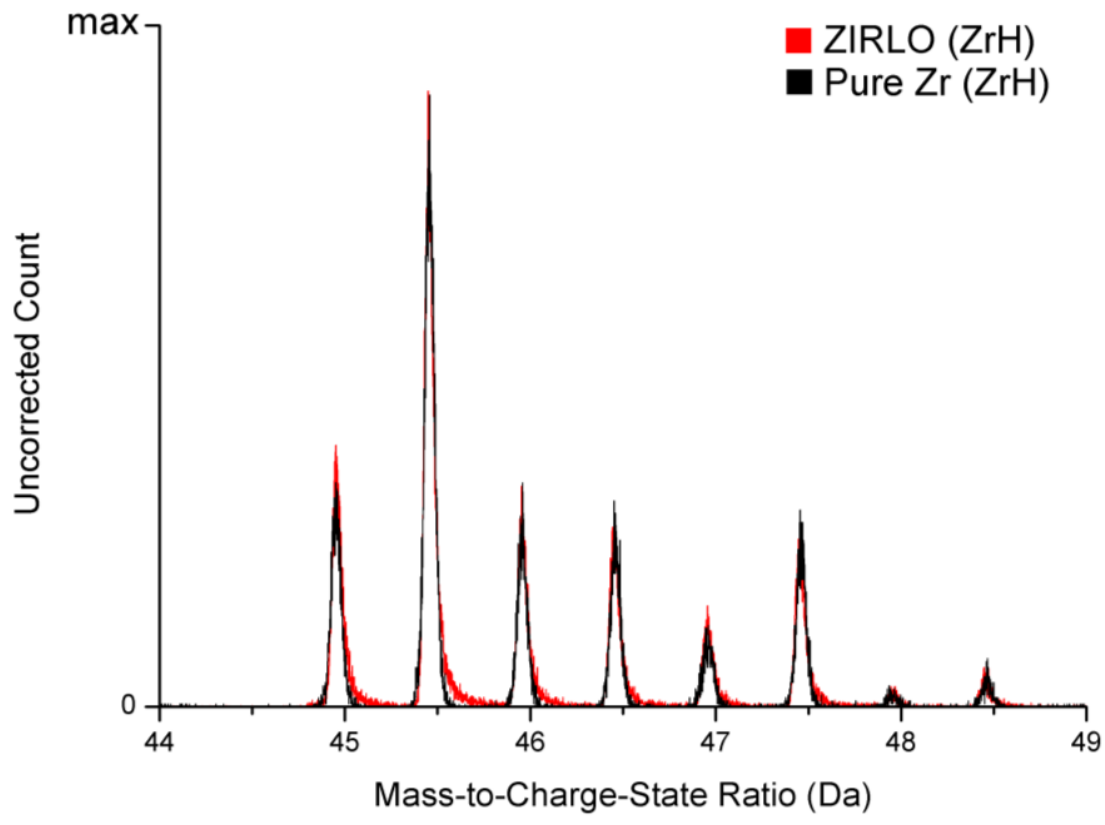

Figure 42: Comparison of the $\mathrm{Zr} / \mathrm{ZrH}(2+)$ region of the mass-to-charge-state spectrum of hydride regions in commercially pure zirconium and ZIRLO specimens. 


\subsection{Laser-pulsed mass spectra}

\subsubsection{Zirconium}

The zirconium-ion containing mass spectra peaks are similar to those in voltage pulsed data (4.2.1), with the exception that the dominant charge state is at the $2+$ position and a minor contribution can be found at the $4+$ charge state. The zirconium-containing ionic species apparent in laser-pulsed data are listed in Table 17.

\subsubsection{Oxygen}

The distribution of oxygen amongst ionic species detected from specimens analysed using laserpulsed 3DAP is more complex than in the case seen in voltage mode. These species are also listed in Table 17.

\begin{tabular}{|c|c|c|c|c|c|c|c|c|}
\hline Species & $\mathrm{Zr}$ & $\mathrm{ZrO}$ & $\mathrm{ZrO}_{2}$ & $\mathrm{O}$ & $\mathrm{O}_{2}$ & $\mathrm{Zr}_{2} \mathrm{O}_{2}$ & $\mathrm{Zr}_{2} \mathrm{O}_{3}$ & $\mathrm{Zr}_{2} \mathrm{C}$ \\
\hline Charge state & $\begin{array}{c}1,2, \\
3,4\end{array}$ & $\begin{array}{c}1,2, \\
3\end{array}$ & 1 & 1 & 1 & 3 & 1 & 3 \\
\hline $\begin{array}{c}\text { Relative } \\
\begin{array}{c}\text { abundance (\%) } \\
\text { in metal matrix }\end{array}\end{array}$ & $\begin{array}{c}\sim 97 \\
\text { in }\end{array}$ & $\sim 1$ & $<1$ & $<1$ & $\sim 0$ & $\begin{array}{c}< \\
0.05\end{array}$ & $\begin{array}{c}<.05 \\
0.05\end{array}$ \\
\hline
\end{tabular}

Table 17: Zirconium- and oxygen-containing ionic species detected by laser-pulsed 3DAP.

The laser-pulsed 3DAP is capable of analysing ceramic oxides. The ionisation behaviour of oxygen in these regions was very different to that seen in the metal. Singly-charged $\mathrm{O}_{2}$ ions were found in the ceramic oxide region of the specimens and make a large contribute to the species detected. The sum of $\mathrm{ZrO}$ and $\mathrm{O}_{2}$ in this region gives a composition of $\sim \mathrm{ZrO}_{2}$. A typical 
spectrum from a ceramic oxide region is shown in Figure 43. The poor thermal conduction of the ceramic oxide causes peak broadening and 'tails' to appear on the mass peaks. This is because the specimen is held at an elevated temperature for a longer duration during each pulse, increasing the period in which field evaporation is promoted and so increasing the spread in ion time-of-flight.

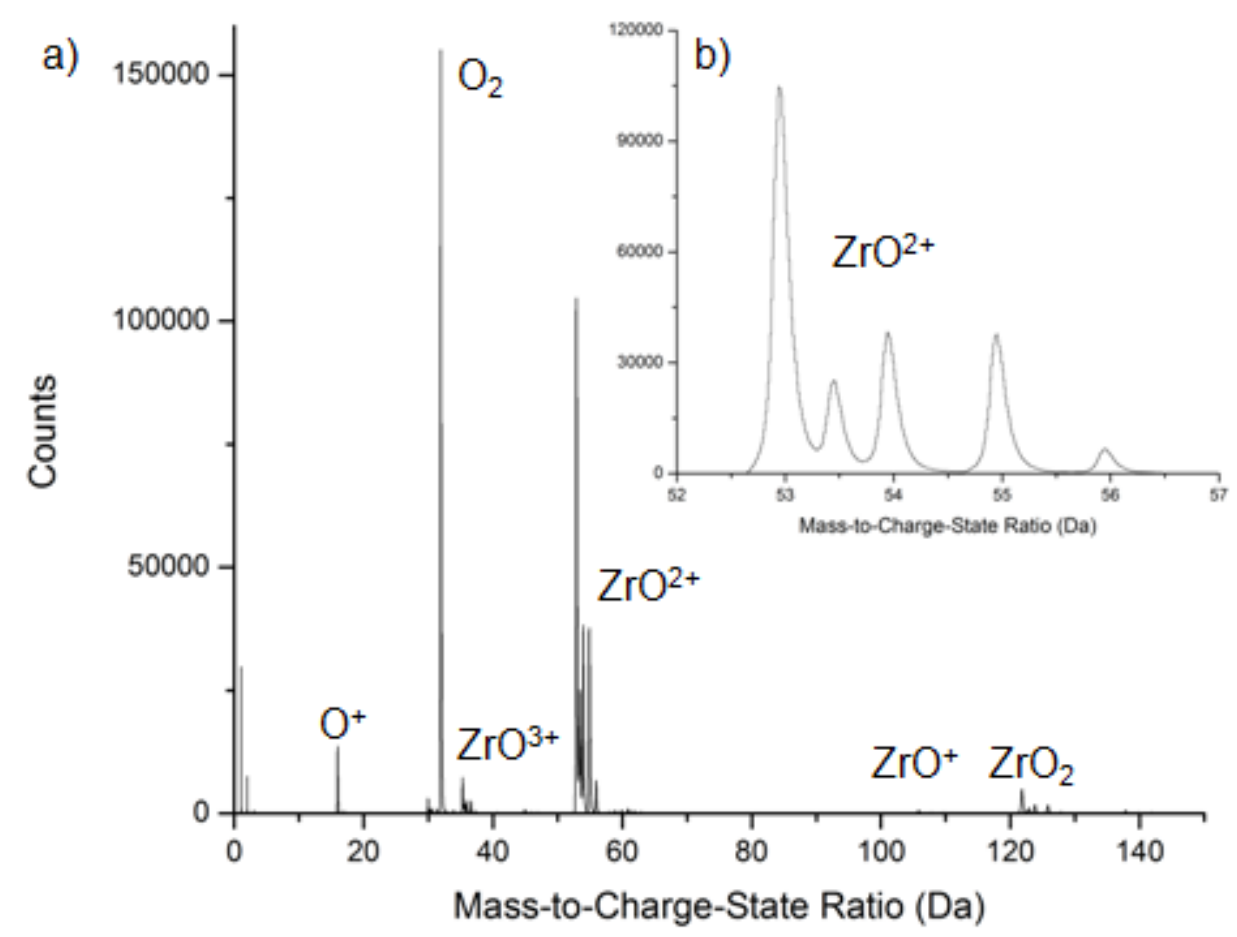

Figure 43: A region of ceramic oxide (of Zircaloy-4). The view of the whole mass spectrum (a) shows the main constituents are detected as singularly charged $\mathrm{O}_{2}$ at $32 \mathrm{Da}$ and doubly charged $\mathrm{ZrO}$ around $53 \mathrm{Da}$. The mass spectrum (b) displays $52-57 \mathrm{Da}\left(\mathrm{ZrO}^{2+}\right)$ and demonstrates the inferior mass resolution that is often achieved by analysis of the oxide. 


\subsubsection{Hydrogen}

The analysis of hydrogen containing peaks is the same for laser-pulsed data as that discussed for the voltage-pulsed data in section 4.2.3. The only noticeable difference is that in voltage pulsed data $\mathrm{ZrH}$ ions were not seen in metallic specimens, only in hydride regions. In laser-pulsed analyses of metallic specimens around $10 \%$ of zirconium-containing ions are evaporated as a $\mathrm{ZrH}$ species.

\subsubsection{Tin}

Tin was detected as a doubly-charged ion in all instances. Of the ten naturally occurring tin isotopes, listed in Table 18, seven are clearly visible in Figure 44. Doubly-charged tin is subject to interference from the tail from the $\mathrm{ZrO}^{2+}$ peaks around $56 \mathrm{Da} .{ }^{114} \mathrm{Sn}^{2+}$ and ${ }^{115} \mathrm{Sn}^{2+}$ are barely distinguishable from this noise level in the metallic region (a) of Figure 44. In order to reduce the effect of overlap of delayed ions from $\mathrm{ZrO}^{2+}$ on the measured tin content, only the two most significant tin-containing peaks are used to calculate the tin concentration. ${ }^{118} \mathrm{Sn}^{2+}$ and ${ }^{120} \mathrm{Sn}^{2+}$ are found at $59 \mathrm{Da}$ and $60 \mathrm{Da}$ and contain $24.3 \%$ and $32.4 \%$ of the total tin content of the mass spectra, respectively. The extent of overlap of $\mathrm{ZrO}^{+}$ions at these positions in the Mass-to-chargestate ratio spectra can be estimated by taking a range window of equal width directly ahead of the mass positions, at around 58.75 Da and 59.75 Da. 


\begin{tabular}{|c|c|c|}
\hline Isotope & $\begin{array}{c}\text { Atomic mass } \\
(\mathrm{Da})\end{array}$ & $\begin{array}{c}\text { Relative } \\
\text { abundance } \\
(\%)\end{array}$ \\
\hline${ }^{112} \mathrm{Sn}$ & 111.9048 & 1.01 \\
${ }^{114} \mathrm{Sn}$ & 113.9028 & 0.67 \\
${ }^{115} \mathrm{Sn}$ & 114.9033 & 0.38 \\
${ }^{116} \mathrm{Sn}$ & 115.9017 & 14.8 \\
${ }^{117} \mathrm{Sn}$ & 116.9029 & 7.75 \\
${ }^{118} \mathrm{Sn}$ & 117.9016 & 24.3 \\
${ }^{119} \mathrm{Sn}$ & 118.9033 & 8.6 \\
${ }^{120} \mathrm{Sn}$ & 119.9022 & 32.4 \\
${ }^{122} \mathrm{Sn}$ & 121.9034 & 4.56 \\
${ }^{124} \mathrm{Sn}$ & 123.9052 & 5.64 \\
\hline
\end{tabular}

Table 18: The naturally occurring isotopes of tin [160].

a)

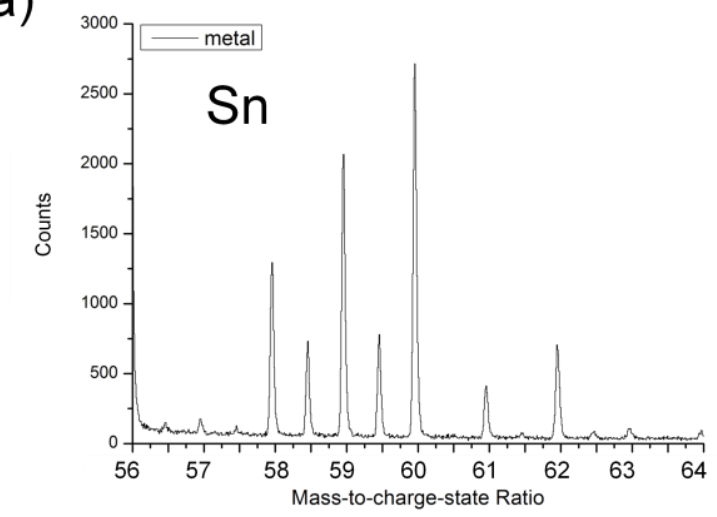

b)

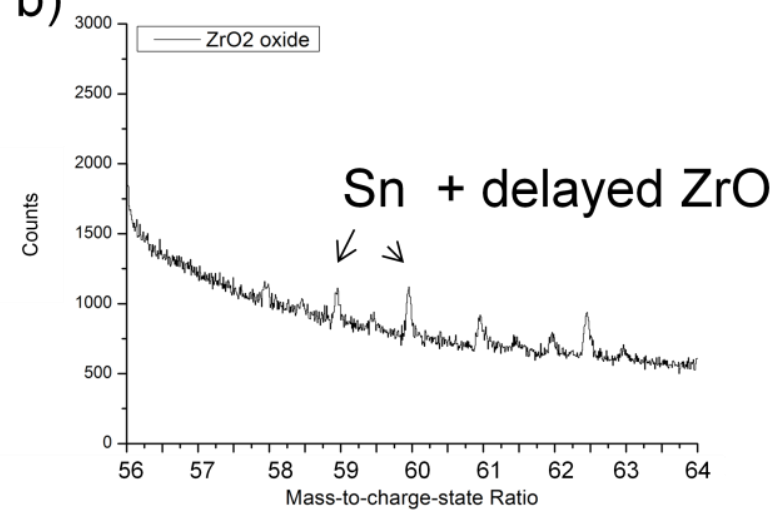

Figure 44: The ten isotopes of tin in the 2+ charge state and $\mathrm{ZrO}^{2+}$ at $56 \mathrm{Da}$. Data is taken from regions of (a) metal and (b) oxide at a metal oxide interface (later reproduced in Figure 123). The higher noise at $55-58 \mathrm{Da}$ is due to delayed ions from the comparatively large ZrO peaks around 53 - 56 Da. Although this noise is noticable on the linear scale in (a), the signal to noise ratio is severly degraded in the oxide region (b). Both regions contain a similar number of ions. 
Figure 44 also shows a difficulty that is encountered when analysing the ceramic $\mathrm{ZrO}_{2}$. The background count due to the extended tail of the $\mathrm{ZrO}$ peak increases greatly. However, the above method of measuring the tin concentration and correcting for this species overlap is efficient at providing an accurate description of the tin concentration in such conditions, albeit with reduced precision. Example spectra are given where necessary in the analysis of metal-oxide interfaces (chapter 6) in order to explain the findings and their limitations.

\subsubsection{Niobium}

Niobium ions are detected in $2+, 3+$ and $1+$ charge states under typical analysis conditions. Niobium is monoisotopic. The niobium peak at the $2+$ state is subject to interference from $\mathrm{ZrH}^{2+}$ in specimens where hydrogen adsorption on the surface of the needle causes these species to be field evaporated. As $\mathrm{ZrH}$ species are found exclusively in the $2+$ state, other niobium charge states are not subject to this overlap. $\mathrm{Nb}^{2+}$ is detected at the same position as ${ }^{92} \mathrm{ZrH}^{2+}$. Eq. 11 and 12 cannot be used to estimate the hydrogen content of the $\mathrm{Zr} / \mathrm{ZrH}^{2+}$ spectra for niobium containing alloys. The $\mathrm{Zr} / \mathrm{ZrH} / \mathrm{Nb}$ species at $2+$ positions in the mass charge spectra are shown for a ZIRLO specimen in Figure 45 (semi-log scale). There are several methods that may be used to deconvolute the niobium content from the $\mathrm{ZrH}$ species. ${ }^{92} \mathrm{Zr}$ represents $17.1 \%$ of the relative isotopic abundance of zirconium, and so ${ }^{92} \mathrm{ZrH}$ will represent $17.1 \%$ of the total amount of the $\mathrm{ZrH}$ ionic species present. A simple method of compensating for the added $\mathrm{ZrH}$ is to deduct the peak at $47.5 \mathrm{Da}$, which is entirely ${ }^{94} \mathrm{ZrH}$, multiplied by a small correction factor to account for the difference in the abundance of ${ }^{94} \mathrm{Zr}$ to ${ }^{92} \mathrm{Zr}$. ${ }^{94} \mathrm{Zr}$ represents $17.4 \%$ of the relative isotopic abundance of zirconium, so:

$$
N_{N b}=N_{46.5 a m u}-\frac{171}{174} N_{47.5 a m u}
$$


This has the advantage of being relatively simple to apply and can be used to correct proxigram and 1D concentration profiles with separately ranged 46.5 and 47.5 Da peaks. This correction also removes the equivalent of the overlap of the tail from the peak at $46 \mathrm{Da}$ from the count at 46.5 Da, as this overlap is similar in form to the overlap of $47 \mathrm{Da}$ that is contained within the count of 47.5 Da. This correction is also relatively insensitive to the ranging metric applied.

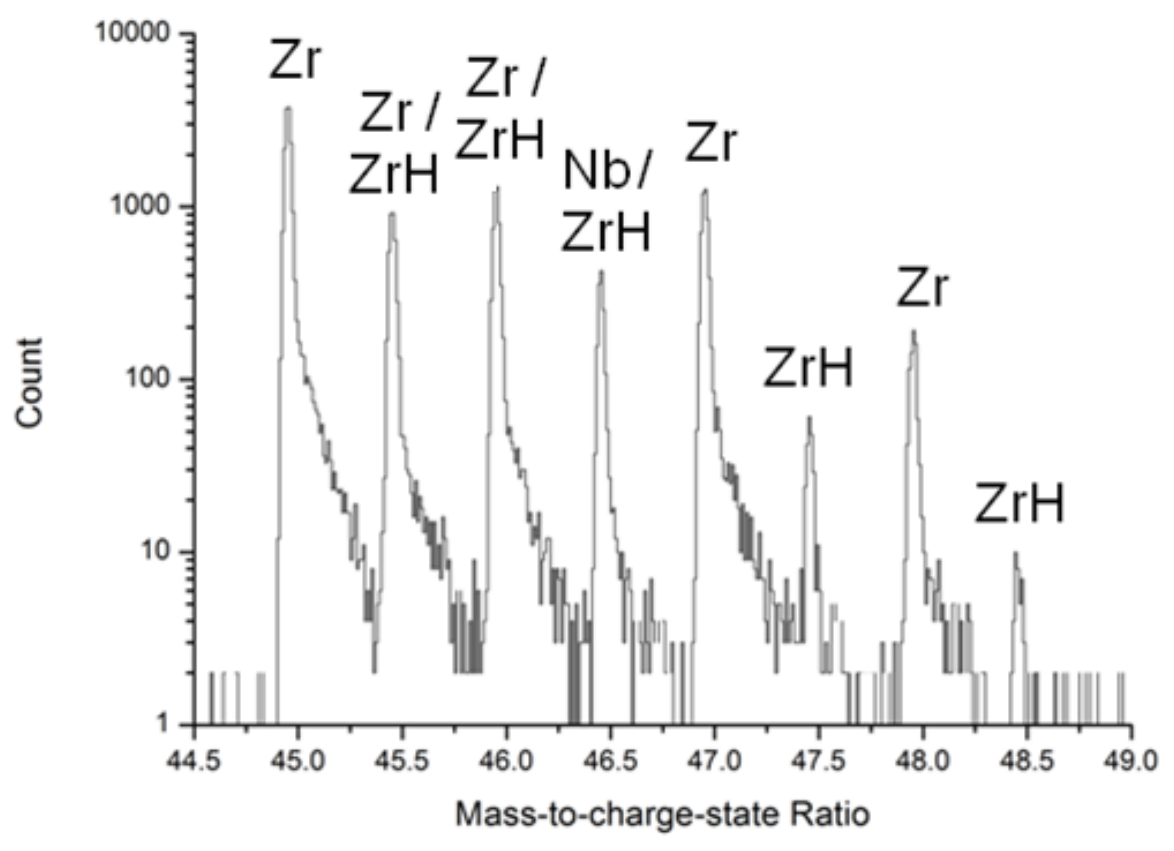

Figure 45: $\mathrm{Zr}^{2+}$ section of the mass-spectrum of a ZIRLO alloy.

A limitation of this correction method is that in situations where the abundance of the $\mathrm{ZrH}$ ionic species is high, the uncertainty in the estimate of niobium content is strongly affected.

$$
\sigma_{N b}=\sqrt{\sigma_{46.5 a m u}^{2}+\sigma_{47.5 a m u}^{2}}
$$

The relative uncertainty in the niobium observed in a variety of sample volumes is given in Table 19. As the $\mathrm{Nb} /(\mathrm{ZrH}+\mathrm{Nb})$ ratio tends to 1 the analysis volume would contain a large proportion of $\mathrm{Nb}$ ions compared to $\mathrm{ZrH}$ ions. There is a fixed relative increase in the uncertainty 
irrespective of the datasets size (the ratio between the uncertainty in $\mathrm{Nb}$ at high and low $\mathrm{ZrH}$ overlap). At low sample counts, such as in proxigrams or concentration profile samples, there is a large uncertainty in the niobium concentration. For a 10,000 ion sample this would be $14.1 \%$ without the extra complications caused by the $\mathrm{ZrH}$ overlap. This increases to an uncertainty of $42.4 \%$ when $80 \%$ of the ions at $46.5 \mathrm{Da}$ are due to $\mathrm{ZrH}$ species.

\begin{tabular}{|c|c|c|}
\hline $\begin{array}{c}\text { Analysis volume } \\
\text { (ions) }\end{array}$ & $\begin{array}{c}\mathrm{Nb} /(\mathrm{ZrH}+\mathrm{Nb}) \\
\text { ratio }\end{array}$ & $\begin{array}{c}\text { 1 standard deviation } \\
\text { uncertainty in } \mathrm{Nb}(\%)\end{array}$ \\
\hline 10000000 & 0.2 & 1.34 \\
\hline 10000000 & 0.5 & 0.77 \\
\hline 10000000 & 0.8 & 0.55 \\
\hline 1000000 & 0.2 & 4.24 \\
\hline 1000000 & 0.5 & 2.45 \\
\hline 1000000 & 0.8 & 1.73 \\
\hline 100000 & 0.2 & 13.42 \\
\hline 100000 & 0.5 & 7.75 \\
\hline 100000 & 0.8 & 5.48 \\
\hline 10000 & 0.2 & 42.43 \\
\hline 10000 & 0.5 & 24.49 \\
\hline 10000 & 0.8 & 17.32 \\
\hline
\end{tabular}

Table 19: Uncertainty in the concentration of niobium (nominally 0.5 at.\%) deconvoluted from ZrH at 46.5 Da with various proportions of ZrH ions and different analysis volume sizes.

In samples with higher oxygen content, niobium is also field evaporated with oxygen atoms. As well as the $\mathrm{Nb}^{1+}, \mathrm{Nb}^{2+}$ and $\mathrm{Nb}^{3+}$ charge states normally observed, $\mathrm{NbO}^{1+}, \mathrm{NbO}^{2+}$ and $\mathrm{NbO}_{2}{ }^{1+}$ are also found in these regions of material. These additional charge states tend to represent very small proportions of the total niobium content found in metal matrix material.

In regions where the level of niobium is high, such as inside some intermetallic precipitates, a higher than expected single peak at $62 \mathrm{Da}$ was found. This was interpreted as $\mathrm{Nb}_{2}{ }^{3+}$, rather than ${ }^{124} \mathrm{Sn}^{2+}$. This unusual species is likely to be an effect of the laser-initiated field evaporation process, similar to the formation of $\mathrm{Zr}_{2} \mathrm{C}$ and $\mathrm{Zr}_{2} \mathrm{O}_{2}$ species described later in sections 4.5 .9 and 4.6.8. 


\subsubsection{Iron}

The majority of the iron present in the zirconium alloy matrix is detected as $\mathrm{Fe}^{2+}$. This is fortunate, as these peaks are not interfered with by any of the other alloying additions, as shown in Figure 46. There is overlap in the spectrum between $\mathrm{ZrO}^{+}$and $\mathrm{Fe}^{+}$at $56 \mathrm{Da}$. The amount of iron that should be present as $\mathrm{Fe}^{+}$was calculated for the dataset from which Figure 46 was produced. This was achieved by comparing the count within the peak at $56 \mathrm{Da}$ with the count that would be expected due to ${ }^{96} \mathrm{ZrO}^{2+}$ based on the isotopic abundance of $\mathrm{Zr}$ and the counts within the other $\mathrm{ZrO}^{2+}$ peaks. The relative isotopic abundance of iron is given in Table 20. $28 \pm 9$ iron ions were found in the $1+$ charge state. This is about $2 \%$ of the level found at $28 \mathrm{Da}\left(\mathrm{Fe}^{2+}\right)$. This uncertainty is usually less than that relating to the counting statistics of the observation of $\mathrm{Fe}^{2+}$. The charge state distribution of iron is very different in second phase particles, where a significant amount of iron is found at the $1+$ charge state, rather than at the $2+$ charge state seen in the alloy matrix. The $1+$ state is included in the analysis of such features in section 5.2.1.

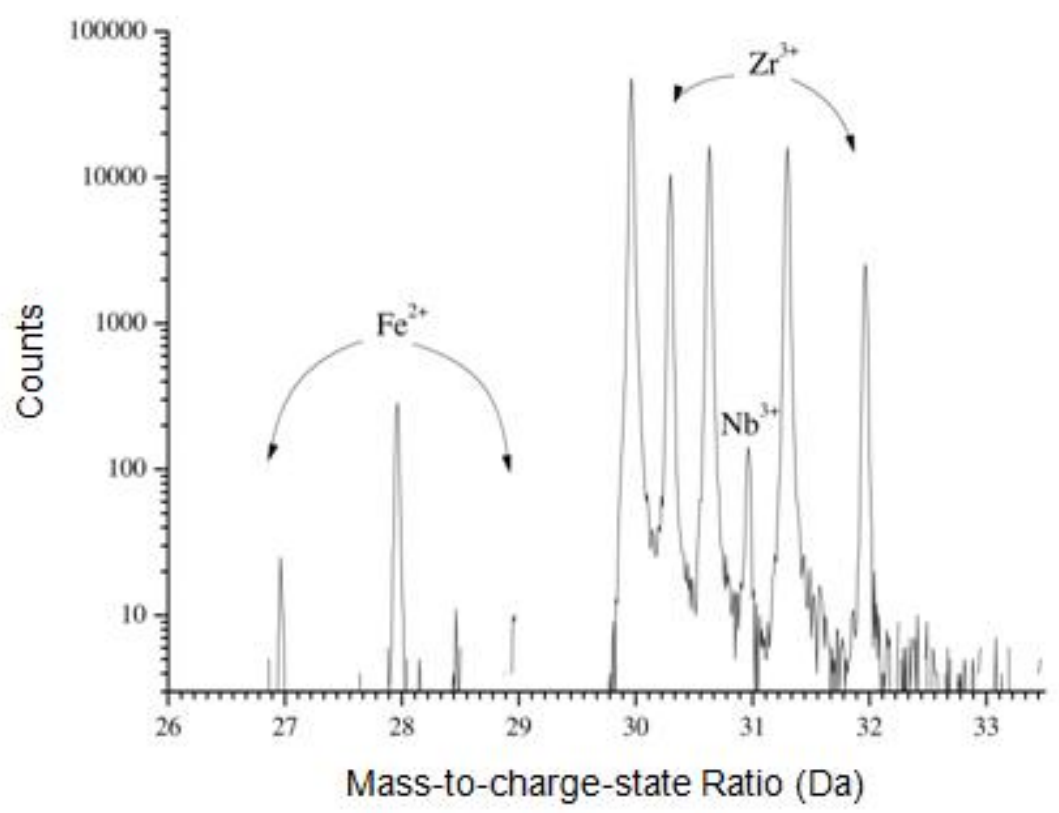

Figure 46. A semi-log scale spectra showing the iron and niobium peaks due to $\mathrm{Zr}^{3+}, \mathrm{Fe}^{2+}$ and $\mathrm{Nb}^{3+}$ in uncorroded ZIRLO alloy matrix. Rendered using 1000 mass bins per Da. 
Unfortunately, although none of the other alloying additions share mass-charge positions with $\mathrm{Fe}^{2+}$, other overlapping of spectrum peaks does occur. In some datasets the mass peak at $28 \mathrm{Da}$ contains both iron and $\mathrm{CO}$ molecules that are adsorbed onto the atom probe specimen from the vacuum system and subsequently field desorbed. The ions due to iron can often be at least partially recovered from those due to $\mathrm{CO}^{+}$. The $\mathrm{CO}^{+}$events are usually homogeneously distributed throughout a dataset; whereas iron may decorate any microstructural features present (discussed in detail in chapters 5 and 6). The $\mathrm{CO}^{+}$counts are also more prominent in the tail of the mass peak, with iron taking the shape of a normal mass peak (Gaussian, with a quicker decaying exponential tail than $\mathrm{CO}^{+}$). This allows the leading edge of the mass peak to be used to give a qualitative impression of the iron distribution in a dataset. An example of these differences in spatial distribution is given in Figure 47.

Dividing the detector hit events into those where multiple ions arrive at the detector during a single pulse, and those where only a single ion arrives, demonstrates that a greater proportion of iron ions are evaporated as multiple hit events than $\mathrm{CO}^{+}$ions. This is determined by comparing the spatial distribution of the two types of event, similar to the process of comparing the leading and trailing edges of the 28 Da peak with ionic spatial distribution shown in Figure 47.

\begin{tabular}{|c|c|c|}
\hline Isotope & $\begin{array}{c}\text { Atomic mass } \\
\text { (Da) }\end{array}$ & $\begin{array}{c}\text { Relative } \\
\text { abundance } \\
(\%)\end{array}$ \\
\hline${ }^{54} \mathrm{Fe}$ & 53.9396 & 5.8 \\
${ }^{56} \mathrm{Fe}$ & 55.9349 & 91.8 \\
${ }^{57} \mathrm{Fe}$ & 56.9353 & 2.15 \\
${ }^{58} \mathrm{Fe}$ & 57.9332 & 0.29 \\
\hline
\end{tabular}

Table 20: The naturally occurring isotopes of iron [160]. 


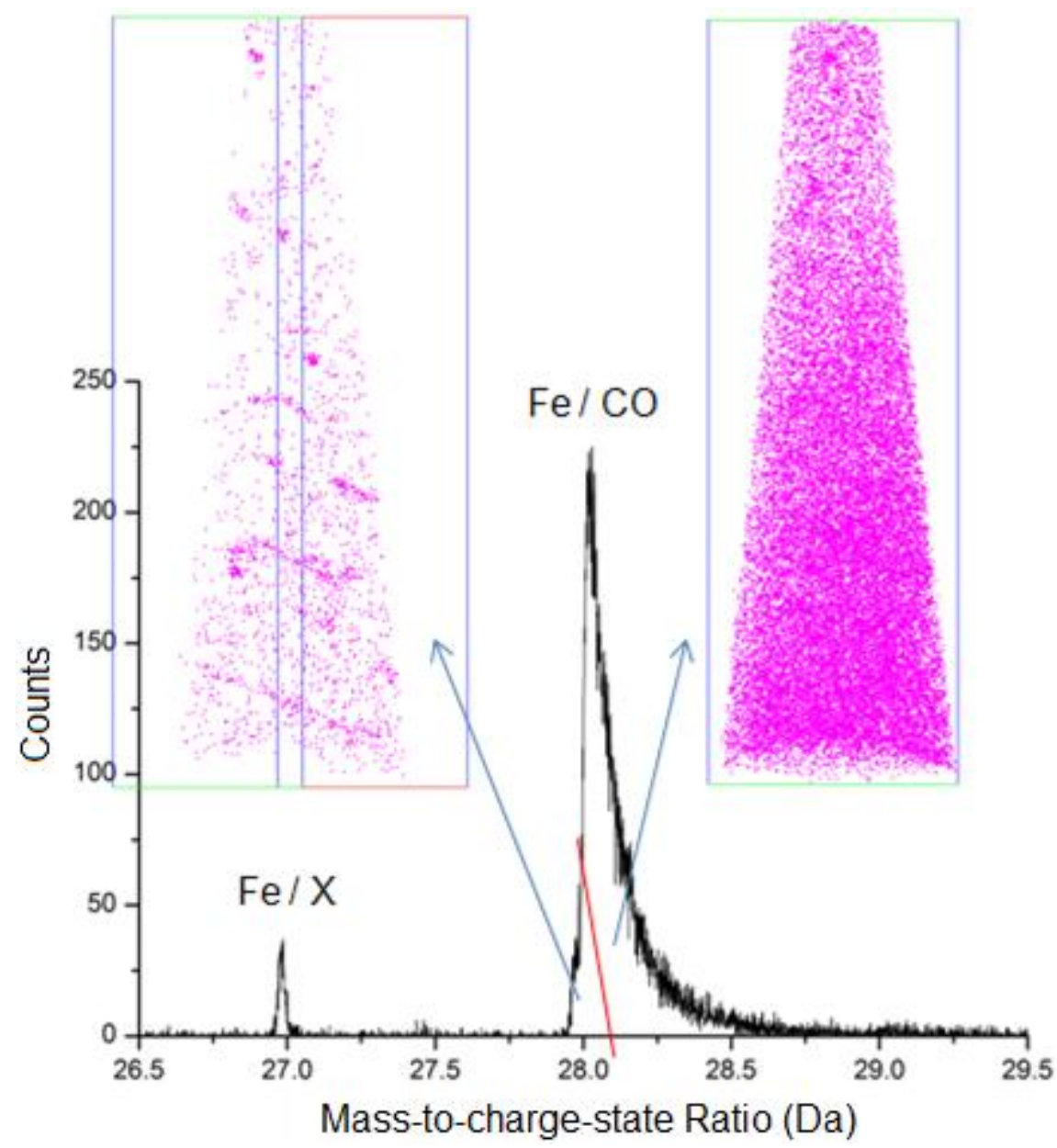

Figure 47: Comparison of the spatial distribution of irons at the leading and trailing edge of the 28 Da mass peak. This corroded ZIRLO specimen is later reproduced as Figure 120.

A comparison of the single hit event and multi-hit event spectra around $28 \mathrm{Da}$ is shown in Figure 48. Some of the single hit species at $28 \mathrm{Da}$ will be due to iron, but might also include a contribution from $\mathrm{CO}$ from the vacuum system. From Figure 48 it can be seen that a higher proportion of the leading edge of the peak at $28 \mathrm{Da}$ is due to the multiple hit (iron, very little noise) species than the single hit (some iron, some $\mathrm{CO}$, some noise) species. This validates the method of using the leading edge of 28 to demonstrate the qualitative distribution of iron in such datasets. 


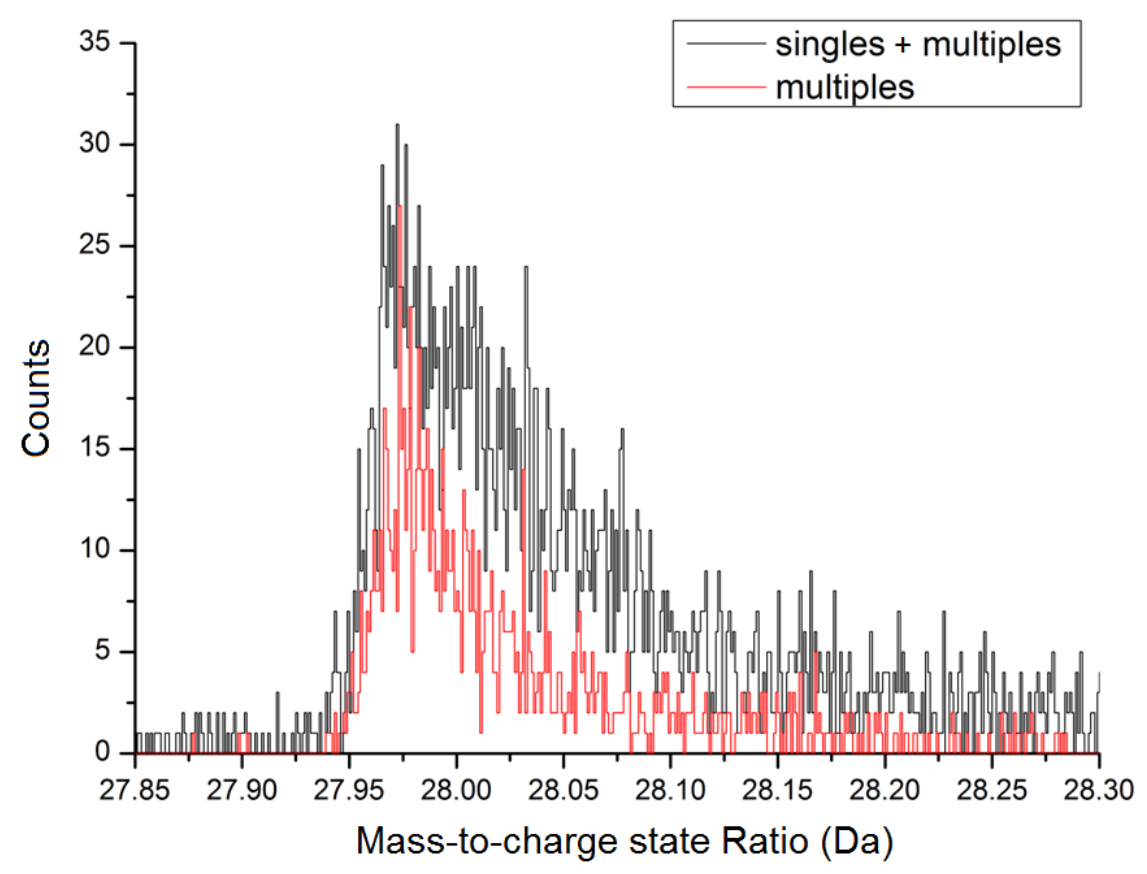

Figure 48: Mass-to-charge-state spectrum for multiple and single hit events around at 28 Da. The multiple hits are considered to be iron and very little detector noise. The single hit events are a mixture of iron and $C O$ and detector dark current noise.

Another method that could be usefully employed to determine the iron concentration would be to use the peak at $27 \mathrm{Da}(5.8 \%$ relative isotopic abundance) and ignore $28 \mathrm{Da}$. Unfortunately, as well as being a minor isotope, this peak is more prone to interference from other minor species than $28 \mathrm{Da}$. Additional counts found at $27 \mathrm{Da}$ cause a discrepancy between the experimentally observed isotopic ratio of $27 \mathrm{Da} / 28 \mathrm{Da}$ seen and that which would be expected of iron (5.8 / 91.8). This additional contribution could come from $\mathrm{Al}^{+}$, although this would lead to the expectation of a peak at $13.5 \mathrm{Da}\left(\mathrm{Al}^{2+}\right)$ which is not observed.

Figure 49 (a) shows an example where the isotopic ratio $27 \mathrm{Da} / 28 \mathrm{Da}$ does not match that expected for iron. This is due to a larger number of counts being observed at $27 \mathrm{Da}$ than would be expected. Figure 49 (b) shows the same data using only the multiple hit events. In this case the isotopic ratios do match, suggesting that these counts are due to iron. However, this method of determining the iron counts from the impurity species counts is unreliable, as in a second 
example, Figure 49 (c) and (d), the counts do not match the expected isotopic ratio using either single or multiple hit events. The full peak anomaly at 27 Da therefore remains to be established.
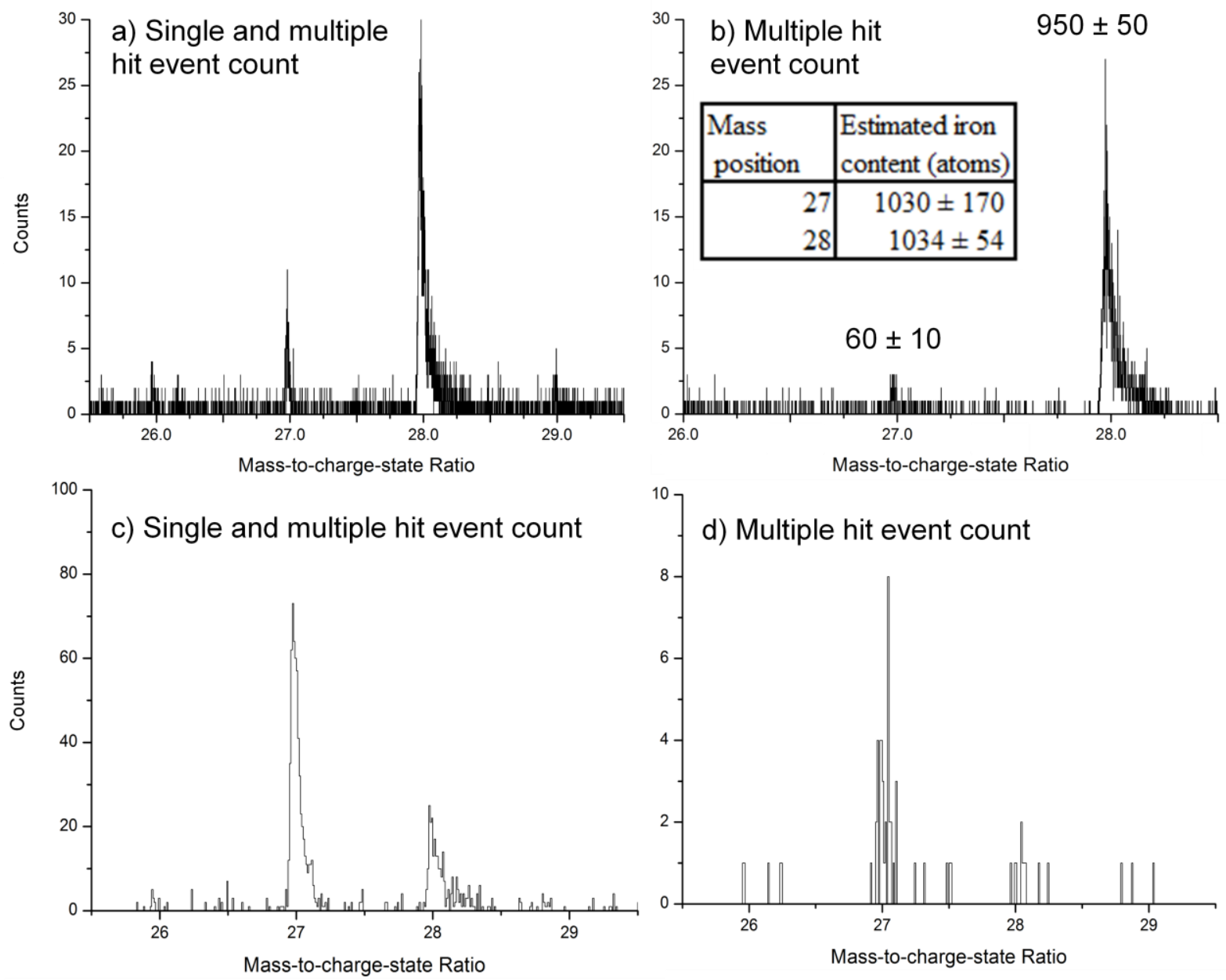

Figure 49: Comparison of the 25.5 - 29.5 Da mass-to-charge spectra from two specimens, the first shown in (a) and (b); and the second in (c) and (d). (a) and (c) display all of the detector hit events and (b) and (d) display only multiple hits. Note the difference in vertical scale between the spectra. The isotopic abundance of iron would lead to $5.8 \%$ of the Fe ${ }^{2+}$ counts at $27 \mathrm{Da}$ and $91.8 \%$ at $28 \mathrm{Da}$.

In the following results chapters a variety of these methods have been used to determine the iron concentration or, where this is not possible, its relative spatial distribution. The process used is recapped briefly, where necessary, to provide validation of these results. 


\subsubsection{Chromium and nickel}

Chromium is a very minor solute in most zirconium alloys. The largest peak occurs at occurs at $52,26,17.33$ Da positions in the $1+, 2+$ and $3+$ charge states, respectively. The relative isotopic abundance of chromium (and nickel) is given in Table 21. A peak is observed in experimental data at $52 \mathrm{Da}$, but is attributed to the presence of the $\mathrm{ZrN}$ and $\mathrm{ZrC}$ species. A peak at $26 \mathrm{Da}$ in some datasets and is attributed to ${ }^{52} \mathrm{Cr}^{2+}$. Figure 50 shows an example spectrum containing transition metal alloying additions at the $2+$ charge state.

${ }^{58} \mathrm{Ni}$ and ${ }^{60} \mathrm{Ni}$ make up around $95 \%$ of the isotopic abundance of nickel (see Table 21). This overlaps with tin at both positions, meaning that no reliable results can be obtained for zirconium alloys containing tin if tin were to be present at this charge state. The isotopic abundance of these peaks agrees well with tin, which would suggest that there is no nickel present in the $1+$ charge state, although the expected level of nickel in the metal matrix is very low. At the doubly charged state, $29 \mathrm{Da}$, there is no overlap $(68.3 \%)$ with other alloying additions. A peak is often seen at the very beginning of analyses together with gallium damage and native oxide formation, but this is taken to be an impurity from the atmosphere.

\begin{tabular}{|c|c|c|}
\hline Isotope & $\begin{array}{c}\text { Atomic mass } \\
(\mathrm{Da})\end{array}$ & $\begin{array}{c}\text { Relative } \\
\text { abundance } \\
(\%)\end{array}$ \\
\hline${ }^{50} \mathrm{Cr}$ & 49.9460 & 4.35 \\
${ }^{52} \mathrm{Cr}$ & 51.9405 & 83.75 \\
${ }^{53} \mathrm{Cr}$ & 52.9406 & 9.5 \\
${ }^{54} \mathrm{Cr}$ & 53.9389 & 2.36 \\
${ }^{58} \mathrm{Ni}$ & 57.9353 & 68.3 \\
${ }^{60} \mathrm{Ni}$ & 59.9308 & 26.1 \\
${ }^{61} \mathrm{Ni}$ & 60.9311 & 1.13 \\
${ }^{62} \mathrm{Ni}$ & 61.9283 & 3.59 \\
${ }^{64} \mathrm{Ni}$ & 63.9280 & 0.91 \\
\hline
\end{tabular}

Table 21: The naturally occurring isotopes of chromium and nickel [160]. 


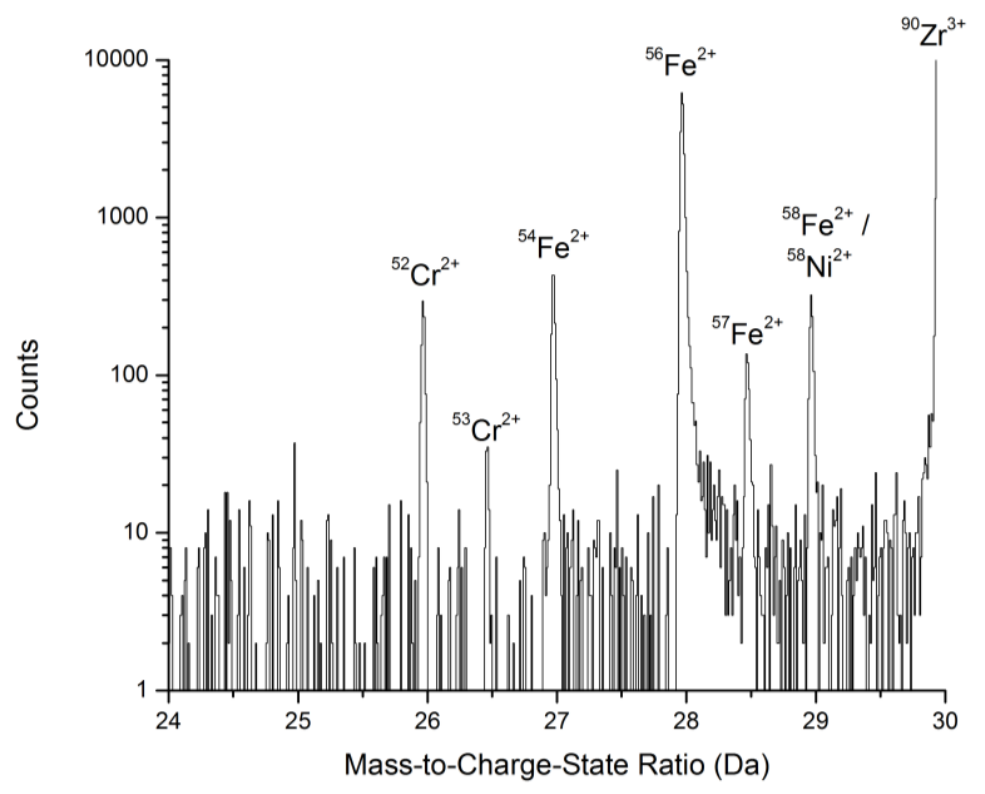

Figure 50: Mass spectrum showing the 2+ charge state of the transition metal solute for a corroded Zircaloy-4 specimen (further analysed in section 6.3.1).

\subsubsection{Titanium}

The expected order of precedence for the evaporation of titanium ions is in the $2+, 3+$ and $1+$ charge states [131]. Quantifying the level of titanium at 3+ and 1+ could be complicated by peak overlap. $73.7 \%$ of titanium occurs at $48 \mathrm{Da}, 24 \mathrm{Da}, 16 \mathrm{Da}$ positions in the $1+, 2+$ and $3+$ charge states, respectively. The other titanium isotopes each contain 5-9 \% of the relative isotopic abundance (see Table 22). Oxygen is also present at $16 \mathrm{Da}$ and $\mathrm{Zr}^{2+}$ overlaps with $48 \mathrm{Da}$. It is fortunate, then, that in practice the only observed peak is seen at the expected, $2+$ position using pulsed laser atom probe. $\mathrm{Zr}^{4+}$ would also overlap with $\mathrm{Ti}^{2+}$ at $24 \mathrm{Da}$, but this problem is overcome by selecting analysis conditions that do not lead to such a high field at the tip that zirconium becomes field ionised in the $4+$ charge state. The titanium content in the $\mathrm{Zr}-\mathrm{Nb}-\mathrm{Ti}$ alloy can be clearly resolved using $\mathrm{Ti}^{2+}$, as shown in Figure 51. 


\begin{tabular}{|c|c|c|}
\hline Isotope & $\begin{array}{c}\text { Atomic mass } \\
(\mathrm{Da})\end{array}$ & $\begin{array}{c}\text { Relative } \\
\text { abundance } \\
(\%)\end{array}$ \\
\hline${ }^{46} \mathrm{Ti}$ & 45.9526 & 8.2 \\
${ }^{47} \mathrm{Ti}$ & 46.9518 & 7.4 \\
${ }^{48} \mathrm{Ti}$ & 47.9479 & 73.7 \\
${ }^{49} \mathrm{Ti}$ & 48.9479 & 5.4 \\
${ }^{50} \mathrm{Ti}$ & 49.9447 & 5.2 \\
\hline
\end{tabular}

Table 22: The naturally occurring isotopes of titanium [160].

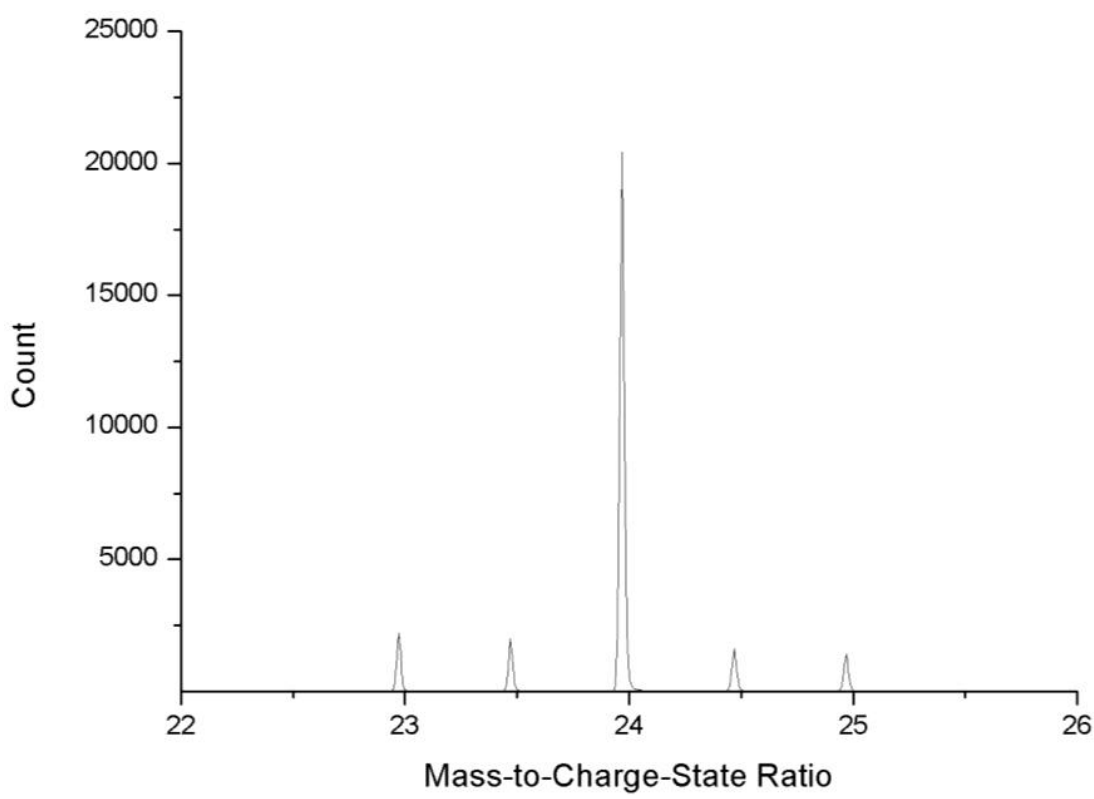

Figure 51: The Ti ${ }^{2+}$ spectrum from within the metal matrix of a $\mathrm{Zr}$-Nb-Ti alloy between 22 and $26 \mathrm{Da}$. All five peaks are due entirely to the presence of titanium. 


\subsubsection{Ion complexes formed by laser pulsing}

Three ionic species containing multiple $\mathrm{Zr}$ atoms have been observed in the ZIRLO metal matrix, these are $\mathrm{Zr}_{2} \mathrm{C}, \mathrm{Zr}_{2} \mathrm{O}_{2}$ and $\mathrm{Zr}_{2} \mathrm{O}_{3}$. These were identified by comparison with calculated isotopic distributions. Data for $\mathrm{Zr}_{2} \mathrm{O}_{2}$ is given as an example in Figure 52. These species are very low in abundance, and this abundance is heavily dependent upon the analysis field conditions. This is discussed in detail in section 4.6.8.
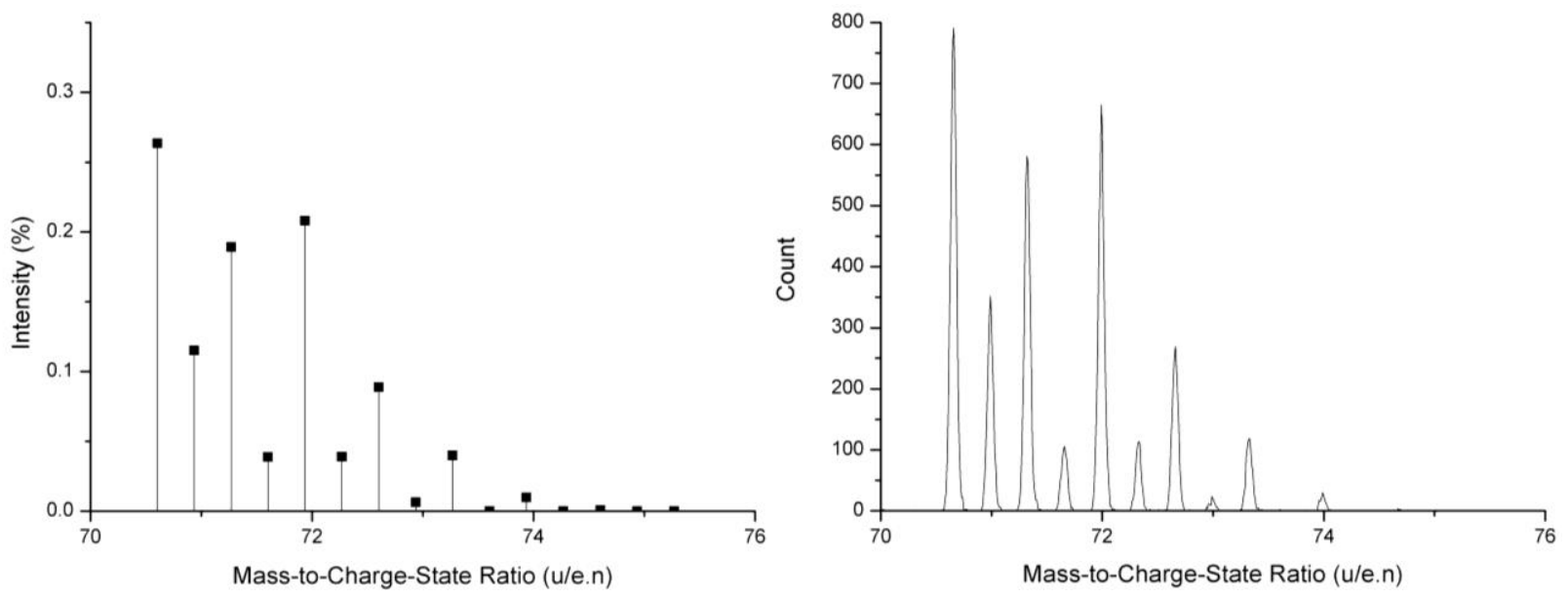

Figure 52: A comparison of the calculated isotopic distribution of $\mathrm{Zr}_{2} \mathrm{O}_{2}$ triply charged ions and a partial mass spectrum showing the corresponding mass-to-charge spectrum obtained by $3 D A P$. 


\subsection{Determining suitable analysis conditions for laser-pulsed LEAP}

The criteria for selecting suitable experimental conditions for laser pulsed acquisitions are different to those for voltage pulsing. Temperatures between $30-90 \mathrm{~K}$ were trialled in the LEAP. It was found that a very high incidence of fracture occurred with specimens probed at 30 $\mathrm{K}$ and $40 \mathrm{~K}$ without the acquisition of useful quantities of data. Above this range the rate of fracture became tolerable. Temperatures in the range 50-60 K were used for all experiments.

The target evaporation rate used fell within two regimes. For data where the aim was to study a microstructural feature, the evaporation rate was kept low, $0.2-0.5 \%$. For specimens where the aim was to examine a (supposedly) homogeneous region in order to make high accuracy compositional calculations, a rate, around $0.5-1 \%$ was used. This equates to a detection rate of up to 2000 ions per second. In this way, datasets comprising millions of ions can be captured in a single LEAP session. 
Calibration experiments, analysing a single specimen with a variety of laser energies, were conducted in order to ascertain the sensitivity of compositional measurement to changes in experimental conditions. A ZIRLO specimen was prepared for a calibration experiment using the electropolshing method and FIB finishing described in 3.5.2. ZIRLO provides information on the evaporation behaviour of all of the solute found in Zircaloy-4, as well as niobium. The reproducibility of specimen preparation was found to be good. This is important as there is a lack of understanding regarding laser interaction with APT specimens and this is itself a very active area of research [161-162]. Limiting the variation in specimen geometry removes uncertainty regarding the change in the laser interaction properties with temperature. Figure 53 shows the specimen examined in the calibration study. The diameter of the end of the specimen is (130 \pm 20) $\mathrm{nm}$ and it has a very low shank angle, around $3^{\circ}$.

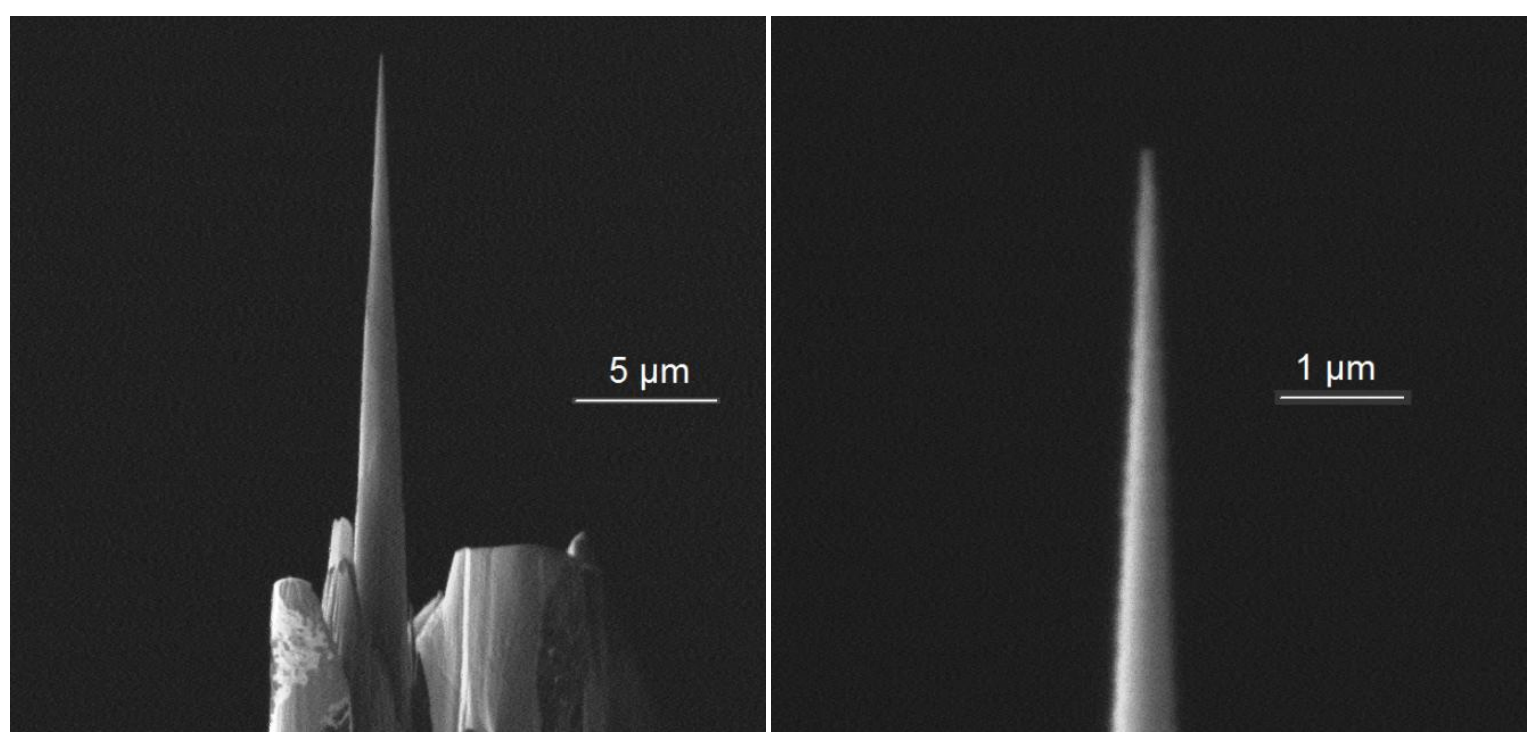

Figure 53: FIB images of a ZIRLO specimen prepared for calibration experiments. The end diameter of the needle is $(130 \pm 20) \mathrm{nm}$ and has a very low shank angle of $(3 \pm 1)^{\circ}$. 


\subsubsection{Evaporation field variation}

Samples of roughly 1 million ions were taken at various laser energies between $0.2 \mathrm{~nJ}$ and $0.6 \mathrm{~nJ}$. Increasing the laser energy causes the control software to drop the DC potential on the specimen in order to maintain the evaporation rate $(0.2 \%$, in this case $)$, as can be seen in Figure 54 . This figure also shows that there is a slight deviation in the laser energy during the run. This is due to the variation in laser coolant temperature. The range of this deviation is of the order of $0.02 \mathrm{~nJ}$.

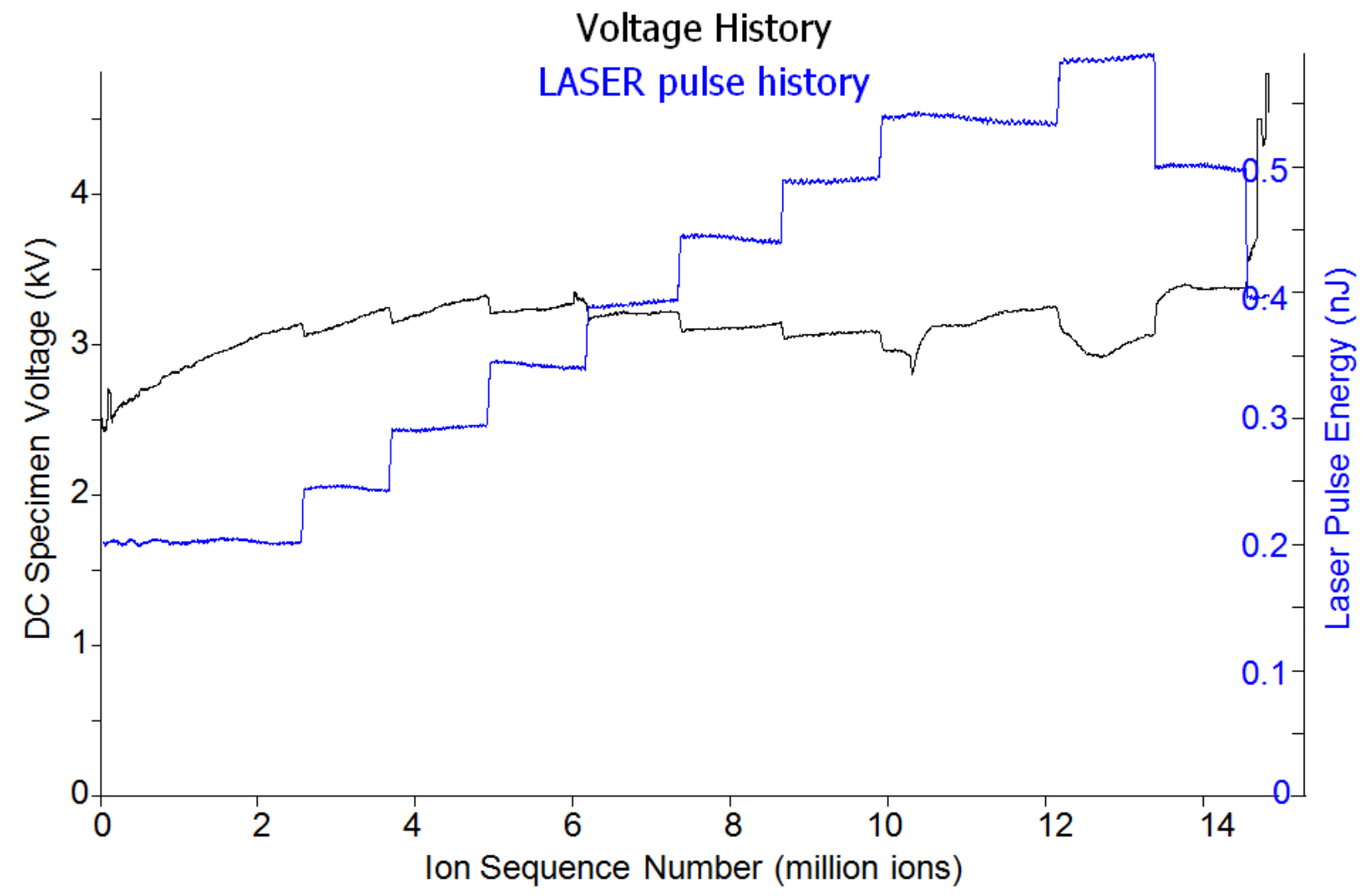

Figure 54: Comparison of laser energy and specimen DC potential during acquisition of the calibration experiment. Specimen temperature: 55 K.

An estimate of the field strength at the apex of the tip can be obtained by comparing the proportion of ions detected at different charge states with theoretical predictions. The majority of 
zirconium species are detected as $\mathrm{Zr}^{2+}$ in all the experimental conditions tested. The $\mathrm{Zr}^{3+} / \mathrm{Zr}^{\text {all }}$ ratio is a useful comparator to find the field at the apex of a specimen. An experimental curve obtained for the ZIRLO specimen has been fitted to the theoretical model proposed by Kingham [163], as shown in Figure 55. Note that the laser energy axis increases from right to left as a lower laser energy corresponds to a higher standing field. The graph has been constructed by superimposing a section of the theoretical Correlation between charge state ratio and evaporation field onto the experimental curve plotting charge state ratio against laser energy. The excellent fit is thus a result of choice of scaling; the graph allows a specimen evaporation field to be estimated for each of the laser energies, it does not prove the relationship between the laser energy and evaporation field.

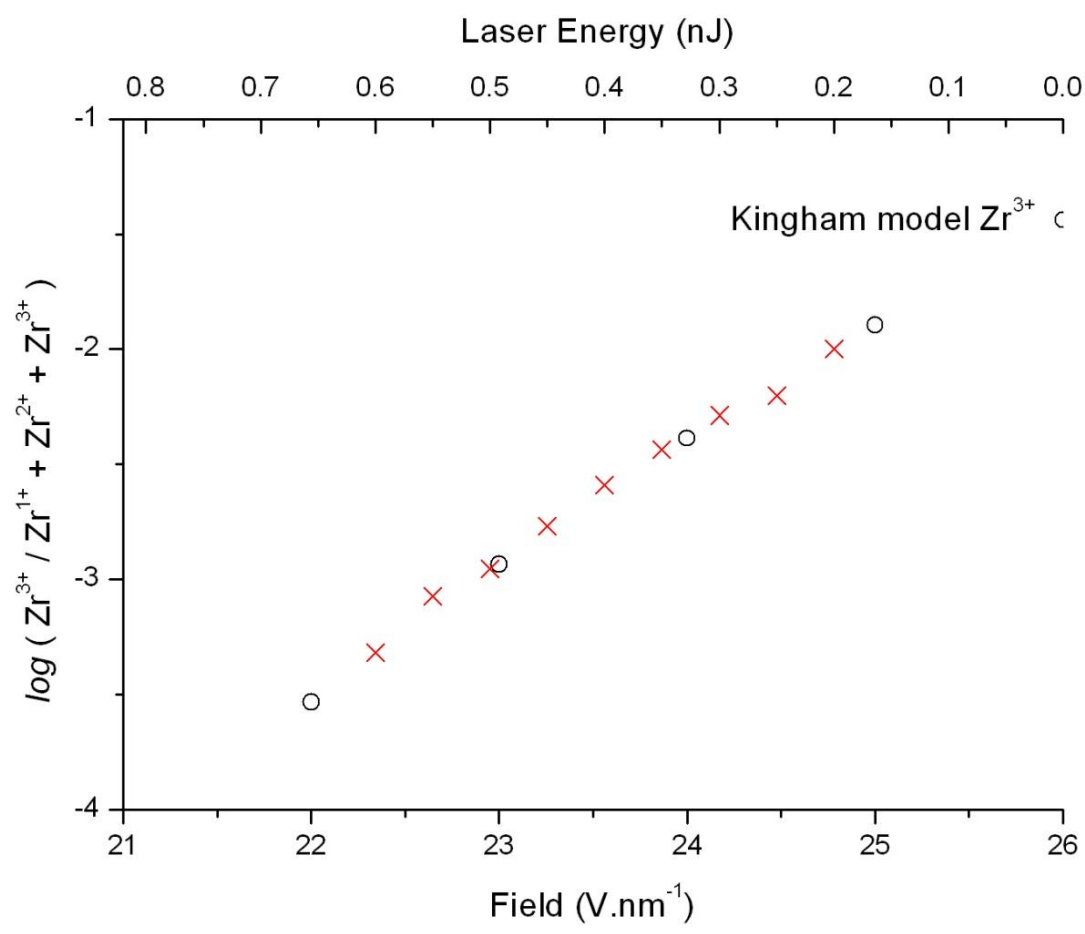

Figure 55: Estimating the field strength at the apex of the tip using various intensities of laser illumination. $55 \mathrm{~K}$ specimen base temperature. Red crosses mark experimental observations $(\sim 1$ $m$ ions) of the charge state ratios at differing laser energies. Black circles are theoretical predictions of the evaporation field that would cause a particular charge state ratio. 
For the specimen tested, which had a similar geometry to all of the metal matrix specimens used in the course of this work, varying the laser energy for a fixed evaporation rate of $0.2 \%$ yields a reduction in calculated specimen field from $24.8 \mathrm{Vnm}^{-1}$ at $0.2 \mathrm{~nJ}$ to $22.4 \mathrm{Vnm}^{-1}$ at $0.6 \mathrm{~nJ}$. The field changes by $\sim 10 \%$ between these laser energies. By extrapolation, evaporation would occur at $26 \mathrm{Vnm}^{-1}$ with negligible laser energy at $55 \mathrm{~K}$. The predicted evaporation field at $77 \mathrm{~K}$ is given as $28 \mathrm{Vnm}^{-1}[131]$.

The variation in field intensity over the surface of the specimen in reconstruction-space is shown for the various laser energies tested in Figure 56. This shows the spatial distribution of the field that is represented by a mean value in Figure 55. A crystallographic pole is visible at $(-10,0)$ with an associated zone line roughly defined by the line $[(-10,-10),(0,15)]$. As a general trend, the appearance of the crystallographic features is more apparent at higher laser energies, where the overall evaporation field is lower. A larger proportion of ions field evaporated at the pole are so at a $3+$ charge state than elsewhere on the sample. The rate of ions evaporated at the pole is also lower, as shown in the density profile of the reconstruction-space, Figure 57 . As the laser energy increases, the probability of field evaporation increases more for the regions away from the pole than at the pole, increasing the difference in evaporation rates of the two regions.

At the higher laser energies, $0.5-0.6 \mathrm{~nJ}$, the laser beam heating experienced by the specimen is non-uniform. The energy deposited in each pulse is too large - it increases the ionisation potential for the atoms with which the laser initially interacts by an amount greater than a threshold that would allow conduction of the heat to occur through electrons within the skin depth of the material and increase the evaporation rate of the areawhich the beam is initially incident upon. This increase in the local evaporation rate leads to a reduction in the local curvature of the specimen, as seen by other workers in low thermal conductivity steels [162]. The higher local radius of curvature leads to a reduced local magnification of this region of the specimen and so its apparent density as seen by 3DAP increases. The effect of this non-uniform 
heating can be seen in the $0.6 \mathrm{~nJ}$ example shown in Figure 57, where the laser beam is incident on the $(+x,-y)$ corner of the profile.
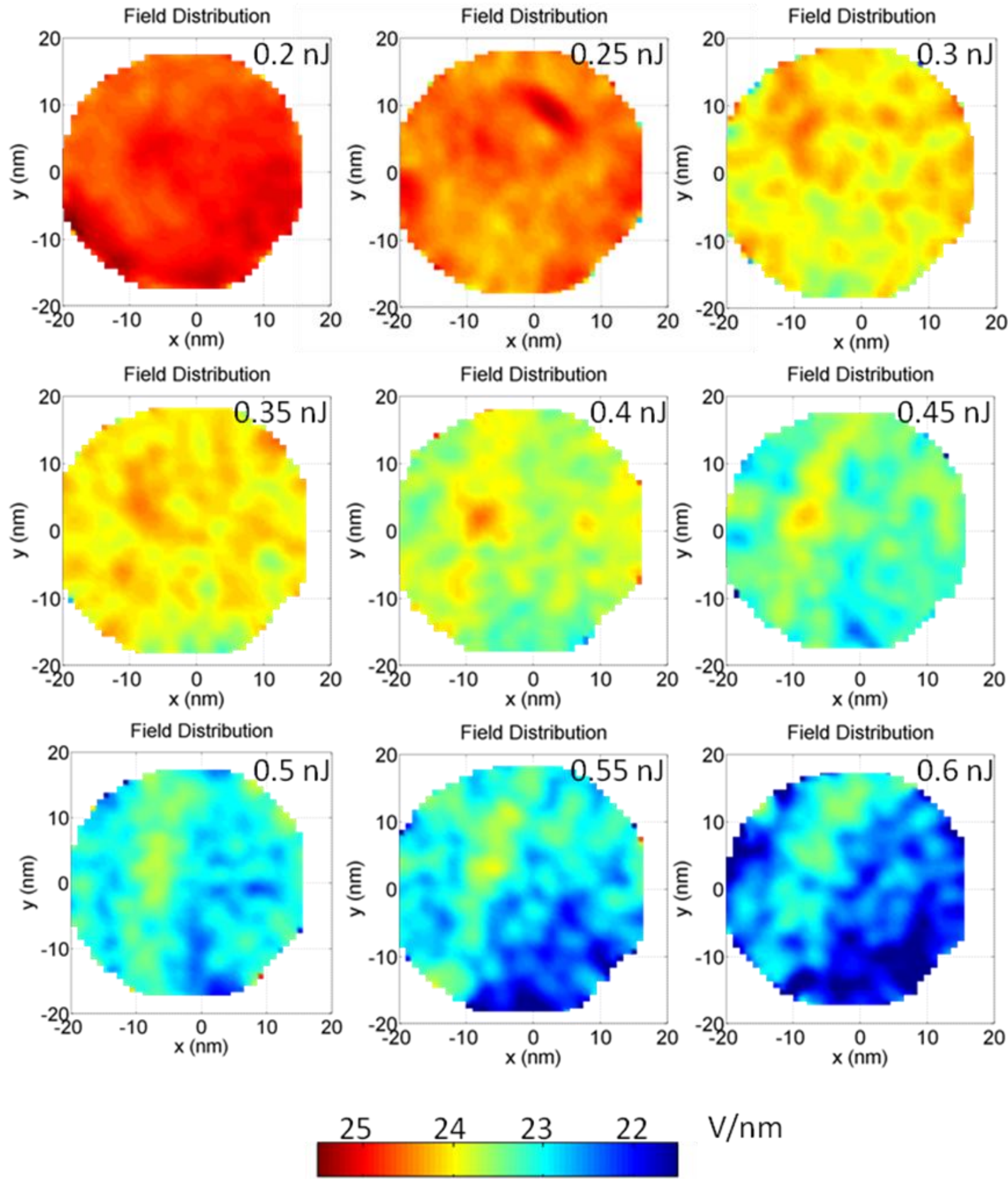

$\mathrm{V} / \mathrm{nm}$

Figure 56: 2D profiles showing the field distribution inferred from the local $\mathrm{Zr}$ charge state ratio at various laser energies. Profiles are shown in the plane of the specimen axis. A single colour scale is used for all of the profiles. 

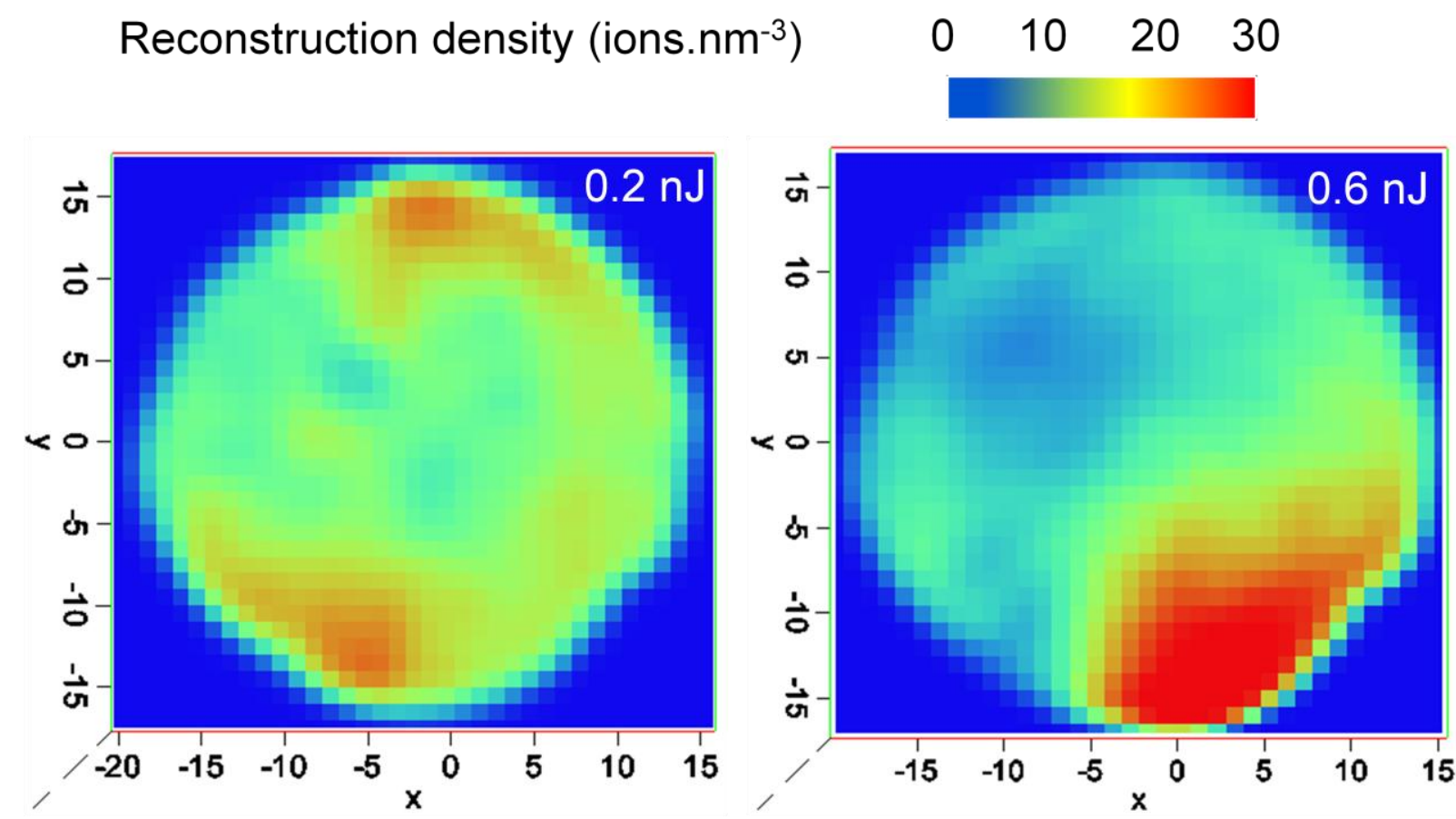

Figure 57: 2D density profiles of the extremes of the laser energies tested. The laser is incident on the specimen at $(+x,-y)$.

\subsubsection{Oxygen}

Figure 58 shows the apparent oxygen concentration against laser pulse energy. The apparent oxygen concentration is stable between $0.3-0.6 \mathrm{~nJ}$ at $\sim 0.65$ at.\%. The detected oxygen concentration increases sharply from 0.7 at $\%$ to 2 at. $\%$ at low laser energy. It is also noted that this datapoint is taken from the start of the run. Although care was taken not to include any native surface oxide in the volume analysed, it appears that native surface oxidation might be the cause of this additional oxygen, although the volume analysed contained no discernable oxygen density variation visible by eye so this is unlikely to be the cause of this variation. The influence of native oxides formed on atom probe specimens is examined in further detail in section 5.1.2. The low laser energy corresponds to a high field; in such circumstances evaporation of low field 
species between pulses is increased. If $\mathrm{ZrO}$ is a higher field species than $\mathrm{Zr}$, the DC evaporation of $\mathrm{Zr}$ between pulses would cause a rapid increase in the apparent oxygen concentration at low laser energy, similar to that seen in Figure 58.

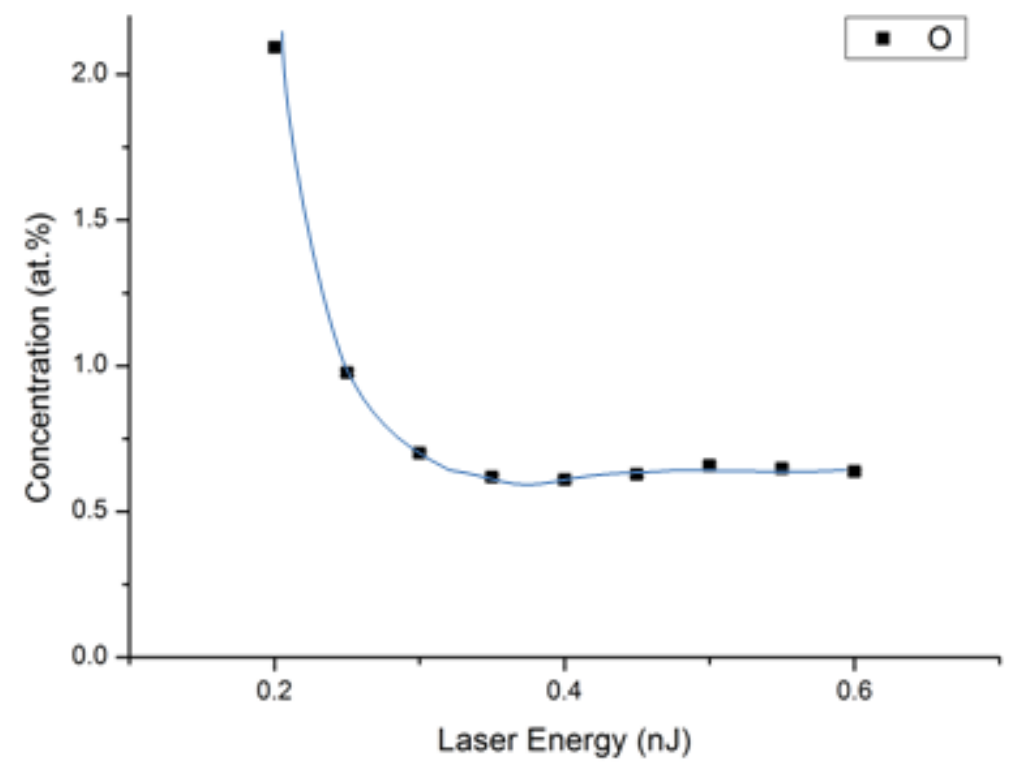

Figure 58: Apparent concentration of oxygen against laser pulse energy. 


\subsubsection{Hydrogen}

The adsorption and removal of hydrogen from the surface of atom probe specimens are field dependent processes [131]. Increasing the laser pulse energy increases the thermal heating applied to the specimen, but it also reduces the evaporation field (as seen in Figure 54) as the automated control software attempts to maintain a constant evaporation rate of ions by varying the standing potential applied to the specimen.

The proportion of the dataset detected as hydrogen in the form of $\mathrm{H}^{+}$increases with laser energy between $0.2-0.4 \mathrm{~nJ}$ and remains stable between $0.4-0.55 \mathrm{~nJ}$. Figure 59 demonstrates how the abundance of hydrogen-containing ionic species evaporation on laser energy. These data are plotted in Figure 59 along with the variation in concentration of two hydrogen complex ions, $\mathrm{H}_{2}^{+}$ and $\mathrm{ZrH}^{2+}$. These three species account for the vast majority of hydrogen detected by the 3DAP. The behaviour of the concentration of hydrogen-complex ions is very different to that of the $\mathrm{H}^{+}$ species. The molecular ion species are seen in greater proportions in lower energy datasets. The concentration of $\mathrm{H}_{2}$ falls from a maximum value at low energy to be almost absent from the data at high laser energy. The rate of hydrogen evaporated or desorbed as $\mathrm{H}^{+}$and $\mathrm{H}_{2}{ }^{+}$compared to the rate of zirconium evaporation changes little as the laser conditions (and so electric field) are varied. The number of $\mathrm{H}_{\mathrm{x}}{ }^{+}$ions detected is $\sim 3 \%$ of all ions counted in all conditions. There is a small increase, $\sim 10 \%$, in the proportion of light hydrogen species detected at low laser energy; where the standing field is higher. The data point for $0.6 \mathrm{~nJ}$ is off-trend. 

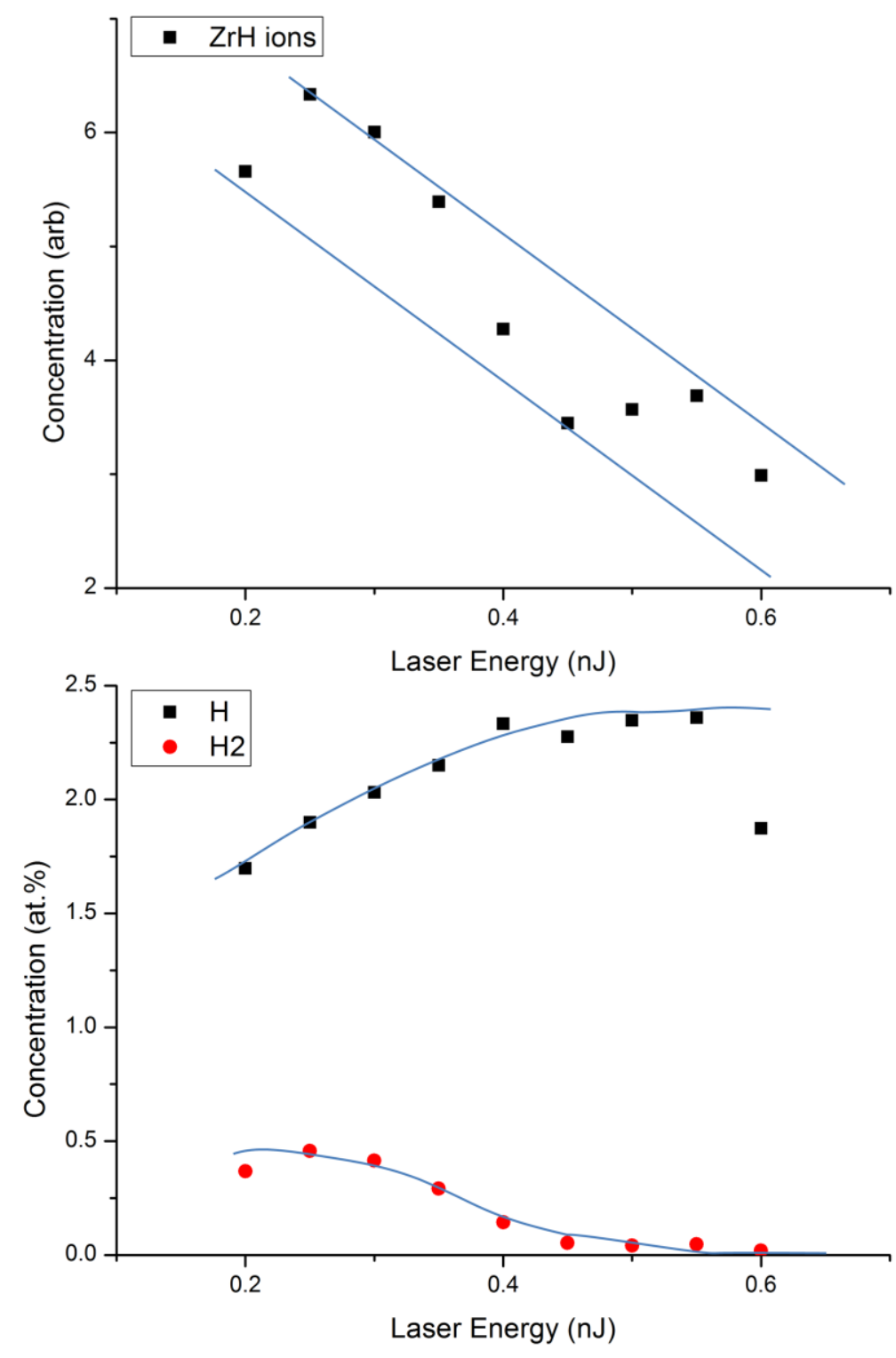

Figure 59: Detected concentration of hydrogen-containing species against laser energy.

The amount of hydrogen detected as $\mathrm{ZrH}$ complex ions is much greater than the amount detected as $\mathrm{H}^{+}$and $\mathrm{H}_{2}^{+}$. Although the scale of Figure $59(\mathrm{ZrH})$ is in arbitrary units (the magnitude of which is equal to approximately 10 at.\% at low laser energy) the trend is one of decreasing $\mathrm{ZrH}$ detection with increasing laser energy, as was seen with $\mathrm{H}_{2}{ }^{+}$complex ions. Unlike $\mathrm{H}_{2}{ }^{+}$, the concentration of $\mathrm{ZrH}$ does not reach zero in this analysis. The evaporation rate of $\mathrm{ZrH}$ compared to $\mathrm{Zr}$ is increased at higher fields and lower temperatures. 
The supply of hydrogen from the vacuum system is likely to be far higher than the zero-field adsorption/desorption rate [164]. There are two mechanisms by which hydrogen can arrive at the apex of the tip. Hydrogen gas from the vacuum system is polarised by the high electric field near the specimen apex and the dipole is attracted to the surface of the needle. The strength of this dipole is proportional to the field strength. At lower laser energies the field strength at the apex of the tip was increased in order to maintain a constant evaporation rate. In the direct case these hydrogen molecule are adsorbed onto the apex surface, some of which are immediately field desorbed and some of which become dissociated into two protons and desorbed. This offers an explanation for the greater number of $\mathrm{ZrH}$ ions (one $\mathrm{Zr}$ ion and a proton) at lower laser energies, but doe not explain why the number of ions detected as protons is lower at lower laser energies. Howe, it should be noted that this mechanism is only valid if the extent of dissociation of hydrogen molecules into individual atoms on the specimen surface increases with increasing holding field.

Hydrogen can also become physisorbed onto the specimen shank and undergo field-enhanced surface migration to the apex. This second process is thermally activated. This mechanism offers an explanation as to why the apparent concentration of $\mathrm{H}_{2}$ is laser energy (temperature) dependent.

It is assumed that hydrogen has a much lower evaporation field than zirconium, as hydrogen ions are seen in greater proportions while specimens are ramping up and in low field regions. The 'best' desorption field for hydrogen is given as $22 \mathrm{~V} \mathrm{~nm}^{-1}$ in the literature [164]. This value is very similar to the evaporation field for zirconium at all of the laser energies (although most similar to the higher energy values). Hydrogen atoms are field desorbed by the potential at the specimen. The mechanism for the desorption of light species may differ significantly from heavier species. For the metallic species, thermal activation 'over' the energy barrier dominates over quantum-mechanical tunnelling 'through' the barrier. However, for light species such as $\mathrm{H}^{+}$ 
and $\mathrm{H}_{2}{ }^{+}$, quantum mechanical tunnelling may be the dominant mechanism below about $55 \mathrm{~K}$ [131].

\subsubsection{Tin}

The apparent tin concentration increases linearly with laser energy. This can be seen in Figure 60. The expected tin concentration in this sample is 0.76 at.\%. Figure 60 also shows that laser energy of $0.3-0.4 \mathrm{~nJ}$ at $55 \mathrm{~K}$ using a specimen of similar geometry would be expected to yield a reasonable value for the overall tin content. $\mathrm{Zr}-\mathrm{Sn}$ bond strength data are not forthcoming from the literature. However, the addition of tin to zirconium results in a drop in melting point from $1855{ }^{\circ} \mathrm{C}$ for pure zirconium to $1592{ }^{\circ} \mathrm{C}$ for $\mathrm{Zr}-21 \mathrm{wt} . \% \mathrm{Sn}$ (the limit of the $\beta$-phase field) [165], which qualititatively indicates the reduced strength of the Zr-Sn metallic bond compared with that of the Zr-Zr metallic bond.

The lower tin concentration found at lower laser energies suggests that tin is preferentially evaporated at these energies. Tin evaporates at a lower field than zirconium due to the lower activation energy required to break $\mathrm{Zr}$-Sn bonds than $\mathrm{Zr}$ - Zr bonds. This leads to a greater proportion of tin atoms than zirconium atoms becoming evaporated between pulses. As only the species evaporated during pulses are counted, this lowers the apparent tin concentration. As the laser energy is increased the standing field falls and the proportion of tin ions lost to preferential evaporation decreases. 


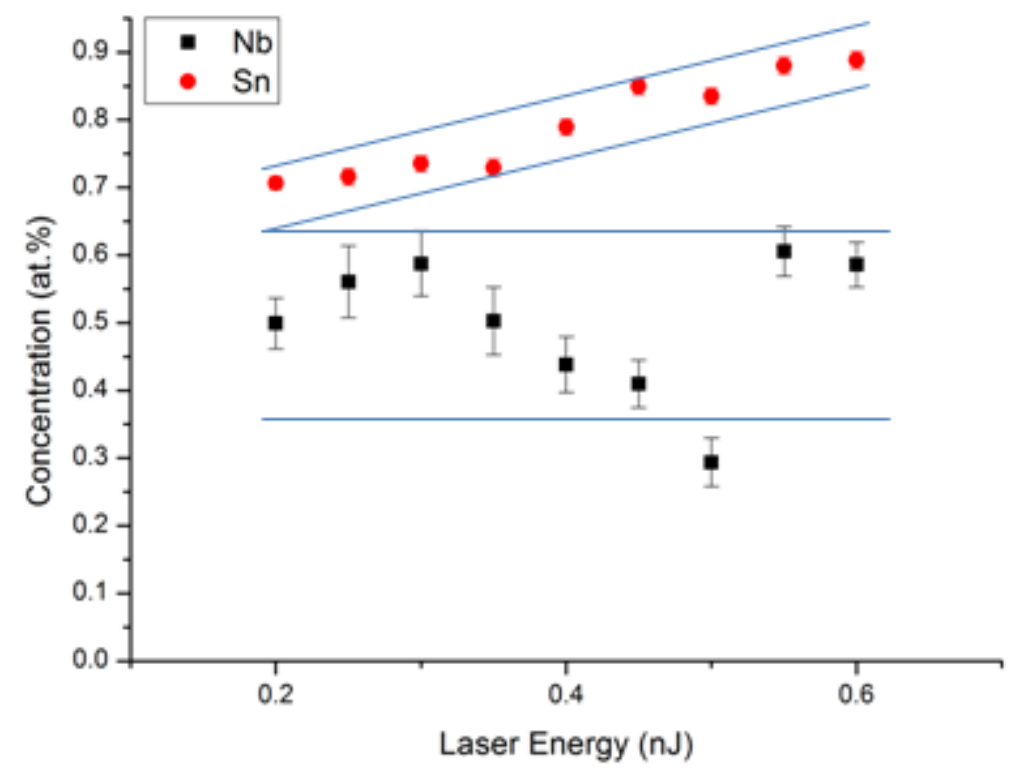

Figure 60: Apparent concentration of niobium and tin against laser pulse energy.

\subsubsection{Niobium}

The niobium concentration compared to laser pulse energy is also shown in Figure 60. There is no clear trend in these data. The uncertainty in the niobium concentration measurement is higher than that seen for tin even though they are at a similar concentration. The reason for this is that niobium must be deconvoluted from other species, as was discussed in detail in section 4.5.5. This increase in uncertainty does not account fully for the variation. The variation in the level of niobium is likely due to a physical variation in the niobium concentration within the material, and so this method is not appropriate for evaluating suitable analysis conditions for niobium. Niobium is more refractory than zirconium. The addition of niobium to zirconium has less of an effect on the melting point than was seen with tin. $\mathrm{Zr}-22 \mathrm{wt} . \% \mathrm{Nb}$ melts at $1743{ }^{\circ} \mathrm{C}$, suggesting that $\mathrm{Zr}-\mathrm{Nb}$ bonds are stronger than $\mathrm{Zr}-\mathrm{Sn}$, although not as strong as $\mathrm{Zr}-\mathrm{Zr}$ bonds. This also suggests that niobium will be underestimated at lower field conditions, but that the behaviour of 
niobium will not differ from that of zirconium as much as is seen for tin; the niobium concentration verses laser energy curve is relatively insensitive to laser energy over this range.

\subsubsection{Iron}

The uncertainty in each observation of iron taken from these 1 million ion samples is high due to the low matrix concentration of iron. Increasing the size of each sample is not practical as a tenfold increase in sample size would reduce the uncertainty by a factor of less than 3.2. Only a limited amount of data can be captured from a specimen before the inevitable fracture occurs. Figure 61 shows the data that is available for the apparent iron level sampled at various laser energies. The observations fall within a band and do not appear to be particularly sensitive to analysis conditions.

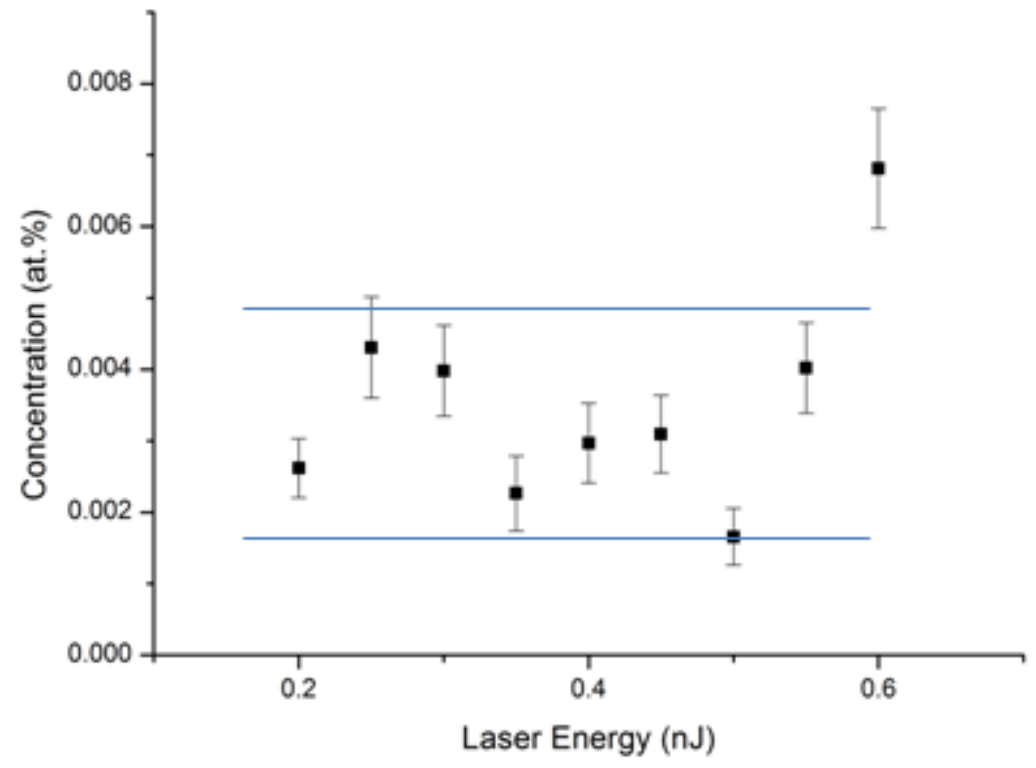

Figure 61: Apparent concentration of iron against laser pulse energy. 


\subsubsection{Titanium}

A similar calibration experiment was conducted for the titanium-contain $\mathrm{Zr}-\mathrm{Nb}-\mathrm{Ti}$ test alloy. The voltage and laser energy curve for this experiment are shown in Figure 62. A narrower range of laser energies was used in this study over the interval $0.3-0.55 \mathrm{~nJ}$. The detected titanium concentration is stable with laser energy over this interval (Figure 63). The apparent concentration of titanium should not be affected by small variations in analysis condition. This is unsurprising as the chemical similarity of zirconium and titanium would be expected to result in $\mathrm{Zr}-\mathrm{Ti}$ and $\mathrm{Zr}-\mathrm{Zr}$ bonds being similar in strength.

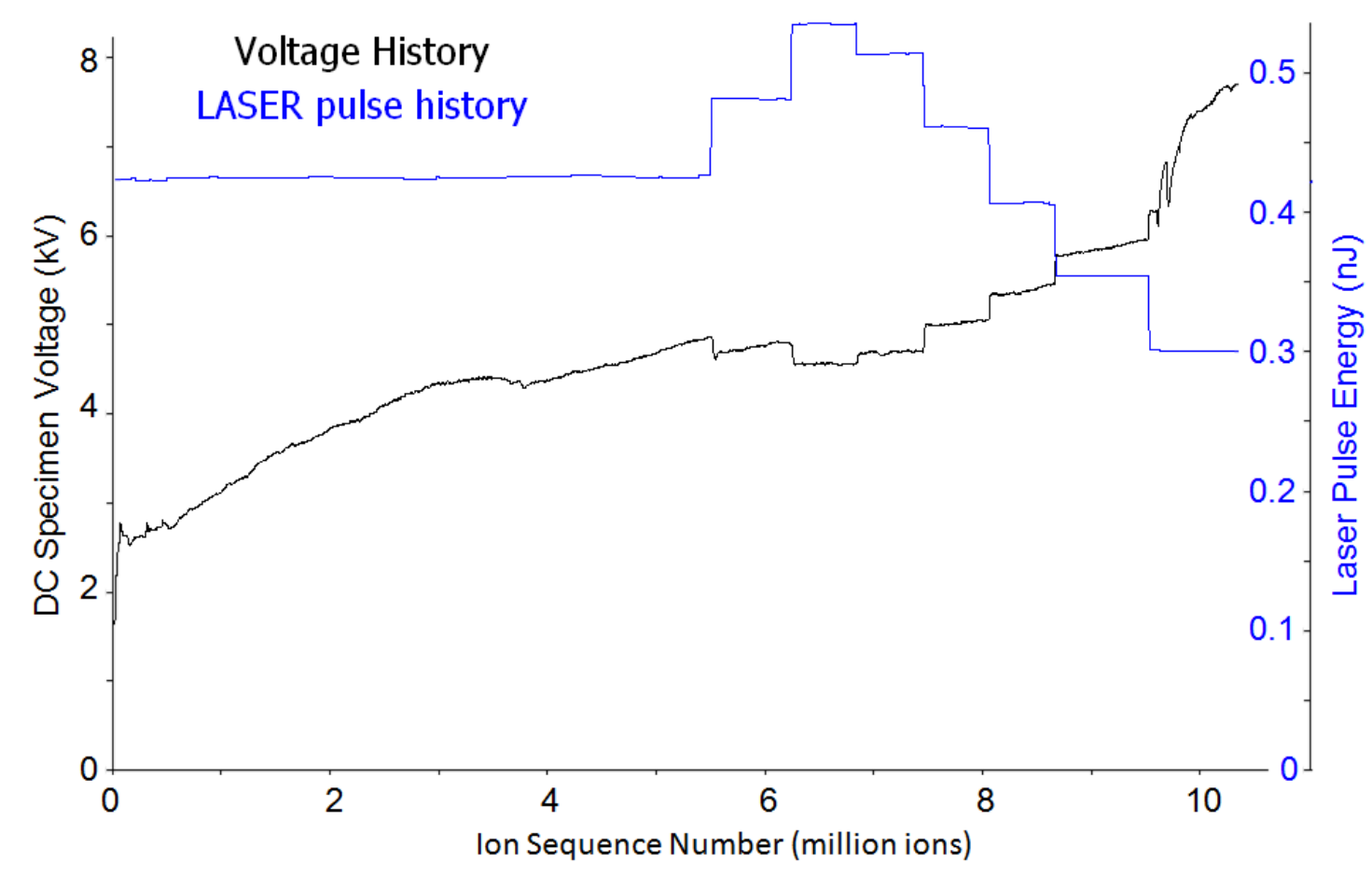

Figure 62: Comparison of laser energy and specimen DC potential during acquisition of the Zr-

Nb-Ti calibration experiment. Specimen temperature: $55 \mathrm{~K}$. 


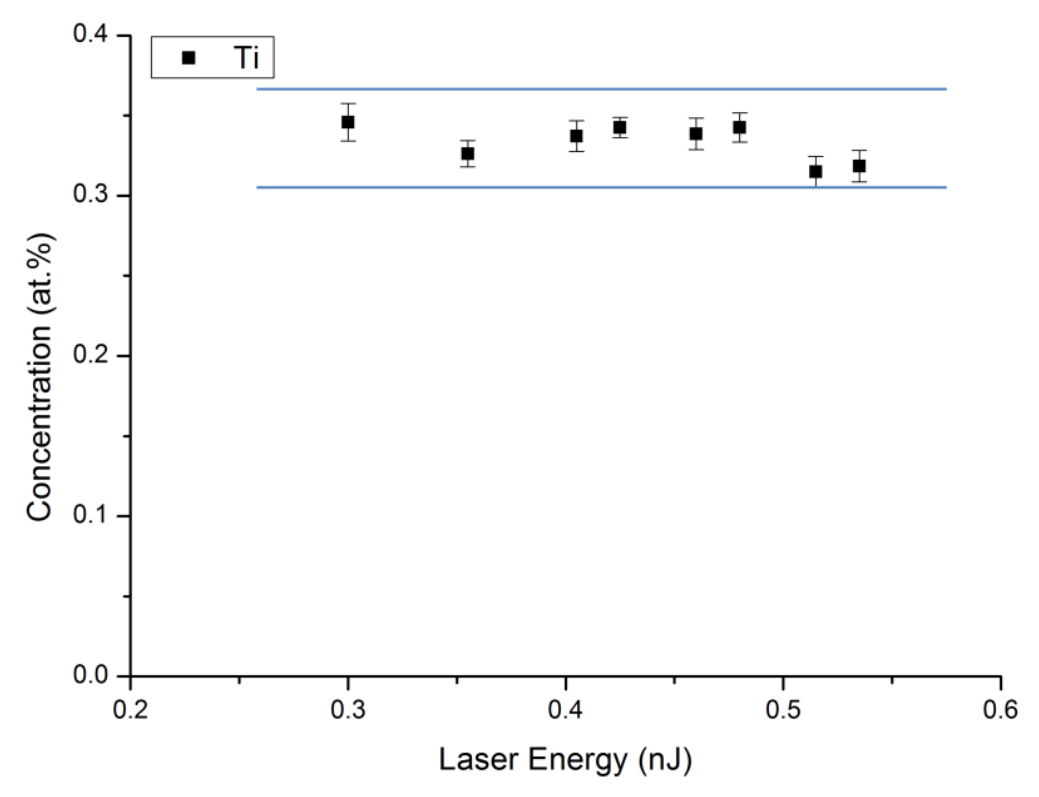

Figure 63: Apparent concentration of titanium against laser pulse energy.

\subsubsection{Ion complexes formed by laser pulsing}

The occurrence of unusual complex ions such as $\mathrm{Zr}_{2} \mathrm{C}$ is an effect of laser pulsing; such species are not seen in voltage pulsed datasets. Figure 64 demonstrates the relationship between laser energy and the apparent composition of a dataset with regard to $\mathrm{Zr}_{2} \mathrm{C}$. Each data point is created from 500,000 ions. Two regimes are apparent. At low laser energy, below $0.35 \mathrm{~nJ}$, there is a very slight linear increase in level of $\mathrm{Zr}_{2} \mathrm{C}$ ions detected. The level is so low that it could be ignored for many purposes. Above $0.35 \mathrm{~nJ}$ the rate of increase of detected $\mathrm{Zr}_{2} \mathrm{C}$ increases dramatically with laser energy. At this level thermal heating is promoting the formation of complex ions at or above the surface of the tip. The step change in the increase of this species at $0.35 \mathrm{~nJ}$ can be explained by the mechanism by which additional carbon are made available for ion formation. The decomposition of a $\mathrm{CO}$ molecule from the vacuum system would require a fixed amount of thermal energy in order to break the $\mathrm{CO}$ bond. Above this threshold, additional energy promotes the formation of the $\mathrm{Zr}_{2} \mathrm{C}$ species, but without this energy there is little carbon in the system with which this species can be formed. 


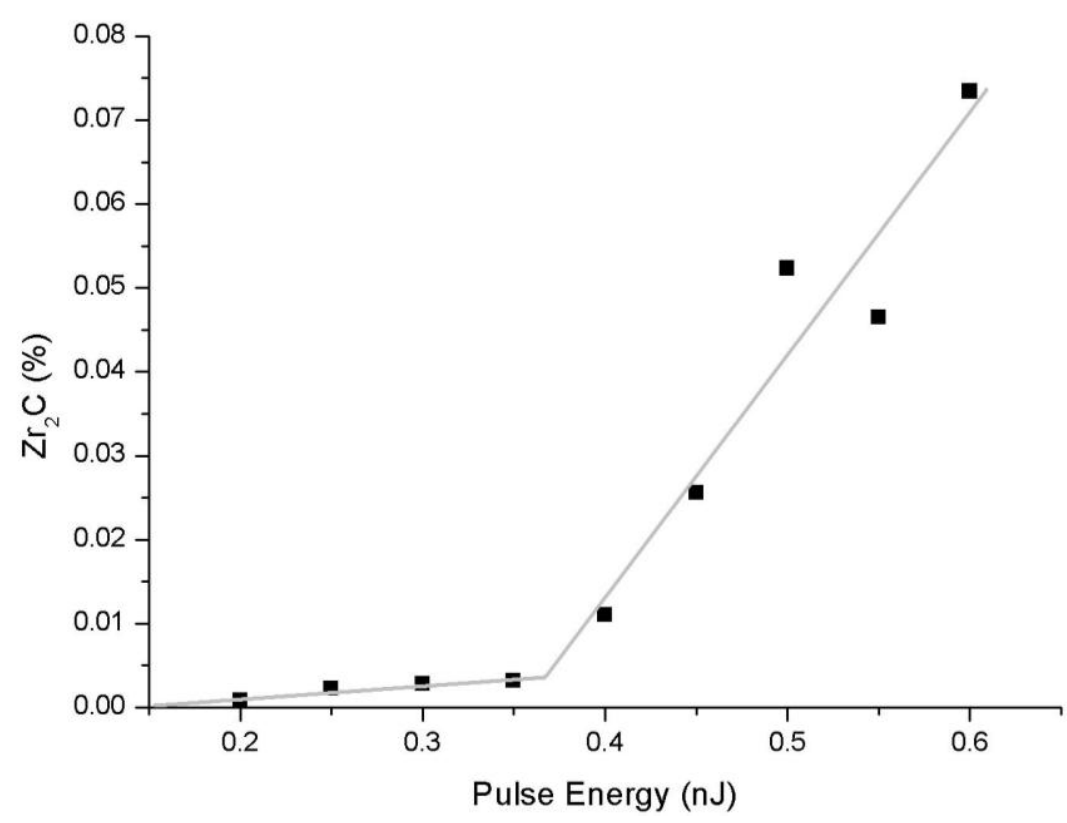

Figure 64: Apparent level of $\mathrm{Zr}_{2} \mathrm{C}$ (in at.\%) as a function of laser energy.

$\mathrm{Zr}_{2} \mathrm{O}_{2}$ was not detected at any of the laser intensities used in this calibration experiment. This ionic species has only been seen in large ( $>10 \mathrm{~m}$ ion) high laser energy $(0.6 \mathrm{~nJ})$ datasets.

\subsubsection{Summary}

A $0.35 \mathrm{~nJ}$ laser pulse is a suitable energy for analysing a $\sim 70 \mathrm{~nm}$ radius zirconium alloy specimen at a base temperature of $55 \mathrm{~K}$. At $0.2 \%$ evaporation rate this will ensure that the correct proportion of tin and oxygen will be detected, many complex ion species will be avoided and the niobium, titanium and iron concentration will be analysed in a regime that is insensitive to variation in the field conditions. This laser energy also provides a pulse of sufficient magnitude that DC evaporation of species between pulses is avoided. 


\section{Examination of 'as-received' alloys}

\subsection{Alloy matrix chemistry}

\subsubsection{Overview}

\subsubsection{Introduction}

The alloy matrix chemistry of all of these materials is very poorly described in the existing literature. The data that is available is often inconsistent or imprecise. Atom probe analysis of metal matrix material has been conducted to provide a baseline with which to compare materials sporting various levels of corrosion, as well as to examine the fundamental chemical properties of the alloy matrix.

There are two issues of interest regarding the behaviour of solute in the alloy matrices. These are the absolute level of solute in solution, and the variation of this level as a function of distance. This second point is a multi-length-scale problem, in that locally the distribution may be homogeneous, but the solute level can vary between local concentration measurements made at random macroscopic distances. The modern generation of $3 \mathrm{D}$ atom probe, after the considerations outlined in sections 4.5 and 4.6 , can be used to determine both of these parameters. The main limitation that the instrument places on this description is the amount of good quality information that can be obtained over a sensible timescale.

The rest of this overview contains tabulated data detailing the concentration of solute in the metal matrix found within a number of atom probe specimens. This gives both the mean concentration of solute and a description of its variation over a limited number of points in the 
material (section 5.1.1.2). Analysis of the distribution of solute on a macroscopic scale, the variation over each dataset has been characterised and compared to that which would be expected of a random solid solution (see section 5.1.1.3). The remainder of selection 5.1 discusses these results for the individual solutes.

\subsubsection{Matrix solute concentration}

ZIRLO

A large number of ZIRLO specimens were analysed in order to characterise the elemental distribution of alloying element additions in as-received material. Of the $\sim 50$ atom probe specimens studied in this condition, 19 of these yielded useful data for this purpose using the analysis conditions selected in section 4.6.9. The compositional information derived from these specimens is summarised in Table 23. Several of the analysed volumes reported in Table 23 contain large amounts of hydrogen, and the majority of samples contain $\sim 10$ at.\% hydrogen. This is likely due to the adsorption of gas from the vacuum system. These data are more sensibly interpreted by comparing the concentration of the major alloying additions $(\mathrm{Nb}, \mathrm{Sn}, \mathrm{O})$ without this additional hydrogen, as summarised in the columns of Table 23. 


\begin{tabular}{|c|c|c|c|c|c|c|c|c|c|c|}
\hline \multirow{2}{*}{$\begin{array}{l}\text { Dataset } \\
\text { size } \\
\text { (10 at.) }\end{array}$} & \multicolumn{2}{|c|}{ Zirconium } & \multicolumn{2}{|c|}{ Niobium } & \multicolumn{2}{|c|}{ Tin } & \multicolumn{2}{|c|}{ Oxygen } & \multicolumn{2}{|c|}{$\begin{array}{c}\text { Gross } \\
\text { Hydrogen }\end{array}$} \\
\hline & $\begin{array}{l}\text { Conc. } \\
\text { (at.\%) }\end{array}$ & $\begin{array}{l} \pm 1 \sigma \\
\text { (at.\%) }\end{array}$ & $\begin{array}{l}\text { Conc. } \\
\text { (at.\%) }\end{array}$ & $\begin{array}{l}1 \text { s.d. } \\
\text { (at.\%) }\end{array}$ & $\begin{array}{l}\text { Conc. } \\
\text { (at.\%) }\end{array}$ & $\begin{array}{l}\text { 1 s.d. } \\
\text { (at.\%) }\end{array}$ & $\begin{array}{l}\text { Conc. } \\
\text { (at.\%) }\end{array}$ & $\begin{array}{l}\text { 1 s.d. } \\
\text { (at.\%) }\end{array}$ & $\begin{array}{l}\text { Conc. } \\
\text { (at.\%) }\end{array}$ & $\begin{array}{l}1 \text { s.d. } \\
\text { (at.\%) }\end{array}$ \\
\hline 0.84 & 98.3 & 0.014 & 0.39 & 0.04 & 0.71 & 0.01 & 0.6 & 0.01 & 24.37 & 0.05 \\
\hline 6.02 & 97.39 & 0.006 & 0.42 & 0.01 & 6 & 0.01 & 1.59 & 0.01 & 8.49 & 0.01 \\
\hline 1.07 & 96.75 & 0.017 & 0.29 & 0.04 & 0.65 & 0.01 & 2.31 & 0.01 & 31.06 & 0.04 \\
\hline 0.65 & 97.32 & 0.020 & 0.48 & 0.05 & 0.38 & 0.01 & 1.82 & 0.02 & 44.41 & 0.06 \\
\hline 2.08 & 97.51 & 0.011 & 0.33 & 0.02 & 0.71 & 0.01 & 1.46 & 0.01 & 12.53 & 0.02 \\
\hline 0.34 & 97.68 & 0.026 & 0.29 & 0.01 & 0.58 & 0.01 & 1.46 & 0.02 & 11.21 & 0.05 \\
\hline 0.16 & 97.08 & 0.042 & 0.36 & 0.11 & 0.69 & 0.02 & 1.86 & 0.03 & 44.4 & 0.1 \\
\hline 0.28 & 97.1 & 0.032 & 0.41 & 0.06 & 0.76 & 0.02 & 1.74 & 0.02 & 3.46 & 0.03 \\
\hline 0.08 & 95.21 & 0.076 & 0.22 & 0.08 & 0.67 & 0.03 & 3.9 & 0.07 & 14.8 & 0.1 \\
\hline 2.04 & 97.93 & 0.010 & 0.43 & 0.01 & 0.78 & 0.01 & 0.86 & 0.01 & 2.04 & 0.01 \\
\hline 0.55 & 97.98 & 0.019 & 0.38 & 0.02 & 0.66 & 0.01 & 0.98 & 0.01 & 7.08 & 0.03 \\
\hline 0.86 & 96.9 & 0.019 & 0.38 & 0.02 & 0.74 & 0.01 & 1.97 & 0.02 & 13.04 & 0.04 \\
\hline 1.02 & 98.13 & 0.013 & 0.43 & 0.03 & 0.68 & 0.01 & 0.76 & 0.01 & 19.65 & 0.04 \\
\hline 0.72 & 98.24 & 0.015 & 0.27 & 0.01 & 0.65 & 0.01 & 0.84 & 0.01 & 2.57 & 0.02 \\
\hline 45.94 & 97.52 & 0.002 & 0.41 & 0.01 & 0.85 & 0.01 & 1.22 & 0 & 10.51 & 0.01 \\
\hline 1.91 & 97.63 & 0.011 & 0.34 & 0.01 & 0.78 & 0.01 & 1.24 & 0.01 & 7.69 & 0.02 \\
\hline 2.13 & 97.72 & 0.010 & 0.33 & 0.01 & 0.74 & 0.01 & 1.21 & 0.01 & 10.2 & 0.02 \\
\hline 6.1 & 97.98 & 0.006 & 0.3 & 0.01 & 0.75 & 0.01 & 0.98 & 0.01 & 11.74 & 0.01 \\
\hline 1.99 & 93.5 & 0.017 & 0.76 & 0.01 & 0.66 & 0.01 & 5.08 & 0.02 & 1.52 & 0.01 \\
\hline \multicolumn{3}{|c|}{ Unweighted mean value } & 0.36 & 0.06 & 0.72 & 0.08 & 1.30 & 0.36 & & \\
\hline
\end{tabular}

Table 23: Compositional information regarding sub-volumes (devoid of any surface contaminants or surface oxides) of several (19) ZIRLO 3DAP specimens. The light grey highlighted regions show the composition of specimens with low hydrogen and 'normal' oxygen content. The summary at the bottom of the table contains a simple (not weighted) arithmetic mean and standard deviation of the highlighted data. 
The issues involved with the chemical analysis of Zircaloy-4 alloy matrix using 3DAP are the same as those involved with the analysis of ZIRLO, albeit without the complications associated with the measurement of niobium concentration. The local chemical compositions of four Zircaloy-4 specimens containing regions of alloy matrix are given in Table 24 . The size of the datasets (in terms of atoms contained) varies greatly between the examples shown and this is not accounted for in the simple mean value. This value considers the individual samples as separate zero-dimensional points of fixed composition, the standard deviation of which gives an estimate of the variation over position within the material.

\begin{tabular}{|c|cc|cc|cc|}
\hline Dataset size & \multicolumn{2}{|c|}{ Tin } & \multicolumn{2}{c|}{ Oxygen } & \multicolumn{2}{c|}{ Iron } \\
& Conc. & $\pm 1 \sigma$ & Conc. & $\pm 1 \sigma$ & Conc. & $\pm 1 \sigma$ \\
$\left(10^{6}\right.$ atoms $)$ & $($ at. $\%)$ & $($ at. $\%)$ & $($ at. $\%)$ & $($ at. $\%)$ & (at.\%) & $($ at. $\%)$ \\
\hline 0.53 & 0.94 & 0.02 & 0.78 & 0.01 & 0.0035 & 0.0010 \\
0.37 & 1.03 & 0.02 & 0.99 & 0.02 & 0.004 & 0.002 \\
5.00 & 1.08 & 0.01 & 0.732 & 0.004 & 0.0038 & 0.0005 \\
5.02 & 1.02 & 0.01 & 0.829 & 0.004 & 0.0035 & 0.0006 \\
\hline Mean value & 1.02 & 0.06 & 0.83 & 0.11 & 0.0037 & 0.0004 \\
\hline
\end{tabular}

Table 24: Chemical composition of four regions of the alloy matrix of as-received Zircaloy-4. Note that regions 3 and 4 contain an order of magnitude more atoms than regions 1 and 2. The mean value gives equal weighting to all of the data points irrespective of their size.

$\mathrm{Zr}-\mathrm{Nb}-\mathrm{Ti}$

Several uncorroded $\mathrm{Zr}-\mathrm{Nb}$-Ti alloy specimens were analysed using 3DAP. The concentration of the major alloying additions $\mathrm{Ti}, \mathrm{Nb}$ and $\mathrm{O}$ are given for four specimens in Table 25 . The mean value given is calculated in the same way as that shown for Zircaloy-4 and ZIRLO. 


\begin{tabular}{|c|cc|cc|cc|}
\hline Dataset size & \multicolumn{2}{|c|}{ Tin } & \multicolumn{2}{c|}{ Niobium } & \multicolumn{2}{c|}{ Oxygen } \\
& Conc. & $\pm 1 \sigma$ & Conc. & $\pm 1 \sigma$ & Conc. & $\pm 1 \sigma$ \\
$\left(10^{6}\right.$ atoms $)$ & (at.\%) & (at.\%) & (at.\%) & (at.\%) & (at.\%) & (at.\%) \\
\hline 9.95 & 0.339 & 0.002 & 0.453 & 0.002 & 0.792 & 0.003 \\
1.35 & 0.34 & 0.01 & 0.378 & 0.002 & 0.971 & 0.008 \\
0.20 & 0.35 & 0.01 & 0.420 & 0.005 & 1.09 & 0.02 \\
6.65 & 0.343 & 0.002 & 0.463 & 0.001 & 1.024 & 0.004 \\
\hline Mean value & 0.343 & 0.005 & 0.43 & 0.04 & 0.97 & 0.12 \\
\hline
\end{tabular}

Table 25: Concentration of major alloying additions within the metal matrix of Zr-Nb-Ti in four specimens. The uncertainty given is 1 standard deviation based on the counting statistics of all of the ranges used to create a given concentration value.

\subsubsection{Matrix solute distribution}

As well as the variation in concentration taken at random points over a macroscopic distance, the nanoscopic variation of solute concentration within a sub-set of these samples has been measured. For each alloy, several datasets were inspected to remove any surface oxide and divided into 250 ion blocks. A histogram of the number of blocks containing a given level of solute was produced, and this distribution compared to a series of binomial frequency distributions with the same mean as the individual solutes. Conventional $\chi^{2}$ statistics and significance testing was performed on the correlation between the experimentally determined data and the theoretical distributions.

The $\chi^{2}$ value, with $v$ degrees of freedom, has been compared with statistical tables [131] to give a $\mathrm{p}$-value, the probability that the $\chi^{2}$ value obtained by randomly sampling $\mathrm{N}$ observations from a Gaussian distribution is larger than the value that would be obtained by fitting the experimental data with the binomial frequency distribution. If the p-value is low, less than 0.01 , then we must reject the hypothesis that the distribution is that of a random solid solution. The converse, that a 
high p-value proves the distribution is a random solid solution, is not true, but can be used to support that assertion while considering this limitation.

The Pearson coefficient, $\mu$, is a better metric than $\chi^{2}$ with which to compare the correlation of different solutes between specimens with different volumes. This is because $\chi^{2}$ scales with the number of ions in the sample. The Pearson co-efficient aims to normalise the $\chi^{2}$ coefficient for the number of ions [166]. This value can range between 0 and 1 . A value of 0 being a perfect correlation between the observed distribution and a random solid solution. A value greater than 0.05 indicates that the distribution deviates greatly from that of a random solid solution.

Table 26, Table 27 and Table 28 give the parameters that describe the correlation between these distributions and those of random solid solutions for ZIRLO, Zircaloy-4 and Zr-Nb-Ti alloys, respectively. All of the data available is presented for Zr-Nb-Ti and Zircaloy-4. The four ZIRLO samples chosen (row 2, 5, 10, 18 of Table 23) contain $2-7$ million ions. 


\begin{tabular}{|c|c|c|c|c|}
\hline Element & $\begin{array}{c}\text { Reduced } \\
x^{2}\end{array}$ & $\mathrm{~N}$ & $p$-value & $\mu$ \\
\hline \multirow[t]{4}{*}{$\mathrm{O}$} & 0.016 & 2 & 0.9844 & 0.0016 \\
\hline & 0.037 & 1 & 0.8481 & 0.0024 \\
\hline & 0.007 & 1 & 0.9327 & 0.001 \\
\hline & 0.001 & 1 & 0.9696 & 0.0005 \\
\hline \multirow[t]{4}{*}{$\mathrm{ZrO}$} & 0.274 & 8 & 0.9746 & 0.0132 \\
\hline & 18.125 & 8 & $<0.0001$ & 0.1504 \\
\hline & 40.431 & 8 & $<0.0001$ & 0.2023 \\
\hline & 10.213 & 9 & $<0.0001$ & 0.1235 \\
\hline \multirow{4}{*}{$\begin{array}{c}\mathrm{Nb}^{2+}+ \\
\mathrm{ZrH}^{2+}\end{array}$} & 79.706 & 15 & $<0.0001$ & 0.1954 \\
\hline & 2.678 & 6 & 0.0134 & 0.0506 \\
\hline & 27.062 & 9 & $<0.0001$ & 0.1628 \\
\hline & 216.26 & 11 & $<0.0001$ & 0.535 \\
\hline \multirow[t]{4}{*}{$\mathrm{ZrH}^{2+}$} & 100.92 & 14 & $<0.0001$ & 0.2116 \\
\hline & 16.018 & 4 & $<0.0001$ & 0.1006 \\
\hline & 27.062 & 9 & $<0.0001$ & 0.1765 \\
\hline & 179.42 & 9 & $<0.0001$ & 0.4626 \\
\hline \multirow[t]{4}{*}{$\mathrm{Nb}^{3+}$} & 0.068 & 2 & 0.934 & 0.0063 \\
\hline & 0.465 & 2 & 0.6284 & 0.0122 \\
\hline & 0.061 & 2 & 0.941 & 0.004 \\
\hline & 0.016 & 1 & 0.8995 & 0.0016 \\
\hline \multirow[t]{4}{*}{ Sn } & 78.996 & 6 & $<0.0001$ & 0.1245 \\
\hline & 1.168 & 5 & 0.322 & 0.0305 \\
\hline & 9.919 & 5 & $<0.0001$ & 0.0806 \\
\hline & 11.783 & 4 & $<0.0001$ & 0.0888 \\
\hline \multirow[t]{4}{*}{ C } & 0.013 & 2 & 0.9873 & 0.0014 \\
\hline & 0.057 & 1 & 0.8116 & 0.003 \\
\hline & 0.005 & 1 & 0.9435 & 0.0008 \\
\hline & 0.071 & 1 & 0.7894 & 0.0035 \\
\hline \multirow[t]{4}{*}{$\mathrm{Fe}$} & 0.175 & 2 & 0.8399 & 0.0053 \\
\hline & 0.006 & 1 & 0.938 & 0.001 \\
\hline & 0.004 & 1 & 0.9491 & 0.0007 \\
\hline & 0.005 & 1 & 0.9438 & 0.0009 \\
\hline $\mathrm{Cr}$ & \multicolumn{4}{|c|}{ None present } \\
\hline $\mathrm{Ni}$ & \multicolumn{4}{|c|}{ None present } \\
\hline
\end{tabular}

Table 26: Statistics relating to the agreement between the distribution of solute in ZIRLO and that expected for a random solid solution. The p-value is the probability level for the hypothesis that the distribution is random. $\mu$ is derived from $X^{2}$, but reduces the dependence on the dataset size, which hampers the comparison of samples using $X^{2}$. 


\begin{tabular}{|c|cccc|}
\hline Element & $\begin{array}{c}\text { Reduced } \\
\mathrm{X}^{2}\end{array}$ & $\mathrm{~V}$ & $\mathrm{p}$-value & $\mu$ \\
\hline $\mathrm{O}$ & 0.141 & 1 & 0.7077 & 0.0127 \\
& 0.236 & 1 & 0.627 & 0.0127 \\
& 0.153 & 1 & 0.6955 & 0.0036 \\
& 0.006 & 1 & 0.9363 & 0.0009 \\
\hline ZrO & 3.997 & 7 & 0.0002 & 0.1764 \\
& 6.624 & 7 & $<0.0001$ & 0.1757 \\
& 0.752 & 7 & 0.6276 & 0.0211 \\
& 3.815 & 7 & 0.0004 & 0.0609 \\
\hline $\mathrm{Sn}$ & 5.038 & 5 & $<0.0001$ & 0.1676 \\
& 6.637 & 6 & $<0.0001$ & 0.1623 \\
& 122.527 & 7 & $<0.0001$ & 0.26 \\
& 7.531 & 7 & $<0.0001$ & 0.0853 \\
\hline $\mathrm{C}$ & 0.078 & 1 & 0.0095 & 0.78 \\
& 0.002 & 1 & 0.0013 & 0.9617 \\
& 0.113 & 1 & 0.0031 & 0.7365 \\
& 0.014 & 1 & 0.0006 & 0.9064 \\
\hline $\mathrm{Fe}$ & 0.052 & 1 & 0.962 & 0.0016 \\
& 0.005 & 1 & 0.9781 & 0.0019 \\
& 0.018 & 1 & 0.8932 & 0.0012 \\
& 0.002 & 1 & 0.9638 & 0.0005 \\
\hline $\mathrm{Cr}$ & None present \\
\hline $\mathrm{Ni}$ & No analysable quantity \\
\hline
\end{tabular}

Table 27: Statistics relating to the agreement between the distribution of solute in Zircaloy-4 and that expected for a random solid solution. The order of the examples is the same as the order of samples in Table 24. The p-value is the probability level for the hypothesis that the distribution is random. $\mu$ is derived from $X^{2}$, but reduces the dependence on the dataset size, which hampers the comparison of samples using $X^{2}$. 


\begin{tabular}{|c|c|c|c|c|}
\hline Element & $\begin{array}{c}\text { Reduced } \\
x^{2}\end{array}$ & v & $p$-value & $M$ \\
\hline \multirow[t]{4}{*}{ O } & 0.504 & 2 & 0.6044 & 0.0045 \\
\hline & 1.026 & 2 & 0.3586 & 0.0086 \\
\hline & 0.028 & 1 & 0.8675 & 0.0058 \\
\hline & 0 & 1 & 0.9963 & 0.0001 \\
\hline \multirow[t]{4}{*}{$\mathrm{ZrO}$} & 2.339 & 8 & 0.0165 & 0.0194 \\
\hline & 37.702 & 9 & 0.0007 & 0.11 \\
\hline & 4.004 & 6 & 0.0005 & 0.1666 \\
\hline & 84.061 & 8 & $<0.0001$ & 0.4895 \\
\hline \multirow{4}{*}{$\begin{array}{l}\mathrm{Nb}^{2+}+ \\
\mathrm{ZrH}^{2+}\end{array}$} & 337.608 & 21 & $<0.0001$ & 0.3559 \\
\hline & 171.494 & 20 & $<0.0001$ & 0.3318 \\
\hline & 1.436 & 16 & 0.1144 & 0.1731 \\
\hline & 87.475 & 16 & $<0.0001$ & 0.6294 \\
\hline \multirow[t]{4}{*}{$\mathrm{ZrH}^{2+}$} & 386.932 & 20 & $<0.0001$ & 0.3675 \\
\hline & 155.602 & 17 & $<0.0001$ & 0.2951 \\
\hline & 0.905 & 16 & 0.5626 & 0.1301 \\
\hline & 81.462 & 14 & $<0.0001$ & 0.5902 \\
\hline \multirow{4}{*}{$\mathrm{Nb}^{3+}$} & 1.873 & 2 & 0.1536 & 0.0088 \\
\hline & 0.263 & 2 & 0.7688 & 0.0044 \\
\hline & 0.011 & 1 & 0.9166 & 0.0038 \\
\hline & 0.044 & 1 & 0.8335 & 0.0046 \\
\hline \multirow[t]{4}{*}{$\mathrm{Ti}$} & 0.477 & 6 & 0.8258 & 0.0076 \\
\hline & 1.436 & 9 & 0.1553 & 0.0740 \\
\hline & 0.519 & 3 & 0.6695 & 0.043 \\
\hline & 1.055 & 3 & 0.3669 & 0.0385 \\
\hline \multirow[t]{4}{*}{ C } & 0.381 & 2 & 0.6831 & 0.0039 \\
\hline & $<0.001$ & 1 & 0.9881 & 0.0001 \\
\hline & 0.002 & 1 & 0.9607 & 0.0017 \\
\hline & 0.065 & 1 & 0.7985 & 0.0055 \\
\hline \multirow[t]{4}{*}{$\mathrm{Fe}$} & 2.807 & 3 & 0.038 & 0.013 \\
\hline & $<0.001$ & 1 & 0.9953 & $<0.0001$ \\
\hline & 0.012 & 1 & 0.9133 & 0.0038 \\
\hline & 0.007 & 1 & 0.9357 & 0.0017 \\
\hline \multirow[t]{4}{*}{$\mathrm{Cr}$} & 0.002 & 1 & 0.9678 & 0.0002 \\
\hline & $<0.001$ & 1 & 0.9925 & 0.0001 \\
\hline & - & - & - & - \\
\hline & & & & \\
\hline \multirow[t]{4}{*}{$\mathrm{Ni}$} & $<0.001$ & 1 & 0.9925 & 0 \\
\hline & 0.007 & 1 & 0.9331 & 0.0001 \\
\hline & $<0.001$ & 1 & 0.9955 & 0.0001 \\
\hline & - & - & - & - \\
\hline
\end{tabular}

Table 28: Statistics relating to the agreement between the distribution of solute in $\mathrm{Zr}-\mathrm{Nb}$-Ti and that expected for a random solid solution. The order of the examples is the same as the order of samples in Table 25. The pvalue is the probability level as described for Table 27. 


\subsubsection{Oxygen}

\section{Macroscopic variation}

Studies of second phase particles have shown that they occupy less that $1 \%$ volume fraction and contain little, if any, oxygen (see section 2.4.2). The level of oxygen present in the bulk material would be expected to be similar to that found in solid solution within the alloy matrix. Table 29 compares the mean of the datapoints given in Table 23 (ZIRLO), Table 24 (Zry-4) and Table 25 ( $\mathrm{Zr}-\mathrm{Nb}-\mathrm{Ti})$. In all cases care was taken not to include regions of native surface oxide in the analysis of the specimen. The bulk level of oxygen introduced in the processing of the $\mathrm{Zr}-\mathrm{Nb}-\mathrm{Ti}$ test alloy is not known, but it would be expected that it would be similar to that seen in ZIRLO, as the same processing route was employed in the manufacture of this test ingot.

ZIRLO and Zircaloy-4 alloy matrix oxygen levels both agree within one standard deviation with the expected composition (the bulk composition). The level of oxygen in the metal matrix of the $\mathrm{Zr}-\mathrm{Nb}$-Ti alloy is similar to that seen in ZIRLO. The local oxygen concentration in all three of the uncorroded materials is seen to vary with position. There is no discernable difference between the variation in oxygen concentration in the uncorroded matrix of the alloys that exhibit good corrosion resistance (ZIRLO, Zircaloy-4) and Zr-Nb-Ti.

\begin{tabular}{|c|c|c|c|c|}
\hline \multirow[t]{2}{*}{ Alloy } & \multirow{2}{*}{$\begin{array}{l}\text { Bulk Oxygen } \\
\text { content }\end{array}$} & \multicolumn{2}{|c|}{ Oxygen } & Number of \\
\hline & & $\begin{array}{l}\text { Conc. } \\
\text { (at.\%) }\end{array}$ & $\begin{array}{c} \pm 1 \sigma \\
\text { (at.\%) }\end{array}$ & observations \\
\hline ZIRLO & 1.10 & 1.30 & 0.36 & 12 \\
\hline Zry-4 & 0.74 & 0.83 & 0.11 & 4 \\
\hline $\mathrm{Zr}-\mathrm{Nb}-\mathrm{Ti}$ & NR & 0.97 & 0.12 & 4 \\
\hline
\end{tabular}

Table 29: Comparison between the expected level of oxygen in solution and the mean level seen over a number of experimentally obtained 3DAP specimens. The concentration given is the arithmetic mean of the atom probe analyses in Table 23, Table 24 and Table 25. 


\section{Nanoscopic variation}

The nanoscopic distribution of solute within each sample was described in Table 26, Table 27 and Table 28, and the information regarding oxygen-containing ions is reproduced below in Table 30 and Table 31.

\begin{tabular}{|c|cccc|}
\hline \multicolumn{5}{|c|}{ Distribution of O ions within the alloy matrix } \\
\hline Alloy & Reduced $x^{2}$ & $\mathrm{v}$ & $\mathrm{p}$-value & $\mu$ \\
\hline ZIRLO & 0.016 & 2 & 0.9844 & 0.0016 \\
& 0.037 & 1 & 0.8481 & 0.0024 \\
& 0.007 & 1 & 0.9327 & 0.001 \\
& 0.001 & 1 & 0.9696 & 0.0005 \\
\hline Zircaloy-4 & 0.141 & 1 & 0.7077 & 0.0127 \\
& 0.236 & 1 & 0.627 & 0.0127 \\
& 0.153 & 1 & 0.6955 & 0.0036 \\
& 0.006 & 1 & 0.9363 & 0.0009 \\
\hline Zr-Nb-Ti & 0.504 & 2 & 0.6044 & 0.0045 \\
& 1.026 & 2 & 0.3586 & 0.0086 \\
& 0.028 & 1 & 0.8675 & 0.0058 \\
& 0 & 1 & 0.9963 & 0.0001 \\
\hline
\end{tabular}

Table 30: The quality of fit between experimentally observed distributions of $O$ ions in ZIRLO, Zircaloy-4 and Zr-Nb-Ti with those expected for a random solid solution. Data summarised from Table 26, Table 27 and Table 28. 


\begin{tabular}{|c|cccc|}
\hline \multicolumn{5}{|c|}{ Distribution of ZrO ions within the alloy matrix } \\
\hline Alloy & Reduced $\mathrm{X}^{2}$ & $\mathrm{v}$ & $\mathrm{p}$-value & $\mu$ \\
\hline ZIRLO & 0.274 & 8 & 0.9746 & 0.0132 \\
& 18.125 & 8 & $<0.0001$ & 0.1504 \\
& 40.431 & 8 & $<0.0001$ & 0.2023 \\
& 10.213 & 9 & $<0.0001$ & 0.1235 \\
\hline Zircaloy-4 & 3.997 & 7 & 0.0002 & 0.1764 \\
& 6.624 & 7 & $<0.0001$ & 0.1757 \\
& 0.752 & 7 & 0.6276 & 0.0211 \\
& 3.815 & 7 & 0.0004 & 0.0609 \\
\hline Zr-Nb-Ti & 2.339 & 8 & 0.0165 & 0.0194 \\
& 37.702 & 9 & 0.0007 & 0.11 \\
& 4.004 & 6 & 0.0005 & 0.1666 \\
& 84.061 & 8 & $<0.0001$ & 0.4895 \\
\hline
\end{tabular}

Table 31: The quality of fit between experimentally observed distributions of $\mathrm{ZrO}$ ions in ZIRLO,

Zircaloy-4 and $\mathrm{Zr}-\mathrm{Nb}$-Ti with those expected for a random solid solution. Data summarised from Table 26, Table 27 and Table 28.
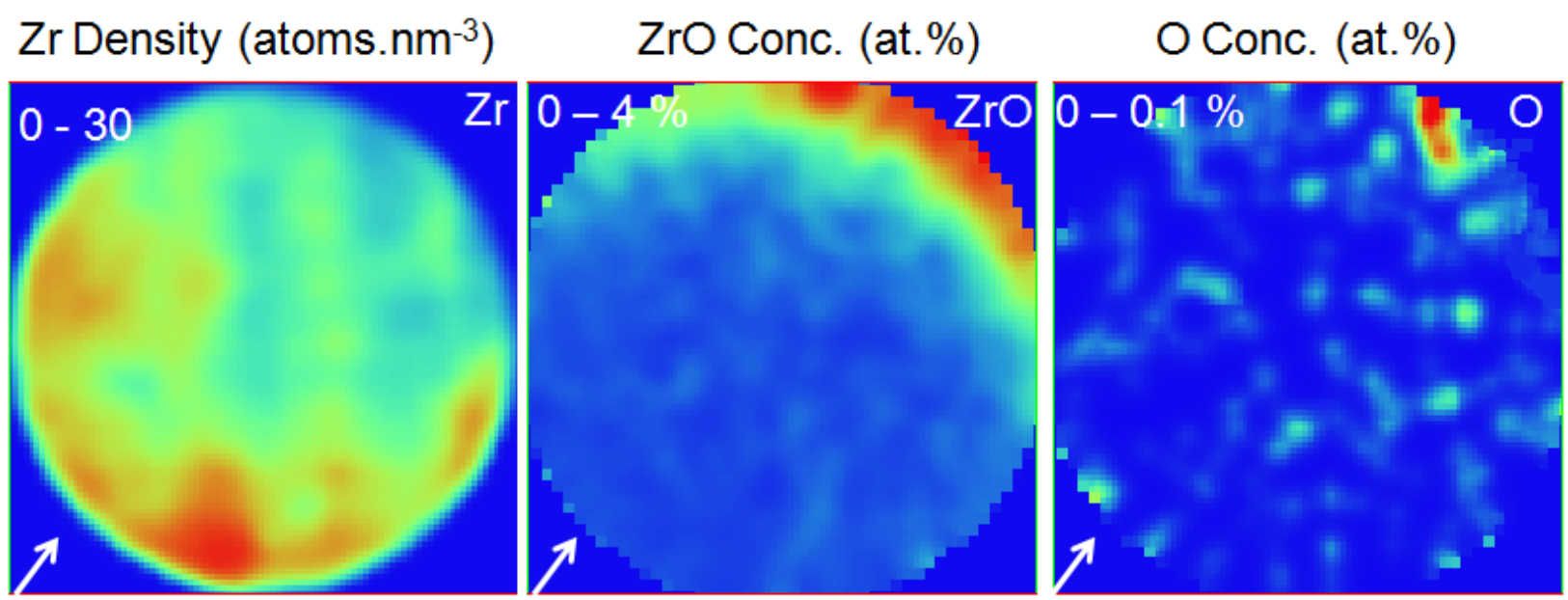

Laser incident direction

Figure 65: 2D density and concentration profiles showing the effect of the laser incidence position on the observed local concentration of $\mathrm{ZrO}$ ions and the distribution of $O$ ions. 


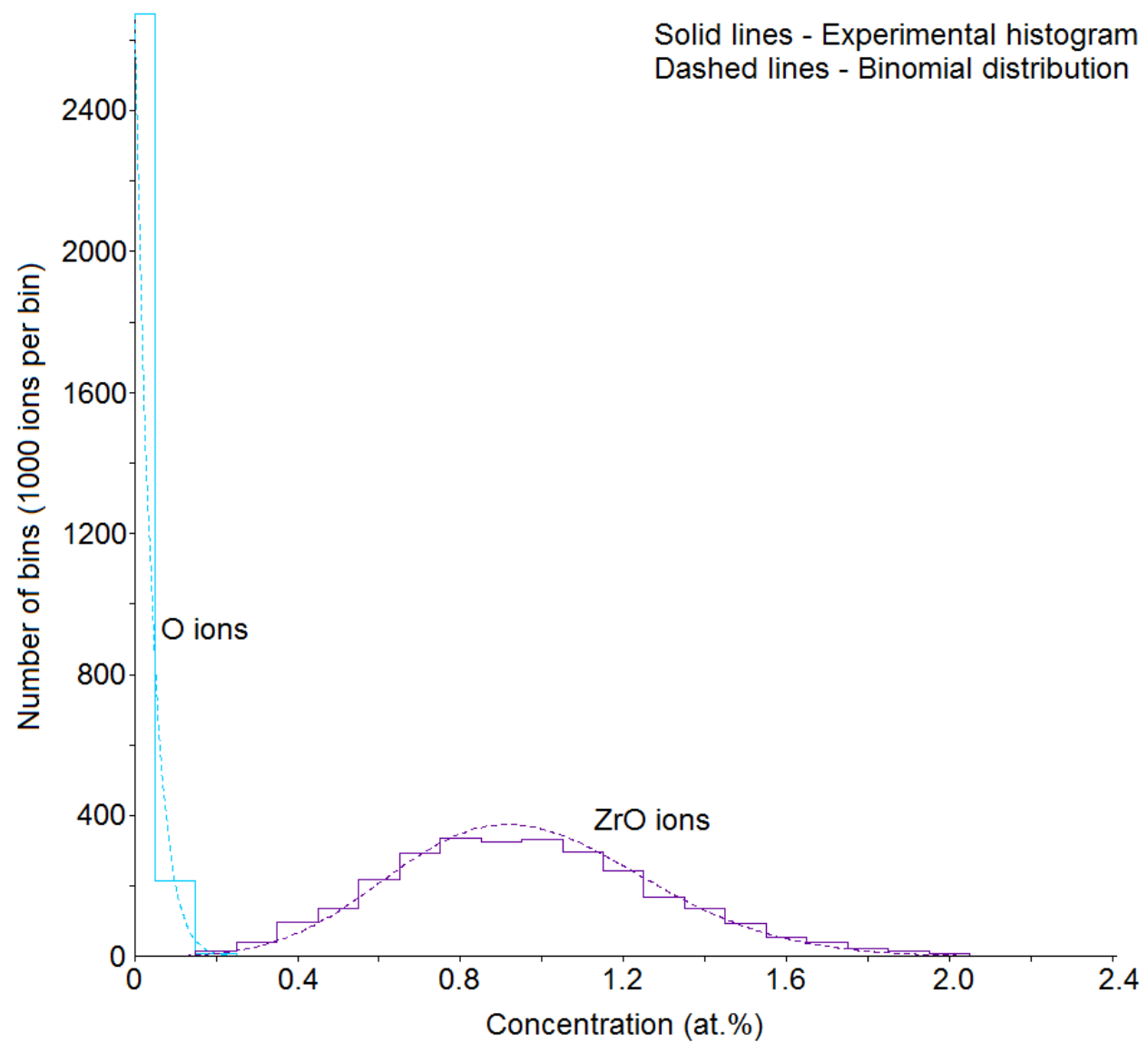

Figure 66: Comparison of binomial distributions (which represent the distribution of solute with a random solid solution) and histograms of the number of blocks of 1000 ions with a certain $\mathrm{ZrO}$ and $O$ composition. The region of interest contains 2895 blocks.

The distribution of the two major oxygen containing species, $\mathrm{ZrO}$ and $\mathrm{O}$, are treated separately in order to identify non-random behaviour due to crystallographic, or specimen specific, effects that lead to an altering of the distribution of some ionic species and not others. This prevents such artefacts being interpreted as non-random intrinsic behaviour of a solute species. The distribution of $\mathrm{O}$ ions agrees well with that expected of a random distribution of solute, however the comparison of the distribution of the $\mathrm{ZrO}$ ionic species with a binomial suggests that the distribution is non-random. Figure 65 compares the ionic density of zirconium with 2D profiles of the concentration of $\mathrm{ZrO}$ and $\mathrm{O}$ species for a dataset. The dataset is typical of many of those studied in that a sub-oxide $\mathrm{ZrO}$ layer is apparent on an outer face of the field of view. This 
region is excluded from the analysed region of interest, but this exclusion may not fully remove a slight gradient in the $\mathrm{ZrO}$ profile running into the material. This variation in this concentration gradient is of the order of 0.1 at.\% and although it does not affect the qualitative distribution of $\mathrm{ZrO}$ species, presented in the histogram Figure 66, it causes the residuals between the distributions to increase and for the correlation to be rejected below the significance threshold, as seen in Table 31. The distribution of $\mathrm{O}$ ions appears to be less dependent on the native oxide (see Figure 65). As such, the distribution of $\mathrm{O}$ ions is not perturbed by this effect, and agrees well with that expected of a random solid solution, as is seen for all the alloys listed in Table 30 .

\subsubsection{Tin}

\section{Macroscopic variation}

The tin concentration within the ZIRLO and Zircaloy- 4 datasets in Table 23 and Table 24 vary considerably between samples. Although the simple mean of each set of datapoints from these tables does yield tin concentrations that agree within 1 standard deviation with the expected value, as summarised below in Table 32, the individual points do not all agree within this uncertainty with the expected composition. This would normally suggest that tin is nonrandomly distributed on a macroscopic scale. However, the reason for the manner in which tin is apparently distributed is that tin is seen to undergo surface diffusion on atom probe specimens; Figure 67 compares the atomic density reconstructed across the plane of the detector for all atoms (a) and tin atoms (b) within one of the ZIRLO specimens. Tin 'hotspots' are present at crystallographic orientations where the overall evaporation rate is low. 


\begin{tabular}{|c|c|cc|c|}
\hline Alloy & $\begin{array}{c}\text { Bulk tin } \\
\text { content }\end{array}$ & $\begin{array}{c}\text { Tonc. } \\
\text { (at.\%) }\end{array}$ & $\begin{array}{c}\text { Tin } \\
\text { (at.\%) }\end{array}$ & observations \\
\hline ZIRLO & 0.74 & 0.72 & 0.08 & 12 \\
Zry-4 & 0.98 & 1.02 & 0.06 & 4 \\
\hline
\end{tabular}

Table 32: Comparison between the expected level of tin in solution and the mean level seen over a number of experimentally obtained 3DAP specimens. Data from Table 23 and Table 24.

The crystallography of the specimen, and its orientation with respect to the region of interest, results in a different measured tin concentration depending upon the level of inclusion of tindecorated poles. This effect means that the overall matrix tin concentration from these crystalline materials can be determined, but only by looking at many random orientations and effectively averaging over all of them.

In the preliminary experiments, performed to find the optimal analysis conditions for tin (section4.6.4), it was seen that the measured tin concentration was a function of laser energy. This can now be better understood by comparison of the 2D density profiles shown in Figure 68, where the total reconstructed density and tin density are shown at various laser energies $(0.2,0.4$, .6) $\mathrm{nJ}$. Sections at 0.3 and $0.5 \mathrm{~nJ}$ are similar in appearance to 0.2 and $0.4 \mathrm{~nJ}$, respectively. 


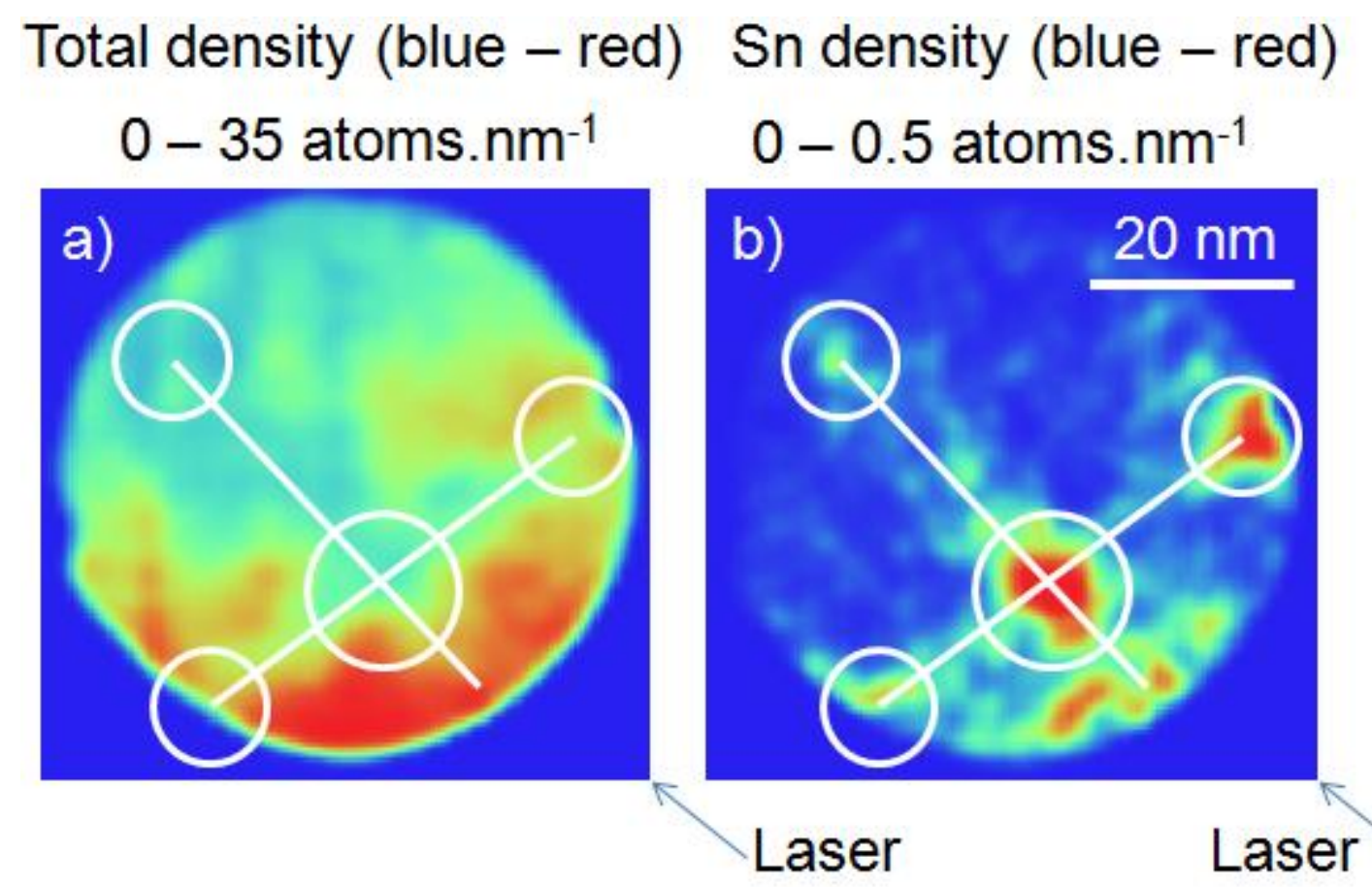

Figure 67: 2D density profiles taken normal to the specimen axis showing a) total reconstruction density between the limits of 0 (blue) to 35 atoms.nm-1 (red) and b) tin atoms reconstructed with the density 0 (blue) to 0.5 atoms. $\mathrm{nm}^{-1}$ (red). Specimen analysed using a laser energy of $0.6 \mathrm{~nJ}$ with a base temperature of $55 \mathrm{~K}$.

The density of zirconium around the central pole decreases as the laser energy is increased. There is segregation of tin to specific crystallographic features at all laser energies. At $0.6 \mathrm{~nJ}$ the laser produces non-uniform heating of the sample and the apparent density of atoms within the specimen is much greater at the area where the laser is incident. The evaporation of tin is also much greater in this region, and this appears to be at the expense of tin evaporation from the poles. 


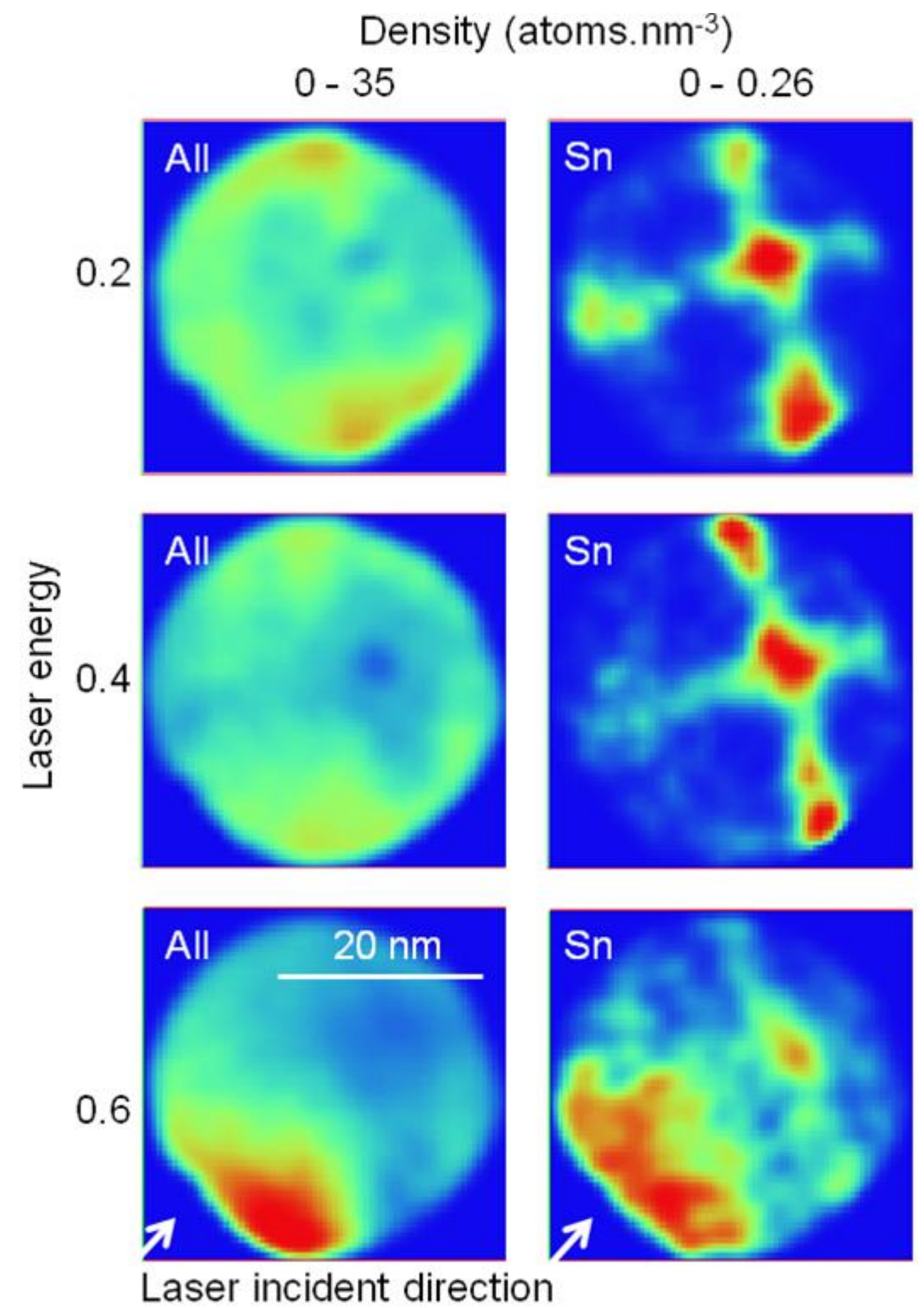

Figure 68: Comparison of the detected atomic density of atoms for all species and tin at various laser energies. Profiles at $0.3 \mathrm{~nJ}$ and $0.5 \mathrm{~nJ}$ are qualitatively similar to $0.2 \mathrm{~nJ}$ and $0.4 \mathrm{~nJ}$. 


\section{Nanoscopic distribution}

Laser pulsed 3DAP is not a suitable tool for studying the local, nanoscopic, distribution of tin because of the artefacts related to field enhance diffusion. Although averaging over many samples yield valid compositional information, the solute distribution is affected by the surface migration problem. This produces the artefact of tin apparently being non-uniformly distributed on a nano-scale.

\begin{tabular}{|c|cccc|}
\hline \multicolumn{5}{|c|}{ Distribution of Sn ions within the alloy matrix } \\
\hline Alloy & Reduced $x^{2}$ & $v$ & $p$-value & $\mu$ \\
\hline ZIRLO & 78.996 & 6 & $<0.0001$ & 0.1245 \\
& 1.168 & 5 & 0.322 & 0.0305 \\
& 9.919 & 5 & $<0.0001$ & 0.0806 \\
& 11.783 & 4 & $<0.0001$ & 0.0888 \\
\hline Zircaloy-4 & 5.038 & 5 & $<0.0001$ & 0.1676 \\
& 6.637 & 6 & $<0.0001$ & 0.1623 \\
& 122.527 & 7 & $<0.0001$ & 0.26 \\
& 7.531 & 7 & $<0.0001$ & 0.0853 \\
\hline Zr-Nb-Ti & \multicolumn{5}{|c}{ Not applicable } \\
\hline
\end{tabular}

Table 33: The quality of fit between experimentally observed distributions of tin in ZIRLO and Zircaloy-4 with those expected for a random solid solution. Data summarised from Table 26 and Table 27.

Table 33 summarises the matrix solute analysis data for ZIRLO and Zircaloy-4, and demonstrates that in almost all cases the hypothesis that tin is distributed as a random solid solution must be rejected using this method because of preferential evaporation from some crystallographic orientations. This artefact may well have influenced the results of Sano and Takeda [130] who observed clustering in high-tin Zr alloys (although not Zircaloy-4) using voltage pulsed 1DAP. The methods employed by these workers also involved aligning their instrument around high evaporation crystallographic orientation to maximise their ion yield. As 
the yield of this species in laser pulsed analysis appears to be dependent on the orientation analysed, this could have the affect of skewing the experimental result. The amount of experimental evidence the conclusions are based upon is unclear, only a single dataset (a few thousand ions) is presented for each case. In order to further assess the likely impact of this form of atom probe analysis on the field enhanced surface migration of tin, the LEAP was used to analyse ZIRLO using voltage pulsing. The effect of segregation to pole is more subtle in data captured using voltage pulsing. Despite this, segregation is still present, as seen in the central spot in Figure 69. Less data are present in this profile than the profiles shown for laser pulse spectra; this has the effect of accentuating other variation that is due to the random distribution of tin within the specimen. Although the spatial distribution of tin is perturbed by the artefacts introduced by the analysis technique, the overall compositional detected by voltage pulsed 3DAP is accurate. This is demonstrated by the good correlation between the measured values of $\mathrm{A}$ and B away from the poles with their respective values produced over the whole dataset.

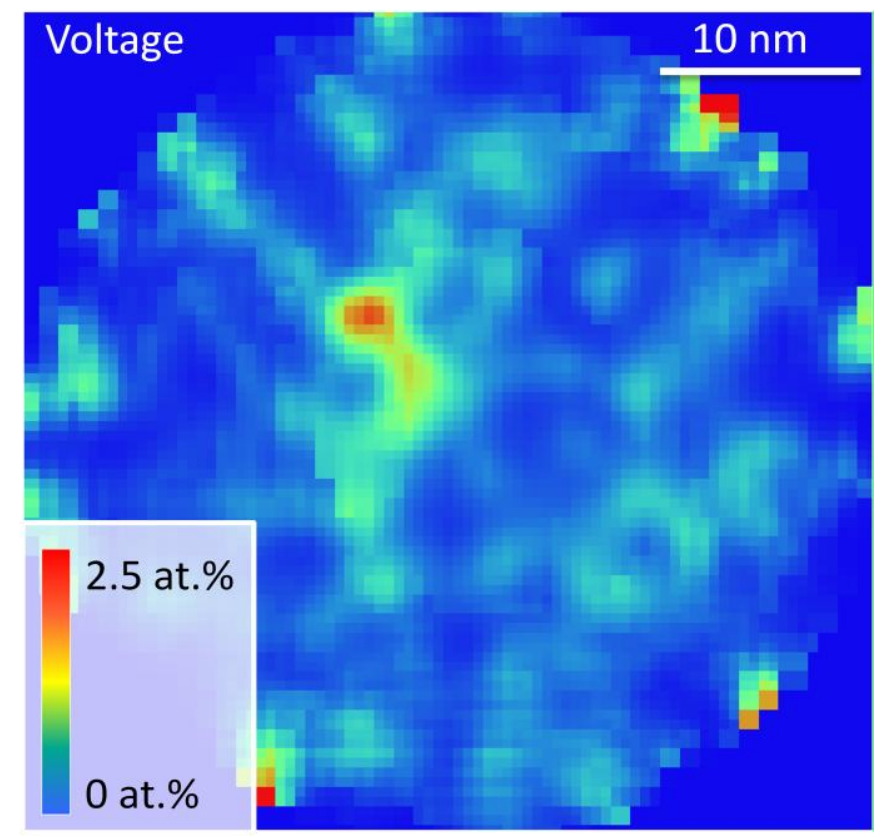

Figure 69: 2D concentration profile of voltage pulsed recrystallised ZIRLO sheet analysed by voltage pulsed LEAP (15\% pulse fraction, $55 \mathrm{~K})$. 


\begin{tabular}{|ccccc|}
\hline $\begin{array}{c}\text { Table } \\
\text { 34Exp. } \\
\text { I.D. }\end{array}$ & $\begin{array}{c}\text { Temp. } \\
(\mathrm{K})\end{array}$ & $\begin{array}{c}\text { Pulse fraction } \\
(\%)\end{array}$ & $\begin{array}{c}\text { Analysis } \\
\text { volume (ions) }\end{array}$ & $\begin{array}{c}\text { Apparent tin } \\
\text { conc. (at.\%) }\end{array}$ \\
\hline \multicolumn{5}{c|}{ Composition away from low-index poles } \\
A & 55 & 18 & 60,000 & $0.77 \pm 0.04$ \\
B & 55 & 15 & 119,000 & $0.63 \pm 0.02$ \\
C & 90 & 15 & 610,000 & $0.77 \pm 0.01$ \\
D & 90 & 15 & 133,000 & $0.73 \pm 0.01$ \\
\hline \multicolumn{5}{c}{ Entire volume including pole regions } \\
A & 55 & 18 & 98,000 & $0.79 \pm 0.03$ \\
B & 55 & 15 & 237,000 & $0.65 \pm 0.02$ \\
\hline
\end{tabular}

Table 34: A comparison of the apparent tin concentration in ZIRLO as found using different voltage pulsed analysis conditions. The expected composition is 0.78 at.\%. Regions taken over the whole specimen and regions away from high-tin concentration poles are compared for samples $A$ and $B$.

\subsubsection{Niobium}

\section{Macroscopic distribution}

The concentration of niobium, and its variation with respect to position, was given in Table 23 for ZIRLO and Table 25 for $\mathrm{Zr}-\mathrm{Nb}$-Ti. These values are summarised here in Table 35 . The level of niobium in solid solution is similar in both of the materials, agreeing within 1 standard deviation from the mean values.

\begin{tabular}{|c|c|cc|c|}
\hline Alloy & Bulk & \multicolumn{2}{|c|}{ Niobium } & Number of \\
niobium & Conc. & $\pm 1 \sigma$ & observations \\
content & (at.\%) & $($ at. \%) & \\
\hline ZIRLO & 0.96 & 0.43 & 0.04 & 12 \\
Zr-Nb-Ti & 0.96 & 0.36 & 0.06 & 4 \\
\hline
\end{tabular}

Table 35: Comparison between the mean niobium concentration seen over a number of experimentally obtained 3DAP specimens of ZIRLO and Zr-Nb-Ti. Data from Table 23 and Table 25 . 
This level of niobium, $\sim 0.4$ at.\%, represents a supersaturated solid solution both at room and reactor temperature, as seen in the $\mathrm{Zr}-\mathrm{Nb}$ phase diagram in section 2.6.2. The expected level in solution at $350{ }^{\circ} \mathrm{C}$ is less than 0.1 at.\%. The $\sim 0.4$ at.\% value does, however, appears sensible, given that the maximum solubility of $\mathrm{Nb}$ in low oxygen concentration $\alpha-\mathrm{Zr}$ is $\sim 0.6$ at. $\%$ and that the $\beta \rightarrow \alpha$ quenching processes employed in the fabrication of this material causes the majority of this niobium to be retained in solution. The limited thermal processing after the last $\beta$-quench, and the sluggish diffusion and precipitation kinetics of zirconium appears to do little to reduce this concentration.

\section{Nanoscopic distribution}

The overlap of $\mathrm{ZrH}^{2+}$ with $\mathrm{Nb}^{2+}$ complicates the analysis of the nanoscopic distribution of niobium as the effect of this species must be accounted for. There are three methods that can be used to distinguish the distribution of niobium from the $\mathrm{ZrH}$ contribution. These are described in Table 36 .

\begin{tabular}{|c|l|l|}
\hline Case & \multicolumn{1}{|c|}{ Method } & \multicolumn{1}{c|}{ Advantage / disadvantage } \\
\hline 1 & $\begin{array}{l}\text { Ignore the presence } \\
\text { of ZrH at the same } \\
\text { position as Nb2+. }\end{array}$ & $\begin{array}{l}\text { The concentration of Nb2+ > } \mathrm{ZrH} 2+(46.5 \mathrm{Da} \\
\text { contains many more counts than 47.5 Da). }\end{array}$ \\
\hline 2 & $\begin{array}{l}\text { Separate dataset into } \\
\text { blocks and perform } \\
\text { deconvolution on } \\
\text { each block. }\end{array}$ & $\begin{array}{l}\text { If the blocks are small (i.e. 1000 ions) then the } \\
\text { statistics of the correction are small. E.g. 46.5 Da = 10 } \\
\text { atoms, 47.5 Da = 5 atoms, then Nb = (5.0 } 13.9) \\
\text { atoms. } \\
\text { If the block size is increased to correct for this then the } \\
\text { number of blocks must be reduced and so the } \\
\text { distribution becomes less well defined. }\end{array}$ \\
\hline 3 & $\begin{array}{l}\text { Use only Nb3+ to } \\
\text { determine the } \\
\text { distribution of niobium }\end{array}$ & $\begin{array}{l}\text { Lose a large proportion of the niobium counts, but the } \\
\text { entire peak is due to niobium alone. } \\
\text { This assumes that the proportion of niobium } \\
\text { evaporated in the 3+ charge state remains constant } \\
\text { over the volume. Variation in the evaporation field } \\
\text { could cause apparently non-random distribution of } \\
\text { niobium. }\end{array}$ \\
\hline
\end{tabular}

Table 36: Methods of determining the $\mathrm{Nb}$ distribution within an analysis volume without including the contribution from $\mathrm{ZrH}$. 
$\mathrm{The} \mathrm{ZrH} / \mathrm{Nb}$ ratio of the experimental data was too high to allow method 1 from Table 36 to be used with confidence. The second method is also less appropriate when the $\mathrm{ZrH} / \mathrm{Nb}$ ratio is high, and so this was also discounted for use in this section. The appearance of the $\mathrm{ZrH}$ ionic species is highly dependent on local field conditions, the result of this being that its distribution is non uniform, as seen in summary Table 37.

\begin{tabular}{|c|cccc|}
\hline \multicolumn{5}{|c|}{ Distribution of Nb-containing ions within the alloy matrix } \\
\hline Species & Reduced $\mathrm{X}$ & $\mathrm{v}$ & $\mathrm{p}$-value & $\mu$ \\
\hline \multicolumn{5}{|c|}{ ZIRLO } \\
\hline $\mathrm{Nb}^{2+}+$ & 79.706 & 15 & $<0.0001$ & 0.1954 \\
$\mathrm{ZrH}^{2+}$ & 2.678 & 6 & 0.0134 & 0.0506 \\
& 27.062 & 9 & $<0.0001$ & 0.1628 \\
& 216.26 & 11 & $<0.0001$ & 0.535 \\
\hline \multirow{4}{*}{$\mathrm{ZrH}^{2+}$} & 100.92 & 14 & $<0.0001$ & 0.2116 \\
& 16.018 & 4 & $<0.0001$ & 0.1006 \\
& 27.062 & 9 & $<0.0001$ & 0.1765 \\
& 179.42 & 9 & $<0.0001$ & 0.4626 \\
\hline $\mathrm{Nb}^{3+}$ & 0.068 & 2 & 0.934 & 0.0063 \\
& 0.465 & 2 & 0.6284 & 0.0122 \\
& 0.061 & 2 & 0.941 & 0.004 \\
& 0.016 & 1 & 0.8995 & 0.0016 \\
\hline \multicolumn{5}{c}{$\mathrm{Zr}-\mathrm{Nb}-\mathrm{Ti}$} \\
$\mathrm{Nb}^{2+}+$ & 337.608 & 21 & $<0.0001$ & 0.3559 \\
$\mathrm{ZrH}^{2+}$ & 171.494 & 20 & $<0.0001$ & 0.3318 \\
& 1.436 & 16 & 0.1144 & 0.1731 \\
& 87.475 & 16 & $<0.0001$ & 0.6294 \\
\hline \multirow{4}{*}{$\mathrm{ZrH}^{2+}$} & 386.932 & 20 & $<0.0001$ & 0.3675 \\
& 155.602 & 17 & $<0.0001$ & 0.2951 \\
& 0.905 & 16 & 0.5626 & 0.1301 \\
& 81.462 & 14 & $<0.0001$ & 0.5902 \\
\hline $\mathrm{Nb}^{3+}$ & 1.873 & 2 & 0.1536 & 0.0088 \\
& 0.263 & 2 & 0.7688 & 0.0044 \\
& 0.011 & 1 & 0.9166 & 0.0038 \\
& 0.044 & 1 & 0.8335 & 0.0046 \\
\hline
\end{tabular}

Table 37: The quality of fit between experimentally observed distributions of niobium-containing ions in ZIRLO and Zr-Nb-Ti with those expected for a random solid solution. 
$\mathrm{The} \mathrm{Nb}^{3+}$ distribution agrees well with that of a random solid solution in the majority of the samples listed in Table 37, and so it is concluded both that the field conditions do not vary greatly within the sub-volumes analysed and that niobium, as determined from this charge state, is distributed in a way that is consistent with a random solid solution.

\subsubsection{Titanium}

\section{Macroscopic variation}

Titanium is only present in the Zr-Nb-Ti alloy. The titanium concentration varies very little between the different regions examined in Table 25 . The concentration of titanium is $(0.343 \pm$ 0.005 ) at. $\%$, homogeneously distributed within the alloy matrix. This value seems reasonable, given that it is less than the concentration of titanium within the bulk, 0.4 at.\%. It suggests that $15 \%$ of the bulk titanium is accommodated within second phase particles or at grain boundaries.

\section{Nanoscopic variation}

Comparison of the distribution of titanium ions within the metal matrix and the binomial distribution shows good agreement between the experimental and theoretical profiles, the correlations of which are summarised below in Table 38 for the data listed in Table 25.

\begin{tabular}{|c|cccc|}
\hline \multicolumn{5}{|c|}{ Distribution of Ti ions within the alloy matrix } \\
\hline Alloy & Reduced $\mathrm{X}^{2}$ & $\mathrm{v}$ & $\mathrm{p}$-value & $\mu$ \\
\hline Zr-Nb-Ti & 0.477 & 6 & 0.8258 & 0.0076 \\
& 1.436 & 9 & 0.1553 & 0.0740 \\
& 0.519 & 3 & 0.6695 & 0.0430 \\
& 1.055 & 3 & 0.3669 & 0.0385 \\
\hline
\end{tabular}

Table 38: The quality of fit between experimentally observed distributions of niobium-containing ions in ZIRLO and Zr-Nb-Ti with those expected for a random solid solution. 


\subsubsection{Iron}

\subsubsection{Overview}

The iron solute concentration within the alloy matrices was analysed using the automated method described in Appendix A with background correction method B1 and ranging metric R3. R3 was chosen because this metric had been shown to provide the least fluctuation in performance. Based on the simulated data findings, the concentration figures obtained for the experimental data are assumed to have an additional uncertainty of $4.6 \%$, added in quadrature to the statistical uncertainty due to population sampling. A summary of the concentrations of iron seen in the uncorroded alloy matrices is given in Table 39.

\begin{tabular}{|c|c|cc|c|}
\hline Alloy & Iron level & \multicolumn{2}{|c|}{ Iron } & $\begin{array}{c}\text { Number of } \\
\text { observations }\end{array}$ \\
\hline ZIRLO & & Conc. (at.\%) & $\pm 1 \sigma$ (at. \%) & 'base' \\
& 0.003 & 0.0005 & 4 \\
& 'enhanced' & 0.018 & 0.001 & 3 \\
\hline Zircaloy-4 & 'base' & 0.0026 & 0.0003 & 4 \\
\hline Zr-Nb-Ti & 'base' & 0.0027 & 0.0004 & 2 \\
& 'enhanced' & $0.004-0.04$ & - & 2 \\
\hline
\end{tabular}

Table 39: Summary of the iron concentration of the metal matrix of ZIRLO, Zircaloy-4 and Zr$\mathrm{Nb}$-Ti alloys measured using 3DAP. The local concentration is separated into two groups, those with 'base' and enhanced iron concentration, discussed in sections 5.1.6.2 - 5.1.6.4.

The variation of the iron concentration with distance was determined by comparing the iron concentration within blocks of 1 million ions taken from within a number of ZIRLO specimens. The Imago IVAS distribution analysis tool was also used to compare the iron solute distribution, and the data describing these correlations are summarised in Table 40. The iron distribution 
within the three alloys shows different patterns of behaviour, and so in this section (5.1.6) the different alloys are discussed separately.

\begin{tabular}{|c|cccc|}
\hline \multicolumn{5}{|c|}{ Distribution of Fe ions within the alloy matrix } \\
\hline Alloy & Reduced $x^{2}$ & $\mathrm{v}$ & $\mathrm{p}$-value & $\mu$ \\
\hline ZIRLO & 0.175 & 2 & 0.8399 & 0.0053 \\
& 0.006 & 1 & 0.938 & 0.001 \\
& 0.004 & 1 & 0.9491 & 0.0007 \\
& 0.005 & 1 & 0.9438 & 0.0009 \\
\hline Zircaloy-4 & 0.052 & 1 & 0.962 & 0.0016 \\
& 0.005 & 1 & 0.9781 & 0.0019 \\
& 0.018 & 1 & 0.8932 & 0.0012 \\
& 0.002 & 1 & 0.9638 & 0.0005 \\
\hline Zr-Nb-Ti & 2.807 & 3 & 0.038 & 0.013 \\
& $<0.001$ & 1 & 0.9953 & $<0.0001$ \\
& 0.012 & 1 & 0.9133 & 0.0038 \\
& 0.007 & 1 & 0.9357 & 0.0017 \\
\hline
\end{tabular}

Table 40: The quality of fit between experimentally observed distributions of iron ions in ZIRLO,

Zircaloy-4 and Zr-Nb-Ti with those expected for a random solid solution.

\subsubsection{ZIRLO}

Macroscopic variation

Data acquired from the analysis of 7 specimens (with suitably low levels of hydrogen and an absence of surface oxidation) is shown in Figure 70. The left hand profile shows the contributions of the individual datasets to the profile. The right hand profile plots these data points as a histogram. The distribution of iron falls roughly into two groups, as summarised in Table 39. The proportion of the material with an 'enhanced' iron concentration (around 0.018 at.\%) and a 'base' concentration (around 0.003 at.\%) cannot be determined from these data, and would required hundreds of additional atom probe analyses to answer this question with 
confidence. Of the specimens analysed a roughly equal number contain the base level and enhanced levels of iron. It should be noted that the local iron distribution in all of the regions analysed appeared to be disordered by a visual inspection (no segregation to intragranular features).
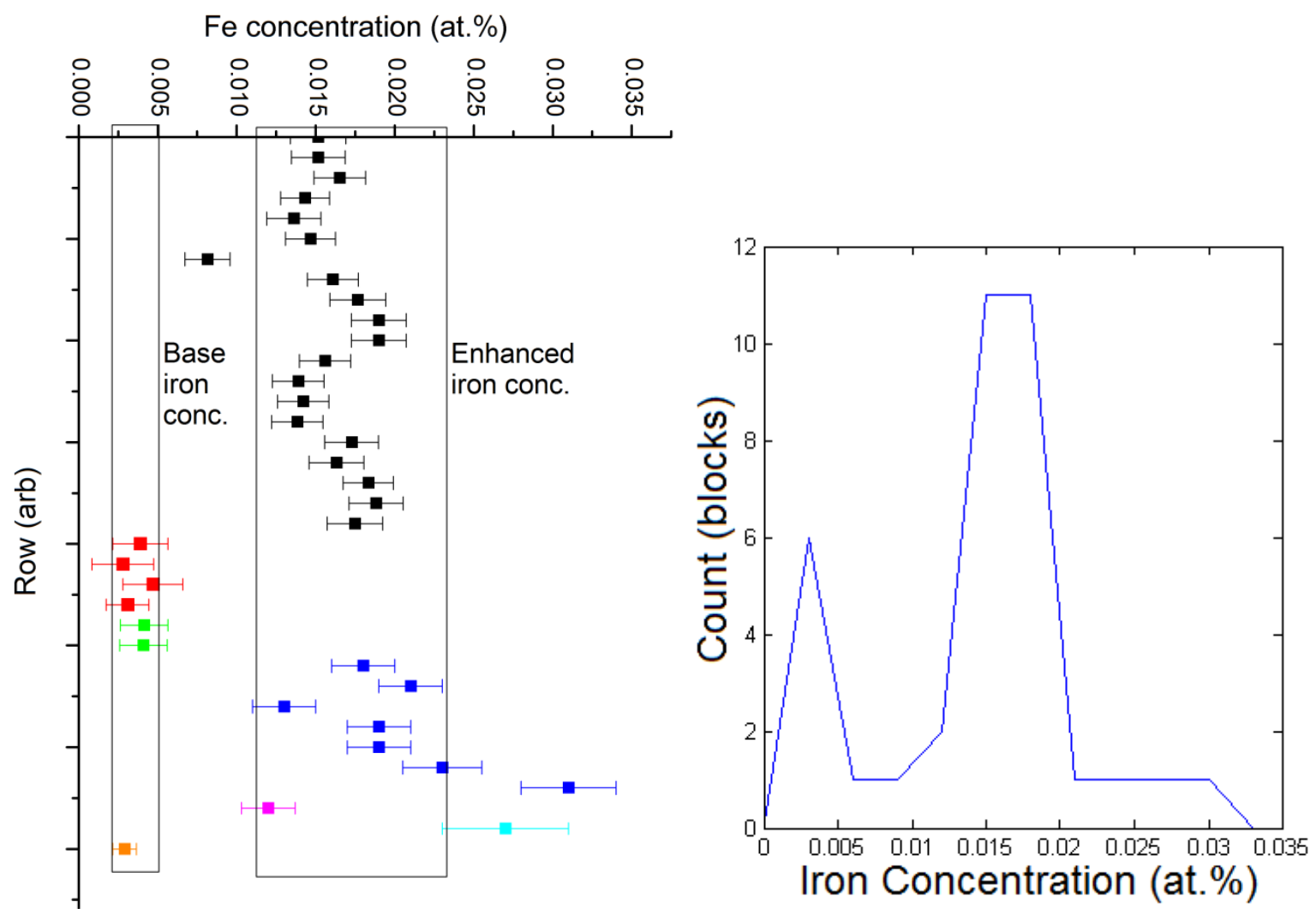

Figure 70: Profiles showing the distribution of iron concentration within 7 separate specimens (each with a different marker). The left hand profile shows the contributions of the individual datasets to the profile. The histogram on the right hand side shows that the distribution of these points using bins of width 0.003 at.\%.

The largest dataset (enhanced iron - black markers in Figure 70) is displayed as a 3D atom map in Figure 71. The dataset contains a grain boundary, and although this region was excluded from the analysis of the metal matrix, it is helpful in that the distribution of the leading edge and 
trailing edge of the peak at $28 \mathrm{Da}$ can be compared. There is no difference in the distribution of the species at the leading and trailing edges of the peak, suggesting that the whole of this enhanced iron level peak is attributable to iron and does not contain a contribution from the $\mathrm{CO}$ impurity species, which would not be segregated to the grain boundary.

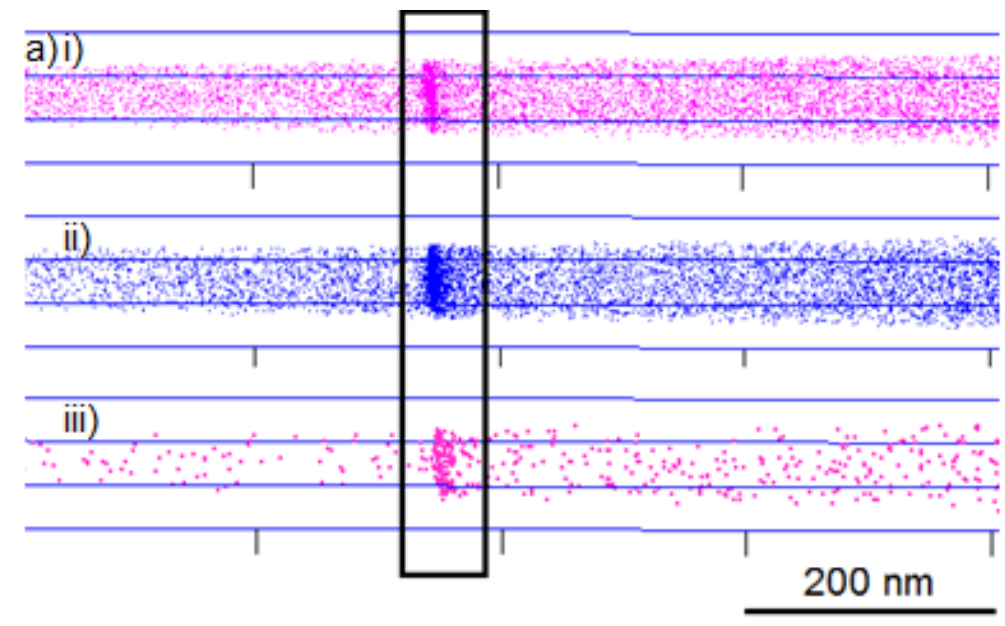

b)

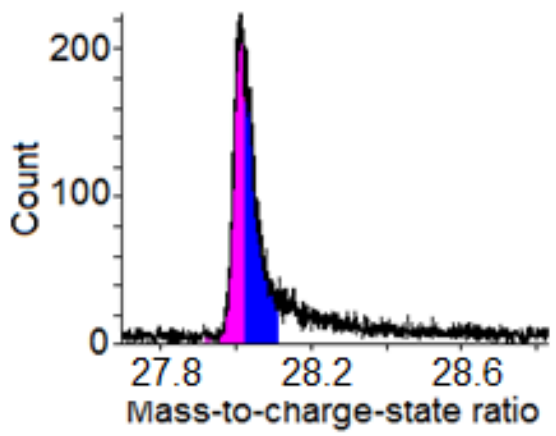

Figure 71: 3D atom maps showing the distribution of iron from i) the leading edge and ii) trailing edge of $28 \mathrm{Da}$, iii) Cr at $26 \mathrm{Da}$. The section of the mass spectrum where these ranges are defined is shown in (b). The Cr ions in (iii) are shown using a larger pixel size to emphasise their position.

\section{Nanoscopic variation}

Figure 72 compares the experimentally obtained histogram of iron distribution within the alloy matrix of the dataset shown in Figure 71 dataset with an approximation of the binomial function. By comparison with the theoretical curve it is shown that the distribution of iron within the matrix of 'as-received' recrystallised ZIRLO alloy is that of a random solid solution. The analysis of the four ZIRLO datasets listed in Table 26 (summarised above in Table 40) also showed a very good correlation between the experimental distributions and the binomial distribution for both 'base' and 'enhanced' iron concentration matrix. 


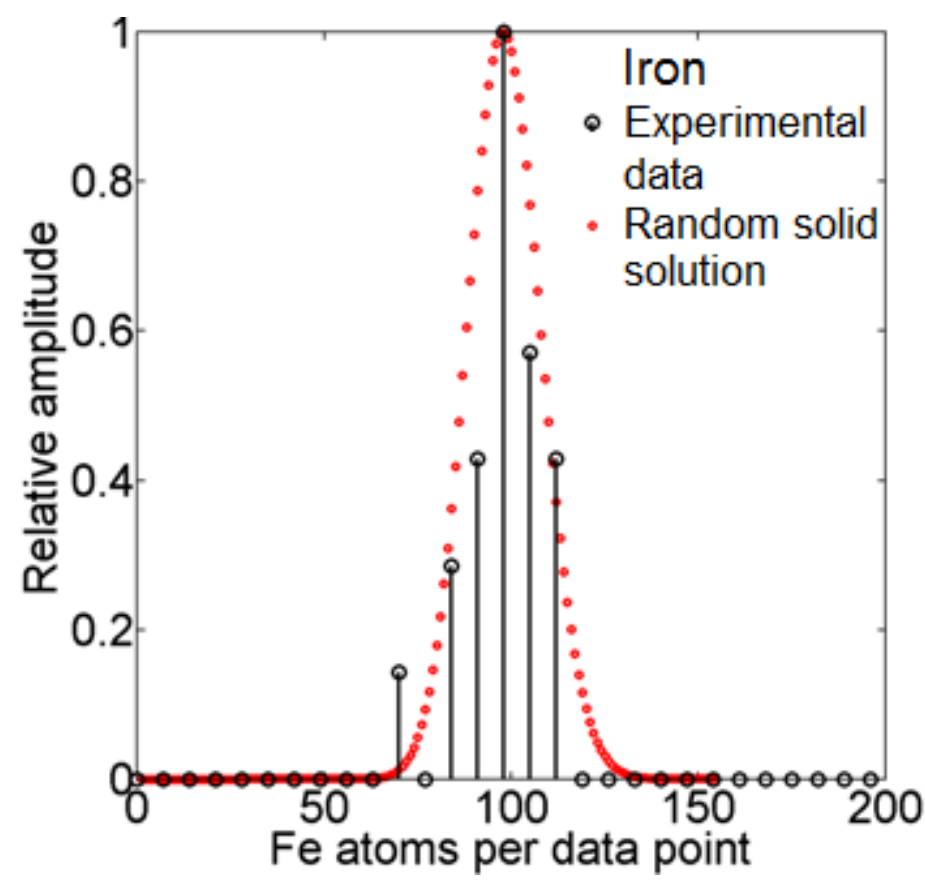

Figure 72: A histogram showing the distribution of iron atoms per data point. Each blue data point is 1 million 'raw' ions, including un-ranged events such as background noise. The data points displayed in bins with a width of $10 \mathrm{Fe}$ atoms. The red curve is what would be expected of a random solid solution distributed about the mean concentration.

\subsubsection{Zircaloy-4}

Macroscopic variation

The iron concentration in the metal matrix of Zircaloy- 4 was found to be $(0.0026 \pm 0.0003)$ at. $\%$ using the automated ranging method from Appendix A. The $3^{\text {rd }}$ and $4^{\text {th }}$ Zircaloy- 4 datasets listed in Table 24 were further subdivided into blocks and analysed using the same the automated techniques. The level of iron seen in these four samples is consistent and similar to the level seen in the regions of ZIRLO matrix with base iron concentration. Within this limited number of observations, no enhanced iron concentration regions of metal matrix were observed. The iron concentrations within the regions described in Table 24 agree within 1 standard deviation. 


\section{Nanoscopic variation}

A histogram showing the distribution of iron concentration within $1 \mathrm{~m}$ ion raw data blocks is shown in Figure 73. The experimental data (blue curve) are compared with the distribution that would be expected of a random solid solution (red curve). The number of blocks is very small, in an ideal situation a factor of 10 times more data points would provide a better comparison between the distributions, but this is not practical given the current generation of atom probe instrumentation. Reducing the block size by a factor of ten would skew the distribution towards a mean of 1.8 atoms per block, hampering visual assessment of the symmetry and spread of the distribution, but still not providing enough data to perform meaningful statistical analysis. The distribution of the experimental histogram occupies a similar range to the binomial and is symmetric about the mean value. Excellent agreement between the 250 ion block size experimental and binomial distributions was seen for Zircaloy-4 in Table 40.

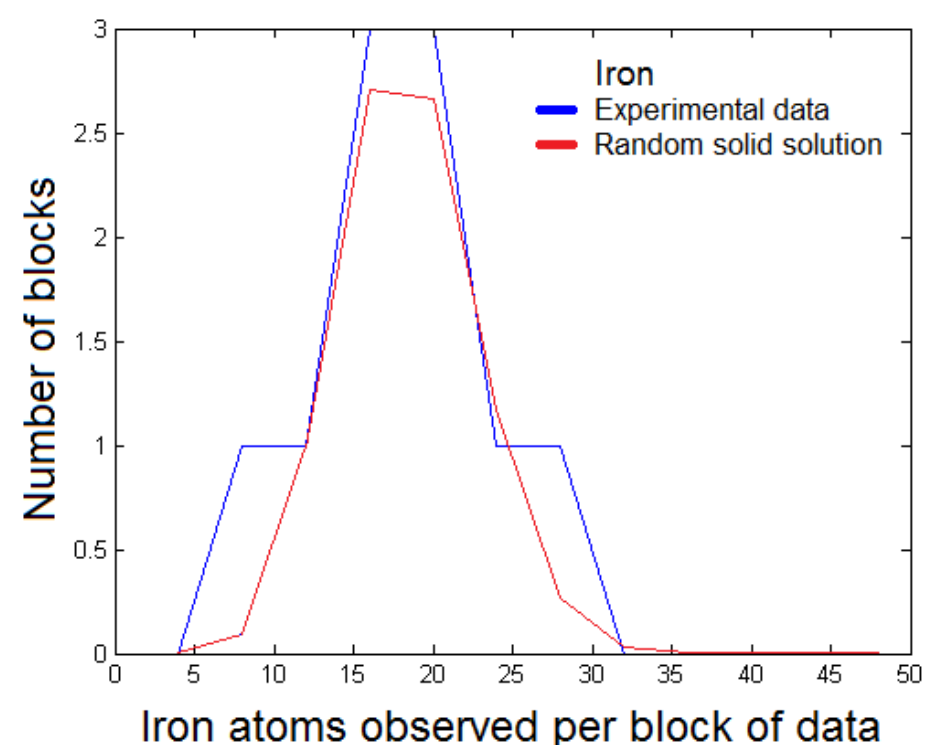

Figure 73: Histogram of number of blocks containing a specific amount of iron atoms in Zircaloy-4 alloy matrix. Experimental data (10 blocks of $1 \mathrm{~m}$ raw ions of which $\sim 600,000$ are ranged within the FWHM) is shown by the blue curve, an approximation of the Poisson distribution is shown by a red curve. The step size of the histogram is 4 atoms. 


\subsubsection{Zr-Nb-Ti}

\section{Macroscopic variation}

The iron distribution in the as-received $\mathrm{Zr}-\mathrm{Nb}$ - Ti alloy exhibits a more complicated behaviour than was found for the ZIRLO and Zry-4 alloys. Figure 74 shows the distribution of iron solute within $\mathrm{Zr}-\mathrm{Nb}$-Ti metal matrix taken from two specimens. Comparison between the two regions allows us to determine if the iron concentration varies macroscopically within the material, the distributions themselves describe the microscopic distribution of solute locally. The iron concentration in specimen (a) in Figure 74 is consistent with the base iron level. The enhanced iron concentration seen in matrix (b) is around 3 times this level. Other local iron concentration measurements were performed on a specimen with iron concentration as high as $(0.0443 \pm$ $0.0006)$ at.\% over a volume of several million ions.

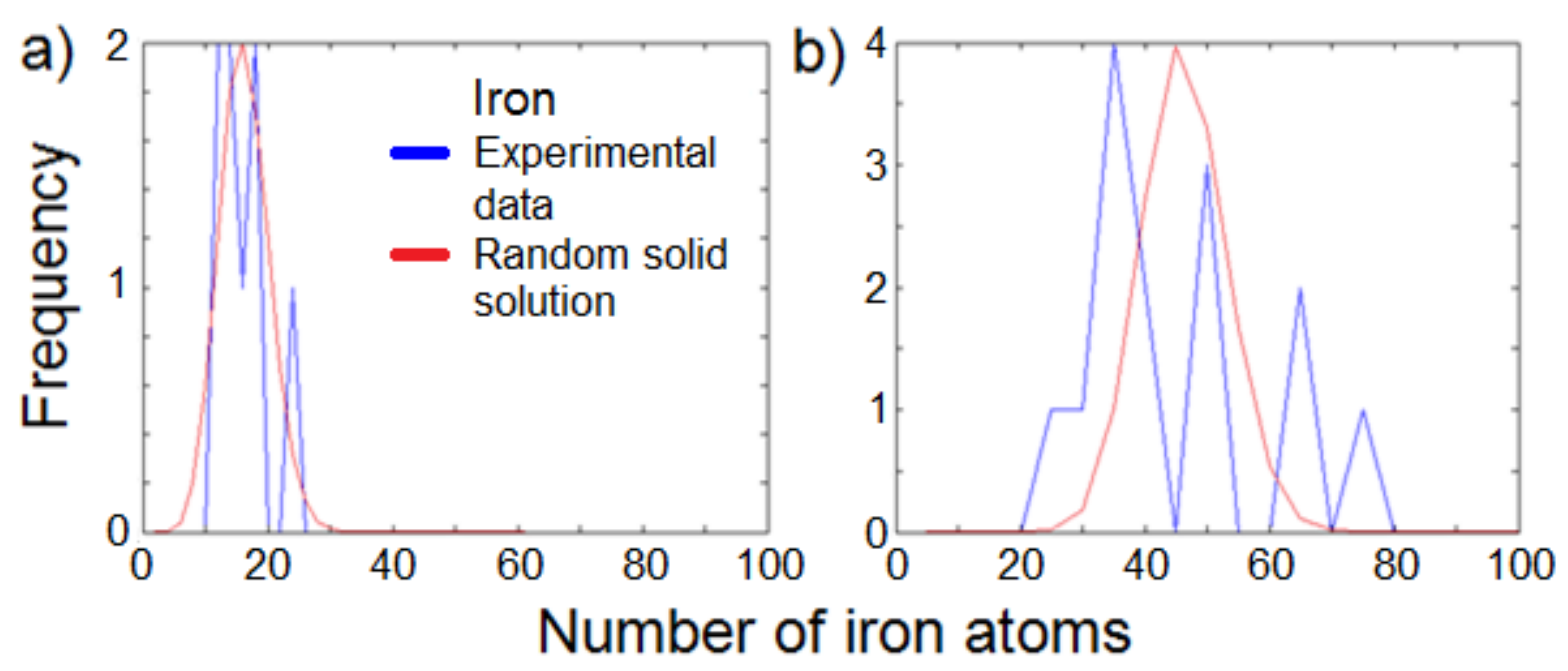

Figure 74: Histograms showing frequency of blocks containing a given number of iron atoms. Histograms are produced using 1 million ion blocks of raw data ranged using the FWHM metric R1 and background correction method B1. Plots (a) and (b) are taken from large datasets from different specimens. Histogram bin size: a) 2 atoms, $b$ and c) 5 atoms. 


\section{Nanoscopic variation}

The spread of observations for specimen (a) is consistent with that of a random solid solution. The distribution of iron within specimen (b) of Figure 74 is not consistent with that of a random solid-solution, this was also seen in Table 40. The distribution is both skewed towards lower concentration than the local mean and the range of the distribution is greater than would be expected. This local mean value is also much higher; approximately three times that of specimen (a).

\subsubsection{Chromium and nickel}

Macroscopic variation

\begin{tabular}{|c|c|c|c|}
\hline Alloy & $\begin{array}{c}\text { Bulk } \\
\text { (at.\%) }\end{array}$ & $\begin{array}{c}\text { Simple mean } \\
\text { (at.\%) }\end{array}$ & $\begin{array}{c}\text { No. } \\
\text { Observations }\end{array}$ \\
\hline \multicolumn{3}{|c|}{ Chromium } \\
\hline ZIRLO & $<0.02$ & $\begin{array}{c}0.0004-0.0005 \\
\text { None detected }\end{array}$ & 1 \\
Zry-4 & 0.19 & None detected & 4 \\
Zr-Nb-Ti & NR & 0.005 & 2 \\
& \multicolumn{3}{|c|}{ None detected } \\
\hline \multicolumn{2}{|c|}{ Nickel } \\
\hline ZIRLO & $<0.02$ & None detected & 7 \\
Zry-4 & NR & Trace & 1 \\
& & None detected & 3 \\
Zr-Nb-Ti & NR & 0.003 - 0.006 & 3 \\
& & None detected & 1 \\
\hline
\end{tabular}

Table 41:Summary of the chromium and nickel content present in the metal matrix of ZIRLO, Zircaloy-4 and Zr-Nb-Ti.

Chromium and nickel were found either not to be present, or to be in solution at very low solute concentrations. The observation of this solute differed for ZIRLO and Zircaloy-4 than the Zr-Nb- 
Ti material. Fifteen specimens were suitable for examining the concentration of chromium and nickel, of these seven were ZIRLO, four Zircaloy-4 and four Zr-Nb-Ti. The results are summarised in Table 41.

Three of the four Zr-Nb-Ti alloy specimens contained detectable levels of nickel and chromium in solution, where as two specimens of the eleven ZIRLO and Zircaloy-4 samples contained detectable quantities of chromium or nickel.

In the ZIRLO specimen that contained a detectable amount of chromium, a difference in chromium concentration was seen in two neighbouring grains. This dataset, containing the grain boundary is shown in Figure 71. The left hand grain in Figure 71 (iii) has a chromium concentration of $(0.00036 \pm 0.00007)$ at.\% based on $9.2 \mathrm{~m}$ atoms. The right hand grain contains $(0.00054 \pm 0.00006)$ at. $\%$ chromium based on a volume of $16.9 \mathrm{~m}$ atoms. Background correction of the data was conducted by subtracting a mass area of equal size ahead of the 26 Da peak from its count.

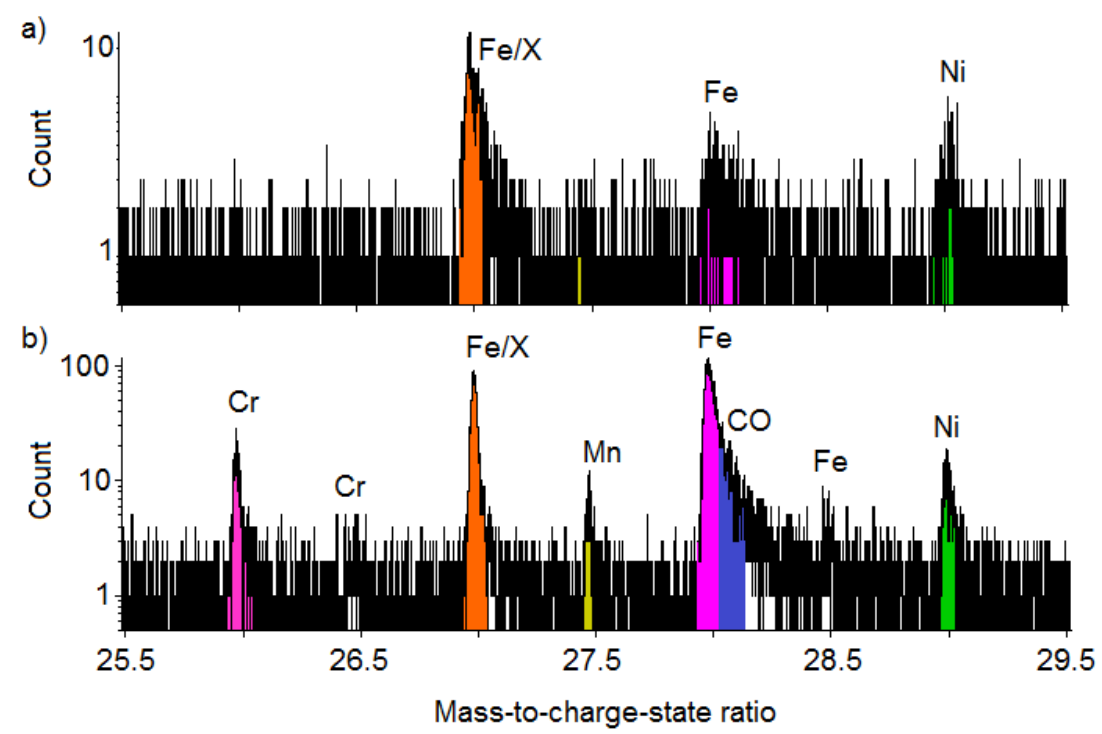

Figure 75: Mass-to-charge-state ratio spectra showing Zr-Nb-Ti matrix regions with a) $\sim 0.003$ iron and nickel content and b) 0.04 at.\% Fe, 0.01 at.\% $(\mathrm{Cr}+\mathrm{Ni})$ and 0.001 at.\% Mn $(27.5$ $D a)$. 
The mass spectra showing the high nickel and chromium regions of $\mathrm{Zr}-\mathrm{Nb}$ - $\mathrm{Ti}$ analysed are shown in Figure 75. Figure 75 (a) is a dataset containing 'base' level iron and some nickel. Figure 75 (b) shows material containing 'enhanced' iron as well as chromium and nickel. Figure 75 (b) also shows a low concentration species at $27.5 \mathrm{Da}(0.0013 \pm 0.0001)$ at. $\%$. This has been attributed to manganese $\left(\mathrm{Mn}^{2+}\right)$ in the figure. This peak could also be due to a complex ion species $\mathrm{Al}_{2} \mathrm{H}^{2+}$, but the formation of such an ion at $0.35 \mathrm{~nJ}$ laser energy in a metal tip of $\sim 80 \mathrm{~nm}$ radius seems unlikely. The count at 28 and $28.5 \mathrm{Da}$ are not high enough to explain the peak at 27.5 Da as ${ }^{54} \mathrm{FeH}^{2+}$.

\section{Nanoscopic variation}

No analysable quantity of chromium and nickel was found in the ZIRLO or Zry-4 material analysed in Table 26 and Table 27. The distribution of these solutes in the $\mathrm{Zr}-\mathrm{Nb}$-Ti material is summarised in Table 42. All of the solute distributions agree very well with those of a random solid solution. These results were retested with a larger ion per block count (1000 ions), with similar results. An example of this agreement is shown in Figure 76, a log-log scale frequency distribution of iron, chromium and nickel with the metal matrix of a $\mathrm{Zr}-\mathrm{Nb}-\mathrm{Ti}$ sample.

\begin{tabular}{|c|cccc|}
\hline Element & $\begin{array}{c}\text { Reduced } \\
\mathrm{x}^{2}\end{array}$ & $\mathrm{v}$ & $\mathrm{p}$-value & $\mu$ \\
\hline $\mathrm{Cr}$ & 0.002 & 1 & 0.9678 & 0.0002 \\
& $<0.001$ & 1 & 0.9925 & 0.0001 \\
& - & - & - & - \\
& - & - & - & - \\
\hline $\mathrm{Ni}$ & $<0.001$ & 1 & 0.9925 & $<0.0001$ \\
& 0.007 & 1 & 0.9331 & 0.0001 \\
& $<0.001$ & 1 & 0.9955 & 0.0001 \\
& - & - & - & - \\
\hline
\end{tabular}

Table 42: The quality of fit between experimentally observed distributions of chromium and nickel ions in $\mathrm{Zr}-\mathrm{Nb}-\mathrm{Ti}$ with those expected for a random solid solution. 


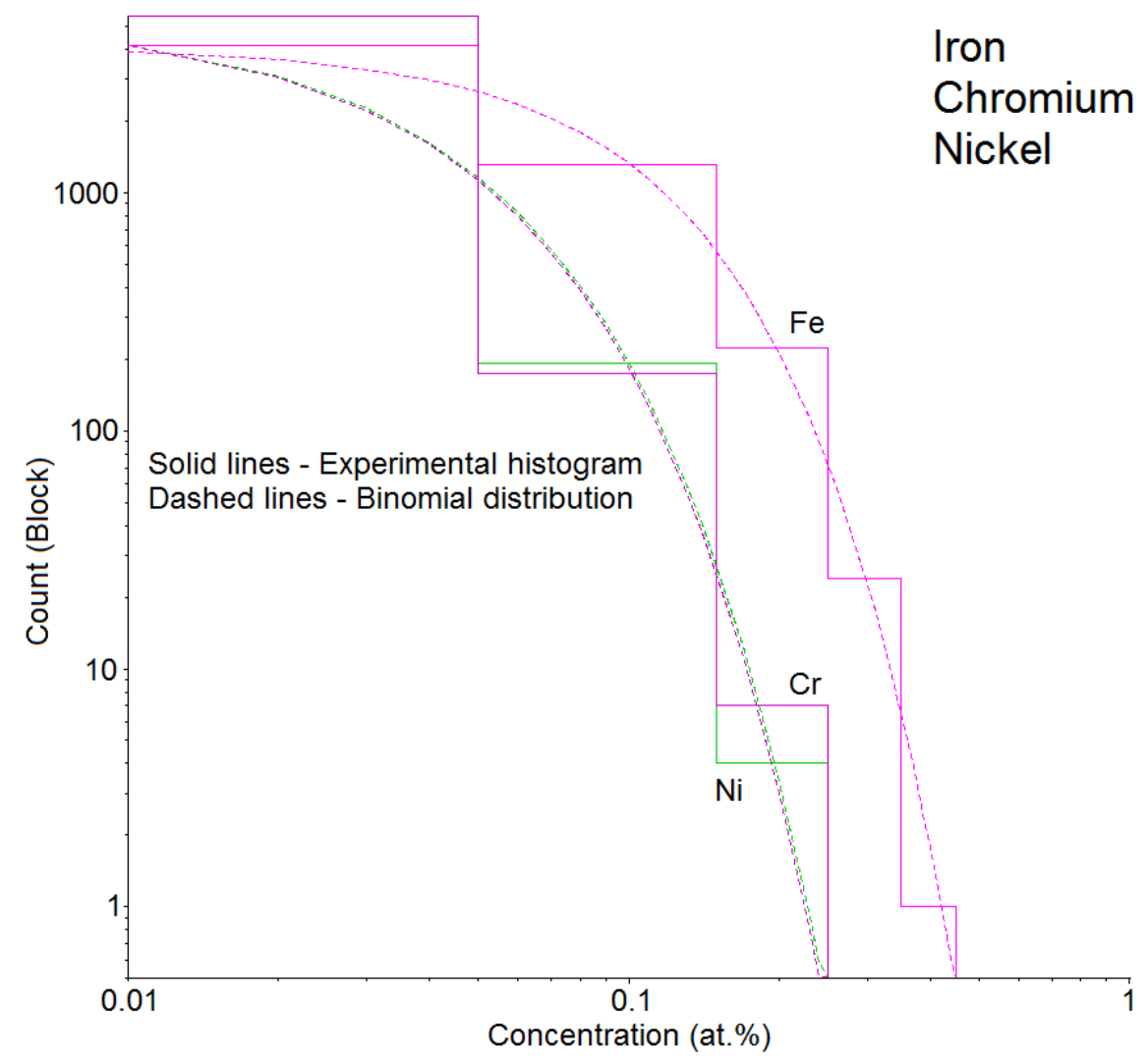

Figure 76: Comparison of binomial distributions (which represent the distribution of solute with a random solid solution) and histograms of the number of blocks of 1000 ions with a certain concentration of a number of transition metals (logarithmic scale). The region of interest contains 5729 blocks. The iron frequency distribution has been created for this plot using tools within the Imago IVAS program, not the ranging methods described by section Appendix A. 


\subsubsection{Summary}

The distribution of solutes within the metal matrix of these uncorroded materials is summarised in Table 43.

\begin{tabular}{|c|c|c|c|}
\hline & $\overline{\mathrm{ZIRLO}}$ & Zircaloy-4 & Zr-Nb-Ti \\
\hline $\mathrm{Nb}$ & $\begin{array}{l}\text { Non-equilibrium } \\
\text { concentration distributed } \\
\text { inhomogeneously on a } \\
\text { microscopic scale, with } \\
\text { some evidence of } \\
\text { conforming to a random } \\
\text { solid solution on the } \\
\text { nanoscopic scale. ( } 0.39 \pm \\
0.06) \text { at. } \% \text { of the } 1 \text { at. } \% \\
\text { bulk niobium is present in } \\
\text { solution in the matrix. }\end{array}$ & Not present & $\begin{array}{l}\text { Non-equilibrium } \\
\text { concentration distributed } \\
\text { inhomogeneously on a } \\
\text { microscopic scale, with } \\
\text { some evidence of } \\
\text { conforming to a random } \\
\text { solid solution on the } \\
\text { nanoscopic scale. ( } 0.43 \pm \\
0.04) \text { at. } \% \text { of the } 1 \text { at.\% } \\
\text { bulk niobium is present in } \\
\text { solution in the matrix. }\end{array}$ \\
\hline Sn & $\begin{array}{l}\text { Crystallographic } \\
\text { dependence of tin } \\
\text { concentration prevents } \\
\text { distribution analysis. The } \\
\text { average matrix } \\
\text { composition is }(0.72 \pm \\
0.08) \text { at.\%, which } \\
\text { accounts for the entire } \\
\text { bulk content. }\end{array}$ & $\begin{array}{l}\text { Crystallographic } \\
\text { dependence of tin } \\
\text { concentration prevents } \\
\text { distribution analysis. } \\
\text { The average matrix } \\
\text { composition is }(1.02 \pm \\
0.06) \text { at. } \% \text {, which } \\
\text { accounts for the entire } \\
\text { bulk content. }\end{array}$ & Not present \\
\hline $\mathrm{Ti}$ & Not present & Not present & $\begin{array}{l}\text { Titanium is present } \\
\text { homogeneously within } \\
\text { the metal matrix on a } \\
\text { microscopic lengthscale. } \\
(0.343 \pm 0.005) \text { at. } \% \text { of } \\
\text { the } 0.4 \text { at. } \% \text { bulk titanium } \\
\text { is present in solid solution } \\
\text { within the metal matrix. } \\
\text { On a local nanoscopic } \\
\text { level, the titanium is } \\
\text { distributed as a random } \\
\text { solid solution within the } \\
\text { metal matrix. }\end{array}$ \\
\hline
\end{tabular}

Table continued overleaf. 


\begin{tabular}{|c|c|c|c|}
\hline 0 & $\begin{array}{l}\text { The oxygen } \\
\text { concentration varies } \\
\text { considerably on a } \\
\text { microscopic scale. The } \\
\text { concentration of oxygen } \\
\text { is }(1.30 \pm 0.31) \text { at. } \% \text {. The } \\
\text { local solute distribution } \\
\text { agrees well with that of a } \\
\text { random solid solution. }\end{array}$ & $\begin{array}{l}\text { The oxygen } \\
\text { concentration varies } \\
\text { considerably on a } \\
\text { microscopic scale. The } \\
\text { concentration of oxygen } \\
\text { is }(0.83 \pm 0.11) \text { at. } \% \text {. } \\
\text { The local solute } \\
\text { distribution agrees very } \\
\text { well with that of a } \\
\text { random solid solution. }\end{array}$ & $\begin{array}{l}\text { The oxygen } \\
\text { concentration varies } \\
\text { considerably on a } \\
\text { microscopic scale. The } \\
\text { concentration of oxygen } \\
\text { is }(0.97 \pm 0.12) \text { at. } \% \text {. The } \\
\text { local solute distribution } \\
\text { agrees very well with that } \\
\text { of a random solid } \\
\text { solution. }\end{array}$ \\
\hline $\mathrm{Fe}$ & $\begin{array}{l}\text { Iron is distributed } \\
\text { inhomogeneously within } \\
\text { the metal matrix and } \\
\text { follows a bi-modal } \\
\text { distribution with means at } \\
(0.0035 \pm 0.0007) \text { at. } \% \\
\text { and }(0.018 \pm 0.01) \text { at. } \% \text {. } \\
\text { Locally the iron is } \\
\text { distributed as a random } \\
\text { solid solution. }\end{array}$ & $\begin{array}{l}\text { Of the limited number of } \\
\text { datapoints obtained ( } 4) \text {, } \\
\text { iron was seen only at } \\
\text { the concentration of } \\
(0.0037 \pm 0.0004) \text { at. } \% \text {. } \\
\text { There is limited } \\
\text { evidence that the iron is } \\
\text { distributed as a random } \\
\text { solid solution. }\end{array}$ & $\begin{array}{l}\text { Of the limited number of } \\
\text { datapoints obtained (4), } \\
\text { iron was seen to occupy } \\
\text { a wide range of } \\
\text { concentrations at } \\
\text { localised points } \\
\text { (comparatively). At some } \\
\text { points the iron distribution } \\
\text { was random and at } \\
\text { others it was non- } \\
\text { random. A proportion of } \\
\text { the material analysed } \\
\text { contained (0.0031 } \pm \\
0.0003 \text { ) at. } \% \text { Fe, but } \\
\text { other sites contained } \\
\text { several times this } \\
\text { amount. }\end{array}$ \\
\hline $\mathrm{Cr}$ & $\begin{array}{l}\text { Chromium seen in the } \\
\text { metal matrix at the } ~ 5 \text { at. } \\
\text { ppm level. Limited } \\
\text { evidence that Cr is } \\
\text { distributed } \\
\text { inhomogeneously } \\
\text { between grains. }\end{array}$ & $\begin{array}{l}\text { Matrix chromium below } \\
\text { detection threshold (< } \\
10 \text { at. ppm.). }\end{array}$ & $\begin{array}{l}\text { Matrix concentration } \\
\text { varies greatly over } \\
\text { microscopic distances } \\
\text { between }<0.0001 \text { at. } \% \\
\text { and } \sim 0.005 \text { at. } \% \\
\text { (observed). }\end{array}$ \\
\hline $\mathrm{Ni}$ & $\begin{array}{l}\text { Matrix nickel below } \\
\text { detection threshold }(<10 \\
\text { at. ppm.). }\end{array}$ & $\begin{array}{l}\text { Matrix nickel below } \\
\text { detection threshold (< } \\
10 \text { at. ppm.). }\end{array}$ & $\begin{array}{l}\text { Matrix concentration } \\
\text { varies greatly over } \\
\text { microscopic distances } \\
\text { between }<0.0001 \text { at. } \% \\
\text { and } \sim 0.005 \text { at. } \% \\
\text { (observed). }\end{array}$ \\
\hline
\end{tabular}

Table 43: Summary of the distribution of solute within the 'as-received' zirconium alloys studied. 


\subsection{Microstructural features}

During the characterisation of the alloy matrix chemistry several microstructural features (second phase particles and grain boundaries) were observed. This information, although insufficient to be described as a thorough or complete description of the microscopic scale chemistry of such features, does allow a limited description of the behaviour of solute at these sites in as-received material and serve as a basis for comparison with large-scale changes that occur after corrosion in autoclave environments.

\subsubsection{Second phase particles}

\subsubsection{ZIRLO}

A second phase particle (SPP) embedded in a ZIRLO alloy matrix was analysed by 3DAP. The particle has an estimated diameter of $100 \mathrm{~nm}$. As such, it was wider that the LEAP field of view. Figure 77 shows a schematic of the SPP within the atom probe specimen. The composition of the particle, detailed in Table 44, corresponds roughly with that of the intermetallic phase $\mathrm{Zr}(\mathrm{Nb}, \mathrm{Fe})_{2}$ identified in ZIRLO by Liu et al. [75] (see section 2.4.2). However, the particle also contains $\mathrm{Cr}$, and so is better described as $\mathrm{Zr}(\mathrm{Nb}, \mathrm{Fe}, \mathrm{Cr})_{2}$, which was identified in a different $\mathrm{Zr}$ $\mathrm{Nb}-\mathrm{Sn}-\mathrm{Fe}-\mathrm{O}$ alloy by Liu et al. [79]. 


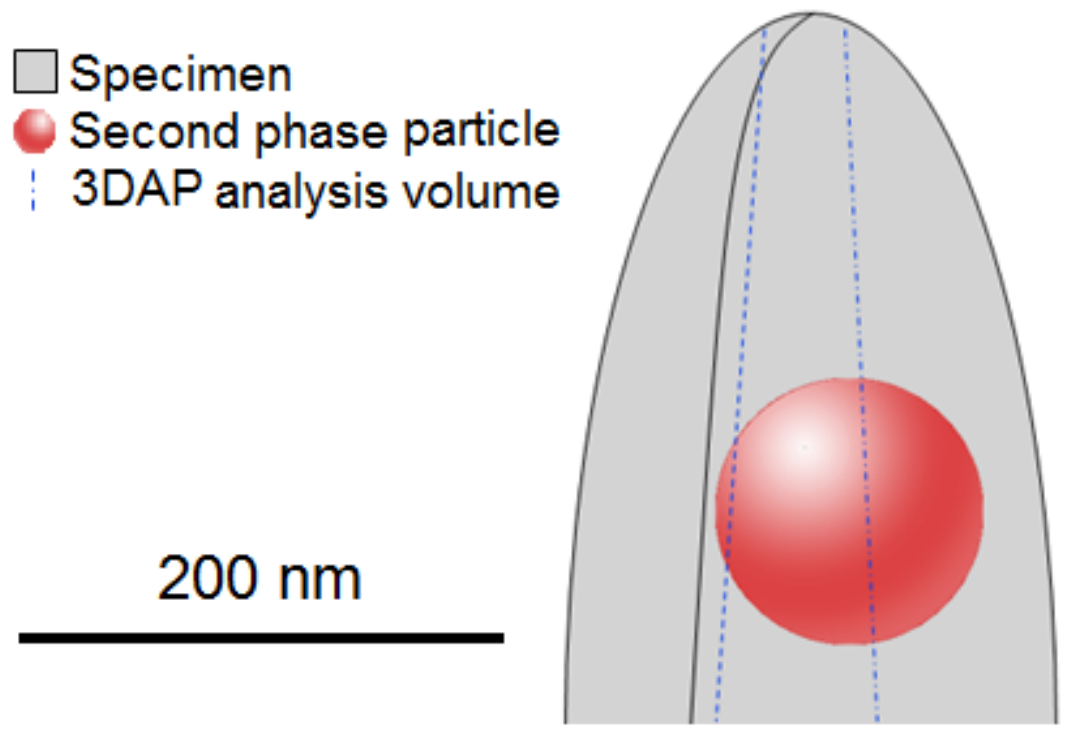

Figure 77: Schematic view of an intermetallic particle within a 3DAP specimen needle.

A $2 \mathrm{D}$ concentration profile across the particle/matrix interface is shown in Figure 78 . There is a sharp interface, which is at the limit of the atom probe spatial resolution. Each element is shown using a different colour scale, as described within the figure. Oxygen and tin are seen in their usual concentration in the alloy matrix but at a very low, or zero level within the particle. No segregation of any solute to the particle matrix/interface is observed, which would have been expected to have ramifications for the creep resistance of the material. The concentration of niobium and iron appears to vary within the particle. In Figure 78 the niobium concentration appears greater (more red) towards the surface of the particle, whereas the iron concentration increases towards the core. This behaviour is also apparent from the 1D concentration profile across the interface, shown in Figure 79. This behaviour could be real, or a possible artefact, caused by the change in field conditions altering the iron and niobium evaporation ratio as more of the particle enters the field of view. Although this is a possibility, it was seen in the preliminary work (4.6.5 and 4.6.6) that the apparent concentration of iron and niobium in the ZIRLO metal matrix was not strongly affected by changes in the evaporation field over quite a large variation of field conditions, so the result is probably reliable. 


\begin{tabular}{|c|c|cc|}
\hline Element & Number & \multicolumn{2}{|c|}{ Composition } \\
& of atoms & Conc. & \pm 1 o \\
(atoms) & (at.\%) & (at.\%) \\
\hline $\mathrm{Zr}$ & 334,433 & 38.96 & 0.053 \\
$\mathrm{O}$ & 1,421 & 0.17 & 0.004 \\
$\mathrm{Nb}$ & 287,418 & 33.48 & 0.06 \\
$\mathrm{Sn}$ & 93 & 0.01 & 0.001 \\
$\mathrm{Fe}(28)$ & 217,227 & 25.30 & 0.05 \\
$\mathrm{Cr}(29)$ & 17,891 & 2.08 & 0.02 \\
\hline Total & 858,483 & & \\
\hline
\end{tabular}

Table 44: Composition of the intermetallic precipitate shown in Figure 78.
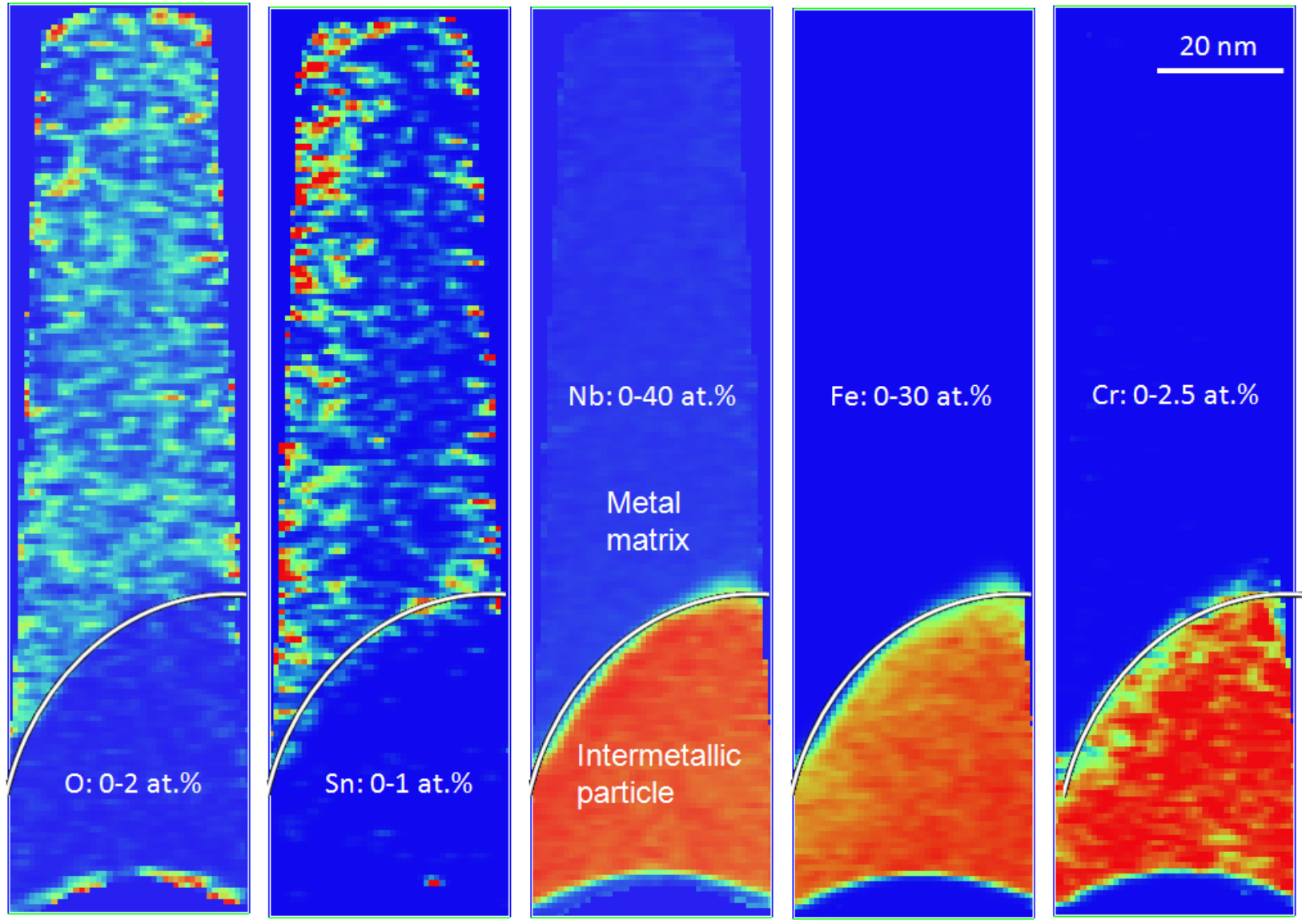

Figure 78: 2D concentration profiles taken through a second phase particle in the metal matrix.

Profiles show the distribution of various elements using alternative colour scales (labelled in figure) ranging from blue (zero) to red (highest concentration). 


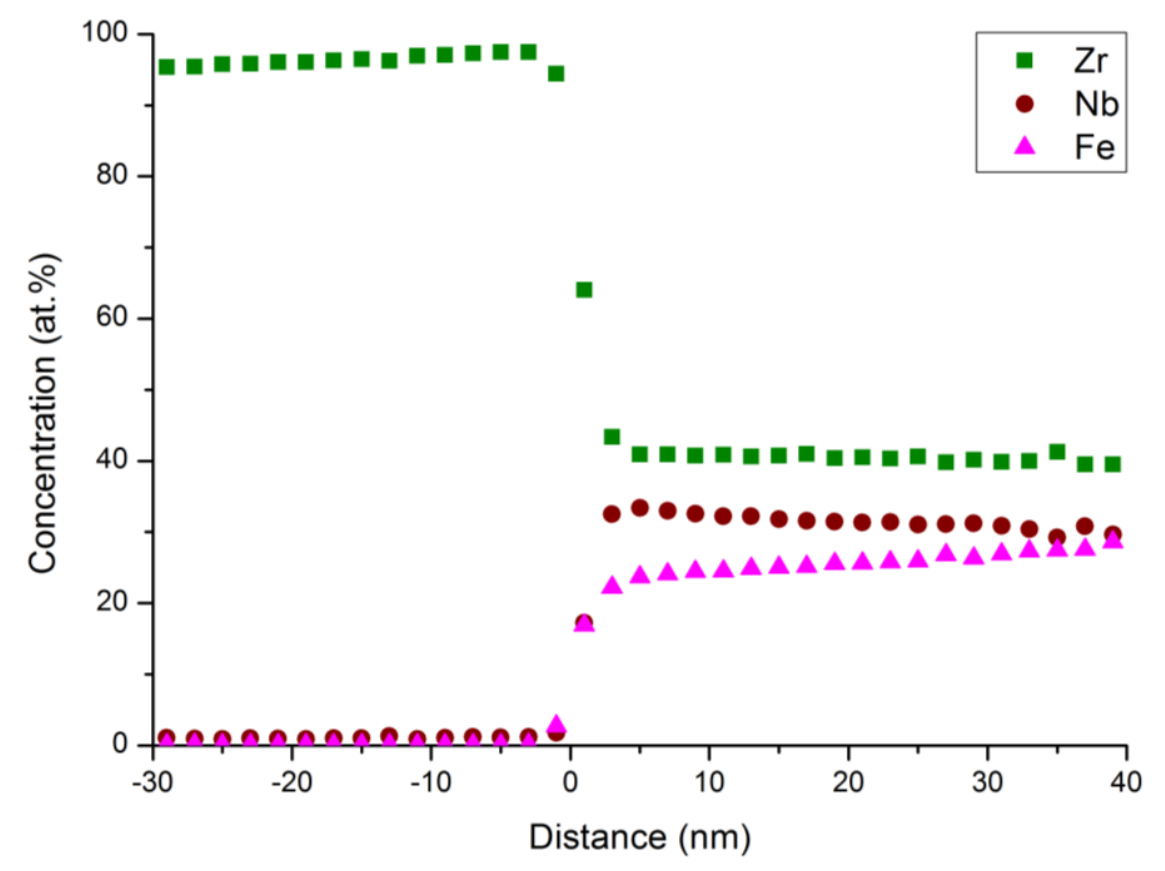

Figure 79: $1 D$ concentration profile produced using a proxigram across the alloy matrix/particle interface. The surface of the particle is at zero distance. The distance becomes positive (RHS) towards the centre of the particle. Although the sampling error increases towards the centre, it is not resolvable on this scale of this figure.

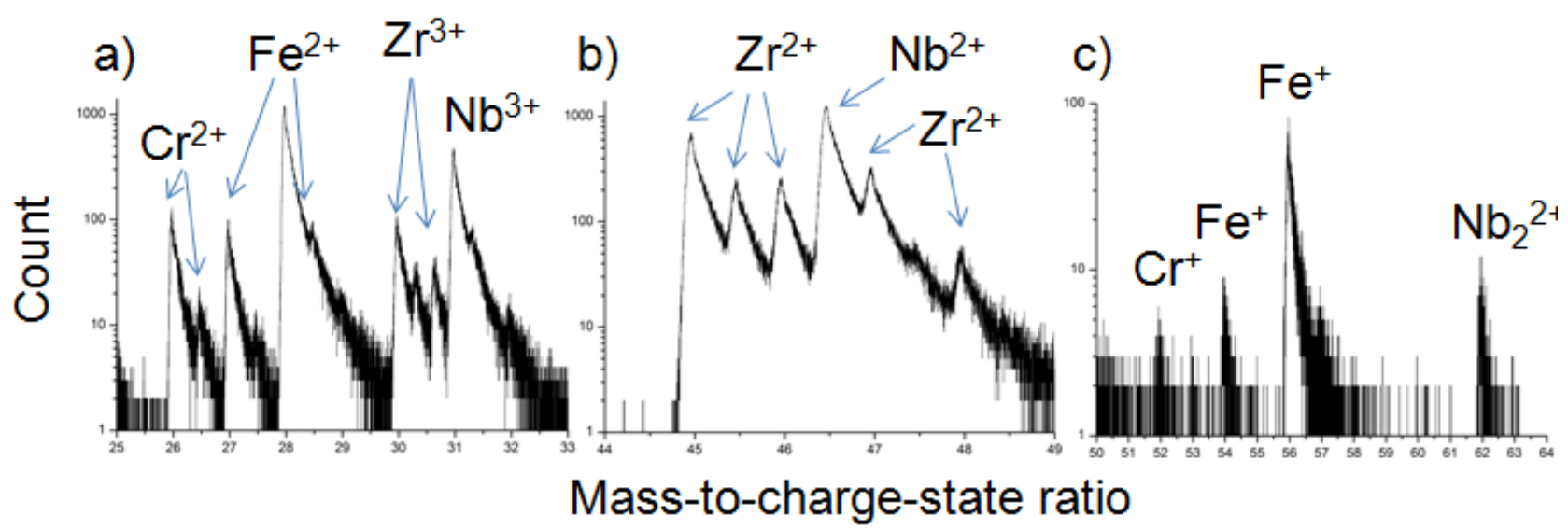

Figure 80: Mass-to-charge state spectra showing the chemical composition within the SPP. Note the semi-logarithmic scale and that (a) and (b) have a different vertical scale to (c). 
Figure 80 shows three sections of the relevant mass-to-charge-spectrum. The mass resolution, related to the thermal conductivity of the material, was poor within the SPP. Several unusual charge states were observed within the particle. The fourth period transition metals present at the $2+$ charge state are visible in (a). The three most significant iron isotopes in the $2+$ charge state are discernible. $0.29 \%$ of iron is also present at 29 Da but cannot be distinguished from the

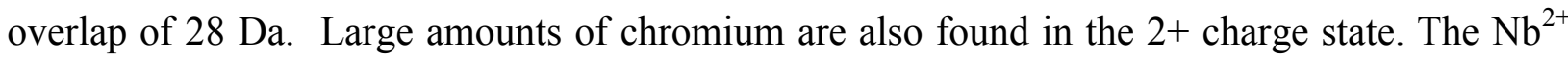
and $\mathrm{Nb}^{3+}$ remain the major niobium-containing peaks, seen in (a) and (b). However, within the particle other $\mathrm{Nb}$ containing charge states are also found. $\mathrm{Nb}_{2}{ }^{2+}$, seen in (c), and $(\mathrm{NbFe})^{2+}$ at 74.5 Da. $\mathrm{Fe}^{+}, \mathrm{Cr}^{+}$are also visible in spectrum (c). This accounts for a very small proportion of the overall iron and chromium content.

\subsubsection{Zr-Nb-Ti}

The $\mathrm{Zr}-\mathrm{Nb}$-Ti test alloy has not been extensively covered by previous publish literature in the same way that ZIRLO has. TEM and EDX have been used to characterise a sample of second phase particles [167]. Seven particles, with diameter ranging from $40-100 \mathrm{~nm}$, a similar distribution as seen in ZIRLO, were characterised. Structural and chemical information regarding these particles is detailed in Table 45. A TEM micrograph and diffraction pattern for SPP \#5 is also given in Figure 81. Atom probe tomography was used to characterise a precipitate with similar composition to SPP \#5, a 3D atom map of which is shown in Figure 82. The diameter of this precipitate is estimated at $40-50 \mathrm{~nm}$. Nickel, albeit at a very low concentration $(0.09 \pm 0.01$ at.\%), chromium and iron are seen to be segregated to the SPP along with iron and niobium. The atom probe allows more precise quantification of the composition than EDX. The concentration of a $25 \mathrm{~nm}$ diameter region of the core of the SPP is shown in Table 46. 


\begin{tabular}{|c|c|c|c|}
\hline \# & $\begin{array}{l}\text { Crystal structure } \\
\text { type identified by } \\
\text { diffraction }\end{array}$ & $\begin{array}{l}\text { Composition measured by EDX } \\
\text { (at. \%) }\end{array}$ & $\begin{array}{l}\text { Diameter } \\
\quad(\mathrm{nm})\end{array}$ \\
\hline 1 & BCC beta-Nb & $\begin{aligned} \mathrm{Zr}(47.5)- & \mathrm{Nb}(51.7)-\mathrm{Fe}(0.3) \\
- & \mathrm{Ti}(0.5)\end{aligned}$ & 40 \\
\hline 2 & $\begin{array}{l}\text { Hex. } \mathrm{Zr}(\mathrm{Fe}, \mathrm{Nb})_{2} \\
\text { or bcc beta- } \mathrm{Nb}\end{array}$ & Not recorded & 70 \\
\hline 3 & $\begin{array}{l}\text { Hexagonal } \\
\mathrm{Zr}(\mathrm{Fe}, \mathrm{Nb})_{2}\end{array}$ & $\begin{array}{c}\mathrm{Zr}(48.6)-\mathrm{Nb}(49.2)-\mathrm{Fe}(0.7)- \\
\mathrm{Ti}(1.0)-\mathrm{Cr}(0.5)\end{array}$ & 40 \\
\hline 4 & $\begin{array}{l}\text { Hexagonal } \\
\mathrm{Zr}(\mathrm{Fe}, \mathrm{Nb})_{2}\end{array}$ & $\begin{array}{c}\mathrm{Zr}(71.6)-\mathrm{Nb}(17.7)-\mathrm{Fe}(8.5)- \\
\operatorname{Cr}(1.1)\end{array}$ & 50 \\
\hline 5 & $\begin{array}{l}\text { Hexagonal } \\
\mathrm{Zr}(\mathrm{Fe}, \mathrm{Nb})_{2}\end{array}$ & $\begin{array}{c}\mathrm{Zr}(52.3)-\mathrm{Nb}(29.3)-\mathrm{Fe}(14.1) \\
-\mathrm{Ti}(2.4)-\mathrm{Cr}(1.8)\end{array}$ & 70 \\
\hline 6 & $\begin{array}{l}\text { Hexagonal } \\
\mathrm{Zr}(\mathrm{Fe}, \mathrm{Nb})_{2}\end{array}$ & Not recorded & 60 \\
\hline 7 & $\begin{array}{l}\text { Hexagonal } \\
\mathrm{Zr}(\mathrm{Fe}, \mathrm{Nb})_{2}\end{array}$ & Not recorded & 100 \\
\hline
\end{tabular}

Table 45: Analysis of intermetallic compounds within the metal matrix of the as-received $\mathrm{Zr}-\mathrm{Nb}$ -

Ti alloy, courtesy of Na Ni, Oxford Materials.
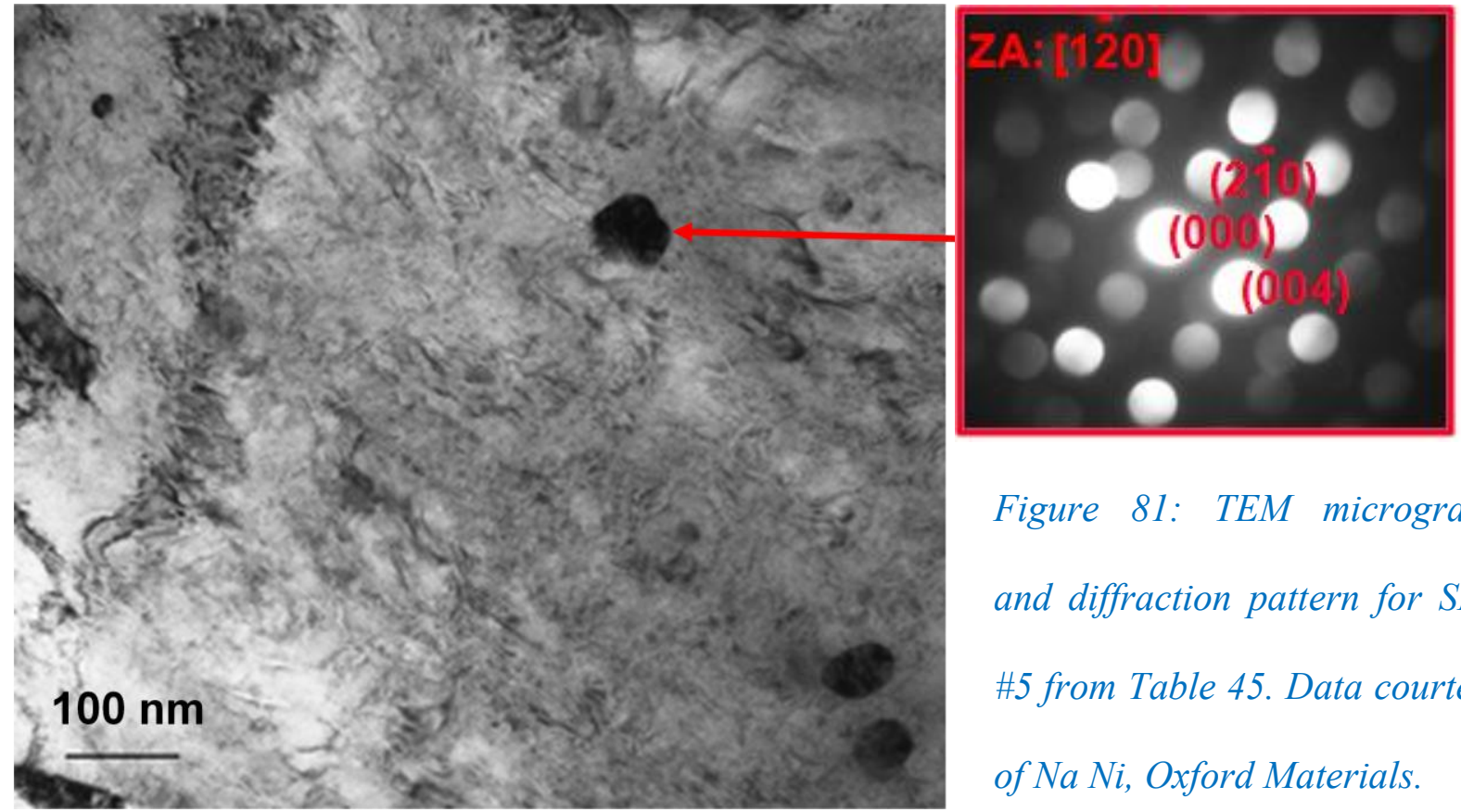

Figure 81: TEM micrograph

and diffraction pattern for SPP

\#5 from Table 45. Data courtesy

of Na Ni, Oxford Materials. 

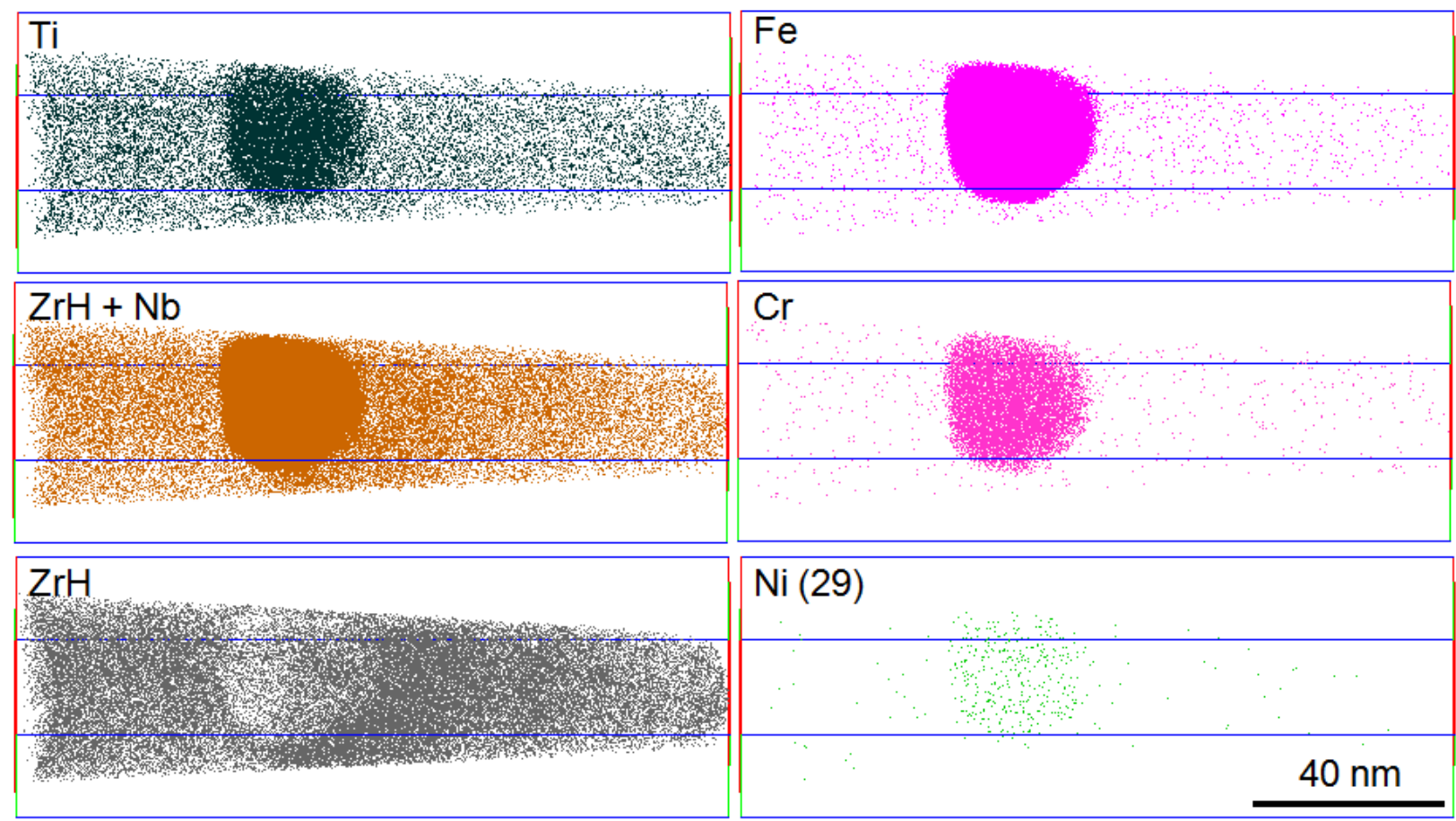

Figure 82: Atom map of a $\mathrm{Zr}$-Nb-Ti specimen containing a second phase particle (SPP).

\begin{tabular}{|c|c|cc|}
\hline Element & $\begin{array}{c}\text { Number } \\
\text { of atoms } \\
\text { (atoms) }\end{array}$ & $\begin{array}{c}\text { Composition } \\
\text { Conc. } \\
\text { (at.\%) }\end{array}$ & $\begin{array}{c} \pm \text { (at.\%) } \\
\text { (at. }\end{array}$ \\
\hline $\mathrm{Zr}$ & 40,838 & 36.63 & 0.144 \\
$\mathrm{O}$ & 517 & 0.46 & 0.01 \\
$\mathrm{Nb}$ & 42,919 & 38.5 & 0.12 \\
$\mathrm{Ti}$ & 2,958 & 2.65 & 0.03 \\
$\mathrm{Fe} \mathrm{(all)}$ & 21,430 & 19.22 & 0.08 \\
$\mathrm{Ni}(29)$ & 95 & 0.09 & 0.01 \\
$\mathrm{Cr}($ all) & 2,717 & 2.44 & 0.03 \\
\hline Total & 111,474 \\
\hline
\end{tabular}

Table 46: Composition of a region at the core of the SPP shown in Figure 82.

The distribution of species within the mass spectra of this particle is similar to that described for ZIRLO in the previous section (5.2.1.1) with the addition of titanium in the $2+$ charge state. From the 1D concentration profile the composition within the precipitate seems stable over its radius and no segregation of solute is seen at the alloy/particle interface. Like the SPP seen in 
ZIRLO, this particle showed no interfacial segregation of any species. However, in another dataset where the specimen appears to have been affected by surface oxidation segregation of iron to a niobium-rich precipitate was observed. This segregation is shown in Figure 84, and the concentration of the core of the particle is given in Table 47. The particle in Figure 82 is a very low iron content $\mathrm{Zr}(\mathrm{Nb}, \mathrm{Fe})_{2}$ type.
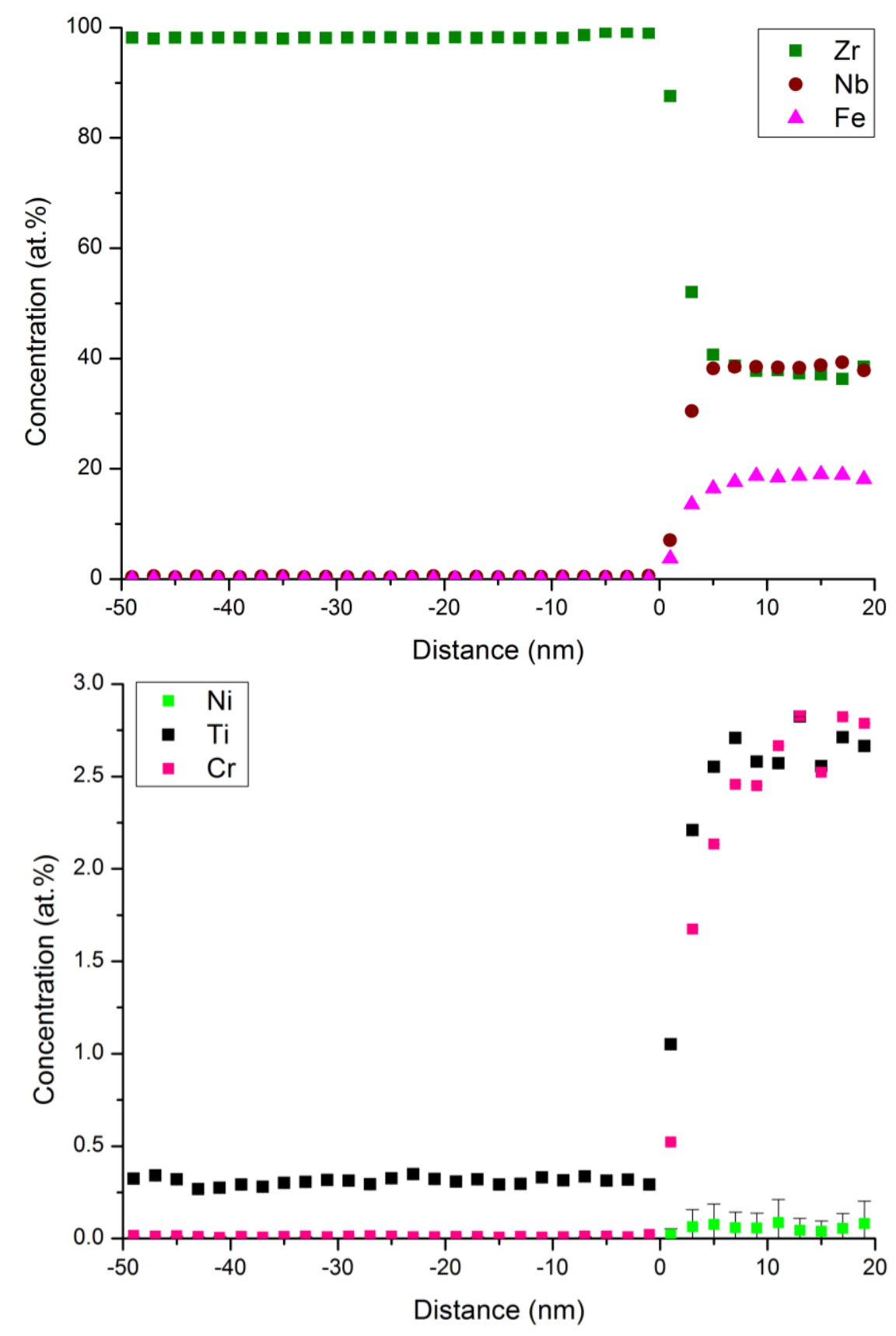

Figure 83: Concentration profile generated by a proxigram across the alloy/particle interface. The particle core is towards the right of the profile. 


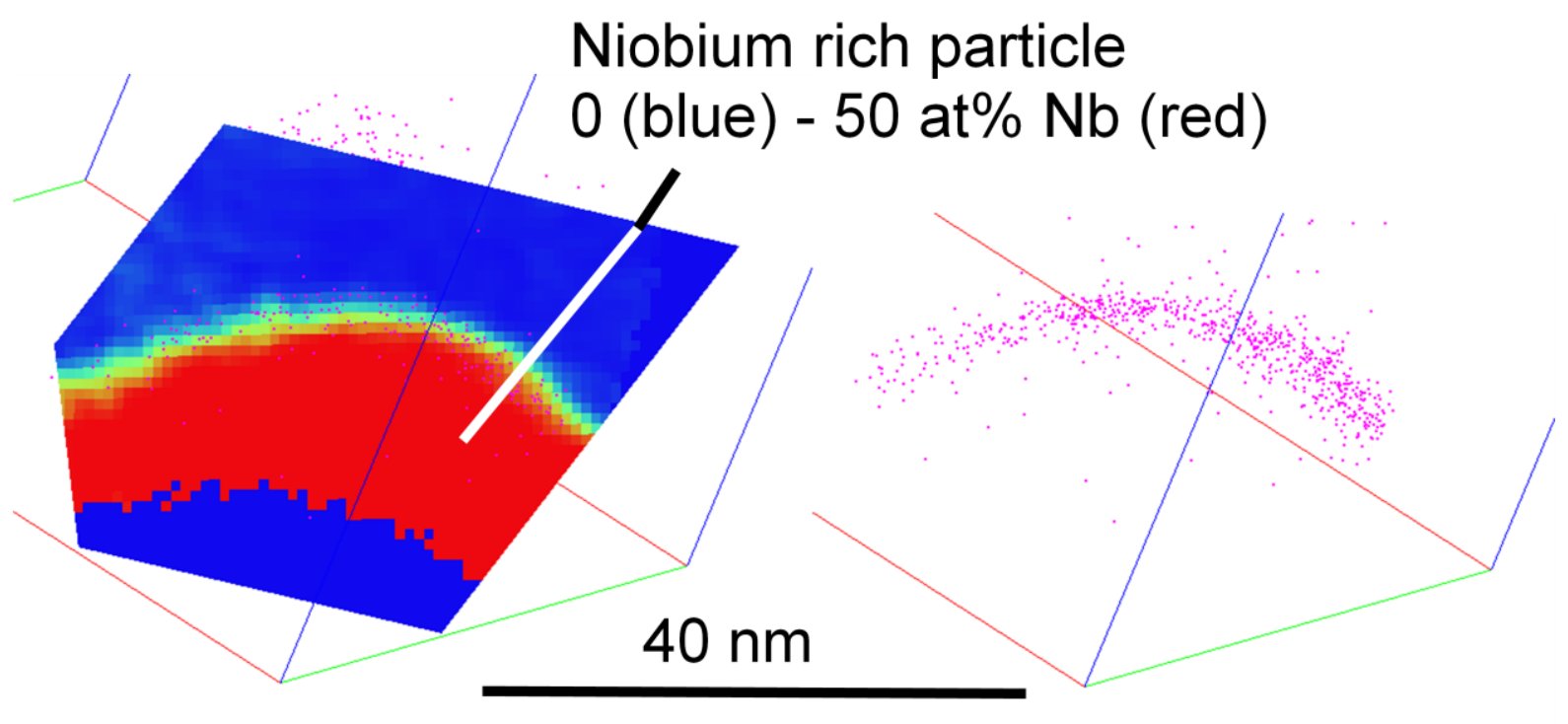

Figure 84: 2D Left) niobium concentration profile and right) an iron 3D atom map (pink dots are $\mathrm{Fe})$. The atom map displays a thin slice $(\sim 10 \mathrm{~nm})$ through the dataset, orientated in plane with the page. This removes the effect of viewing the iron distributed across the entire curved surface of the hemisphere.

\begin{tabular}{|l|r|rr|}
\hline Element & $\begin{array}{c}\text { Number } \\
\text { of atoms } \\
\text { (atoms) }\end{array}$ & $\begin{array}{c}\text { Composition } \\
\text { Conc. } \\
\text { (at.\%) }\end{array}$ & $\begin{array}{r} \pm 1 \sigma \\
\text { (at.\%) }\end{array}$ \\
\hline $\mathrm{Zr}$ & 8,684 & 32.5 & 0.3 \\
$\mathrm{Nb}$ & 17,457 & 65.3 & 0.5 \\
$\mathrm{Ti}$ & 221 & 0.83 & 0.06 \\
$\mathrm{O}$ & 337 & 1.26 & 0.07 \\
$\mathrm{Fe}$ & 16 & 0.06 & 0.02 \\
\hline Total & 26,715 & & \\
\hline
\end{tabular}

Table 47: Concentration within the niobium rich precipitate shown in Figure 83.

3DAP analysis of this type of particle provides additional information that is not available from conventional microscopy. Precise chemical analysis of individual particles provides a basis for analysing corroded specimens and gives information regarding the location of very low concentration solute (e.g. Ni) and the nature of the particle/matrix interface. 


\subsubsection{Grain boundaries}

\subsubsection{Introduction}

The chemical sensitivity and high spatial resolution of the atom probe is capable of detecting segregation of alloying additions to interfaces that might not be possible using conventional electron microscopy. Analysis of grain boundaries in zirconium alloys and the variation in their chemistry has not been directly studied and so there is very little understanding of their effect on corrosion resistance, mechanical creep, or irradiation stability. In this section, a handful of grain boundaries in as-received materials are analysed to act both as a benchmark for comparison with behaviour in corroded materials, and as a early step in characterising the chemical behaviour at these interfaces.

The analysis methodology used to analyse grain boundaries studied using $3 \mathrm{D}$ atom probe is introduced in 5.2.2.2. This describes the important features of the 3D atom map and cumulative concentration profile of the first example, a ZIRLO grain boundary, in a generalised way. The specific chemical properties of this boundary are described in 5.2.2.3, along with a second example of a ZIRLO grain boundary with different interfacial characteristics. A Zr-Nb-Ti boundary has also been examined, and these data are presented in 5.2.2.4. These results are compared and discussed in 5.2.2.5. Section 5.2.2.6 summarises the properties of the grain boundaries analysed in 'as-received' material.

\subsubsection{Grain boundary analysis}

Figure 85 shows 3D atom maps of a specimen containing a grain boundary. The feature is unambiguously identifiable as a boundary as its image is two-dimensional, as opposed to dislocation networks that might be present in the material. Solute segregated to dislocations 
would be visible in the atom probe as distinct one-dimensional arrangements. The grain boundary maps in Figure 83 have been oriented so that the view is along the plane of the grain boundary. Local magnification effects occur at interfaces observed in the atom probe [168] which limits the spatial resolution that can be achieved mapping species in these regions. For example, field-focussing can occur in the region of interfaces, leading to an apparent increase in total atom density in that region. This is evident in Figure 85 (a), which shows a map of all of the ions detected, regardless of their identity. This acts as a further confirmation of the presence of the boundary.

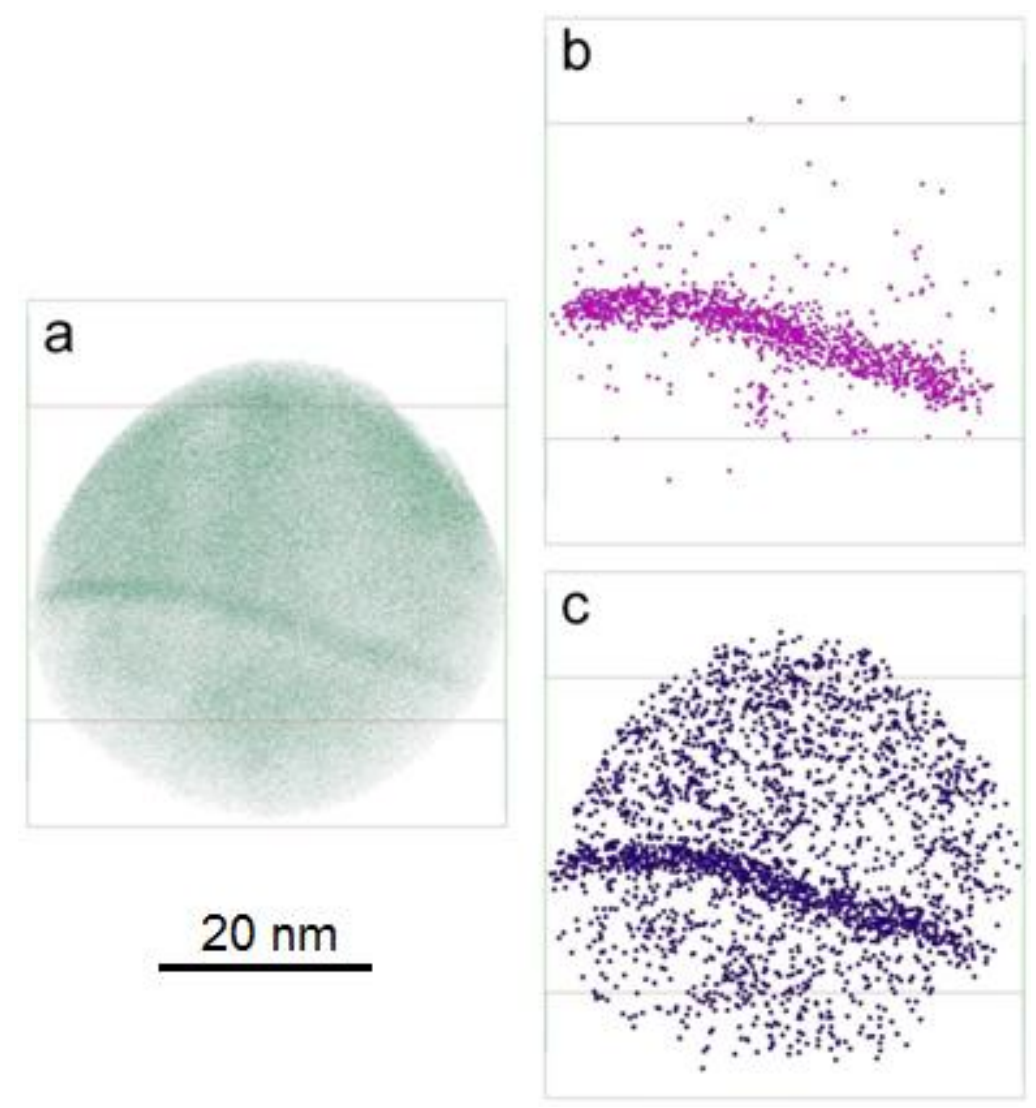

Figure 85: 2D view along a grain boundary from 3DAP analysis of ZIRLO showing a) all atoms, b)iron atoms and c) niobium atoms. The higher apparent density of atoms in (a) is due to local magnification effects encountered by 3DAP at boundaries. 
The relative proportions of the various atomic species present at different locations across the boundary can be determined using cumulative concentration profiles [169-170]. Cumulative profiles are calculated across the grain boundary by selecting cylindrical regions perpendicular to the interface within the $3 \mathrm{D}$ atom map reconstruction. These profiles are plots of the cumulative number of atoms of a particular solute against the total number of atoms detected along this region, normal to the interface. The gradient of each curve at a given point describes the instantaneous concentration at that position in the cross section. This method excludes effects relating to the boundary geometry. The disadvantage of this approach is that it reduces the sample size and so increases the statistical uncertainty related to an observation. Cumulative compositional profiles, taken across the boundary from Figure 85, are shown in Figure 86 (found below in section 5.2.2.3).

Quantitative measurement of the number of "equivalent monolayers" of excess solute present at the boundary can be obtained by calculation of the Gibbsian boundary excess, $\Gamma_{\mathrm{I}}$, using these cumulative compositional profiles. Several profiles were taken at different positions on the interface and their results averaged in order to improve the significance of the measurement. For an interface such as a grain boundary we would expect to find that the material in the grains on either side shares a common composition. For such a boundary the number of excess atoms detected at the interface, $N_{\text {excess }}$, can readily be found by measuring the distance between the two parallel regions of the profile that represent the concentration of solute within either grain. An estimate of the amount of solute at the boundary can be made using

$$
\Gamma=\frac{N_{\text {excess }}}{e_{d} A}
$$

where $\Gamma$ is the excess amount of solute in terms of atoms per unit area, $e_{d}$, is the atom probe detector efficiency, and $A$ is the surface area of the region of interest over which the cumulative 
composition profile was taken. For the LEAP $e_{d} \sim 0.38$. For the grain boundary case where a cumulative profile is created with equal distance on either side of the interface, this can be more usefully expressed as

$$
\Gamma_{i}=\frac{N\left(c_{a}-c_{m}\right)}{e_{d} A}
$$

where $N$ is the total number of atoms in the cross section, $C_{a}$ is the average concentration of a solute, $i$, over the entire profile and $C_{m}$ is the concentration of a solute, $i$, in matrix region.

The calculated value for the Gibbsian excess can be related to known properties of the material to give an estimate of the excess solute at the interface in terms of an equivalent number of monolayers of material [131],

$$
\Phi_{i}=\frac{\Gamma_{i}}{\rho \omega}
$$

where $\rho$ is the average density of the material and $\omega$ is the closest matrix plane spacing. These properties are taken to be the density and basal (0001) plane spacing of $\alpha-\mathrm{Zr}, \rho=43.0$ atom.nm ${ }^{-3}$ and $\omega=0.323 \mathrm{~nm}[63]$ respectively.

Enhancement factors for individual solutes at the interface can be estimated by making the simplifying assumption that the excess solute is concentrated in a single atom layer at the plane of the interface. This analysis is best suited to the situation where the enrichment factor is high, i.e. the concentration of solute at the boundary is much higher than that in the matrix, and where the concentration of solute within the grains on either side of the interface is uniform. 


\subsubsection{ZIRLO}

Example 1

The 3D atom maps shown in Figure 85 relate to a grain boundary in ZIRLO reconstructed from voltage-pulsed atom probe data. The 3D atom maps in Figure 85 (b) and (c) demonstrate the spatial distribution of iron and niobium respectively. The extent of the non-uniformity in the distribution of these species is much greater than that due to the field focussing effects seen in Figure 85 (a). Segregation of iron and niobium to the boundary region is clearly evident, as is the niobium present in solid solution. This substantial enrichment of iron and niobium at the boundary is also apparent in the cumulative concentration profile Figure 86 . The other elements ( $\mathrm{Sn}, \mathrm{O}, \mathrm{C}$, and $\mathrm{N}$ ) are not segregated to the boundary. The constant oxygen concentration across the interface suggests that this grain boundary was not acting as a short circuit route for oxidation, despite its proximity to the zirconium-air interface after specimen preparation. 


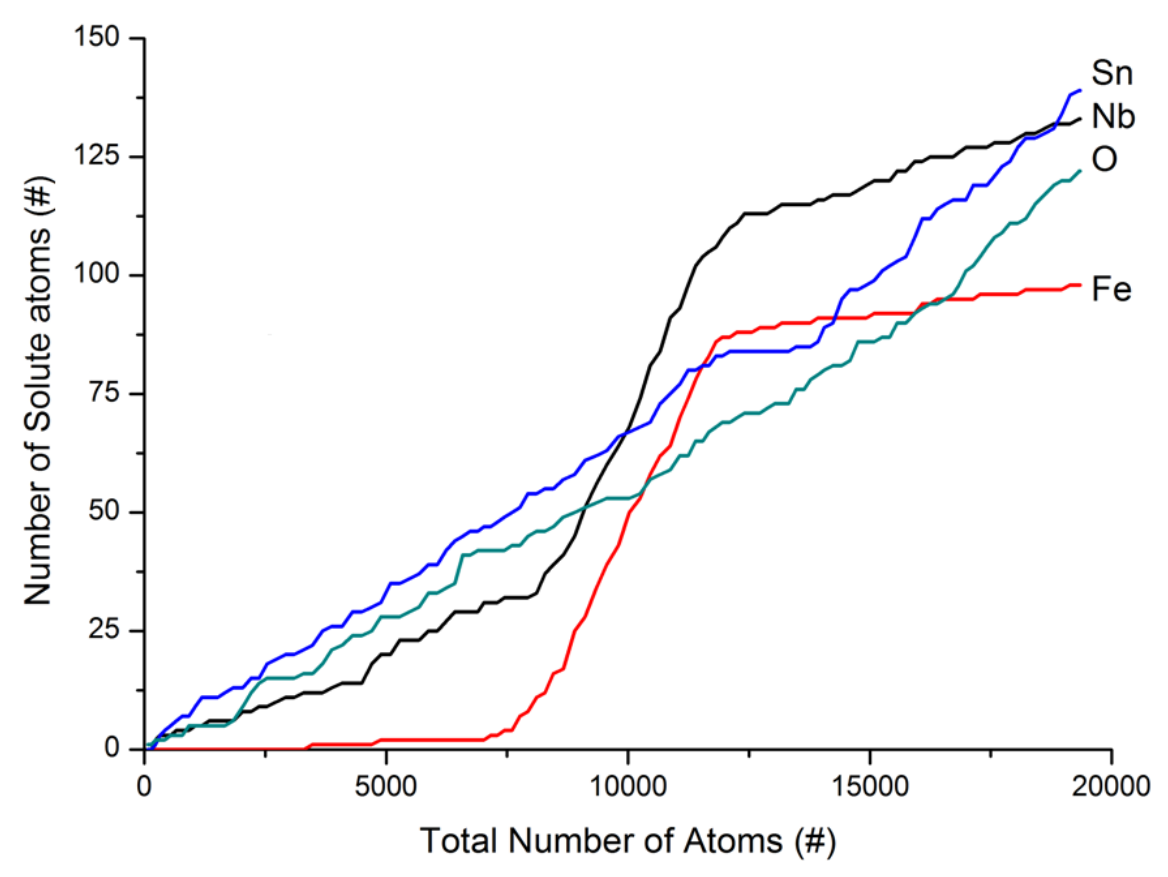

Figure 86: Cumulative composition profile showing the number of solute atoms detected across the ZIRLO grain boundary. A cylindrical region is selected perpendicular to the interface with equal length entering both grains.

Figure 87 demonstrate that the ratios of the peaks at $28 \mathrm{Da}$ and $28.5 \mathrm{Da}$ agree well with the isotopic ratio of iron. No significant amounts of chromium or nickel were detected at or around this particular grain boundary. The main species detected were identified as containing $\mathrm{Zr}, \mathrm{Nb}$, Sn, C, N, O and Fe. The background noise levels seen in the mass spectrum is low. The composition of the matrix (defined as material inside the grain interiors and more than $10 \mathrm{~nm}$ away from the interface) is summarised in Table 48. The observed level of iron in solution agrees well with the base level seen in section 5.1.6.2. As expected, appreciable quantities of niobium, tin and oxygen are found to be in solid solution in this dataset. 


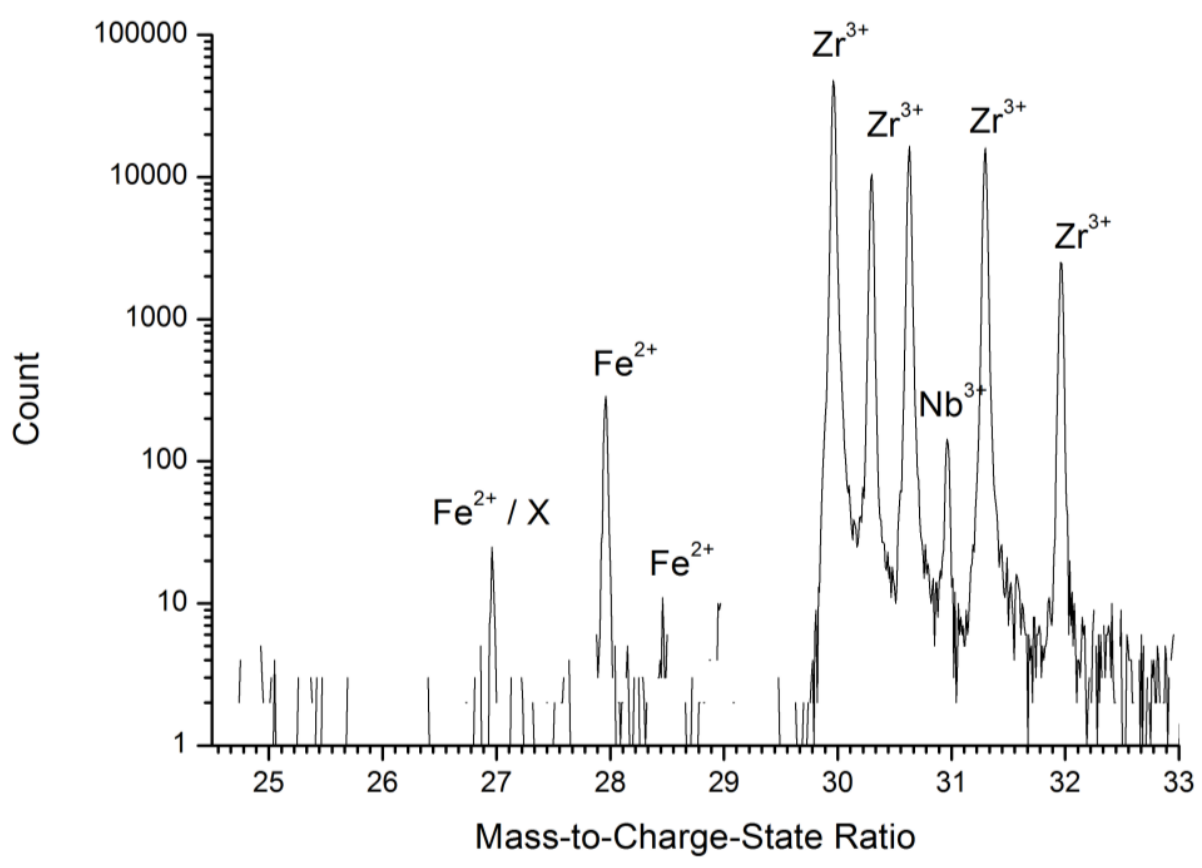

Figure 87: Mass spectrum showing $4^{\text {th }}$ period transition metals in the $2+$ charge state and $\mathrm{Zr}$ and $\mathrm{Nb}$ in the $3+$ charge state in this ZIRLO specimen.

\begin{tabular}{|c|cc|cc|c|}
\hline Element & \multicolumn{2}{|c|}{ Matrix } & \multicolumn{2}{c|}{ Boundary $^{(1)}$} & Boundary $^{(2)}$ \\
& Conc. & $\pm 1 \sigma$ & Conc. & $\pm 1 \sigma$ & Conc. \\
(at.\%) & $($ at.\%) & (at.\%) & $($ at.\%) & (at.\%) \\
\hline $\mathrm{Zr}$ & 98.31 & 0.3 & 93.90 & 0.5 & 95.6 \\
$\mathrm{Nb}$ & 0.52 & 0.02 & 1.83 & 0.07 & 2.1 \\
$\mathrm{Sn}$ & 0.69 & 0.02 & 0.74 & 0.04 & 1.0 \\
$\mathrm{Fe}$ & 0.003 & 0.001 & 2.84 & 0.09 & 0.5 \\
$\mathrm{O}$ & 0.39 & 0.02 & 0.56 & 0.04 & 0.9 \\
$\mathrm{C}$ & 0.055 & 0.006 & 0.051 & 0.011 & 0.02 \\
\hline
\end{tabular}

Table 48: Bulk composition of ZIRLO matrix and grain boundary. The matrix data are taken from a region more than $10 \mu m$ away from the grain boundary. The boundary composition has been calculated using two different methods: 1) The interface region is classed as the volume within an iron concentration isosurface of $0.8 \%$ around the interface, approximately $5 \mathrm{~nm}$ diameter in reconstruction space. The uncertainty quoted is one standard deviation. 2) The boundary concentration has been determined by the maximum gradient of the curves shown in Figure 86. The two metrics agree well, except for the iron concentration. 
It is estimated that the equivalent of $(0.33 \pm 0.03)$ monolayers of iron are segregated to the interface, where the uncertainty in the measurement represents one standard deviation over seven interface cross sections. Niobium segregated to a lesser extent, with $(0.15 \pm 0.04)$ monolayers of excess material at the grain boundary. There was a much greater relative variation in the measured excess niobium than iron.

Two methods of calculating the boundary composition are used in Table 48. Both are subject to limitations. The boundary composition given by boundary ${ }^{(1)}$ calculates the composition of a fixed volume of atoms within isosurfaces defined at the interface. The uncertainty quoted is calculated from the counting statistics associated with this volume, not variation caused by the choice of volume definition. The second method (boundary ${ }^{(2)}$ ) calculates the composition from the gradient of the cumulative concentration profiles in Figure 86. Although this can be achieved by averaging over several points on this section of the curve, the value is very sensitive to noise. The agreement between the two values is good, except for the iron concentration where the measurements differ by a factor of five. This may be due to the lighter species such as iron being affected by the local magnification at the interface more than the heavier elements and so affecting the apparent distance over which the iron atoms are distributed. Enhancement factors produced by comparison of the matrix and boundary composition must be treated with this limitation in mind. The enhancement factor for iron is very sensitive to the uncertainty in the measurement of its matrix and boundary concentration. However, it is of the order of hundreds of times. The enhancement factor for niobium can be estimated with more certainty, and is found to be $3-4$. Qualitatively, the presence of enhancement at the interface can be judged by visual inspection of cumulative concentration profiles. 
Figure 88 shows a cumulative concentration profile across a different grain boundary separating two regions of metal matrix with higher iron content (as described in 5.1.6.2). The oxygen concentration of the metal matrix is also towards the higher end of the range and the niobium matrix concentration is similar to that seen in the previous example. The composition of the matrix and boundary are given in Table 49. The iron is enriched at this interface, but only by a factor of $\sim 20$. This is not only due to the higher matrix concentration. The absolute amount of excess iron segregated to the matrix per unit area is also lower by a factor of ten. Chromium, which was not present in detectable quantities in the grain boundary shown in Figure 85, is seen to be enriched at this interface by a factor of the same order as iron. Niobium, which was also previously seen to be enriched at the grain boundary shown in Figure 85, shows no enrichment at this interface. The interstitial species $\mathrm{O}$ and $\mathrm{C}$ showed no apparent segregation in the previous example. In this case these species demonstrate a very slight preference for grain boundary occupation. Tin is not segregated to any of the boundaries studied.

\begin{tabular}{|c|ccc|c|ccc|}
\hline Element & \multicolumn{3}{|c|}{ LHS Matrix } & Boundary & \multicolumn{3}{c|}{ RHS Matrix } \\
& $\begin{array}{c}\text { No. } \\
\text { (atoms) }\end{array}$ & $\begin{array}{c}\text { Conc. } \\
\text { (at.\%) }\end{array}$ & $\begin{array}{c} \pm \sigma \\
\text { (at.\%) }\end{array}$ & $\begin{array}{c}\text { Conc. } \\
\text { (at.\%) }\end{array}$ & $\begin{array}{c}\text { No. } \\
\text { (atoms) }\end{array}$ & $\begin{array}{c}\text { Conc. } \\
\text { (at.\%) }\end{array}$ & $\begin{array}{c} \pm \sigma \\
\text { (at.\%) }\end{array}$ \\
\hline $\mathrm{Zr}$ & $4,622,816$ & 97.41 & 0.007 & 96.6 & $4,897,776$ & 97.37 & 0.007 \\
$\mathrm{Nb}$ & 22,220 & 0.47 & 0.01 & 0.5 & 25,031 & 0.50 & 0.01 \\
$\mathrm{Sn}$ & 35,841 & 0.755 & 0.004 & 0.8 & 38,402 & 0.763 & 0.004 \\
$\mathrm{Fe}$ & 1,048 & 0.0221 & 0.0007 & 0.4 & 1,217 & 0.0242 & 0.0007 \\
$\mathrm{Cr}$ & 23 & 0.0005 & 0.0001 & 0.01 & 62 & 0.0012 & 0.0002 \\
$\mathrm{O}$ & 63,331 & 1.334 & 0.005 & 1.7 & 66,823 & 1.329 & 0.005 \\
$\mathrm{C}$ & 435 & 0.0092 & 0.0004 & 0.04 & 620 & 0.0123 & 0.0005 \\
\hline
\end{tabular}

Table 49: Compositional information derived from the two grains shown in Figure 71 (section 5.1.6.2) calculated using a bulk region of interest defined at least $10 \mathrm{~nm}$ away from the grain boundary and the local composition at the boundary as determined from the maximum slopes of the cumulative concentration profiles in Figure 88. 

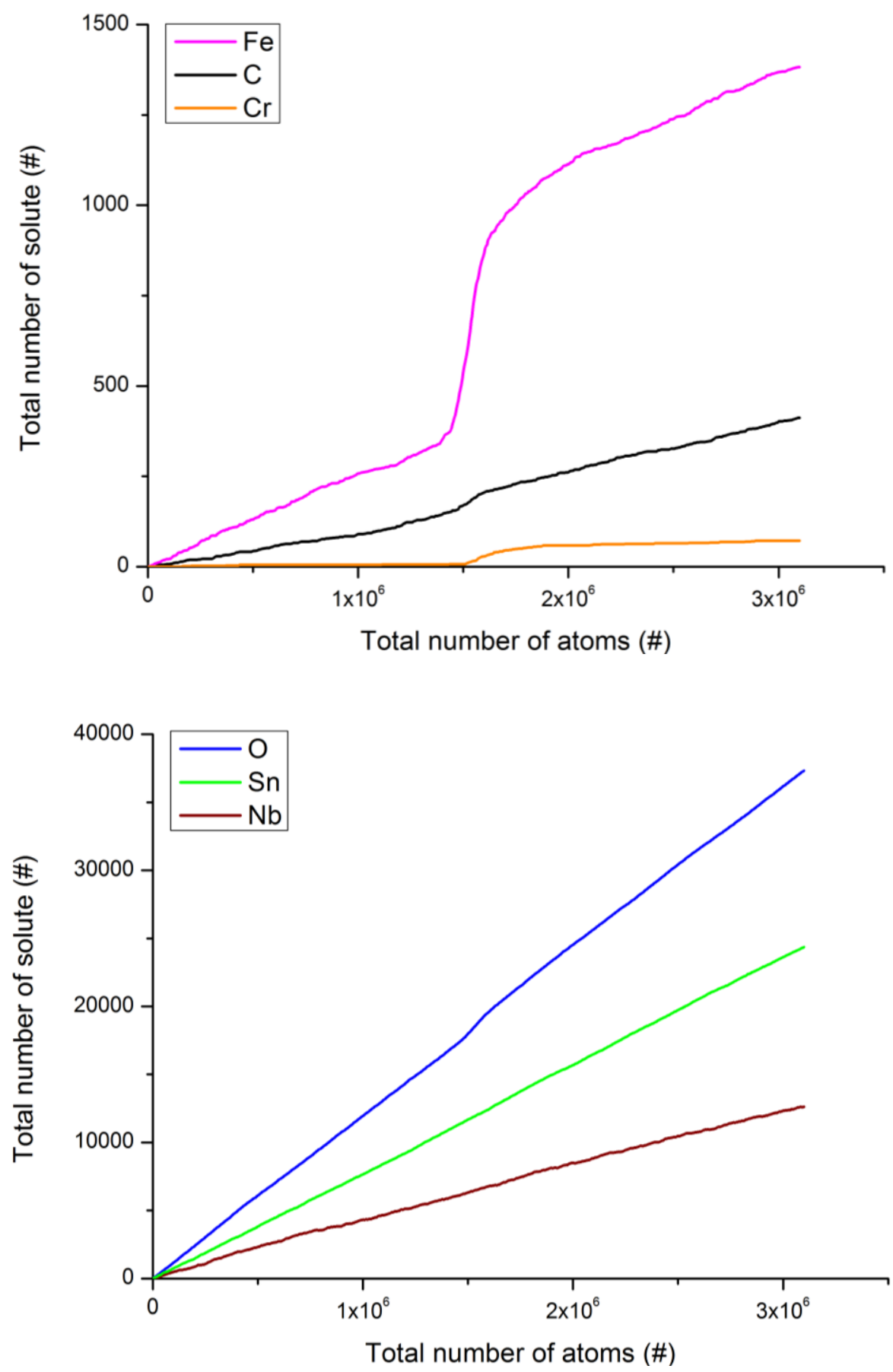

Figure 88: Cumulative composition profile showing the number of solute atoms detected across the grain boundary. A cylindrical region is selected perpendicular to the interface, and extending an equal distance into each grain. 


\subsubsection{4 $\mathrm{Zr}-\mathrm{Nb}-\mathrm{Ti}$}

Two grain boundaries of the $\mathrm{Zr}-\mathrm{Nb}$-Ti test alloy were analysed in the as-received condition in this study, one was corroded by the ambient environment of the needle-like specimen, rather than any intentional treatment. The other did not show signs of corrosion around the grain boundary region, and is described here in comparison to the ZIRLO alloy grain boundary behaviour (5.2.2.3) and the $\mathrm{Zr}-\mathrm{Nb}-\mathrm{Ti}$ alloy matrix chemistry (5.1.8).

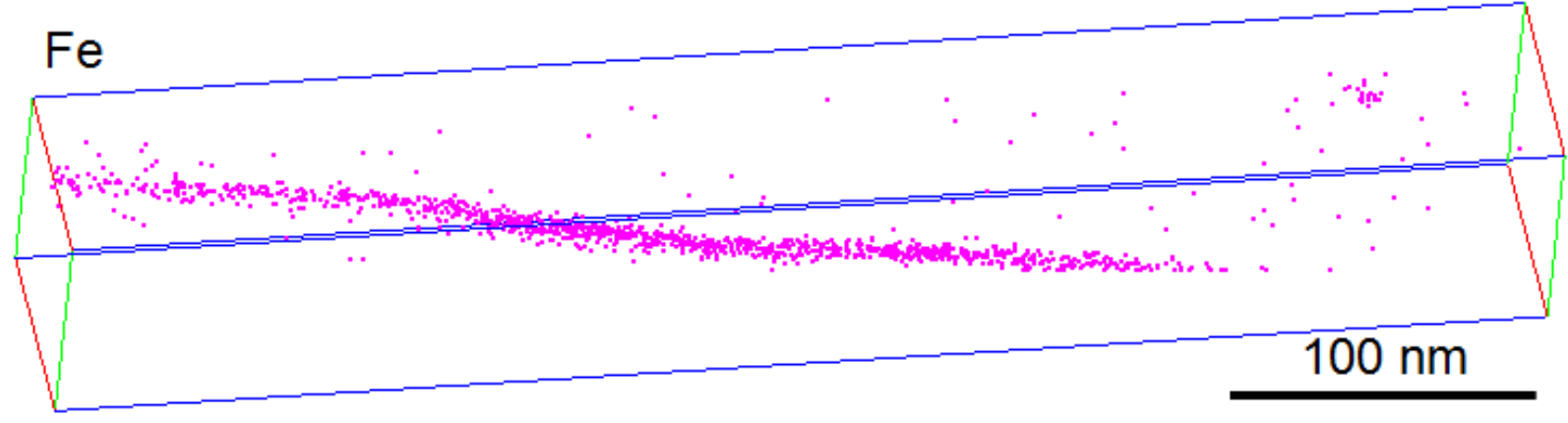

Figure 89: 3D atom map showing the iron distribution in the region of interest.

A $3 \mathrm{D}$ atom map showing the distribution of iron within the dataset from the uncorroded grain boundary is presented in Figure 89. A 2D concentration profile has been prepared from a section of this interface. A cumulative version of this profile is shown in Figure 90 for the solutes $\mathrm{O}, \mathrm{Nb}$, $\mathrm{Ti}$ and Fe. Iron, as seen in the ZIRLO specimens is enriched at the grain boundary. There is slight enrichment of $\mathrm{O}$ and $\mathrm{Nb}$ at the grain boundary and no significant enrichment of titanium at the site studied. The matrix chemistry of the grains on either side of the interface agrees well with the alloy matrix chemistry of $\mathrm{Zr}-\mathrm{Nb}-\mathrm{Ti}$. The composition of the grains, and a local composition at the interface obtained from the gradient of the curves in Figure 90, is shown in Table 50 . 


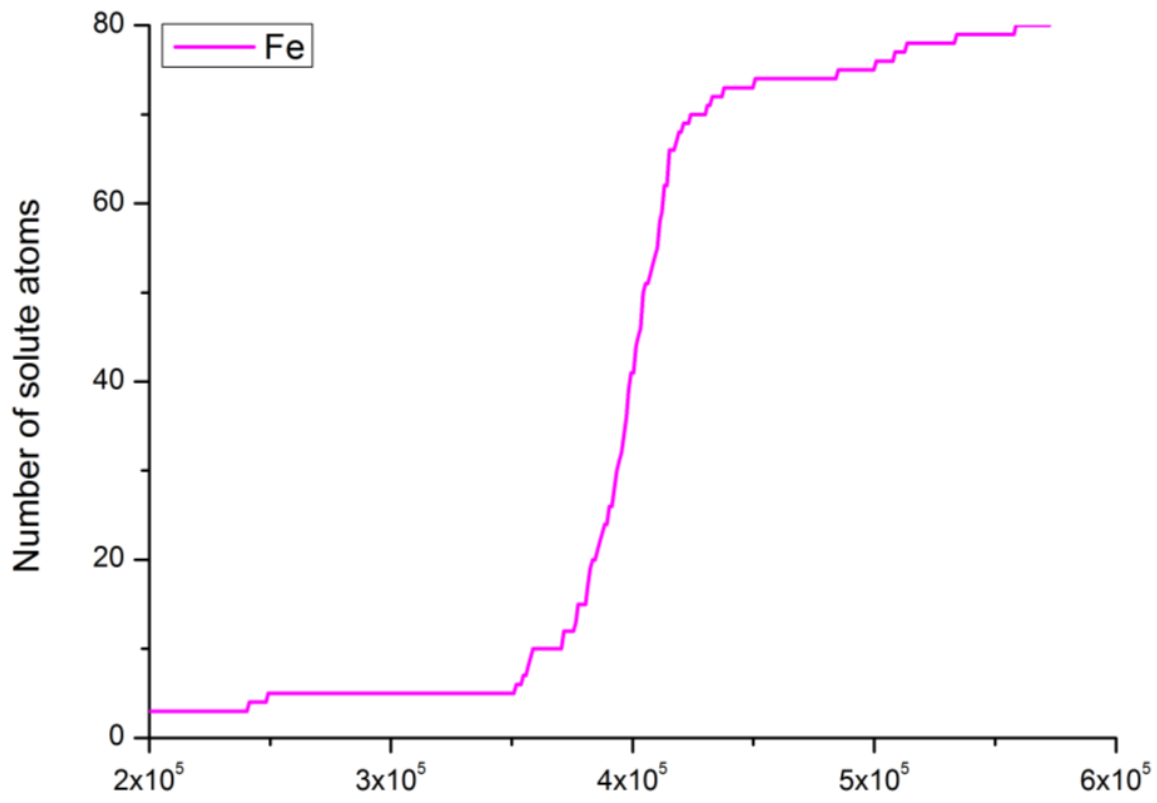

Total number of atoms

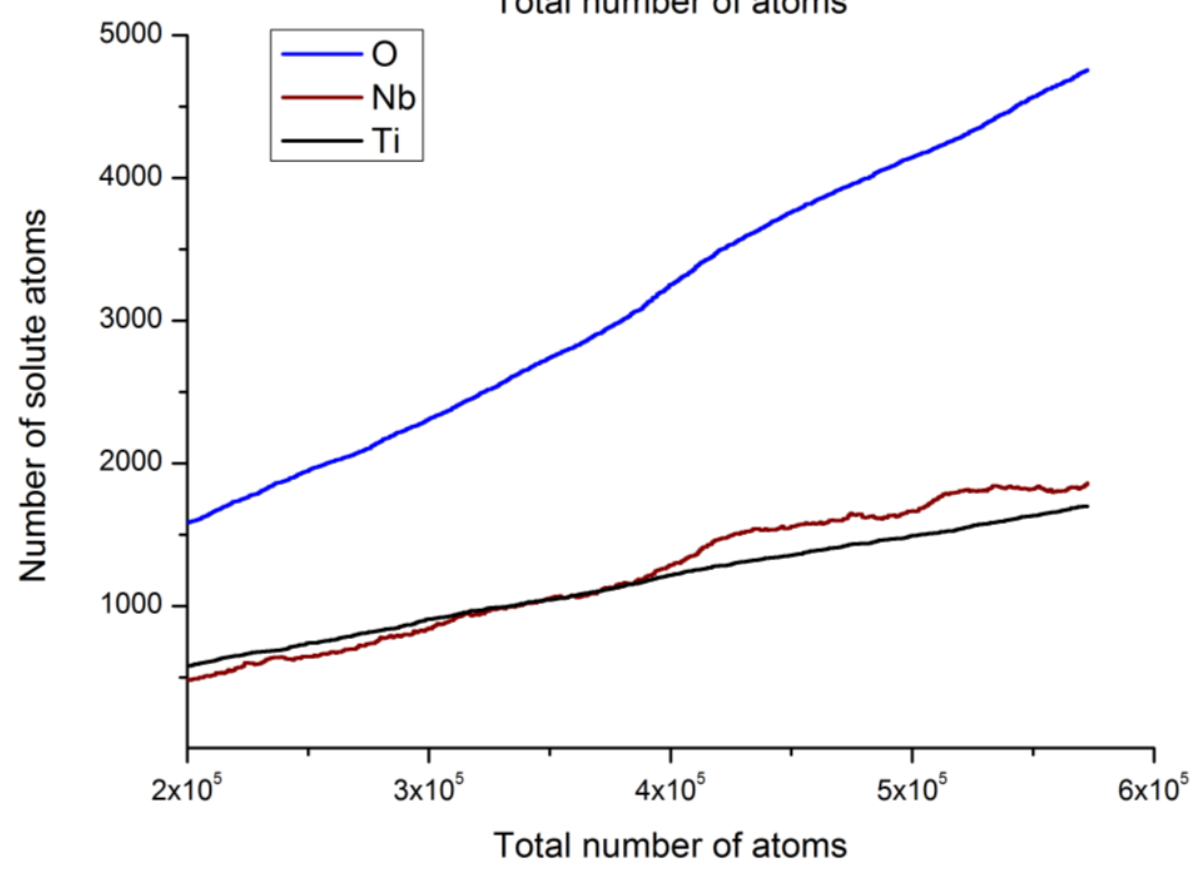

Figure 90: Cumulative composition profiles showing the number of solute atoms detected across the $\mathrm{Zr}-\mathrm{Nb}$-Ti grain boundary. A cylindrical region is selected perpendicular to the interface. An equal distance in each grain is shown. Iron is displayed on a different vertical scale to oxygen, niobium and titanium. Niobium appears noisy due to the on-going correction for $\mathrm{ZrH}$ ions present at $2+$. 


\begin{tabular}{|c|ccc|c|ccc|}
\hline Element & \multicolumn{3}{|c|}{ LHS Matrix } & Boundary & \multicolumn{3}{c|}{ RHS Matrix } \\
\cline { 2 - 8 } & $\begin{array}{c}\text { No. } \\
\text { (atoms) }\end{array}$ & $\begin{array}{c}\text { Conc. } \\
\text { (at.\%) }\end{array}$ & $\begin{array}{c} \pm 1 \sigma \\
\text { (at.\%) }\end{array}$ & & $\begin{array}{c}\text { No. } \\
\text { (atoms) }\end{array}$ & $\begin{array}{c}\text { Conc. } \\
\text { (at.\%) }\end{array}$ & $\begin{array}{c} \pm 1 \sigma \\
\text { (at.\%) }\end{array}$ \\
\hline $\mathrm{Zr}$ & 360,464 & 98.14 & 0.023 & 97.2 & $7,858,423$ & 98.405 & 0.004 \\
$\mathrm{Nb}$ & 1,910 & 0.52 & 0.012 & 0.9 & 24,831 & 0.311 & 0.002 \\
$\mathrm{Ti}$ & 1,218 & 0.33 & 0.01 & 0.4 & 25,992 & 0.325 & 0.002 \\
$\mathrm{Fe}$ & 17 & 0.0046 & 0.0011 & 0.1 & 221 & 0.0028 & 0.0002 \\
$\mathrm{O}$ & 3,861 & 1.051 & 0.017 & 1.4 & 76,232 & 0.955 & 0.003 \\
$\mathrm{C}$ & 11 & 0.003 & 0.0009 & 0.005 & 64 & 0.0008 & 0.0001 \\
\hline
\end{tabular}

Table 50: Compositional information derived from the two grains shown in Figure 89, calculated using a bulk region of interest defined at least $10 \mathrm{~nm}$ away from the grain boundary and the instantaneous composition at the boundary as determined from the cumulative concentration profile in Figure 90.

The iron concentration in the matrix region of both grains correspond with the low $\mathrm{Fe}$ concentration matrix $(\sim 0.003$ at.\%) seen in many ZIRLO and Zr-Nb-Ti specimens. The enrichment of iron to the grain boundary is of the order of a factor of $\sim 40$. This is lower than the factor seen in the ZIRLO material with a similar matrix concentration and the absolute amount of excess iron at the boundary (per unit area) is lower than was seen in either of the ZIRLO examples.

\subsubsection{Discussion}

As might be expected, the behaviour of solute at grain boundaries in the as-received material shows variation depending upon the locality of the boundary studied. From the three examples described we can draw limited but insightful conclusions on the possible range of behaviour of solute at such interfaces in this material. A summary of the data from each boundary is presented in a consistent form in Table 51 (see page 195), giving the chemistry of the grains either side of the interface and the composition at the boundary as determined from cumulative concentration 
profiles. The enhancement factors determined from these data are given, as are relative excess figures for each solute.

Using data from [160] the Goldschmidt atomic radii for atoms in conditions of 12-fold symmetry are $\operatorname{Zr} 1.6 \AA$, Sn $1.58 \AA$, Nb $1.47 \AA$, Fe $1.28 \AA$, Cr $1.28 \AA, \mathrm{C} 0.77 \AA, \mathrm{N} 0.71 \AA$ and $\mathrm{O} 0.60 \AA$. From these data the substitutional atoms with smaller mismatch with zirconium are seen to have lower excess at the boundary.

There is disagreement within the literature as to whether iron is a substitutional or interstitial element in $\alpha-\mathrm{Zr}$, due to its fast diffusion and intermediate size [171]. Based on its atomic radius, iron has a mismatch greater than $20 \%$ with zirconium; therefore one might expect it to be a low solubility substitutional alloying addition. Iron segregates to the boundary more strongly than any other element. The degree of iron segregation is similar in terms of solute concentration (or excess) at the boundary, and this is independent of the matrix chemistry, which was seen to vary between the different examples, as would be expected from the study in 5.1.6. Zirconium alloys are generally resistant to stress-corrosion cracking [172], suggesting that excess iron at the grain boundary does not lead to enhanced corrosion in these systems.

Chromium atoms are approximately the same size as iron and are not enriched at the interface in the first example. However, there is also no chromium in the matrix locally, which limits the local supply of material to segregate.

Niobium has a $7.5 \%$ mismatch with zirconium. In the first ZIRLO example niobium is seen to be segregated to the interface, although to a much less extent than iron. In the second ZIRLO grain boundary niobium shows no segregation. There are two possible mechanisms by which the segregation of niobium (and iron) to the boundary could have occurred, during the $\beta \rightarrow \alpha$ transition or during annealing and cold working within the $\alpha$ phase. The quantities of niobium and iron present in this alloy are both completely soluble in the $\beta$ phase. On quenching this 
material is rejected from the formation of the $\alpha$ matrix. It is likely that much of the iron and niobium found at the boundary was rejected during the allotropic phase transformation. In other systems, local solute redistribution is seen during the $\beta \rightarrow \alpha$ transition, and this could explain the variation in the observed behaviour of niobium. The variation in the behaviour of niobium could also be explained by difference in the boundary energy between the two sets of grains encountered, where the mismatch is greater in the first ZIRLO example and promotes the segregation of niobium and iron to the boundary. If the local degree of disorder between the grains in the second example were less, the driving force for segregation would be reduced, resulting in the lack of niobium segregation. For the $\mathrm{Zr}-\mathrm{Nb}-\mathrm{Ti}$ alloy, the low (but detectable) segregation of niobium to the interface is also between the values seen in the two ZIRLO examples, which could suggest the interfacial energy at this interface is greater than that seen in the second ZIRLO grain boundary. It is not possible to determine the relative grain orientation from this atom probe data as the spatial resolution of the instrument is insufficient. Field ion microscopy of such specimens would give the required information, but this method results in a very high specimen failure rate.

Interstitial solutes such as carbon, nitrogen and oxygen show very limited segregation to homophase interfaces. This is presumably related to their high solid solubility in alpha zirconium, and is in marked contrast to their behaviour in BCC metals such as iron, where segregation of interstitial elements to grain boundaries is very pronounced. The segregation of these interstitial elements is the reverse of that seen for the substitutional elements; no segregation is seen in the 'high energy' ZIRLO grain boundary ( $1^{\text {st }}$ example) and a small degree of segregation was seen in the 'low energy' ZIRLO grain boundary ( $2^{\text {nd }}$ example). In the $\mathrm{Zr}-\mathrm{Nb}-$ Ti sample, segregation of interstitial oxygen to the grain boundary is observed, but only to a very slight degree (seen in Table 50 and Figure 90). No significant carbon segregation is detected in this example. 
Tin, which has only a very small difference in radius from zirconium (1\%), is completely solubility in zirconium (see section 2.6.1) at the concentration present in ZIRLO; tin does not show any interfacial segregation in either ZIRLO example.

Titanium, because of its chemical similarity to zirconium, is soluble in zirconium at all concentrations [173] and so there is a low driving force for rejection of this solute from the alloy matrix despite titanium having an atomic radius of $1.47 \AA$ [160], similar to niobium and representing a $7.5 \%$ mismatch with zirconium. Segregation of titanium to the metal oxide interface is not observed. 


\begin{tabular}{|c|c|c|c|c|c|c|c|c|c|}
\hline \multirow[t]{2}{*}{ Element } & \multicolumn{2}{|c|}{ LHS grain } & \multicolumn{2}{|c|}{ RHS grain } & \multirow{2}{*}{$\begin{array}{l}\text { Boundary } \\
\text { Conc. } \\
\text { (at.\%) }\end{array}$} & \multicolumn{2}{|c|}{$\mathrm{N}_{\text {excess }}$} & \multicolumn{2}{|c|}{$\begin{array}{c}\text { Enrichment } \\
\text { factor }\end{array}$} \\
\hline & $\begin{array}{l}\text { Conc. } \\
\text { (at.\%) }\end{array}$ & $\begin{array}{l} \pm 1 \sigma \\
\text { (at. \%) }\end{array}$ & $\begin{array}{l}\text { Conc. } \\
\text { (at.\%) }\end{array}$ & $\begin{array}{l} \pm 1 \sigma \\
\text { (at.\%) }\end{array}$ & & $\begin{array}{c}\# \\
\text { (at.) }[x\end{array}$ & $\begin{array}{l}1 \sigma \\
\left.{ }^{16} \mathrm{~m}^{-2}\right]\end{array}$ & $\begin{array}{r}\text { vrs. } \\
\text { LHS } \\
(\#) \\
\end{array}$ & $\begin{array}{c}\text { vrs. } \\
\text { RHS } \\
(\#) \\
\end{array}$ \\
\hline \multicolumn{10}{|c|}{ ZIRLO - high segregation } \\
\hline $\mathrm{Zr}$ & 97.88 & 0.3 & 97.80 & 0.2 & 95.56 & -214 & 200 & 1 & 1 \\
\hline $\mathrm{Nb}$ & 0.55 & 0.03 & 0.53 & 0.02 & 2.06 & 216 & 10 & 4 & 4 \\
\hline Sn & 0.74 & 0.03 & 0.76 & 0.02 & 0.95 & -79 & 20 & 1 & 1 \\
\hline $\mathrm{Fe}$ & \multirow{3}{*}{\multicolumn{2}{|c|}{$\begin{array}{c}\text { Inc. cluster } \\
\text { None detected } \\
\text { None detected }\end{array}$}} & 0.003 & 0.001 & 0.47 & 78 & 5 & - & 155 \\
\hline $\mathrm{Cr}$ & & & \multicolumn{2}{|c|}{ None detected } & 0.02 & 1.5 & 0.8 & High & High \\
\hline $\mathrm{Ni}$ & & & 0.01 & 0.00 & 0.04 & 3.7 & 0.4 & High & 4.4 \\
\hline $\mathrm{O}$ & 0.71 & 0.03 & 0.79 & 0.02 & 0.9 & -3 & 18 & 1 & 1 \\
\hline $\mathrm{C}$ & 0.08 & 0.01 & 0.08 & 0.01 & 0.02 & -2 & 3 & 0.3 & 0.2 \\
\hline \multicolumn{10}{|c|}{ ZIRLO - Iow segregation } \\
\hline $\mathrm{Zr}$ & 97.41 & 0.007 & 97.37 & 0.007 & 96.62 & -74 & 126 & 1 & 1 \\
\hline $\mathrm{Nb}$ & 0.47 & 0.01 & 0.50 & 0.01 & 0.5 & -68 & 9 & 1 & 1 \\
\hline Sn & 0.755 & 0.004 & 0.763 & 0.004 & 0.8 & 55 & 11 & 1 & 1 \\
\hline $\mathrm{Fe}$ & 0.0221 & 0.0007 & 0.0242 & 0.0007 & 0.4 & 49.2 & 1.3 & 17 & 16 \\
\hline $\mathrm{Cr}$ & 0.0005 & 0.0001 & 0.0012 & 0.0002 & 0.01 & 4.7 & -0.1 & 27 & 11 \\
\hline $\mathrm{Ni}$ & \multicolumn{2}{|c|}{ None detected } & \multicolumn{2}{|c|}{ None detected } & 0 & 0 & 0 & 1 & 1 \\
\hline $\mathrm{O}$ & 1.334 & 0.005 & 1.329 & 0.005 & 1.7 & 22 & 14 & 1 & 1 \\
\hline $\mathrm{C}$ & 0.0092 & 0.0004 & 0.0123 & 0.0005 & 0.035 & 11 & 1 & 4 & 3 \\
\hline \multicolumn{10}{|c|}{$\mathrm{Zr}-\mathrm{Nb}-\mathrm{Ti}$} \\
\hline $\mathrm{Zr}$ & 98.41 & 0.004 & 98.14 & 0.023 & 97.2 & -166 & 79 & 1 & 1 \\
\hline $\mathrm{Nb}$ & 0.311 & 0.002 & 0.52 & 0.012 & 0.9 & 54 & 4 & 3 & 2 \\
\hline $\mathrm{Ti}$ & 0.325 & 0.002 & 0.277 & 0.009 & 0.4 & -12 & 5 & 1 & 1 \\
\hline $\mathrm{Fe}$ & 0.0028 & 0.0002 & 0.0046 & 0.0011 & 0.1 & 22 & 2 & 41 & 25 \\
\hline $\mathrm{Ni}$ & 0.0003 & 0.0001 & None d & etected & 0 & 0 & 0 & 1 & 1 \\
\hline $\mathrm{Cr}$ & None c & etected & None d & etected & 0 & 0 & 0 & 1 & 1 \\
\hline $\mathrm{O}$ & 0.955 & 0.003 & 1.051 & 0.017 & 1.4 & 102 & 6 & 2 & 1 \\
\hline $\mathrm{C}$ & 0.0008 & 0.0001 & 0.003 & 0.0009 & 0.005 & 1 & 0 & 6 & 2 \\
\hline
\end{tabular}

Table 51: Summary of the chemistry at and adjacent to the grain boundaries described in section

5.2.2. The boundary concentration is determined by taking the average gradient at the interface of the relevant cumulative concentration profile (this figure is very sensitive). The enrichment factor takes this value as its denominator. The $N_{\text {excess }}$ is based on well defined values, but is still very sensitive to the matrix concentration, as is seen in the quoted uncertainty. 


\subsubsection{Summary}

The 3DAP allows the precise chemical description of grain boundaries segregation and comparison between behaviour observed between different alloy classes. The primary limitation of the technique is the work required in preparing and analysing site specific features using FIB. These observations are believed to be the first direct measurements of grain boundary segregation in zirconium alloys. 


\section{Analysis of corroded material}

\subsection{Introduction}

\subsubsection{Overview}

This chapter describes the nanoscale chemistry at the metal-oxide interface, and in specific cases the wider metal matrix of materials exposed to a variety of periods of corrosion within a static autoclave. The materials analysed are the same ZIRLO, Zircaloy-4 and Zr-Nb-Ti described in section 3.4.1 having undergone the corrosion as described in section 3.4.2. The prior chemistry of these materials was thoroughly examined in chapter 5, and this information is frequently used as a point of reference in this chapter.

The metal oxide interface of ZIRLO (§ 6.2) was examined after 4 corrosion periods, 3 before and 1 after the transition in oxidation rate. The behaviour of oxygen, niobium, tin and iron (and chromium and nickel where present) was characterised and the mechanisms controlling the redistribution of solute discussed. Pre-transition Zircaloy-4 (§6.3) was also examined and the behaviour of the solutes in this material is described, and compared with that of ZIRLO.

The Zr-Nb-Ti test alloy ( $§ 6.4)$, with awful corrosion resistance, has also been studied by TEM and EDX, as this material has not been characterised in the prior literature. The metal-oxide interface of this material was analysed by 3DAP after much post transition oxide had developed (if indeed this alloy has pre-transition oxidation regime) and the solute distribution is put in the context of what is known about this oxide microstructure of the alloy. 


\subsubsection{Notes on reproducibility of results}

Multiple specimens were prepared and analysed for each of the materials and corrosion conditions described in this section. The data presented is representative of the all of the data available for each point, or if it is not, any variation is described in the text, or additional examples are provided. As an indicator of the reproducibility of these results, Table 52 lists the successful experimental results drawn upon in the following sections.

\begin{tabular}{|c|c|c|c|}
\hline Material & 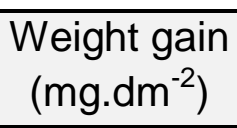 & Section & Number and type of observations \\
\hline ZIRLO & 18.3 & 6.2 .1 & $\begin{array}{l}2 \text { good quality metal-oxide interface transition } \\
\qquad 4 \text { oxide only }\end{array}$ \\
\hline ZIRLO & 25.7 & 6.2 .2 & $\begin{array}{c}2 \text { adequate quality metal-oxide interface transitions } \\
1 \text { partial interface } \\
1 \text { oxide only }\end{array}$ \\
\hline ZIRLO & 28.5 & $\begin{array}{l}6.2 .3 \\
\text { and } \\
6.2 .4\end{array}$ & $\begin{array}{c}3 \text { good quality metal-oxide interface transitions } \\
1 \text { large precipitate at interface engulfing ROI } \\
1 \text { region of metal adjacent to interface } \\
1 \text { partial interface }\end{array}$ \\
\hline ZIRLO & 45.9 & 6.2 .5 & $\begin{array}{c}1 \text { good quality metal-oxide interface transition } \\
2 \text { adequate quality metal-oxide interface transitions } \\
2 \text { oxide only }\end{array}$ \\
\hline Zry-4 & 15.3 & 6.3 .1 & 1 good quality metal-oxide interface transition \\
\hline Zry-4 & 23.4 & 6.3 .2 & $\begin{array}{l}2 \text { good quality metal-oxide interface transitions } \\
\quad 3 \text { partial interface } \\
2 \text { region of metal adjacent to interface }\end{array}$ \\
\hline $\mathrm{Zr}-\mathrm{Nb}-\mathrm{Ti}$ & 1189 & $\begin{array}{l}6.4 .2 \\
\text { and } \\
6.4 .3\end{array}$ & $\begin{array}{c}2 \text { adequate quality metal-oxide interface transitions } \\
1 \text { poor quality metal-oxide interface transition } \\
11 \text { partial interface } \\
1 \text { region of metal near to interface }\end{array}$ \\
\hline
\end{tabular}

Table 52: A description of the atom probe tomography data available and an indicator of the number and type of observation made of each material condition. A partial interface means that not all of the metal-outer oxide transition is within the ROI. Adequate quality means that either the dataset is a small volume captured before fracture or only a small amount of one of the interfacial phases is present within the ROI. 


\subsection{ZIRLO}

\subsubsection{Pre-transition metal-oxide interface: $18.3{\mathrm{mg} . \mathrm{dm}^{-2}}^{-2}$}

After 34 days in the static autoclave, recrystallised ZIRLO sheet had developed an oxide film with an average weight gain of $18.3 \mathrm{mg} \cdot \mathrm{dm}^{-2}$.

\subsubsection{Oxygen}

2D oxygen concentration profiles across atom probe datasets produced from metal oxide interfaces in this condition are shown in Figure 91. The interface morphology is a simple junction between $\mathrm{ZrO}_{2}$ (oxide) and $\mathrm{Zr}$ metal. The interface is planar on the length-scale of tens of nanometres as viewed by 3DAP. Figure 91 (b) use an alternative colour scale to Figure 91 (a) which better displays variation in oxygen concentration in the range $33-66$ at.\%. There is a region of $1-5 \mathrm{~nm}$ on the oxide side of the interface that appears to be deficient in oxygen. The region is approaching the limit of resolution of the instrument for such an interface. The apparent low oxygen content could be due to ion trajectory aberrations, or it could indicate the presence of a very thin region of intermediate composition between the alloy and $\mathrm{ZrO}_{2}$. An alternative view of the interface in Figure 91 is given by the 3D representation presented in Figure 92. This figure uses surfaces of constant concentration (blue: 45 at.\% oxygen, and red: 55 at.\% oxygen) to represent the appearance of the interface. The orange interfacial region in Figure 91 is shown here by the small void region between the red and blue surfaces that effectively demarcate the boundaries of the $\mathrm{ZrO}_{2}$ and $\mathrm{Zr}$ metal regions. There is no evidence of an intermediate layer when viewing this information as a $1 \mathrm{D}$ concentration profile, as shown in Figure 93. This region is shown as a gradual transition between $\mathrm{ZrO}_{2}$ and 50 at.\%, followed by a sharp step change to the matrix oxygen concentration. The composition of the outer oxide agrees well with that of $\mathrm{ZrO}_{2}$. 
The level of oxygen in the metal is seen to be $\sim 20$ at.\% in the first few nanometres of metal and decays to $\sim 10$ at.\% within $50 \mathrm{~nm}$.
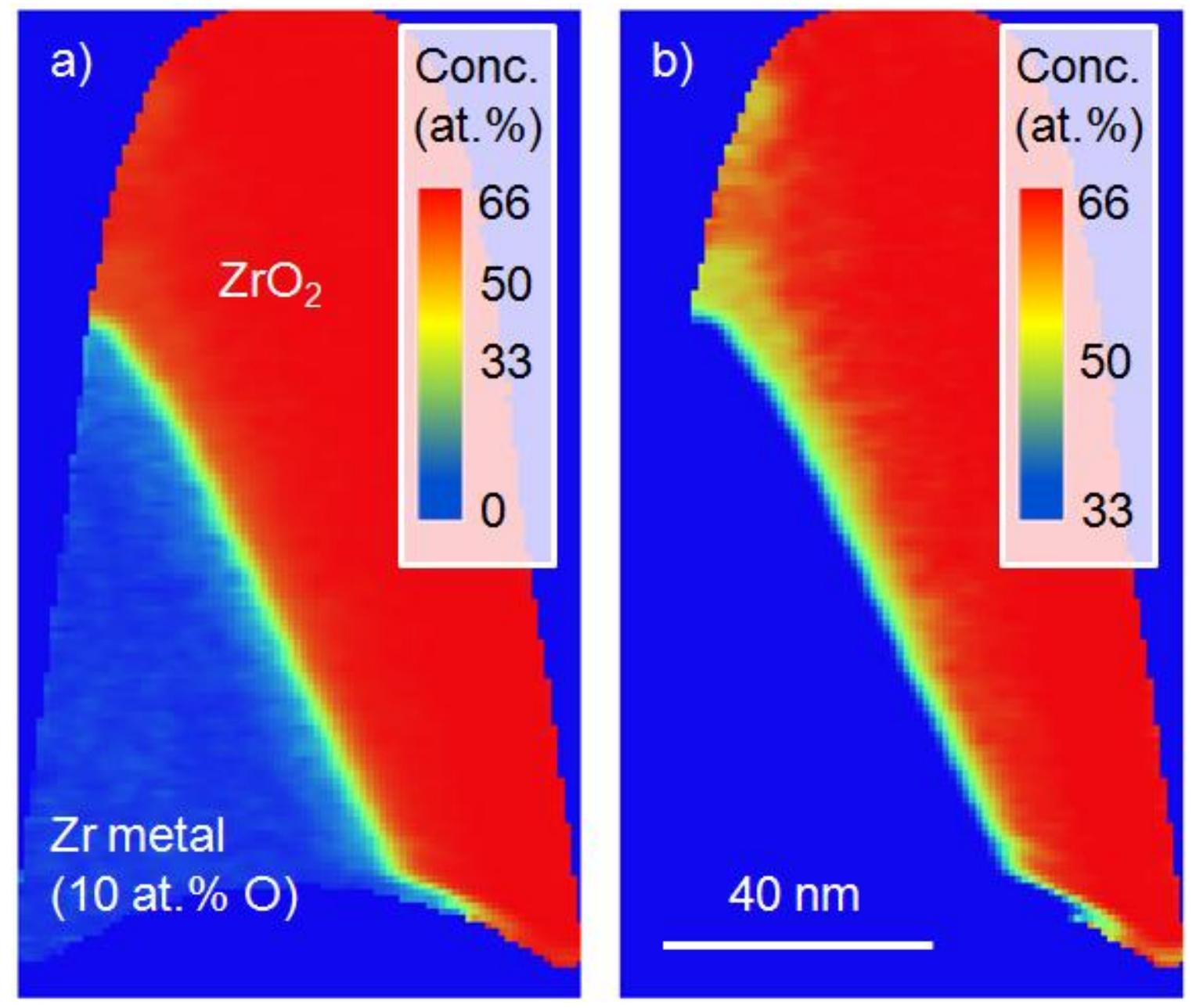

Figure 91: 2D oxygen concentration profiles taken across the metal-oxide interface of 18.3 $m g . \mathrm{dm}^{-2}$ corroded pre-transition ZIRLO. The profile plane perpendicular to the tip axis. The oxygen profile in (a) shows that there is some oxygen in solid solution adjacent to the metal oxide interface. This is more clearly, and quantitatively described in the accompanying $1 D$ profile, Figure 93. The metal-oxide interface concentration profile (b) is displayed with the colour scale from 33-66 at.\% oxygen and shows that there is a region at the interface with intermediate composition that is very thin $(1-5 \mathrm{~nm})$. 


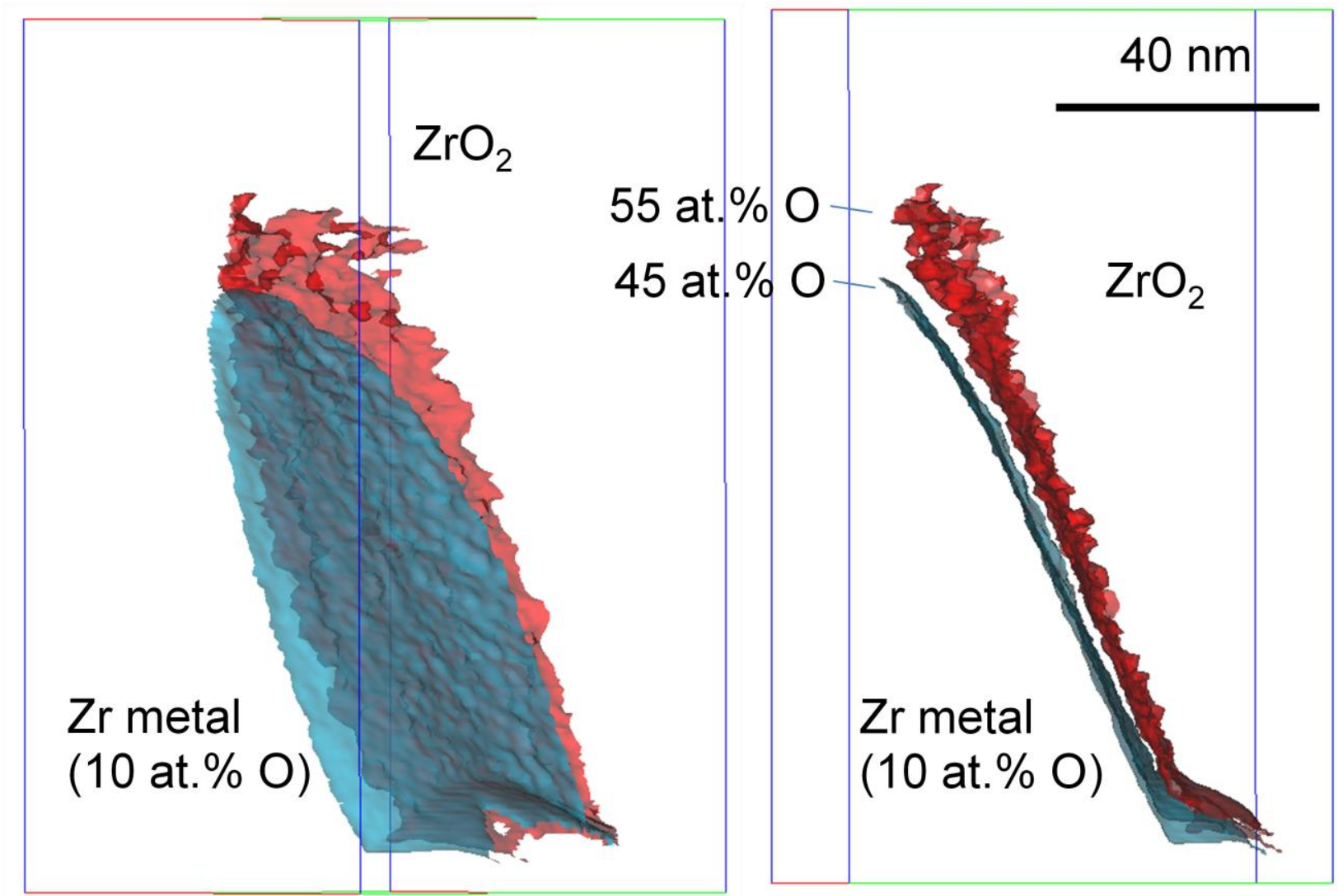

Figure 92: Isoconcentration surfaces calculated for this dataset $\left(18.3 \mathrm{mg} . \mathrm{dm}^{-2}\right.$ corroded pretransition ZIRLO) at 45 and 55 at.\% O, effectively showing the boundaries of the metal phase and of $\mathrm{ZrO}_{2}$.

\begin{tabular}{|c|r|rr|}
\hline Element & \multicolumn{2}{|c|}{$\begin{array}{c}\text { Number } \\
\text { of atoms }\end{array}$} & \multicolumn{2}{|c|}{$\begin{array}{c}\text { Composition } \\
\text { Conc. }\end{array}$} & $\begin{array}{r} \pm 1 \\
\text { (atoms) }\end{array}$ \\
(at.\%) & (at.\%) \\
\hline $\mathrm{Zr}$ & $1,432,862$ & 84.8 & 0.03 \\
$\mathrm{Nb}$ & 13,704 & 0.81 & 0.01 \\
$\mathrm{Sn}$ & 6,139 & 0.36 & 0.005 \\
$\mathrm{O}$ & 235,288 & 13.9 & 0.03 \\
$\mathrm{Fe}(28)$ & 1,578 & 0.093 & 0.002 \\
$\mathrm{Fe}(27)$ & 3,172 & 0.19 & 0.01 \\
\hline Total & & & \\
atoms & $1,689,572$ & & \\
\hline
\end{tabular}

Table 53: Composition of the alloy matrix adjacent to the metal-oxide interface of $18.3 \mathrm{mg} \cdot \mathrm{dm}^{-2}$ corroded pre-transition ZIRLO. 


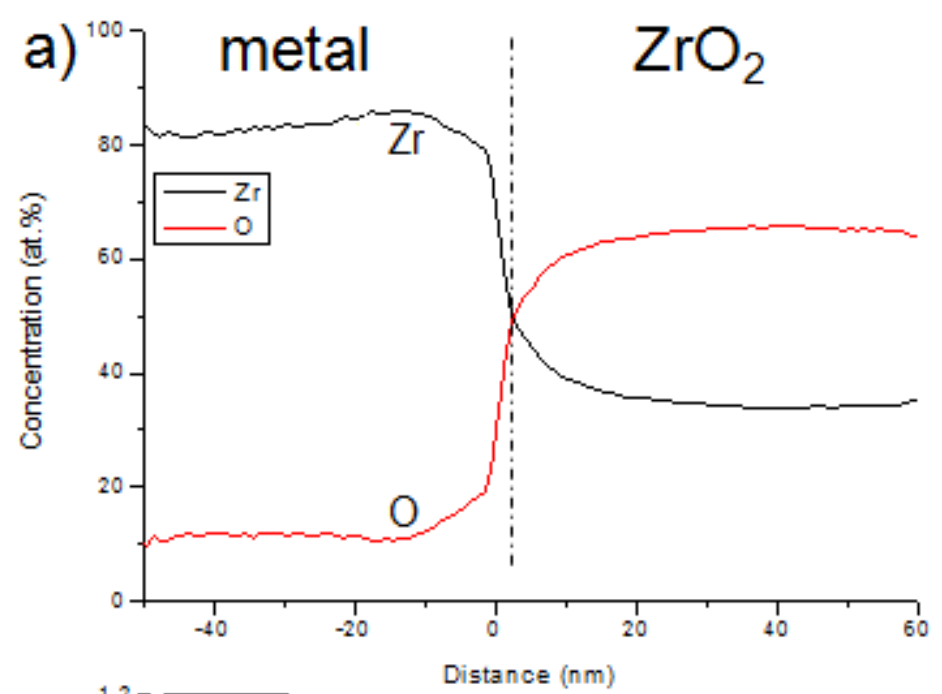

Figure 93: 1D concentration profile across the metal-oxide interface of 18.3 mg.dm ${ }^{-2}$ corroded pretransition ZIRLO showing the distribution of a) $\mathrm{Zr}$ and $\mathrm{O}$; b) $\mathrm{Nb}$ (taking into account all Nbcontaining species and compensating for peak overlap and $\mathrm{ZrH}$ convolution) and Sn (calculated from 59 and 60 Da peaks and correcting for overlap from $\mathrm{ZrO}^{+}$; c) Three methods of estimating the Fe distribution: Using 27 Da peak only (poor counting statistics), the leading edge of 28 (likely to include a high proportion of iron but does not allow for an estimate of the absolute value); and the whole peak at $28 \mathrm{Da}$ (which might contain CO).

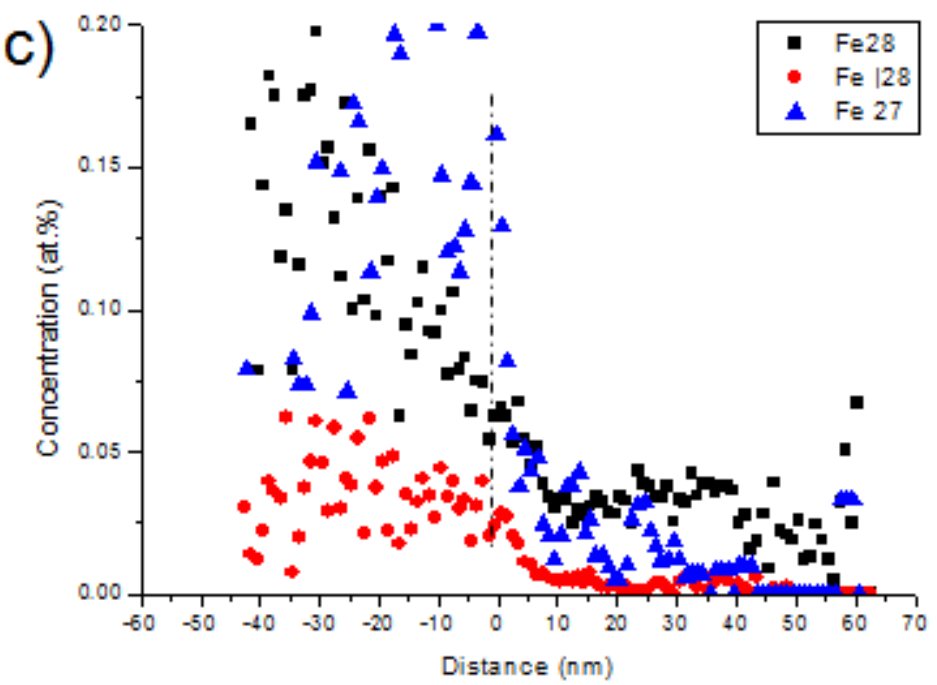




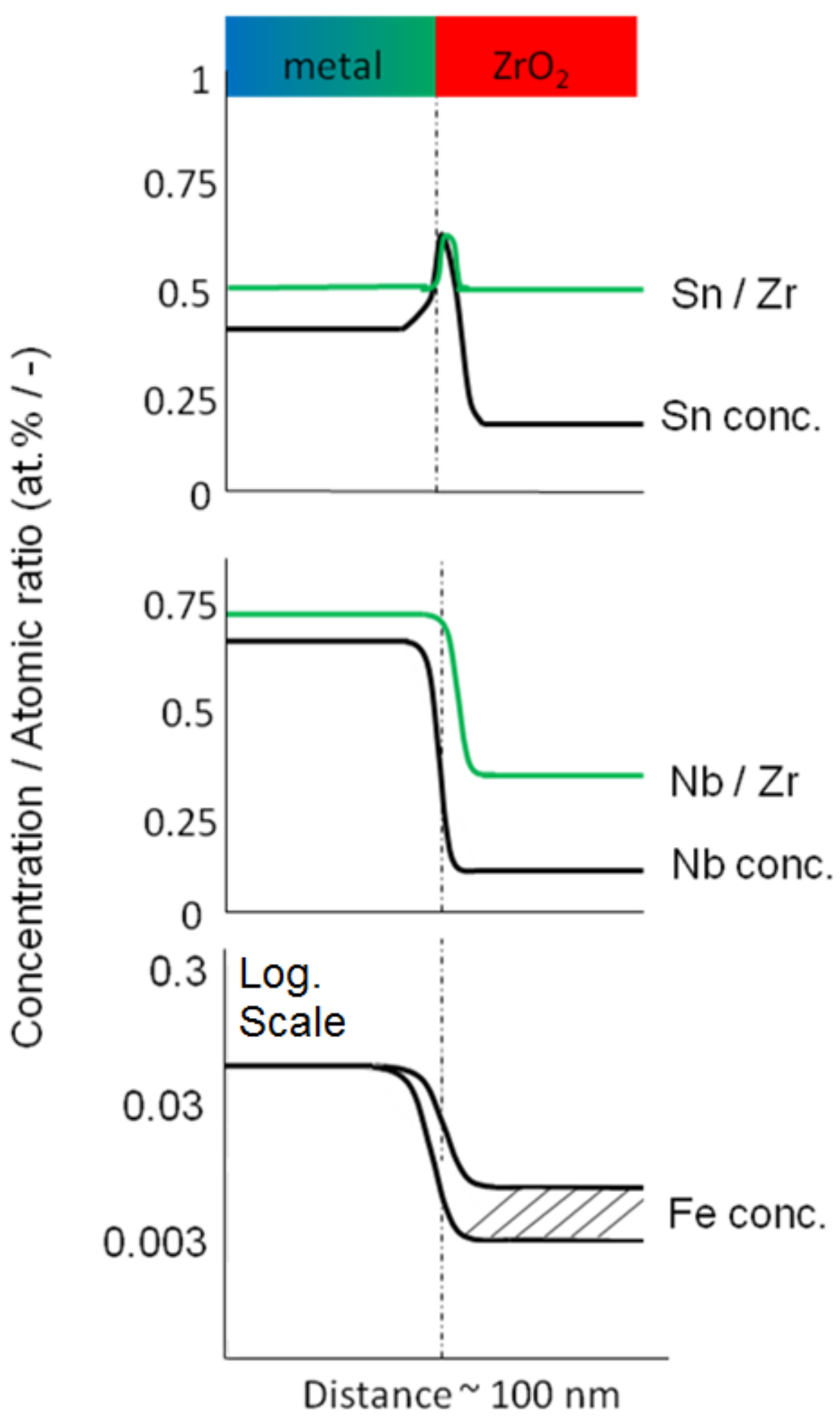

Figure 94: Idealised solute concentration and solute/Zr profiles across the metal-oxide interface of $18.3 \mathrm{mg} \cdot \mathrm{dm}^{-2}$ corroded pre-transition ZIRLO. The tin and niobium profiles are on a linear scale, the iron profile is on a logarithmic scale. 


\subsubsection{Tin}

A step change in the tin concentration is seen at the metal-oxide interface. This is readily apparent in both the 1D concentration profile in Figure 93 and the tin atom map in Figure 95. From these data it would seem that there is more tin in the metal adjacent to the interface than in the oxide. A more useful view of the situation can be obtained by considering the ratio of tin atoms to zirconium atoms, this removes the effective change in solute concentration due to the addition of oxygen. This is shown along with a schematic of the original tin concentration profile in Figure 94. Here we see that the ratio $\mathrm{Sn} / \mathrm{Zr}$ remains constant between the metal and oxide. The tin present in the metal is taken into the newly formed oxide without redistribution.

Another feature apparent in Figure 93 and Figure 95, and also represented in the schematic view Figure 94, is an apparent excess of tin at the interface. There are two possible explanations for this observation; that the mismatch between atomic planes at the interfaces accommodates tin, or that the apparent excess tin found here is due to field enhanced migration. The former of these, that the observation is a physically significant manifestation of enhanced $\mathrm{Sn}$ concentration at the interface, seems less plausible as the solubility of tin in zirconium is high compared to the other solutes present. No other solutes are not seen to be segregated in such a way here. Tin has also not been observed to be preferentially segregated to grain boundaries in virgin ZIRLO. On the other hand, tin has been seen to be sensitive to field-enhanced migration to an extent that is not observed for the other solute. The overall level of tin in the metal in this spectrum is low compared to the expected matrix level. This seems to be a sample specific problem, and other examples of this data point (which have inferior count statistics and / or ROI positioning to the example shown) do not show this discrepancy. This is attributed to the sensitivity of tin to evaporation field, as has previously been discussed in section 4.6.4, causing the preferential retention of tin given the field conditions present during this experiment. 


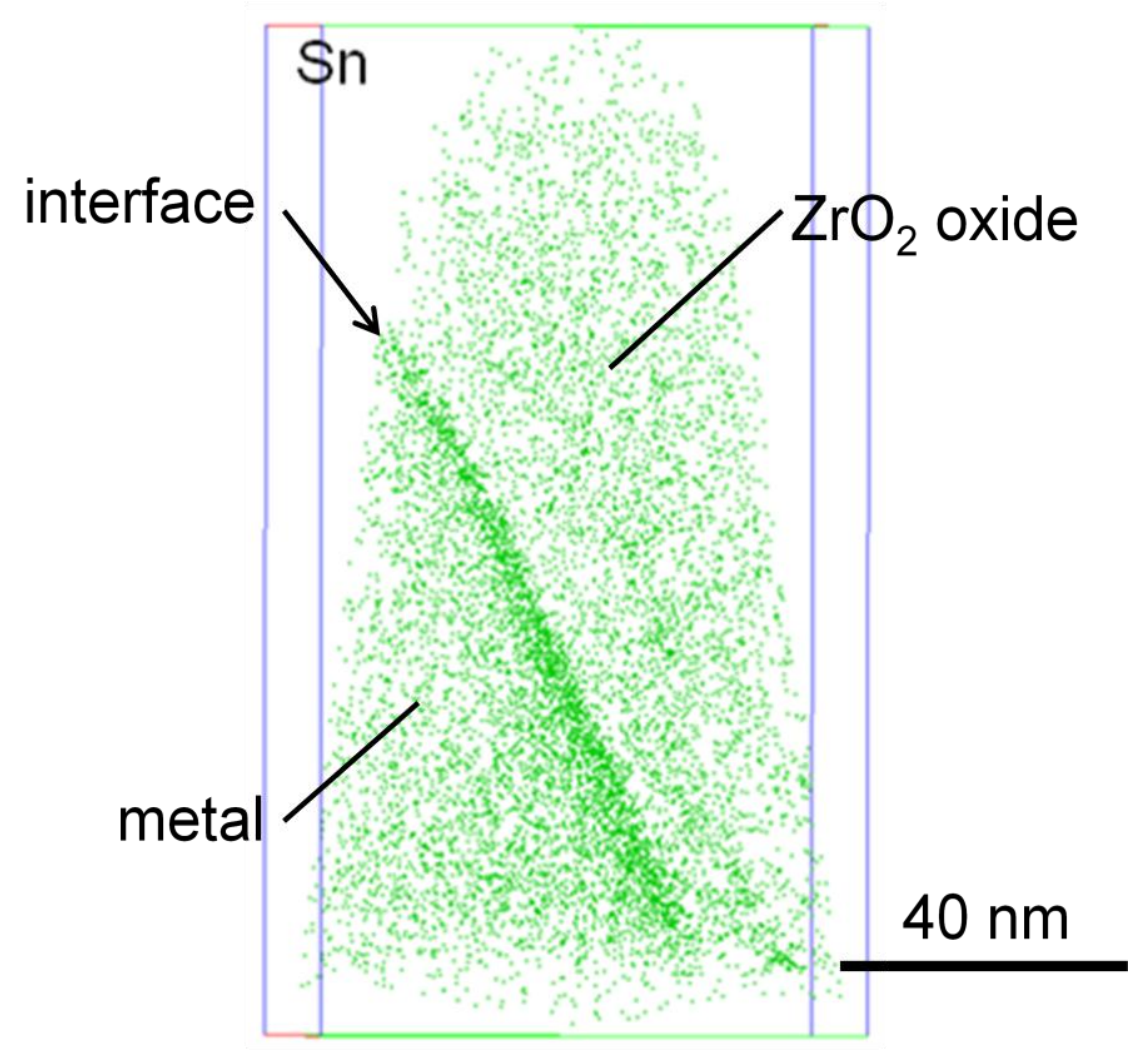

Figure 95: 3D atom map of the $S n(a t 59$ and $60 \mathrm{Da}$ ) distribution across the metal-oxide interface of $18.3 \mathrm{mg} \cdot \mathrm{dm}^{-2}$ corroded pre-transition ZIRLO.

\subsubsection{Niobium}

The niobium concentration has been established using all of the niobium containing ions, correcting for $\mathrm{Nb} / \mathrm{ZrH}$ peak overlap and the additions of delayed ions from other nearby species, namely $\mathrm{Zr}$ and $\mathrm{ZrO}$. The $1 \mathrm{D}$ concentration profile taken across the interface in Figure 93 (b) shows a region of enhanced niobium ahead of the metal-oxide interface. This profile, taken perpendicular to the interface, averages over a smaller volume at its extremities due to the shape of the dataset. This increases the uncertainty in the observations of individual points and so increases the scatter seen at the ends of the profiles. Averaging over a large (1.7 million atom) region of metal adjacent to the interface gives an estimate of the average niobium concentration 
as $0.81 \pm 0.01$ at.\%, which is greater than the usual matrix concentration of 'as-received' material which in section 5.1 .4 was shown to be $(0.36 \pm 0.06)$ at.\%. The niobium level in the oxide is much lower than that in the metal adjacent to the interface, and it appears that niobium rejected from the progressing oxide front is the cause of this supersaturation of niobium at the interface. As is described later in section 6.2.3, this supersaturated niobium matrix ahead of the interface is not maintained during the entire pre-transition oxidation process as other factors reduce the level of niobium in solution.

The niobium concentration of the 'as-received' ZIRLO was itself supersaturated. There must, therefore, be a considerable driving force towards precipitation of niobium out of solution, a slow process due to the slow diffusion and precipitation kinetics of niobium. Apart from the $\sim 50$ $\mathrm{nm}$ scale precipitate observed in the virgin material due to processing treatments, no smaller scale nucleation of niobium has been observed in material subjected to this level of corrosion or in the virgin material. Other authors have conducted simulations of the precipitation of nanoscale niobium precipitates in Zr-Nb alloys [174]. This work modelled a higher Nb-content alloy, $\mathrm{Zr}-2.5 \mathrm{Nb}$. It predicts the precipitate distribution that would be expected in an alloy subjected, as the ZIRLO was, to several hours final annealing at $\sim 580{ }^{\circ} \mathrm{C}$. The model predicts a fine distribution of nanometre scale particles. However, the number density of the particles is $\sim 1$ per $100 \mu \mathrm{m}^{3}$. This density is too low to expect significant observations by 3DAP as it suggests only 0.1 precipitate per $100 \mathrm{~nm}^{3}$ volume. This explains why no small scale precipitates are seen in the homogeneous regions of uncorroded metal matrix examined.

There is evidence that niobium is partially soluble in tetragonal $\mathrm{ZrO}_{2}$ at $1500{ }^{\circ} \mathrm{C}$ [175-176]. However, lower temperature studies at $1000{ }^{\circ} \mathrm{C}$ found the niobium solubility to be vanishingly small [177]. Low temperature phase diagrams of zirconia are an unusual research interest for ceramicists, and no information regarding the solubility of niobium in $\mathrm{ZrO}_{2}$ could be found on the exhaustive reference database Phase Equilibria Diagrams Database version 31 by the 
American Ceramics Society [178]. The level of niobium found in this early pre-transition oxide, which is usually found to have a relatively high volume fraction of the tetragonal phase, suggests that the solubility of niobium in this phase is very low.

\subsubsection{Iron and Chromium}

Several alternative methods have been used to analyse the iron distribution at the metal-oxide interface. This is necessary to avoid incorrectly attributing the behaviour of other species that occupy the same, or similar, mass-to-charge states with those of iron, as was described in section 4.5.6. Three 'iron' profiles are plotted in Figure 93 (c). These show the iron concentration as calculated from the peak at $27 \mathrm{Da}(5.8 \%$ relative isotopic abundance), $28 \mathrm{Da}(91.8 \%$ r.i.a. $)$ and the leading edge of $28 \mathrm{Da}$ (not quantitative). All three iron estimate profiles show good agreement in that there is a step change at the interface, with there being a much greater concentration of iron on the metal side compared to the oxide. The Table 53 gives the concentration within the metal as $(0.093 \pm 0.002)$ at. $\% \mathrm{Fe}($ from 28$)$ and $(0.19 \pm 0.01)$ at. $\% \mathrm{Fe}$ (from 27). The lower of these figures provides an upper bound for the Fe concentration within the material, as the additional counts in the higher of the values must be due to the presence of another species. The lower value, 0.93 at.\% iron, is well in excess of the iron concentration seen in the virgin ZIRLO material, was seen to be around either 0.003 or 0.01 at.\%.

When considering the behaviour of iron at the interface it seems sensible, given the issues regarding estimation uncertainties and possible artefacts, to consider the concentration to be within ranges, rather than to give an absolute value. The idealised schematic iron profile given in Figure 94 describes the concentration of iron on a semi-log scale. The iron concentration is at a supersaturated level within the metal matrix and seen at a lower concentration $(<0.02$ at.\%) within the $\mathrm{ZrO}_{2}$ oxide. The source of this additional iron was not immediately apparent; rejection 
of iron at the interface would enrich the matrix, but it would be expected that the anomalously fast diffusing iron [171] would redistribute within the metal, rather than form a thermodynamically unstable locally supersaturated solution. Similarly, additional iron introduced into the specimen from the autoclave environment does not provide a satisfactory solution to this problem because of the water chemistry used in the experiment (PWR-type), which has not been shown to cause this effect [41], as well as the observation that there is not additional iron in the oxide through which the iron must be transported to reach the metal. Analysis of the behaviour of iron within the bulk of corroded material provides a more compelling explanation of these observations, and is described in detail in section 6.2.4.

A very minor peak was seen at the 26 Da position in a mass spectrum from the metal oxide interface and is attributed to chromium, of which $83.75 \%$ of the relative isotopic abundance at $2+$ charge state would be present at $26 \mathrm{Da}$. The concentration of this solute is very low, and should be considered to be at a 'trace' level in the metal matrix adjacent to the interface. The distribution of chromium at the interface is shown in Figure 97. This distribution is similar to that seen for iron, which is unsurprising, as chromium has been seen to behave in a similar way to iron at microstructural features in uncorroded ZIRLO, segregating to grain boundaries and second phase particles. Chromium was only detected in one metal matrix specimen of asreceived ZIRLO. 


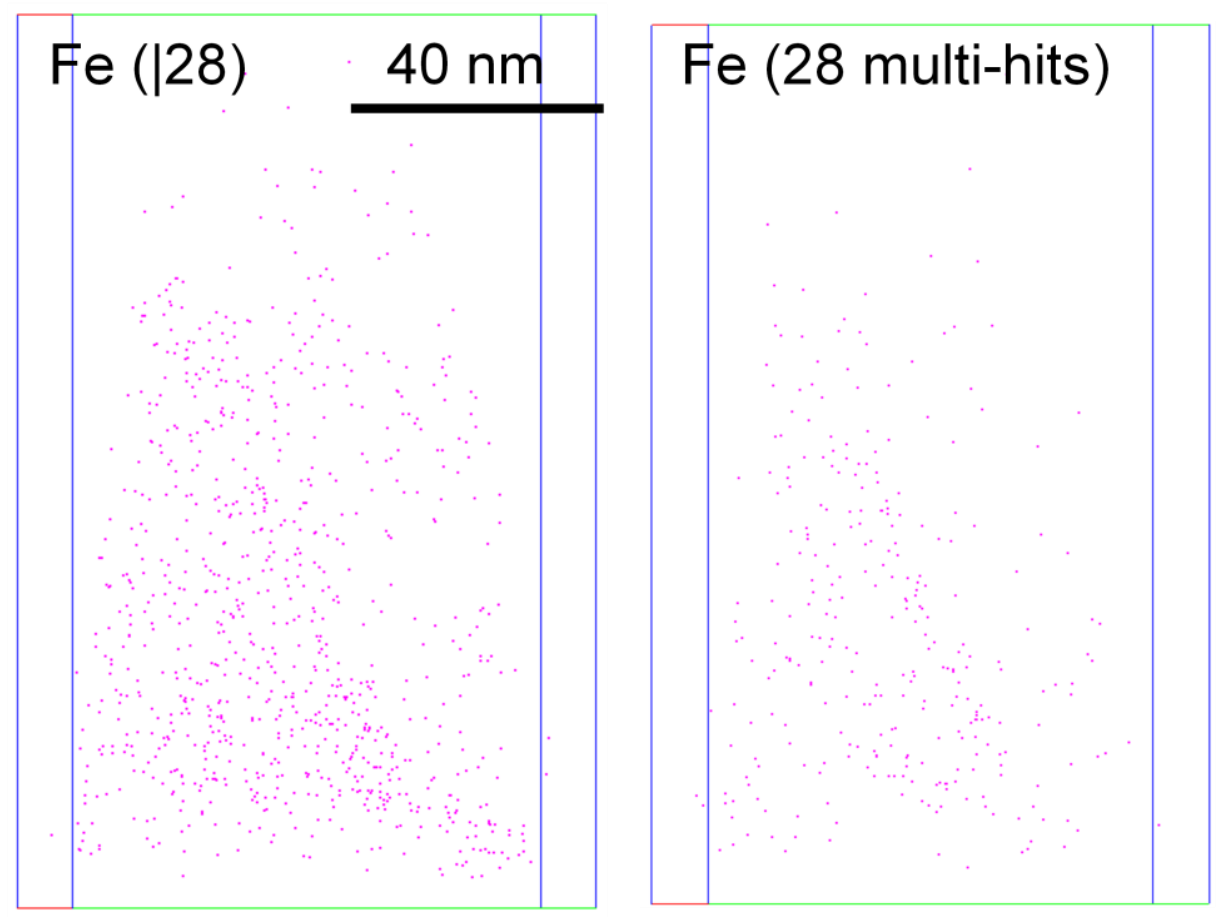

Figure 96: 3D atom maps showing the distribution of Fe from the leading edge of $28 \mathrm{Da}$ and multi-hit event iron ions at 28 Da for $18.3 \mathrm{mg} \cdot \mathrm{dm}^{-2}$ corroded pre-transition ZIRLO.

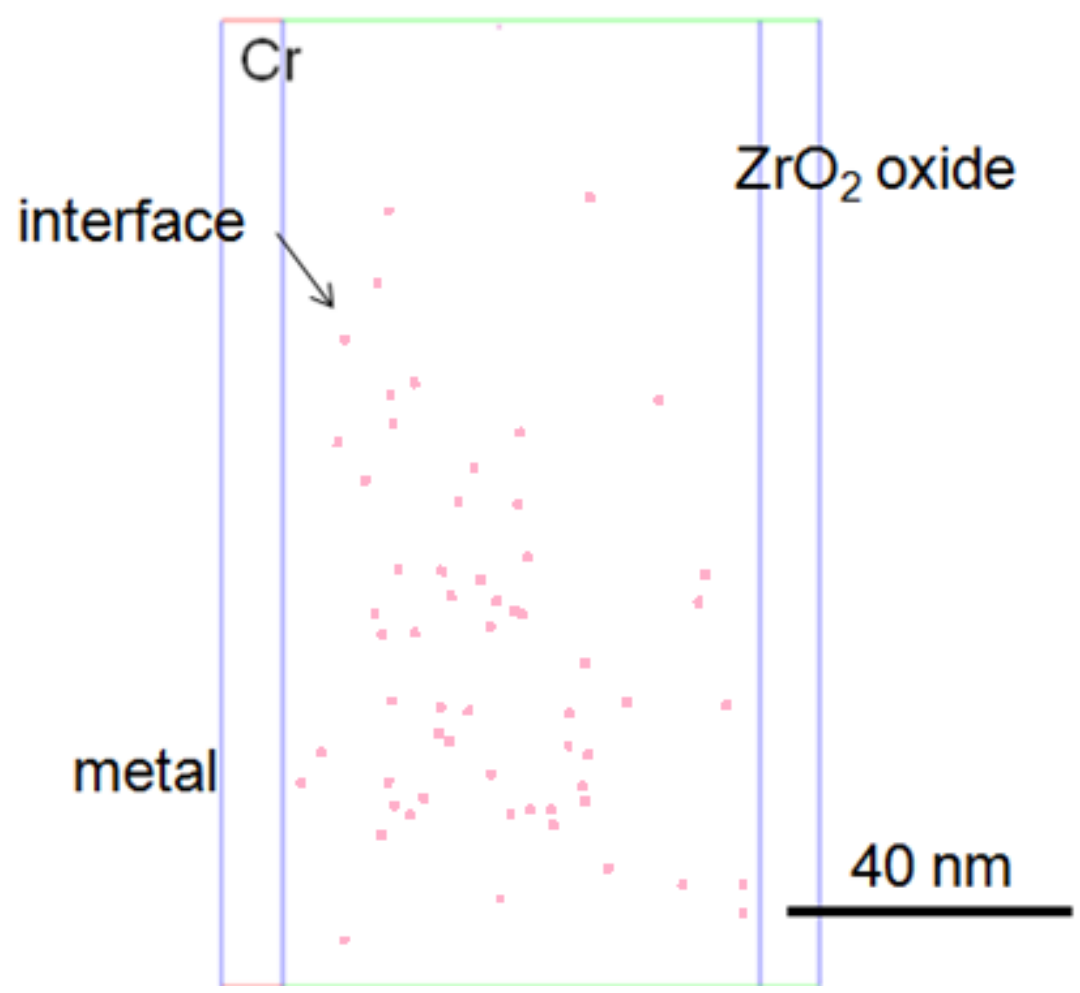

Figure 97: $3 D$ atom maps of the $C r(26 \mathrm{Da}, \mathrm{s} / \mathrm{n}$ ratio of $\sim 10)$ distribution across the metaloxide interface of $18.3 \mathrm{mg} \cdot \mathrm{dm}^{-2}$ corroded pre-transition ZIRLO. Dot size increased for contrast. 


\subsubsection{Pre-transition metal-oxide interface: $25.7{\mathrm{mg} . \mathrm{dm}^{-2}}^{-2}$}

This material had been in the static autoclave for 114 days, but showed below average weight gain for that time period. The average weight gain was more consistent with material that had been subjected to 75 - 80 days exposure.

\subsubsection{Oxygen}

2D oxygen concentration profiles of the metal-oxide interface of pre-transition material with higher average weight gain are shown in Figure 98. The oxygen concentration in the metal adjacent to the interface is at the level expected for a saturated solid solution of oxygen in zirconium, 30 at.\% oxygen. A sandwich layer structure is visible in these oxygen profiles; a layer of constant oxygen concentration separates the zirconium metal phase from the $\mathrm{ZrO}_{2}$ oxide. There are relatively sharp boundaries between these three layers. The intermediate layer is of the order of 10 nanometres thick, although precise values cannot be determined from the 3DAP measurements as the reconstruction algorithm used to create these representations makes the assumption of uniform density across the dataset. In this case the three layers all have different atomic densities, and the density of the intermediate layer is not known. It would be surprising if this density differed from the assumed value by more than $30 \%$, and is probably much less than this value.

The measurement of the oxygen concentration in the outer oxide, $\sim 63$ at. $\% \mathrm{O}$, agrees fairly well with the expected value for $\mathrm{ZrO}_{2}$, as shown in Figure 99. Figure 99 is a profile across a thin region of interest chosen so that both boundaries are roughly parallel with each other and perpendicular to the axis of the profile. This reduces the concentration blurring effect seen at boundaries when the profile is taken off-normal. The boundaries of the interfaces are quite sharp, 
less than $5 \mathrm{~nm}$. The concentration of the intermediate region can be seen to settle at a constant level in Figure 99. This level is at $\sim 50$ at.\% O. This phase has been labelled as $\mathrm{ZrO}$ in Figure 98.
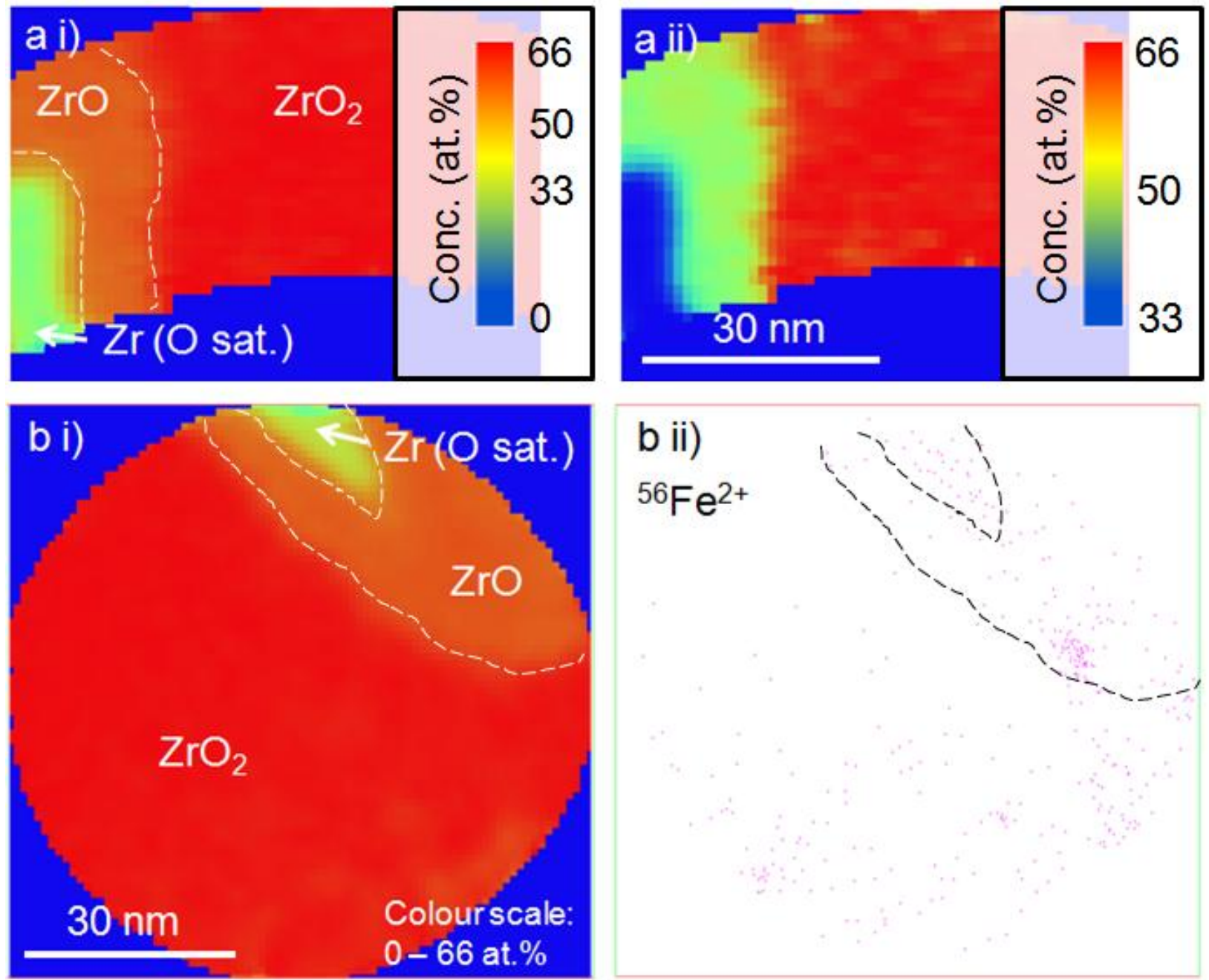

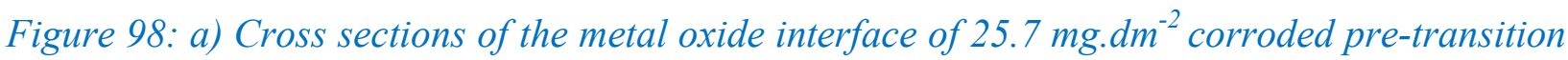
ZIRLO, demonstrating 'thin' $(10 \mathrm{~nm})$ intermediate layer of uniform oxygen concentration (ZrO) with sharp (<2 nm) interfaces. Colour scale i) 0-66 at.\% oxygen, ii) 33-66 at.\% oxygen. An alternative view is shown in ' $b$,' where the metal-oxide interface is viewed normal to the axis of the specimen. $b$ i) 2D oxygen concentration profile with colour scale $0-66$ at.\% $O$ (blue to red, see key). ii) Atom map showing Fe ions from the same perspective as (b i). Dashed lines represent the boundaries of phases. 

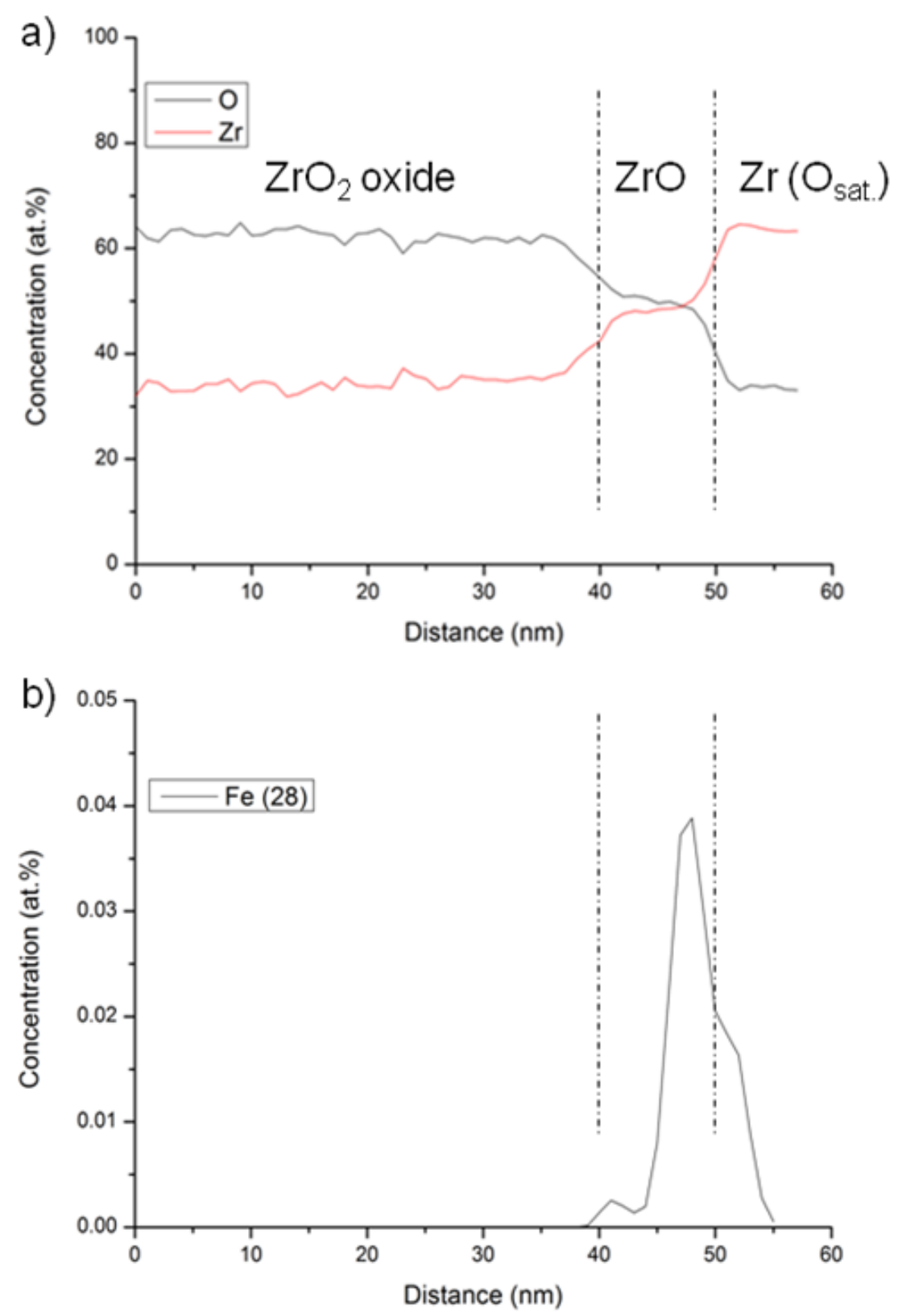

Figure 99: $1 D$ concentration profile across the metal-oxide interface of $25.7 \mathrm{mg} . \mathrm{dm}^{-2}$ corroded pre-transition ZIRLO. Profile is taken across a very small cross section of the region of interest. 


\subsubsection{Solute distribution}

All of the data collected for this condition predominantly contained the oxide phase within the analysed regions of interest. It is unfortunate that it is not possible to draw many conclusions regarding the behaviour of solute near this interface due to this, because of lack of evidence. Despite this, it is still possible to highlight two general observations regarding iron at this interface.

Iron appears to be inhomogeneously distributed within the oxide. Localised clustering of iron at the $\mathrm{ZrO}-\mathrm{ZrO}_{2}$ boundary is seen in Figure 98 . The iron profile in Figure 99 is over a very small region of interest and cannot be used to draw conclusions about the average level of iron in the oxide (although it is clearly non-zero) or the metal, where only a few cubic nanometres of material are analysed. The profile suggests that there is an excess of iron segregated to the $\mathrm{Zr}$ $\mathrm{ZrO}$ boundary. Iron is dissimilar to tin in that it has not been shown to be subject to field enhanced surface migration on atom probe needles. Iron has also been seen to be enhanced at grain boundaries within uncorroded material.

\subsubsection{Pre-transition metal-oxide interface: $28.5 \mathrm{mg.dm}^{-2}$}

This material had been exposed to 100 days of corrosion in the static autoclave, and the weight gain experienced by this specimen is consistent with the overall corrosion curve.

\subsubsection{Oxygen}

The metal-oxide interfaces of the specimens examined in this corrosion condition are more complicated than the structures previously seen. Like the previous corrosion condition, an interfacial layer is seen between the $\mathrm{Zr}$ metal and $\mathrm{ZrO}_{2}$ oxide. In this corrosion condition a 
thicker $\mathrm{ZrO}$ layer with a complicated morphology is seen at the interface. Figure 100 shows a 2D oxygen concentration profile at the metal-oxide interface showing an example of this complicated structure. The intermediate layer can be further sub-divided into $\mathrm{ZrO}$ ' and $\mathrm{ZrO}$ ', where $\mathrm{ZrO}$ ' is richer in $\mathrm{Zr}$ and $\mathrm{ZrO}$ ' ' is richer in $\mathrm{O}$. The geometry of the $\mathrm{ZrO}_{2}-\mathrm{ZrO}$ ' ' boundary is relatively simple. Interestingly, the ZrO', layer, which has slight surplus of O similar to that seen in the $\mathrm{ZrO}$ layer in the less corroded pre-transition condition, is also of a similar thickness to the layer previously seen. Below this layer is a new $\mathrm{ZrO}$ ' layer that has highly variable thickness and protrudes into the alloy matrix. The length-scale of these observations is sub-granular, so the reason for these protrusions is unlikely to be crystallographic. A possible explanation of the observed behaviour of these $\mathrm{ZrO}$ 'fingers' is given in the discussion of niobium solute at the interface, later in this chapter, in sub-section 6.2.3.3.

There is a sharp interface between the $\mathrm{ZrO}$ ' and $\mathrm{ZrO}$ ', layers seen in the 2D profile Figure 100, and also the 1D profile in Figure 101. However, it should also be noted in this figure that the level of oxygen seen in the outer oxide is inexplicably low, $\sim 60$ at. $\%$, so this could indicate that there is a possible issue regarding loss of oxygen-containing species for this data set. However, the oxygen concentration in the metal corresponds well with the saturation level of oxygen in solution in zirconium. The geometry of the sample and variation in thermal conductivity between regions could cause the proportion of oxygen in low conductivity regions to appear lower, as a single ranging metric must be applied to all regions with different local mass resolution. The lower thermal conductivity effectively reduces the mass resolution for a localised region of the material. This would explain the slightly anomalous reading for the outer oxide, and why this is a problem identified with this sample and few others. It also suggests that the oxygen concentration readings within the $\mathrm{ZrO}$ layers are not affected by this issue, as these regions tend to have a mass resolution similar to that of the metal substrate. 


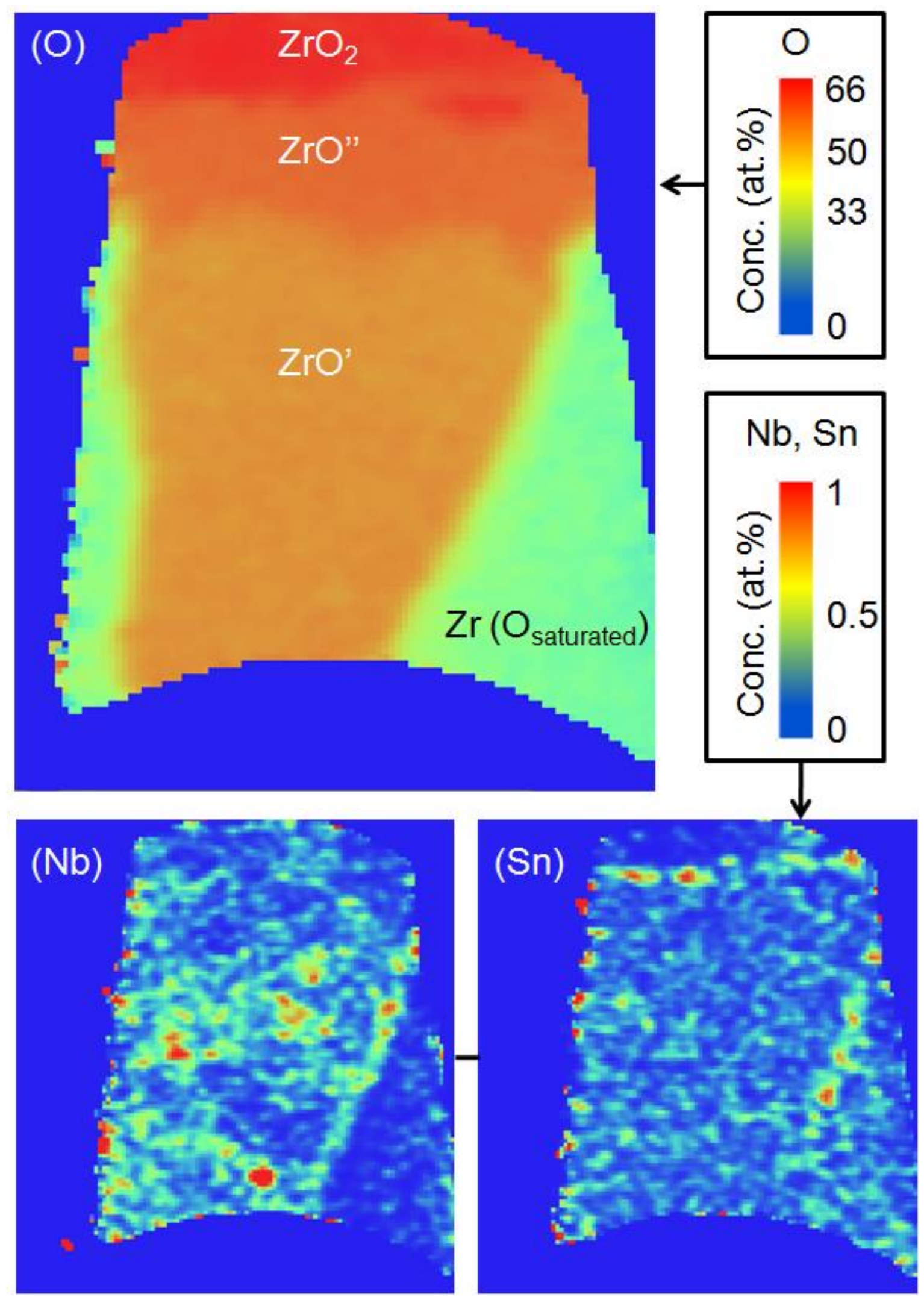

Figure 100: 2D composition profiles for $\mathrm{O}$, Sn and $\mathrm{Nb}$ taken across the metal-oxide interface of $28.5 \mathrm{mg} \cdot \mathrm{dm}^{-2}$ corroded pre-transition ZIRLO. The oxygen profile shows two separate homogeneous $\mathrm{ZrO}$ layers between the $\mathrm{ZrO}_{2}$ and metal. The metallic region is oxygen saturated. 

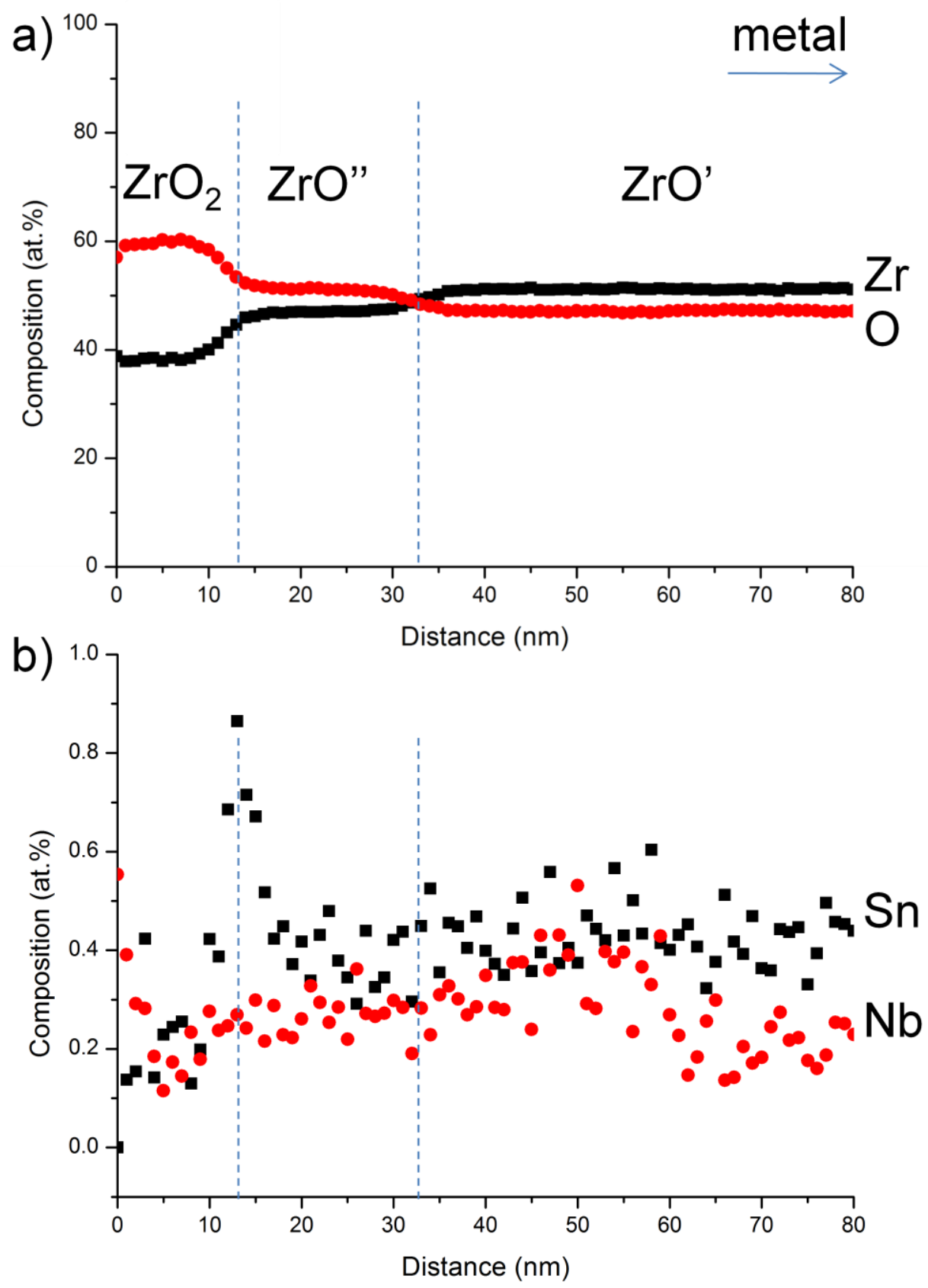

Figure 101: $1 \mathrm{D}$ concentration profiles across $\mathrm{ZrO}_{2}-\mathrm{ZrO}{ }^{\prime \prime}-\mathrm{ZrO}^{\prime}$ interface of $28.5 \mathrm{mg} \cdot \mathrm{dm}^{-2}$ corroded pre-transition ZIRLO. Plotting a) Zr and O; and b) Sn and Nb. A step change in the oxygen concentration profile is seen at the $\mathrm{ZrO}$ ' '-ZrO' interface. 
a i)
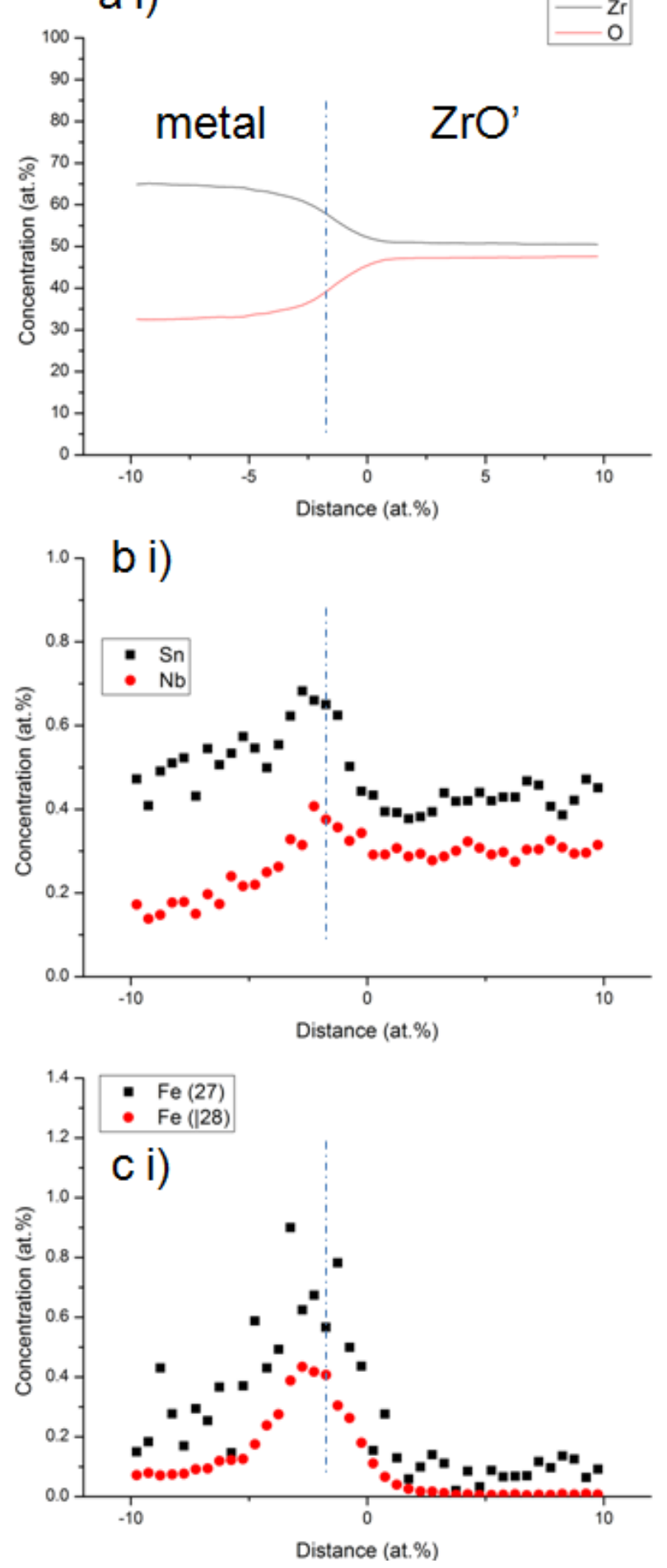

a ii)
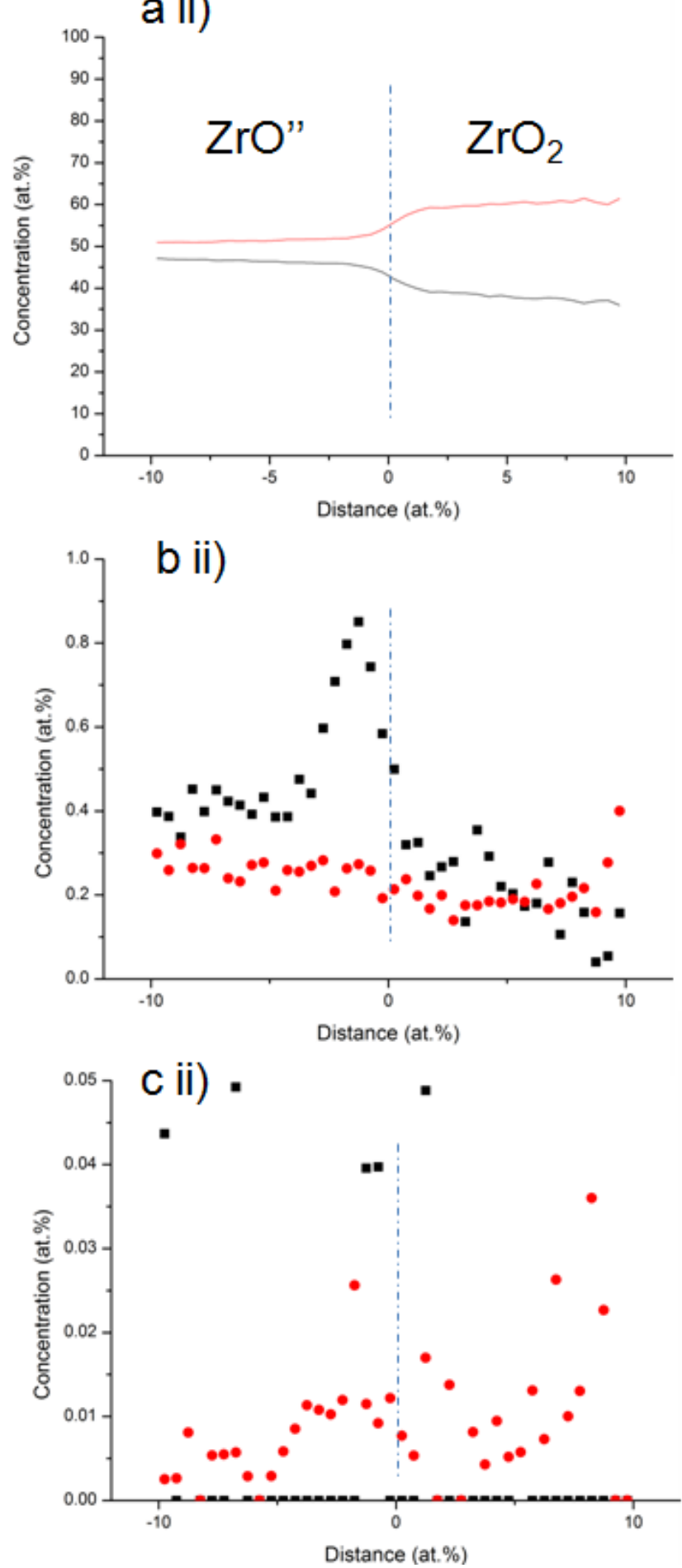

Figure 102: $1 \mathrm{D}$ concentration profiles across the i) metal- $\mathrm{ZrO}$ and ii) $\mathrm{ZrO}-\mathrm{ZrO}_{2}$ interfaces of $28.5 \mathrm{mg} . \mathrm{dm}^{-2}$ corroded pre-transition ZIRLO. Plotting a) $\mathrm{Zr}$ and $\mathrm{O}$; b) Sn and $\mathrm{Nb}$; and two estimates of the Fe distributions using only the peak at 27 to give a low precision estimate of the total iron concentration, and the leading edge of 28 , which provides a qualitative (not absolute) description of the distribution. Note the difference in concentration scale between ci and cii. 


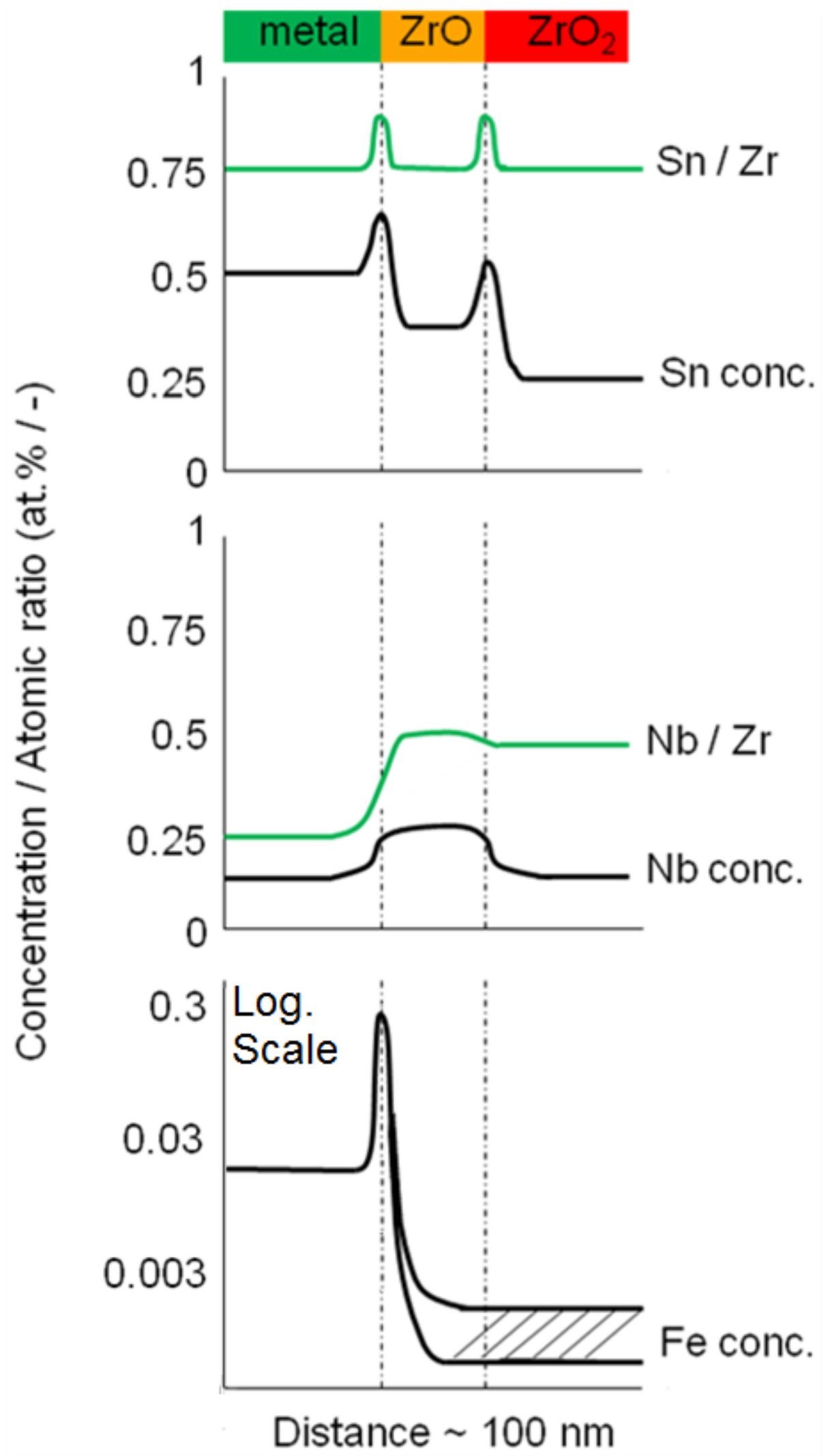

Figure 103: Idealised schematics of the solute profiles across the metal-oxide interface of 28.5 $m g . d m^{-2}$ corroded pre-transition ZIRLO. The tin and niobium profiles are on a linear scale, the iron profile is on a semi-logarithmic scale. 


\subsubsection{Tin}

The idealised tin profiles in Figure 103 show apparent peaks in the tin concentration at the $\mathrm{Zr}$ $\mathrm{ZrO}$ and $\mathrm{ZrO}-\mathrm{ZrO}_{2}$ interfaces, with step changes in the tin concentration between the different regions. This detected enhancement in the tin level is also seen graphically in the $2 \mathrm{D}$ tin concentration profile in Figure 100 and is likely the result of field enhanced migration of tin. The $\mathrm{Sn} / \mathrm{Zr}$ ratio, which ignores the effect that adding oxygen has on the tin concentration, is the same within all three regions. The Sn concentration, ignoring the effect of oxygen, is $\sim 0.75$ at.\%, which compares well with the expected level of $\mathrm{Sn}$ in the $\mathrm{Zr}$ matrix, 0.76 at.\% (1 wt.\%). The behaviour of tin appears to be the same as seen in the previous corrosion condition; tin is taken into the oxide as the metal is oxidised without redistribution.

\subsubsection{Niobium}

Figure $100(\mathrm{Nb})$ shows the niobium distribution across a 2D slice of the metal-oxide interface. The distribution is inhomogeneous. It should be noted that the red 'hot spots' on this profile within the $\mathrm{ZrO}$ ' region suggest the existence of niobium-rich regions within this intermediate layer. The niobium concentration at the core of these volumes is many standard deviations from that expected of a random solid solution. The 1D concentration profiles in Figure 102 are prepared, as far as possible, to exclude these niobium-rich regions from the analysed volume so as to only show the matrix concentration within the separate regions. The concentration of niobium in the alloy matrix adjacent to the interface is now much lower than was seen in the early pre-transition condition and lower than the level expected in as-received material. This difference is attributed to the presence of these niobium-rich regions, taking niobium out of the majority of the matrix adjacent to the interface. A 3D view of another example interface is shown in Figure 104. The isosurfaces in this representation of the reconstructed dataset delineate the boundaries of the metal (blue) and $\mathrm{ZrO}_{2}$ (red) phases. 
a)
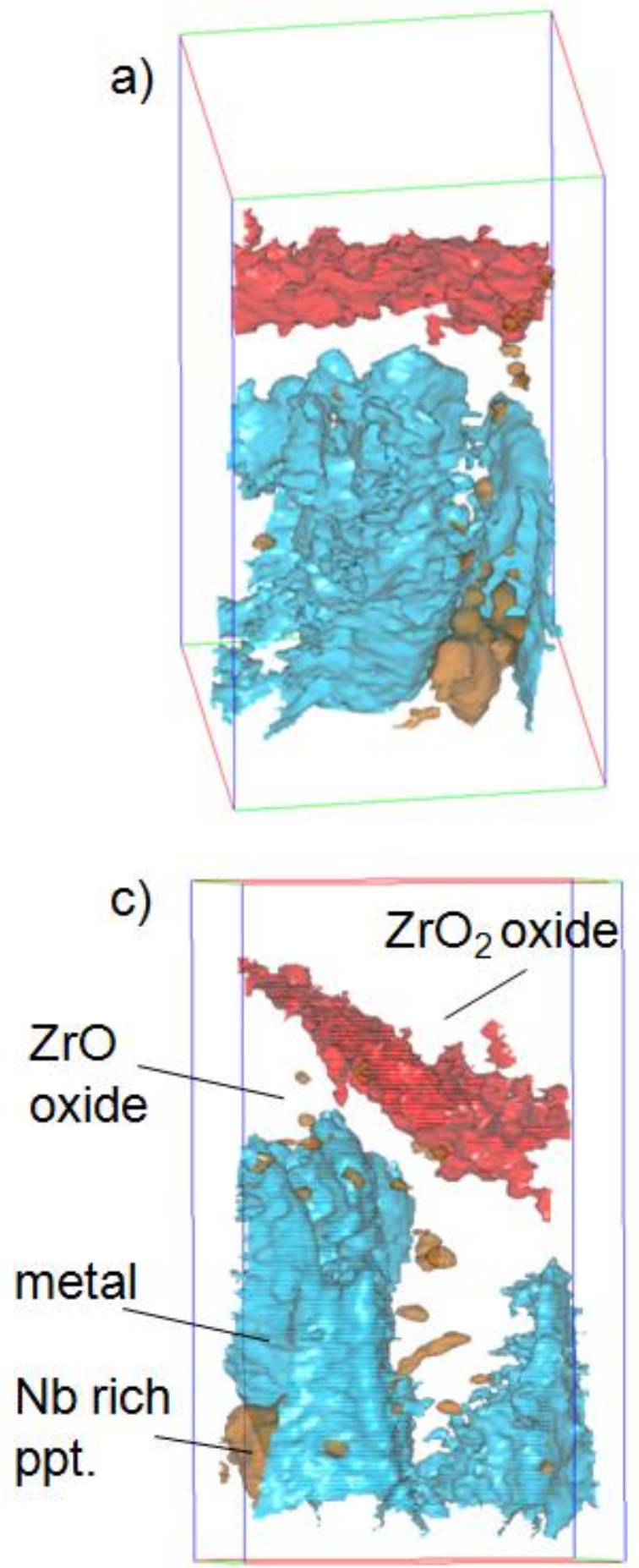

b)

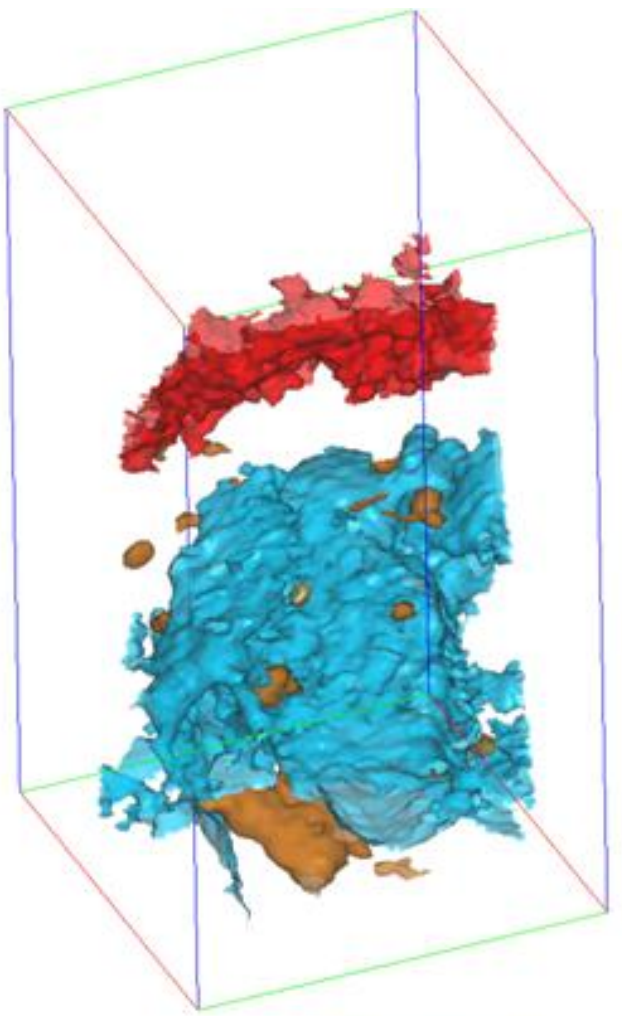

d)

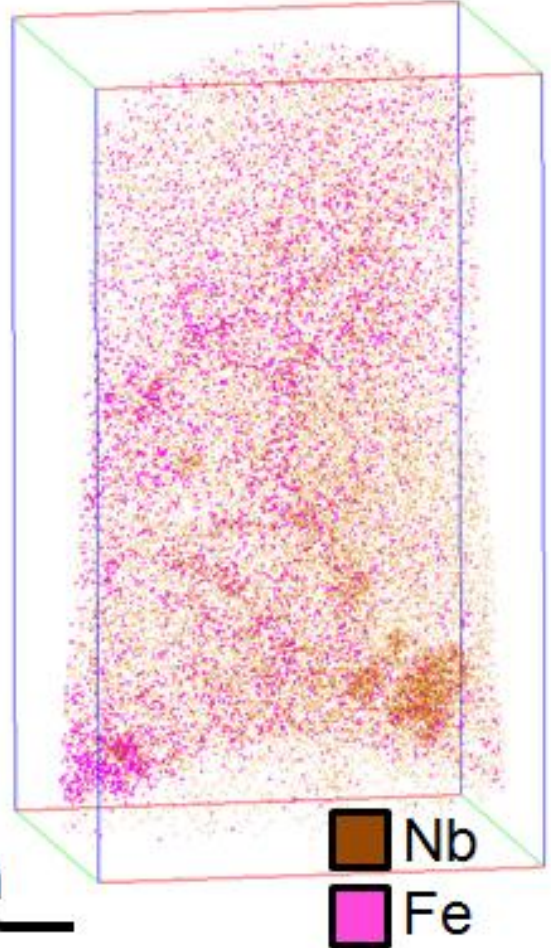

Figure 104: 3D view of the metal oxide interface of $28.5 \mathrm{mg}^{-\mathrm{dm}^{-2}}$ corroded pre-transition ZIRLO, showing $a-c)$ three views of the interface plotting a red surface at 55 at.\% oxygen to mark the $\mathrm{ZrO}-\mathrm{ZrO}_{2}$ interface and a very complicated blue surface at 45 at.\% oxygen marking the $\mathrm{Zr}-\mathrm{ZrO}$ boundary. A brown surface at 2 at.\% $\mathrm{Nb}$ defines Nb-rich regions. $d)$ A tilted forward $3 \mathrm{D}$ atom map of (c) rotated by 180 degrees, showing $\mathrm{Fe}$ (pink) and $\mathrm{Nb}$ (brown) atoms. 


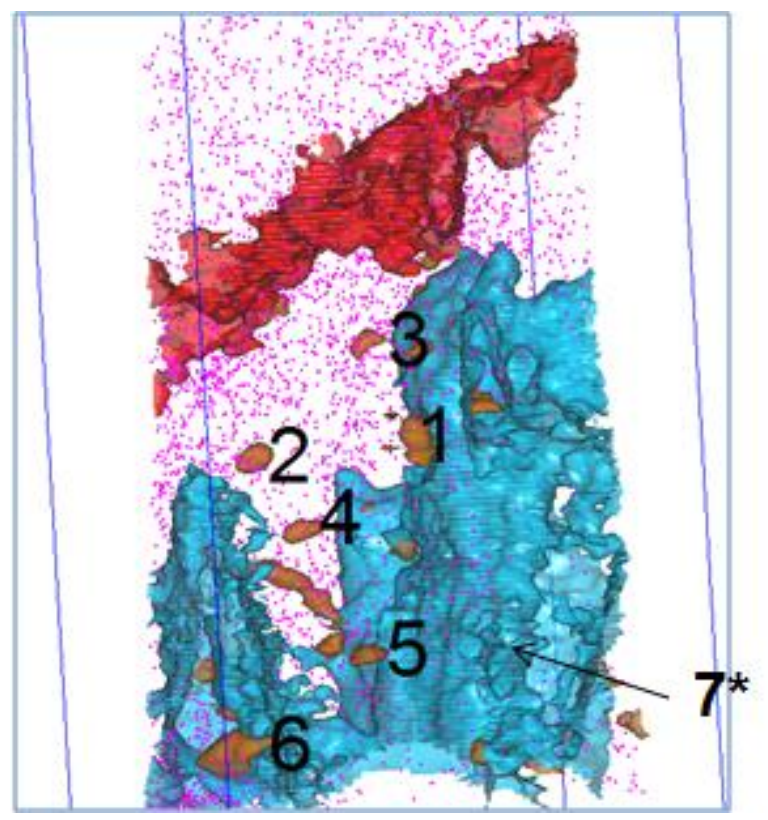

Figure 105: key to Table 54. Nb-rich regions in

the metal and $\mathrm{ZrO}$ oxide. Red surface defines

55 at.\% oxygen ( $\mathrm{ZrO}-\mathrm{ZrO}_{2}$ boundary), blue

surface defines 4 at.\% oxygen $(\mathrm{Zr}-\mathrm{ZrO}$

boundary), and brown surface defines 2 at.\%

Nb surfaces. Iron atoms are also shown as pink

dots. ${ }^{*} 7$ is hidden behind the isosurface.

\section{$50 \mathrm{~nm}$}

\begin{tabular}{|c|cc|cc|cc|cc|c|}
\hline I.D. & \multicolumn{2}{|c|}{ Zr } & \multicolumn{2}{c|}{ Nb } & \multicolumn{2}{c|}{ O } & \multicolumn{2}{c|}{ Fe (28) } & Total \\
(\#) & No. & Conc. & No. & Conc. & No. & Conc. & No. & Conc. & No. \\
(atoms) & (at.\%) & (atoms) & (at.\%) & (atoms) & (at.\%) & (atoms) & (at.\%) & (atoms) \\
\hline $1-\mathrm{m}$ & 736 & 47.3 & 68 & 4.4 & 741 & 47.6 & 11 & 0.7 & 1,556 \\
$2-\mathrm{m}$ & 967 & 43.8 & 116 & 5.3 & 1,116 & 50.5 & 9 & 0.4 & 2208 \\
$3-\mathrm{m}$ & 290 & 42.9 & 28 & 4.1 & 349 & 51.6 & 9 & 1.3 & 676 \\
$4-\mathrm{m}$ & 295 & 50.6 & 31 & 5.3 & 250 & 42.9 & 7 & 1.2 & 583 \\
$5-\mathrm{m}$ & 285 & 45.1 & 27 & 4.3 & 313 & 49.5 & 7 & 1.1 & 632 \\
$6-\mathrm{O}$ & 1,980 & 54.5 & 176 & 4.8 & 1,390 & 38.2 & 90 & 2.5 & 3,636 \\
$7-\mathrm{O}$ & 28,790 & 60.3 & 3672 & 7.7 & 14,837 & 31.1 & 445 & 0.9 & 47,744 \\
\hline
\end{tabular}

Table 54: Comparison of region compositions. Regions are enumerated in the key in Figure 105.

The region in which the niobium-rich region is found is labelled as ' $m$,' metal, or ' $O$,' $Z r O$. The precipitate volume is defined as that within a 2 at.\% $\mathrm{Nb}$ isosurface. The concentration at the core of the precipitate is higher than this average figure.

The surfaces marking the bounds of the niobium-rich regions are set, rather arbitrarily, at 2 at.\% niobium (a value chosen to be substantially higher than the matrix level). These regions are described chemically in Table 54, aided by the key Figure 105. The niobium concentration at the core of these regions is at a substantially higher level than the boundary level, as can be seen in analysis later in this section. In general, the volumes contain an average of around 1 at.\% iron 
and several at.\% niobium. The clustering of iron with some of the niobium-rich regions is also demonstrated by Figure 104. These regions appear to be no nobler than the material that surrounds them; the regions in the metal and the $\mathrm{ZrO}$ contain the same amount of oxygen as the phase in which they reside. The niobium-rich regions in the metal occupy a larger volume than the particles observed in the $\mathrm{ZrO}$ oxide. The majority of the smaller regions are observed in the ZrO' oxide (oxygen deficient). It is noted that $\mathrm{NbO}$ exists as a stable (cubic) phase [101] and so niobium, in the form of $\mathrm{NbO}$ may be easily accommodated by a $\mathrm{ZrO}$ oxide layer. No particles are seen in the $\mathrm{ZrO}_{2}$ layer in any of the samples studied; niobium is present in solution in this phase. The presence of these particles in the $\mathrm{ZrO}^{\prime}$ sub-layer, the lower density of observations in the outer $\mathrm{ZrO}$ " sub-layer, the absence of the regions in the oxide, together with the observation that these nano-scale regions are no more noble than their surrounding environment suggests that they are oxidised within the ZrO' sub-layer and dissolve completely within the outer ZrO', sublayer.

Figure 106 represents a section of the metal-oxide interface where a $\mathrm{ZrO}$ ' 'finger' protruding into the metal substrate has less chaotic interfaces than the previous example shown in Figure 104. This could be an insignificant variation in interface morphology, but it is interesting to note the lack of niobium-rich regions in this dataset, only one has been identified. These clusters might act as pinning sites for the progression of the Zr-ZrO' boundary. Although the chemistry of these regions shows that they are no more noble than their surroundings, these datasets are snapshot views and do not give detail about the time taken for oxidation of the cluster to occur. From the macroscopic weight gain data it is known that the average oxide thickness increases from 1.8 to 2.2 microns between 34 and 114 days. This gives a rough guide to the progression of the oxide front as being of the order of $5 \mathrm{~nm}$ per day during the majority of the pre-transition period. It is possible that the larger niobium-rich particles, such as particles \#6 and \#7 listed in 
Table 54 and displayed at the Zr-ZrO' boundary in Figure 104 could act locally as a drag on the progression of the slowly progressing oxide.

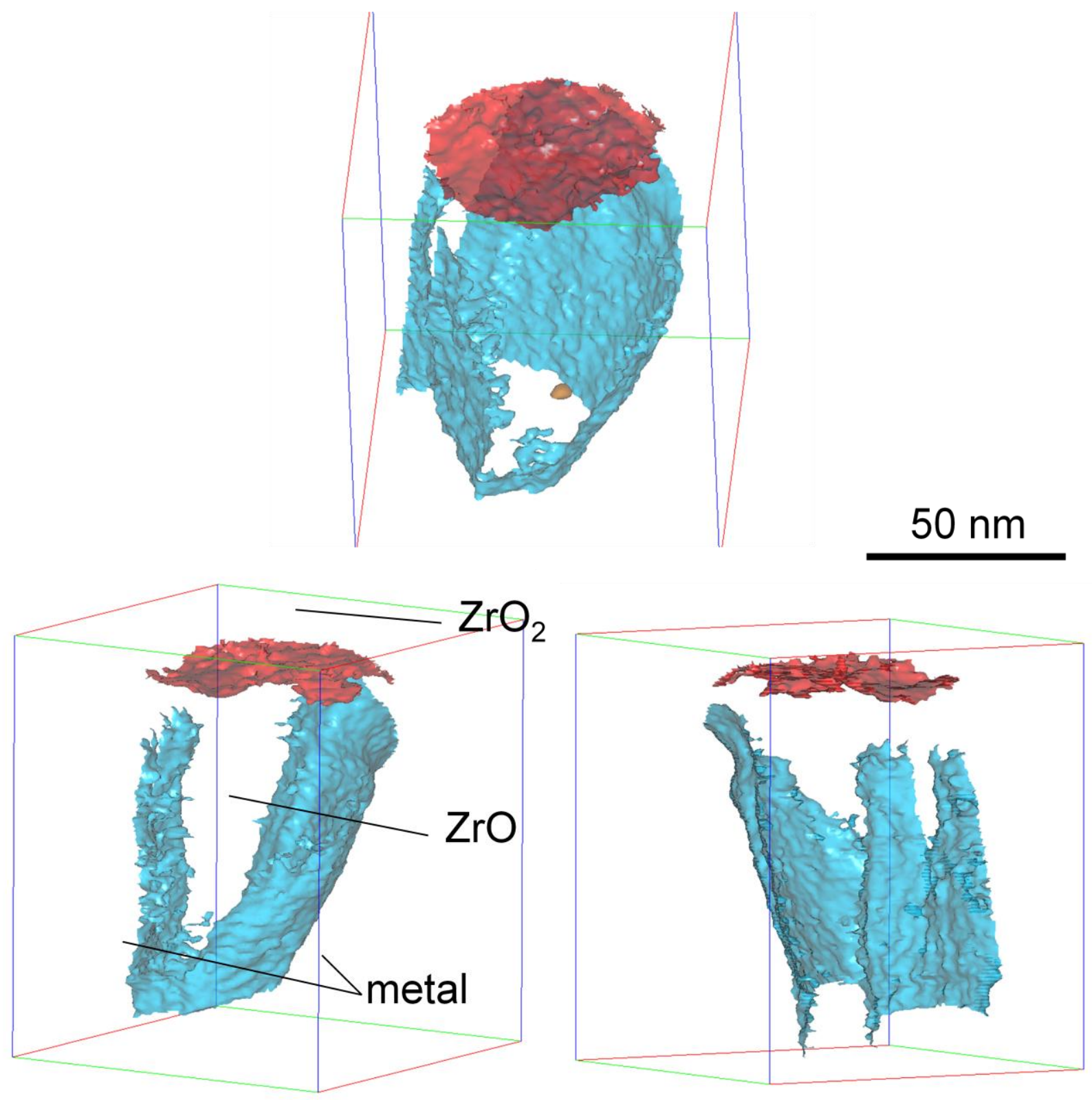

Figure 106: 3D reconstruction of a different metal-oxide interface of $28.5 \mathrm{mg} . \mathrm{dm}^{-2}$ corroded pretransition ZIRLO, showing three views of the interface. A red surface at 55 at.\% oxygen marks the interface between $\mathrm{ZrO}-\mathrm{ZrO}_{2}$ and a very complicated blue surface at 45 at.\% oxygen marks the $\mathrm{Zr}$-ZrO boundary. A brown surface with 2 at.\% $\mathrm{Nb}$ concentration defines a niobium-rich region within the $\mathrm{ZrO}$ layer. 
Figure 107 shows a dataset where only a section of the $\mathrm{ZrO}$ ' finger extending into the metal matrix is visible. The view in this profile is taken roughly perpendicular to the metal-oxide interface, rather than the profile views displayed previously. This $2 \mathrm{D}$ oxygen concentration profile demonstrates the bounds of a $\mathrm{Zr}-\mathrm{ZrO}$ ' interface. A niobium-rich area can be seen at this interface from the atom map. Comparison with the iron atom map shows that this region also has an unusually high iron concentration. The $\mathrm{ZrH}$ profile is given to show that the $\mathrm{Nb}$ atoms are properly identified and are not erroneously labelled $\mathrm{ZrH}$ ions, either from a real hydride particle or from field adsorbed hydrogen. Table 55 compares the composition defined within a 2 at.\% $\mathrm{Nb}$ surface surrounding this precipitate with the metal matrix adjacent to the interface. Figure 108, which is a $1 \mathrm{D}$ profile, shows that the concentration of niobium at the core is much higher, 20 30 at.\% $\mathrm{Nb}$. As has previously been seen, the particle has the same oxygen content as its surroundings and the particle iron concentration is $\sim 20 \mathrm{x}$ greater than in the enriched matrix (using 27 Da peak as an indicator). 


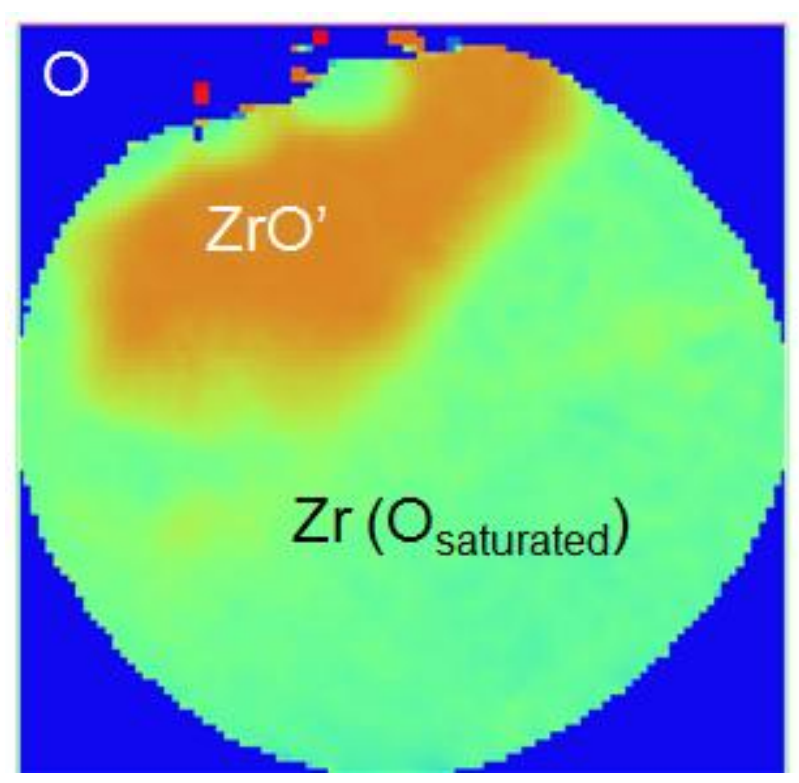

Figure 107: Left) 2D oxygen concentration profile shows the position of the $\mathrm{Zr}$ - $\mathrm{ZrO}$ interface of $28.5 \mathrm{mg} \cdot \mathrm{dm}^{-2}$ corroded pretransition ZIRLO (0 [blue] - 66 at\% [red]). Below) Atom maps showing the distribution of $\mathrm{Fe}$, at 27 and $28 \mathrm{Da}$, and $\mathrm{Nb}$ containing ions, which also contain $\mathrm{ZrH}$ and delayed ions from $\mathrm{ZrO}$ species within the sub-oxide layer.
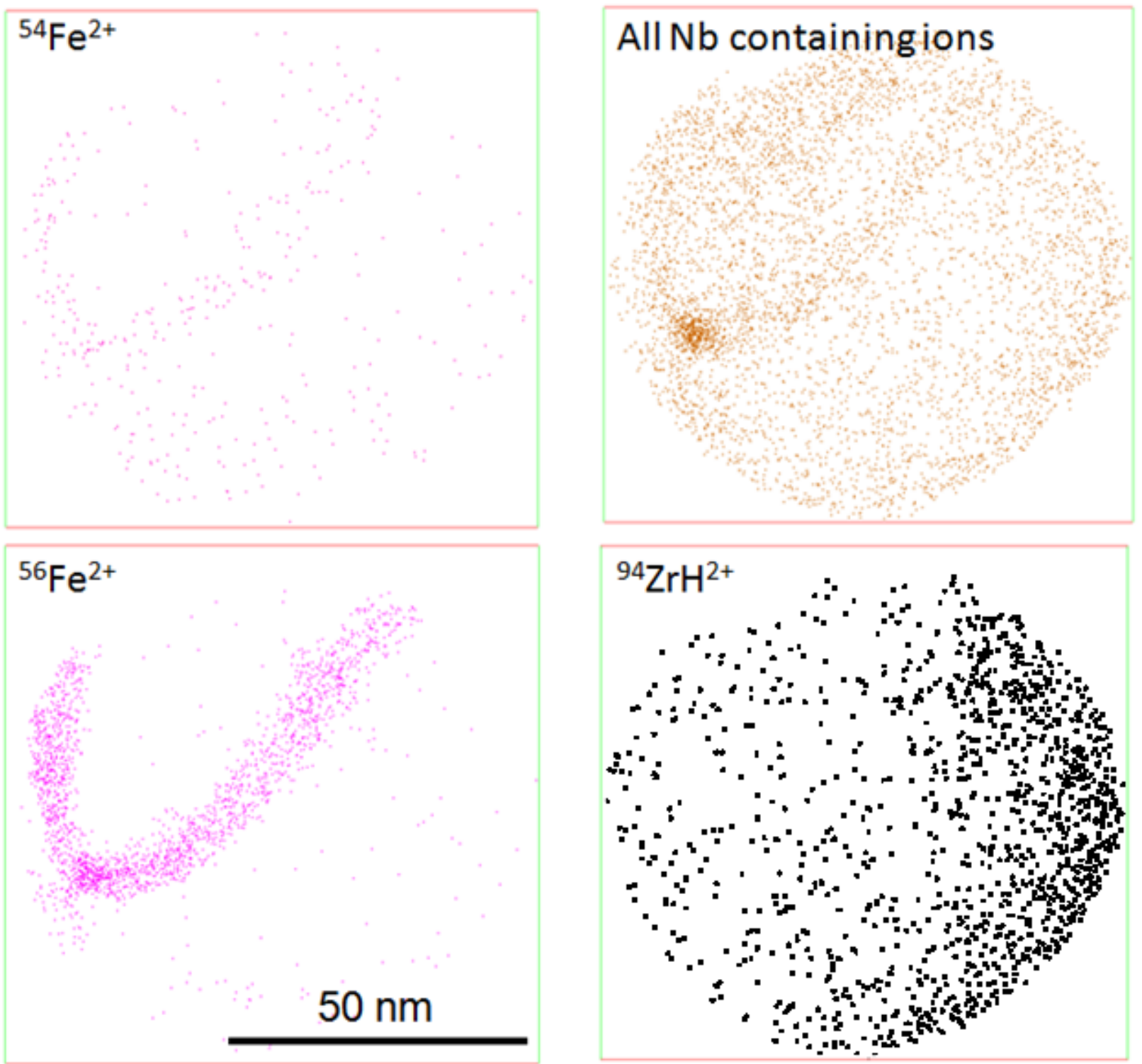


\begin{tabular}{|c|c|c|c|c|c|c|}
\hline \multirow[t]{2}{*}{ Element } & \multicolumn{3}{|c|}{ SPP } & \multicolumn{3}{|c|}{ Matrix } \\
\hline & $\begin{array}{c}\text { No. } \\
\text { (atoms) }\end{array}$ & $\begin{array}{l}\text { Conc. } \\
\text { (at.\%) }\end{array}$ & $\begin{array}{r} \pm 1 \sigma \\
\text { (at.\%) } \\
\end{array}$ & $\begin{array}{c}\text { No. } \\
\text { (atoms) }\end{array}$ & $\begin{array}{l}\text { Conc. } \\
\text { (at.\%) }\end{array}$ & $\begin{array}{l} \pm 1 \sigma \\
\text { (at.\%) } \\
\end{array}$ \\
\hline $\mathrm{Zr}$ & 3,597 & 62 & 0.6 & 976,934 & 67.49 & 0.038 \\
\hline $\mathrm{Nb}$ & 274 & 4.8 & 0.3 & 797 & 0.055 & 0.004 \\
\hline Sn & - & & & 6,506 & 0.45 & 0.01 \\
\hline 0 & 1,789 & 31.1 & 0.7 & 457,756 & 31.62 & 0.05 \\
\hline $\mathrm{Fe}(28)$ & 98 & 1.7 & 0.2 & 5,475 & 0.38 & 0.01 \\
\hline $\mathrm{Fe}(27)$ & 121 & 2.1 & 0.2 & 1,448 & 0.10 & 0.01 \\
\hline Total & 5,758 & & & $1,447,468$ & & \\
\hline
\end{tabular}

Table 55: Overall composition within the niobium-rich region adjacent to the metal-oxide interface and the surrounding region of $28.5 \mathrm{mg} \cdot \mathrm{dm}^{-2}$ corroded pre-transition ZIRLO alloy matrix shown in Figure 120. Boundary chosen to be at 2 at.\% Nb

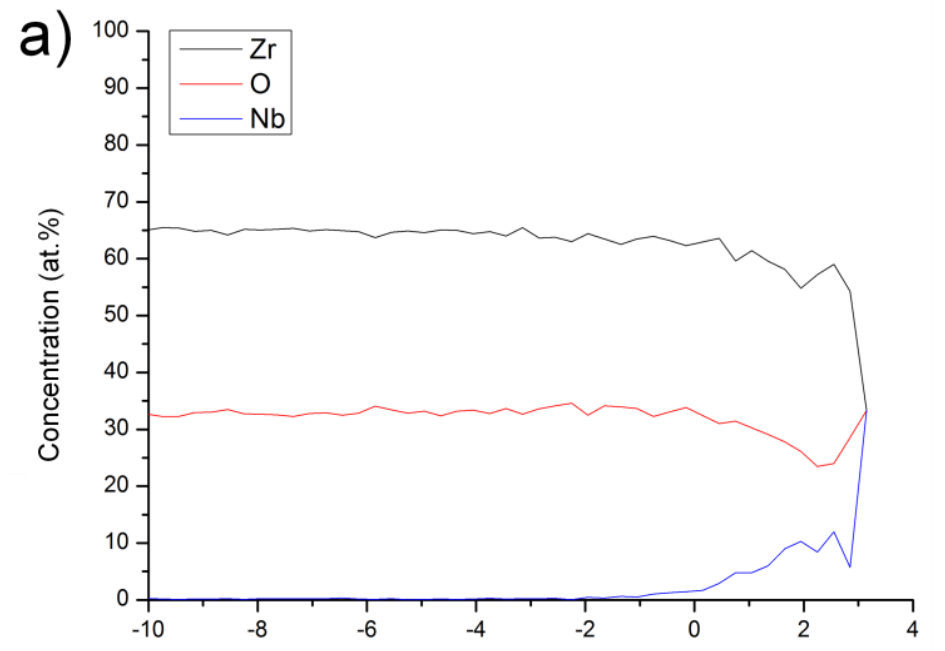

Figure 108: Composition profiles across the interface of the niobiumrich particle seen adjacent to the metal-oxide interface of $28.5 \mathrm{mg} \cdot \mathrm{dm}^{-2}$ corroded pre-transition ZIRLO in Figure 120. Showing a) $\mathrm{Zr}, \mathrm{O}$ and $\mathrm{Nb}$ profiles and b) three different

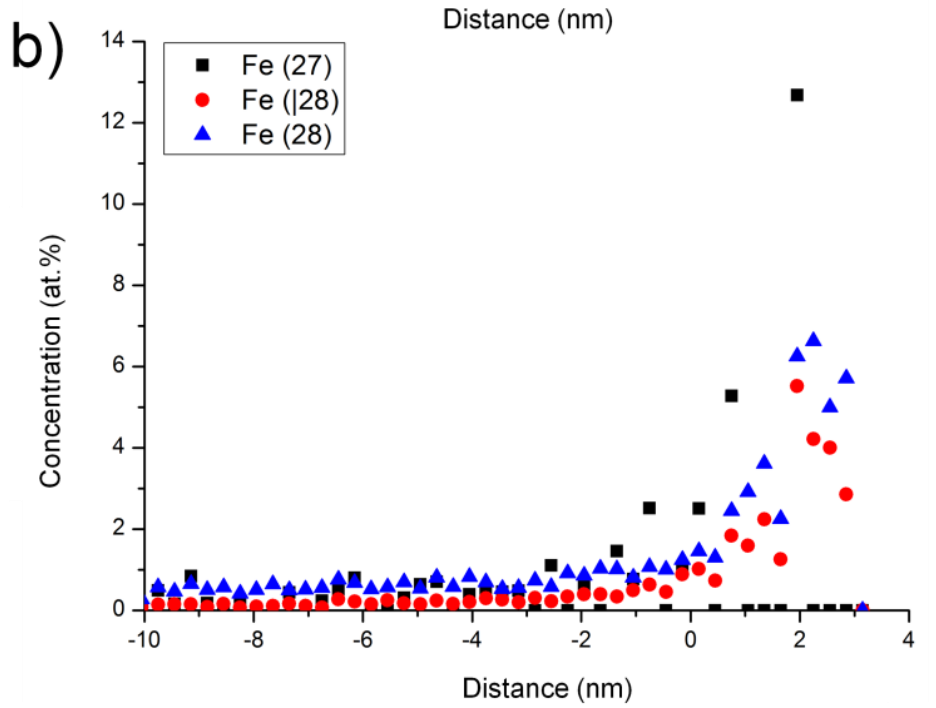
methods of describing the iron concentration at the interface using the peak at 27 Da (27 - black squares), the leading edge of the peak at $28 \mathrm{Da}(128-$ red circles) and all of 28 Da (28 - blue triangles). 
The niobium concentration at the core of the niobium-rich region shown in the 1D profile Figure 121 is high, $20-30 \%$. This suggests that these regions are the early stages of precipitation of a separate phase. The stable niobium-containing intermetallic phases in this system are $\beta-\mathrm{Nb}$ and $\mathrm{Zr}(\mathrm{Nb}, \mathrm{Fe})_{2}$. The composition of the core of this particle $(\mathrm{Zr}-30$ at.\% $\mathrm{Nb}-30$ at.\% $\mathrm{O}-5$ at.\% Fe) does not conform to either of these phases, suggesting that the nanoscale precipitates that form adjacent to the interface are meta-stable phases. More detailed analysis and interpretation of these precipitates within the metal matrix adjacent to the metal-oxide interface is described in section 6.2.4.1, which described the behaviour of solutes within the bulk and at grain boundaries affected by the corrosion process.

A large (many tens of nanometres diameter) niobium-rich precipitate was also analysed. The precipitate was within $\sim 100$ nanometres of the metal-oxide interface, although its precise location with regard to the interface is not known. The precipitate filled the field of view of the LEAP detector and the analysis run started and ended within it. The mass resolution within this large particle was poor compared to that attained in the alloy matrix. A large amount of chromium (1.4 at.\%) was detected within the precipitate, along with iron and niobium as is normally seen. The light transition metal $2+$ region of the mass charge spectra is shown in Figure 109 (a) and the chemical composition of the precipitate is given in Table 56. The standard LEAP conditions, when applied to the $\mathrm{Zr}(\mathrm{Nb}, \mathrm{Fe})_{2}$ particle, produced field conditions that evaporated most of the $\mathrm{Zr}$ as a 3+, rather than 2+ species, as shown in Figure 109 (b) and (c).

There is very little oxygen at the core of this precipitate. Unlike the particles that nucleate in the region close to the metal-oxide interface during corrosion, these large, stable, precipitates are nobler than their surroundings; they are not oxidised at or near the metal-oxide interface. The composition of this precipitate is similar to that seen in the uncorroded material, described in section 5.2.1.1. This observation is also consistent with a large body of work by other authors who have analysed the behaviour of precipitates of this scale within the oxide layer $[70,115]$. 

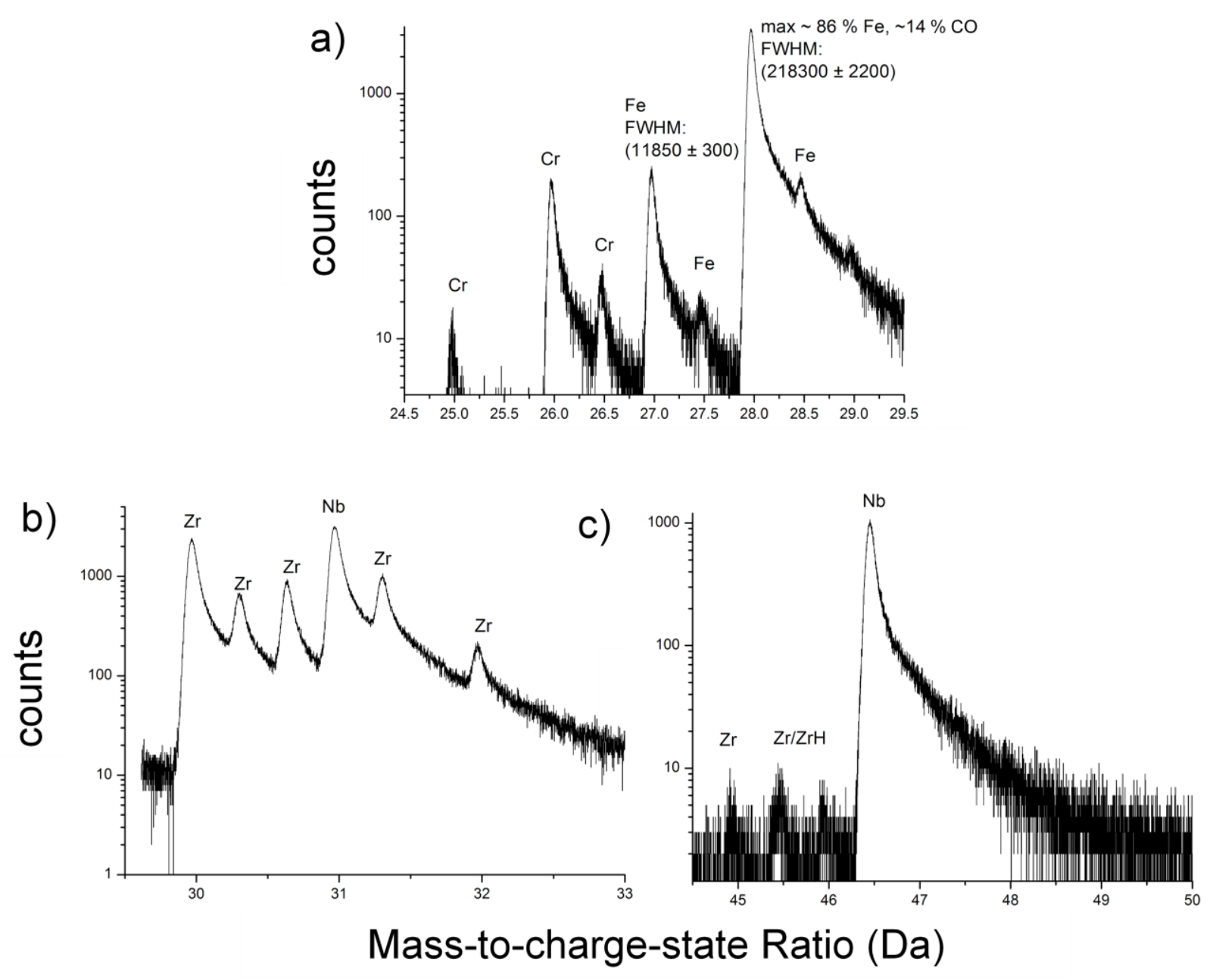

Figure 109: Mass spectra taken from within the $\mathrm{Zr}(\mathrm{Nb}, \mathrm{Fe})_{2}$ precipitate analysed nearlat the metal-oxide interface of $28.5 \mathrm{mg} . \mathrm{dm}^{-2}$ corroded pre-transition ZIRLO, showing the region of the mass spectrum around i) $\mathrm{Fe}^{3+}, \mathrm{Cr}^{3+}$, ii) $\mathrm{Zr}^{3+}, \mathrm{Nb}^{3+}$, and iii) $\mathrm{Zr}^{2+}$ and $\mathrm{Nb}^{2+}$.

\begin{tabular}{|c|c|c|c|}
\hline Element & $\begin{array}{c}\text { Number of } \\
\text { atoms } \\
\text { (atoms) }\end{array}$ & $\begin{array}{l}\text { Comr } \\
\text { Conc. } \\
\text { (at.\%) }\end{array}$ & $\begin{array}{l}\text { osition } \\
\pm 1 \sigma \\
\text { (at.\%) }\end{array}$ \\
\hline $\mathrm{Zr}$ & 708,508 & 41.20 & 0.04 \\
\hline $\begin{array}{l}\mathrm{Nb} \\
\mathrm{Sn}\end{array}$ & 540 & 31.41 & 0.04 \\
\hline 0 & 6,6 & 0.387 & 0.005 \\
\hline $\mathrm{Fe}$ (all) & 440,283 & 25.60 & 0.04 \\
\hline Cr (all) & 24,279 & 1.41 & 0.01 \\
\hline Total atoms & $1,719,842$ & & \\
\hline
\end{tabular}

Table 56: Bulk composition of a large $\operatorname{Zr}(\mathrm{Nb}, \mathrm{Fe})_{2}$ precipitate found at/very close to the metal oxide interface of $28.5 \mathrm{mg} . \mathrm{dm}^{-2}$ corroded pre-transition ZIRLO. 


\subsubsection{Iron}

The distribution of iron within the niobium-rich precipitates has already been discussed in detail in section 6.2.3.3. This section describes the distribution of iron across the metal-oxide interface.

In general, iron is seen to be enhanced in the metal matrix adjacent to the metal oxide interface in the same way that it was in the less corroded pre-transition material (section 6.2.1.4). The level in the matrix in Figure 102 is supersaturated, about tenfold above the expected level, measured using $27 \mathrm{Da}$ as a rather noisy quantitative description of the iron distribution. Qualitatively, the distribution of the peak at $27 \mathrm{Da}$ and the leading edge of $28 \mathrm{Da}$ are similar, suggesting that the peak at 27 can safely be attributed to iron, and not an impurity species. The level of iron in the $\mathrm{ZrO}$ ' and $\mathrm{ZrO}_{2}$ is much lower, below the saturation level of oxygen in virgin ZIRLO alloy matrix. Figure 103 represents this information in an idealised schematic form. There is also an excess of iron seen at the $\mathrm{Zr}-\mathrm{ZrO}$ ' interface, as seen previously in section 0 for less corroded pretransition ZIRLO. The segregation of iron at the interface can also be seen graphically in Figure 107 with both $27 \mathrm{Da}\left({ }^{54} \mathrm{Fe}^{2+}\right)$ and $28 \mathrm{Da}\left({ }^{56} \mathrm{Fe}^{2+}\right)$.

\subsubsection{Pre-transition corroded alloy matrix: $28.5{\mathrm{mg} . \mathrm{dm}^{-2}}^{-2}$}

Material that had undergone $28.5 \mathrm{mg} \cdot \mathrm{dm}^{-2}$ oxide growth after 100 days autoclave exposure was analysed by 3DAP. Specimens were prepared from the metal-oxide interface, as described above in section 6.2.3, as well as from regions of metal near to the interface and from the bulk of the corroded metal (away from the interface). This analysis is described here, and allows changes in microstructure due to the conditions at the interface to be distinguished from those produced purely by thermal aging. In this section the role of oxygen and niobium become interlinked to an extent where it is more sensible to consider them together, previous these elements have been considered separately in the description of chemistry at the metal-oxide interfaces. 


\subsubsection{Niobium}

\section{Material near the interface}

Figure 110 shows surfaces of constant concentration of 2 at.\% niobium in a region of metal within $200 \mathrm{~nm}$ of the metal-oxide interface of ZIRLO. In Figure 110 (a) all niobium-rich regions are shown. Niobium is seen to have precipitated out of the matrix. By applying a filter based on the size of the precipitates (Figure 110 (b)) the larger precipitates are seen to be aligned along a planar feature, marked by blue arrows. This is consistent with conventional nucleation and growth preferentially occurring at grain boundary sites, with further nucleation occurring later intragranularly. A large proportion of the intragranular precipitates also seem to be aligned along linear features. This suggests that dislocations within the grain are also preferred sites for nucleation.

Figure 111 shows a one-dimensional concentration profile taken normal to the grain boundary. As would be expected from Figure 110, there is a pronounced increase in the average niobium concentration at the boundary due to the precipitates. This enrichment is much greater than the fractional mono-layer level of excess niobium seen at the grain boundaries of uncorroded material and so require depletion of the surrounding matrix, rather than just redistribution of material already at the boundary. This reduces the matrix niobium concentration, in the case of the material in Figure 110, to 0.3 at.\%. This is marginally lower than the level seen in 'as received' material which is 0.36 at.\% in the matrix.

Radial concentration profiles have been taken across the interfaces of individual precipitates visible in Figure 110. Profiles for the large and small precipitates are shown in Figure 112 (a) and (b), respectively. It was seen that the chemistry of the individual particle types is consistent, and it is different for the large $(>10 \mathrm{~nm}$ diameter) intergranular precipitates and the small $(<5 \mathrm{~nm}$ diameter) intragranular precipitates. 
Figure 110: Isosurfaces at 2 at.\% niobium. Displaying all surfaces containing more than a) 100 polygons and $b$ ) 1000 polygons. Blue arrows marks large (> $10 \mathrm{~nm}$ diameter) $\beta-N b$ precipitates aligned along a planar feature. Red arrows mark strings of smaller precipitates $\left(<\begin{array}{lll}5 & \mathrm{~nm}\end{array}\right)$ decorating linear features.

c) The feature in (b) viewed along its plane after a 90 degree rotation.

d) Region of interest rotated $\sim 40^{\circ}$ to show linear features where iron atoms (pink dots) decorate dislocations. Features marked with green arrows. Localised nonlinear iron rich features circled.

e) Niobium isosurfaces as shown in (a) with Fe atoms and identifying markers described in superimposed .

Region of interest in relation to oxide/metal interface.
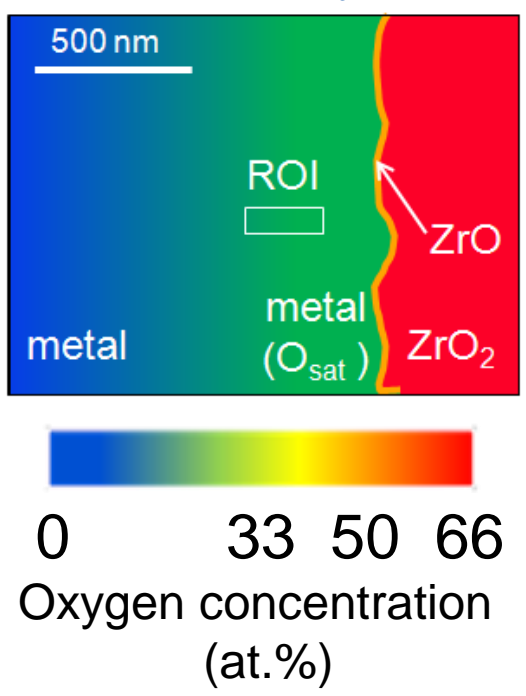
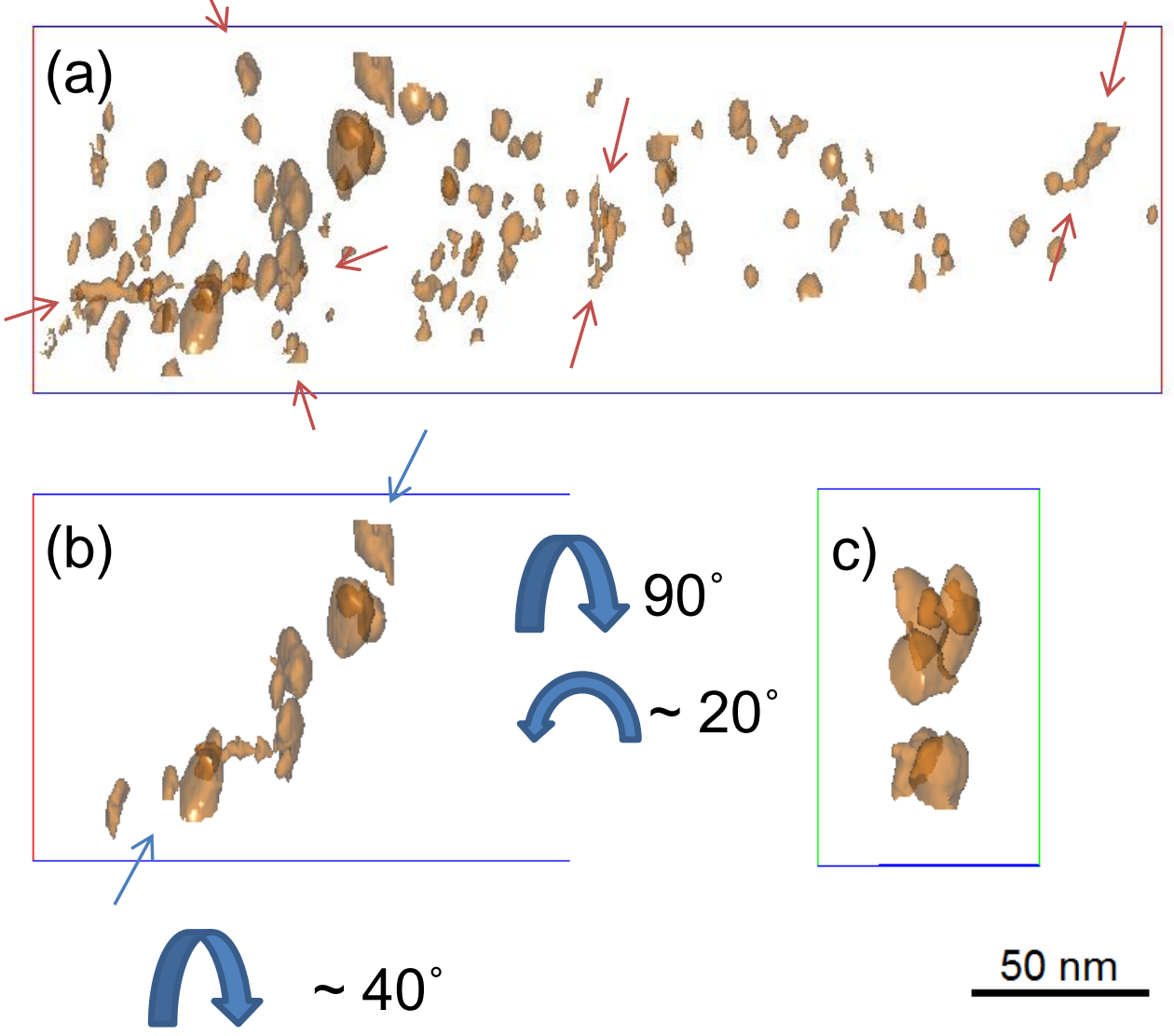

$50 \mathrm{~nm}$

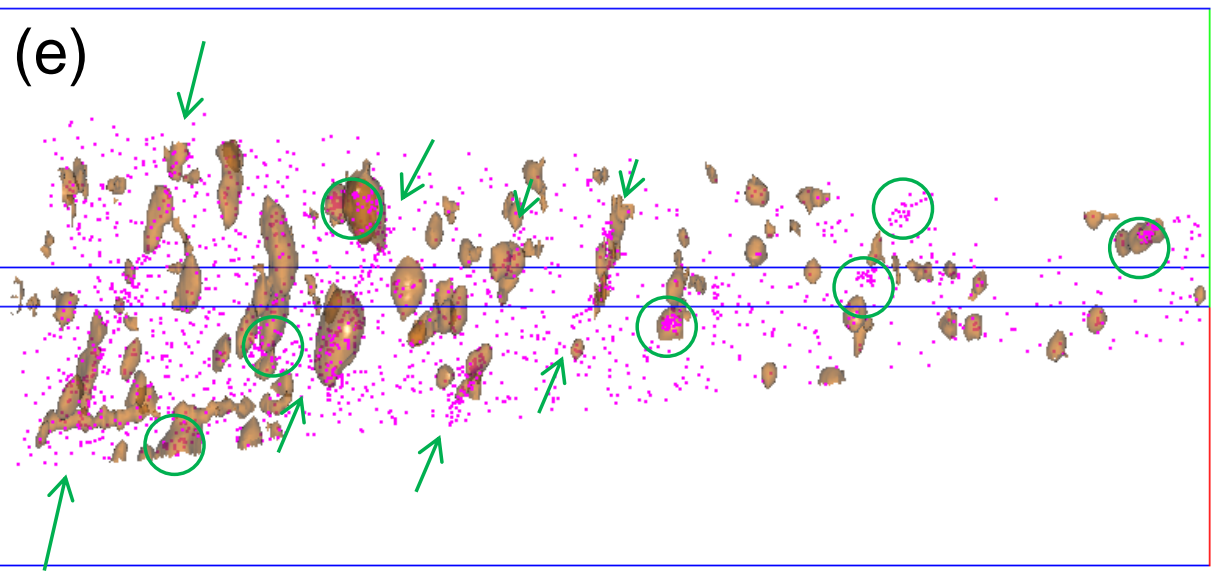



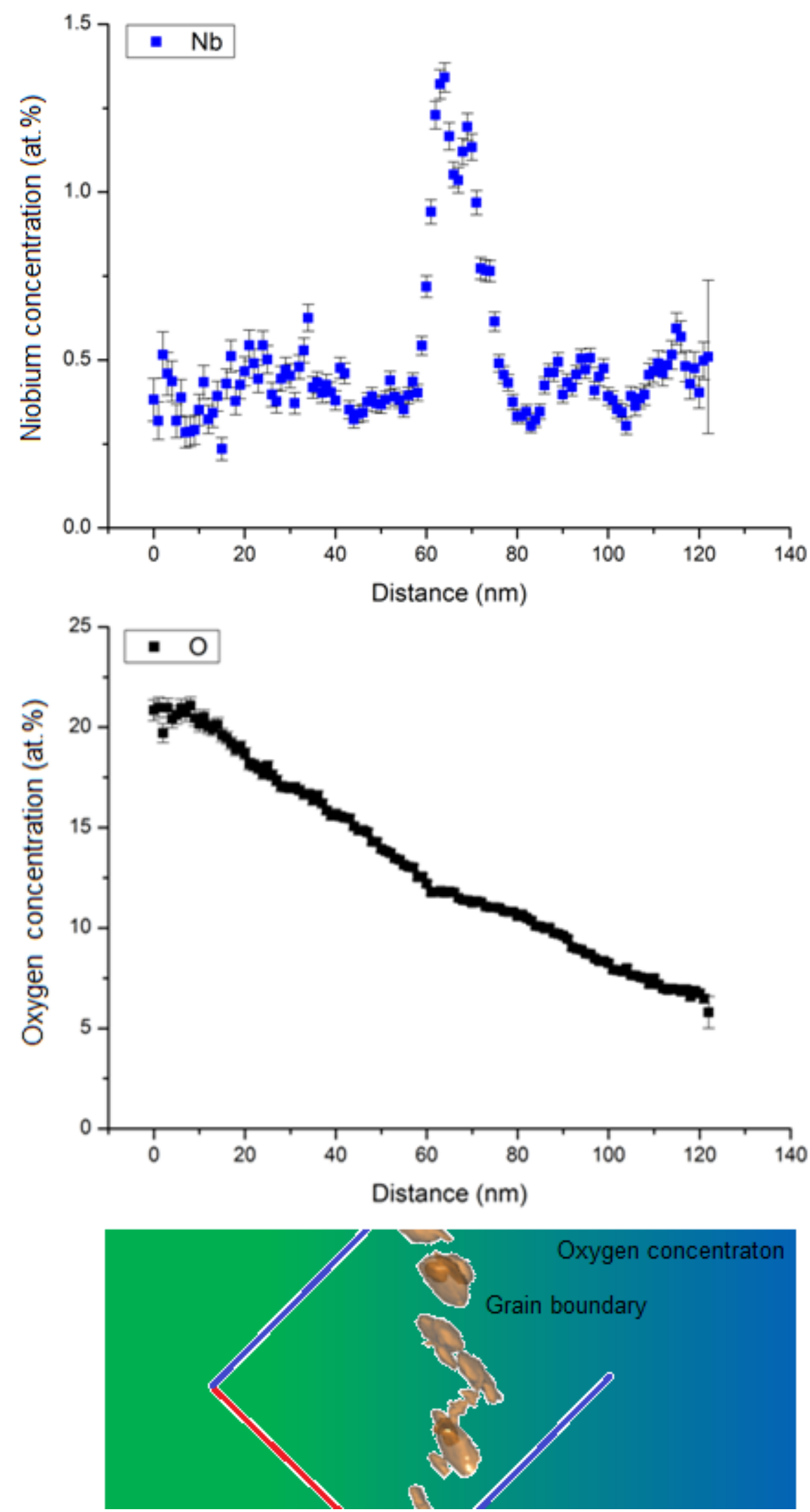

Figure 111: 1D concentration profiles across the planar feature seen in Figure 110 (b) in 28.5 $m g . d m^{-2}$ corroded pre-transition ZIRLO. Nb profile is not adjusted for ZrH overlap (15\% of the level in the metal matrix is attributed to $\mathrm{ZrH}$ ). 

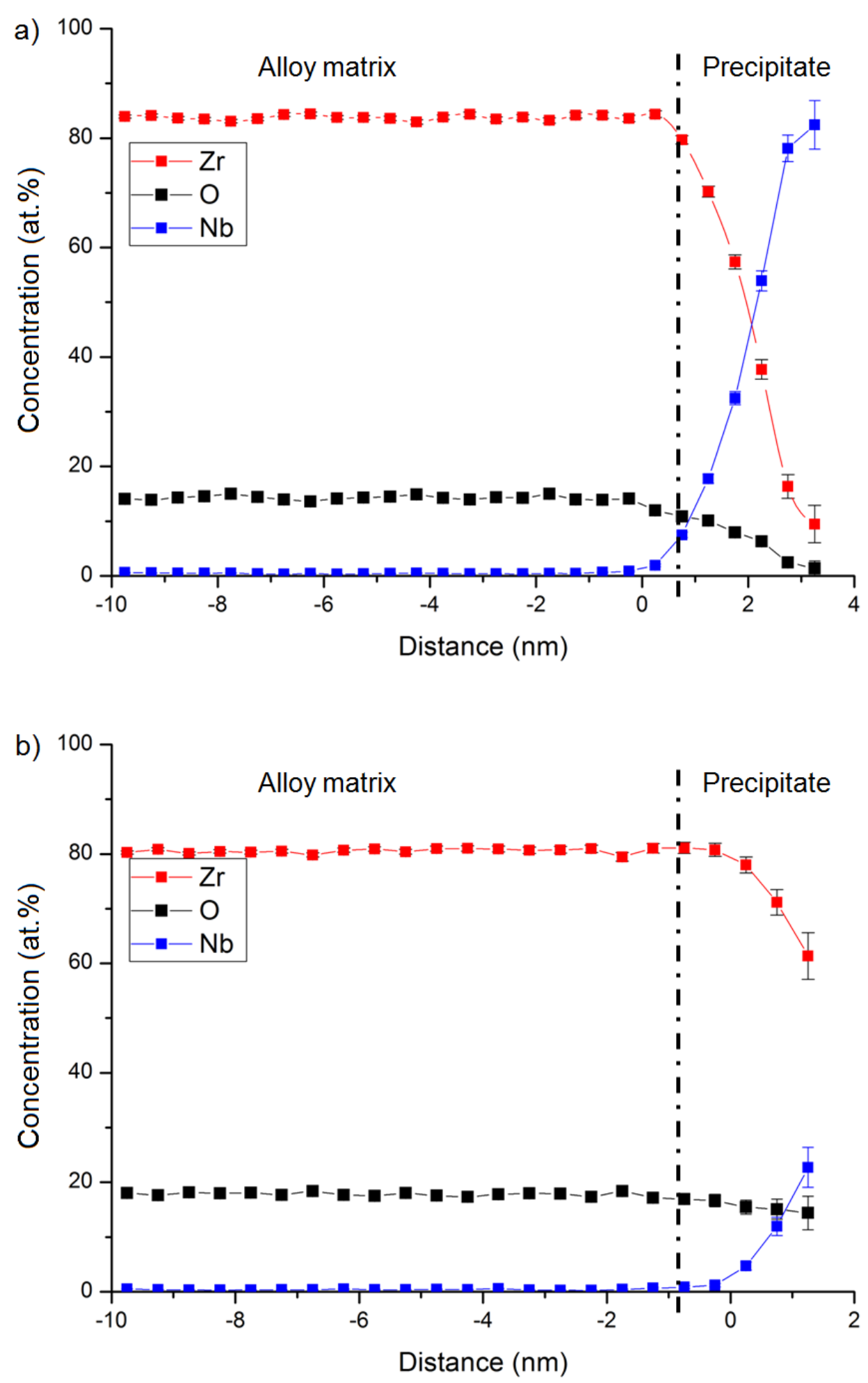

Figure 112: Radial concentration profile taken around the core of a) a large (>10 $\mathrm{nm}$ diameter), stable beta-niobium precipitates; and b) small $(<5 \mathrm{~nm}$ diameter $)$, meta-stable niobium precipitates. 
The large precipitates (Figure 112 (a)) are almost entirely pure niobium at the core, consistent with the beta-niobium phase. The small precipitates are approximately 20 at.\% niobium at their core (Figure 112 (b)), with zirconium being the main element in the particle. The particles with composition tending towards $100 \%$ niobium contain a low oxygen concentration at their core, whereas the $\mathrm{Zr}-20$ at.\% $\mathrm{Nb}$ particles contain the same level of oxygen as their surroundings, as was seen in the particles analysed in section 6.2.3.3.

$\mathrm{Zr}-20$ at.\% $\mathrm{Nb}$ is not a stable phase at room temperature, but is consistent with a suggested meta-stable beta-niobium phase. The $\mathrm{Zr}-\mathrm{Nb}$ phase diagram, shown in Figure 113, has a miscibility gap within the bcc phase [179] with a monotectoid point at 20 at.\% $\mathrm{Nb}$, corresponding with a local minima of the Gibbsian free energy. This phase is stable only above the monotectoid temperature, $\sim 895 \mathrm{~K}$ [101]. However, in a dilute $\mathrm{Zr}-\mathrm{Nb}$ alloy it may be easier for particles to initially form as meta-stable beta-niobium. For precipitates of this scale $(<5 \mathrm{~nm})$ the Gibbs-Thompson (capillary) effect will also have the effect of stabilising particles with composition other than those that would be thermodynamically stable on a macroscopic scale $[180]$.

The two distinct types of niobium precipitate seen at the two different defect sites in this study could be due to one of two mechanisms; either all of the particles originally nucleate as the metastable version and as they grow convert to become stable beta-niobium once they reach a critical size, or alternatively a different mechanism of growth operates for particles that grow at intragranular defects rather than intergranular sites. A reason for the latter mechanism is not immediately apparent. It might be expected that in fact all of the particles were originally metastable beta-niobium and are converted to a stable chemical form by further growth, which in turn has the effect of reducing the Gibbs-Thompson effect. A critical particle size must exist (between 5 and $10 \mathrm{~nm}$ ) where this conversion becomes necessary because of these thermodynamic considerations. 


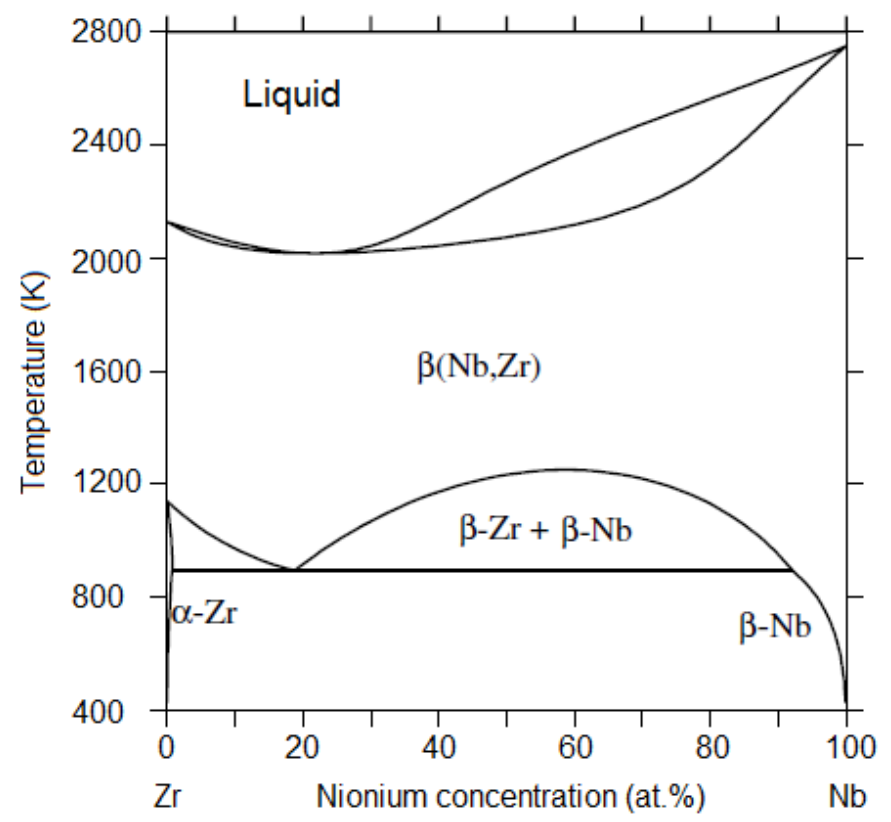

Figure 113: Zr-Nb phase diagram between 400 and $2800 \mathrm{~K}$ [101]. The $\beta$-(Nb,Zr) phase has a monotectoid at $20 \mathrm{wt} \% \mathrm{Nb}$.

The radial profiles in Figure 112 (a) and (b) have an ever decreasing sample size for the data points approaching the core of the precipitates. This is why the uncertainty in measurement is seen to increase left-to-right on all of the plot points. The lateral resolution of the instrument can be expected to be $0.5-1 \mathrm{~nm}$ around interfaces and so some 'spreading' of solute occurs which is significant when making observations of very small particles. This can significantly affect the composition measured near a particle/matrix interface. However, in this case, the particles are sufficiently large to be able to make clear statements about their core composition relative to the external matrix.

Material away from the interface (aged but not corroded)

The material processing route creates a supersaturated solid solution of niobium, so there is a driving force from the outset for the precipitation of niobium out of solution during thermal 
aging. However, the diffusion and kinetics of precipitation of niobium are very slow, and after 100 days of exposure in the autoclave precipitation is not seen in the alloy bulk.

Niobium is found at the $\mathrm{Nb} 1+, 2+$ and $3+$ charge states, as well as at $\mathrm{NbO}^{2+} . \mathrm{Nb}^{3+}$ is clearly resolved, as seen in Figure $114 \mathrm{a}$, as is $\mathrm{NbO}^{2+}$. Unfortunately, most of the detected $\mathrm{Nb}$ is derived from the peak at $46.5 \mathrm{Da}$, which is convoluted with a $\mathrm{ZrH}$ species peak. $15 \%$ of the peak at 46.5 $\mathrm{Da}$ is due to $\mathrm{ZrH}$. By ranging the $47.5 \mathrm{Da}$ species separately it can be seen that $\mathrm{ZrH}$ ions are distributed homogeneously in this dataset. This means that the measured composition of $\mathrm{Nb}$ in the metal region is really $\sim 85 \%$ of the directly measured value. In niobium-rich precipitates the proportion of $\mathrm{ZrH}$ to $\mathrm{Nb}$ is reduced and the measured composition converges on the true composition.

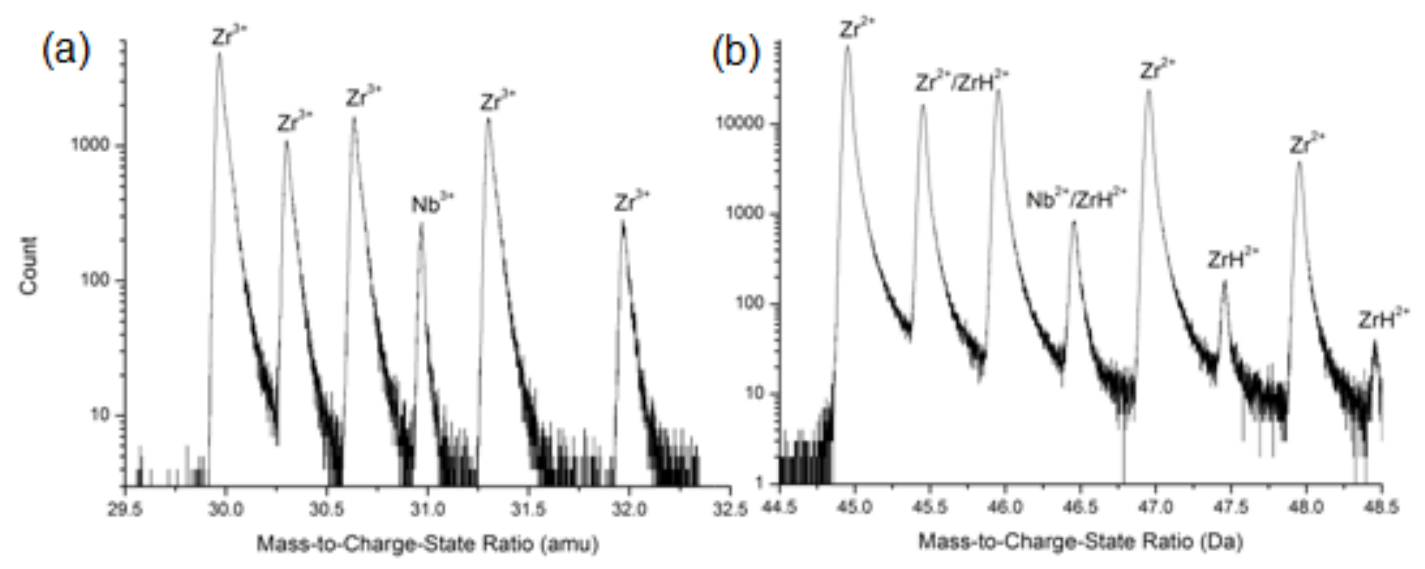

Figure 114: Mass-to-charge-state ratio spectra at a) $29.5-32.5 \mathrm{Da}$ showing $\mathrm{Zr}^{3+}$ and $\mathrm{Nb}^{3+}$, and b) $44.5-48.5$ Da showing $\mathrm{Zr}^{2+}, \mathrm{ZrH}^{2+}$ and $\mathrm{Nb}^{2+}$.

Figure 115 shows the distribution of $46.5 \mathrm{Da}(\mathrm{Nb}+\mathrm{ZrH})$ in 45,000 100 ion blocks of metal matrix in 100 day aged material. The chemistry of this region of the matrix is shown in Table 57. The oxygen level is consistent with that which is expected in the bulk metal matrix (away from the metal-oxide interface). The niobium concentration has been calculated over the whole of the region ( $4.5 \times 10^{6}$ atoms) and compensates for the overlap of $\mathrm{ZrH}$ with $\mathrm{Nb}$. Unfortunately a great 
deal of precision is lost as in this dataset the peak at 46.5 contains a large quantity of $\mathrm{ZrH}$ and only $\sim 10 \% \mathrm{Nb}$. The distribution in Figure 115 (a) shows that the peak at $46.5 \mathrm{Da}$ has a similar distribution to a binomial distribution, therefore the niobium that is present does not significantly perturb the distribution from that of a random solution. This region of matrix is also shown as an atom map in Figure 115(b). No niobium (or $\mathrm{ZrH}$ ) ion clustering is visible by inspection of this map.
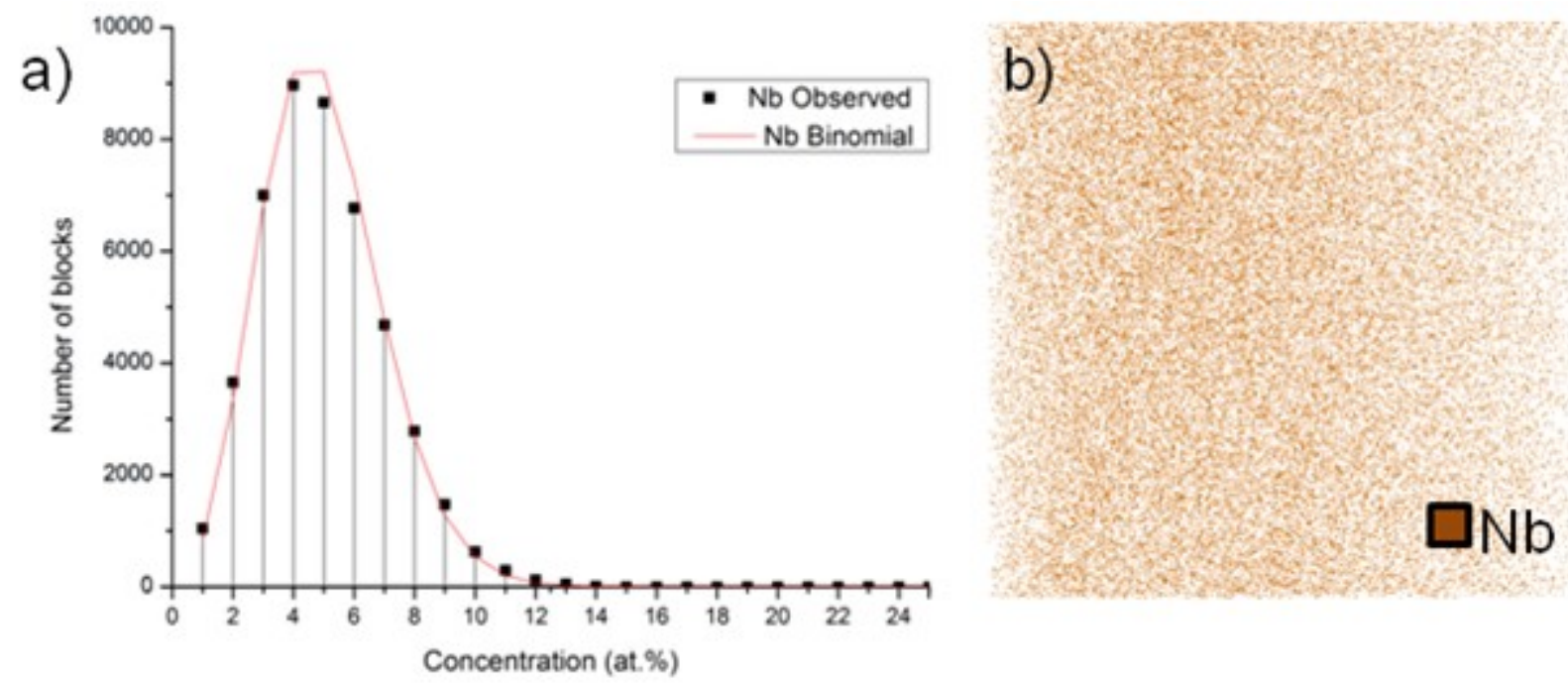

Figure 115: a) Comparison between a Poisson distribution (red curve) and the experimentally observed distribution of the number of 100 ion blocks of solute containing a certain percentage of $\mathrm{ZrH}$ and $\mathrm{Nb}(46.5 \mathrm{Da})$ ions. The 4.5 million ion dataset is split into 45,000 blocks. The good agreement between the experimental and theoretical curve suggests that ions are distributed as a random solid solution. b) A region of alloy matrix subjected to 100 days of autoclave exposure in a different dataset containing 6 million ions. No clustering of solute or precipitation can be observed visually. The apparent horizontal density variation is because the volume is cylindrical, and is being viewed normal to the axis of the cylinder. 


\begin{tabular}{|c|c|cc|}
\hline Element & $\begin{array}{c}\text { Number } \\
\text { of atoms }\end{array}$ & \multicolumn{2}{|c|}{$\begin{array}{cc}\text { Composition } \\
\text { Conc. }\end{array}$} \\
& $\begin{array}{c} \pm 1 \sigma \\
\text { (atoms) }\end{array}$ & (at.\%) & $($ at. $\%)$ \\
\cline { 2 - 4 } $\mathrm{Zr}$ & 4391653 & 97.80 & 0.007 \\
$\mathrm{Nb}$ & 18090 & 0.40 & 0.01 \\
$\mathrm{Sn}$ & 37106 & 0.826 & 0.006 \\
$\mathrm{O}$ & 42997 & 0.958 & 0.005 \\
$\mathrm{Fe}(28)$ & 426 & 0.0095 & 0.0005 \\
Total & 4490272 & & \\
\hline
\end{tabular}

Table 57: Solute concentration in the bulk alloy matrix away from the metal-oxide interface or any microstructural features in $28.5 \mathrm{mg} \cdot \mathrm{dm}^{-2}$ corroded pre-transition ZIRLO.

Comparison of niobium behaviour in near the surface and the bulk

Precipitation is only seen at and near the metal-oxide interface. Here the nucleation of particles occurs primarily at dislocations and grain boundaries, but is also seen in the matrix adjacent to the interface. Niobium precipitation has not been observed in bulk material having undergone the same level of corrosion, and is also not seen in less corroded material, or indeed in as-received ZIRLO. This effect is attributed to the presence of high levels of oxygen, introduced into the metal matrix by the corrosion process, as well as the higher dislocation density caused by interfacial stresses creating heterogeneous nucleation sites within the matrix. Thermodynamic considerations show that the solubility of niobium in alpha-zirconium is reduced by the addition of oxygen to the solution [95]; an extended version of Figure 8 is reproduced as Figure 116 demonstrating the effect of small oxygen additions on the niobium solubility. The observed oxygen concentration in the metal adjacent to the interface is consistent with the accepted value 
for the solubility of oxygen in zirconium, 28 - 35 at.\% depending upon chemistry and temperature $[19,101]$.

Both the oxygen concentration in the layer ahead of the interface, which increases the driving force for niobium precipitation, as well as the length of time this layer has been present, are important factors in promoting the precipitation of niobium in regions adjacent to the metaloxide interface.

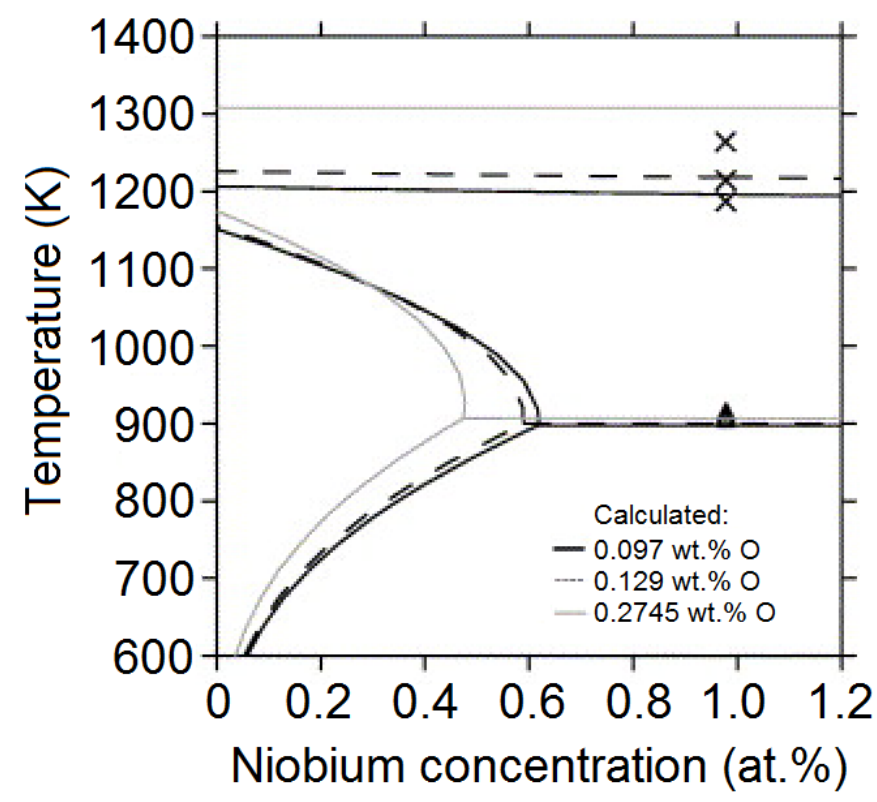

Figure 116: Partial Zr-Nb phase diagram showing the effect of oxygen additions on the solubility of $\mathrm{Nb}$ in $\alpha-\operatorname{Zr}[95]$. 


\subsubsection{Iron}

Correlation of iron concentration with local oxygen level

The distribution of the local concentration of iron against that of oxygen is plotted in Figure 117. These data combine results from several different specimens, prepared from various locations within corroded material; a key for where these regions are found in the material is shown in Figure 118. The metal regions nearer to the surface contain additional oxygen that has been introduced into the metal by the corrosion process [181]. The bulk of the material has not been affected in this way and continues to contain around $0.7-1.2$ at.\% oxygen, introduced by the original fabrication process. In datapoints (i) the level of oxygen is consistent with the background level seen in as-received material and represents regions of metal matrix in corroded material that are remote from the metal-oxide interface. The iron concentration in this region, (i), occupies a lower range than the iron distribution observed in the as-received condition (described previously in section 5.1.6.2).

The general trend in the iron concentration in regions (i), (ii) and (iii) from Figure 117 (b) is that the iron concentration increases with the local oxygen concentration. Analysis of the metal oxide interface of pre-transition material, described previously in sections 6.2.2 and 6.2.3, showed that iron is rejected from the formation of the metal oxide interface, and this could partially explain the enhanced iron concentration seen in the oxygen-rich regions. However, it is known that iron diffuses anomalously quickly in the matrix of $\alpha-\mathrm{Zr}$ (and indeed $\beta-\mathrm{Zr}$ ) [171] and so the rejected iron might be expected to become redistributed within the bulk of the material. 

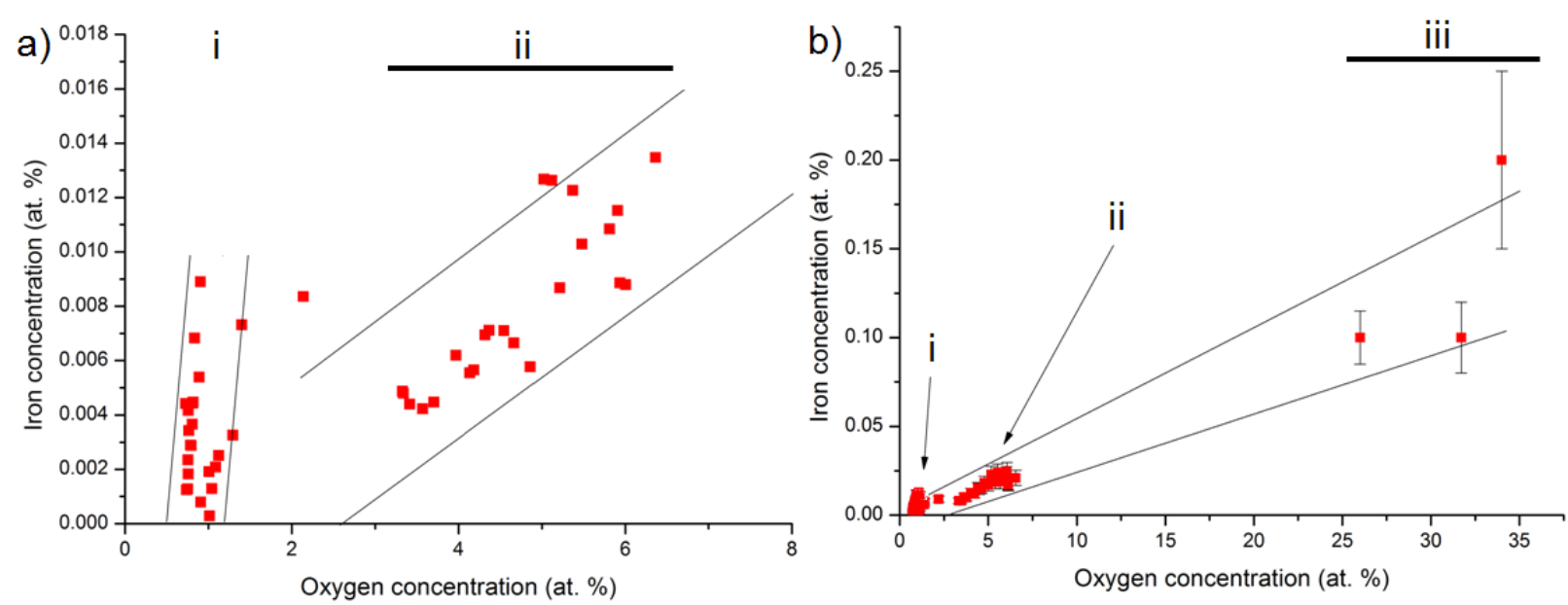

Figure 117: Plots of measured iron composition against measured oxygen composition of $1 m$ ion blocks of experiment data. Red squares markers represent data obtained after 94 days exposure in an autoclave (360 C, $18 \mathrm{MPa}$ primary water). a) Profile shows low oxygen concentration data. b) Profile includes regions from near the metal-oxide interface (iii). Relative position of data points with regard to the metal-oxide interface of 94 day exposed material is shown in key Figure 118.

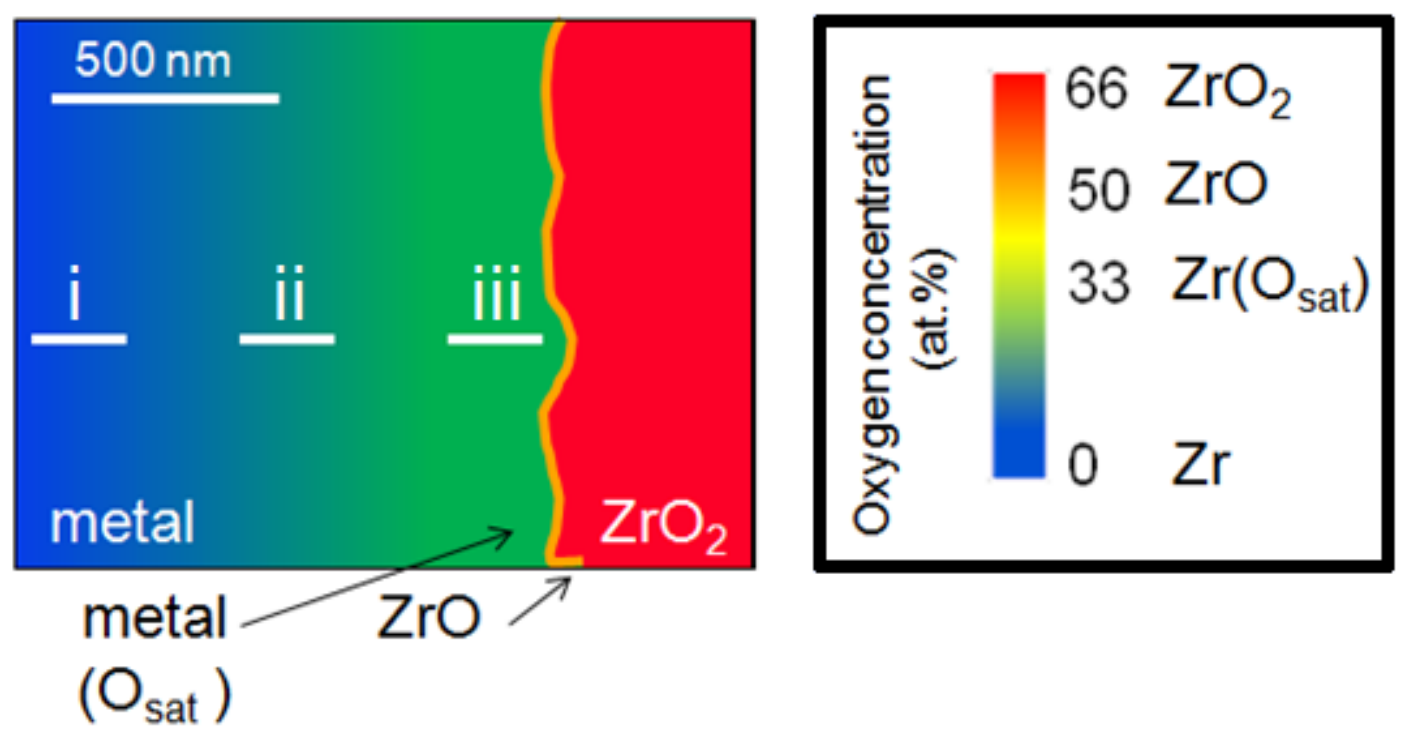

Figure 118: Key to the location of data points seen in Figure 117 with respect to the metal-oxide interface in $28.5 \mathrm{mg} . \mathrm{dm}^{-2}$ corroded pre-transition ZIRLO. 
These observations suggests that the iron solubility within the matrix material is proportional to the oxygen concentration, and that the additional solute in oxygen rich regions appears to be from iron that has migrated from the matrix that is not enriched with oxygen. Increased oxygen concentration may induce disorder in the matrix lattice and so reduce the strain of accommodating iron atoms interstitially. Other authors have suggested mechanisms whereby FeO pairs occupy interstitial sites in the $\mathrm{Zr}$ lattice [182], which would also suggest that additional oxygen could create low-energy intragranular sites for iron. It has not been established whether iron acts as either an interstitial or substitutional solute in alpha-zirconium, due to its intermediate size (atomic radius in situations of 12-fold symmetry; Zr: $1.6 \AA$, Fe: 1.28, O: 0.60 [160]). 
Figure 119 show the mass-to-charge-state spectrum of a combination of many of the data points used to measure the Fe/O ratio at mid-range (ii) oxygen concentrations in Figure 117. In this dataset the peak at 28.5 Da is discernible from the tail of $28 \mathrm{Da}$, and the isotopic ratio is in good agreement with that of iron. In contrast, the ratio of $27 \mathrm{Da}$ to $28 \mathrm{Da}$ suggests that 27 is overlapping with another species, as has been described previously for other datasets. The automated method used to inspect the iron concentration of the matrix (see Appendix A and B) used only the higher signal/noise ratio peak at $28 \mathrm{Da}$.

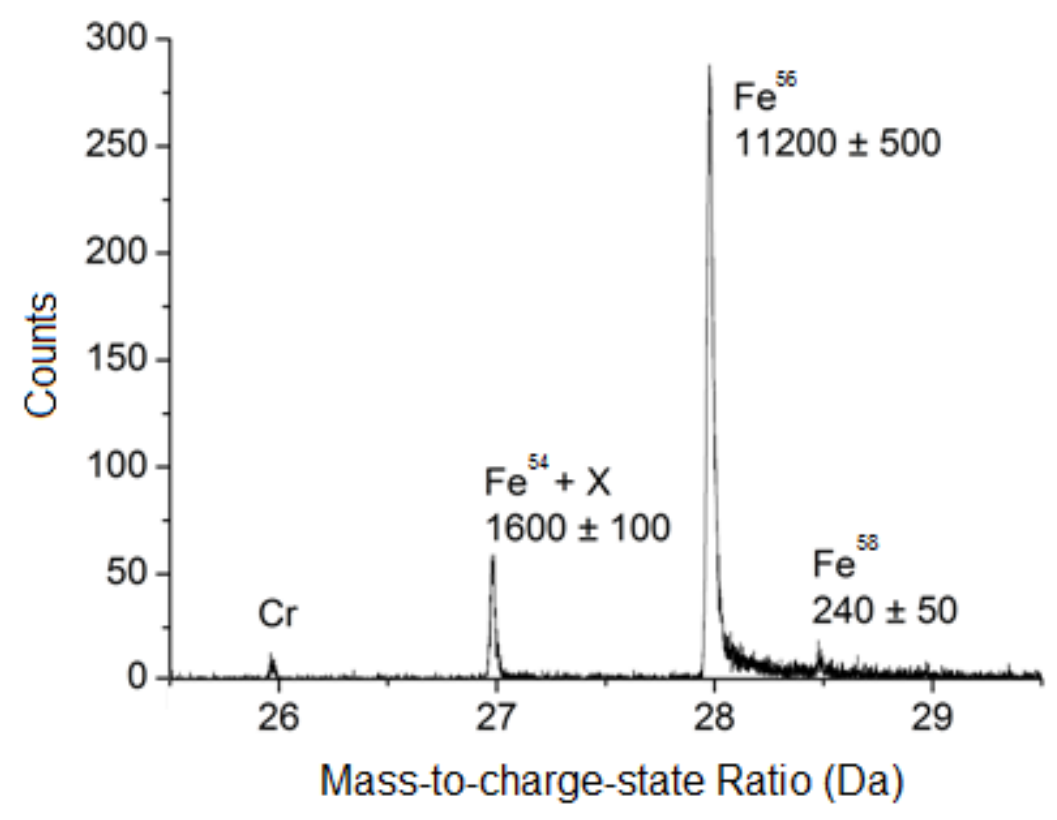

Figure 119: Mass-to-charge state ratio showing the region around $\mathrm{Fe}^{2+}$ in $28.5 \mathrm{mg} \cdot \mathrm{dm}^{-2}$ corroded pre-transition ZIRLO. Peak area values determined manually for the whole visible area of background corrected data. Plot shown is not background corrected. 
$\mathrm{CO} / \mathrm{Fe}$ overlap was found to be an issue with the niobium precipitate data described in Figure 110. The distribution of 27 and $28 \mathrm{Da}$ is shown in Figure 120 and shows how the iron distribution can be described quantitatively when both 27 and $28 \mathrm{Da}$ are subject to unknown levels of contamination, removing the ability to quantitatively describe the iron concentration. The distribution of iron is still well described qualitatively by the left-hand atom map taken from the leading edge of $28 \mathrm{Da}$.

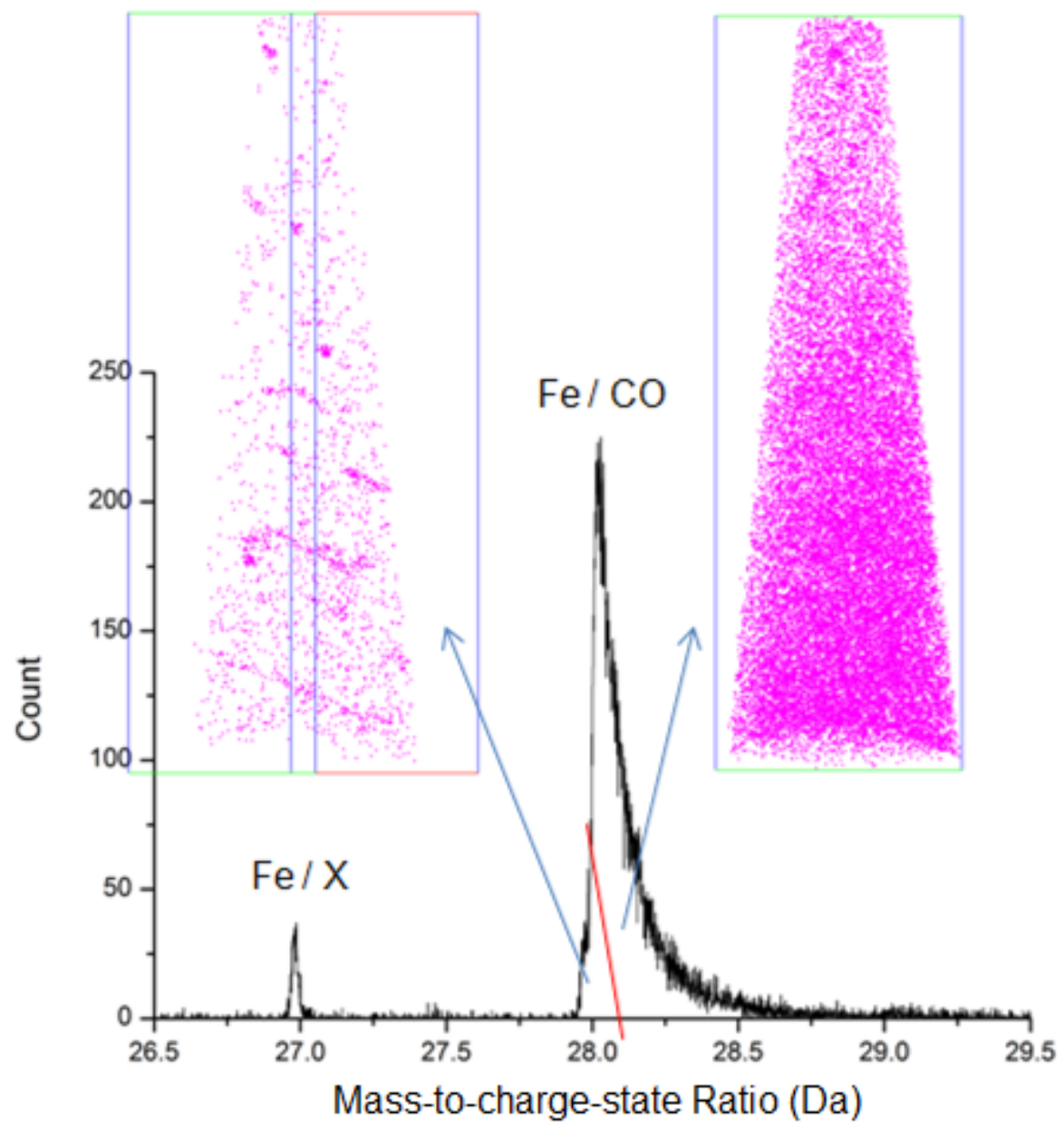

Figure 120: Mass-to-charge-state spectrum at 26.5 - $29.5 \mathrm{Da}$. Windowing the leading edge of the peak at 28 shows solute to be strongly segregated to linear features in $28.5 \mathrm{mg} . \mathrm{dm}^{-2}$ corroded pre-transition ZIRLO near the metal-oxide interface. Ranging the whole peak gives an apparently homogeneous distribution. 


\subsubsection{Post-transition metal-oxide interface: $45.9{\mathrm{mg} . \mathrm{dm}^{-2}}^{-2}$}

After 140 days the oxidation weight gain kinetics has departed from the pre-transition cubic/quadratic relationship and oxidation begins to proceed at an elevated rate (see Figure 29 in section 3.4.2.2). The sample analysed had an average weight gain consistent with the general trend after this period of oxidation. From the weight gain data, it was determined that the sample analysed had undergone break-away. However, the samples taken out of the autoclave after this period of time do not sport the mottled grey/black patches often associated with the breakaway process. The surface of the oxide appears black and is fully adherent to the substrate.

\subsubsection{Oxygen}

Figure 121 (a) shows a 2D oxygen concentration profile for a specimen having undergone transition. The sketch in Figure 121 (b) describes the specimen geometry and shows why the oxide region is towards the lower left hand side of the dataset, rather than running parallel with the metal vertically or forming an oxide cap, as in previous examples. Figure 121 (a) shows a sharp interface between $\mathrm{ZrO}_{2}$ oxide and oxygen saturated metallic zirconium, with no $\mathrm{ZrO}$ interfacial layer. The oxygen saturated region appears to be quite thin, and its bounds are not entirely parallel to the interface. The blue region towards the top of the region of interest is metal matrix containing only a few atomic percent oxygen.

During the transition in oxidation kinetics many hundreds of nanometres of metal are consumed by rapid oxide growth. It appears that the $\mathrm{ZrO}$ layer that develops during the slow pre-transition oxidation is completely engulfed by this process. The oxide/metal interface now appears to have a very similar morphology to the early pre-transition material that had undergone an initial period of rapid growth and then a short period of slower growth. The chief difference between 
the two situations is that a very thin region of oxygen enriched metal is either retained at the post transition interface or develops within a few days of the transition event.

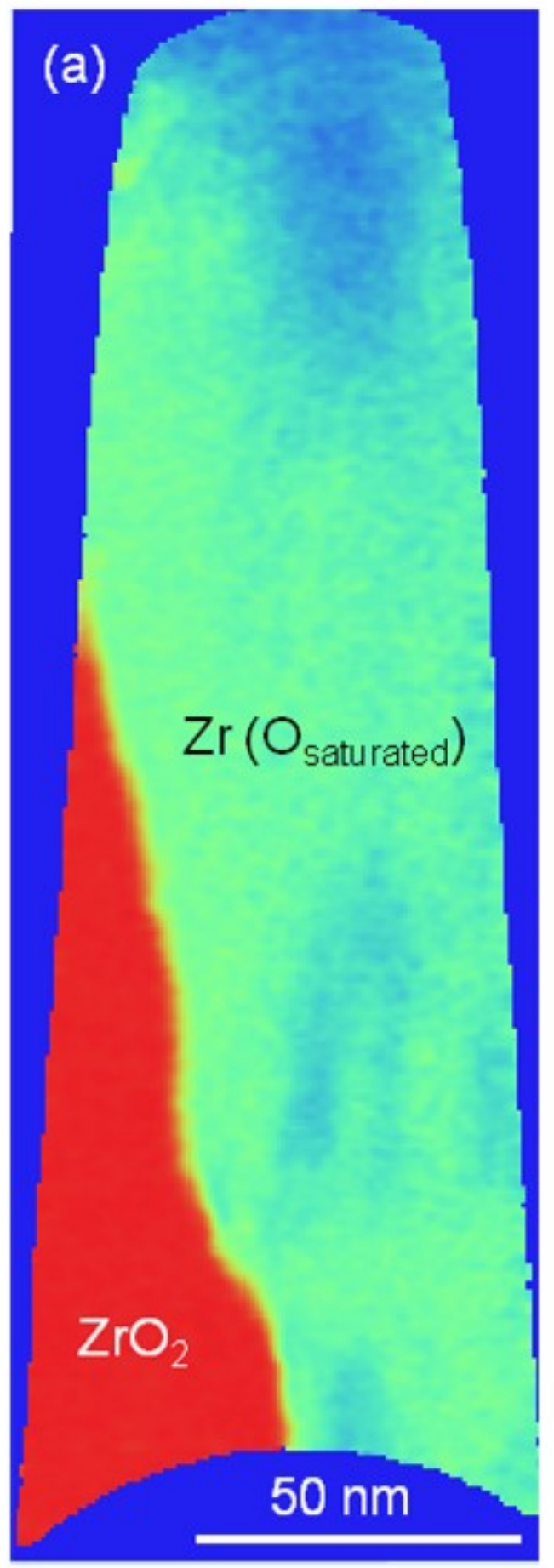

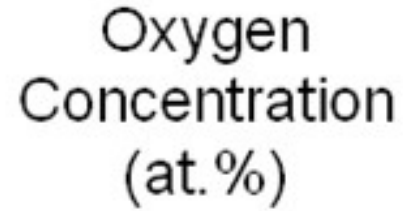

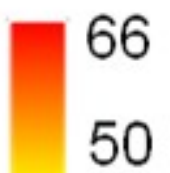

33

Region of interest

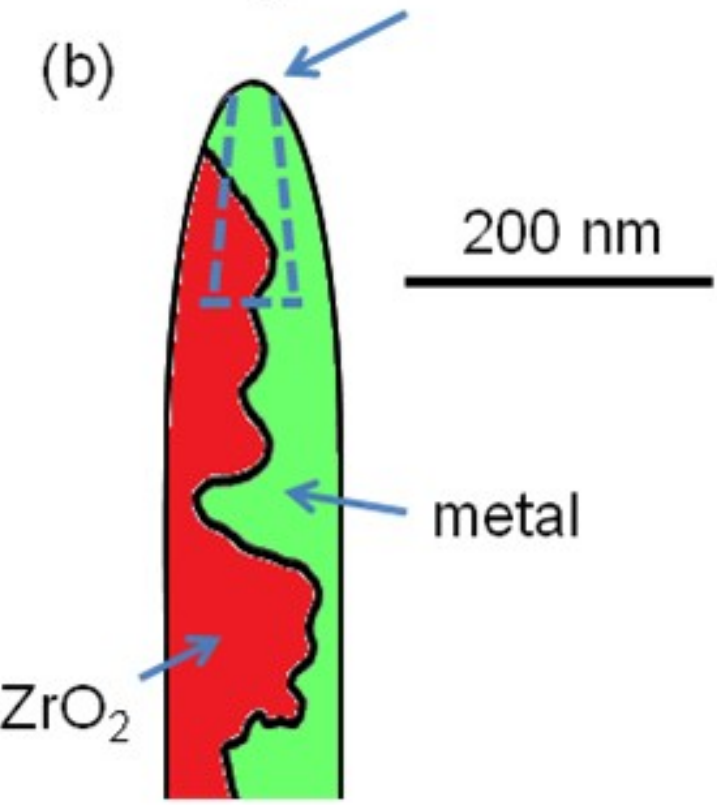

Figure 121: a) 2D oxygen concentration profile across the metal-oxide interface of $45.9 \mathrm{mg}^{-\mathrm{dm}^{-2}}$ corroded post-transition ZIRLO with b) explanation of tip geometry used to produce this specimen. 


\subsubsection{Tin}

The 1D tin profile across the metal-oxide interface of the specimen of Figure 121 is shown in Figure 122 (a). The mass spectra of the metal and oxide regions of the Figure 122 profile are displayed in Figure 123 (a) and (b). The majority of the 10 tin isotopes can be resolved within the metal region, (a), although only the two most significant are used to calculate the tin content in order to improve the signal to noise ratio $(\mathrm{S} / \mathrm{N})$. The spectrum of the oxide region, $(\mathrm{b})$, has a large overlap of the delayed $\mathrm{ZrO}^{+}$ions. The $\mathrm{S} / \mathrm{N}$ of ${ }^{120} \mathrm{Sn}^{2+}(60 \mathrm{Da})$ is reduced from $\sim 50$ in the metal to $2-3$ in the oxide. The additional counts from the $\mathrm{ZrO}$ overlap are corrected in this profile by deducting an equal area ahead of the peaks at 59 and $60 \mathrm{Da}$. The profile in Figure 122 (a) also has a higher uncertainty at its extremities, which can be understood by inspection of Figure 121; the volume of slices taken parallel to the interface decreases as the distance from the boundary increases due to the geometry of the dataset.

The tin concentration profile in Figure 122 (a) is at a constant level in the metal, $\sim 0.5$ at.\%, and undergoes a change at the interface to another roughly constant level, $\sim 0.3$ at.\%. There is also an apparent excess of tin at the interface, as has been seen previously. The idealised profile Figure 124 shows the behaviour of tin in terms of the $\mathrm{Sn} / \mathrm{Zr}$ ratio, rather than looking at these absolute tin concentrations. The tin concentration on the metal side agrees well with the expected level of tin in solid solution, 0.76 at.\%, after this correction for added oxygen. It should be noted that in order for this flat profile to be plotted, the tin content in the oxide would need to be 0.25 at. $\%$, rather than the 0.3 at. $\%$ observed. This difference is accounted for by the high level of noise in the oxide region, shown in Figure 123 (b), reducing the accuracy of the measurement attained. 

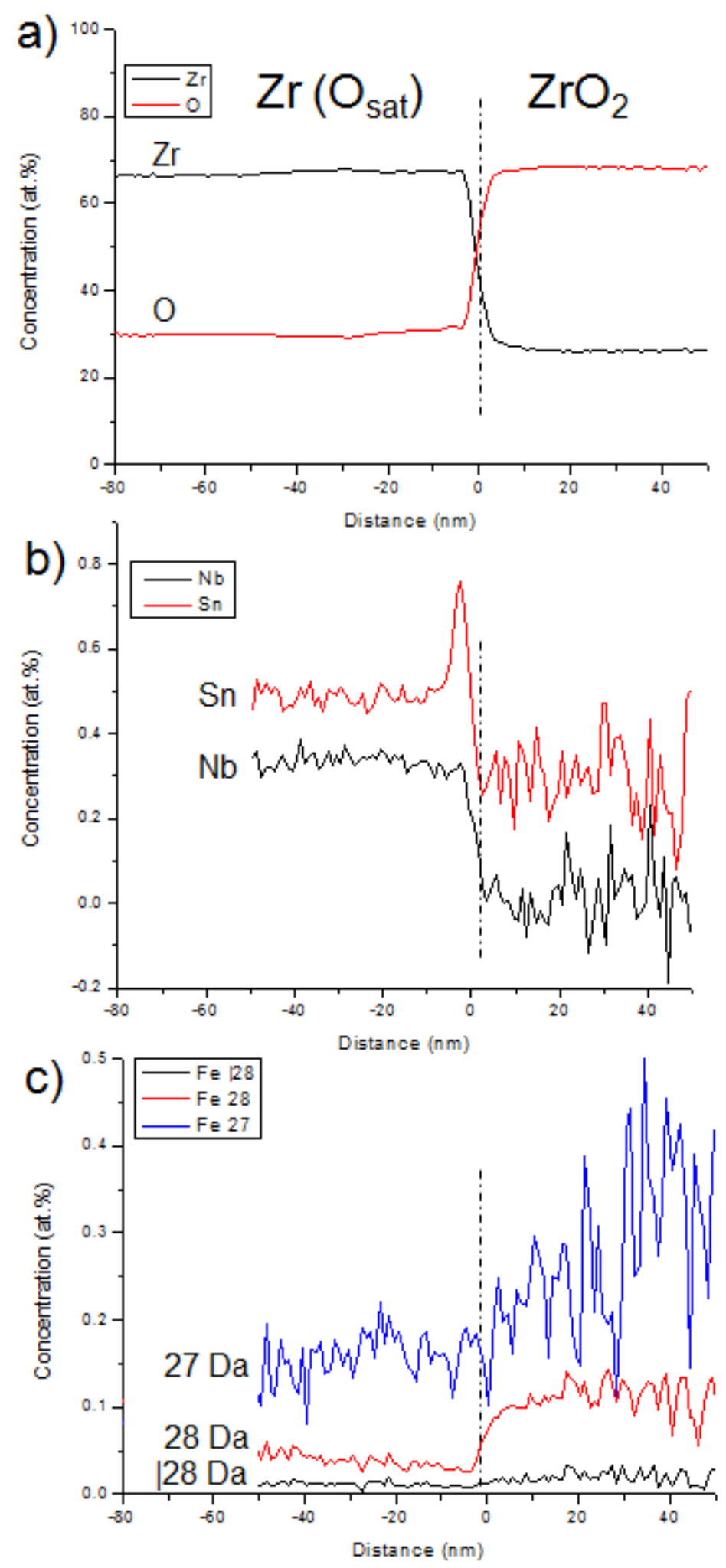

Figure 122: $1 D$ concentration profiles across the metal-oxide interface of $45.9 \mathrm{mg} . \mathrm{dm}^{-2}$ corroded post-transition ZIRLO. Elements plotted: a) Zr and O; b) Sn and Nb; and three different methods of estimating Fe distributions as previously described for Figure 93 and Figure 108. 
a)

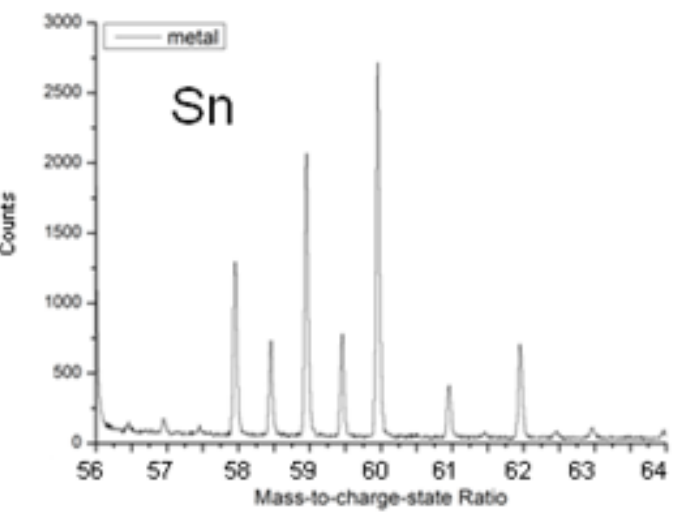

c)

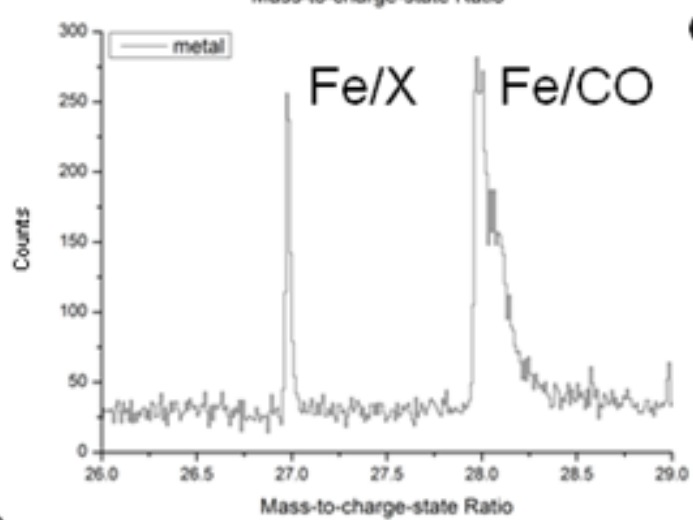

e)

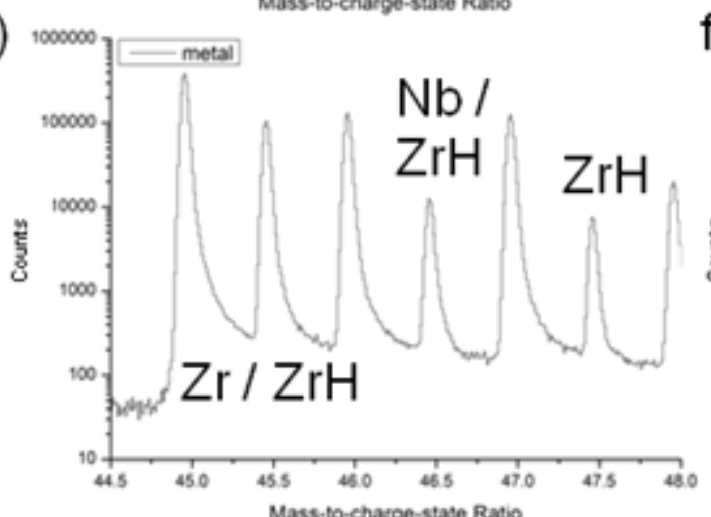

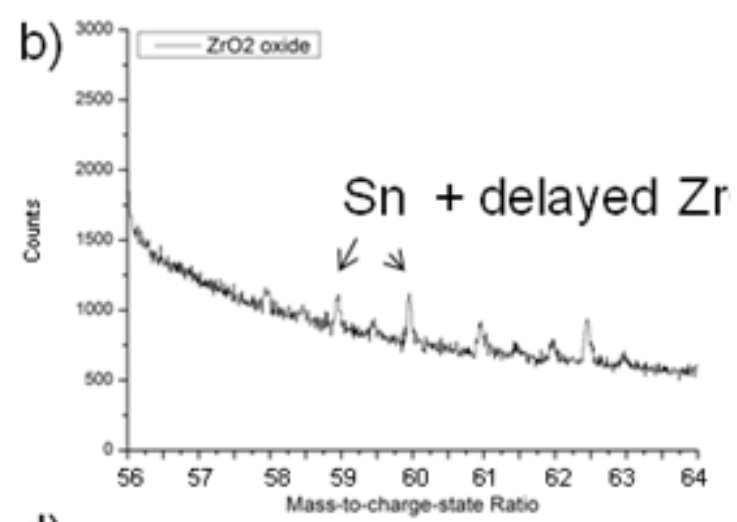

d)

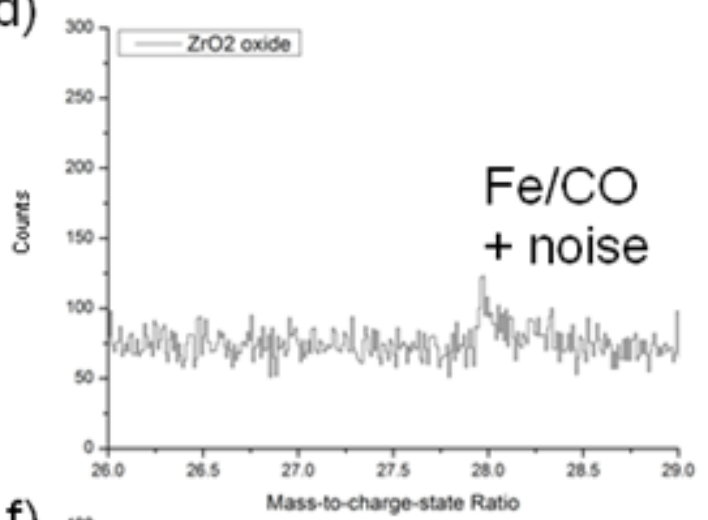

f)

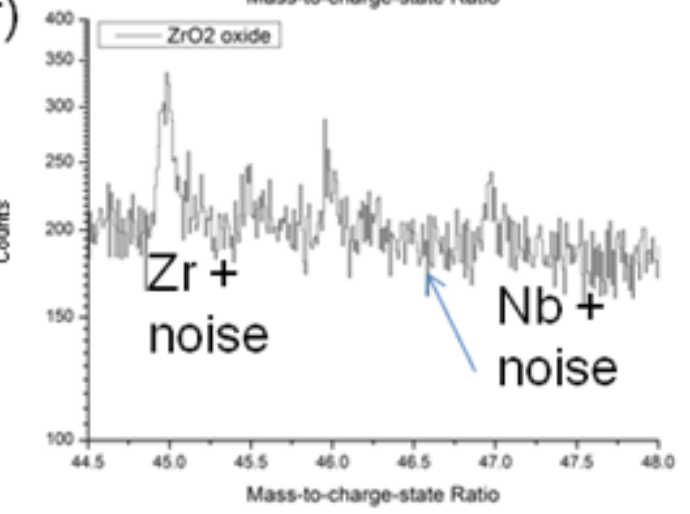

h)

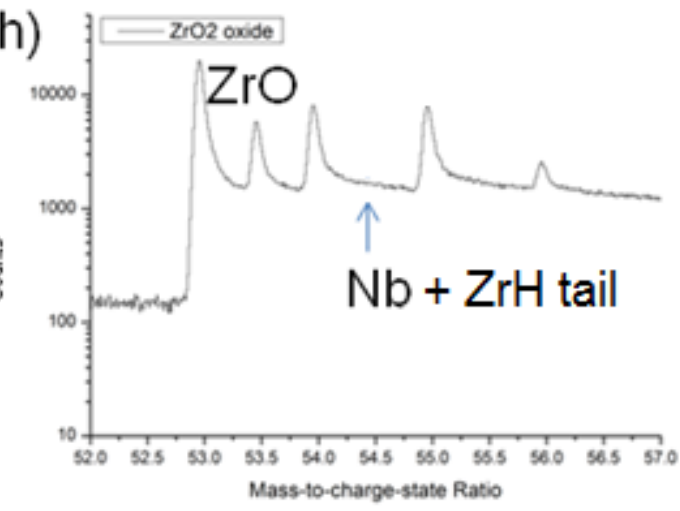

Figure 123: Mass-to-charge-state ratio spectra demonstrating the difficulties associated with making quantitative estimates of solute concentration at this $45.9 \mathrm{mg} . \mathrm{dm}^{-2}$ corroded posttransition ZIRLO interface. Elements shown: Sn (a: metal, b: oxide), Fe (c: metal, d: oxide), $\mathrm{Zr}^{2+}$ (e: metal, f: oxide), $\mathrm{ZrO}^{2+}$ (g: metal, h: oxide). 


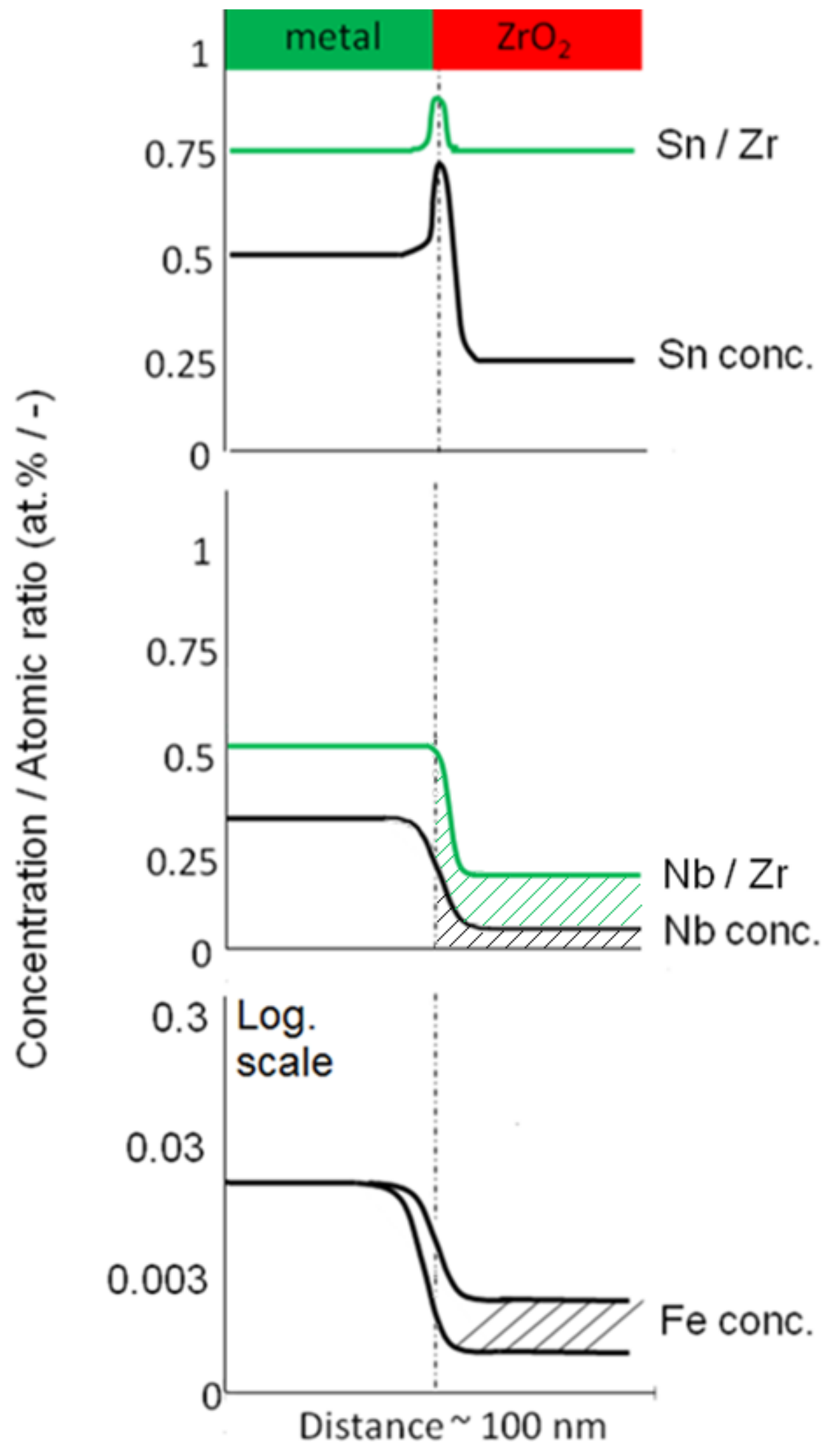

Figure 124: Idealised solute concentration profiles across the metal-oxide interface of 45.9 $m g . \mathrm{dm}^{-2}$ corroded post-transition ZIRLO. The tin and niobium profiles are on a linear scale, the iron profile is on a logarithmic scale. 


\subsubsection{Niobium}

The niobium profile across the metal-oxide interface is shown in Figure 123. The noise level in this profile is higher in the oxide than in the metal. The reason for this is apparent from Figure 122, which displays the region of the mass spectra where the $\mathrm{Nb}^{2+}$ and $\mathrm{NbO}^{2+}$ species at are found in the metallic, Figure 122 (e), (g), and oxide, Figure 122 (f), (h), regions of the dataset. In the metallic region these two niobium containing ionic species are clearly resolvable from the delayed $\mathrm{ZrH}$ and $\mathrm{ZrO}$ ions. This is not the case in the oxide region, where the peaks at $46.5 \mathrm{Da}$ and 54.5 $\mathrm{Da}$ are engulfed by delayed ions from $\mathrm{Zr}$-containing ionic species. The contribution of the $\mathrm{Nb}$ ionic species that are expected at these mass positions cannot be determined from the background of delayed ions. It is not possible to make a reliable estimate of the amount of solute in the oxide adjacent to the interface in this specimen. Similar problems were encountered when attempting to use data containing only oxide to make a precise estimate of the niobium level, and other specimens given the same treatment that contain the entire metal-oxide interface contain most of the region of interest in the metal region, with only a very small analysable volume of oxide (see notes on reproducibility Table 52 in section 6.1.2).

The idealised niobium profile Figure 124 in the metal is similar in appearance to that seen for early pre-transition material. As with the early pretransition case, niobium rejection occurs at the $\mathrm{Zr}-\mathrm{ZrO}_{2}$ interface. This was not seen to be the case with the $\mathrm{ZrO}$ ' $-\mathrm{ZrO}_{2}$ interface in the more advanced pre-transition material. In that case, the niobium present in the $\mathrm{ZrO}$ oxide was incorporated into the newly formed $\mathrm{ZrO}_{2}$. Approximately 1 micron of alloy substrate was consumed between the observations at 114 and 140 days. As breakaway may be considered to be a sudden process, it is likely that this consumption happens within a much shorter period of time between the two observations. This caused the high-oxygen concentration, nano-scale niobium precipitate-rich band of metal which was shown to be built up below the metal-oxide interface during pre-transition oxidation to be consumed by the breakaway oxidation process. 
There is some variation in the available diffusion data for niobium in $\alpha-\mathrm{Zr}$, however from data tabulated by [183] niobium may diffuse at a rate of the order of $100 \mathrm{~nm}$ per day at $360{ }^{\circ} \mathrm{C}$. It appears that this is not fast enough to diffuse ahead of this oxide wave front as the niobium rich layer is consumed by the progressing oxide, rather than being redistributed within the substrate.

The nano-scale particles formed in the metal were seen to have partially dissolved within the outer $\mathrm{ZrO}$ layer present in the later-stage pre-transition material. This solute must be accommodated within the $\mathrm{ZrO}_{2}$ layer in some way, but the manner in which this occurs, or the distance of the boundary of this region from the metal-oxide interface (within the oxide) has not been established in this study. The atom probe would not be a suitable instrument with which to perform such measurement due to the increase in delayed ion emission in the oxide regions because of poor conductivity, which can make the accurate measurement of niobium concentration within the oxide impossible.

There are conflicting mechanisms influencing the niobium concentration in the metal at this early post-transition stage in the oxidation process. The rejection of niobium ahead of the, now slowly moving (compared to the breakaway process), oxide front increases the local niobium concentration. However, thermal ageing and the high level of oxygen in solution in the region directly ahead of the interface increase the driving force for precipitation of niobium out of solution. As the oxygen-rich region near the interface is entirely consumed during oxidation, as must be the case due to the increment of oxide thickness produced during the breakaway process, the oxygen-rich region seen as the interface must have developed since breakaway. This means that the material in this region has undergone 140 days of thermal aging, but only perhaps 20 days at the saturated oxygen level seen at the interface.

A non-uniform distribution of niobium is seen in the metal adjacent to the metal-oxide interface in some of the material of this corrosion condition. Defect sites near the interface remain 
important for the heterogeneous nucleation of niobium clustering after transition. The clustering observed is less developed and frequent than the niobium rich precipitates seen to form near the metal oxide interface of the more exposed pre-transition material (see section 6.2.3.3). The niobium concentration at the core of these clusters is less than 3 at.\%, whereas the majority of the precipitates described in the previous corrosion conditions contained around 20 at.\% niobium, with some tending towards $\beta-\mathrm{Nb}$. A slice of atom map data is shown in Figure 125. These data are collected from a region with a low, and uniform, $\mathrm{ZrH}$ ion distribution to avoid potential misidentification of this impurity species as niobium. The iron distribution is also shown for the same slice of data, and is also non-randomly distributed, although there is not good agreement between the position of the niobium and iron clusters.

The observed clustering of niobium adjacent to the interface of post transition material is less developed than that seen adjacent to the metal-oxide interface of pre-transition material in section 6.2.4, and is due to less than 20 days aging in the highly stressed, oxygen-rich, matrix that develops at the metal-oxide interface. 


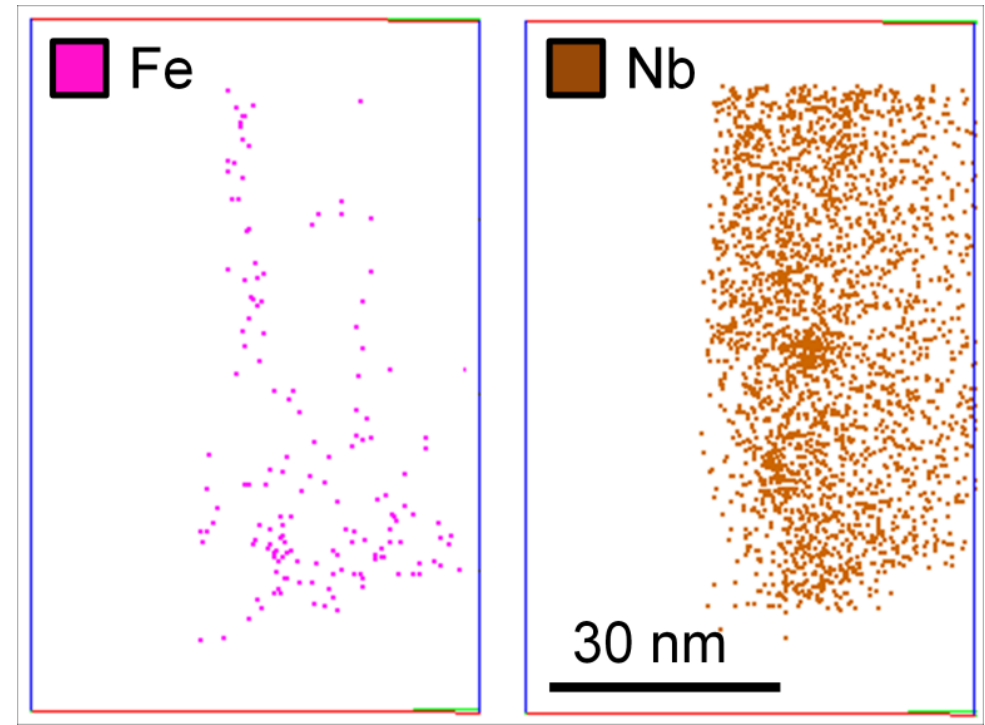

Figure 125: $3 D$ atom maps of a region of the alloy matrix near to the oxide interface of $45.9 \mathrm{mg} \cdot \mathrm{dm}^{-2}$ corroded post-transition ZIRLO (the interface is $\sim 20 \mathrm{~nm}$ from the lower left corner of the diagram, roughly horizontal and out of plane with the page). The data have been sectioned so as to remove high ZrH regions from the field of view. The slice is about $10 \mathrm{~nm}$ thick and the area displayed is most easily visualised by reference to the niobium atom map. Niobium and iron are both non-uniform.
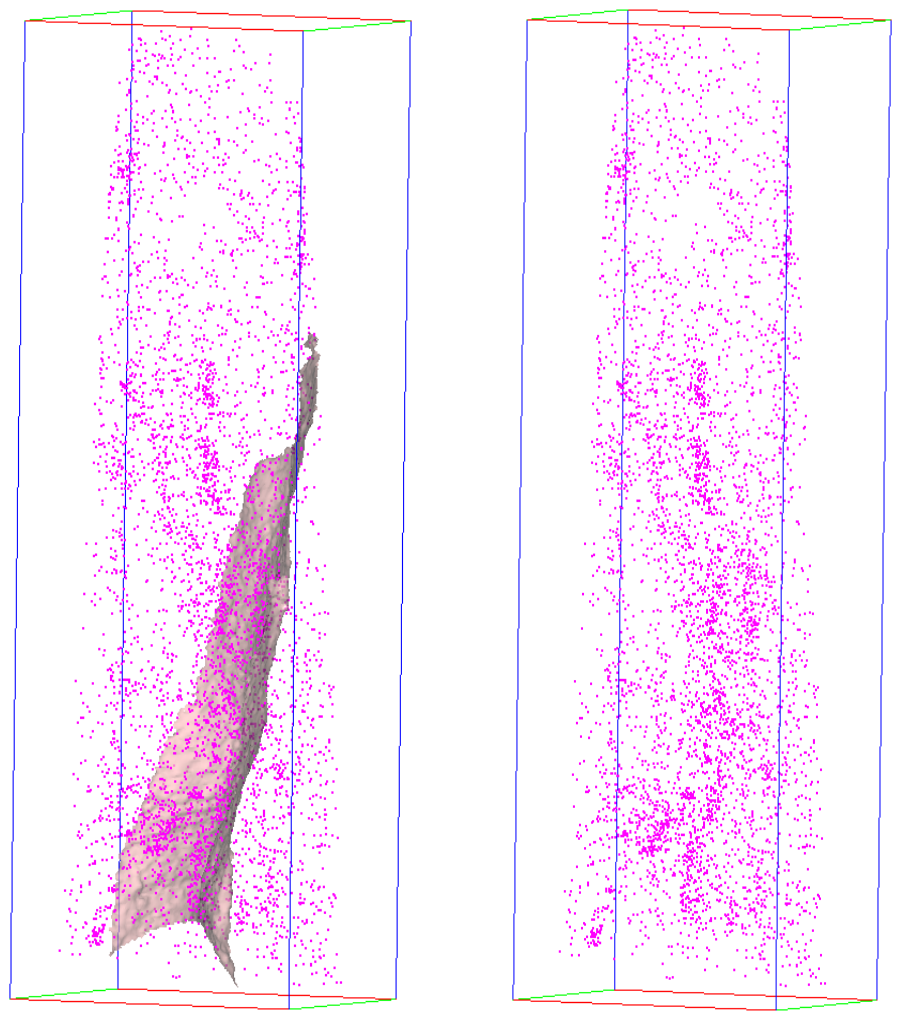

Figure 126:3D iron atom maps from the dataset shown in Figure 121 (45.9 mg. $\mathrm{dm}^{-2}$ corroded post-transition ZIRLO). Iron atoms (pink dots) shown left) with an isosurface at 50 at.\% oxygen defining the boundary between the metal and oxide and right) without the surface. 


\subsubsection{Iron}

The iron profiles in Figure 122 are all affected by the discontinuity in the background noise level at the interface. The effect of this can be seen more clearly from the fourth period transition metal spectra in regions of metal and oxide, Figure 123 (c) and (d), respectively. There are the usual problems in the metal region with other species overlapping with the iron peaks at both 27 and 28 Da.

The distributions of the $28 \mathrm{Da}$ 'iron' ions in Figure 125 and Figure 126 have a non-random component. The majority of ions appears to be clustered in the high oxygen region closest to the interface, and at the interface itself. The majority of dots on the lower side of the isosurface (the oxide) are noise, as can be seen from the spectrum of this region shown in Figure 124 (d). Given this clustering near the interface, and treating the lowest estimate of the iron concentration measured in the metal adjacent to the interface from Figure 123 with a generous uncertainty of $50 \%$, it would appear that the iron concentration adjacent to the interface is supersaturated with iron. The level of iron in the oxide seen in Figure 124 (d) is within the solubility level of iron within the metal phase.

The idealised profile shown in Figure 124 displays these qualitative statements about the level of iron across the interface. The profile is essentially the same as that seen in the early pretransition material, where iron was seen at a high level in the oxygen-rich matrix on the metal side of the interface and appears to be largely rejected from the progressing oxide front. Some iron was seen to be taken into the oxide, both in the early pre-transition material, and in material at this stage of corrosion. Analysis of specimens created from the oxide near the interface shows that the iron is distributed non-randomly once it is incorporated into the oxide. Figure 127 shows a region of the oxide where a plane decorated with iron can be seen. This planar feature is most convincingly explained as being a grain boundary between two $\mathrm{ZrO}_{2}$ grains. The grain boundary shows a high 
enrichment factor for iron compared to the intragranular level in the oxide, although quantification of this level is not appropriate, given the mass resolution of some of the $\mathrm{Zr}$ and $\mathrm{O}$ containing mass peaks. Iron clustering is also apparent intragranularly. This is the first time in which iron segregation to grain boundaries within the oxide of 'real' materials has been shown directly by experiment. Indirect observations of iron decorating grain boundaries of surface oxidised alloys has previously been shown using cathodoluminescence [59], as discussed earlier in section 2.7.3.

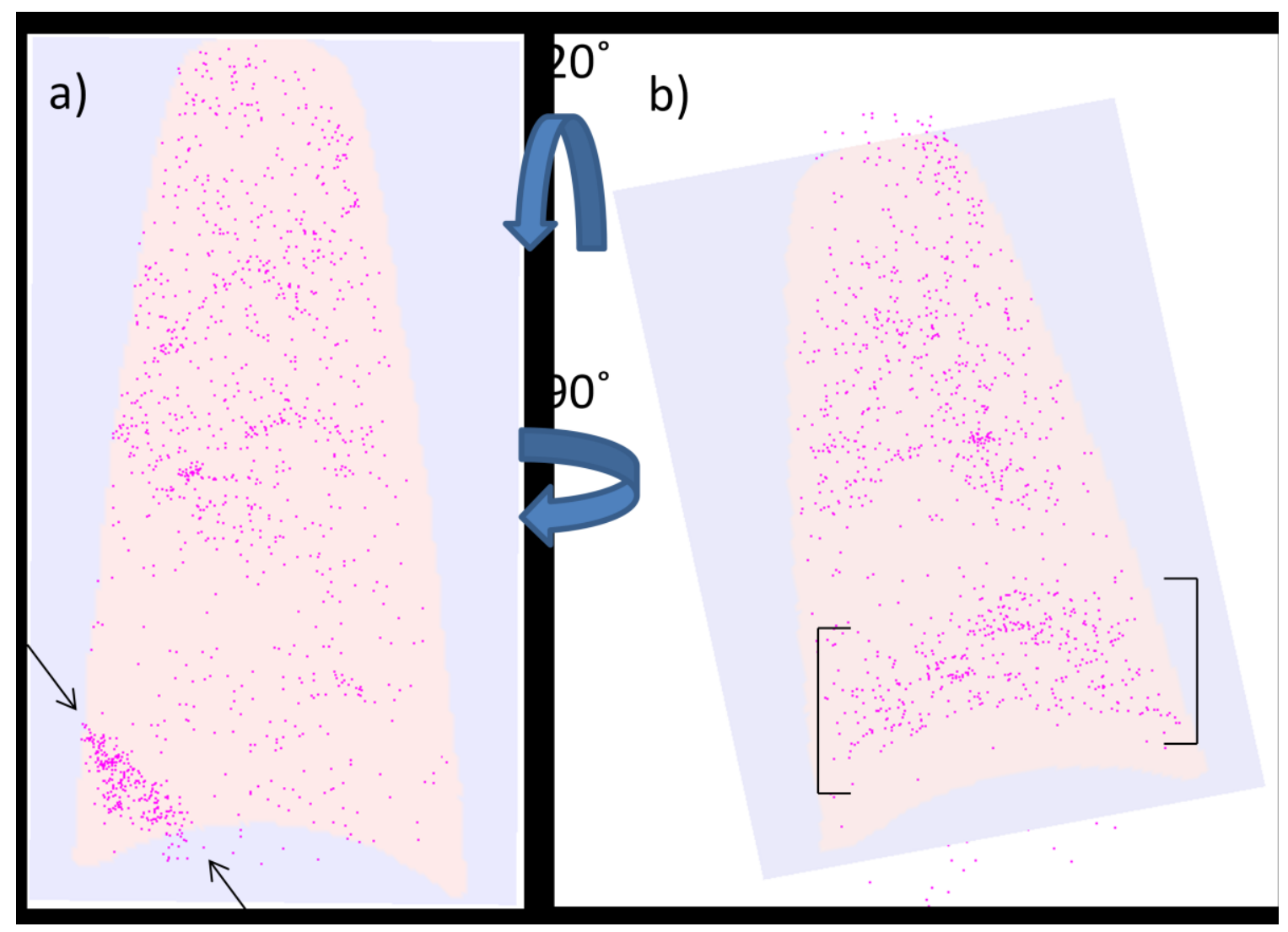

Figure 127: $45.9 \mathrm{mg} \cdot \mathrm{dm}^{-2}$ corroded post-transition ZIRLO $\mathrm{ZrO}_{2} / \mathrm{ZrO}_{2}$ boundary. The pink background is a $90 \%$ transparent $2 D$ oxygen concentration profile with colour scale $0-66$ at. $\%$ $O$ (blue to red); the entirety of the dataset consists of $\mathrm{ZrO}_{2}$ oxide (light red). Iron (at $28 \mathrm{Da}$ ) is seen to be non-randomly distributed within the oxide. A planar feature has been identified in the lower left-hand portion of (a), and is shown in planar view in (b). 


\subsection{Zircaloy-4}

\subsubsection{Pre-transition metal-oxide interface: $\sim 15.3{\mathrm{mg} . \mathrm{dm}^{-2}}^{-2}$}

Zircaloy-4 material subjected to 7 days exposure in a static autoclave environment developed an early pre-transition oxide layer with an average thickness of approximately $1 \mu \mathrm{m}$. Of the many attempts to produce specimens for this corrosion condition, only one sample was successfully prepared and analysed by 3DAP. The discussion of this result is considered with this limitation in mind.

\subsubsection{Oxygen}

Figure 128 shows the 2D oxygen concentration profile across the metal-oxide interface. On this length-scale the morphology of the metal-oxide interface is relatively simple. There is a region at the interface where the oxygen concentration deviates from that of $\mathrm{ZrO}_{2}$. Figure 128(c) uses an alternative colour scale to highlight variation between oxygen-saturated matrix and $\mathrm{ZrO}_{2}$ oxide. This demonstrates that this is in fact a thin transitional region, rather than an area of constant composition. The 1D oxygen/zirconium concentration profile in Figure 129 uses an alternative representation to show the transition between the metal and oxide. There is no evidence of a transition layer, such as $\mathrm{ZrO}$, in this sample. This is consistent with the observations of early pretransition ZIRLO material described in section 6.2. A marked difference between the early-stage ZIRLO material and Zircaloy-4 is the level of oxygen in solid solution in the matrix. Even after only 7 days exposure, much less than the 34 day exposed ZIRLO, this Zircaloy-4 sample exhibits an oxygen-saturated region many tens of nanometres thick ahead of the interface. This is the light green area in Figure 128 (a) and (b). 


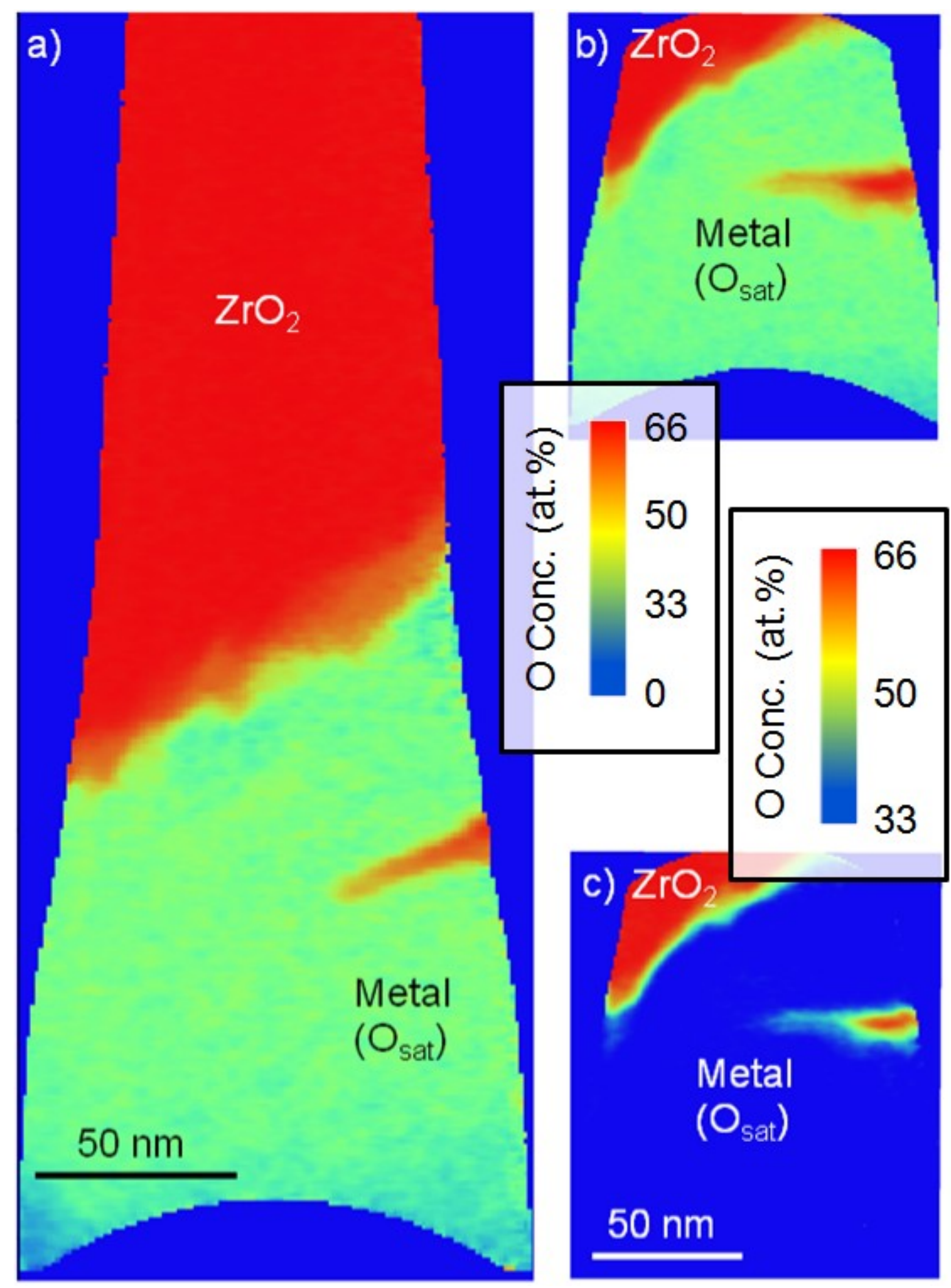

Figure 128: 2D oxygen concentration profiles taken across the metal-oxide interface of $\sim 15.3$ $m g . d m^{-2}$ corroded pre-transition Zircaloy-4. The profiles are shown using two alternative colour scales: (a,b) have limits between 0 at.\% oxygen and $\mathrm{ZrO}_{2}$; (c) has limits around the solid solution saturation level of oxygen in alpha zirconium (33 at.\%), and $\mathrm{ZrO}_{2}$. 

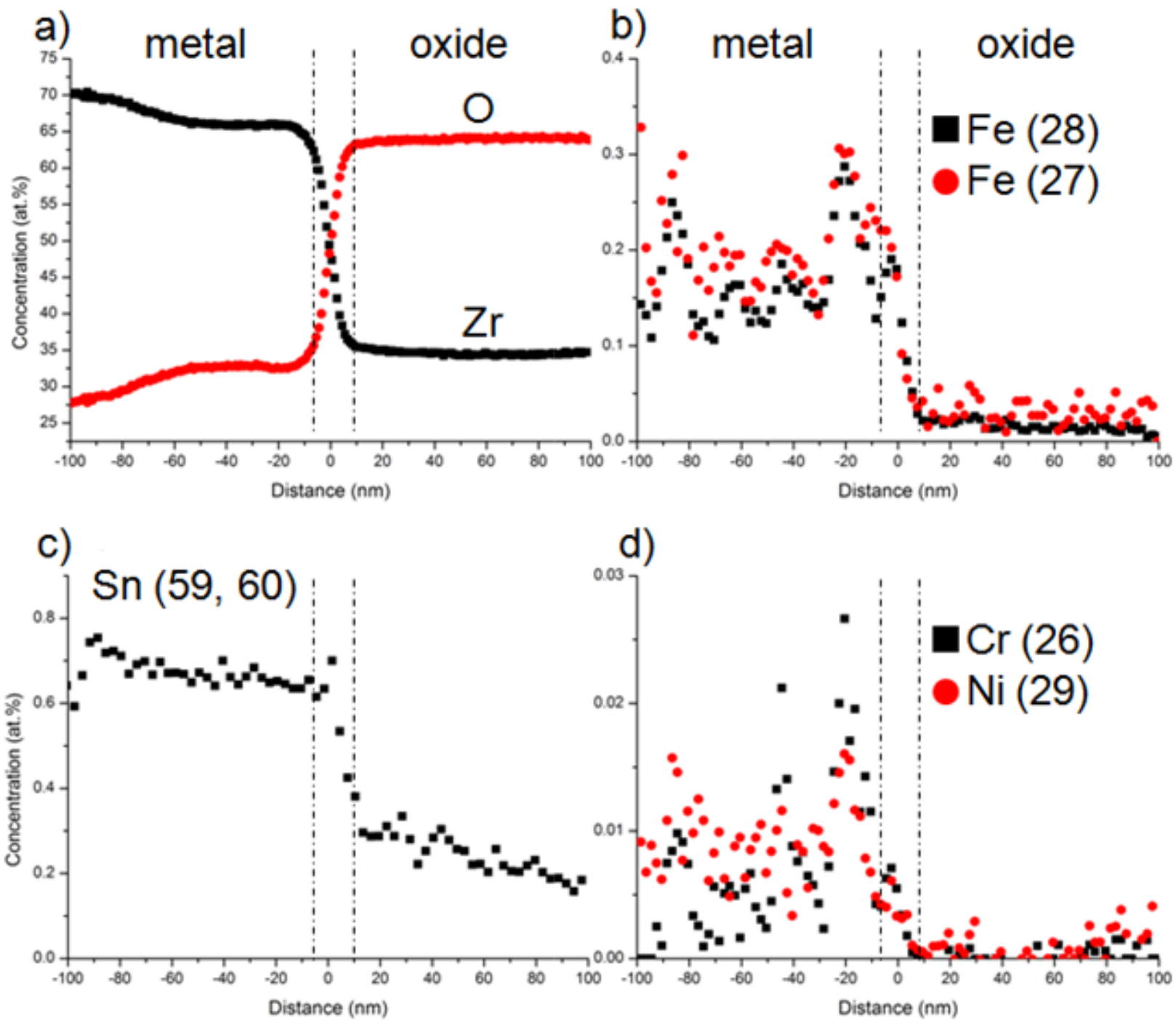

Figure 129: $1 D$ concentration profiles across the metal-oxide interface of $\sim 15.3 \mathrm{mg} \cdot \mathrm{dm}^{-2}$ corroded pre-transition Zircaloy-4. All profiles cover the same region, and displaying the change in concentration of a) zirconium and oxygen, b) iron as calculated using the isotopes ${ }^{54} \mathrm{Fe}^{2+}$ and ${ }^{56} \mathrm{Fe}^{2+}$ separately, c) tin and d) chromium and nickel calculated from the ionic species ${ }^{52} \mathrm{Cr}^{2+}$ and ${ }^{58} \mathrm{Ni}^{2+}$.

The additional oxygen-saturated matrix region found in the Zircaloy-4 can be understood with reference to the behaviour of niobium within these materials. In ZIRLO, niobium acts as a betastabiliser, lowering the solubility of oxygen in solution and retarding the diffusion of oxygen into the material ahead of the interface. 


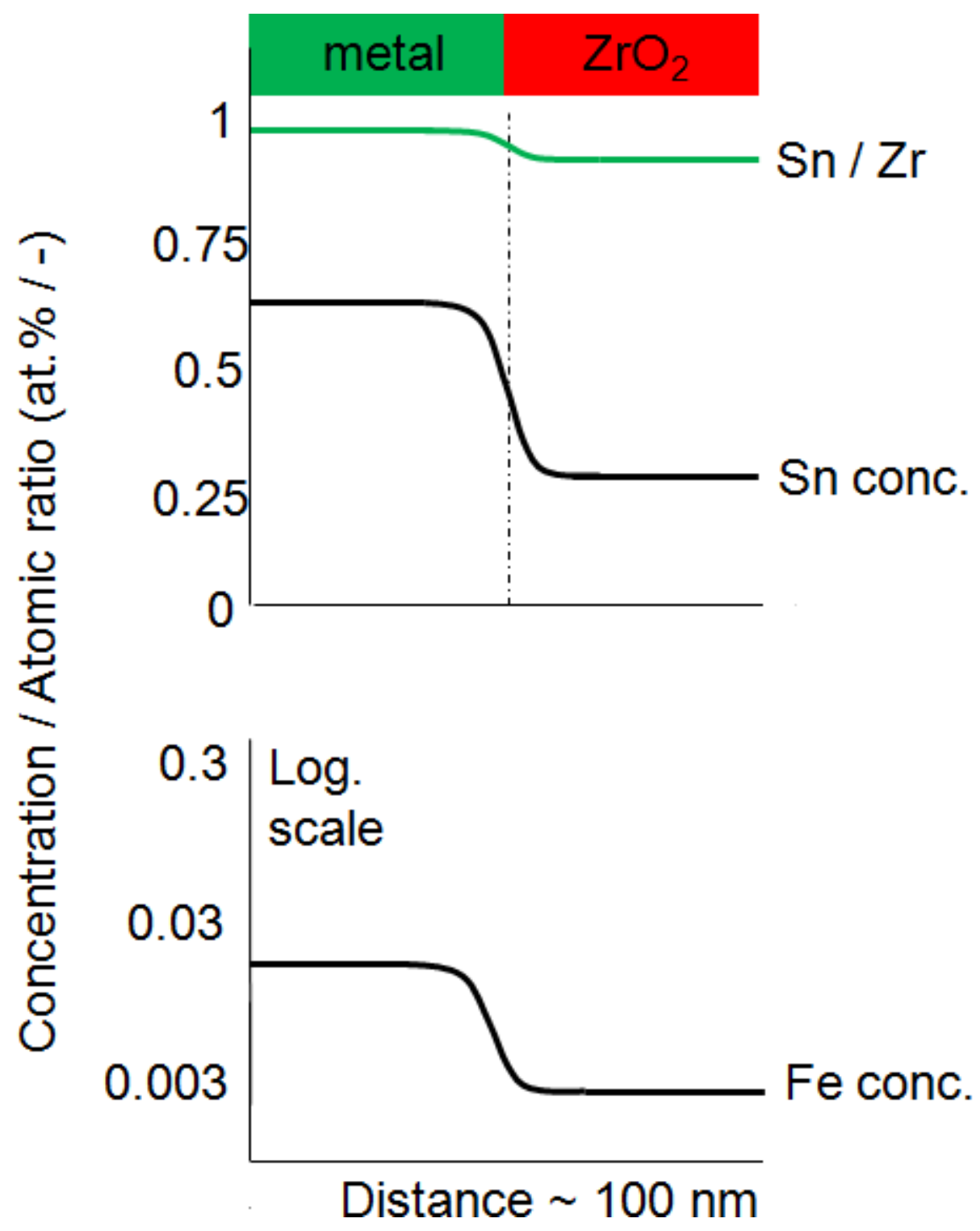

Figure 130: Idealised profiles of tin and iron concentration at the metal-oxide interface of $\sim 15.3$ $m g . d m^{-2}$ corroded pre-transition Zircaloy-4 based on data in Figure 129. The tin profile is on a linear plot, the iron profile is on a semi-log. scale.

\subsubsection{Tin}

Figure 129 (c) shows the tin concentration profile across the metal oxide interface. The profile follows the same step-like function at the interface as has previously been seen in the ZIRLO samples. Figure 130 shows this profile in terms of the $\mathrm{Sn} / \mathrm{Zr}$ ratio, rather than the tin concentration. The $\mathrm{Sn} / \mathrm{Zr}$ ratio remains roughly constant across the metal-oxide interface. This is 
consistent with the observations in ZIRLO that tin is accepted into the oxide as it forms and does not become redistributed. It should be noted that the $\mathrm{Sn} / \mathrm{Zr}$ ratio is more sensitive to noise within the oxide as the scaling factor applied to account for the additional oxygen also magnifies the uncertainty. The measured concentration of tin is around 0.3 at. $\%$ within the oxide, compared with 0.33 at.\% the expected value.

Figure 130 shows the spectrum from within the metal and oxide around the mass positions of $\mathrm{Sn}^{2+}$. In this sample there is not a problem with delayed $\mathrm{ZrO}$ ions overlapping with $\mathrm{Sn}$, as is sometimes the case. The tin spectrum peaks within the oxide are broader than in the metal. This change in mass resolution causes a different proportion of the peaks in the metal and oxide to be included within a fixed ranging window (which must remain constant over the whole profile). This could well lead to the apparent loss of $10 \%$ of the tin seen in the oxide region.

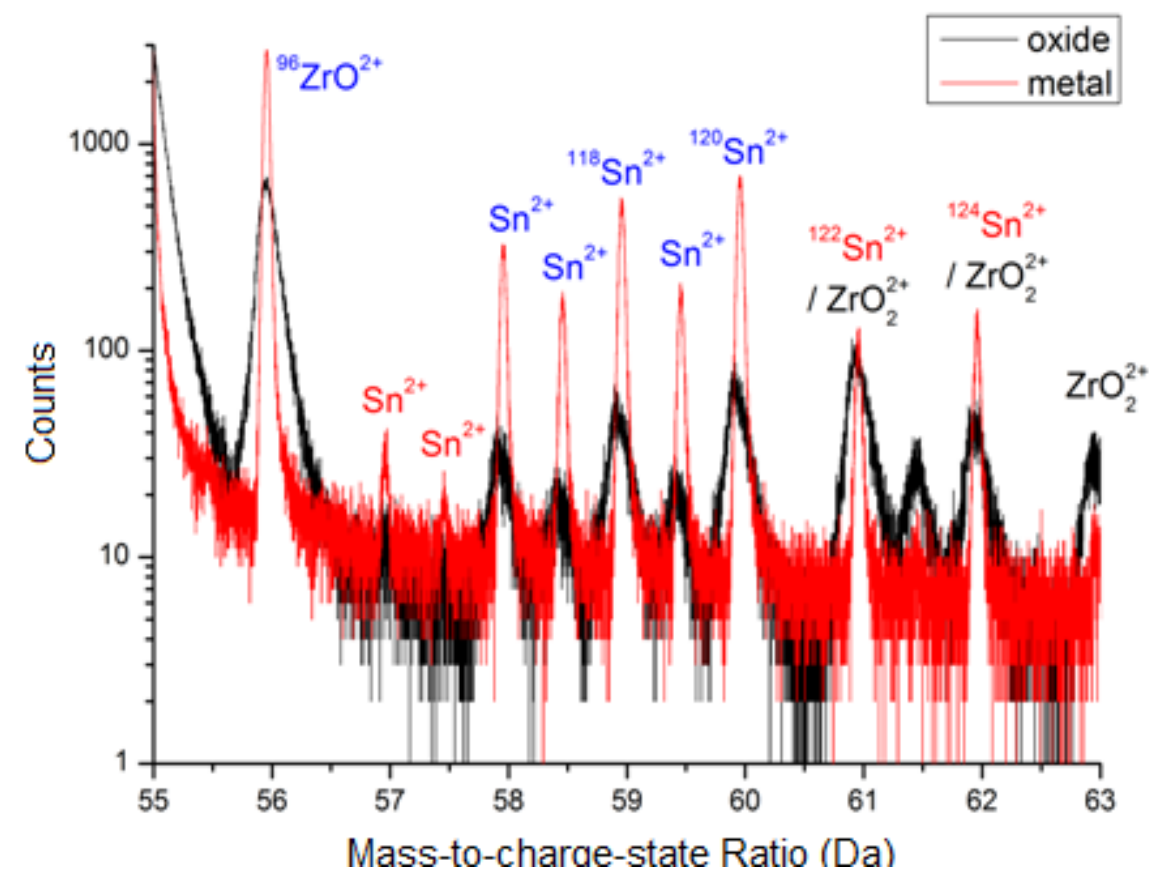

Figure 131: Mass-to-charge state spectra from the metal and $\mathrm{ZrO}_{2}$ oxide regions adjacent to the interface of $\sim 15.3 \mathrm{mg} \cdot \mathrm{dm}^{-2}$ corroded pre-transition Zircaloy-4, showing the area of the spectrum around $\mathrm{Sn}^{2+}$. 
The chemical composition of a region of alloy matrix adjacent to the interface is presented in Figure 52. The niobium concentration measured using the methods used in the previous section relating to ZIRLO is also included even though this alloy should contain no niobium. No niobium was detected, further validating the $\mathrm{ZrH}$ correction method used in sections 5 and 6.2.

\begin{tabular}{|c|c|cc|}
\hline Element & Number & \multicolumn{2}{|c|}{ Composition } \\
& of atoms & $\begin{array}{c}\text { Conc. } \\
\text { (atoms) }\end{array}$ & $\begin{array}{c} \pm \sigma \\
\text { (at. \%) } \\
\text { (at.\%) }\end{array}$ \\
\hline $\mathrm{Zr}$ & $6,543,547$ & 68.03 & 0.015 \\
$\mathrm{O}$ & $2,991,117$ & 31.10 & 0.02 \\
$\mathrm{Nb}$ & $|94|$ & $|<0.001|$ & 0.001 \\
$\mathrm{Fe}(27)$ & 17,672 & 0.184 & 0.006 \\
$\mathrm{Fe}(28)$ & 16,934 & 0.176 & 0.001 \\
$\mathrm{Fe}(28.5)$ & 15,209 & 0.158 & 0.009 \\
$\mathrm{Fe}($ all) & 16,897 & 0.176 & 0.001 \\
$\mathrm{Sn}$ & 65,115 & 0.677 & 0.003 \\
$\mathrm{Ni}(29)$ & 1,035 & 0.011 & 0.0004 \\
$\mathrm{Cr}(26)$ & 925 & 0.010 & 0.0003 \\
\hline $\mathrm{Total}$ & $9,618,636$ & & \\
\hline
\end{tabular}

Table 58: Chemical composition of the metal matrix region adjacent to the metal-oxide interface of $\sim 15.3 \mathrm{mg} \cdot \mathrm{dm}^{-2}$ corroded pre-transition Zircaloy-4. The quoted uncertainty is one standard deviation due to counting statistics; it does not include additional uncertainty related to ranging methodology. Total ion count disregards ' $\mathrm{Nb}$ ' and uses only $\mathrm{Fe}$ (all) iron content estimate. Figure in parenthesis details the mass peak from which the total number of solute atoms has been derived.

The tin concentration within the metal matrix is $(0.677 \pm 0.003)$ at.\%. Correction for the additional oxygen in solution, $\mathrm{C}_{\text {add }}$, can be applied (accounting for the original amount of oxygen in the fabricated material, $\mathrm{C}_{\mathrm{O}}$, which was shown in Table 8 to be 0.74 at.\%).

$$
C_{S n_{-} \text {corr }}=\frac{C_{S n}}{1-\left(C_{a d d}-C_{O}\right)}
$$


This yields a tin concentration of $(0.983 \pm 0.003)$ at.\%, which agrees well with the bulk content of the material, 0.98 at.\% tin. This bulk value is expected to be distributed uniformly in the matrix, which occupies almost all of the material volume, and to be rejected from second phase particles which occupy a small volume fraction of the material $(\sim 0.3 \%)$. This figure also agrees well with the measured value in as-received Zircaloy-4 material in section 5.1.3.

\subsubsection{Iron, chromium and nickel}

The fourth period transition metal $2+$ charge-state region of the mass-to-charge spectrum is shown on a semi-log scale Figure 132. Three chromium and three iron peaks are visible and agree well with the expected isotopic abundance of these species. There is also a single peak at $29 \mathrm{Da}$, which is attributed to nickel.

The 1D profile Figure 129 demonstrates a step change in the iron, chromium and nickel concentrations at the interface. The concentrations of these transition metals in the alloy matrix adjacent to the interface is much higher than in the oxide. The iron concentration is approximately 0.18 at.\%, around sixty times the level seen in uncorroded Zircaloy-4 matrix material. Figure 133 shows the same section of the mass-to-charge spectra displayed in Figure 132 , but for the oxide region, rather than the metal. Only the iron peak at $28 \mathrm{Da}$ is visible in this spectrum, and the counts $(\max \sim 22)$ are much lower than counts seen in the metal region ( $\max \sim$ 800). The volume of the ROI, in terms of the number of atoms detected, is similar in both of these examples and both figures are displayed using 1000 bins per atomic mass unit. Figure 133 suggests that the small $\mathrm{Cr}$ and $\mathrm{Ni}$ content visible in the oxide in the 1D profile (Figure 129) is due to detector noise. 


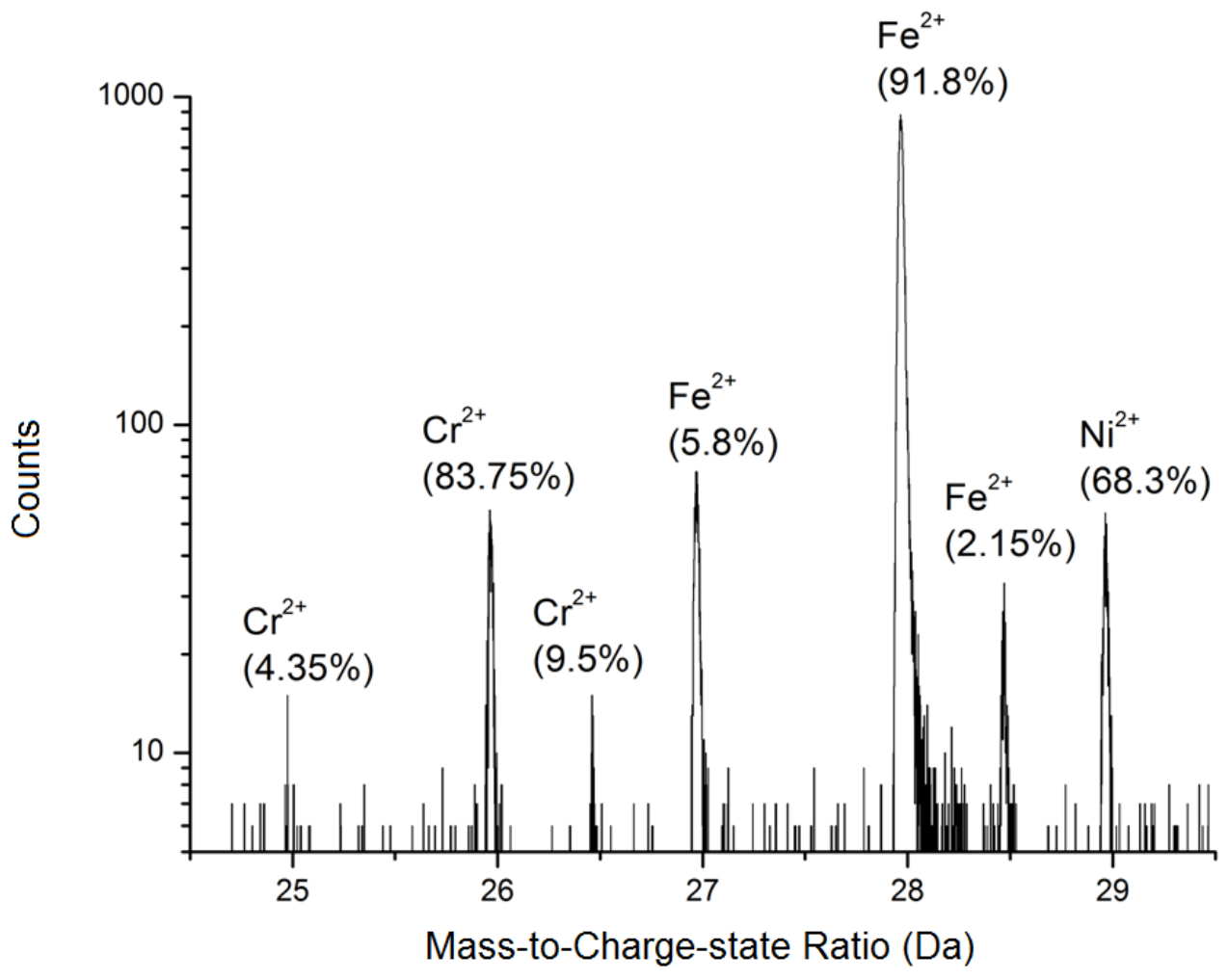

Figure 132: Mass-to-charge state ratio spectrum from the metal region adjacent to the interface of $\sim 15.3 \mathrm{mg} \cdot \mathrm{dm}^{-2}$ corroded pre-transition Zircaloy-4 showing the $2+$ charge state of the fourth period transition metal species. Cr, Fe and Ni can all be clearly resolved.

The spatial distribution of $\mathrm{Fe}, \mathrm{Cr}$ and $\mathrm{Ni}$ species in relation to the metal-oxide interface is shown in Figure 134. Iron forms clusters, and also heavily decorates linear features adjacent and parallel to the interface. This is consistent with iron segregation to dislocations, with additional iron present due to rejection from the newly formed oxide. In ZIRLO, the metal matrix of corroded material away from the interface was seen to be deficient in iron compared to uncorroded material where oxygen has been injected into the alloy matrix. Similar information is not available here, but it is envisaged that much of the additional iron seen here originates from within the bulk of the material, as well as solute rejected from the oxide. 


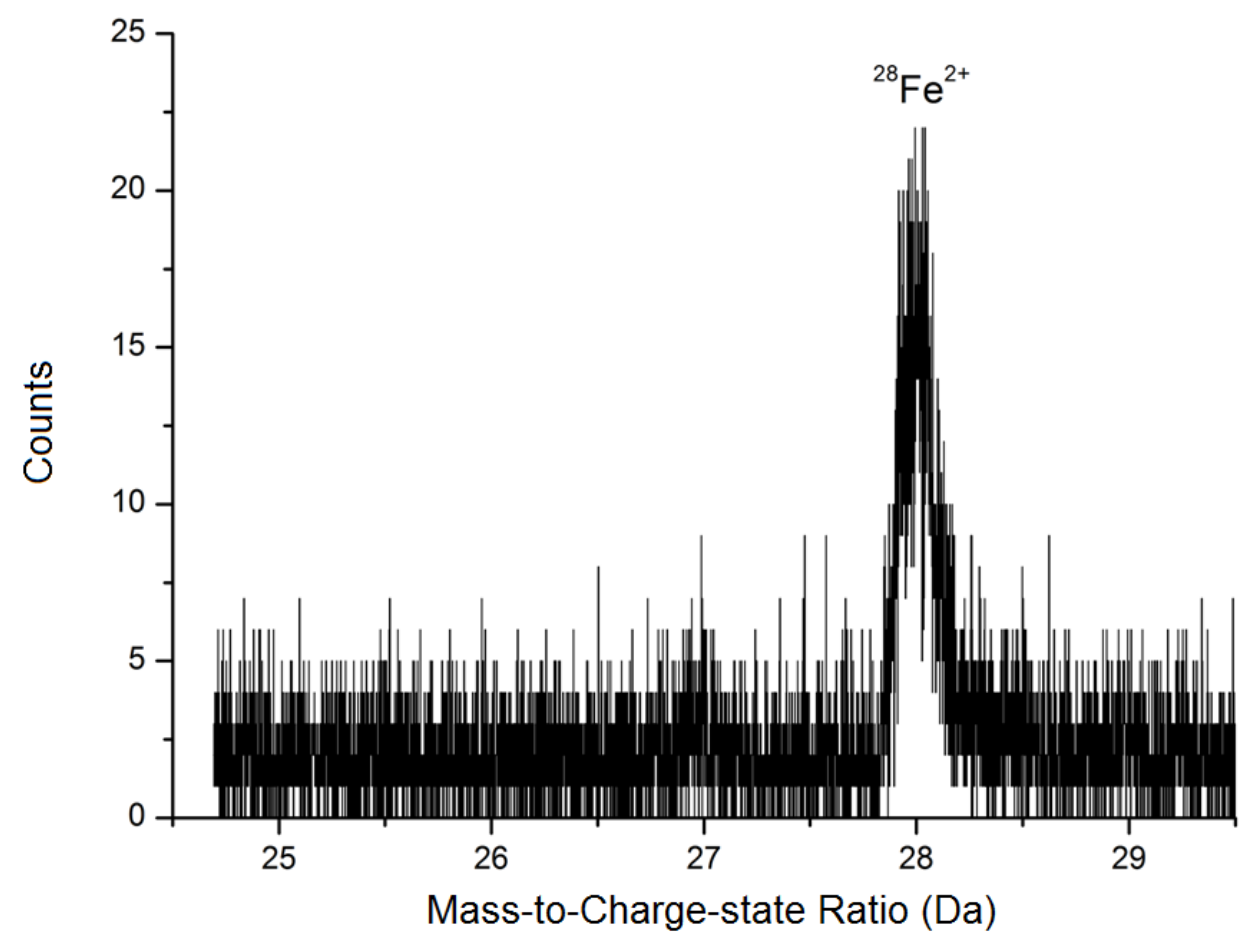

Figure 133: Mass-to-charge state ratio spectrum from the $\mathrm{ZrO}_{2}$ oxide region adjacent to the interface of $\sim 15.3 \mathrm{mg} \cdot \mathrm{dm}^{-2}$ corroded pre-transition Zircaloy-4, showing the $2+$ charge state of the fourth period transition metal species. Only the peak at $28 \mathrm{Da}$ can be resolved from the background noise. The signal / noise ratio of this peak is $\sim 4$.

Chromium and nickel are also seen in much higher concentration in the metal adjacent to the interface than in the oxide. Chromium forms clusters, many of which are also the site of iron rich clusters. Nickel and chromium are seen to be higher in concentration in the region with the most iron decorated dislocations, although not to the extent whereby these features could be identified only with reference to the $\mathrm{Cr}$ or $\mathrm{Ni}$ atom maps. Nickel was seen as a trace element in some of the regions of uncorroded Zircaloy-4 matrix. Chromium was not detected in those experiments. 


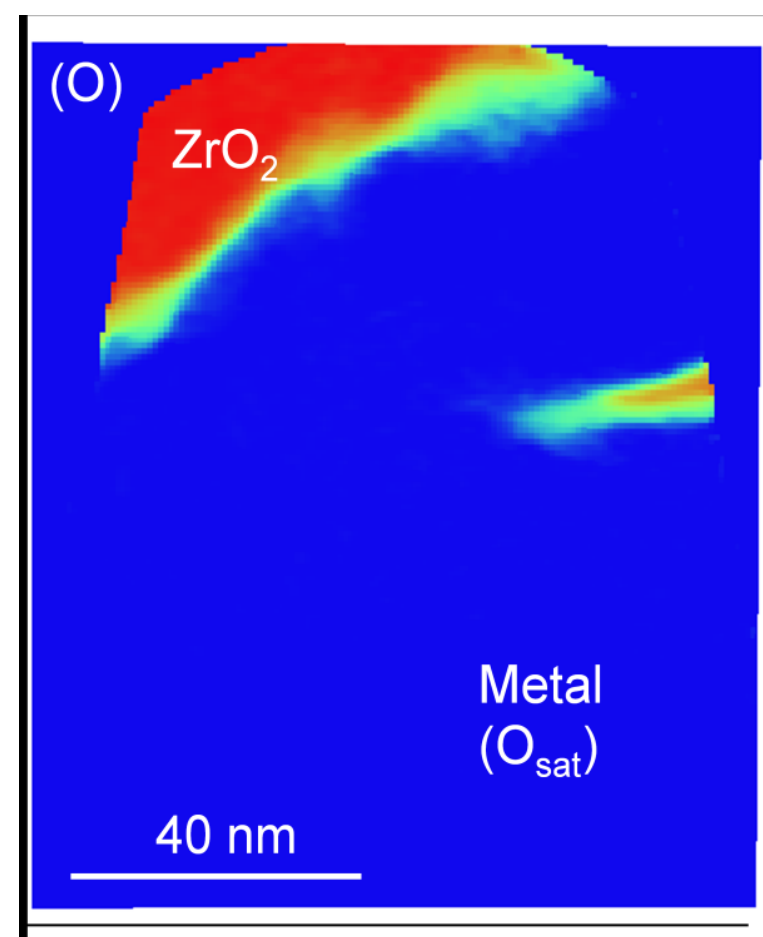

$(\mathrm{Ni})$

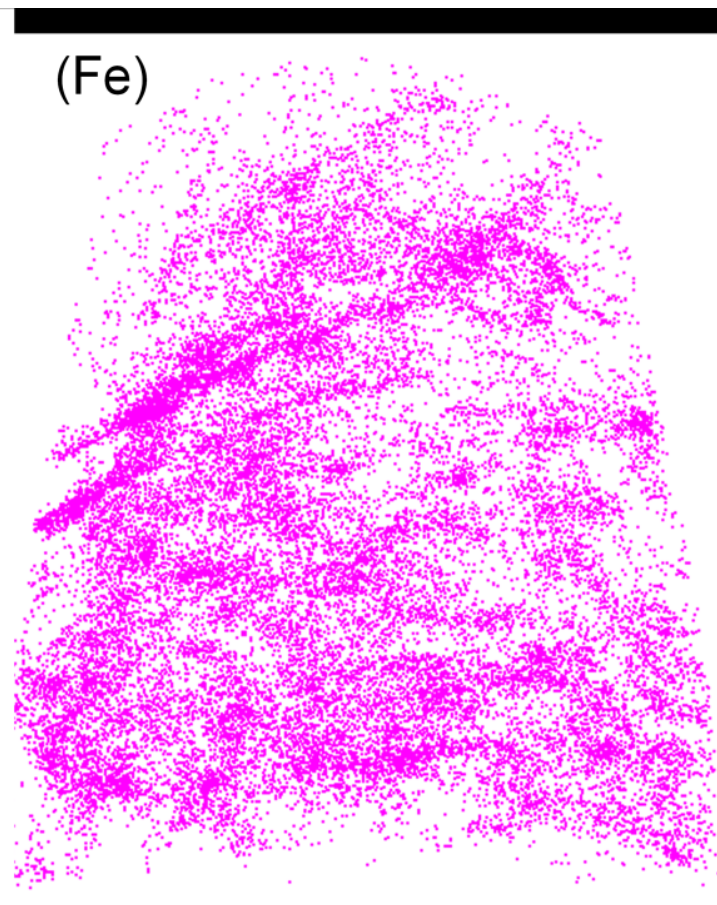

$(\mathrm{Cr})$

Figure 134:O) 2D oxygen concentration profile cross section of the metal oxide interface of $15.3 \mathrm{mg} \cdot \mathrm{dm}^{-2}$ corroded pre-transition Zircaloy-4. Colour scale 33 (blue) - 66 (red) at.\% oxygen. $\mathrm{Fe}, \mathrm{Ni}$ and $\mathrm{Cr}$ views are shown using the same perspective. Fe is plotted using all of the $28 \mathrm{Da}$ peak, Ni is plotted using $29 \mathrm{Da}$ and $\mathrm{Cr}$ is displayed using $26 \mathrm{Da}$. The mass spectrum for the metal volume within this region is shown in Figure 132. 


\subsubsection{Pre-transition metal-oxide interface: $23.4 \mathrm{mg.dm}^{-2}$}

After 114 days autoclave exposure the Zircaloy-4 material had undergone a weight gain of 23.4 $\mathrm{mg} \cdot \mathrm{dm}^{-2}$. Both the weight gain and appearance of the corroded material suggest that it was pretransition. Two of the analysed atom probe specimens contained the entire metal-oxide interface, several others contain part of the interface and a region of adjacent material.

\subsubsection{Oxygen}

A 2D oxygen concentration profile taken across the metal oxide interface is shown in Figure 135. There is a $\mathrm{ZrO}$ layer between the metal and the $\mathrm{ZrO}_{2}$ oxide which varies in thickness considerably over the volume examined. In general, the thickness is of the order of tens of nanometres. The intermediate layer is homogeneous in composition and does not appear to contain two separate sub-layers. The morphology of the interfacial layer is relatively simple, similar to the same corrosion condition seen in ZIRLO in areas with little niobium precipitation. The metal adjacent to the interface is saturated with oxygen (light green region in Figure 135). Figure 136 demonstrates the three-dimensional structure of the interface described in 2D by Figure 135. Surfaces of constant oxygen concentration mark the boundaries of the metal (blue surface) and $\mathrm{ZrO}_{2}$ oxide (red surface). 

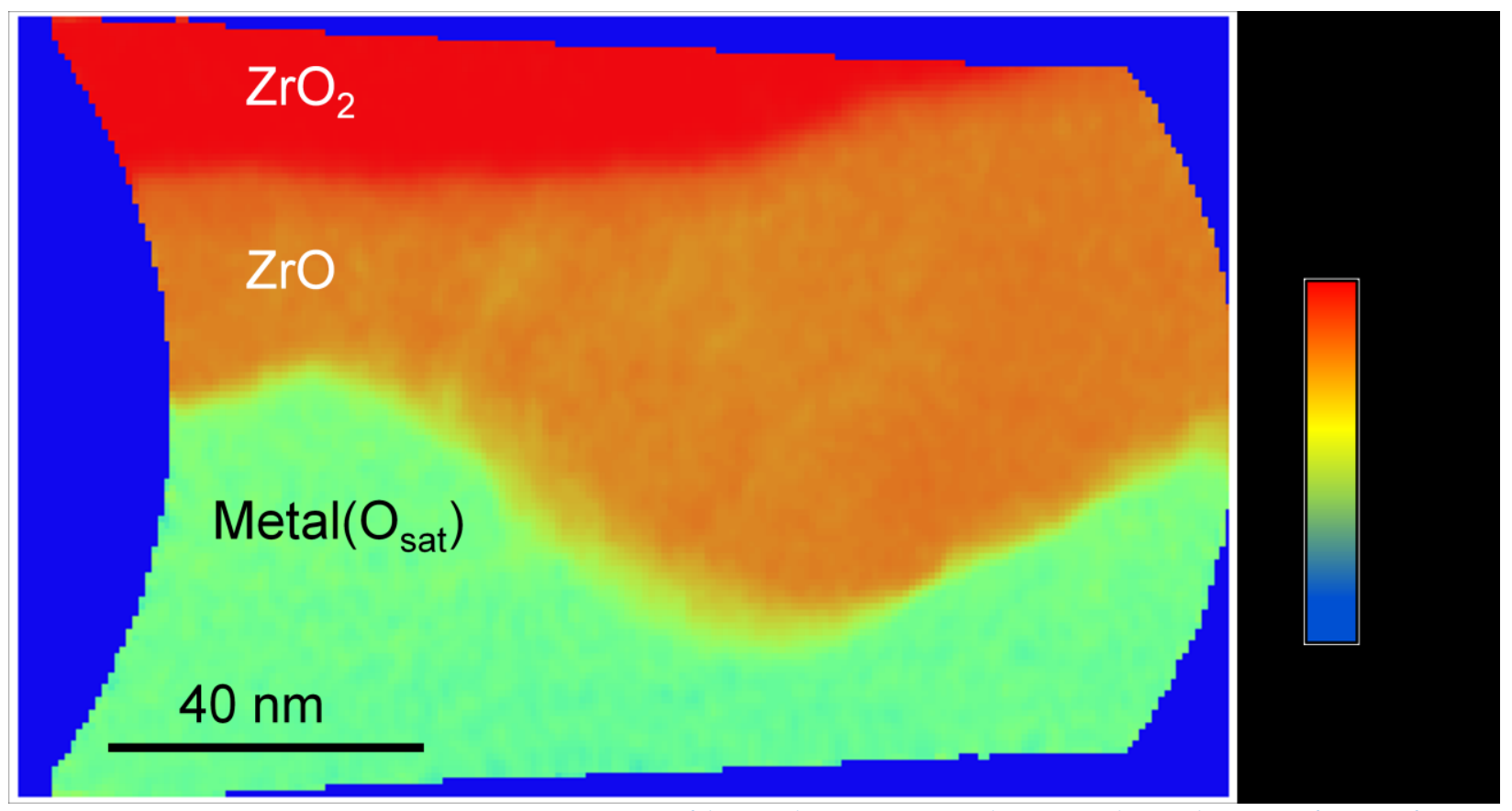

Figure 135: 2D oxygen concentration profiles taken across the metal-oxide interface of 23.4 $m g . d m^{-2}$ corroded pre-transition Zircaloy-4. The specimen needle axis is horizontal (purely for layout purposes). Colour scale limits are 0 at.\% oxygen and 66 at.\% oxygen $\left(\mathrm{ZrO}_{2}\right)$.

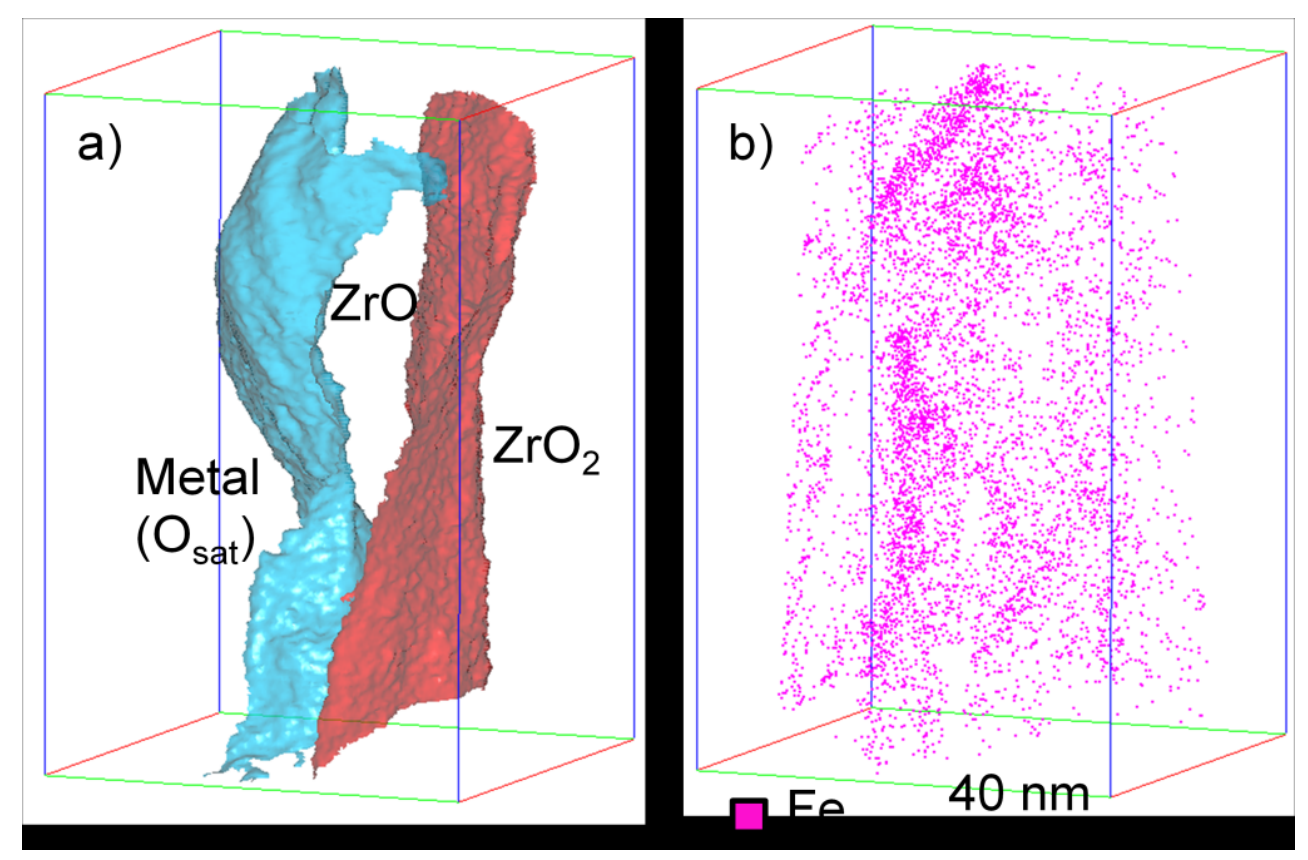

Figure 136: a) 3D view of the metal-oxide interface shown in Figure 135 (23.4 mg. $\mathrm{dm}^{-2}$ corroded pre-transition Zircaloy-4) plotted using a red surface at 55 at.\% oxygen to mark the $\mathrm{ZrO}-\mathrm{ZrO}_{2}$ interface and a blue surface at 45 at.\% oxygen marking the Zr-ZrO interface. b) 3D atom map showing the iron distribution at the metal-oxide interface viewed in the same orientation to (a). 


\subsubsection{Tin}

The concentration of tin undergoes a step change at the $\mathrm{Zr}-\mathrm{ZrO}$ and $\mathrm{ZrO}-\mathrm{ZrO}{ }_{2}$ interfaces, as shown in the 1D profile in Figure 137. The distribution of tin across the metal-oxide interface is similar to that seen in late pre-transition ZIRLO and is again explained with reference to the $\mathrm{Sn} / \mathrm{Zr}$ ratio, removing the effect of additional oxygen added to the matrix during corrosion. As with ZIRLO, there are apparent peaks in the tin concentration at the interface that are likely due to tin surface migration on the surface of the specimen near regions of high electric field gradient, and might not be physically representative of tin segregation to these interfaces. The $\mathrm{Sn} / \mathrm{Zr}$ ratio is seen to remain constant across the interface, as shown in Figure 138. The tin concentration at the interface agrees well with the expected matrix value, 0.98 at.\%, after the additional oxygen in solution has been accounted for. Four regions of metal adjacent to the interface have also been analysed, and after correction for the oxygen added to solution during the corrosion process. The tin concentration in these regions also agrees well with the expected value. These data are presented in Table 59. 


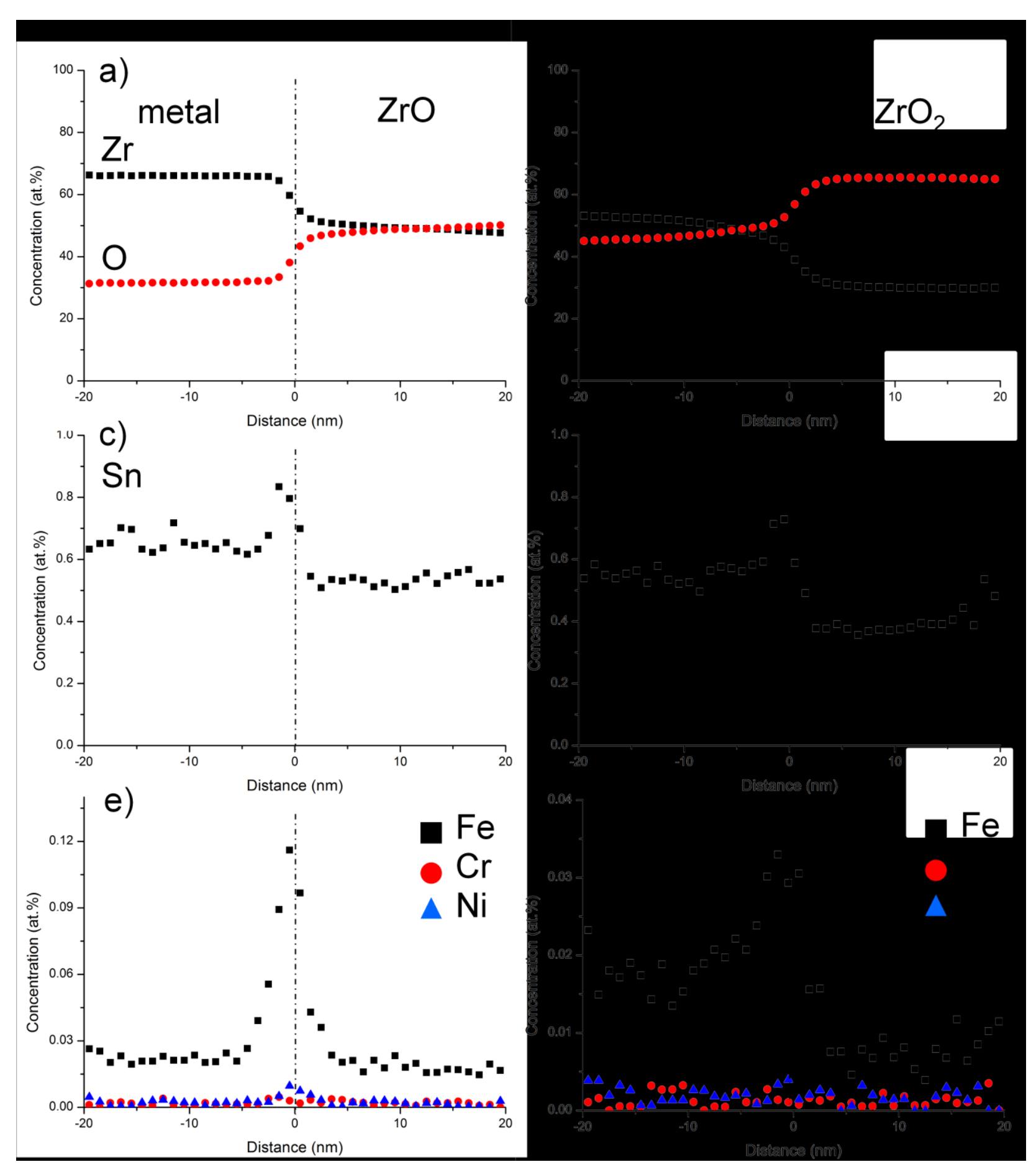

Figure 137: Profiles showing the i) $\mathrm{Zr}$ - $\mathrm{ZrO}$ and ii) $\mathrm{ZrO}-\mathrm{ZrO}_{2}$ interfaces across the metal-oxide and oxide/oxide interfaces of $23.4 \mathrm{mg} \cdot \mathrm{dm}^{-2}$ corroded pre-transition Zircaloy-4. Various species shown: a/b) zirconium and oxygen; c/d) tin; and e/f) iron (28 Da), chromium (26 Da) and nickel (29 Da). The fourth period transition metals are plotted using the charge states shown in parenthesis. In this case, the deviation from $\mathrm{ZrO}$ stoichiometry in (b) is due to the variation in ZrO layer thickness, as seen in Figure 136 (a). 


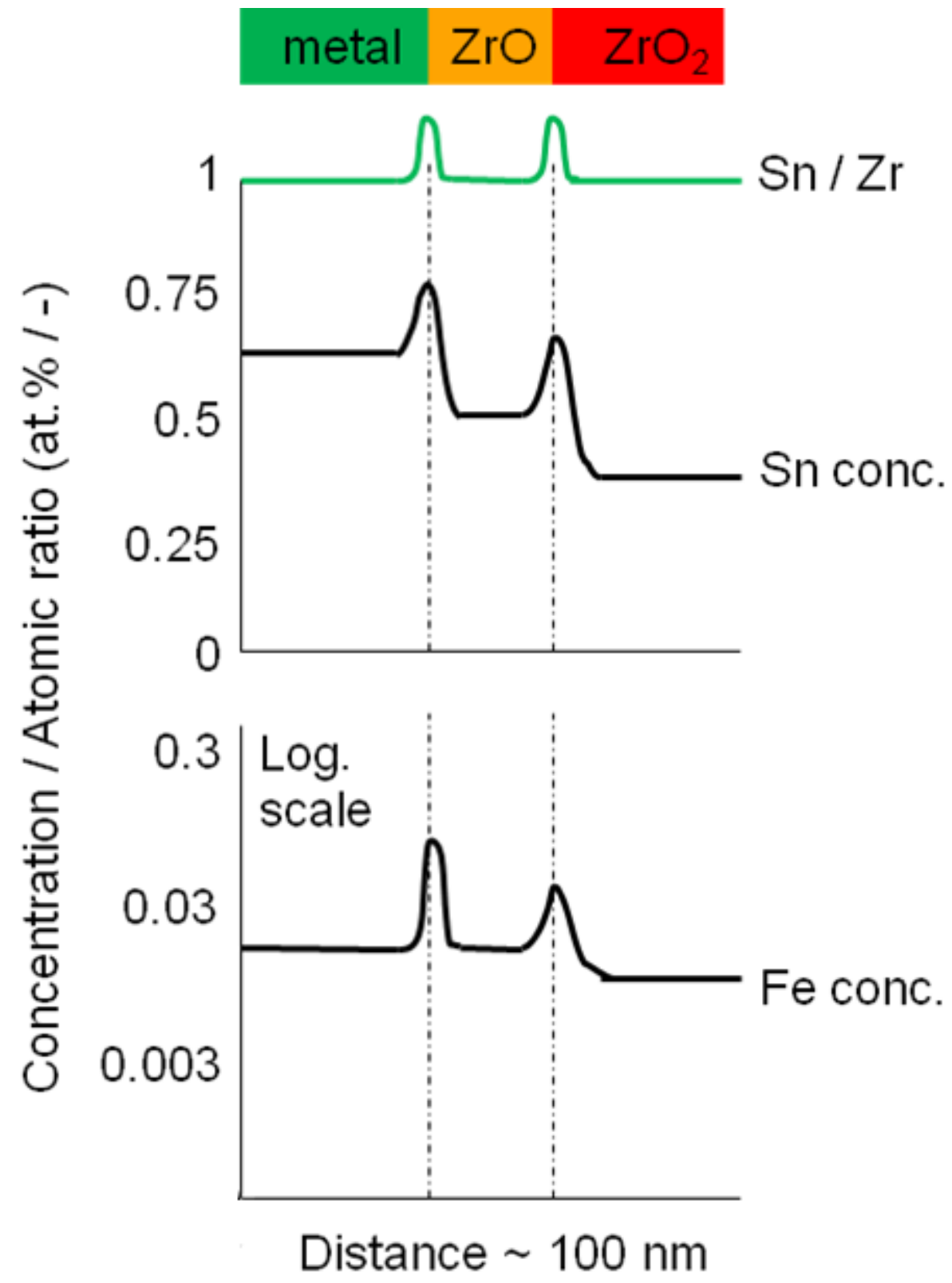

Figure 138: Idealised concentration and solute/solvent profiles across the metal-oxide interface of $23.4 \mathrm{mg} \cdot \mathrm{dm}^{-2}$ corroded pre-transition Zircaloy-4 for tin and iron. 


\begin{tabular}{|c|c|c|c|}
\hline $\begin{array}{c}\text { Volume } \\
\left(10^{6} \text { atoms }\right)\end{array}$ & $\begin{array}{l}\text { Detected } \\
\text { tin conc. } \\
\text { (at. \%) }\end{array}$ & $\begin{array}{c}\mathrm{C}_{\text {Snd }} \\
/\left(1-\mathrm{C}_{\mathrm{Od}}+\mathrm{C}_{\mathrm{Ob}}\right)\end{array}$ & $\begin{array}{l}\text { Tin conc. accounting } \\
\text { for oxygen (at. \%) }\end{array}$ \\
\hline 8.8 & 0.671 & $\begin{array}{c}0.671 \text { at. } \% \\
/(1-0.322+0.0074)\end{array}$ & 0.98 \\
\hline 7.9 & 0.679 & $\begin{array}{c}0.679 \text { at. } \% \\
/(1-0.3272+0.0074)\end{array}$ & 1.00 \\
\hline 16.8 & 0.809 & $\begin{array}{c}0.809 \text { at. } \% \\
/(1-0.1864+0.0074)\end{array}$ & 0.99 \\
\hline 32.1 & 0.791 & $\begin{array}{c}0.79 \text { at. } \% \\
/(1-0.2125+0.0074)\end{array}$ & 0.99 \\
\hline
\end{tabular}

Table 59: Calculated tin concentration in metal matrix adjacent to the metal-oxide interface of $23.4 \mathrm{mg} \cdot \mathrm{dm}^{-2}$ corroded pre-transition Zircaloy-4 with correction for the oxygen added due to corrosion. $C_{S n d}$ and $C_{O d}$ are the detected concentrations of tin and oxygen, respectively. $C_{O b}$ is the background level of oxygen introduced during the original fabrication of the material, (1.02 $\pm 0.06)$ at. $\%$.

\subsubsection{Iron, chromium and nickel}

The transition metal solute concentrations of the four regions of alloy matrix adjacent to the metal-oxide interface described in Table 59 with regard to tin are listed in Table 60. Specimens 1 and 2 have a mean oxygen concentration of $\sim 30$ at.\%, specimens 3 and 4 contain around 20 at.\% oxygen. The trend seen in ZIRLO, that the oxygen rich region adjacent to the metal-oxide interface contains a much higher concentration of iron compared to the normal metal matrix, appears also to be the case in Zircaloy-4, although differences were seen in the amount of iron in solution in Zircaloy-4 and ZIRLO. In the highest oxygen concentration regions the local iron concentration is almost tenfold that seen in the metal matrix of uncorroded material. A range of transition metal solute segregation behaviour was observed in this material. This is described below for the four datasets listed in Table 60 . 


\begin{tabular}{|c|c|c|c|c|}
\hline \multirow[t]{2}{*}{ Specimen } & \multirow[t]{2}{*}{ Element } & \multirow{2}{*}{$\begin{array}{c}\text { Number of } \\
\text { atoms } \\
\left(10^{6} \text { atoms }\right)\end{array}$} & \multicolumn{2}{|c|}{ Composition } \\
\hline & & & $\begin{array}{l}\text { Conc. } \\
\text { (at.\%) }\end{array}$ & $\begin{array}{c} \pm 1 \sigma \\
\text { (at. } \%)\end{array}$ \\
\hline \multirow[t]{3}{*}{1} & $\mathrm{Fe}$ & 8.8 & 0.029 & 0.001 \\
\hline & $\mathrm{Ni}$ & & 0.0012 & 0.0001 \\
\hline & $\mathrm{Cr}$ & & 0.0006 & 0.0001 \\
\hline \multirow[t]{3}{*}{2} & $\mathrm{Fe}$ & 7.9 & 0.038 & 0.001 \\
\hline & $\mathrm{Ni}$ & & 0.0031 & 0.0002 \\
\hline & $\mathrm{Cr}$ & & 0.0021 & 0.0001 \\
\hline \multirow[t]{3}{*}{3} & $\mathrm{Fe}$ & 16.8 & 0.0157 & 0.0002 \\
\hline & $\mathrm{Ni}$ & & $<$ trace & - \\
\hline & $\mathrm{Cr}$ & & 0.0022 & 0.0002 \\
\hline \multirow[t]{3}{*}{4} & $\mathrm{Fe}$ & 32.1 & $\mathrm{NR}$ & - \\
\hline & $\mathrm{Ni}$ & & $<$ trace & - \\
\hline & $\mathrm{Cr}$ & & $<$ trace & - \\
\hline
\end{tabular}

Table 60: The concentration of transition metal solutes seen in volumes of oxygen-rich alloy matrix adjacent to the metal-oxide interface of four specimens.

A $3 \mathrm{D}$ iron atom map is featured in Figure 136 (specimen 1 in Table 60). By comparison with the accompanying isosurface view in Figure 136 it can be seen that the majority of iron appears to be located at the boundary between the alloy and the $\mathrm{ZrO}$ layer. A set of $1 \mathrm{D}$ concentration profiles across this metal-oxide interface is shown in Figure 137. The fourth period transition metal solute profiles are across the $\mathrm{Zr}-\mathrm{ZrO}$ interface in Figure 137 (e) and the $\mathrm{ZrO}-\mathrm{ZrO}{ }_{2}$ interface in (f). Note the difference in vertical scale between (e) and (f). Chromium and nickel are at a very low concentration in all regions, although generally higher in the metal adjacent to the interface than within the bulk. In this example, nickel enrichment is seen at the $\mathrm{Zr}-\mathrm{ZrO}$ interface. The iron profile across the interface is also plotted as an idealised profile in Figure 138 on a semi-log scale. Iron segregation to the boundary of the $\mathrm{ZrO}$ phase was seen in this sample, and this is consistent with enrichment of iron seen at other heterogeneities within the material. The iron concentration within the metal layer adjacent to the interface is similar to that in the $\mathrm{ZrO}$. It 
appears there is partial rejection of iron at the metal-oxide interface. The apparent concentration of iron seen in the oxide region of Figure 137 is also subject to a higher background noise level than within the metal, so the value displayed is somewhat of an over estimate.

A set of 3D atom maps showing the position of the metal-oxide interface using isosurfaces (a) and the distribution of iron ( $a$ and b), chromium (c) and nickel (d) are shown in Figure 139 (specimen 2 from Table 60). The relevant region of the mass-to-charge spectrum of this metal region is shown in Figure 140. The peak at $29 \mathrm{Da}$ is higher than the level that would be expected due to the presence of iron, and is attributed to nickel. As with Figure 136, iron appears to be primarily located at linear features that run parallel with the plane of the $\mathrm{Zr}-\mathrm{ZrO}$ interface. Iron is also seen to be non-uniformly distributed in the metal adjacent to the metal-oxide interface. The high density and proximity of these linear features to the interface hampers quantitative analysis, however, similar characteristics are seen in subsequent examples that do lend themselves to such analysis.

Figure 139 (c) and (d) show a tendency for nickel to be co-segregated with iron along these features. There is also a small, concentrated, point-cluster of chromium atoms (circled) in the centre of the image (c) that is not part of any of the linear arrays of solute decorated dislocations. Comparison with (b) shows that iron is also clustered at this point and is circled in (b) and (c). This chromium cluster is remote enough from other dislocations to allow a more rigorous analysis. It is useful to get an idea of the probability of occurrence of a region of chromium concentration of the scale observed that might be expected due to a random solid solution. Figure 141 compares the number of regions in this dataset that have a chromium concentration lower than a threshold level for two different minimum sizes of cluster. 


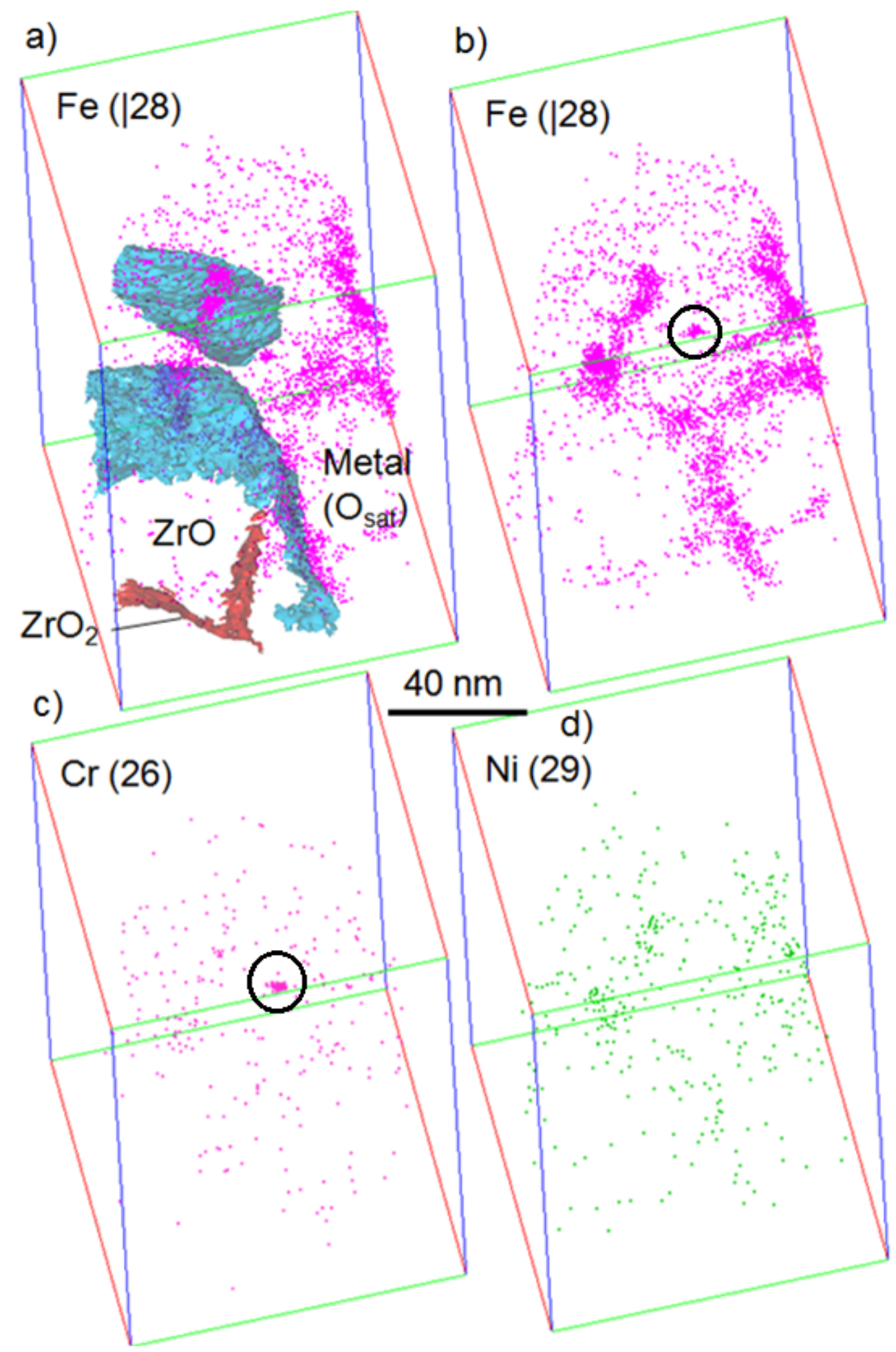

Figure 139: 3D atom maps displaying solute distribution around the metal-oxide interface of $23.4 \mathrm{mg} . \mathrm{dm}^{-2}$ corroded pre-transition Zircaloy-4 for a) iron with isosurfaces marking the bounds of the metal (blue) and $\mathrm{ZrO} 2$ oxide (red), b) iron from the leading edge of the peak at $28 \mathrm{Da}, \mathrm{c}$ ) chromium (at $26 \mathrm{Da}$ ) and d) nickel (at $29 \mathrm{Da}$ ). The circled region marks the position of a chromium cluster. 


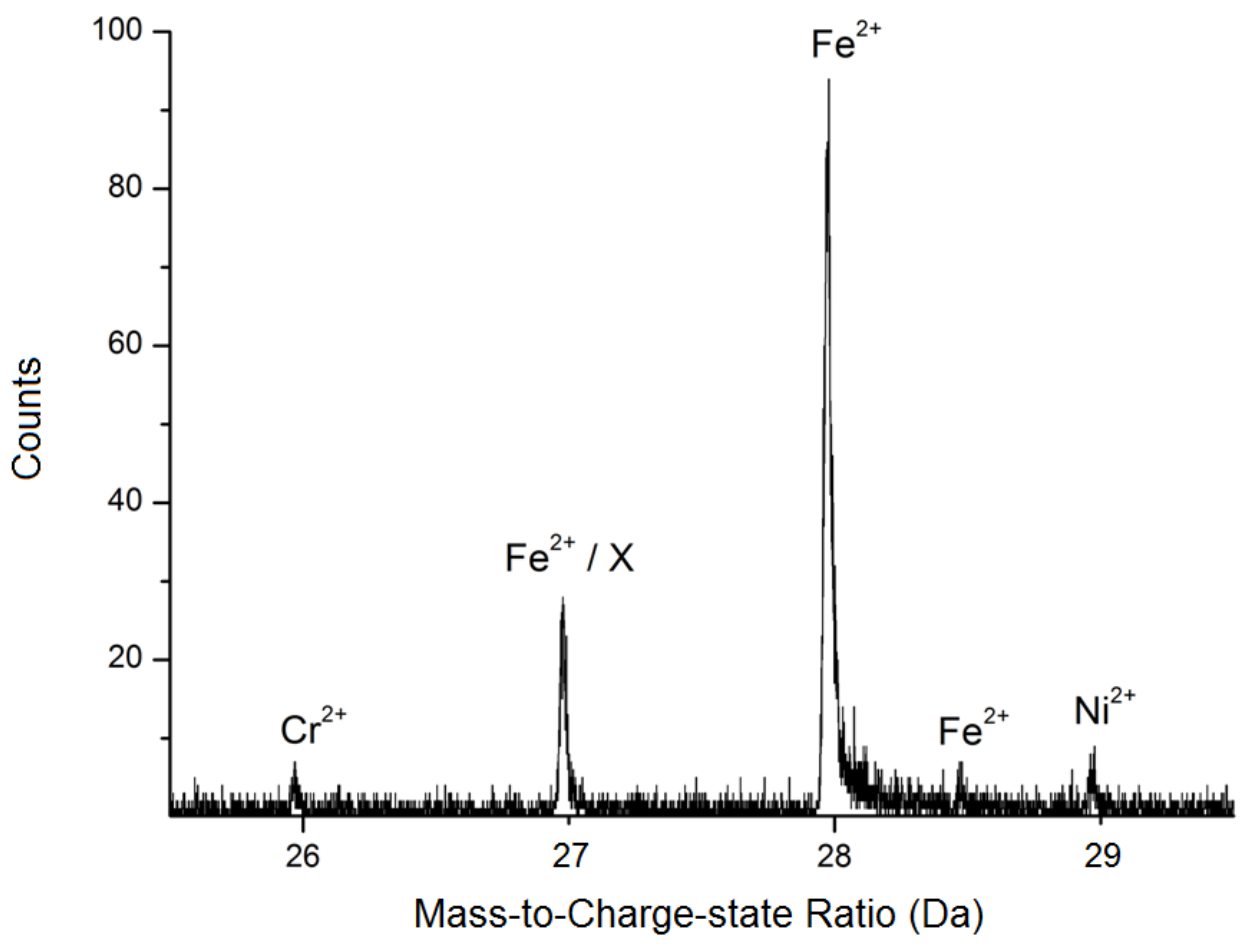

Figure 140: Mass-to-charge state ratio spectra of the 2+ charge-state fourth period transition metals for the metal region (including dislocations) of the dataset displayed in Figure 139 (23.4 $m g . d m^{-2}$ corroded pre-transition Zircaloy-4). The spectrum uses 1000 bins per Da.

Threshold sizing is performed by filtering out regions that require less than a prescribed number of polygons to draw them. From Figure 141 it is seen that where the threshold size of a 'cluster' is kept small, a large number of regions are see due to random local chromium concentration fluctuations, even at high concentrations $(0.6$ at.\% $\mathrm{Cr}$ is of the order of 1000 times the matrix concentration seen in the bulk material). If the minimum size of the regions is increased by an order of magnitude then only the single region shown in Figure 139 is detected. The volume (or radius) of the cluster is, to a large extent, a function of the defining criteria. Figure 142 compares the apparent volume of this cluster as defined by various threshold levels. The volume tends towards $\sim 50 \mathrm{~nm}^{3}$ at high concentration, corresponding to a cluster radius of $\sim 3.5 \mathrm{~nm}$. A proxigram across the surface of the cluster is shown in Figure 143. At its core the combined iron and chromium concentration of the cluster is around 18 at.\%. This increase is at the expense of zirconium and oxygen. 


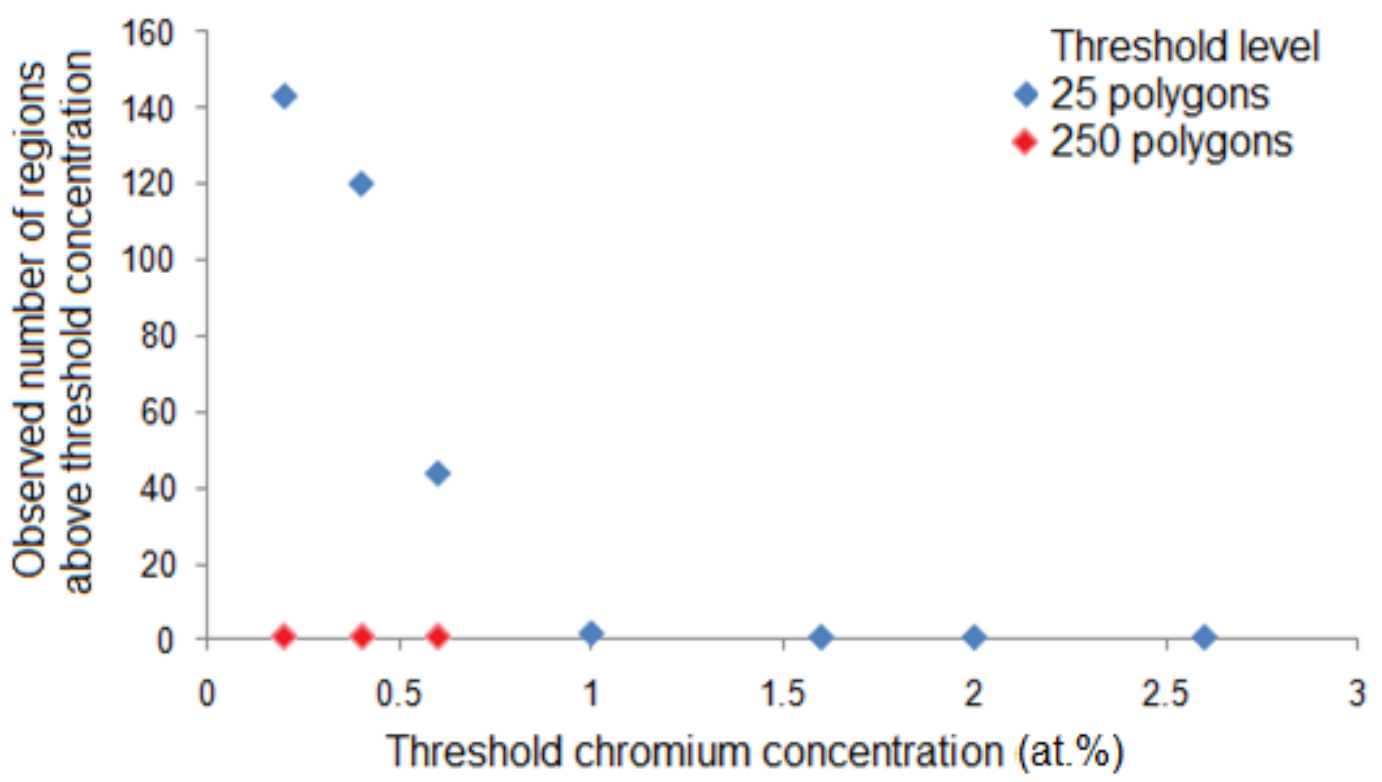

Figure 141: The number of regions within the dataset shown in Figure 139 that contain a chromium concentration higher than a threshold level using two. Two minimum sizes of volume (in terms of the number of polygons used to render) are compared. A high observed number of regions shows the effect of random fluctuations of chromium concentration on the apparent number of clusters.

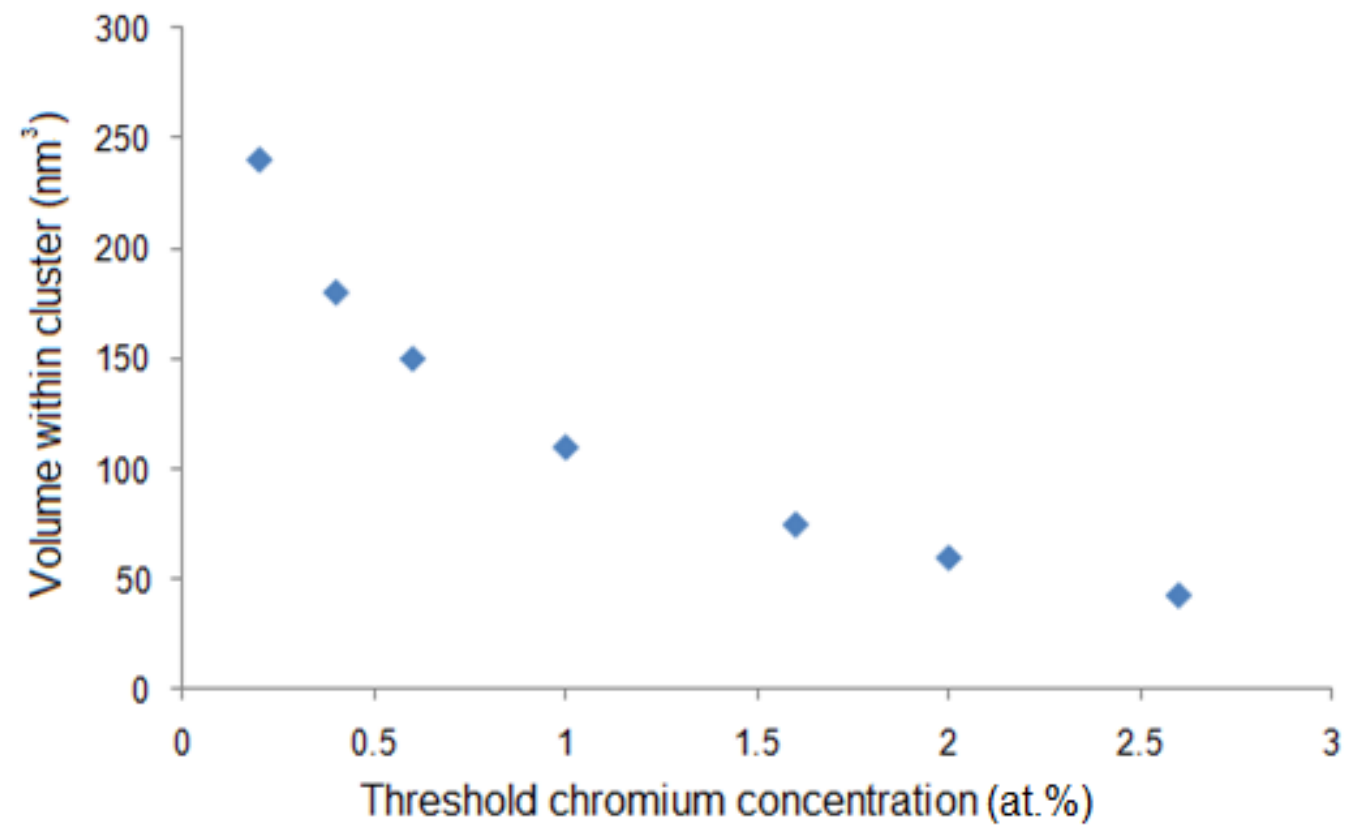

Figure 142: The volume contained the cluster as defined by isosurfaces of differing threshold concentration. 

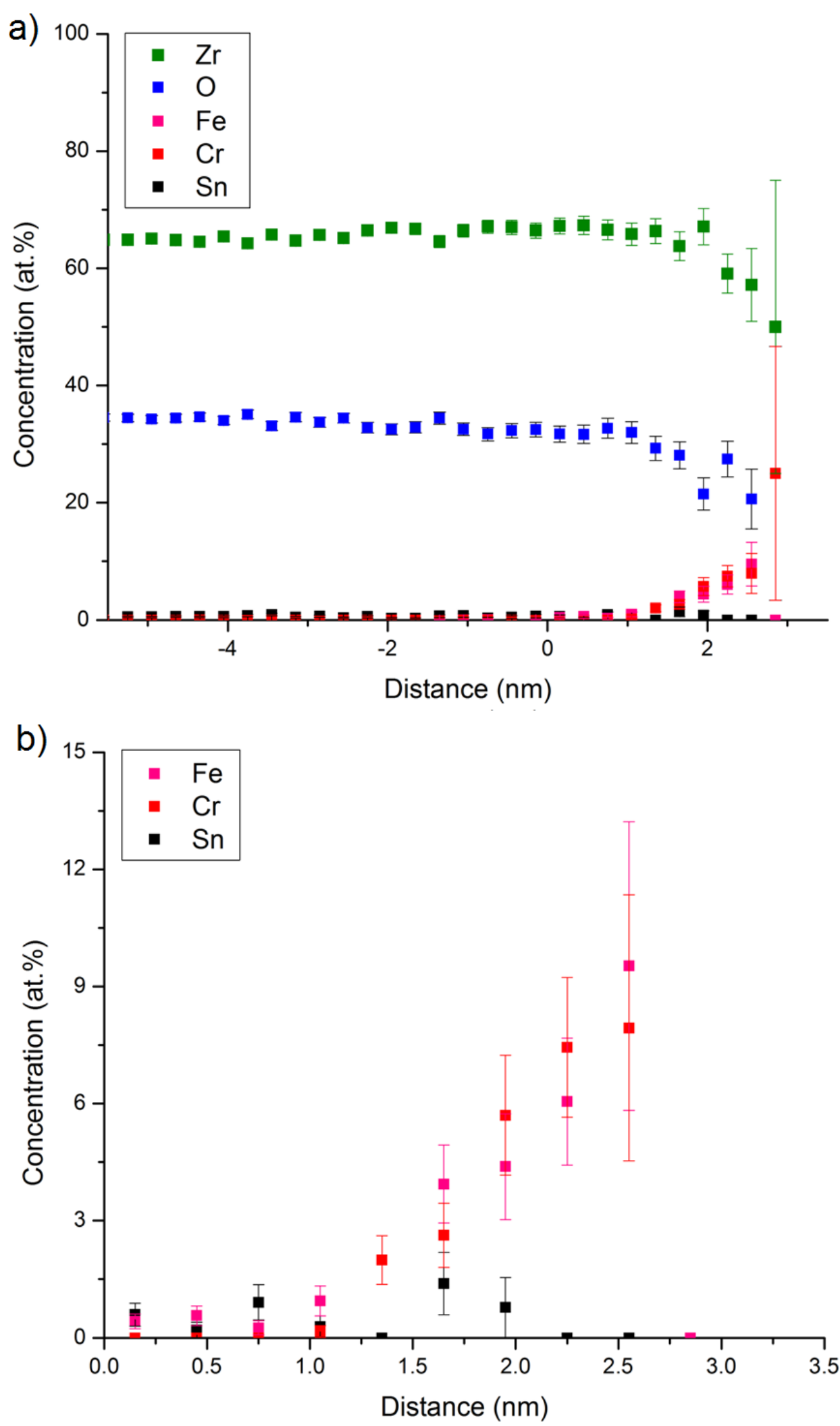

Figure 143: Proxigram across the surface of the chromium point-cluster shown in Figure 139 showing a) $\mathrm{Zr}, \mathrm{O}, \mathrm{Fe}, \mathrm{Cr}$ and Sn between 0 and 100 at.\% concentrations within the matrix (LHS) and the cluster (RHS); and b) highlighting the behaviour of the minor elements (0-15 at.\%). 


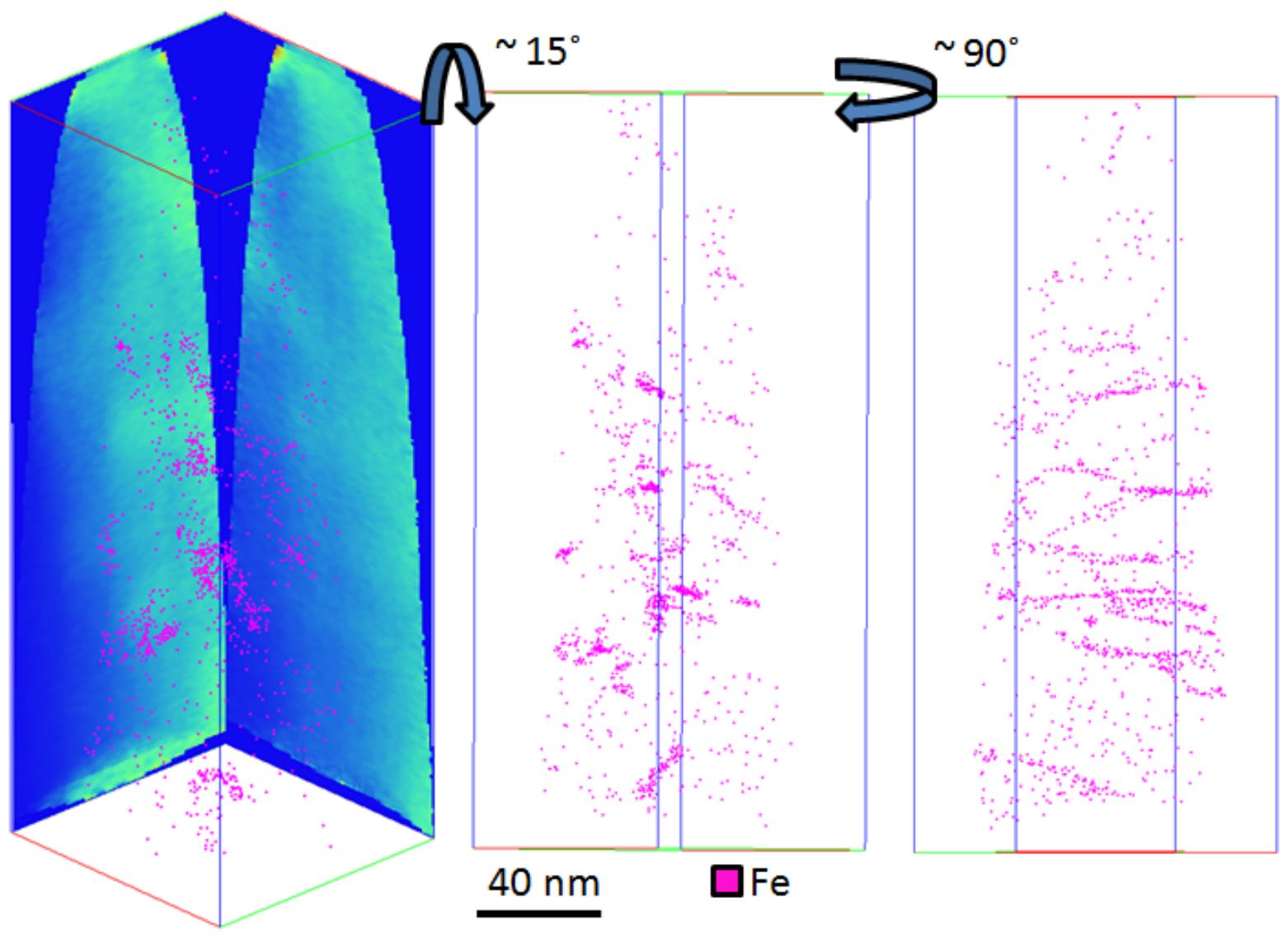

Figure 144: 3D atom maps of the iron distribution in a region of metal near to the metal oxide interface (within $200 \mathrm{~nm}$ ) of $23.4 \mathrm{mg} \cdot \mathrm{dm}^{-2}$ corroded pre-transition Zircaloy-4. The 2D oxygen concentration profiles shown in the far left frame display the oxygen concentration using the colour scale 0 at.\% O (metal - blue) to 66 at.\% $\mathrm{O}\left(\mathrm{ZrO}_{2}\right.$ - red). Iron atoms are displayed as pink dots and are preferentially aligned along co-linear features.

For another example, labelled specimen 3 in Table 60, 2D oxygen concentration profiles are shown in Figure 144. The planes of these profiles are orthogonal to each other. The area of highest oxygen concentration is at the right hand side of the analysis volume. From this perspective, the dislocations are viewed along their axes. Rotating the dataset by 90 degrees allows a profile view of the dislocation network. The majority of the iron-decorated dislocations are aligned parallel to the metal-oxide interface (normal to the oxygen concentration gradient). Isosurfaces at 0.2 at.\% iron were applied to this dataset in order to define the iron-decorated 
dislocations. These isosurfaces are shown in Figure 145. The individual features can be isolated and proxigrams (see section 3.3.5) computed around separate dislocations. Four unconnected surfaces have been selected, labelled $\mathrm{a}-\mathrm{d}$ in Figure 145. The iron concentrations at the core of these dislocations as determined by a $0.3 \mathrm{~nm}$ bin proxigram are listed in Table 61 . There is little variation in the chemistry at the dislocations in this sample. The mean iron concentration at the dislocation core defined by this method is $(2.7 \pm 0.2)$ at.\%. The concentration of oxygen and tin was not seen to vary significantly within the dislocations compared to the surrounding matrix.
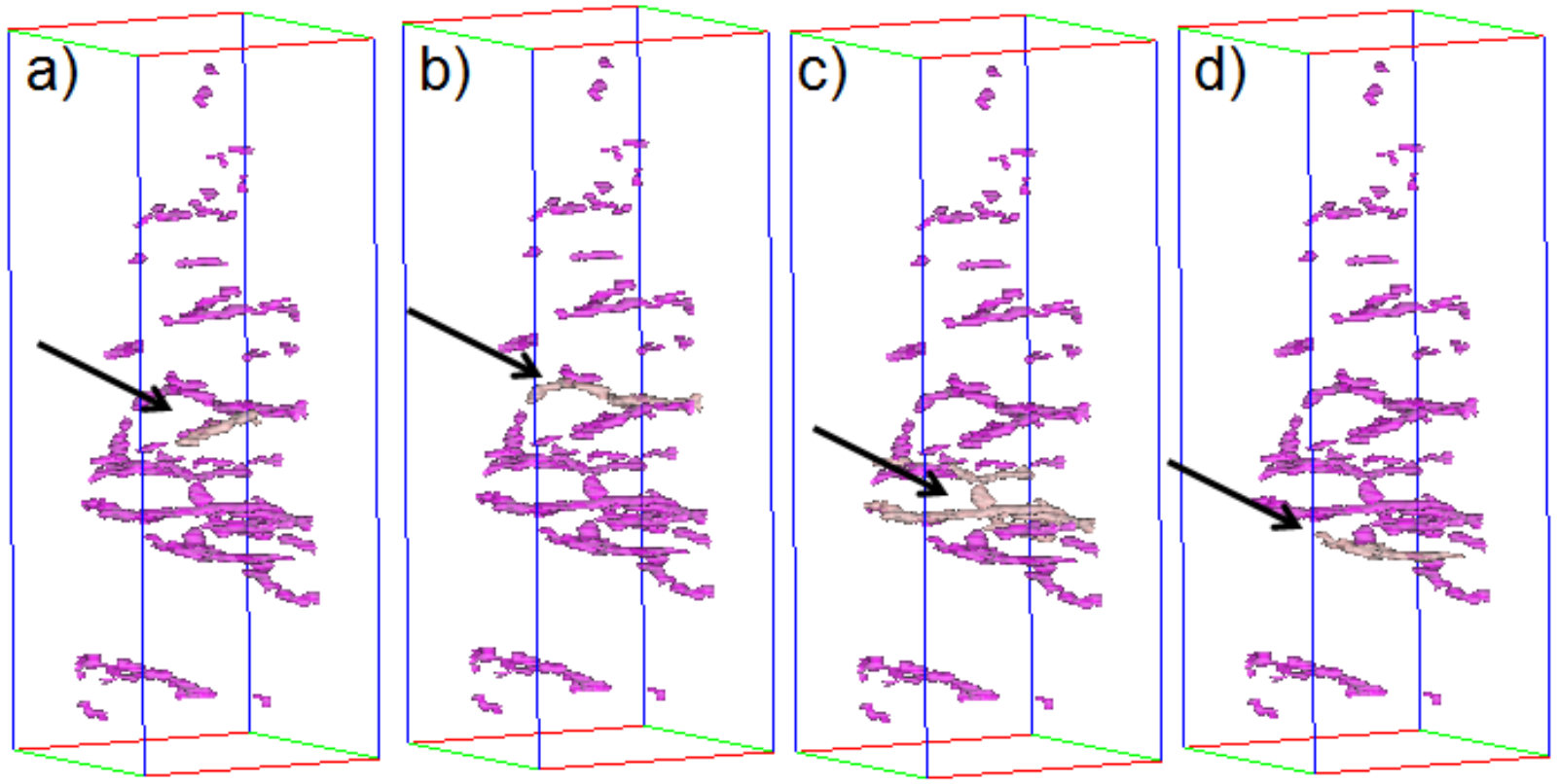

Figure 145: Isosurfaces plotted at 0.2 at.\% iron for the dataset shown in Figure 144 (23.4 $m g . d m^{-2}$ corroded pre-transition Zircaloy-4). Separate isosurface are selected in frames $a-d$. These are a lighter shade than the other surfaces and indicated by an arrow.

\begin{tabular}{|c|ccccc|}
\hline Dislocation (see Figure 145) & a & b & C & d & mean \\
\hline Iron conc. at core (at.\%) & 2.8 & 2.6 & 2.8 & 2.4 & 2.7 \\
Uncertainty (at.\%) & 0.3 & 0.2 & 0.2 & 0.3 & 0.2 \\
\hline
\end{tabular}

Table 61: Iron concentrations at the core of the dislocations highlighted in fames a-d of Figure 145 (above) as determined from a proxigram taken across these features. 
Nickel is not resolvable from the background noise level in specimen 3 (see Table 60) shown in Figure 144. It should be noted that the apparent concentration of minor solutes in these inhomogeneous regions of metal matrix is highly dependent upon the volume fraction of dislocations within the region of matrix analysed.

The 2D oxygen concentration profile in Figure 146 (specimen 4 from Table 60) depicts another dataset where the region of interest intersects with the edge of the $\mathrm{ZrO}$ region. The majority of the volume is occupied by the metal region adjacent to the interface. The oxygen-rich region close to the interface is less than $100 \mathrm{~nm}$ in thickness here. The iron and chromium atom maps in Figure 146 are viewed from the same perspective as the $2 \mathrm{D}$ oxygen profile. The majority of the iron-decorated dislocations are in the lower-oxygen content region of this material. The iron concentration at the core of the linear features is $2-3$ at. $\%$, as was seen in the previous example. The oxygen gradient in the dataset drops from the saturation level of oxygen to $\sim 5$ at. $\% \mathrm{O}$ over $\sim 200 \mathrm{~nm}$. As in the previous example, the dislocations seem to be aligned with the plane of the interface (out of plane with the page, normal to the oxygen concentration gradient).

Chromium clustering is seen to be co-segregated with iron, but is not primarily found along linear features. The cluster highlighted by the black rectangle in Figure $146(\mathrm{Fe})$ is shown enlarged in Figure 147. Two views of the iron decorated feature are shown. The distribution of solute appears less, even along the dislocation, than that seen in the previous example. What from some angles appears to be a linear feature could be equally well described as an aligned series of clusters, terminating in a loop-like arrangement of solute. Chromium is also segregated to this loop and to the nearby cluster visible in the top right of the right-hand atom maps in Figure 147. 

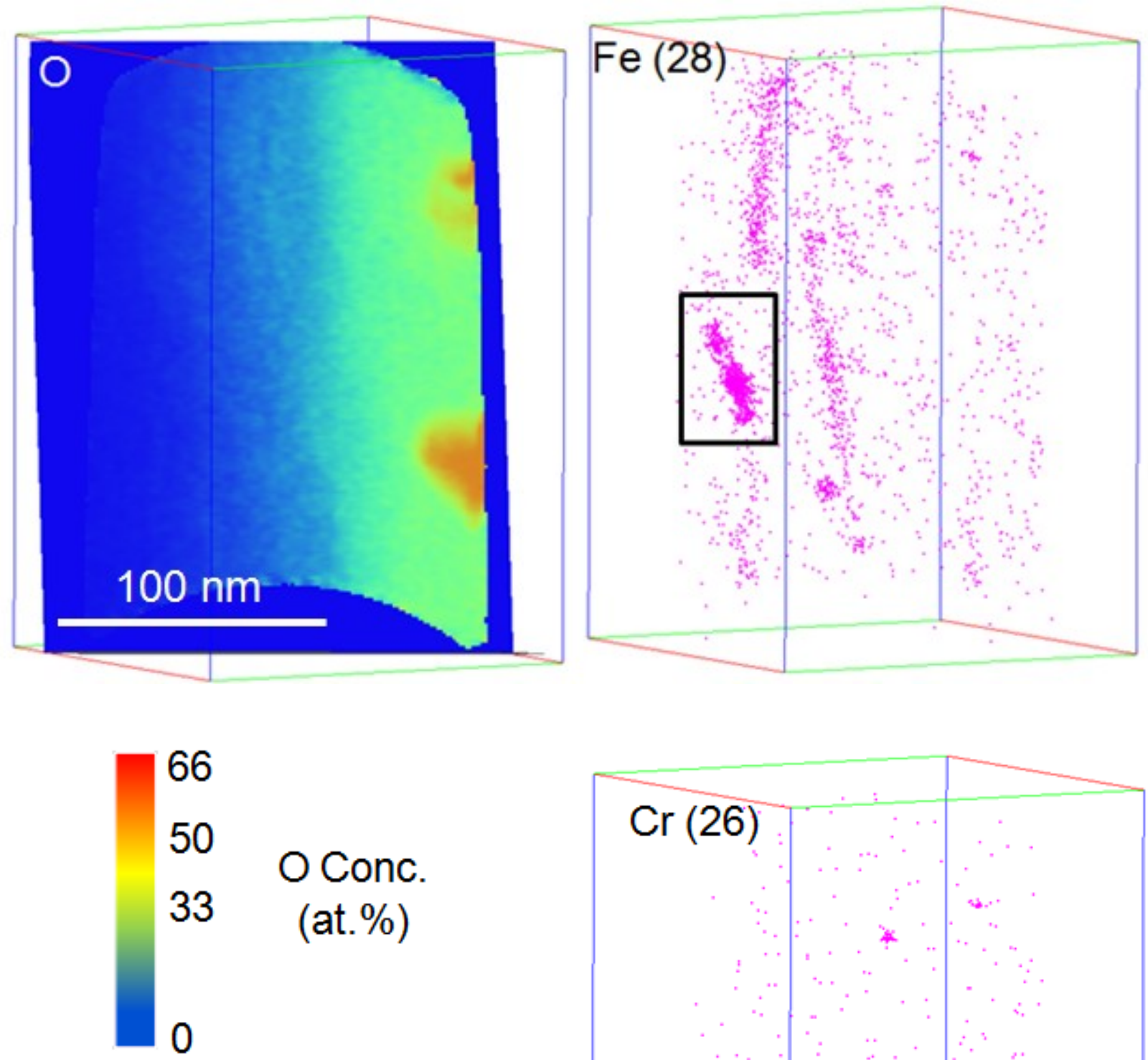

Figure 146: O) $2 D$ oxygen concentration profile at a metal-oxide interface of 23.4 $m g . d m^{-2}$ corroded pre-transition Zircaloy-4. The region shown touches on a $\mathrm{ZrO}$ layer but

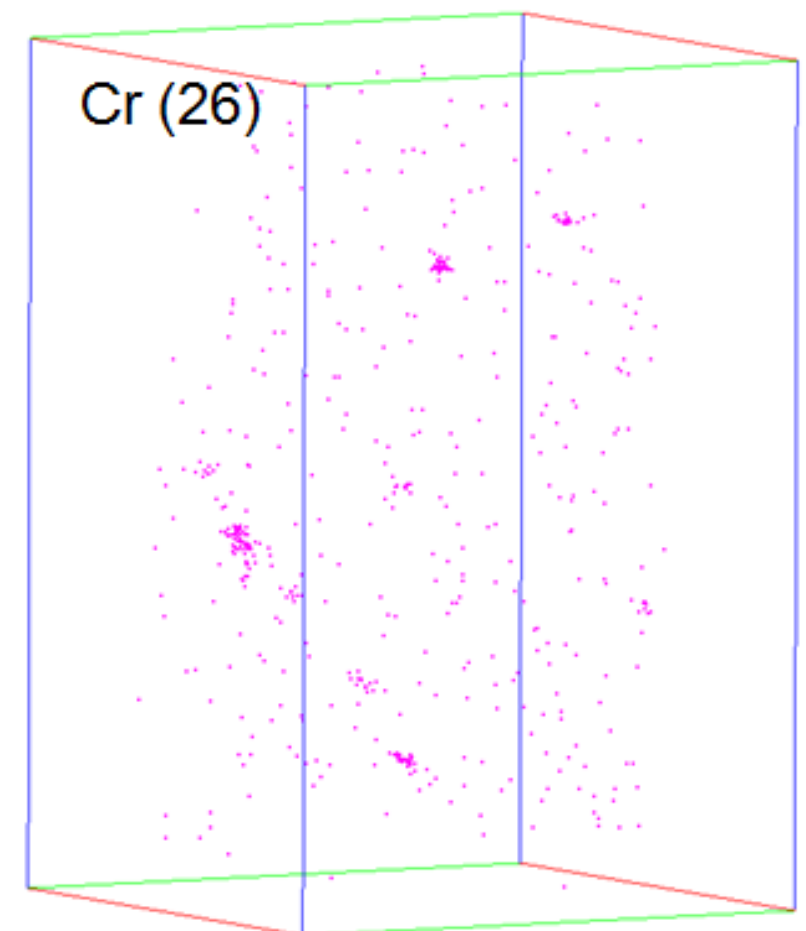
is mainly metal, and an oxygen rich metal region adjacent to the interface. The $3 D$ atom maps are viewed from the same perspective and show the distribution of iron (at $28 \mathrm{Da}$ ) and chromium (at $26 \mathrm{Da}$ ). The highlighted black rectangular region in the iron atom map is shown in further detail in Figure 147. The apparently linear alignment of chromium clusters (bottom left) is due to perspective only. The clusters are not aligned in depth (into the page). 

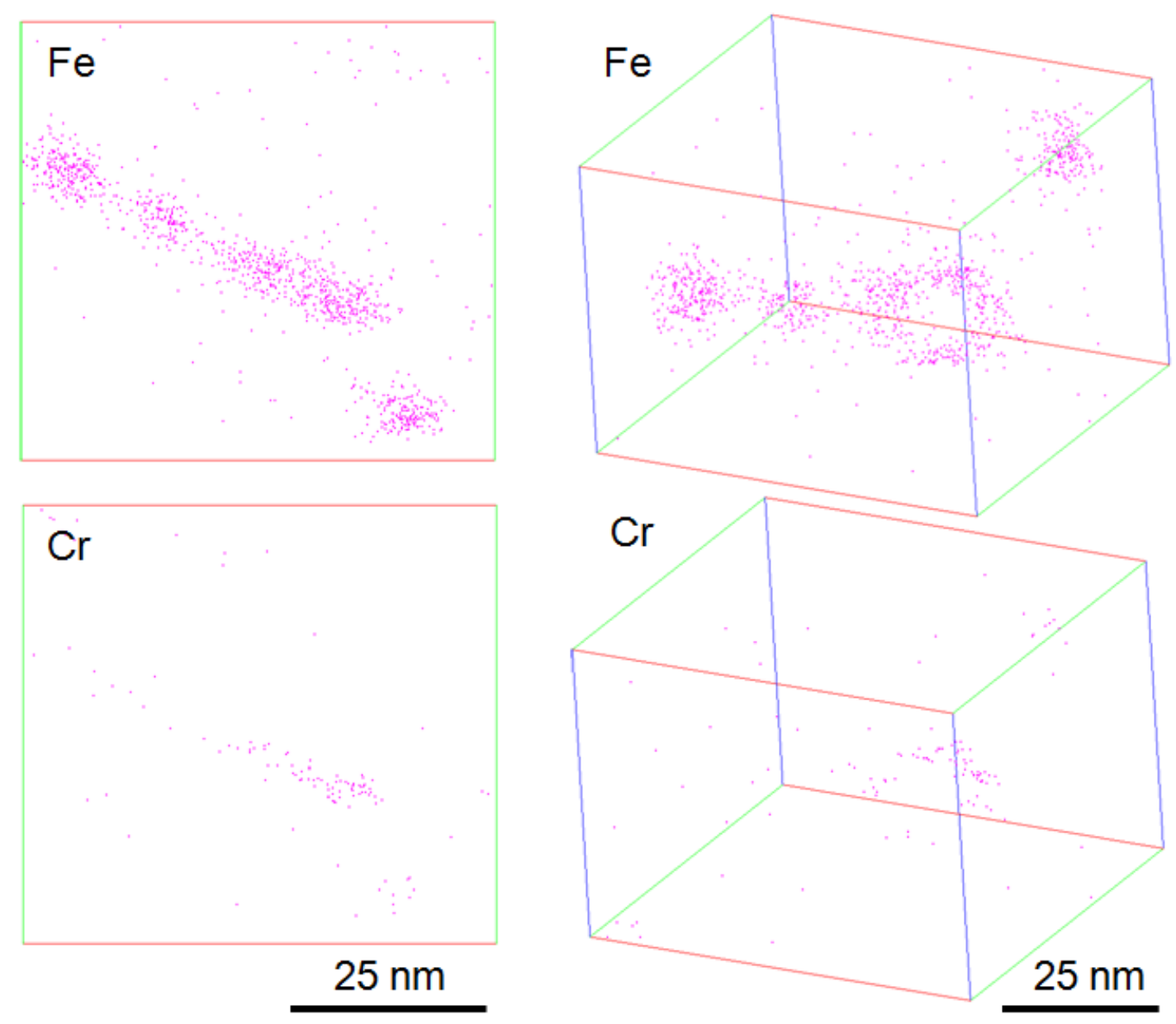

Figure 147: Highlighted region from the 3D atom map in Figure 146. Two views of minor solute decorating a linear feature and additional large cluster not in line with the feature. The feature solute terminates at a loop (most clearly seen Fe top right).

The solute decorated dislocations in Figure 147 were analysed in a similar way as for the previous example; by creating proxigrams across the defined surfaces of the features and comparing the core values. As the chemistry of the loop feature is evidently inhomogeneous a separate surface was created around this sub volume, in order to ascertain the chromium value at the core of this region. These surfaces are shown in Figure 148. The iron concentration at the core of this line of point features is much higher than that seen distributed relatively evenly along the linear features in the previous example (Figure 145). Table 62 shows that the iron concentration at the core of these features is around 7 at.\%. The increase in iron and chromium 
concentration is at the expense of the oxygen and zirconium concentration at the dislocation. The tin concentration at the dislocation may also be lower than in the matrix, but this difference is difficult to quantify.

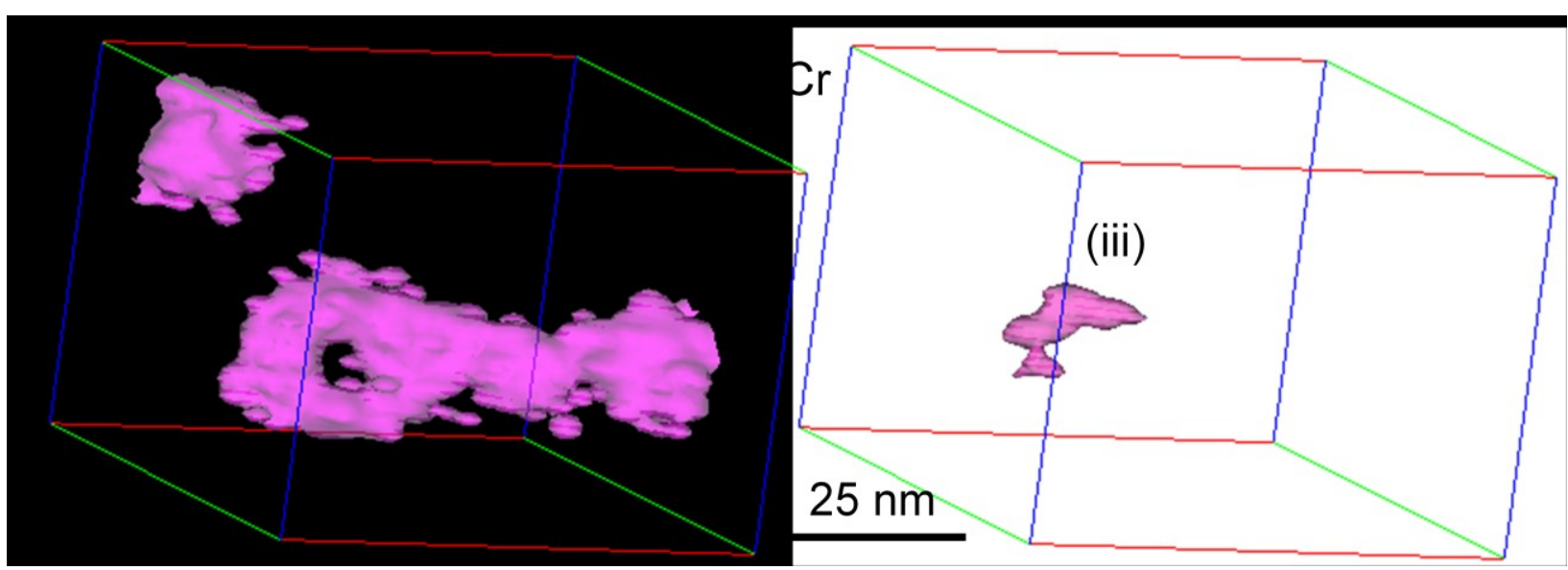

Figure 148: Isosurfaces plotted at 0.2 at.\% iron ( $i$ and ii) and chromium (iii) for the dataset shown in Figure 147 (23.4 mg.dm ${ }^{-2}$ corroded pre-transition Zircaloy-4).

\begin{tabular}{|c|ccc|}
\hline Dislocation (see Figure 148) & i & ii & iii \\
\hline Iron conc. at core (at.\%) & $15 \pm 5$ & $7 \pm 2$ & $7 \pm 2$ \\
Chromium conc. at core (at.\%) & $0.3 \pm 0.3$ & $0.8 \pm 0.2$ & $4 \pm 1$ \\
$\begin{array}{c}\text { Difference between dislocation } \\
\text { and matrix oxygen conc. (at.\%) }\end{array}$ & $-3.3 \pm 1$ & $-4.2 \pm 0.5$ & $-3.5 \pm 0.5$ \\
$\begin{array}{c}\text { Difference between dislocation } \\
\text { and matrix zirconium conc. (at.\%) }\end{array}$ & $-9 \pm 5$ & $-3 \pm 1.5$ & $-8 \pm 0.5$ \\
\hline
\end{tabular}

Table 62: The concentration of iron and chromium at the iron decorated dislocations shown in

Figure 148, along with the difference between the dislocation and matrix concentration of oxygen (a negative value indicates that there is less oxygen at the dislocation than in the metal matrix). 
A wide range of minor alloying element transition element behaviour has been observed at dislocations in the proximity of the metal/oxide interface of pre-transition Zircaloy-4. These are summarised as follows:

- All five specimens analysed show iron-decorated linear features in proximity to the metal-oxide interface.

- These iron-decorated dislocations have an iron concentration of $(2.7 \pm 0.2)$ at.\% at their core.

- The nucleation of iron and chromium rich clusters is also seen along some of these features. These tend to have a higher core iron and chromium concentration, $\sim 10$ at.\% combined, the majority of which is iron.

- Chromium is seen to cluster at point-features. A chromium rich cluster with radius $\sim 3.5$ $\mathrm{nm}$ and core chromium concentration of $\sim 9$ at. $\%$ was analysed. 


\section{$1.3 \mathrm{Zr}-\mathrm{Nb}-\mathrm{Ti}$}

The $\mathrm{Zr}-\mathrm{Nb}$-Ti test alloy was subjected to one day of exposure to a $360{ }^{\circ} \mathrm{C}$ pure water autoclave environment. During this time an $80 \mu \mathrm{m}$ thick oxide layer $\left(1189 \mathrm{mg} \cdot \mathrm{dm}^{-2}\right)$ developed on the substrate. The appearance of the layer was that of a white, adherent, post-transition oxide. The oxidation rate of this material is several hundred times greater than the commercially deployed alloys studied. All of the material described in this section is from this advanced corrosion condition.

A large body of literature describing the metallography, performance and oxide microstructure of ZIRLO and Zircaloy-4 was discussed in chapter 2. The same level of information is not available in the open literature for the $\mathrm{Zr}-\mathrm{Nb}-\mathrm{Ti}$ test alloy. Characterisation of the alloy matrix, grain boundary chemistry and second phase particles distribution within the alloy using 3DAP, TEM and EDX was described in sections 3.4.1.4, 5.2.1.2 and 5.2.2.4. The oxide morphology was investigated using electron microscopy by $\mathrm{Na} \mathrm{Ni}$, Oxford Materials. This work is described in section 6.4.1 and provides a context for the 3DAP observations of the metal-oxide interface ( $\S$ 6.4.2) and surrounding material $(\S 6.4 .3)$ that follow. 


\subsubsection{Overview of test alloy corrosion: Electron microscopy}

\subsubsection{Oxide microstructure}

The oxide morphology of the corroded material was characterised using TEM by Na Ni, Oxford Materials. A TEM micrograph of a section of the metal-oxide interface is shown in Figure 149, along with a schematic diagram of the interface structure. Small, $\sim 50 \mathrm{~nm}$ diameter, equiaxed oxide grains were seen right up to the metal oxide interface. A larger proportion of the oxide consisted of the tetragonal phase than was seen in the oxide of better performing alloys, such as Zircaloy-4. Large numbers of cracks, parallel with each other and the metal-oxide interface, can be seen in Figure 149. These cracks have limited development in the direction normal to the interface (vertical cracks), and in the 2D cross sectional view horizontal crack layers are not seen to be interconnected by vertical cracks. Connected porosity is seen near the interface, and this could offer a path from the free surface to the metal-oxide interface [14]. 

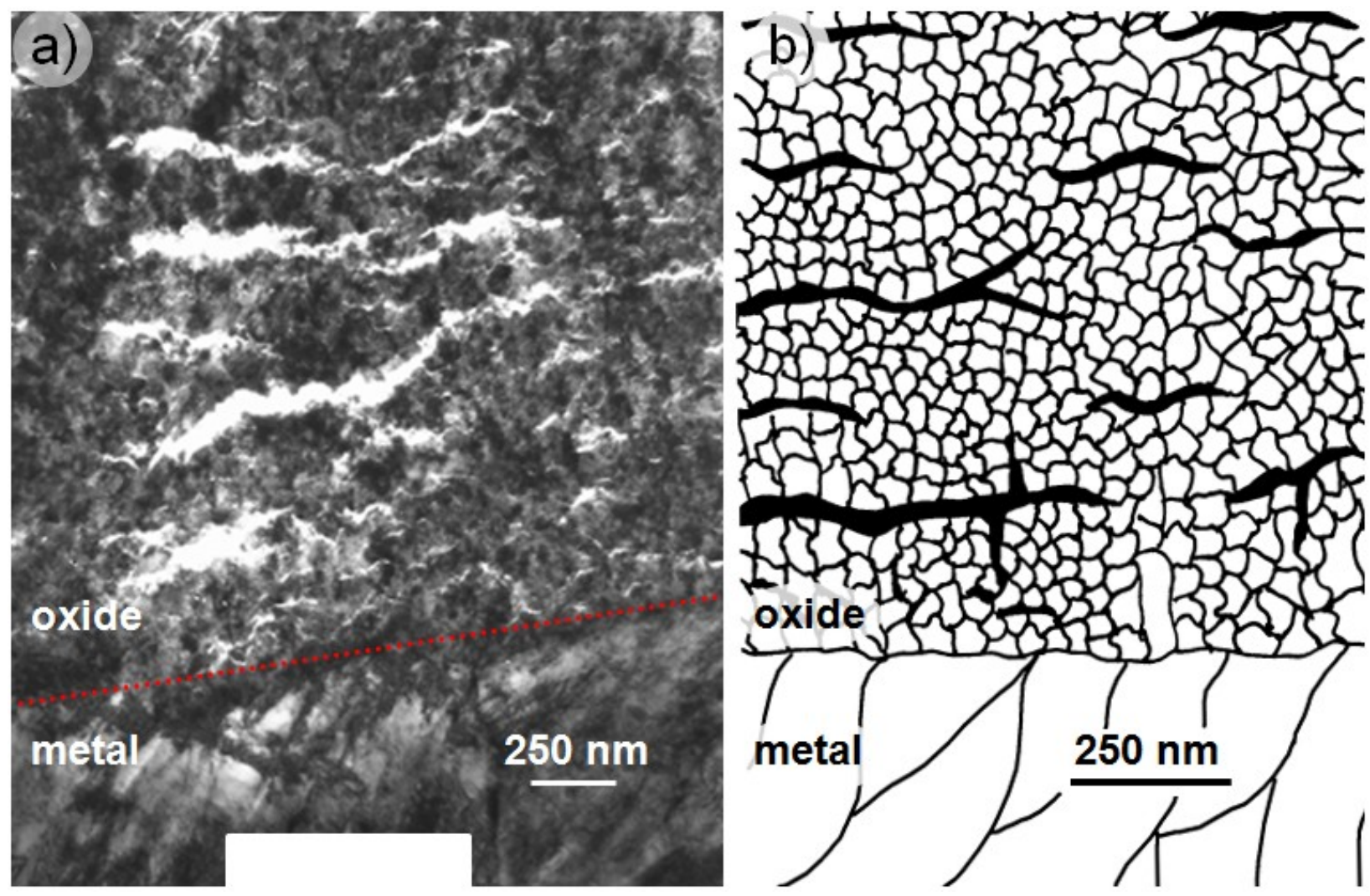

Figure 149: a) TEM micrograph of the 1 day corroded Zr-Nb-Ti metal-oxide interface, courtesy

of $\mathrm{Na} \mathrm{Ni}$, Oxford Materials. Cracks are shown in white. The interface is highlighted by a dotted red line. b) Schematic of the metal-oxide interface emphasising oxide morphology. Cracks and grain boundaries are shown in black. The full thickness of the oxide is $80 \mu \mathrm{m}$.

\subsubsection{Chemical mapping}

EDX mapping across the metal-oxide interface has also been conducted for the heavily corroded 1 day autoclave sample. Chemical maps of a region of $20 \mu \mathrm{m}$ of oxide and metal either side of the interface are shown in Figure 150. The cracks parallel with the interface can be clearly resolved in the $\mathrm{O}$ and $\mathrm{Zr}$ profiles. The concentration of titanium appears greater (brighter) within the metal than the oxide, but it should be noted that there appears to be a general gradient in intensity (bottom left to top right) in all of the profiles, as seen most easily in the $\mathrm{Zr}$ profile. It is also difficult to separate the effect of the reduced metal atom concentration due to the addition of 2 oxygen atoms for every cation in the oxide layer. 

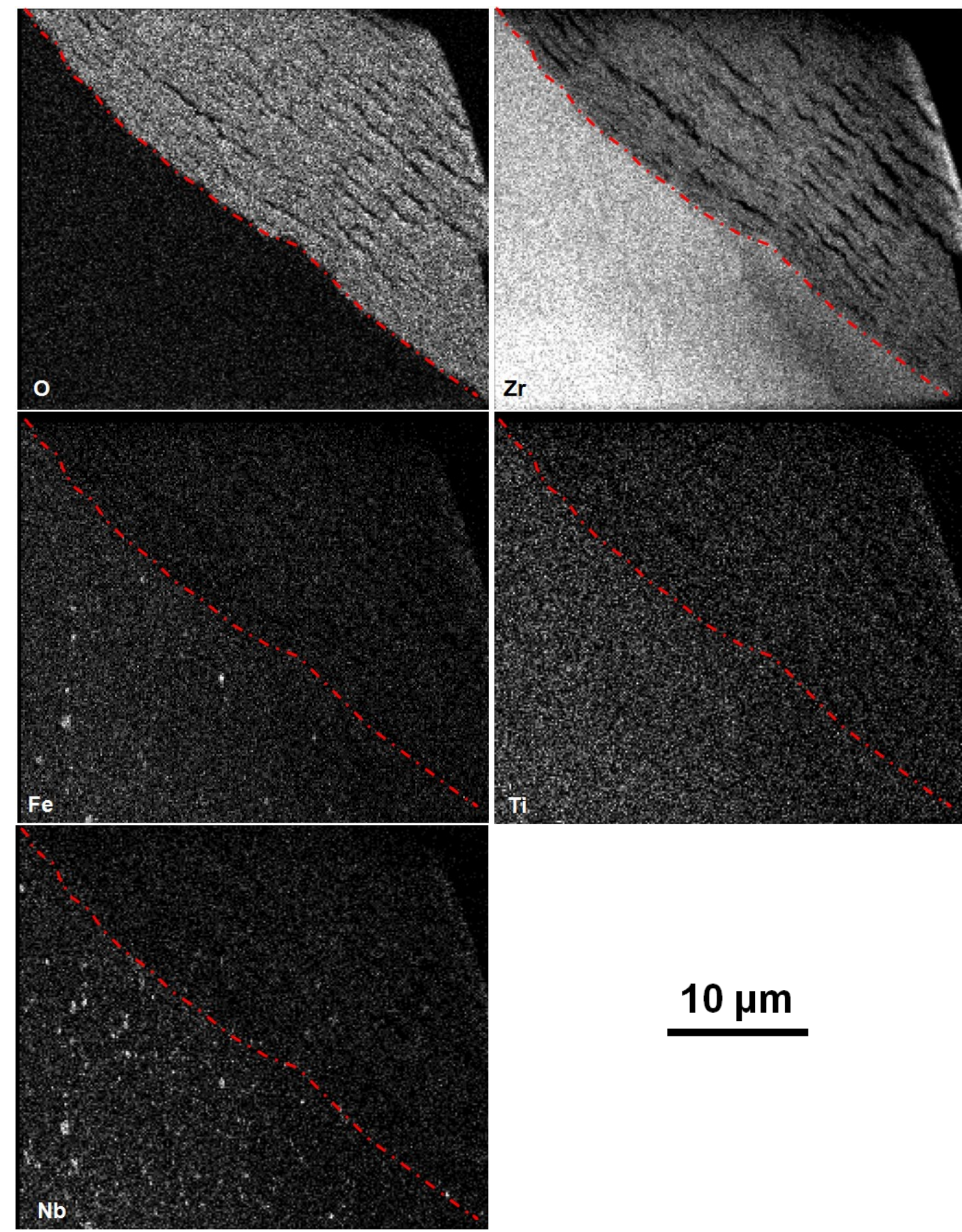

\section{$10 \mu \mathrm{m}$}

Figure 150: EDX profiles of the 1 day corroded Zr-Nb-Ti metal-oxide interface (shown by red dotted line) for $\mathrm{Zr}, \mathrm{O}, \mathrm{Nb}, \mathrm{Ti}$ and $\mathrm{Fe} .20 \mu \mathrm{m}$ of the $80 \mu \mathrm{m}$ oxide is shown. Micrographs courtesy of Na Ni, Oxford Materials. 


\subsubsection{Second phase particle distribution}

Brightly imaging iron- and niobium-containing particles are visible within the metal in Figure 150. Similar particles are harder to resolve within the oxide layer, although some niobium-rich particles are visible within the oxide in this profile. Although they suffer the same issues described above for titanium, the niobium and iron profiles seem generally brighter within the metal than the oxide. It is important to establish whether the lower observed particle density in the oxide is due to difficulty in resolving the features within the oxide using EDX scanning or if it is due to a rapid dissolution of these particles within the oxide.

Particles have been observed within the oxide of corroded $\mathrm{Zr}-\mathrm{Nb}$-Ti using TEM at $250 \mathrm{~nm}$ and $1.5 \mu \mathrm{m}$ from the metal-oxide interface, summarised in Table 63. Figure 151 is a micrograph showing a particle $1.5 \mu \mathrm{m}$ from the interface. The compositions of both of these particles are consistent with those studied in as received material (see 5.2.1.2) and so it does not appear that rapid dissolution of these precipitates occurs within the oxide. 


\begin{tabular}{|c|c|c|c|}
\hline Location & $\begin{array}{c}\text { Crystal structure type } \\
\text { identified by diffraction }\end{array}$ & $\begin{array}{c}\text { Composition measured by EDX } \\
\text { (at. \%) }\end{array}$ & $\begin{array}{c}\text { Diameter } \\
\text { (nm) }\end{array}$ \\
\hline $\begin{array}{c}\text { In oxide, } 1.5 \mu \mathrm{m} \\
\text { from interface }\end{array}$ & Hexagonal $\mathrm{Zr}(\mathrm{Nb}, \mathrm{Fe})_{2}$ & $\mathrm{Zr}(76.1)-\mathrm{Nb}(21.7)-\mathrm{Fe}(2.2)$ & 50 \\
\hline $\begin{array}{c}\text { In oxide, } 250 \mathrm{~nm} \\
\text { from interface }\end{array}$ & Hexagonal $\mathrm{Zr}(\mathrm{Nb}, \mathrm{Fe})_{2}$ & $\mathrm{Zr}(76.8)-\mathrm{Nb}(20.3)-\mathrm{Fe} \mathrm{(2.9)}$ & 40 \\
\hline
\end{tabular}

Table 63: Structural and chemical information relating to two second phase particles observed in the oxide formed on 1 day corroded Zr-Nb-Ti. Table provided by $\mathrm{Na} N \mathrm{~N}$, Oxford Materials.

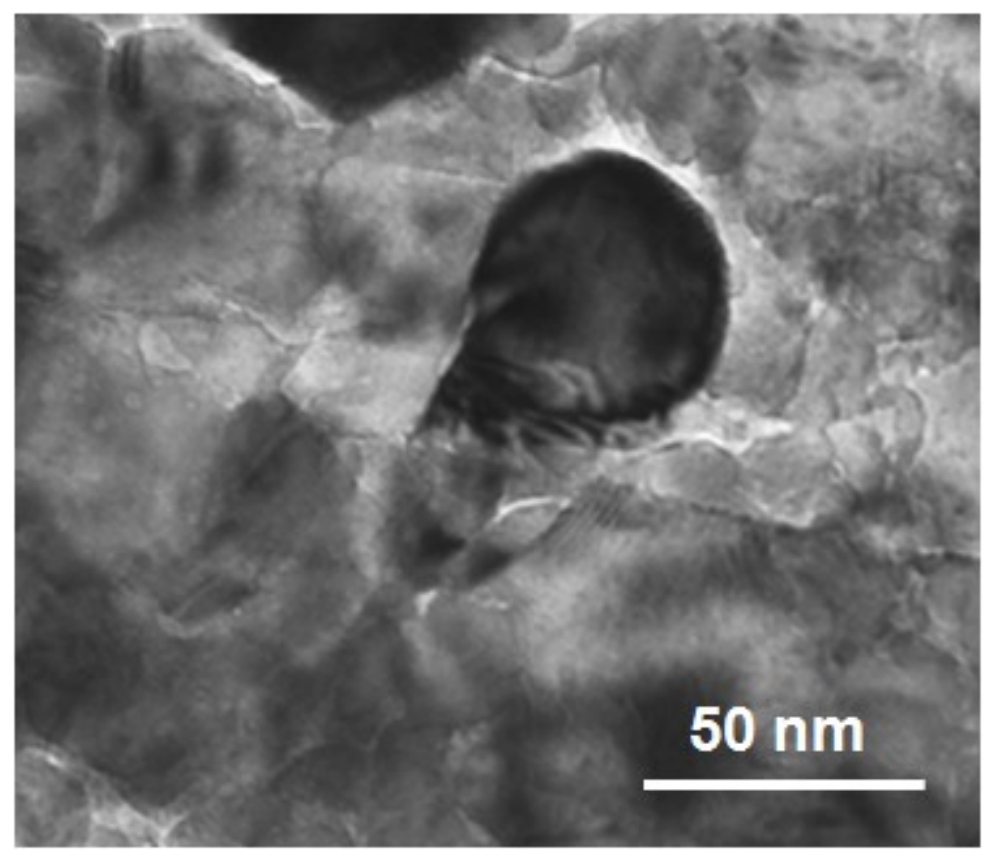

Figure 151: TEM micrograph of a $\mathrm{Zr}(\mathrm{Nb}, \mathrm{Fe})_{2}$ precipitate within the oxide formed on 1 day corroded Zr-Nb-Ti, $1.5 \mu \mathrm{m}$ from the metal-oxide interface. Courtesy of Na Ni, Oxford Materials. 


\subsubsection{Summary}

These electron microscopy observations are summarised as follows:

- Oxidation occurs at a rate of $70 \mathrm{~nm}$ per minute.

- The oxide appears white.

- The oxide has a fine, equiaxed structure right up to the metal interface. The grains have a diameter of a few tens of nanometres.

- Cracking is seen parallel to the interface with a period of submicron periodicity.

- There is a greater concentration of alloy element additions within the metal than the oxide, although the ratio of solute/zirconium is not well described.

- Second phase particles do not appear to be rapidly dissolved within the oxide.

These observations, along with the characterisation of the base material described in sections 3.4.1.4 and 5.2 provide an excellent framework within which to consider the 3DAP analysis of the metal oxide interface of this corroded material. This is described in the following sections, 6.4.2 and 6.4.3. 


\subsubsection{Metal-oxide interface: $3 D A P$}

\subsubsection{Oxygen}

Two 2D oxygen concentration profiles of highly corroded Zr-Nb-Ti material are shown in Figure 152. There is a sharp interface between $\mathrm{Zr}$ metal and $\mathrm{ZrO}_{2}$ oxide. There is also a low level of oxygen in solid solution in region of the metal adjacent to the interface. The rate of oxide growth is very rapid, so it is unsurprising that a $\mathrm{ZrO}$ layer, which has previously been seen to develop in other materials during the slow growth phases of the oxidation process, is absent. The formation of new $\mathrm{ZrO}_{2}$ oxide is evidently more rapid than the diffusion of oxygen from the interface into the bulk matrix.
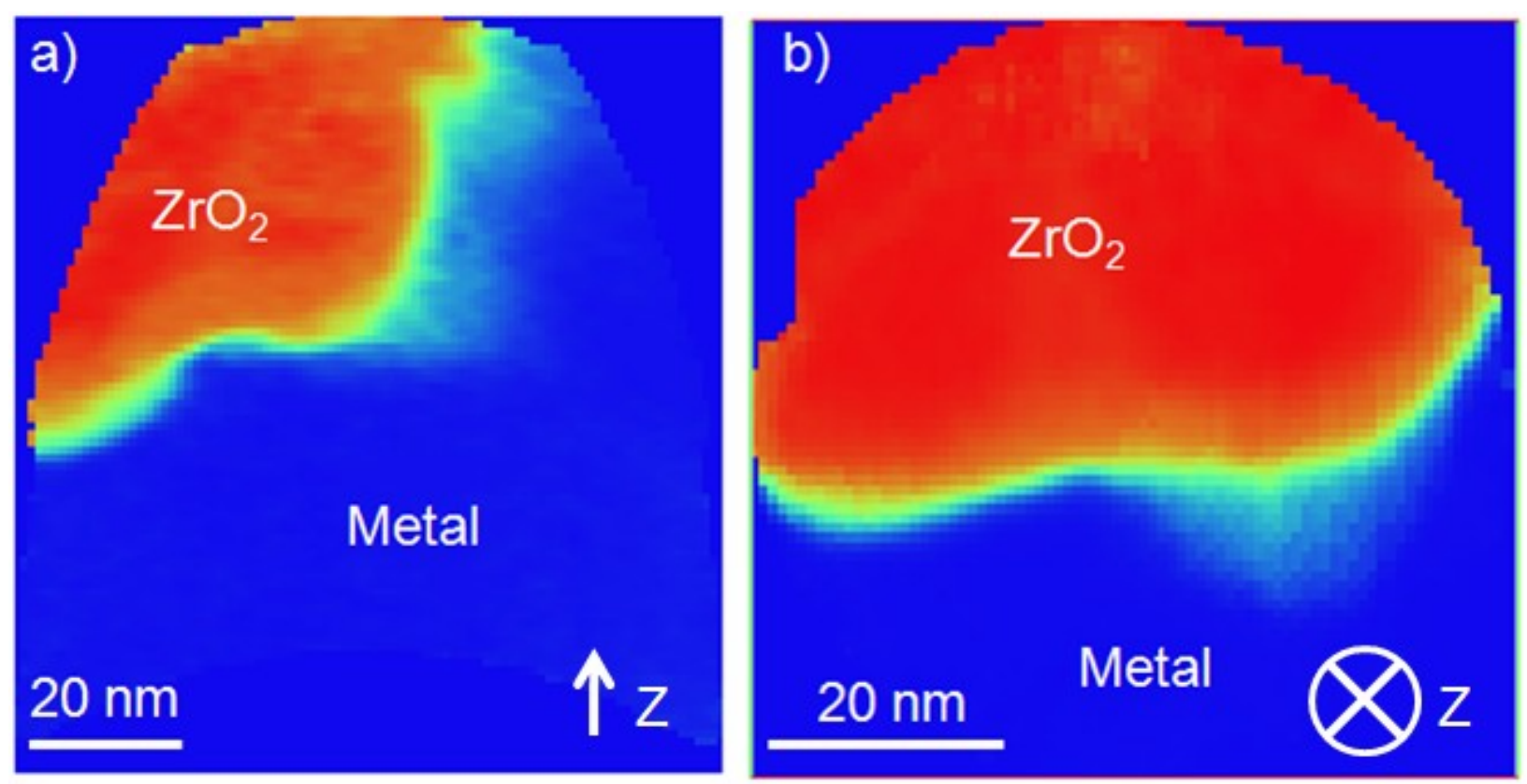

Figure 152: 2D Oxygen concentration profiles across the metal oxide interface of two different specimens prepared from 1 day corroded $\mathrm{Zr}$-Nb-Ti. Profiles are orientated so that the profile plane is co-linear with the specimen axis (a) and normal to the axis (b). 
a)

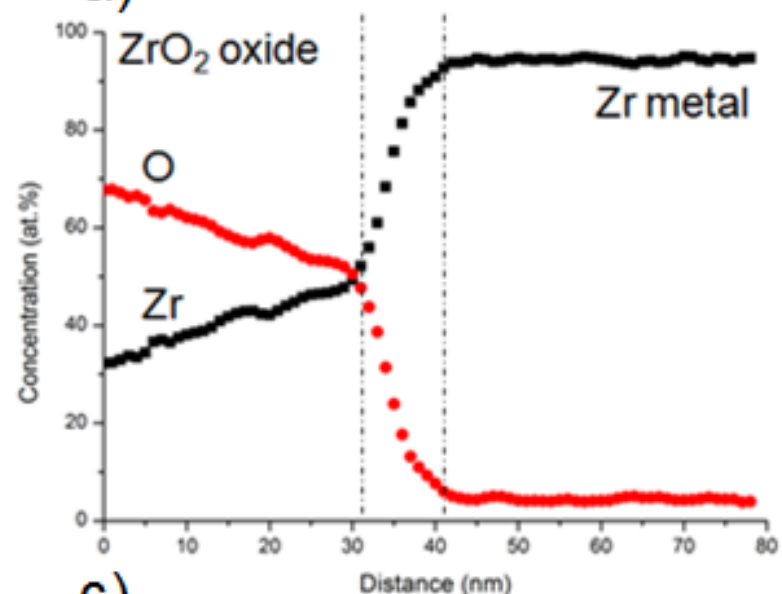

c)

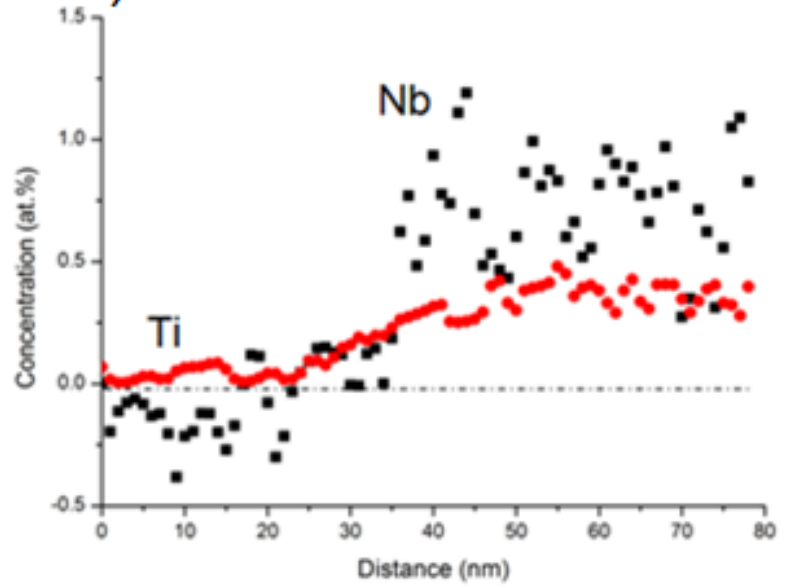

b)

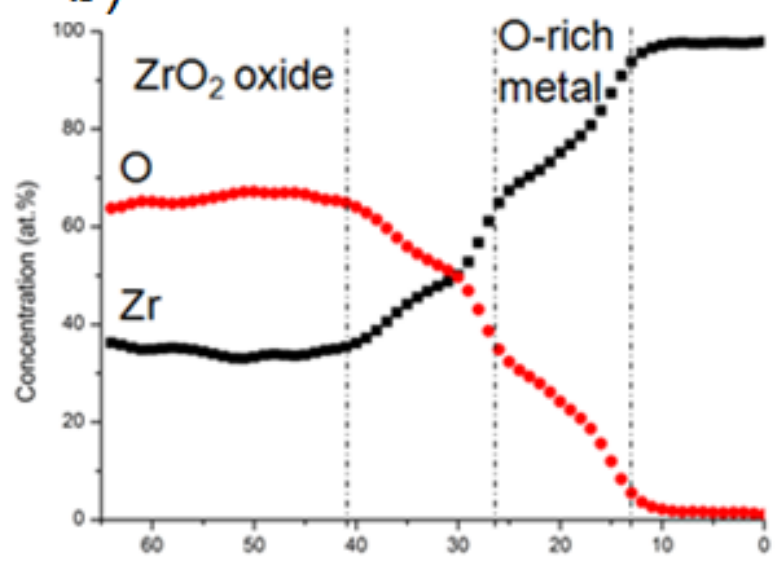

d)

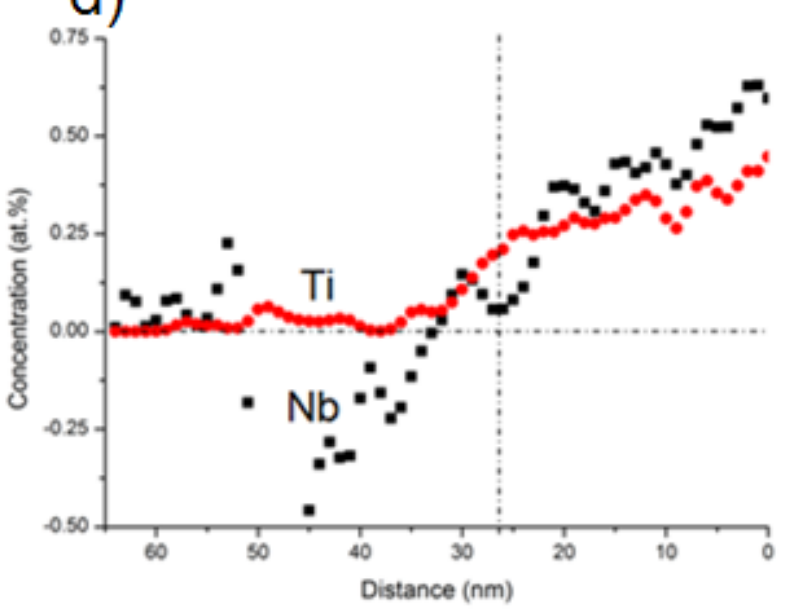

Figure 153: Profiles showing the oxide/metal interface across two specimens $(a, c$ and $b, d)$ prepared from 1 day corroded $\mathrm{Zr}$-Nb-Ti. Specimen $(a, c)$ shows a larger region of metal adjacent to the interface. Specimen $(b, d)$ has a larger region of oxide demonstrating approximately the expected stoichiometry for $\mathrm{ZrO}_{2} . \mathrm{Zr}$ and $\mathrm{O}$ profiles shown in (a) and (b), $\mathrm{Nb}$ and Ti profiles displayed by (c) and (d). The Nb concentration is calculated by deducting $\mathrm{ZrH}$ and overlapping species and noise from the $\mathrm{Nb}$ profile.

1D profile across the interfaces shown in Figure 152 is presented in Figure 153. The oxygen profile within the oxide of Figure 153 (a) deviates from the expected stoichiometry. A large amount of hydrogen (not included in the profile) was field evaporated from these specimens, mainly as multiple hit detector events. The desorption of this species could have affected the proportion of other species lost in detector dead time. The source of this hydrogen is not clear, 
but could have been induced by the focussed ion beam milling process used to fabricate these tips, although this problem has not be encountered during other similar analyses. The oxygen concentration in the oxygen-enriched matrix region adjacent to the interface decays to near the background level of oxygen in the material within a few nanometres.

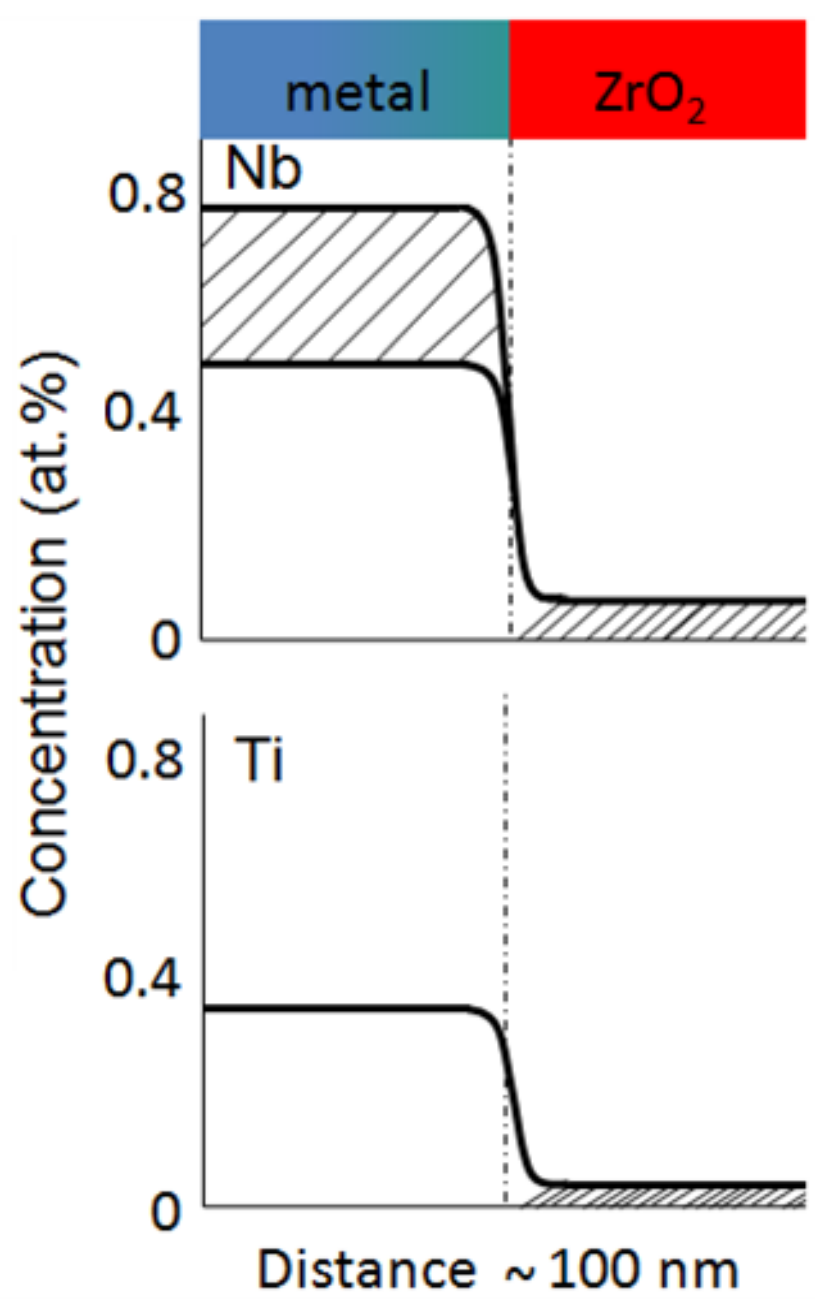

Figure 154: Idealised concentration profile based on the metal-oxide interfaces of 1 day corroded Zr-Nb-Ti described in Figure 153. Shaded regions show a range of possible values with limits defined by thick lines. Both niobium and titanium profile are displayed on a linear scale. 


\subsubsection{Niobium}

The niobium profile across the metal-oxide interface of these examples is also shown in Figure 153 (b). Hydrogen was also field evaporated combined with zirconium ions as $\mathrm{ZrH}$ and $\mathrm{ZrH}_{2}$ ionic species. A relatively large amount of $\mathrm{ZrH}$ ions were seen in both these datasets. This makes a quantitative analysis of the niobium content of these specimens difficult as $\mathrm{Nb}^{2+}, \mathrm{ZrH}^{2+}$ and $\mathrm{ZrH}_{2}{ }^{2+}$ are all found at $46.5 \mathrm{Da}$, and this is reflected in the scatter of datapoints for $\mathrm{Nb}$ seen in Figure 153.

The niobium concentration undergoes a step change at the interface. The level of niobium seen in the metal ahead of the interface, $0.4-0.8$ at.\%, is higher than the range seen in uncorroded material, $(0.43 \pm 0.04)$ at.\%, although perhaps not significantly so. As niobium is apparently rejected from the oxide, the niobium concentration in the metal immediately ahead of the interface would be expected to be larger than in the base material. However, the rate of oxidation is much greater than the rate of diffusion of niobium, and so niobium rejection at the metal-oxide interface cannot happen. Other sinks exist for this excess niobium. Both the metal and oxide grains are very fine, with diameters of $250 \mathrm{~nm}$ and $\sim 50 \mathrm{~nm}$ respectively, creating a relatively large volume of grain boundary which would be energetically favourable for niobium to occupy.

\subsubsection{Titanium}

At high temperatures, titanium additions to $\mathrm{ZrO}_{2}$ acts as a partial tetragonal phase stabiliser [184]. Titanium is not as effective as a phase stabiliser as yttrium, cerium or calcium, but significant additions (several percent) have been seen to stabilise the tetragonal phase down to $650{ }^{\circ} \mathrm{C}$. However, the required concentration of titanium and the lower temperature found in the autoclave do not provide the conditions required for titanium to stabilise $\mathrm{t}-\mathrm{ZrO}_{2}$ in this system. On the contrary, the solubility of $\mathrm{Ti}$ in $\mathrm{m}-\mathrm{ZrO}_{2}$, the stable phase in these conditions, is 
vanishingly small and a solid solution of $\mathrm{m}-\mathrm{ZrO}_{2}$ and $\mathrm{ZrTiO}_{4}$ would be expected [185]. This thermodynamic consideration explains why no titanium is seen in the oxide at the metal-oxide interface.

Like niobium, the titanium profile ahead of the metal-oxide interface also suggests that it is rejected from the fast progressing oxide front. Also like niobium, the level ahead of the interface is not dissimilar to the level seen in the matrix of uncorroded material, and like niobium, the diffusion of titanium in alpha-zirconium is sluggish. In order to explain these observations of the interface, it is suggested that titanium is also rejected to grain boundaries within the oxide and metal in the region of the metal-oxide interface in a similar manner postulated for niobium.

\subsubsection{Iron, chromium and nickel}

The distribution of minor transition metals at the interface cannot be determined in this case as in all of the datasets available there is substantial interference from impurity species. Figure 155 is an example section of such a mass spectrum. The spatial distribution of the peaks at 27 and 28 Da do not agree, and no peak is visible at $28.5 \mathrm{Da}$, suggesting that these counts are not due to iron. The ratios of 27, 28 and $29 \mathrm{Da}$ were also different in the separate specimens.

The peak at $29 \mathrm{Da}$ is also probably not due to nickel as it is seen only at the surface layer at the start of the data acquisition and is seen both on the surface of the metal and oxide phases. This would suggest that this is due to an adsorbed surface contaminant that has not previously been seen during the atom probe analysis of ZIRLO or Zircaloy-4. 


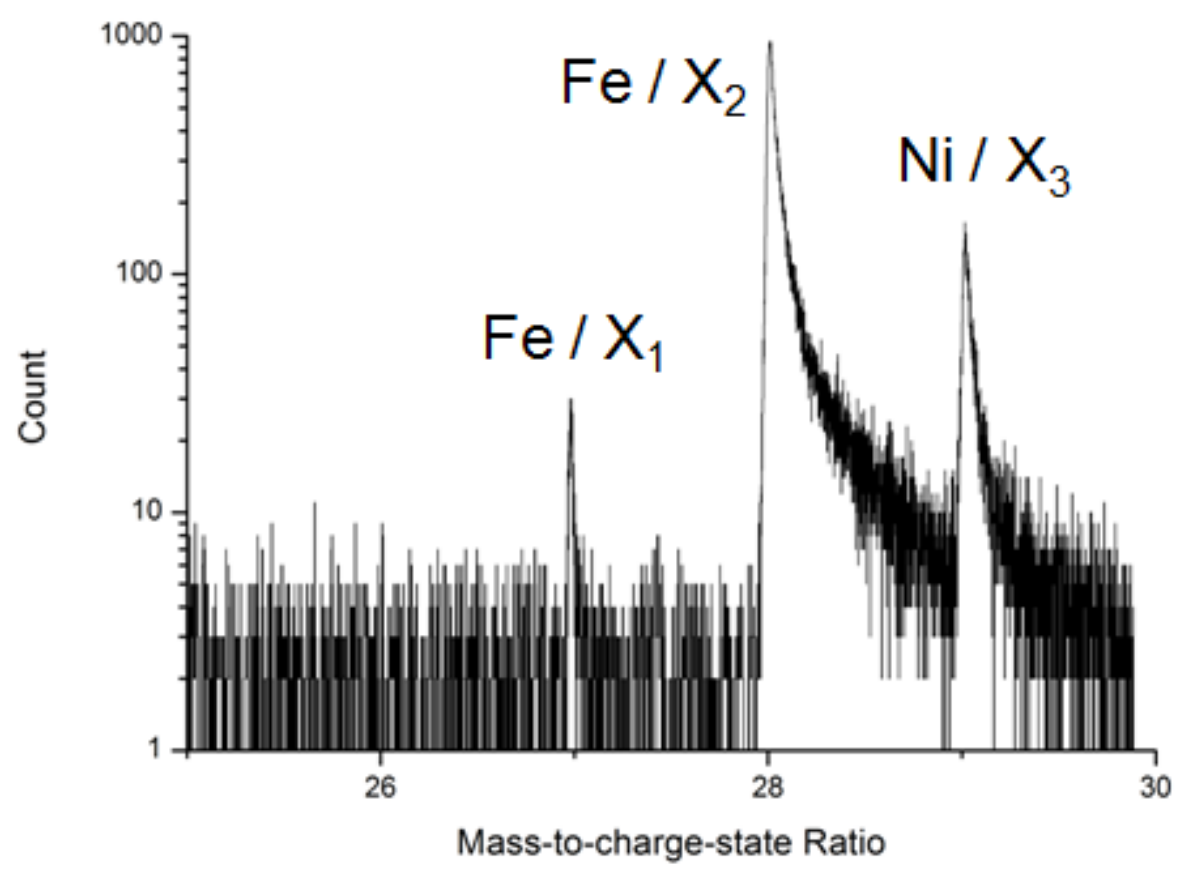

Figure 155: The fourth period transition metal $(2+$ charge state) section of the Mass-to-chargestate ratio spectra for one of the datasets shown in Figure 152 (1 day corroded Zr-Nb-Ti).

\subsubsection{Material adjacent to interface: $3 D A P$}

A section of alloy matrix was prepared from material adjacent to the metal-oxide interface. This region is shown in Figure 156. The metal-oxide interface was situated $200-500 \mathrm{~nm}$ above the top of the dataset in Figure 156. This region, although in very close proximity to the interface, can be expected to show the signs of corrosion that were seen in corroded ZIRLO.

$\mathrm{Nb}, \mathrm{Ti}, \mathrm{Fe}$ and $\mathrm{O}$ are all enriched at this grain boundary. The relative segregation is given in Figure 58 and also presented graphically by the cumulative concentration profile Figure 157. 


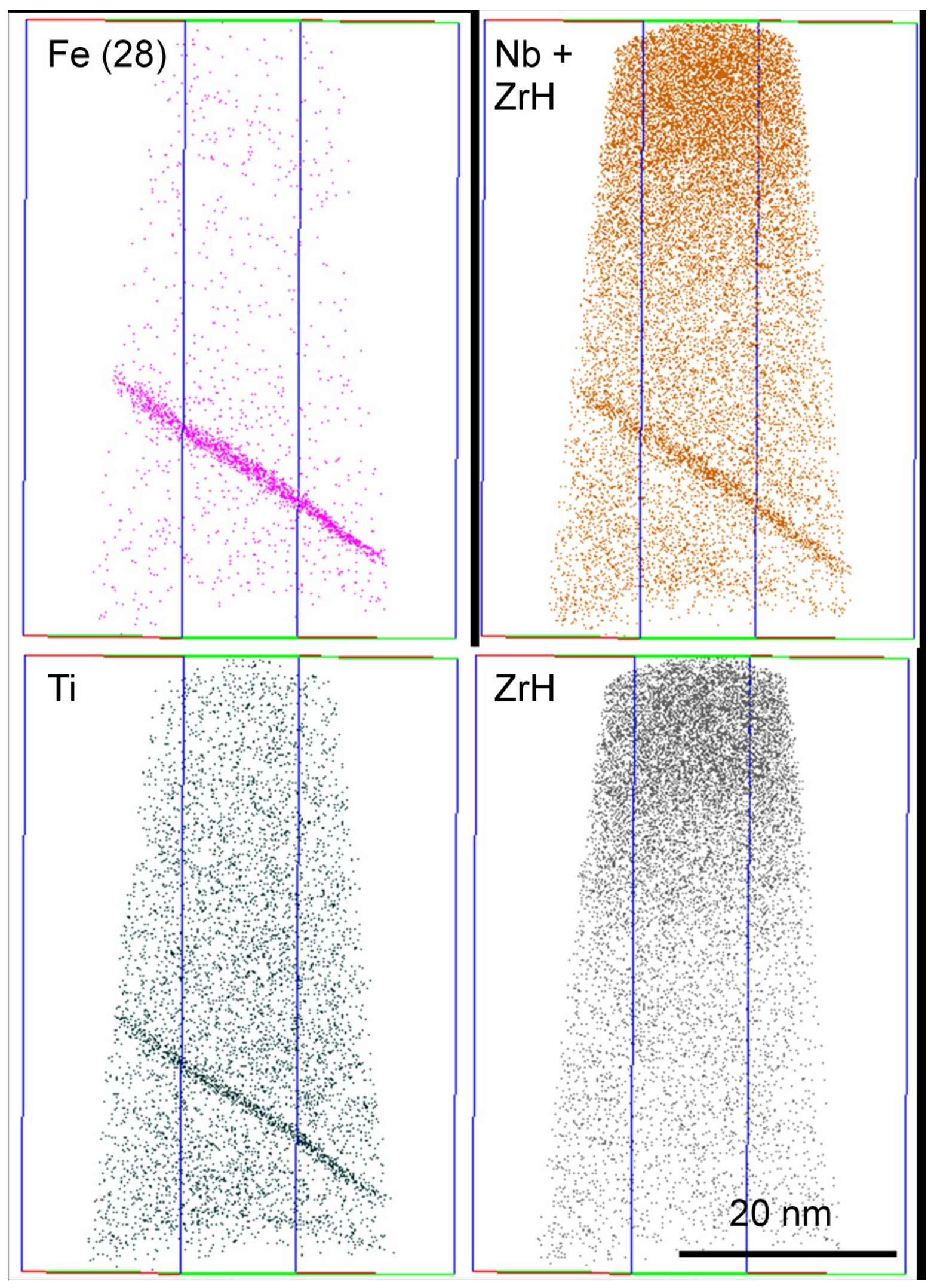

Figure 156: 3D atom maps showing the distribution of iron (at $28 \mathrm{Da}$ ), $\mathrm{Nb}+\mathrm{ZrH}$ (at $46.5 \mathrm{Da}$ ) and a similar amount of $\mathrm{ZrH}$ (at $47.5 \mathrm{Da}$ ) and titanium of a region including a grain boundary in the material adjacent to $(200-500 \mathrm{~nm})$ the metal-oxide interface of 1 day corroded $\mathrm{Zr}-\mathrm{Nb}-\mathrm{Ti}$. 


\begin{tabular}{|c|c|c|c|c|c|c|c|}
\hline \multirow[t]{2}{*}{ Element } & \multicolumn{3}{|c|}{ Lower matrix grain } & Boundary & \multicolumn{3}{|c|}{ Upper matrix grain } \\
\hline & $\begin{array}{c}\text { No. } \\
\text { (atoms) }\end{array}$ & $\begin{array}{l}\text { Conc. } \\
\text { (at.\%) }\end{array}$ & $\begin{array}{l} \pm 1 \sigma \\
\text { (at.\%) }\end{array}$ & $\begin{array}{l}\text { Conc. } \\
\text { (at.\%) }\end{array}$ & $\begin{array}{c}\text { No. } \\
\text { (atoms) }\end{array}$ & $\begin{array}{l}\text { Conc. } \\
\text { (at.\%) }\end{array}$ & $\begin{array}{c}\sigma \\
(\text { at. \%) }\end{array}$ \\
\hline $\mathrm{Zr}$ & 395,523 & 98.2 & 0.2 & 92.77 & 420,906 & 98.3 & 0.2 \\
\hline $\mathrm{Nb}$ & 992 & 0.25 & 0.01 & 1.42 & 1,226 & 0.29 & 0.02 \\
\hline $\mathrm{Ti}$ & 1,213 & 0.30 & 0.01 & 1.25 & 1,504 & 0.35 & 0.01 \\
\hline $\mathrm{Fe}$ & 209 & 0.052 & 0.004 & 1.79 & 153 & 0.036 & 0.003 \\
\hline 0 & 4,760 & 1.18 & 0.02 & 2.45 & 4,574 & 1.07 & 0.02 \\
\hline C & 75 & 0.019 & 0.002 & 0.01 & 50 & 0.012 & 0.002 \\
\hline
\end{tabular}

Table 64: Compositional information for the 'lower' and 'upper' matrix regions with reference to the grain boundary in Figure 156 (1 day corroded $\mathrm{Zr}$-Nb-Ti). The composition at the boundary is calculated using the gradient at the boundary.

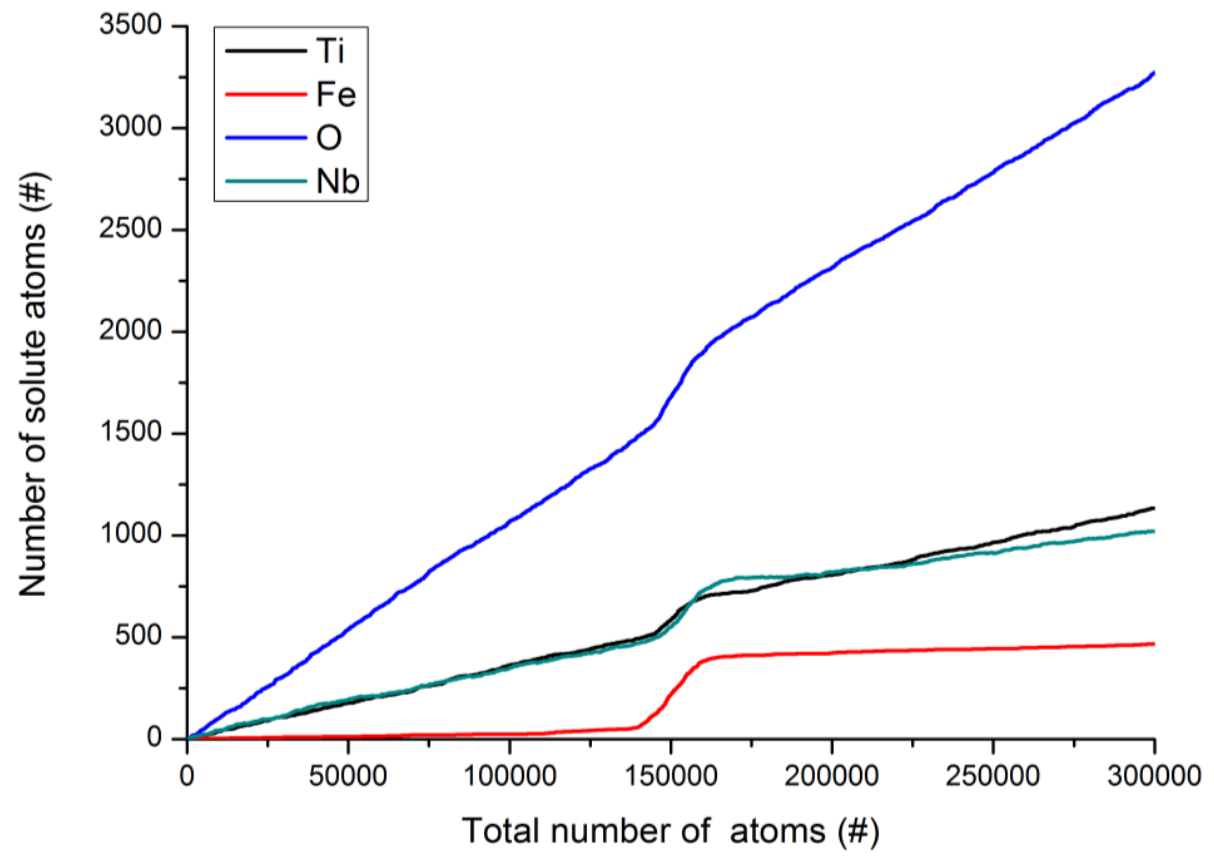

Figure 157: Cumulative concentration profile across a grain boundary in a corroded region of metal matrix adjacent to the metal-oxide interface of 1 day corroded $\mathrm{Zr}$-Nb-Ti. 


\subsubsection{Oxygen}

The oxygen concentration within the grains is the same as that seen in uncorroded material. This is unsurprising as the observations of the interface do not show enhanced oxygen concentration within the metal ahead of the interface.

The level of oxygen at this grain boundary is higher than the range of values seen at the grain boundaries in un-corroded $\mathrm{Zr}-\mathrm{Nb}$-Ti or ZIRLO. This suggests that oxygen is transported along grain boundary paths ahead of the oxide front during the corrosion process. This observation also suggests that the rapid growth of the oxide occurs as a broad front of the progressing oxide/metal interface, rather than oxidation via metallic grain boundaries. This boundary is less than half a micron from the metal-oxide interface it would be consumed by the advancing oxide within ten minutes at $360{ }^{\circ} \mathrm{C}$. If oxidation proceeded from grain boundary sites it would be expected that the morphology of this site would be markedly different.

\subsubsection{Niobium}

The excess niobium at the grain boundary is not dissimilar to that seen at the "high energy" grain boundary seen in ZIRLO. Compositional information regarding the grains adjacent to the boundary is given in Table 64 . Niobium diffuses too slowly within the metal matrix to be segregated in this region due to rejection of niobium at the metal-oxide interface. Indeed, the niobium concentration in both of these grains is towards the lower limit of the level seen in solution. Niobium precipitation was not seen in this sample or in observations of the metal-oxide interface. Precipitation would not be expected in the matrix of this material as it has only been subjected to aging at $360{ }^{\circ} \mathrm{C}$ for 1 day, rather than the $\sim 100$ days where it was seen in ZIRLO. 


\subsubsection{Titanium}

Titanium is also not seen in enhanced quantities in the metal matrix; titanium has a similar diffusion coefficient to niobium in $\alpha-\operatorname{Zr}[186]$ and so cannot migrate through the bulk from the metal-oxide interface in the time available. However, the enrichment of solute at this grain boundary is likely to be enhanced by the corrosion processes occurring at the metal oxide interface. Titanium is completely soluble within the $\alpha$-Zr matrix, and no segregation was seen in the uncorroded grain boundary example in section 5.2.2.4. Although the additional niobium present could be explained by variation in boundary geometry it is unlikely that this would create the conditions required for the segregation of titanium. Metallic grain boundary diffusion of this solute from the interface along the network of fine metal grains (see Figure 28 in section 3.4.1.4) would allow an alternative route for the removal of this solute, as well as avenue of segregation to the fine oxide/oxide grain boundaries, which were characterised by TEM in section 3.4.1.4.

\subsubsection{Iron}

The level of iron seen within these grains is towards the upper end of the scale seen in the asreceived material, but still within that range. The segregation of iron to the boundary is similar to the range seen in ZIRLO. The 'high' matrix concentration $\sim 0.04$ at.\% reduces the enrichment factor at this boundary to $\sim 40$. This is a similar enrichment factor as that seen in the uncorroded Zr-Nb-Ti example. 


\subsection{Summary}

Metal-oxide interfaces and the surrounding regions of matrix material have been investigated for a variety of alloys in several corrosion conditions. The role of oxygen in creating an interface with graded mechanical properties, and the development of a $\mathrm{ZrO}$ sub-oxide phase has been demonstrated for alloys with good corrosion resistance, and not observed in the post-breakaway Zr-Nb-Ti alloy. The development of these interfaces, and the redistribution of alloying additions during the corrosion process is summarised in detail in section 7.4 of the conclusions, chapter 7 . 


\section{Summary and conclusions}

\subsection{Overview of the contribution of this work}

A systematic comparison of the nanoscale chemistry of the metal-oxide interface of ZIRLO at different stages in the corrosion cycle both pre- and post-transition has been conducted. Exhaustive analysis of the alloy matrix of un-corroded ZIRLO provides a benchmark to describe the chemical distribution of solute on a nanoscopic scale before corrosion. The formation and consumption of an intermediate oxide layer, $\mathrm{ZrO}$, has been characterised and its impact on the redistribution of solute elucidated. Possible correlation between solute distributions within the matrix has also been examined. In corroded material the local iron concentration is a function of the matrix oxygen concentration. There is also interplay between niobium and high oxygen concentration regions of the matrix in corroded material. The precipitation of nanoscale niobiumrich precipitates near the metal-oxide interface has been seen in ZIRLO aged at reactor temperatures for 100 days, and this is taken to be due to the presence of a high level of oxygen and a high dislocation density in this region. This offers a mechanism for additional precipitation hardening in ZIRLO during corrosion, and also appears to alter the geometry of the $\mathrm{ZrO}$ interfacial layer due to pinning.

The behaviour of Zircaloy-4 metal-oxide interface chemistry has been compared to that of ZIRLO, and many similarities are found. The differences in chemical behaviour between the systems are attributable to the absence of niobium.

An alloy with remarkably bad corrosion resistance ( $\mathrm{Zr}-\mathrm{Nb}-\mathrm{Ti})$ has been characterised, using several techniques, both before and after corrosion. This characterisation explains the behaviour of solute during corrosion, and the observed structure of the oxide agrees well with the literature 
view of corrosion resistance, yet a firm conclusion as to the cause of the enhanced corrosion has not been provided by his work.

\subsection{Preliminary experiments}

\subsubsection{Native oxides $(\$ 4.3)$}

- Analysis of zirconium-based atom probe specimens using LAR-3DAP confirmed the presence of a native oxide film with the composition $\mathrm{ZrO}$, previously reported by Wadman and Andrén [157].

- The same layer was formed irrespective of the specimen fabrication method (electropolishing or FIB) or the environment to which the specimen was exposed $\left(150{ }^{\circ} \mathrm{C}\right.$ air, $100{ }^{\circ} \mathrm{C}$ water, ambient electrochemical solution).

- The early stage oxidation kinetics of the formation of this layer was studied by repeatedly oxidising and analysing the same sample. After an initial discontinuous (immediate) formation of a surface oxide layer of a few monolayers of $\mathrm{ZrO}$, the growth kinetics in air at ambient temperature were well characterised by a linear fit, suggesting that at this stage the $\mathrm{ZrO}$ layer is non-protective or that at this stage the rate of oxide growth is not dependent upon the oxide thickness. 


\subsubsection{Hydrides induced by cathodic charging (\$ 4.4)}

○ A very limited-scope analysis of meta-stable hydrides in zirconium alloys was reported. Voltage-pulsed 3DAP analysis of nanoscale hydride platelets formed during electropolishing is possible, and although these specimens were much more prone to fracture than those analysed using laser-pulsing, the limited mass spectra that are produced may be worth the loss of data volume in future studies of these materials.

- About $10 \%$ of zirconium ions were detected as $\mathrm{ZrH}$ ions in metal matrix regions of laserpulsed datasets. This species is not seen in metal matrix regions of voltage pulsed data.

- Hydrogen in the form of $\mathrm{H}$ and $\mathrm{H}_{2}$ ions was more prone to trajectory aberration artefacts at the boundary of $\mathrm{ZrH}$ hydrides studied by 3DAP than $\mathrm{ZrH}$ ions. This is because of their small mass, and causes 2-5 $\mathrm{nm}$ of hydrogen 'blurring' at the $\mathrm{Zr} / \mathrm{ZrH}$ boundary. The enhanced surface diffusion of hydrogen also increases the apparent area from which these species were detected.

- A $\mathrm{ZrH}$ hydride 'fingerprint' of the $\mathrm{Zr} / \mathrm{ZrH}$ ratio was found to be the same for commercially pure zirconium analysed using voltage pulsed LAR-3DAP and a ZIRLO specimen analysed by laser-pulsing LEAP.

\subsubsection{Laser-pulsed 3DAP analysis conditions (\$ 4.6)}

- Laser-pulsing of 3DAP at too high an energy density causes the formation of complex ionic species, as well as non-uniform evaporation from the tip [162]. The relative concentration of these species is negligible below a threshold energy, and then increases at an apparently linear rate with increasing laser energy.

- Suitable conditions for analysing zirconium based 3DAP specimens with a low shank angle $\left(1-3^{\circ}\right)$ and $80 \mathrm{~nm}$ nominal radius were obtained by experiment. These conditions are $50-60$ K specimen temperature and $(0.35 \pm 5) \mathrm{nJ}$ laser-pulse energy for a $10 \mu \mathrm{m}$ spot. 


\subsection{Nanoscale chemistry of the 'as-received' material}

\subsubsection{Alloy matrix chemistry (\$ 5.1)}

- 3DAP has been used to give a precise description of the matrix alloy chemistry for Zircaloy4, ZIRLO and Zr-Nb-Ti. This is summarised in Table 43, section 5.1.8.

$\circ$ The behaviour of niobium, tin and oxygen was found to be similar between the alloys. Iron, which was present in greater concentrations intragranularly than chromium and nickel, was seen to behave differently in the niobium containing alloys ZIRLO and Zr-Nb-Ti compared to Zircaloy-4. The iron concentration in Zircaloy-4 was seen at the level of 0.003 at. $\%$ in all the specimens examined. In ZIRLO, some specimens contained this level of iron, while others were seen to have an enhanced level, around 4 times this value. The range of local iron concentration seen in $\mathrm{Zr}-\mathrm{Nb}$-Ti material appears greater still, ranging between $0.003-0.04$ at. $\%$

- The concentrations of niobium and oxygen were seen to vary between different samples, although there was no correlation between the $\mathrm{Nb}$ and $\mathrm{O}$ concentration on either a macroscopic or nanoscopic level.

- Nanoscale niobium precipitation or intragranular segregation of iron was not observed in any of the materials studied. This is consistent with the expected volume fractions of such features. 


\subsubsection{Second phase particles (\$ 5.2.1)}

○ 3DAP was used to study the nanoscale chemistry around SPPs in as-received ZIRLO material. These results are consistent with previous TEM studies [75, 79], and show no detectable interfacial segregation of solute.

$\circ$ TEM , EDX and 3DAP characterisation of the uncorroded Zr-Nb-TI test alloy was conducted (EM by Na Ni, Oxford Materials). 2 particles were studied. The superior chemical sensitivity of 3DAP to EDX provides a more precise description of the minor solute content.

○ The majority of the $\mathrm{Zr}-\mathrm{Nb}$-Ti SPP sampled in this study are of the $\mathrm{Zr}(\mathrm{Nb}, \mathrm{Fe})_{2}$ type. $\mathrm{A} \mathrm{ZrNb}_{2}$ example studied by 3DAP (partially oxidise by the specimen environment) demonstrated iron segregation at the surface of the SPP.

\subsubsection{Grain boundaries (\$ 5.2.2)}

The chemistry of three grain boundaries within uncorroded material was examined, two from ZIRLO, and one from $\mathrm{Zr}-\mathrm{Nb}$-Ti. These are believed to be the first such direct observations of the solute segregation in zirconium-based alloys.

O This provides the basis for future comparison with boundaries in corroded material.

Iron was seen to be present in excess at the boundaries of all the examples, and the excess atoms per unit area were seen to vary by a factor of two between the two ZIRLO specimens. The relative excess of niobium changed considerable between these two samples, with a large excess of niobium (boundary of 3-4 times matrix concentration) seen at the specimen that contained a higher quantity of iron at the boundary (hundreds of times the matrix concentration), whereas the boundary with less iron ( $\sim 20$ times enrichment factor) was deficient in niobium (similar to matrix concentration). 
The segregation behaviour of substitutional solutes correlates with the degree of atomic radius mismatch with zirconium.

O Niobium enrichment to a grain boundary was observed in $\mathrm{Zr}-\mathrm{Nb}$-Ti alloy. The level of enrichment was approximately twice that seen in the matrix. Iron segregation in this alloy was similar to that seen in ZIRLO. Titanium was not found to be enriched at the interface. Titanium, like tin in ZIRLO, is highly soluble in alpha zirconium and so there is a small driving force for precipitation.

$\bigcirc$ The segregation of solute could occur during the $\beta \rightarrow \alpha$ transition, or during subsequent cold work and annealing. It is unclear if the $\beta \rightarrow \alpha$ treatment caused the variation in observed enrichment behaviour at the interface, or whether this is due to the variation in interfacial energy leading to differing behaviour during subsequent processing.

\subsection{Nanoscale chemical redistribution during corrosion}

\subsubsection{ZIRLO (\$ 6.2)}

Z ZIRLO was examined at various stages in the corrosion cycle, three pre-transition and one post-transition.

O The $\mathrm{Sn} / \mathrm{Zr}$ ratio remained constant in all of the phases present in all of the corrosion conditions examined; tin was not seen to redistribute during the corrosion process.

O The solubility of tin in the oxide is greater than the concentration found in this alloy. Other studies have shown that tin is soluble in $\mathrm{ZrO}_{2}$ up to several atomic percent at $1050{ }^{\circ} \mathrm{C}$, and is more soluble at this temperature than at $1300{ }^{\circ} \mathrm{C}$ [187]. Lower temperature data are not available. 


\section{Pre-transition}

- Niobium was rejected at the metal-oxide interface of early pre-transition material, and was seen as a supersaturated solid solution in the metal adjacent to the interface.

O During pre-transition oxidation a $\mathrm{ZrO}$ layer developed at the interface between the metal and $\mathrm{ZrO}_{2}$ oxide. Growth of this layer occurred during a period of slow overall oxide thickening, suggesting that the low partial pressure of oxygen at the interface favours the formation of the intermediate phase.

O In some cases, this $\mathrm{ZrO}$ layer may be described as having cation deficient (ZrO') and cation surplus sub-layers (ZrO”).

- After further pre-transition corrosion, niobium in the highly-stressed, oxygen-enriched layer of metal adjacent to the interface precipitates out of solution, forming nanoscale particles. These particles appear to dissolve in the $\mathrm{ZrO}$ oxide in the cation enriched $\mathrm{ZrO}$ ', layer next to the $\mathrm{ZrO}_{2}$ oxide. The $\mathrm{ZrO}_{2}$ layer formed from this material was seen to contain more niobium than was the case in the $\mathrm{ZrO}_{2}$ oxide formed directly from the alloy matrix.

○ These precipitates were also seen to form preferentially at grain boundaries, but were not observed in the bulk matrix of corroded materials.

\section{Post-transition}

- After the transition in corrosion kinetics the $\mathrm{ZrO}$ layer is consumed.

- The post-transition interface morphology was similar to the early pre-transition case, with the exception that oxygen is seen at a high level in the alloy matrix adjacent to the interface in all but the earliest pre-transition specimens.

- The behaviour of niobium at the interface was again seen to be similar to the early pre-transition case (before the development of the $\mathrm{ZrO}$ layer).

- The solubility of niobium in zirconia at reactor temperatures was not previously know, although high temperature studies suggest that the solubility in $\mathrm{t}-\mathrm{ZrO}_{2}$ is vanishingly low. 
This work supports this observation, as little to no niobium was seen in solution in the oxide during the stages of corrosion where the tetragonal volume fraction is highest.

- Iron was seen as a supersaturated solid solution in the alloy matrix adjacent to the metal oxide interface in all of the corrosion conditions studied.

- The iron concentration within the metal matrix appeared to be correlated with the oxygen concentration, suggesting that the disordered lattice created by the addition of large amounts of oxygen reduces the strain of accommodating iron within the metal matrix.

- Iron was also accommodated at dislocations generated by the stress field at the metal-oxide interface. The iron concentration at the core of these features was similar to that seen in Zircaloy-4, where more details analysis of data was possible.

- Iron was rejected from the formation of oxide at the metal-oxide interface in all the corrosion conditions studied.

\subsubsection{Zircaloy-4 (\$ 6.3)}

- Pre-transition Zircaloy-4 material was examined after 7 and 90 days of corrosion. Many of the observed chemical properties of the interface were similar to those seen in pre-transition ZIRLO.

- $\mathrm{A} \mathrm{Zr}-\mathrm{ZrO}_{2}$ interface was seen in the 7 day corroded material, as was seen in early pretransition ZIRLO (after 34 days).

$\circ$ The level of oxygen in the metal matrix in the 7 day material was at a solid solution saturation level, which differs from the level seen in ZIRLO. This finding is based on only one observation, so it is possible that it is due to localised differences that might occur along the metal-oxide interface. It is also possible that the addition of niobium in ZIRLO retards the solution of oxygen in the zirconium-based metal matrix, as would be expected from the 
$\mathrm{Zr}-\mathrm{Nb}-\mathrm{O}$ phase diagram, and that this observation in the niobium-free system is the result of normal oxygen injection ahead of the interface in a $\mathrm{Zr}-\mathrm{Sn}$ alloy.

$\circ$ The oxygen was also present as a saturated solid solution at the metal-oxide interface of the further corroded (90 day) pre-transition Zircaloy-4.

- The 90 day corroded material had a $\mathrm{ZrO}$ layer of the order of several tens of nanometres thickness. Less variation was seen in the thickness, or the complexity of geometry of this interface compared to that observed in the ZIRLO material at a similar stage in the corrosion process. This could be due to the removal of the retarding effect of niobium-rich precipitates on the advancement of the $\mathrm{ZrO}$ layer into the metal.

- As in the case of ZIRLO, the tin present in solid solution in the uncorroded metal matrix was not redistributed at the metal-oxide interface of either of the corrosion conditions examined. $\mathrm{The} \mathrm{Sn} / \mathrm{Zr}$ ratio remains constant in the metal and oxide, and the measured value agrees well with the expected value.

- Iron was seen to be heavily segregated to dislocations formed in the stress field in the metal adjacent to the interface. The majority of the iron-decorated dislocations are aligned parallel to the metal-oxide interface, normal to the oxygen concentration gradient. The iron concentration at the core of the dislocations was seen to be $2-3$ at. $\%$.

- The density of iron-decorated dislocation at the interface is orders of magnitude greater than in the bulk metal matrix.

- Chromium and nickel were also seen clustered around point-like features, some of which iron was co-segregated to. The concentration of solute at the core of these clusters was seen to approach 20 at. $\%$.

$\circ$ The fourth period transition metal concentration in the oxygen-rich region ahead of the interface is also an order of magnitude greater than that seen in the bulk metal matrix. 
O It is suggested that a similar correlation between matrix iron concentration and oxygen concentration, as was observed in ZIRLO, is in operation in Zircaloy-4, and zirconium alloys in general.

\subsubsection{Zr-Nb-Ti (\$ 6.4)}

○ The very poorly corrosion resistant $\mathrm{Zr}-\mathrm{Nb}-\mathrm{Ti}$ alloy was examined by 3DAP after 1 day of corrosion in a $360{ }^{\circ} \mathrm{C}$ static autoclave (note: Parallel TEM observations by $\mathrm{Na} \mathrm{Ni}$ (Oxford) have shown this to be an extremely irregular oxide microstructure with a lack of columnar grains.)

- No ZrO layer is seen by 3DAP, which is consistent with the fast pace of the oxidation process. Oxygen is also not injected into solution ahead of the oxide front.

- Niobium and titanium are rejected from the oxide that forms at the metal-oxide interface.

- This solute must be conserved, and the material ahead of the interface does not appear to be enriched in $\mathrm{Nb}$ or $\mathrm{Ti}$. Also, the oxidation proceeds at such a rate $\sim 70 \mathrm{~nm} \cdot \mathrm{min}^{-1}$ that the diffusion of these species away from the interface into the bulk material is not possible.

O It is proposed that this additional solute is accommodated at grain boundaries, both metal/metal and oxide/oxide. The grain boundary volume in this material is very high, with fine grains in both the metal $(250 \mathrm{~nm} \times 40 \mathrm{~nm})$ and oxide (30 nm dia.).

- Both $\mathrm{Nb}$ and $\mathrm{Ti}$ are seen in excess in a metal/metal grain boundary adjacent to the metaloxide interface. This grain boundary chemistry is different to that seen in the uncorroded material where no titanium enrichment was observed.

- It is of particular note that, titanium, which is completely miscible in zirconium, is present in excess at this interface. This would not be expected in bulk material from a thermodynamic consideration, and suggests that additional titanium has been introduced to the boundary, supporting the observation of the behaviour of titanium at the metal-oxide interface. 


\subsubsection{Implications of these findings}

Oxygen was seen to be injected into the metal matrix ahead of the growing oxide. This will have two important effects on the properties of the material. Oxygen at the interface:

- increases the strength of layer next to interface by two mechanisms.

○ The most obvious of these is solid solution strengthening. This effect increases with time, as the oxygen-rich region ahead of the interface is seen increase in thickness with time. The high concentration $\mathrm{Zr}_{0.7}\left(\mathrm{O}_{0.3}\right)$ metal is likely a partially or fully ordered phase.

- For niobium-containing alloys, oxygen promotes niobium precipitation, and so produces precipitation strengthening. The density of the fine, nano-scale, niobium particles in the matrix adjacent to the interface changes by two orders of magnitude between uncorroded and advanced pre-transition ZIRLO (from an expected level of 0.1 per $100 \mathrm{~nm}^{3}$ [174], to an observed density of several particles per $100 \mathrm{~nm}^{3}$ ). The ability of niobium containing alloys to increase the density of these nano-scale particles in the critical region adjacent to the interface helps to explain why the most favourable corrosion resistance for these alloys is where the amount of niobium in solid solution is maximised [76].

- increases the solubility of iron in the metal matrix. This causes redistribution of iron from the bulk towards the metal-oxide interface, producing iron deficient matrix further away from the enriched interface. The matrix iron concentration has previously been correlated with corrosion resistance (less is worse $[1,188]$ ). As oxidation proceeds, this change in matrix chemistry will lead to an increase in the overall corrosion rate, which has been seen in many long term studies (the increase in the linear post transition rate). 
The formation and development of a $\mathrm{ZrO}$ sub-oxide layer during the pre-transition oxidation of commercial alloys in reactor conditions has given insight into a previously unknown phenomenon at work in these systems. The formation of $\mathrm{ZrO}$ layer is probably due to a change in the conditions at the interface during corrosion, as the protective oxide increases in thickness the oxygen partial pressure at the interface decreases, favouring the formation of the $\mathrm{ZrO}$ phase over $\mathrm{ZrO}_{2}$. Although the consumption of this layer after break away is likely an effect of the breakaway process, rather than its cause, the $\mathrm{ZrO}$ layer does have an effect on the redistribution of solute during the corrosion process of niobium containing alloys. The decomposition of metastable, nano-scale, niobium particles within the oxide increases the level of this solute seen in the $\mathrm{ZrO}_{2}$ layer adjacent to the interface. This process happens shortly before breakaway in ZIRLO.

The dislocation density generated at the metal-oxide interface of Zircaloy-4 and ZIRLO is very high. The dislocation density in the processed material before corrosion has not been characterised in this study, but is likely in the range $10^{10}-10^{12} \mathrm{~m}^{-2}(0.0001-0.01$ dislocations per $100 \mathrm{~nm}^{-2}$ ), which is why none are observed in the uncorroded material examined by 3DAP. The density calculated from Figure 141 (pre-transition Zircaloy-4) is of the order of $10^{15} \mathrm{~m}^{-2}$ locally. For comparison, other authors [189] have observed a dislocation density of $3 \times 10^{14} \mathrm{~m}^{-2}$ in E365 alloy subjected to irradiation of $20 \mathrm{dpa}$. In this context the increase in mechanical creep that would be expected in the material adjacent to the interface can be seen to be significant. The iron in solution pays a vital role in pinning these dislocations and maintaining the strength of the substrate. Niobium plays a similar role in the oxygen-enriched matrix for niobium-containing alloys in the more advanced pre-transition period. Dislocation-dislocation (effectively workhardening) interactions at such high dislocation densities must also be expected to increase the strength of the outer substrate.

The high solubility of tin in both zirconium and zirconium dioxide ensures that no redistribution of tin occurs during the corrosion process. This was seen in ZIRLO both pre- and post-transition 
and in the pre-transition Zircaloy-4. The effect of tin on the corrosion resistance of zirconium alloys is likely to be mainly due to its role of a solid solution strengthener of the substrate. Small additions of tin to $\mathrm{ZrO}_{2}$ are unlikely to affect the conductivity of the oxide, as tin and zirconium have similar valance.

Solutes with low solubility in $\mathrm{ZrO}_{2}$ were frequently seen to be rejected at the metal-oxide interface $(\mathrm{Nb}, \mathrm{Fe})$. This necessitates the redistribution of these solutes, either to the metal adjacent to the interface, or to grain boundaries within the metal or oxide. A fine grain structure, as was seen in the poorly performing $\mathrm{Zr}-\mathrm{Nb}-\mathrm{Ti}$ alloy, is detrimental in that it promotes the nucleation of many, small, grains and provides a large sink volume for the excess material, removing it from solution where niobium and iron are seen to perform useful roles, as outlined above. The processing treatment that this alloy underwent was similar to that of the ZIRLO analysed in this study. The fine substrate grain structure is, therefore, due to the addition of this small quantity of titanium.

Overall, it appears that metal-oxide interfaces in zirconium alloys are essentially graded structures, exhibiting a progressive change in composition and microstructure, from pure $\mathrm{ZrO}_{2}$ at one extreme to pure metal at the other. In between there are sub-oxide and oxygen saturated matrix regions, strengthened by precipitate and dislocation-solute interactions. This leads to a gradual and progressive change in mechanical properties across the interface, which is conducive to good adhesion. The problem of breakaway corrosion appears to emanate from further out into the oxide.

\subsection{Future directions}

Hydride analysis, which was only touched upon in the most cursory fashion during the preliminary work in this study, offers another exciting area of research involving zirconium 
alloys and the 3D atom probe. The characterisation of the metal matrix performed in this study, and the description of hydride peak identification would be of real value to future researchers in this field. Yet despite the possibility of high precision chemical analysis of hydrides by this method, the value of this information to furthering understanding of hydrogen-based corrosion appears limited.

Further 3DAP study of post-transition materials, particularly those produced in the EDF autoclave used in this work would be an excellent extension of this work, providing further insight into the nanoscale chemical redistribution that occurs at the metal oxide interface after corrosion. A limited amount of such material became available during the very late stages of this project. Unfortunately, both the difficulty of specimen fabrication and specimen failure rate increase with thicker and more porous oxide, such that the success rate becomes very low, restricting the availability of data and the scope of conclusions that can be drawn from such analysis.

The 3DAP would be a suitable tool to investigate the degree of solute segregation to grain boundaries resulting from various heat treatments. This work could elucidate the role of quenching in the segregation of niobium and iron to such features. This investigation would benefit from only requiring the analysis of metallic material, although it would still require sitespecific specimen fabrication. 


\section{References}

1. B. Cox, Some Thoughts on the Mechanisms of In-Reactor Corrosion of Zirconium Alloys, Journal of Nuclear Materials, (2005) 336 (2-3) 331-368.

2. B. Cox, Effects of Irradiation on the Oxidation of Zirconium Alloys in High Temperature Aqueous Environments : A Review, Journal of Nuclear Materials, (1968) 28 (1) 1-47.

3. F. Lefebvre and C. Lemaignan, Irradiation effects on corrosion of zirconium alloy claddings, Journal of Nuclear Materials, (1997) 248 268-274.

4. A.T. Motta, F. Lefebvre, and C. Lemaignan, Amorphization of Precipitates in Zircaloy under Neutron and Charged-Particle Irradiation, Zirconium in the Nuclear Industry : Ninth International Symposium, (1991) 1132 718-737.

5. R.B. Adamson, Effects of neutron irradiation on microstructure and properties of Zircaloy, Zirconium in the Nuclear Industry: Twelfth International Symposium, ASTM STP 1354, (2000) 15-31.

6. S.A. Nikulin and A.B. Rozhnov, Corrosion cracking of zirconium cladding tubes. A review. 2. Effect of external factors, structure, and properties of the alloys, Metal Science and Heat Treatment, (2005) 47 (9-10) 427-433.

7. K.L. Murty and I. Charit, Texture Development and Anisotropic Deformation of Zircaloys, Progress in Nuclear Energy, (2006) 48 (4) 325-359.

8. M.P. Puls, Review of the thermodynamic basis for models of delayed hydride cracking rate in zirconium alloys, Journal of Nuclear Materials, (2009) 393 (2) 350-367.

9. C.E. Coleman and D. Hardie, Hydrogen Embrittlement of Alpha-Zirconium - a Review, Journal of the Less-Common Metals, (1966) 11 (3) 168-185.

10. Anon. (2005) Nuclear Ship SAVANNAH Decommissioning and Preservation.

11. F.E. Bowman and D.D. Cubicciotti, Use of Zirconium in Liquid-Sodium Systems, American Institute of Chemical Engineers Journal, (1956) 2 (2) 173-176.

12. Pomerance, Thermal Neutron Capture Cross Sections, Physical Review, (1951) 83 (3).

13. M. Atoji, Slow-Neutron Cross Section of Hafnium, Acta Crystal, (1964) 17 1087-1088.

14. N. Ni, et al., Porosity in oxides on zirconium fuel cladding alloys, and its importance in controlling oxidation rates, Scripta Materialia, (2010) 62 (8) 564-567.

15. A.T. Motta, et al. Microstructure and Growth Mechanism of Oxide Layers Formed on $\mathrm{Zr}$ Alloys Studied with Micro-Beam Synchrotron Radiation, in Zirconium in the Nuclear Industry: Fourteenth International Symposium, ASTM STP 1467, American Society for Testing Materials (2004) 205-232.

16. M. Inagaki, M. Kanno, and H. Maki. Effect of Alloying Elements in Zircaloy on PhotoElectrochemical Characteristics of Zirconium Oxide Films, in Zirconium in the Nuclear Industry: Ninth International Symposium, ASTM STP 1132, American Society for Testing Materials (1990) 437-458.

17. H. Frank, Transport Properties of Zirconium Alloy Oxide Films, Journal of Nuclear Materials, (2002) 306 (2-3) 85-98.

18. R.A. Pérez and M. Weissmann, Ab-initio Approach to the Effect of Fe on the Diffusion in HCP Zr, Journal of Nuclear Materials, (2008) 374 (1-2) 95-100.

19. J.P. Abriata and J. Garcés, The O-Zr (Oxygen-Zirconium) System, Bulletin of Alloy Phase Diagrams, (1986) 7 (2) 116-123.

20. X. Guo, Hydrothermal Degradation Mechanism of Tetragonal Zirconia Journal of Materials Science, (2001) 36.

21. R.C. Garvie, Stabilization of the Tetragonal Structure in Zirconia Microcrystals, Journal of Physical Chemistry, (1978) 82. 
22. M. Oskarsson, et al., Characterisation of Pre-Transition Oxides on Zircaloys, Journal of Nuclear Materials, (2001) 297 (1) 77-88.

23. S. Abolhassani, et al. TEM Examinations of the Metal-Oxide Interface of Zirconium Based Alloys Irradiated in a Pressurized Water Reactor, in Zirconium in the Nuclear Industry: Fourteenth International Symposium, ASTM STP 1467, American Society for Testing Materials (2004) 467-493.

24. N. Petigny, et al., In-Situ XRD Analysis of the Oxide Layers Formed by Oxidation at 743 $K$ on Zircaloy-4 and Zr-1NbO, Journal of Nuclear Materials, (2000) 280 195-201.

25. J.B. M. Steinbrück, A.V. Goryachev, M. Grosse, T. J. Haste, Z. Hozer, A.E. Kisselev, V.I. Nalivaev, V.P. Semishkin, L. Sepold, J. Stuckert, N. Vér, M.S. Veshchunov, Status of Studies on High-Temperature Oxidation and Quench Behaviour of Zircaloy-4 and E110 Cladding Alloys, in ERMSAR. (2008). Session S2 Paper 2.

26. P.T. Moseley and B. Hudson, Phases involved in the corrosion of zircaloy by hot water (350 ${ }^{\circ}$ C), Journal of Nuclear Materials, (1981) 99 (2-3) 340-344.

27. X. Iltis and H. Michel, Caractérisation par Microscopie Électronique à Transmission d'une Solution Solide Zr-O Localement Ordonnée Obtenue à l'Issue d'un Traitement d'Oxydation sur un Alliage de Type Zircaloy-4, Journal of Alloys and Compounds, (1991) 177.

28. P. Bossis, et al. Multi-Scale Characterisation of the Metal-Oxide Interface of Zirconium Alloys, in Zirconium in the Nuclear Industry: Twelfth International Symposium, ASTM STP 1354, ASTM (2000) 918-945.

29. S. Banerjee and P. Mukhopadhyay, Phase Transformations: Examples from Titanium and Zirconium Alloys. Pergamon Materials Series. (2007), Oxford: Elsevier.

30. H.I. Yoo, et al., A Working Hypothesis on Oxidation Kinetics of Zircaloy, Journal of Nuclear Materials, (2001) 299 (3) 235-241.

31. D.G. Franklin and C.Y. Li. Zirconium-Alloy Corrosion: A Review Based on an International Atomic Energy Agency (IAEA) Meeting, in Zirconium in the Nuclear Industry: Seventh International Symposium, ASTM STP 939, American Society for Testing Materials (1987) 206-223.

32. A. Grandjean and Y. Serruys, Metal and Oxygen Mobilities During Zircaloy-4 Oxidation at High Temperature, Journal of Nuclear Materials, (1999) 273 (1) 111-115.

33. B. Cox, Y.-M. Wong, and J. Mostaghimi, High Conductivity Porous Oxides Formed on Zr-2.5 wt\% Nb, Journal of Nuclear Materials, (1995) 226272.

34. F. Garzarolli, et al. Oxide Growth Mechanism on Zirconium Alloys, in Zirconium in the Nuclear Industry: Ninth International Symposium, ASTM STP 1132, American Society for Testing Materials (1990) 395-415.

35. Y. Chen, M. Urquidi-Macdonald, and D.D. Macdonald, The Electrochemistry of Zirconium in Aqueous Solutions at Elevated Temperatures and Pressures, Journal of Nuclear Materials (2006) 348 133-147.

36. B. Cox, Rate Controlling Processes During the Pre-Transition Oxidation of Zirconium Alloys, Journal of Nuclear Materials, (1969) 31 (1) 48-66.

37. M.M.R. Howlader, et al., The Electrical Conductivity of Zircaloy Oxide Films, Journal of Nuclear Materials, (1998) 253 149-155.

38. M. Tupin, et al., Differences in Reactivity of Oxide Growth During the Oxidation of Zircaloy-4 in Water Vapour Before and After the Kinetic Transition, Journal of Nuclear Materials, (2003) 317 (2-3) 130-144.

39. M. Tupin, et al., Oxidation Kinetics of ZrNbO in Steam: Differences Between the Preand Post-Transition Stages, Journal of Nuclear Materials, (2005) 342 108-118.

40. D.G. Franklin and P.M. Lang. Zirconium-Alloy Corrosion: A Review Based on an International Atomic Energy Agency (IAEA) Meeting, in Zirconium in the Nuclear 
Industry: Ninth International Symposium, ASTM STP 1132, American Society for Testing Materials (1990) 3-29.

41. C. Lemaignan, Corrosion of Zirconium Alloy Components in Light Water - 13C. ASM Handbook on Corrosion: Enviroments and Industries, ed. S.D. Cramer and B.S. Covino. (2006), Ohio: ASM International.

42. Y. Ishii, A Microstructural Study of Oxidation of Zircaloy-2 in Air, in Department of Materials. (Michaelmas 1998), Pembroke College, University of Oxford: Oxford, UK.

43. O. Kubaschewski and B.E. Hopkins, Oxidation of Metals and Alloys, Second Ed. ed. (1962), London: Butterworths.

44. K. Hauffe, Oxidation of Metals. (1965), New York: Plenum.

45. B. Cox, Are Zirconia Corrosion Film a Form of Partially Stabilized Zirconia (PSZ)? (1987), Chalk River Nuclear Labs.: Chalk River, Ontario.

46. P. Rudling, Corrosion Performance of Zircaloy-2 Cladding. (April 1990), Electric Power Research Institute: Palo Alto, Calif.

47. H. Göhr, et al. Long-Term In Situ Corrosion Investigation of Zr Alloys in Simulated PWR Environment by Electrochemical Measurements, in Zirconium in the Nuclear Industry, Eleventh International Symposium, ASTM STP 1295, America Society for Testing and Materials (1996) 181.

48. T. Arima, et al., Oxidation Kinetics of Zircaloy-2 Between $450^{\circ} \mathrm{C}$ and $600^{\circ} \mathrm{C}$ in Oxidizing Atmosphere, Journal of Nuclear Materials, (1998) 257.

49. J. Godlewski, et al. Raman Spectroscopy Study of the Tetragonal-to-Monoclinic Transition in Zirconium Oxide Scales and Determination of Overall Oxygen Diffusion by Nuclear Microanalysis of $O$, in Zirconium in the Nuclear Industry: Ninth International Symposium, ASTM STP 1132, American Society for Testing Materials (1990) 416-433.

50. M. Harada, M. Kimpara, and KAbe. Effect of Alloying Elements on Uniform Corrosion Resistance of Zirconium-based Alloys in $360^{\circ} \mathrm{C}$ Water and $400^{\circ} \mathrm{C}$ Steam, in Zirconium in the Nuclear Industry: Ninth International Symposium, ASTM STP 1132, American Society for Testing Materials (1990) 368-389.

51. F. Garzarolli, E. Steinberg, and H.G. Weidinger. Microstructure and Corrosion Studies for Optimized PWR and BWR Zircaloy Cladding, in Zirconium in the Nuclear industry, ASTM STP 1023, Philadelphia, American Society for Testing and Materials (1989) 202212.

52. B.H. Lim, H.S. Hong, and K.S. Lee, Measurements of Hydrogen Permeation and Absorption in Zirconium Oxide Scales, Journal of Nuclear Materials, (2003) 312134 140.

53. R.C. Garvie and P.S. Nicholson, Phase Analysis in Zirconia Systems, Journal of the American Ceramic Society, (1972) 55.

54. Accuratus. Zirconium Oxide. (2005) 23/09/08]; Available from: http://www.accuratus.com/zirc.html.

55. M. Rezaei, et al., Synthesis of Pure Tetragonal Zirconium Oxide with High Surface Area, Journal of Materials Science, (2007) 42 1228-1237.

56. A. Yilmazbayhan, et al., Structure of Zirconium Alloy Oxides Formed in Pure Water Studied with Synchrotron Radiation and Optical Microscopy: Relation to Corrosion Rate, Journal of Nuclear Materials, (2004) 324 6-22.

57. J.J. Kearns, Terminal Solubility and Partitioning of Hydrogen in the Alpha Phase of Zirconium, Zircaloy-2 and Zircaloy-4, Journal of Nuclear Materials, (1967) 22 (3) 292303.

58. B. Cox, Pore Structure in Oxide Films on Irradiated and Unirradiated Zirconium Alloys, Journal of Nuclear Materials, (1987) 148 (3) 332-343.

59. H.K. Yueh and B. Cox, Cathodoluminescence Imaging of Oxidised Zirconium Alloys, Journal of Nuclear Materials, (2004) 324 (2-3) 203-214. 
60. B. Cox, Processes Occurring During the Breakdown of Oxide Films on Zirconium Alloys, Journal of Nuclear Materials, (1969) 29 (1) 50-66.

61. D.J. Park, et al., Microstructural Characterization of $\mathrm{ZrO}_{2}$ Layers Formed During the Transition to Breakaway Oxidation, Journal of Nuclear Materials, (2010) 399 (2-3) 208211.

62. G.P. Sabol. ZIRLO An Alloy Development Success, in Zirconium in the Nuclear Industry: Fourteenth International Symposium, ASTM STP 1467, American Society for Testing Materials (2004) 3-24.

63. J.H. Schemel, ASTM Manual on Zirconium and Hafnium. ASTM STP 639. (1977): American Society for Materials Testing.

64. H.-J. Sell, S. Trapp-Pritsching, and F. Garzarolli. Effects of Alloying Elements and Impurities on in-BWR Corrosion of Zirconium Alloys, in Zirconium in the Nuclear Industry: Fourteenth International Symposium, ASTM STP 1467, American Society for Testing Materials (2004) 404.

65. M. Steinbrück, Hydrogen Absorption by Zirconium Alloys at High Temperatures, Journal of Nuclear Materials, (2004) 334 58-64.

66. J.P. Mardon, D. Charquet, and J. Senevat. Influence of Composition and Fabrication Process on Out-of-Pile and In-Pile Properties of M5 Alloy, in Zirconium in the Nuclear Industry, Twelfth International Symposium, ASTM STP 1354, America Society for Testing and Materials (2000) 505.

67. R.J. Comstock, G. Schoenberger, and G.P. Sabol. Influence of Processing Variables and Alloy Chemistry on the Corrosion Behavior of ZIRLO Nuclear Fuel Cladding, in Zirconium in the Nuclear Industry, Eleventh International Symposium, ASTM STP 1295, America Society for Testing and Materials (1996) 710.

68. S.V. Ivanova, Effect of hydrogen on serviceability of zirconium items in VVER and RBMK-type reactors fuel assemblies, International Journal of Hydrogen Energy, (2002) 27 (7-8) 819-824.

69. A. Yilmazbayhan, et al., Transmission Electron Microscopy Examination of Oxide Layers Formed on Zr Alloys, Journal of Nuclear Materials, (2006) 349 265-281.

70. H.G. Weidinger, et al. Corrosion-Electrochemical Properties of Zirconium Intermetallics, in Zirconium in the Nuclear Industry: Ninth International Symposium, ASTM STP 1132, American Society for Testing Materials (1990) 499-533.

71. J.-C. Tedenac and P. Perrot, Iron - Niobium - Zirconium in Iron Systems: Phase Diagrams, Crystallographic and Thermodynamic Data, MSIT, Editor. (2009), Springer Berlin Heidelberg. 101-116.

72. J.-Y. Park, et al., Corrosion and Oxide Characteristics of $\mathrm{Zr}-1.5 \mathrm{Nb}-0.4 \mathrm{Sn}-0.2 \mathrm{Fe}-0.1 \mathrm{Cr}$ Alloys in $360^{\circ} \mathrm{C}$ Pure Water and LiOH Solution, Journal of Nuclear Materials, (2008) 373 (1-3) 343-350.

73. P. Barberis, D. Charquet, and V. Rebeyrolle, Ternary $\mathrm{Zr}-\mathrm{Nb}-\mathrm{Fe}(\mathrm{O})$ System: Phase Diagram at $853 \mathrm{~K}$ and Corrosion Behaviour in the Domain $\mathrm{Nb}<0.8 \%$, Journal of Nuclear Materials, (2004) 326 (2-3) 163-174.

74. C. Toffolon, Etude métallurgique de base et calculs de diagrammes de phases des alliages base zirconium du système : $\mathrm{Zr}-\mathrm{Nb}-\mathrm{Fe}-(\mathrm{O}, \mathrm{Sn}), \mathrm{PhD}$ Thesis. (2000), l'université de Paris 6: Paris.

75. Y.Z. Liu, et al., Study of Microstructure of $\mathrm{Zr}-\mathrm{Sn}-\mathrm{Nb}-\mathrm{Fe}-\mathrm{Cr}$ Alloy in the Temperature Range of 750-820 ${ }^{\circ} \mathrm{C}$, Materials Chemistry and Physics, (2008) 108 534-540.

76. H.G. Kim, et al., Corrosion and Microstructural Characteristics of $\mathrm{Zr}-\mathrm{Nb}$ Alloys with Different Nb Contents, Journal of Nuclear Materials, (2008) 373 429-432.

77. D.O. Northwood, X. Meng-Burany, and B.D. Warr. Microstructure of Zr-2.5Nb Alloy Pressure Tubing, in Zirconium in the Nuclear Industry: Ninth International Symposium, ASTM STP 1132, American Society for Testing Materials (1990) 156-172. 
78. B. Cox, Is Zirconium Oxide Morphology on Fuel Cladding Largely Determined by Lithium Hydroxide Concentration Effects?, Journal of Nuclear Materials, (1997) 249 (1) 87-90.

79. W. Liu, et al., Effect of Heat Treatment on the Microstructure and Corrosion Resistance of a $\mathrm{Zr}-\mathrm{Sn}-\mathrm{Nb}-\mathrm{Fe}-\mathrm{Cr}$ Alloy, Journal of Nuclear Materials, (2005) 341 (2-3) 97-102.

80. P. Barberis, et al. Microstructure and Phase Control in $\mathrm{Zr}-\mathrm{Fe}-\mathrm{Cr}-\mathrm{Ni}$ Alloys: Thermodynamic and Kinetic Aspects, in Zirconium in the Nuclear Industry: Fourteenth International Symposium, ASTM STP 1467, American Society for Testing Materials (2004) 129-156.

81. T. Thorvaldsson, et al. Correlation Between $400^{\circ} \mathrm{C}$ Steam Corrosion Behavior, Heat Treatment, and Microstructure of Zircaloy-4 Tubing, in Zirconium in the Nuclear Industry, Eighth International Symposium, ASTM STP 1023, America Society for Testing and Materials (1989) 128.

82. E.R. Bradley, J.H. Schemel, and A.-L. Nystöm. Influence of Alloy Composition and Processing on the Nodular Corrosion Resistance of Zircaloy-2, in Zirconium in the Nuclear Industry: Ninth International Symposium, ASTM STP 1132, American Society for Testing Materials (1990) 304-315.

83. C.T. Wang, C.M. Eucken, and R.A. Graham. Investigation of Nodular Corrosion Mechanism for Zircaloy Products, in Zirconium in the Nuclear Industry: Ninth International Symposium, ASTM STP 1132, American Society for Testing Materials (1990) 319-345.

84. C. Toffolon-Masclet, et al. Study of $\mathrm{Nb}$ and Fe Precipitation in -Phase Temperature Range (400 to $550^{\circ} \mathrm{C}$ ) in $\mathrm{Zr}-\mathrm{Nb}-(\mathrm{Fe}-\mathrm{Sn})$ Alloys, in Zirconium in the Nuclear Industry: Fourteenth International Symposium, ASTM STP 1467, American Society for Testing Materials (2004) 81-101.

85. R.A. Graham and C.M. Eucken. Controlled Composition Zircaloy-2 Uniform Corrosion Resistance, in Zirconium in the Nuclear Industry: Ninth International Symposium, ASTM STP 1132, American Society for Testing Materials (1990) 279-300.

86. D. Charquet, R. Triot, and T.F. Wadier. Homogeneous Scale Growth During Steam Corrosion of Zircaloy-4 at $500{ }^{\circ} \mathrm{C}$, in Zirconium in the Nuclear Industry: Eighth International Symposium, ATSM STP 1023, American Society for Testing and Materials (1989) 374.

87. E. Tenckhoff. Review of Deformation Mechanisms, Texture, and Mechanical Anisotropy in Zirconium and Zirconium Base Alloys, in Zirconium in the Nuclear Industry: Fourteenth International Symposium, ASTM STP 1467, American Society for Testing Materials (2004) 25-52.

88. Waterside Corrosion of Zirconium Alloys in Nuclear Power Plants. (1998), IAEA: Vienna.

89. T. Kubo and M. Uno. Precipitate Behavior in Zircaloy-2 Oxide Films and Its Relevance to Corrosion Resistance, in Zirconium in the Nuclear Industry: Ninth International Symposium, ASTM STP 1132, American Society for Testing Materials (1990) 473-497.

90. A.R. Massih, et al., Effect of Quenching Rate on the Beta-to-Alpha Phase Transformation Structure in Zirconium Alloy, Journal of Nuclear Materials, (2003) 322 (2-3) 138-151.

91. G. Ökvist and K. Källström, The Effect of Zirconium Carbide on the $\beta \rightarrow a$ Transformation Structur in Zircaloy, Journal of Nuclear Materials, (1970) 35.

92. R.A. Holt, The Beta to Alpha Phase Transformation in Zircaloy-4, Journal of Nuclear Materials, (1970) 35.

93. R.A. Holt, Comments on the Beta to Alpha Phase Transformation in Zircaloy-4, Journal of Nuclear Materials, (1973) 47. 
94. S.L. Wadekar, et al. Correlation of Microstructure and Mechanical Properties of Zr-SN Alloys, in Zirconium in the Nuclear Industry: Ninth International Symposium, ASTM STP 1132, American Society for Testing Materials (1990) 140-154.

95. C. Toffolon, et al. Experimental Study and Preliminary Thermodynamic Calculations of the Pseudo-Ternary Zr-Nb-Fe- $(\mathrm{O}, \mathrm{Sn})$ System, in Zirconium in the Nuclear Industry: Thirteenth International Symposium, STP1423, American Society for Testing and Materials (2002) 361.

96. T. Isobe and Y. Matsuo. Development of Highly Corrosion Resistant Zirconium-Base Alloys, in Zirconium in the Nuclear Industry: Ninth International Symposium, ASTM STP 1132, American Society for Testing Materials (1990) 346-367.

97. D. Charquet, Improvement of the Uniform Corrosion Resistance of Zircaloy-4 in the Absence of Irradiation, Journal of Nuclear Materials, (1988) 160 186-195.

98. H.K. Yueh, et al. Improved ZIRLO Cladding Performance Through Chemistry and Process Modifications, in Zirconium in the Nuclear Industry: Fourteenth International Symposium, ASTM STP 1467, American Society for Testing Materials (2004) 330.

99. S.G. Popov and V.N. Proselkov, The Heat Capacity in Two-phase Regions and the Heat of Phase Transformations of some Alloys in $\mathrm{Zr}-\mathrm{Nb}$ and $\mathrm{Zr}$-Sn Systems, High Temperature, (2009) 47 (4) 514-526.

100. L. Lanzani and M. Ruch, Comments on the Stability of Zirconium Hydride Phases in Zircaloy, Journal of Nuclear Materials, (2004) 324 (2-3) 165-176.

101. R.J. Pérez and A.R. Massih, Thermodynamic Evaluation of the Nb-O-Zr System, Journal of Nuclear Materials, (2007) 360 (3) 242-254.

102. K. Kapoor, et al. On Secondary -Nb Phase Precipitation within Primary -Zr Phase in ZrNb Alloys During Tensile Deformation, in Zirconium in the Nuclear Industry: Fourteenth International Symposium, ASTM STP 1467, American Society for Testing Materials (2004) 102-128.

103. K. Kakiuchi, et al. Role of Iron for Hydrogen Absorption Mechanism in Zirconium Alloys, in Zirconium in the Nuclear Industry: Fourteenth International Symposium, ASTM STP 1467, American Society for Testing Materials (2004) 349.

104. B. Cox, Electron Conduction Through Oxide Films on Zircaloy-2, Journal of Nuclear Materials, (1970) 37.

105. B. Cox, The Effects of Some Alloying Additions on the Oxidation of Zirconium in Steam. (1963), UK Atomic Energy Authority: Harwell.

106. E. Hillner. Corrosion of Zirconium-Base Alloys - An Overview, in Zirconium in the Nuclear Industry: Third International Symposium. ASTM STP 633, American Society for Testing and Materials (1977) 211-235.

107. R.J. Comstock, $\mathrm{Zr}-1 \mathrm{Nb}-0.2$ Ti Weight Gain Data, G.D.W. Smith, Editor. (2009).

108. R.J. Comstock, Zr - 1 Nb Weight Gain Data, G.D.W. Smith, Editor. (2009).

109. G.L. Miller, Zirconium. Metallurgy Of The Rarer Metals. (1954), London: Butterworths Scientific Publications.

110. D. Charquet, Phase Constitution and Steam Corrosion Resistance of Binary Zr-S Alloys, Journal of Nuclear Materials, (2002) 304 (2-3) 246-248.

111. K. Takeda and H. Anada. Microstructure of Oxides on Zircaloy-4, 1.0Nb Zicaloy-4, and Zircaloy-2 Formed in 10.3-MPa Steam at 673K, in Zirconium in the Nuclear Industry: Eleventh International Symposium, American Society for Testing and Materials (1996) $35-54$.

112. R. Kuwae, et al., Mechanism of Zircaloy Nodular Corrosion, Journal of Nuclear Materials, (1983) 119 29-239.

113. R. Ploc. Oxidation Kinetics and Auger Microprobe Analysis of Some Optimised Zirconium Alloys, in Zirconium in the Nuclear Industry: Eighth International Symposium, ATSM STP 1023, American Society for Testing and Materials (1989) 498. 
114. B. Cheng and R.B. Adamson. Mechanistic Study of Zircaloy Nodular Corrosion, in Zirconium in the Nuclear Industry: Seventh International Symposium, ASTM STP 939, American Society for Testing and Materials (1987) 387-416.

115. D. Pêcheur, Oxidation of $\beta-\mathrm{Nb}$ and $\mathrm{Zr} / \mathrm{Fe}, V_{2}$ Precipitates in Oxide Films Formed on Advanced Zr-Based Alloys, Journal of Nuclear Materials, (2000) 278 195-201.

116. Y.P. Lin and O.T. Woo, Oxidation of beta-Zr and Related Phases in Zr-Nb Alloys: An Electron Microscopy Investigation, Journal of Nuclear Materials, (2000) 277 11-20.

117. H. Anada, et al. Effect of Annealing Temperature on Corrosion Behaviour and $\mathrm{ZrO}_{2}$ Microstructure of Zircaloy-4 Cladding Tube, in Zirconium in the Nuclear Industry, Eleventh International Symposium, ASTM STP 1295, America Society for Testing and Materials (1996) 74.

118. H.G. Rickover, L.D. Geiger, and B. Lustman, History of the Development of Zirconium Alloys for use in Nuclear Reactors. (1975), Division of Naval Reactors: Washington DC.

119. B. Cheng, R.M. Kruger, and R.B. Adamson. Corrosion behaviour of Irradiated Zircaloy, in Zirconium in the Nuclear Industry: Tenth International Symposium, ATSM STP, ASTM International (1994) 400.

120. K. Ogata. Effects of Alloying Element Distribution on Nodular Corrosion of Zircaloy-2, in Zirconium in the Nuclear Industry: Eighth International Symposium, ATSM STP 1023, American Society for Testing and Materials (1989) 346-359.

121. P. Rudling, et al. The Influence of Second Phase Particles on Zircaloy Corrosion in BWR Enviroment, in Zirconium in the Nuclear Industry: Seventh International Symposium, ASTM STP 939, American Society for Testing and Materials (1987) 292-306.

122. R.A. Graham, J.P. Tosdale, and P.T. Finden. Influence of Chemical Composition and Manufacturing Variables on Autoclave Corrosion of the Zircaloys, in Zirconium in the Nuclear Industry: Seventh International Symposium, ASTM STP 1023, American Society for Testing and Materials (1989) 334.

123. J.J. Vermoyal, A. Frichet, and L. Dessemond, Contribution to the Understanding of the $\mathrm{ZrNb}(\mathrm{l} \%) \mathrm{O}(0.13 \%)$ Oxidation Mechanism at $500{ }^{\circ} \mathrm{C}$ in Dry Air, Journal of Nuclear Materials, (2004) 328.

124. C.B. Park J., Jo Yoo S., Hwan Jeong Y. , Corrosion and Oxide Properties of HANA Alloys, ASTM International, (2008) 5 (5).

125. R.M. Kruger, R.B. Adamson, and S.S. Brenner, Effects of Microchemistry and Precipitate Size on Nodular Corrosion Resistance of Zircaloy-2, Journal of Nuclear Materials, (1992) 189 193-200.

126. B. Wadman, et al., Microstructural Influence on Uniform Corrosion of Zircaloy Nuclear Fuel Claddings, Journal of Nuclear Materials, (1993) 200 (2) 207-217.

127. B. Wadman and H.-O. Andrén. Microanalysis of the Matrix and the Oxide-Metal Interface of Uniformly Corroded Zircaloy, in Zirconium in the Nuclear Industry: Ninth International Symposium, ASTM STP 1132, American Society for Testing Materials (1990) 461-472.

128. D. Charquet. in Zirconium in the Nuclear Industry: Eighth International Symposium, ATSM STP 1023, American Society for Testing and Materials (1989) 113-127.

129. B. Wadman, H.-O. Andrén, and U. Rolander, Preferential Field Evaporation During Atom Probe Analysis of Zircaloy-4, Journal de Physique, (1988) 49-C6 (11) 323.

130. N. Sano and K. Takeda, Atom Probe Analysis of Sn in Zr-Based Alloys, Journal of Nuclear Materials, (1998) 252 63-70.

131. M.K. Miller, et al., Atom Probe Field Ion Microscopy. Oxford Science Publications. (1996), Oxford: Clarendon Press.

132. B. Wadman, Mechanisms of Uniform Corrosion of Zirconium Alloys in Water and Steam, in Physics. (1993), Chalmers University of Technology: Göteborg. 79. 
133. A. Cerezo and G.D.W. Smith, Field-ion Microscopy: Atom Probe Microanalysis, in Encyclopedia of Materials: Science and Technology K.H.J. Buschow, et al., Editors. (2003), Elsevier Ltd. 3177-3181.

134. P.J. Warren, A. Cerezo, and G.D.W. Smith, Observation of atomic planes in 3DAP analysis, Ultramicroscopy, (1998) 73 (1-4) 261-266.

135. G. Sha and S.P. Ringer, Effect of Laser Pulsing on the Composition Measurement of an Al-Mg-Si-Cu Alloy using Three-dimensional Atom Probe, Ultramicroscopy, (2009) 109 (5) $580-584$.

136. A. Cerezo, T.J. Godfrey, and G.D.W. Smith, Development and Initial Applications of a Position Sensitive Atom Probe, Journal de Physique, (1988) 49 (C3) 25-30.

137. M.J. Fransen, M.H.F. Overwijk, and P. Kruit, Brightness Measurements of a $\mathrm{ZrO} / W$ Schottky Electron Emitter in a Transmission Electron Microscope Applied Surface Science, (1999) 146 (1-4) 357-362.

138. H. Salemink, Scanning tunneling microscopy Microelectronic Engineering, (1986) 5 (14) 501-508.

139. A. Cerezo, et al., Performance of an Energy-Compensated Three-Dimensional Atom Probe, Review of Scientific Instruments, (1998) 69 49-58.

140. T.F. Kelly and M.K. Miller, Atom Probe Tomography, Rev. Sci. Instrum., (2007) 78 031101 .

141. O. Jagutzki, et al., Device and Method for Two-dimensional Detection of particles or Electromagnetic Radiation. (2001).

142. O. Jagutzki, et al., Multiple Hit Readout of a Microchannel Plate Detector with a Threelayer Delay-line Anode, IEEE Transactions on Nuclear Science, (2002) 49 2477-2483.

143. M.K. Miller and R.G. Forbes, Atom Probe Tomography, Materials Characterization, (2009) 60 461-469.

144. G.L. Kellogg and T.T. Tsong, Pulser-Laser Atom-Probe Field-Ion Microscopy, Journal of Applied Physics, (1980) 51 1184-93.

145. M. Yamamoto, D.N. Seidman, and S. Nakamura. in Proceedings of the 27th International Field Emmission Symposium, Tokyo, University of Tokyo Press (1980) 317.

146. B. Gault, et al., Estimation of the Reconstruction Parameters for Atom Probe Tomography, Microscopy and Microanalysis, (2008) 14 296-305.

147. S. Humphry-Baker, in Department of Materials. (2009), University of Oxford.

148. P. Bas, et al., A general protocol for the reconstruction of 3D atom probe data, Appl. Surf. Sciences, (1995) 87 (88) 298-304.

149. O.C. Hellman, et al., Analysis of Three-dimensional Atom-probe Data by the Proximity Histogram, Microscopy and Microanalysis, (2000) 6 437-444.

150. F. Vurpillot, A. Bostel, and D. Blavette, Trajectory Overlap and Local Magnification in Three-Dimensional Atom Probe, Applied Physical Letters, (2000) 76 (21).

151. J.P. Foster, S.A. Worcester, and R.J. Comstock, Zirlo material composition and fabrication processing E.P. Office, Editor. (1991), WESTINGHOUSE ELECTRIC CORPORATION.

152. D.J. Larson, et al., Focused Ion-beam Milling for Field-ion Specimen Preparation: Preliminary Investigations Ultramicroscopy, (1998) 75 (3) 147-159.

153. D.J. Larson, et al., Field-ion Specimen preparation using focused ion-beam milling, Ultramicroscopy, (1999) 79 287-293.

154. M.K. Miller and K.F. Russell, Atom probe specimen preparation with a dual beam SEM/FIB miller, Ultramicroscopy, (2007) 107 (9) 761-766.

155. B. Wadman and H.-O. Andrén. Microanalysis of the Matrix and the Oxide-Metal Interface of Uniformly Corroded Zircaloy, in Zirconium in the Nuclear Industry: Ninth 
International Symposium, ASTM STP 1132, American Society for Testing Materials (1991) 461-472.

156. J.H. Clark, D.C. Bradley, and P. Thornton, The Chemistry of Titanium, Zirconium and Hafnium. (1975): Pergamon Press.

157. B. Wadman, H.-O. Andrén, and L.K.L. Falk, Atom Probe Analysis of Thin Oxide Layers on Zircaloy Needles, Journal de Physique, (1989) 50 (11) C8-303.

158. J.V. Dobson and G. Brimsa, Attempts on the Electrolytic Hydrogen Charging of Zirconium and the Measurements of the pH Response of Surface Oxides Electrochimica Acta, (1986) 31 (7) 887-890.

159. J. Takahashi, et al., Atom probe analysis of titanium hydride precipitates, Ultramicroscopy, (2009) 109 568-573.

160. C.J. Smithells, Smithells Metals Reference Book, 8th ed, ed. W.F. Gale and T.C. Totemeier. (2004), Amsterdam: Elsevier Butterworth Heinemann.

161. B. Gault, et al., Estimation of the tip field enhancement on a field emitter under laser illumination, Applied Physics Letters, (2005) 86.

162. G. Sha, A. Cerezo, and G.D.W. Smith, Field Evaporation Behavior during Irradiation with Picosecond Laser Pulses, Appl. Phys. Lett., (2008) 92.

163. D.R. Kingham, The Post-Ionisation of Field Evaporated Ions: A Theoretical Explanation of Multiple Charge States, Surface Science, (1982) 116 273-301.

164. E.W. Müller and T.T. Tsong, Field-Ion Microscopy: Principles and Application. (1969), New York: Elsevier.

165. J.P. Abriata and J.C. Bolcich, The Sn-Zr system, Bulletin of Alloy Phase Diagrams, (1983) 4 (2).

166. M.P. Moody, et al., Quantitative Binomial Distribution Analyses of Nanoscale LikeSolute Atom Clustering and Segregation in Atom probe Tomography Data, Microscopy Research and Techniques, (2008) 71 542-550.

167. N. Ni, TEM and EDX Study of Zr-Nb-Ti. (2010), Department of Materials, University of Oxford.

168. F. Vurpillot, et al., Modeling Image Distortions in 3DAP, Microscopy and Microanalysis, (2004) 10 (3) 384-390

169. B.W. Krakauer and D.N. Seidman, Absolute Atomic-Scale Measurements of the Gibbsian Interfacial Excess of Solute at Internal Interfaces, Physical Review B, (1993) 48 (9).

170. K. Seto, et al., Grain Boundary Segregation in Boron Added Interstitial Free Steels Studied by 3-Dimensional Atom Probe, Scripta Materialia, (1999) 40 (9) 1029-1034.

171. R. Tendler and J.P. Abriata, Atomic Size and Fast Diffusion of Metallic Impurities in Zirconium, Journal of Nuclear Materials, (1987) 150 251-258.

172. T.-L. Yau, Stress Corrosion Crack of Zirconium Alloys, in Stress Corrosion Cracking: Materials Performance and Evaluation, R.H. Jones, Editor. (1992), ASM International: Michigan.

173. H. Okamoto, Comment on Ti-Zr (Titanium-Zirconium), Journal of Phase Equilibria, (1995) 16 (4).

174. J.D. Robson, Modelling Precipitation in Zirconium Niobium Alloys, Journal of Nuclear Materials, (2008) 377 (3) 415-422.

175. W.C. Wyder and M. Hoch, The system $\mathrm{Nb}-\mathrm{Ti}-\mathrm{Zr}-\mathrm{O}$ at $1500^{\circ} \mathrm{C}$, Trans. Metall. Soc. AIME, (1962) 244 (2) 373-378.

176. R.S. Roth and L.W. Coughanour, J. Res. Natl. Bur. Stand. (U.S.), (1955) 55 (4) 209-213.

177. M.K. Asundi, et al., J. Alloy Phase Diagrams (India), (1986) 2 (2) 141-148.

178. Phase Equilibria Diagrams. NIST Standard reference database 31. Vol. 3.1. (2010): AcerS-NIST.

179. H. Okamoto, $\mathrm{Nb}-\mathrm{Zr}$ (Niobium-Zirconium), Journal of Phase Equilibria, (1992) 13 (5). 
180. D.A. Porter and K.E. Easterling, Phase Transformations in Metals and Alloys. (1987), Wokingham: Van Nostrand Reinhold (UK).

181. S. Abolhassani, G. Bart, and A. Jakob, Examination of the Chemical Composition of Irradiated Zirconium Based Fuel Claddings at the Metal/Oxide Interface by TEM, Journal of Nuclear Materials, (2010) 399 (1) 1-12.

182. P. Merle, et al., Study of the microstructural evolution of a-quenched or cold-rolled zirconium alloys during isothermal agings between 20 and $400^{\circ} \mathrm{C}$, Journal of Nuclear Materials, (1994) 208 135-143.

183. F. Dyment and C.M. Libanati, Self-diffusion of Ti, Zr, and Hf in their hcp phases, and diffusion of ${ }^{95} \mathrm{Nb}$ in hcp Zr Journal of Materials Science, (1968) 3.

184. A.B. Panda, J.C. Roy, and P. Pramanik, Thorium (IV) or titanium (IV) stabilized tetragonal zirconia nanocrystalline powders processed by chemical synthesis, Journal of the European Ceramic Society, (2003) 23.

185. A.E. McHale and R.S. Roth, "Investigation on the Phase Transition in $\mathrm{ZrTiO}_{4}$ and $\mathrm{ZrO}_{2}-$ $\mathrm{SnO}_{4}$ Solid Solutions, J. Am. Ceram. Soc., (1983) 66 (2) C18-C20.

186. G.M. Hood and R.J. Schultz, Tracer diffusion in $\alpha-Z r$ Acta Metall., (1974) 22.

187. S.F. Arico and L.M. Gribaudo, The $\alpha-Z r$ phase field in the $Z r-S n-O$ system, Scr. Mater., (1999) 41 (2) 159-165.

188. W.J.S. Yang, et al., Journal of Nuclear Materials, (1986) 138185.

189. V.N. Shishov, et al., Structure-Phase State, Corrosion and Irradiation Properties of Zr$\mathrm{Nb}-\mathrm{Fe}$-Sn System Alloys, Journal of ASTM International, (2008) 5 (3). 


\section{Appendix A: Automated Ranging Method}

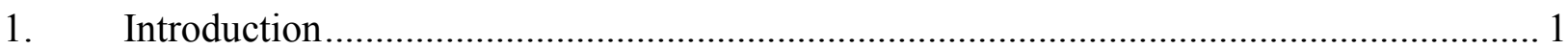

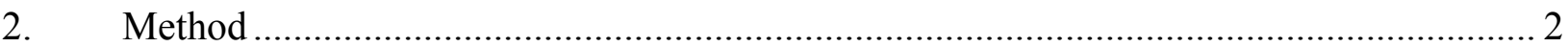

3. Analysis of simulated mass spectra using automated ranging methods ......................... 4

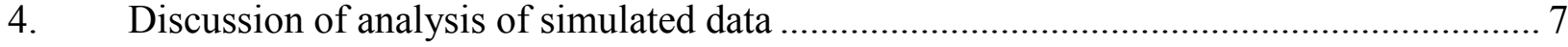

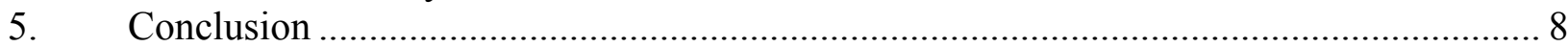

Appendix B: Automated Ranging Method Source Code

Appendix C: Confidential 


\section{Appendix A: Automated ranging methods}

\section{Introduction}

As well as the choice of suitable analysis conditions, the apparent concentration of solute detected using 3DAP varies depending upon the choice of ranging metric applied to the experimental data and the background correction method employed. Much work has been performed in standardising and automating many of the aspects of the reconstruction of atom probe data. The effect of inconsistency in ranging is increase when examining low solute concentration peaks in data with background noise. This area, despite its importance in obtaining accurate and reproducible compositional information from datasets, has received little attention. As an effort to address this problem, an automated method of applying numerous ranges and background correction methods has been developed. The source code for this software is available as Appendix B of this thesis. This allows numerous ranging strategies to be trialled and the best performing methods adopted for use with low concentration solute analysis. This method was used to investigate the concentration of iron solute in a range of alloys in chapters 5.

\section{Method}

Baptiste Gault (formerly of Oxford Materials) produced realistic simulated mass spectra with known composition that imitated the form of experimentally obtained data [1]. A matrix of automated methods of background correction and peak ranging was applied to these simulated datasets in a blind trial. This allowed a systematic comparison between the compositional values obtained by a variety of analysis methodologies. The advantage of this approach is that an exact 'real' composition of the simulated dataset can be known with complete certainty and compared 
with the measured results obtained by the automated ranging methods. The methods and metrics implemented in this code are described in Table 1. These metrics were selected based on common methods that human users from different research groups have been known to attempt to implement in their analyses. The list is not exhaustive, but illustrates many common strategies.

\begin{tabular}{|c|c|}
\hline Identifier & Description \\
\hline \multicolumn{2}{|r|}{ Background correction methods } \\
\hline B0 & No background correction - raw data \\
\hline B1 & Noise fitted by a curve of the form A/SQRT(m) \\
\hline B2 & $\begin{array}{l}\text { Noise corrected by deducting the count } 0.2 \mathrm{u} / \mathrm{e} \\
\text { ahead of the bin from each bin }\end{array}$ \\
\hline B3 & $\begin{array}{l}\text { Noise corrected by deducting the count averaged } \\
\text { over } 5 \text { bins at the closest of a number of key, } \\
\text { manually identified positions containing only noise. }\end{array}$ \\
\hline \multicolumn{2}{|r|}{ Ranging metrics } \\
\hline R1 & $\begin{array}{l}\text { Range is defined using the full width at half maximum } \\
\text { (FWHM). }\end{array}$ \\
\hline $\mathrm{R} 2$ & $\begin{array}{c}\text { Range is defined using the full width at one tenth } \\
\text { maximum (FW1/10M). }\end{array}$ \\
\hline R3 & $\begin{array}{c}\text { Range is defined using the full width at nine tenths } \\
\text { maximum (FW9/10M). }\end{array}$ \\
\hline R4 & $\begin{array}{l}\text { Range is defined using the full width at half maximum } \\
\text { as viewed on a semi-log plot (FWlog10[M]). }\end{array}$ \\
\hline R5 & $\begin{array}{l}\text { Range is defined as containing only the maximum bin } \\
\text { in each peak. }\end{array}$ \\
\hline R6 & $\begin{array}{l}\text { Range looks at the whole visible peak (up to half a } \\
\text { mass unit away) }\end{array}$ \\
\hline
\end{tabular}

Table 1: Descriptions of the automated background correction methods and ranging metrics used to analyse simulated data.

Figure 1 shows the different ranging techniques applied to a single peak without background correction. Peaks such as the FW9/10M (R3) will have a higher signal to noise ratio as they range a smaller number of bins with the highest proportion of signal in them, whereas broader windowing metrics like the FW1/10M (R2) have a lower signal to noise ratio, but sample a 
greater proportion of the population. This analysis will compare the merits of these metrics when applied to well background corrected spectra.
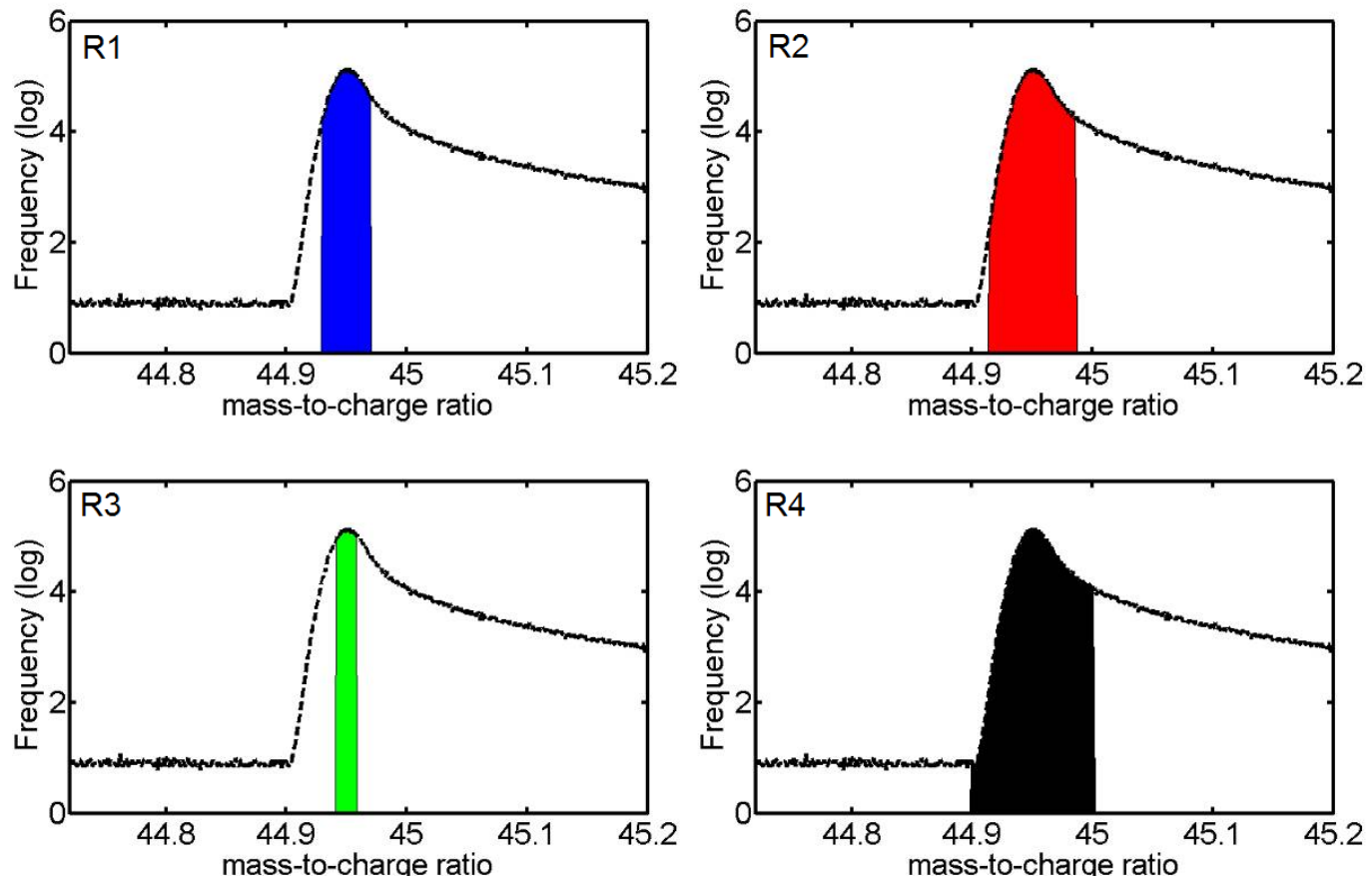

Figure 1: A semi-log plot of a mass spectrum peak displaying various ranging methods: R1 FWHM (blue), R2 - FW1/10M (red), R3 - FW9/10M (green), R4 - FWlog10[M] (black).

\section{Analysis of simulated mass spectra using automated ranging methods}

The success of various background correction methods in reducing the effect of noise on quantitative solute analysis was assessed by comparing the variance between the true compositions of simulated data with the measured compositions using the single ranging metric R3 (FW9/10M). A profile showing the standard deviation of the difference between the true and measured values as a percentage of the true composition is presented in Figure 2 (Note the log scale). This figure gives data for all the correction methods (B0-3) for a variety of different solute additions. Unsurprisingly, the minor solute is vastly overestimated without background 
correction (B0). Although offering large improvements compared to uncorrected data, B2 and B3 were both seen to be ineffective in reducing the effect of background noise. B1 was more successful in removing this noise. From a visual inspection of the mass spectra treated by B1, there appears to be a very low level of discernable noise. The signal-to-noise ratio of the peak at mass $/$ charge $=28$ is a factor of 70 for $\mathrm{B} 1$ and 30 for $\mathrm{B} 2$.

Figure 3 shows the average difference between true and measured values for a variety of solute for all of the ranging metrics. This provides an estimate of the accuracy of the different metrics. The performance of the $\mathrm{FWf}(\mathrm{x}) \mathrm{M}$ ranging metrics (R1-4) is similar. The difference between the measured and true values tended to be in the range of $5-10 \%$ of the concentration of the dilute alloying additions. However, using only the maximum count bin (R5) for each peak tends to exacerbate the overestimate of minor solute. Using a ranging method that included the entire visible peak (as determined algorithmically - R6) appears to give the most accurate result for some of the solutes (at lower concentration). For other solutes it is the least accurate (at higher concentration). Figure 4 shows the standard deviation of the difference between true and measured concentration gathered from six separate simulated datasets. This measure of variance gives an estimate of the reliability of each metric. The greatest variance is seen in R5 and R6, with the trend in R1-4 being that the metrics that discard more information from the extremities of peaks give more reliable performance. The FW9/10M (R3) metric shows the least spread in deviation. 


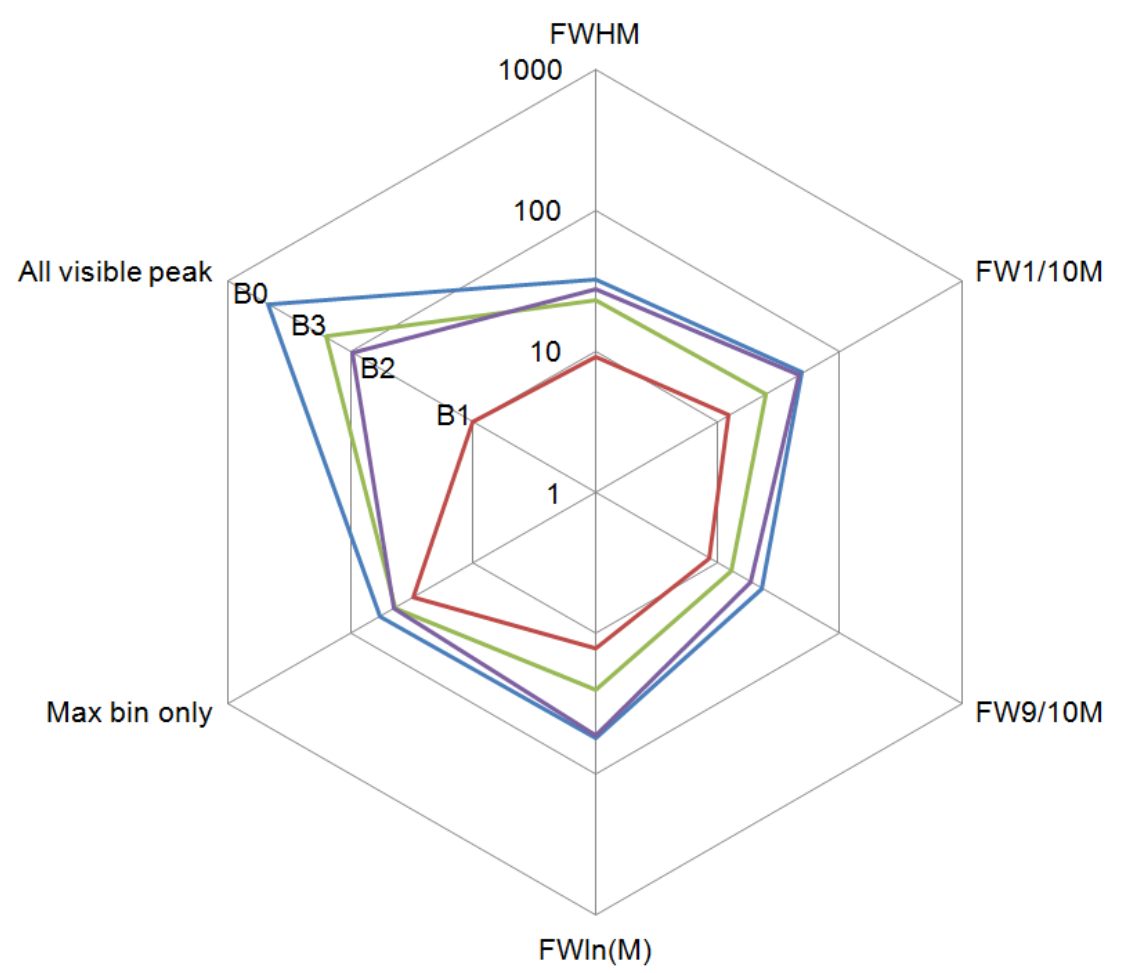

Figure 2: Log-scale standard deviation of the difference between actual and measured composition of simulated data using the FW9/10M ranging metric and various background correction methods 


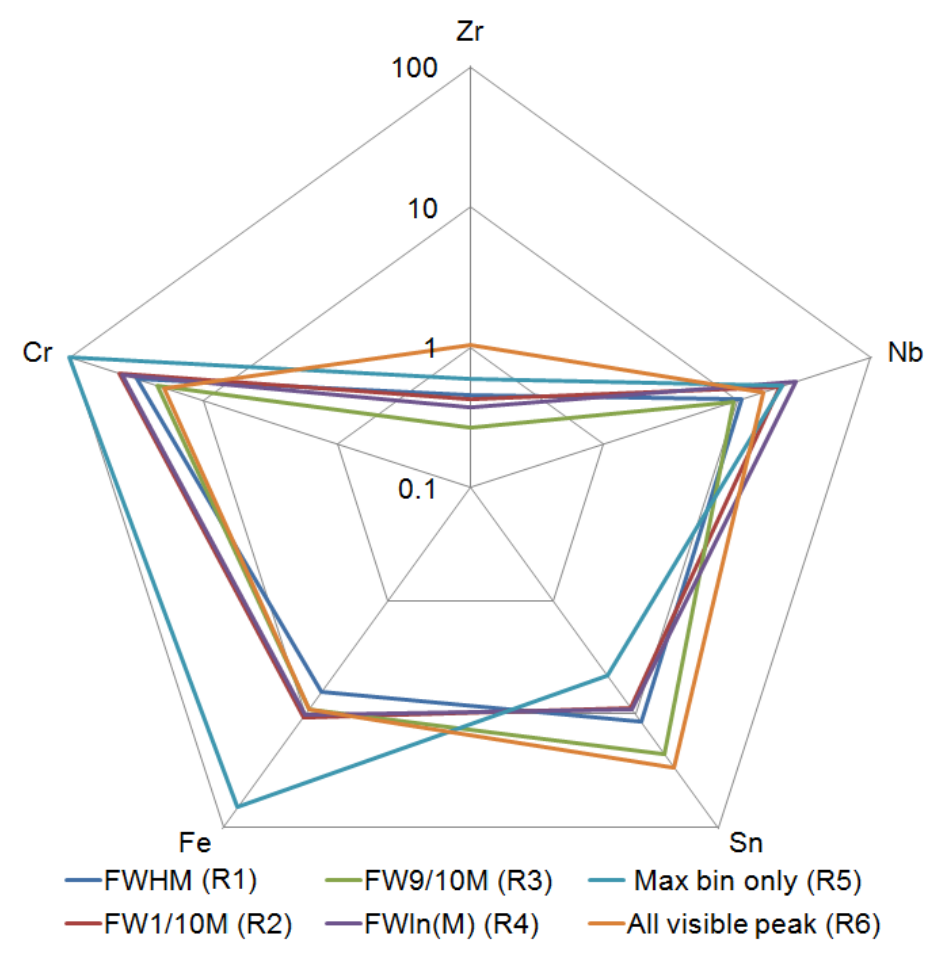

Figure 3: Log-scale percentage difference between the measured and actual composition of a simulated dataset (D1) using B1 background correction.

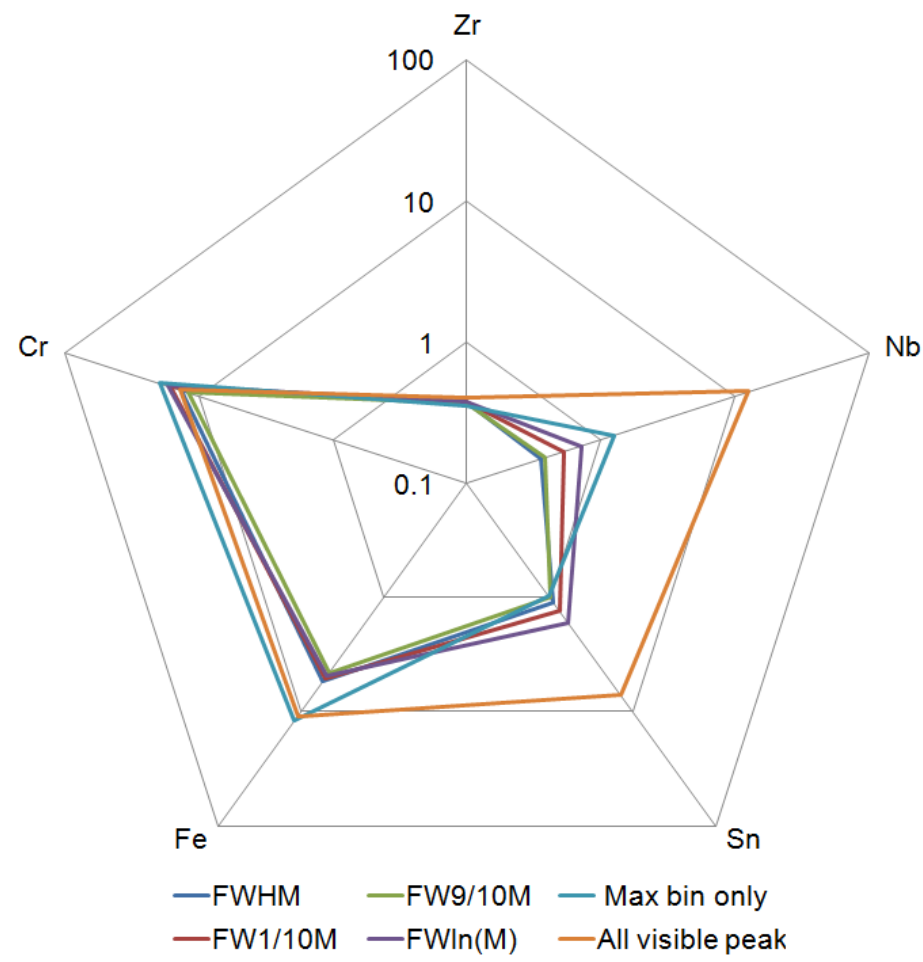

Figure 4: Log-scale standard deviation of the difference between the actual and measured composition of nine separate simulated datasets - using real background correction. 


\section{Discussion of analysis of simulated data}

The variation in performance of a metric is more important than the mean difference between the true and measured concentration of a solute, as this systematic deviation can be adjusted for. This requires that trials such as this are used to assess the performance of the metrics and background correction on simulated data, with known composition, so that solute over/under estimation can be quantified.

Ranging metric R6 aimed to emulate users attempting to window the 'whole' of the visible peak. Like many human users, the algorithm had severe difficulty in determining the limits of very low solute peaks. These, less systematic ranging metrics can appear to give accurate results on individual trials, but over many trials they are seen have a greater variation in performance than the systematic metrics (R1-4). R5 tended to overestimate the minor solute. This is because in ZIRLO these species tend to be at lower positions in the mass spectra. Lower mass peaks contain a greater proportion of ions in the maximum count bin as the resolution of the peak scales with the square root of mass, but the mass-to-charge state bins are of fixed width.

Of the systematic ranging techniques (R1-4), the trend for both accuracy and precision is that performance is improved by the metrics that discard the most data from the peak. This is not as counter-intuitive as it would immediately appear, as these 'narrower' metrics effectively have a higher signal to noise ratio. This reduces the effect of the statistical blurring applied to the signal peaks, and the residual background noise after background correction. The relative increase in the Poisson statistical error induced by the lower measured count is often less important that the improvement gained from the reduced effect of the additional noise and Gaussian position uncertainty. For example the standard deviation due to the Poisson associated error of Fe $(0.02$ at. $\%$ ) in a 10 million atom dataset is $2.2 \%$ of the measured Fe concentration. This rises to $3.2 \%$ after half of the ions are discarded by tighter ranging. The standard deviation due to using the 
automated ranging metrics R5 and R6 would be $\sim 11-12 \%$. R3 (FW9/10M) would result in only a $4.6 \%$ uncertainty.

\section{Conclusion}

Ranging method R3 will be used with background correction method B1 in precise quantification of iron concentration and variation between specimens of uncorroded material in chapter 5 of the main body of the thesis. The source code developed to perform this task is provided below in Appendix B. 
CALL load spectrum(spectra)

PRINT *, "Working..."

CALL get_user_mass_resolution(user_mass_resolution)

PRINT *, " get_user_mass_resolution - ok"

CALL perform_bg_correction(spectra)

PRINT *, " - perform bg correction - ok"

CALL find_max_bin(lookup_peak, spectra)

PRINT *, " find_max_bin ok"

CALL write corrected spectra(spectra, smooth spectra)

PRINT * " " write_corrected_spectra - ok"

!! Main program loop

!! For each different width metric and each different peak of interest sum over that peak and store

!! the area in the peak area array for each background correction method.

DO method $=0,3$

DO lookup_peak_loop $=1,66$

DO metric $=1,6$

PRINT *, " method", method, "metric", metric, "peak", \&

lookup_peak_loop,"- in progress", "u_m_R", user_mass resolution

$$
\text { sum }=0
$$

!!!!!!!!!!!!!!!!!!!!!!!!!!!!!!!!!!!!!

!!! METRIC $5 \quad$ !!

!!!!!!!!!!!!!!!!!!!!!!!!!!!!!!!!!!!!!

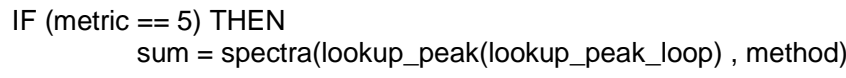

!!!!!!!!!!!!!!!!!!!!!!!!!!!!!!!!!!!

!!! METRIC $6 \quad$ !!

!!!!!!!!!!!!!!!!!!!!!!!!!!!!!!!!!!!!!

ELSE IF (metric $==6)$ THEN

peak_start $=-1$

peak end $=-1$

! IF (method .GT. 0) THEN

! PRINT *, peak start, peak end, lookup peak(lookup peak loop)

! PRINT *, spectra(lookup_peak(lookup_peak_loop), method), \&

! smooth_spectra(lookup_peak(lookup_peak loop), method)

CALL metric_6(peak_start, peak_end, lookup_peak(lookup_peak_loop), \& spectra, smooth_spectra, method)

DO sum loop $=$ peak start, peak end

\section{END DO} sum $=$ sum + spectra(sum_loop, method $)$

! ELSE

!!!!!!!!!!!!!!!!!!!!!!!!!!!!!!!!!!!!!

!!! METRIC $7 \quad$ !!

!!!!!!!!!!!!!!!!!!!!!!!!!!!!!!!!!!!

!!! Requires metric 6 to have been !!!

!!! run on the same peak directly !!!

!! beforehand.

!!!!!!!!!!!!!!!!!!!!!!!!!!!!!!!!!!!!

\section{ELSE IF (metric $==7$ ) THEN}

CALL metric_7(peak_end, smooth_spectra, \&

lookup_peak(lookup_peak_loop), method, correction)

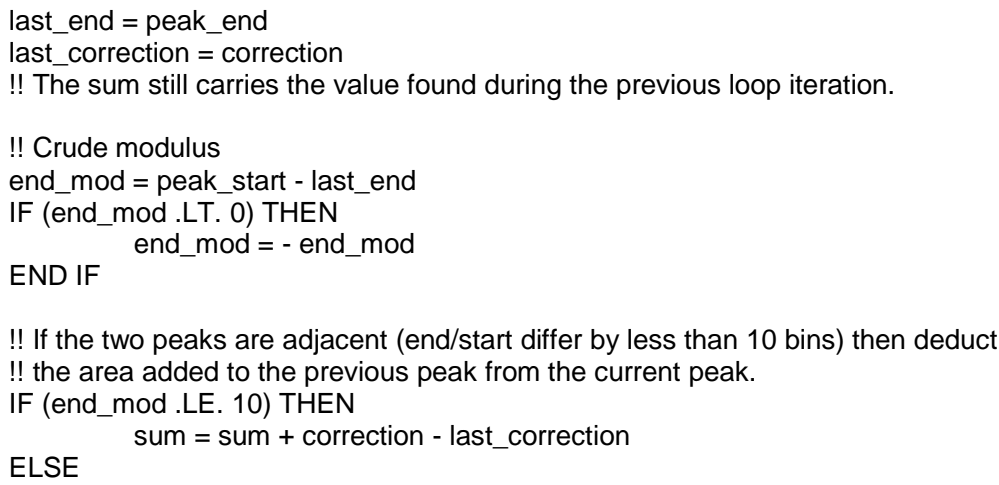

!! If the two peaks are adjacent (end/start differ by less than 10 bins) then deduct !! the area added to the previous peak from the current peak.

IF (end mod .LE. 10) THEN

ELSE sum $=$ sum + correction - last_correction 


\section{END IF \\ sum $=$ sum + correction}

!!!!!!!!!!!!!!!!!!!!!!!!!!!!!!!!!!!!!

!!! METRICS 1, 2, 3, 4

!!!

!!!!!!!!!!!!!!!!!!!!!!!!!!!!!!!!!!!!!

ELSE

DO sum_loop $=$ \&

- peak half width(metric, user mass resolution, lookup peak(lookup peak loop)), \& peak_half_width(metric, user_mass_resolution, lookup_peak(lookup_peak_loop)) sum $=$ sum + spectra(lookup_peak(lookup_peak_loop) - sum_loop, method) !PRINT * method, metric, peak half width (metric, user mass resolution, \& !lookup_peak(lookup_peak_loop)), lookup_peak(lookup_peak_loop) -

END IF

END DO !sum loop, sum

END DO

END DO

peak_area(method, lookup_peak_loop, metric) = sum

IPRINT * lookup_peak(lookup_peak_loop), peak_half_width(metric, user_mass_resolution, \&

!PRINT *, " lookup_peak(lookup_peak_loop))

END DO

PRINT *, " Main program loop - all ok"

CALL calculate_composition(peak_area, lookup_peak)

PRINT *, " calculate composition - ok"

PRINT * "Analysis complete."

END PROGRAM Zr_analysis

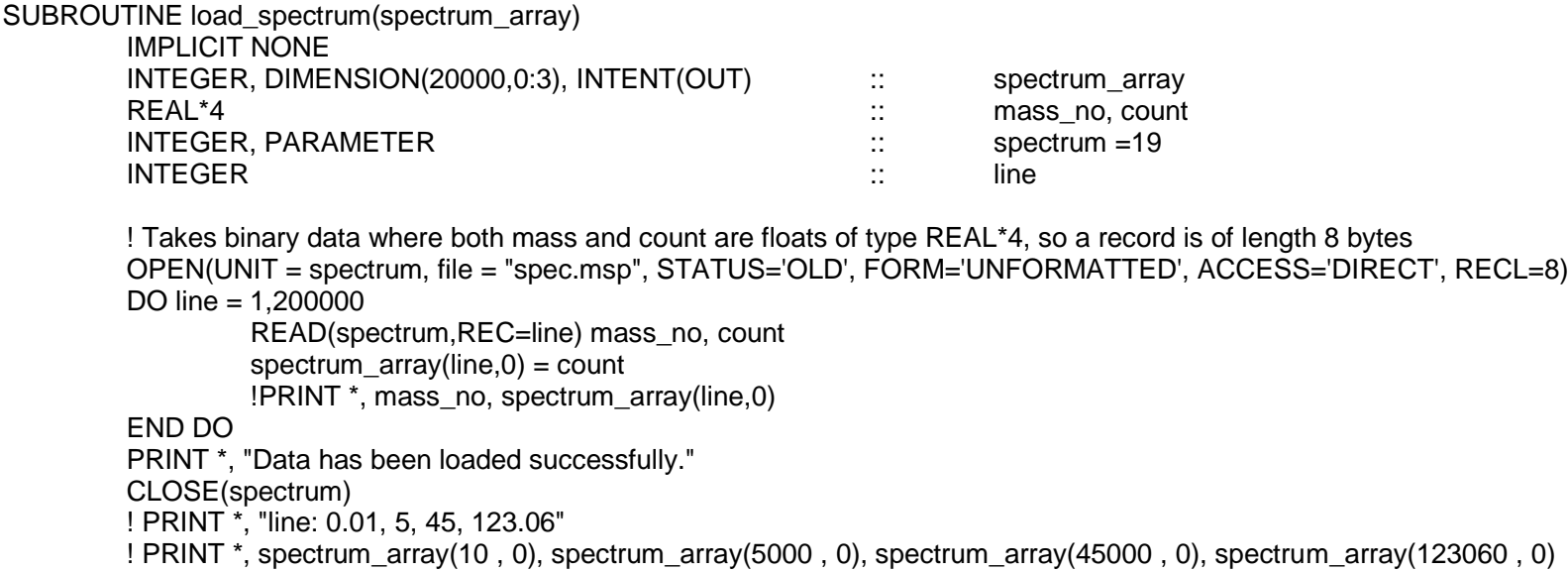

! Takes binary data where both mass and count are floats of type REAL* 4 , so a record is of length 8 bytes OPEN(UNIT = spectrum, file = "spec.msp", STATUS='OLD', FORM='UNFORMATTED', ACCESS='DIRECT', RECL=8) DO line $=1,200000$

$\mathrm{READ}$ (spectrum, REC=line) mass no, count spectrum_array $($ line, 0$)=$ count

END DO !PRINT * ${ }^{*}$ mass_no, spectrum_array(line, 0$)$

PRINT *, "Data has been loaded successfully."

CLOSE(spectrum)

! PRINT *, "line: 0.01, 5, 45, 123.06"

! PRINT *, spectrum array $(10,0)$, spectrum array $(5000,0)$, spectrum $\operatorname{array}(45000,0)$, spectrum $\operatorname{array}(123060,0)$

END SUBROUTINE

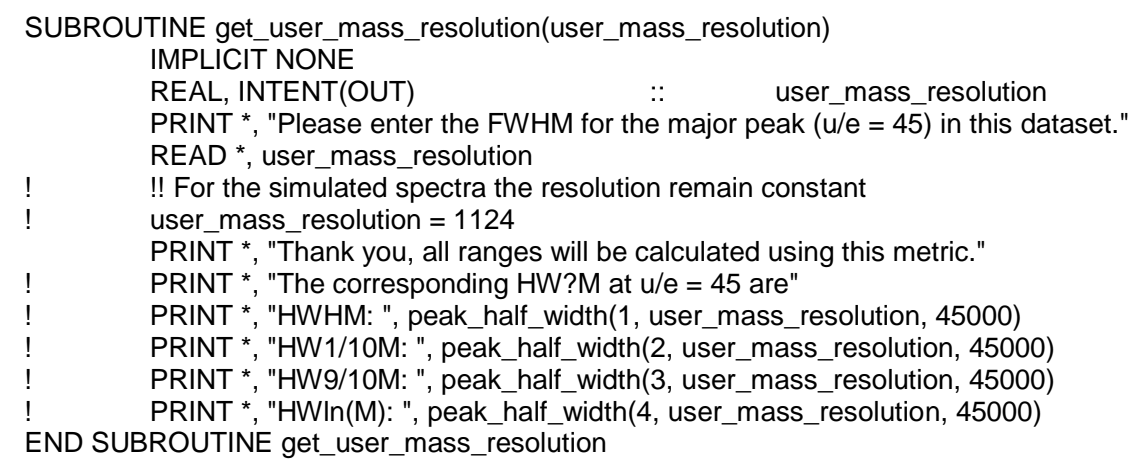

SUBROUTINE perform_bg_correction(spectrum_array)

IMPLICIT NONE

INTEGER

INTEGER, EXTERNAL

correction loop $=0$, current point $=0$, method 3 correction $=0$

REAL, EXTERNAL

background_correction

background_correction_ 
INTEGER, DIMENSION(200000,0:3), INTENT(INOUT) :: spectrum_array

REAL

factor

INTEGER,DIMENSION(1:11) $\quad:: \quad$ correction_point $=(/ 3300,13300,23300,33300,43300,52300, \&$

$63300,73300,83300,100000,201000 /)$

!! Do background correction : spectrum array $(\mathrm{x}, 1)$

!! Correction based on fitting a curve to the background noise

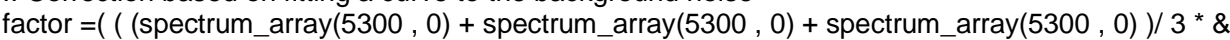

background correction $r(5300,1.0))+\&$

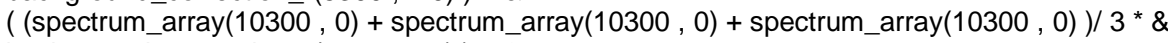

background_correction_r(1030,1.0) ) + \&

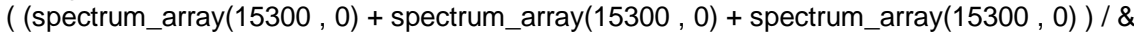

background_correction_r(15300,1.0) ) )/ 3

DO correction_loop $=0,200000$

IF ((spectrum_array(correction_loop , 0) - background_correction(correction_loop, factor)).LT.0) THEN

ELSE spectrum_array(correction_loop, 1) = 0

END DO

spectrum array(correction loop, 1$)=$ spectrum array(correction loop , 0) - \&

END IF background_correction(correction_loop, factor)

!! Do background correction : spectrum array $(x, 2)$

!! Correction based on deducting the equivilent amount of noise from each peak,

!! based on the count $0.2 \mathrm{~m}$ u 920 bins) ahead.

!! The first 20 bins of the correct mass spectra will be set to 0 by the variable definition.

DO correction_loop $=21,200000$

IF ((spectrum_array(correction loop , 0) - spectrum_array(correction loop - 20,0$))$.LT.0) THEN

ELSE spectrum_array(correction_loop , 2) = 0

spectrum_array(correction_loop , 2) = spectrum_array (correction_loop , 0) - \&

END IF spectrum_array(correction_loop - 20,0$)$

END DO

!! Do background correction : spectrum array $(x, 3)$

!! Similar method to '2' except this time the section looks for only a few key points in the spectrum to adjust the !! noise, this avoids the problems that method ' 2 ' encounters with peak tails.

!! These positions are at $\mathrm{m} / \mathrm{u}=3.3,13.3,23.3,33.3,43.3,52.3,63.3,73.3,83.3,100$ in 'correction_points' current_point $=1$

DO correction loop $=335,200000$

IF ((correction_loop).GT.(correction_point(current_point +1$)))$ THEN

END IF current point $=$ current point +1

method3_correction $=($ spectrum_array $($ correction_point(current_point $)-2,0)+\&$

spectrum_array(correction_point(current_point) $-1,0)+8$

spectrum array (correction point(current point), 0$)+\&$

spectrum_array(correction_point(current_point) $+1,0)+\&$

spectrum_array(correction_point(current_point) $+2,0)) / 5$

IF ((spectrum_array(correction_loop , 0) - method3_correction).LT.0) THEN

ELSE

spectrum_array(correction_loop , 3) = 0

END IF

spectrum_array(correction_loop , 3) =spectrum_array(correction_loop , 0) - method3_correction

END DO

RETURN

END SUBROUTINE perform_bg_correction

SUBROUTINE find_max_bin(lookup_peak, spectrum_array)

IMPLICIT NONE

INTEGER, DIMENSION(200000,0:3), INTENT(IN) ::

INTEGER, DIMENSION(66), INTENT(INOUT) INTEGER

max bin count

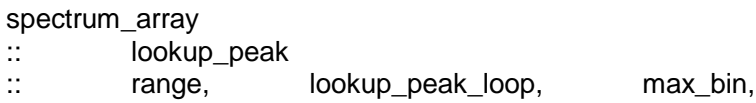

!! Searchs 0.05 mass units either side of the expected peak location and replaces the expected max position with

!! the actual max position within that range. Acts on row ' 0 ' of the spectrum array (the raw data)

!! Don't change the bin if the max count is less than 50 .

DO lookup peak loop $=1,66$

max_bin_count $=0$

max_bin = lookup_peak(lookup_peak_loop) ! Default value to use if the count value is 0 in all the bins tested 
DO range $=-50,50$

IF ( ( spectrum_array(lookup_peak(lookup_peak_loop) + range , 0 ) ).GT.(50)) THEN

IF ( (max_bin_count).LT.(spectrum_array(lookup_peak(lookup_peak_loop) + range , 0 ) ) ) THEN max bin count $=$ spectrum array $($ lookup peak (lookup peak loop $)+$ range, 0$)$

END IF END IF max_bin = lookup_peak (lookup_peak_loop) + range

range, 0 ),

IPRINT *, range, lookup_peak(lookup_peak_loop) + range, spectrum_array( lookup_peak(lookup_peak_loop) +

$\&$

END DO

! max_bin_count, max_bin

END DO

lookup peak(lookup peak loop) $=$ max bin

END SUBROUTINE

SUBROUTINE find max bin_smooth(lookup_peak, spectrum array, method)

IMPLICIT NONE

INTEGER, DIMENSION(200000,1:3), INTENT(IN) ::

INTEGER, DIMENSION(66), INTENT(INOUT)

INTEGER

max bin count

INTEGER, INTENT(IN)

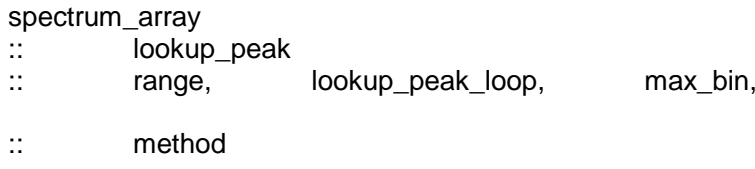

!! Searchs 0.05 mass units either side of the expected peak location and replaces the expected max position with

!! the actual max position within that range. Acts on row ' 0 ' of the spectrum array (the raw data)

!! Don't change the bin if the max count is less than 50

DO lookup_peak_loop $=1,66$

max bin count $=0$

max_bin = lookup_peak(lookup_peak_loop) ! Default value to use if the count value is 0 in all the bins tested

DO range $=-50,50$

IF ( ( spectrum_array( lookup_peak(lookup_peak_loop) + range , method ) ).GT.(50)) THEN

THEN

IF ( (max_bin_count).LT.( spectrum_array( lookup_peak(lookup_peak_loop) + range , method ) ) )

END IF

max_bin_count $=$ spectrum_array $($ lookup_peak(lookup_peak_loop) + range, method $)$

END IF max_bin = lookup_peak(lookup_peak_loop) + range

!PRINT *, range, lookup_peak(lookup_peak_loop) + range, spectrum_array( lookup_peak(lookup_peak_loop) +

range , 0 ),

END ! max_bin_count, max_bin

END DO

lookup_peak (lookup_peak_loop) = max_bin

END SUBROUTINE

SUBROUTINE write_corrected_spectra(spectrum_array, smoothed_spectra)

IMPLICIT NONE

INTEGER, PARAMETER

INTEGER, DIMENSION(200000,0:3), INTENT(IN) :

$: \quad$ output $=9$

INTEGER

smoothed_spectra

$\because \quad$ line $=0$

OPEN(UNIT = output, file = "corr_spec.csv", FORM='FORMATTED')

WRITE(output, *) "Mass / charge state ratio" ,",", "Raw data" ,",", "Not smoothed",",", \&

"Method 1" ,",", "M1 smoothed" ,",", \&

"Method 2" ,",", "M2 smoothed" ,",", \&

"Method 3" ,",", "M3 smoothed"

DO line $=1,200000$

WRITE(output, ${ }^{\star}$ ) line/1000.0, ",", \&

spectrum_array(line, 0), ",", "-", ", \&

spectrum array(line, 1), ",", smoothed spectra(line, 1), ",", \&

spectrum_array(line, 2), ",", smoothed_spectra(line, 2), ",", \&

spectrum_array(line, 3), ",", smoothed_spectra(line, 3)

!!replaced as smoothed spectra is now redundant 
3)

\title{
END DO
CLOSE(output)
}

END SUBROUTINE write_corrected_spectra

\author{
SUBROUTINE calculate_composition(peak_area, lookup_peak) \\ IMPLICIT NONE \\ INTEGER, PARAMETER \\ INTEGER, DIMENSION(0:3, 66, 1:6), INTENT(IN) :: \\ INTEGER \\ INTEGER,DIMENSION(6) \\ INTEGER,DIMENSION(66), INTENT(IN)
}

$:: \quad$ output $=9$

peak_area

$\because: \quad$ method $=0$, metric $=0$, sum_loop $=0$, sum, \&

moop, zirc loop

$\because \quad \quad \mathrm{Zr}, \mathrm{Nb}, \mathrm{Fe}, \mathrm{C}, \mathrm{O}, \mathrm{N}, \mathrm{Sn}, \mathrm{Cr}, \mathrm{Ni}$, total

: $\quad$ lookup_peak

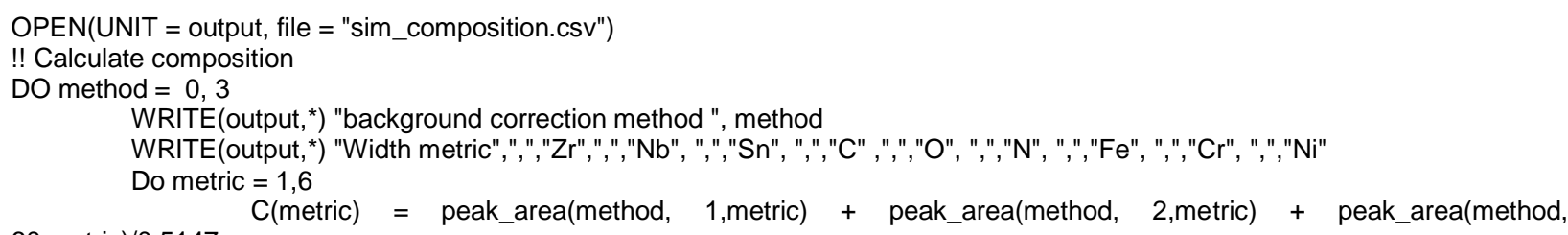
60 ,metric) $/ 0.5147$

$\mathrm{N}($ metric $)=$ peak_area $($ method, 3,metric $)+$ peak_area(method, 4,metric $)+$ peak_area(method, 63,metric) $/ 0.171$

$\mathrm{O}($ metric $)=$ peak_area $($ method, 5,metric $)+$ peak_area(method, 6,metric) + peak_area(method, 7,metric)

DO zirc_loop $=40,54$

END DO

$\mathrm{O}($ metric $)=\mathrm{O}($ metric $)+$ peak_area(method, zirc_loop,metric $)$

DO zirc_loop $=55,59$

END DO

$\mathrm{O}($ metric $)=\mathrm{O}($ metric $)+2^{*}$ peak_area $($ method, zirc_loop, metric $)$

$\operatorname{Cr}($ metric $)=$ peak_area $($ method, 9 ,metric $) / 0.8375$

$\mathrm{Fe}($ metric $)=$ peak_area $($ method, 12 ,metric $) / 0.918$

$\mathrm{Ni}($ metric $)=$ peak_area $($ method, 14, metric $) / 0.683$

$! \mathrm{Sn}($ metric $)=0$

!Do tin_loop $=15,24$

!END DO

Sn $($ metric $)=$ Sn $($ metric $)+$ peak_area(method, tin_loop,metric)

$\mathrm{Sn}($ metric $)=($ peak_area $($ method, 20, metric $)+$ peak_area $($ method, 22, metric $)) / 0.567$

$\mathrm{Zr}($ metric $)=0$

DO zirc loop $=25,63$

END DO

$\mathrm{Zr}($ metric $)=\mathrm{Zr}($ metric $)+$ peak_area $($ method, zirc_loop,metric $)$

$\mathrm{Nb}$ (metric) $=$ peak_area(method, 64,metric) + peak_area(method, 65,metric) + peak_area(method, 66 ,metric)

total $($ metric $)=\mathrm{Zr}($ metric $)+\mathrm{Nb}$ (metric $)+\mathrm{Fe}($ metric $)+\mathrm{C}($ metric $)+\mathrm{O}$ (metric) $+\mathrm{N}$ (metric $)+\&$ $\mathrm{Sn}$ (metric) $+\mathrm{Cr}$ (metric) $+\mathrm{Ni}($ metric $)$

WRITE(output, *) metric,",,",Zr(metric),",,",Nb(metric), ",,"Sn(metric), ",,",C(metric) ,",", \&

$\mathrm{O}$ (metric), ",",N(metric), ",",Fe(metric), ",",Cr(metric), ",",Ni(metric)

WRITE(output, *) metric,", ", 100.000000*Zr(metric)/total(metric),", ",100.0* $\mathrm{Nb}$ (metric)/total(metric), \& ", ",100.000000* $\mathrm{Sn}$ (metric)/total(metric),", ",100.0*C(metric)/total(metric),", ", \&

$100.0^{*} \mathrm{O}$ (metric)/total(metric),", ", 100.0* $\mathrm{N}$ (metric)/total(metric),", ", \&

$100.0^{*} \mathrm{Fe}$ (metric)/total(metric),", ", 100.0* $\mathrm{Cr}$ (metric)/total(metric),", ", \&

END Do $100.0^{*} \mathrm{Ni}($ metric $) /$ total (metric)

WRITE(output,") "1 = FWHM",", ","2 = FW1/10M",", ","3 = FW9/10M",", ","4 = FWIn(M)",", ","5 = Max bin only" \&

WRITE(output, ${ }^{*}$ )

, ",", "6 = All visible peak", ",", "7 = All visible peak correcting for overlapping tails" 
!! Works well only with background corrected spectra.

SUBROUTINE metric_6(peak_start, peak_end, lookup_peak, spectrum_array, smooth_spectra, method)

$\begin{array}{lll}\text { IMPLICIT NONE } & :: & \text { lookup_peak, method } \\ \text { INTEGER, INTENT(IN) } & :: & \text { peak_start, peak_end } \\ \text { INTEGER, INTENT(OUT) } & \text { spectrum_array } \\ \text { INTEGER, DIMENSION(200000,0:3), INTENT(IN) :: } & :: & \text { smooth_spectra } \\ \text { INTEGER, DIMENSION(200000, 1:3), INTENT(OUT) } & :: & \text { line }=0 \\ \text { INTEGER } & :: & \text { set_start, set_end } \\ \text { LOGICAL } & \end{array}$

smooth_spectra $=0$

DO line $=3,199998$

smooth spectra (line, method $)=($ spectrum array $($ line -4 , method $)+$ spectrum array $($ line -3 , method $)+\&$ spectrum_array(line - 2, method) + spectrum_array(line -1 , method $)+$ spectrum_array (line, method $)+\&$ spectrum_array(line +1 , method $)+$ spectrum_array(line +2 , method $)+$ spectrum _array(line +3, method $)+\&$ END DO spectrum_array (line +4 , method) ) / 9

!! Find start of peak.

!! The peak starts when the gradient changes sign or count goes to 0 going left from the max peak of the smoothed spectrum.

line $=0$

set_start $=$.FALSE.

set_end $=$.FALSE.

!! Don't do method 0

IF (method .GT. 0) THEN

!! Only works for reasonable count sizes

IF (smooth_spectra(lookup_peak, method) .GT. 15) THEN

!! Find start of region

DO line $=0,500$

IF (set start .EQV. .FALSE.) THEN

!! LE is "less than or equal to", GT is "less than"

IF (smooth_spectra(lookup_peak - line, method) .LT. \&

smooth spectra(lookup peak - line - 2, method)) THEN peak_start = lookup_peak - line

END IF set start $=$.TRUE

IF (smooth_spectra(lookup_peak - line, method) .EQ. 0) THEN peak start $=$ lookup peak - line

END IF set_start $=$.TRUE.

END DO

END IF

line $=0$

!! Find end of region

DO line $=0,500$

IF (set end .EQV. .FALSE.) THEN

IF (smooth_spectra(lookup_peak + line, method) .LT. \&

smooth spectra(lookup peak + line +2 , method)) THEN

peak_end = lookup_peak + line

END IF set_end $=$.TRUE.

IF (smooth_spectra(lookup_peak + line, method) .EQ. 0) THEN peak end $=$ lookup peak + line

END IF

$$
\text { set_end }=\text {.TRUE. }
$$

END DO

ELSE

!! If the countsize is too low then set:

peak_start $=$ lookup_peak

END IF

peak end $=$ lookup peak 
peak_start $=$ lookup_peak

peak_end = lookup_peak

END IF

! PRINT *, "

method", method, "metric 6: start", peak_start, "end", peak_end

END SUBROUTINE metric_6

!! Requires metric 6 to have been performed first

SUBROUTINE metric 7(intersection, smooth spectra, lookup peak, method, correction)

IMPLICIT NONE

INTEGER, INTENT(IN)

INTEGER, DIMENSION(200000, 1:3), INTENT(IN)

INTEGER, INTENT(OUT)

INTEGER

REAL

REAL, DIMENSION(200000)

INTEGER, EXTERNAL

\author{
lookup_peak, method, intersection \\ smooth_spectra \\ correction \\ bin, $x 1, x 2, c x$ \\ $\mathrm{m}, \mathrm{c}, \mathrm{y} 1, \mathrm{y} 2$ \\ spectra in use \\ peak_half_width
}

!! Take the natural log of the method in use and place it in a real array.

DO bin $=0,200000$

END DO

spectra_in_use $($ bin $)=\operatorname{LOG}\left(1 .{ }^{*}\right.$ smooth_spectra(bin, method $\left.)\right)$

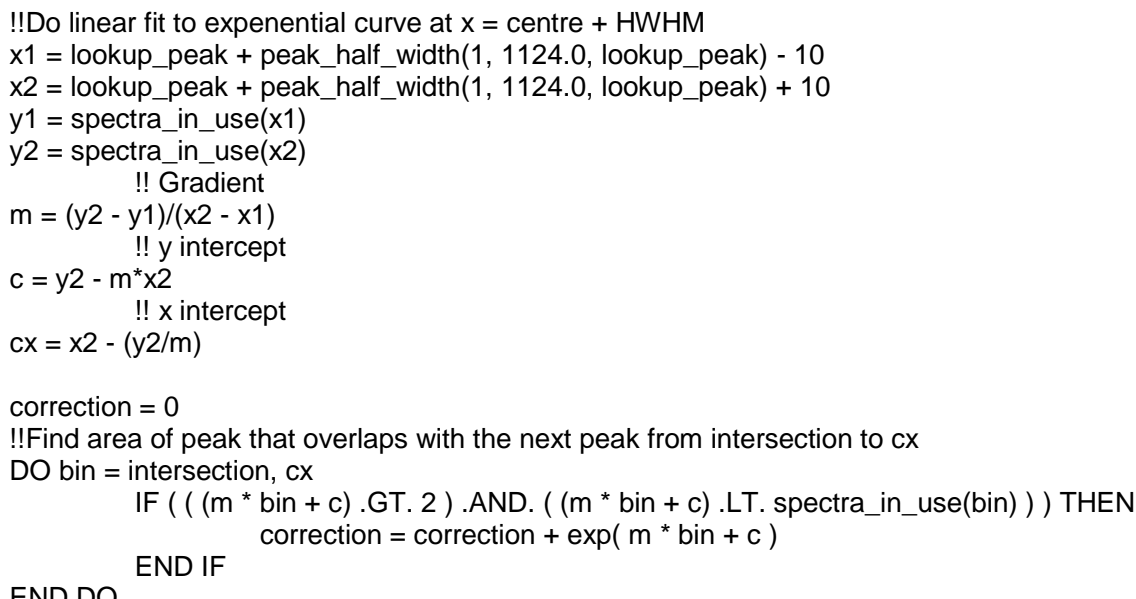

END SUBROUTINE metric_7

INTEGER FUNCTION background_correction(mass_position, factor) INTEGER, INTENT(IN) $\quad:: \quad$ mass_position

REAL, INTENT(IN) :: factor

!! The factor should be set by finding a mass bin with just noise in it, pass in that location with factor $=1$

!! Then the count at that position / the return value is the factor. Do for several positions and average.

END FUNCTION background_correction = factor $/ \mathrm{SQRT}(1.0$ * mass_position $)$

REAL FUNCTION background correction $r$ (mass position, factor)

INTEGER, INTENT(IN) :: mass_position

REAL, INTENT(IN) :: factor

background_correction_r $=$ factor $/ \mathrm{SQRT}\left(1.0^{*}\right.$ mass_position $)$

END FUNCTION 


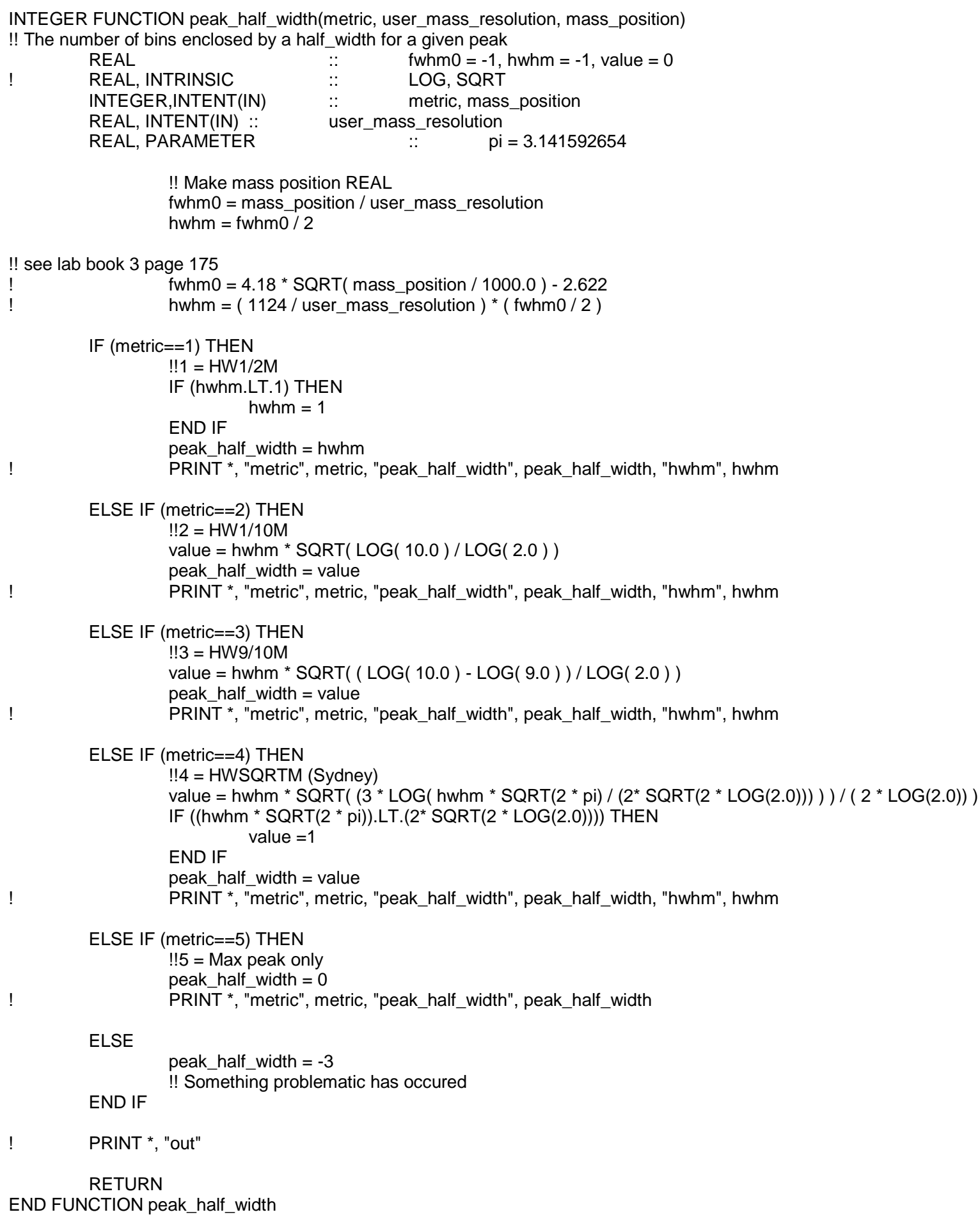

